\title{
ANAEROBIC CO-DIGESTION OF MULTIPLE FEEDSTOCKS FOR BIOMETHANE RECOVERY- THE IMPACT OF LIPIDS:PROTEINS:CARBOHYDRATES RATIO
}

By

\author{
Anahita Rabii \\ Master of Civil Engineering, University of Tehran, 2009 \\ Bachelor of Applied Physics, University of Mazandaran, 2000
}

\author{
A thesis \\ presented to Ryerson University \\ in partial fulfillment of the \\ requirements for the degree of \\ Doctor of Philosophy \\ in the program of Civil Engineering
}

Toronto, Ontario, Canada, 2020

(C) Anahita Rabii, 2020 


\section{Author's Declaration for Electronic Submission of a Dissertation}

I hereby declare that I am the sole author of this dissertation. This is a true copy of the dissertation, including any required final revisions, as accepted by my examiners.

I authorize Ryerson University to lend this dissertation to other institutions or individuals for the purpose of scholarly research.

I further authorize Ryerson University to reproduce this dissertation by photocopying or by other means, in total or in part, at the request of other institutions or individuals for the purpose of scholarly research. I understand that my dissertation may be made electronically available to the public. 


\title{
ANAEROBIC CO-DIGESTION OF MULTIPLE FEEDSTOCKS FOR BIOMETHANE RECOVERY- THE IMPACT OF LIPIDS:PROTEINS:CARBOHYDRATES RATIO
}

\author{
Anahita Rabii, Doctor of Philosophy, 2020 \\ Department of Civil Engineering, Ryerson University
}

\begin{abstract}
Municipalities are facing increasing challenges regarding management and disposal of solid waste. Anaerobic digestion (AD) of municipal biowaste enables waste reduction and biogas production that can be utilized as a renewable source of energy for heat and power generation. Anaerobic co-digestion (AnCoD) enhances the performance of conventional mono-digestion. The mixing ratio of the feedstocks is an important criterion in AnCoD design which is typically determined based on the optimum carbon to nitrogen $(\mathrm{C}: \mathrm{N})$ ratio within the range of 25-30 or COD:N ratio in the range of 50140. However, literature has shown contradictory results for the optimum C:N and COD:N ratios. Therefore, the main objective of this study was to primarily investigate the influence of the mixing ratio of the feedstocks including thickened waste activated sludge (TWAS), manure and source separated organics (SSO) on improving biomethane production and introducing a new methodology for optimizing the mixing ratio in $\mathrm{AnCoD}$ based on the lipids, proteins, and carbohydrates contents as the three main compounds existing in biowaste. The hydrolysis/acidification performance in $\mathrm{AnCoD}$ of manure, TWAS and SSO individually and in different combinations was also investigated. This study has introduced an empirical model to explain the relationship between the biomethane production and lipids: proteins: carbohydrates ratio of the feedstocks in anaerobic co-digestion of TWAS, manure and SSO. Among the binary ad ternary combinations, the ternary co-digestion of TWAS/manure/ SSO at the mixing ratio of 2:4:4 and lipids: proteins: carbohydrates ratio of 1:3:12 resulted in the maximum ultimate methane production. The maximum methane yield of $363 \mathrm{ml} \mathrm{CH}_{4} / \mathrm{g} \mathrm{COD}$ added corresponded to co-digestion of manure/SSO at the mixing ratio of 7:3. The maximum hydrolysis rate corresponded to the co-digestion of TWAS/manure at the ratio of 9:1. Overall, the best performance in both hydrolysis and methanogenesis was achieved by the co-digestion of TWAS with SSO at the ratio of 3:7 as well as TWAS/manure/SSO at the ratio of 2:4:4 compared to other feedstock mixes. It was observed that the proposed second order polynomial model could describe the relationship between biomethane production and lipids, proteins, and carbohydrates content of the feedstock.
\end{abstract}




\section{ACKNOWLEDGMENTS}

I am extremely grateful to almighty God, who has been there every step of the way, for giving me the strength to keep going and for his blessing throughout my life. My doctoral study at Ryerson University was a journey of learning, research, and teaching, full of inspiration. I definitely owe the success of this journey to the people who made it possible for me.

I would like to express my sincere gratitude to my $\mathrm{PhD}$ advisors, Dr. Elsayed Elbeshbishy, Dr. Yaser Dahman, and Dr. Saad Aldin for supporting me during these past few years. I owe a great deal to them for their guidance and support through this process. Their ideas and feedback have been absolutely invaluable.

I am profoundly grateful to my dear supervisor, Dr. Elsayed Elbeshbishy for his invaluable guidance and his continued support. I have had the great privilege to work in his research group; in fact, I have been very fortunate to learn from his knowledge and expertise during my research. While being always supportive and helpful at different stages of my work, he granted me the liberty to express and pursue my own ideas. He has been much more than my supervisor, rather a mentor and a great leader. I hope these precious experiences continue throughout my career as I still have a great deal to explore through collaborations with him.

My sincere thanks go to my committee members, Dr. Jiangning Wu, Dr. Ahmed Shaker, Dr Songnian Li, and Dr. Hisham Hafez. In addition, I also appreciate Dr. Darko Joksimovic and Dr. Grace Luk for their suggestions on my research proposal. I greatly appreciate their support and invaluable suggestions for improving this work. I would like to extend my gratitude to Dr. Younggy Kim at McMaster University for reviewing this dissertation and for providing invaluable comments and suggestions for improving my dissertation.

My teaching experiences as a Teaching Assistant at Ryerson University were gratifying and full of inspiration. I am thankful that I had the opportunity to contribute to the learning of students in the civil engineering department at Ryerson University. I appreciate the help and support of the faculty whom I had the opportunity of working with; many thanks to Dr. Darko Joksimovic, Dr. Soheil Kashi, Dr. Ehsan Hoseini Koupaye, and Dr. Rania Hamza. I also greatly appreciate the Teaching Development team, Michelle Schwarts, Curtis Maloley, and Dr. John Paul Foxe for their support and valuable resources throughout this journey. I would also like to thank my students in 
my tutorial and laboratory sessions, particularly those who inspired me by their enthusiasm, attention, and effort. A genuine thanks go to the administrative staff of the civil engineering department, Rachel Peluso, Kim Kritzer, Helen Rusan, Tamar Svadjian, and Annie Jiang for their continued support throughout these years.

I would like to thank my colleagues in Environmental Research for Resource Recovery (ER3) group, specifically Frances Okoye, Ahmed El Sayed, Devarshi Sevak, Salomeh Chegini, Dr. Valeriy Bekmuradov, and Hassan Mahdi for all their support and assistance. My gratitude extends to my amazing colleagues Farokhlaqa Kakar, Neha Purohit, Umme Sharmeen Hyder, and Abeer Hamzeh.

I would also thank Dr. Amir Bazyar Lakeh and Dr. Ehsan Hoseini Koupaye for their guidance and valuable suggestions during my research. In addition, I truly appreciate Robin Loung for his hard work and for providing continued support in conducting my research.

The financial contribution from the Natural Sciences and Engineering Research Council of Canada (NSERC) to this research is gratefully acknowledged. I would also like to acknowledge the City of Toronto's Ashbridges Bay Treatment Plant and Disco Road Organic Processing Facilty for all their support and collaborations throughout this work.

I would like to appreciate my dear parents who taught me how to work hard, how to be brave and pursue my dreams. Thanks to my sister Alaleh and my brother Joseph who have been always encouraging, supportive and caring during the challenging stages of my life. My dear thanks go to the heavenly gift of my life, who has been always by my side, whose smile has given me the strength to keep going, and who I am always grateful for, my son, Armin. Thank you for being so great, thoughtful, and understanding. 
Q Dedication

To the light of my eyes

Ta my heavenly gift

And to all of my

inspiration

Premix 


\section{Table of Contents}

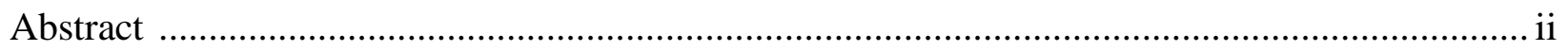

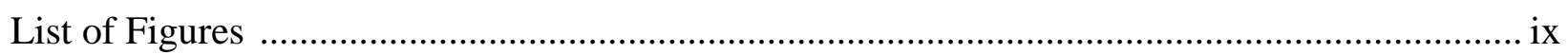

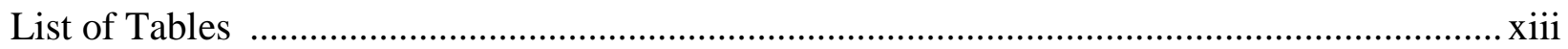

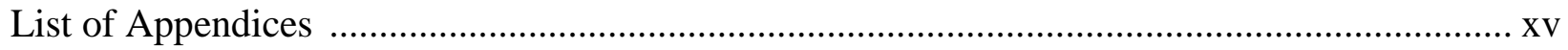

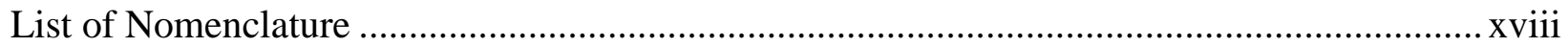

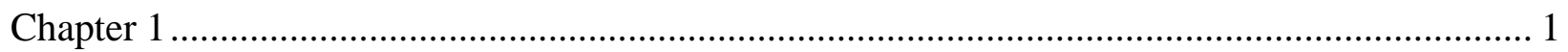

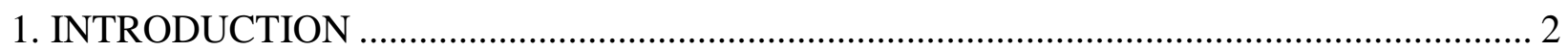

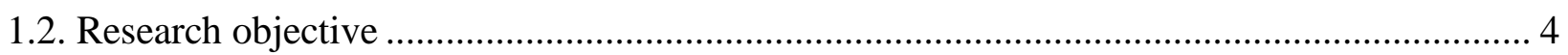

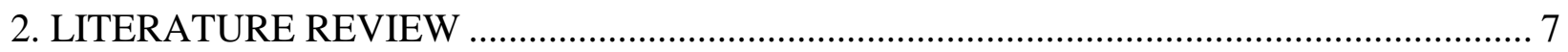

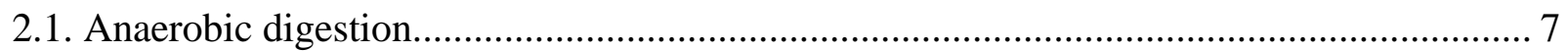

2.2. Processparameters of anaerobic digestion..................................................................... 12

2.2.1. Waste composition and volatile solids ................................................................... 12

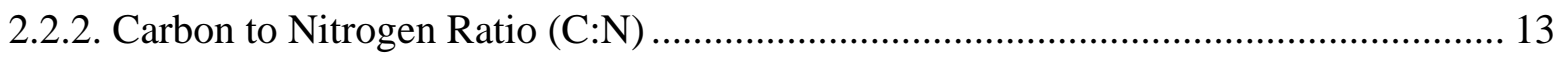

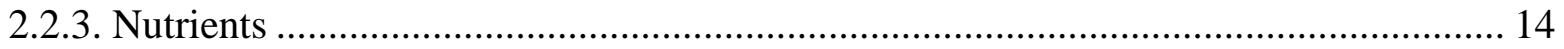

2.2.4. Total solids content (TS)/Organic loading rate (OLR) ................................................. 14

2.2.5. $\mathrm{pH}$, alkalinity and volatile acids/alkalinity ratio...................................................... 15

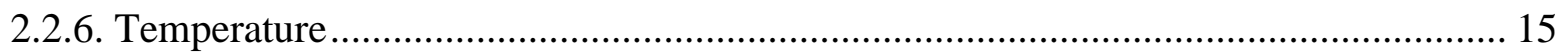

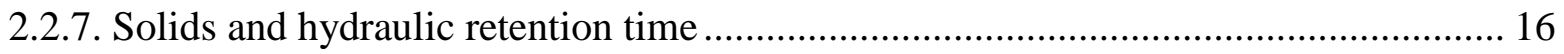

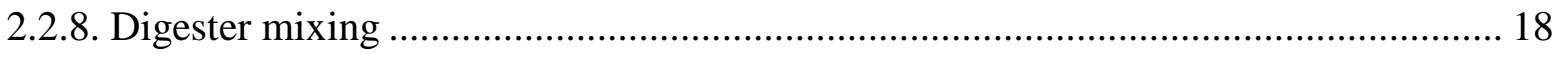

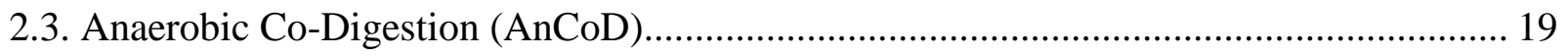

2.3.1. Microbial Diversity and Synergy in AnCoD ............................................................... 26

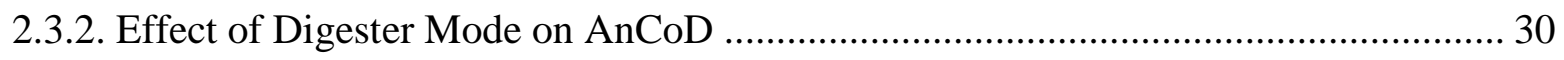

2.4. Pretreatment for Improving AnCoD ................................................................................ 36

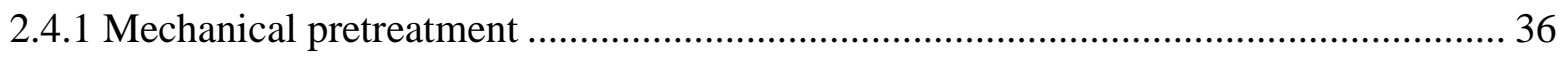

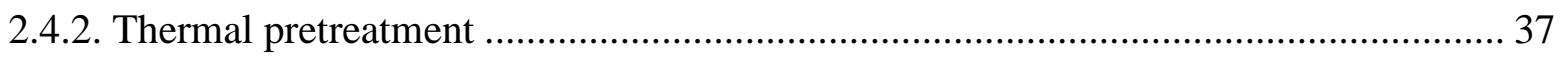

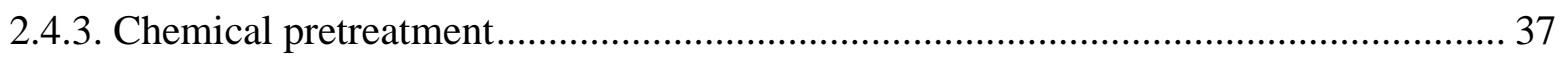




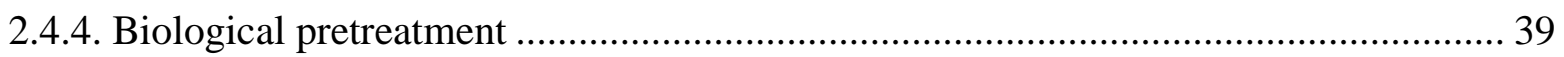

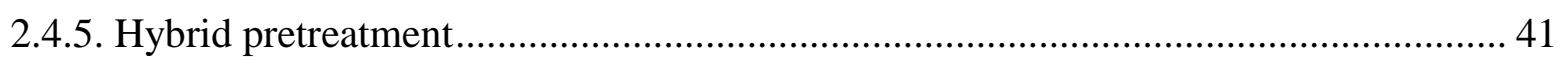

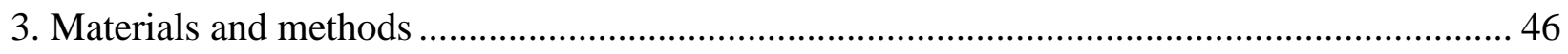

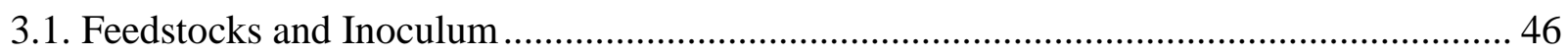

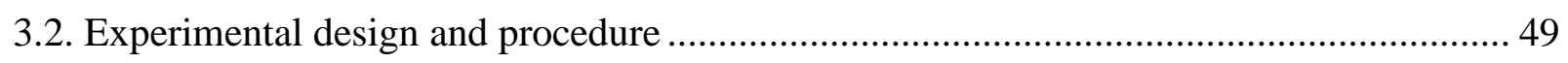

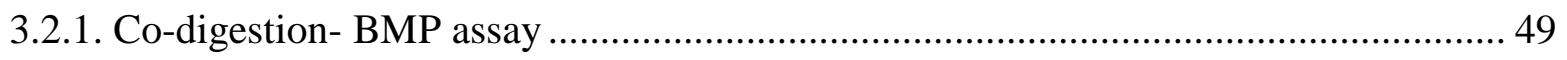

3.2.2. Co-digestion- hydrolysis/acidification experiment ..................................................... 52

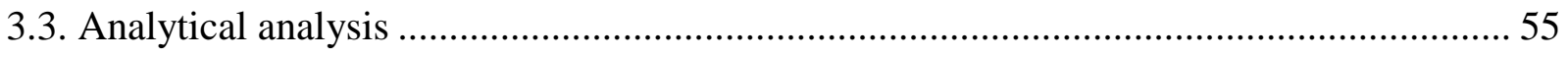

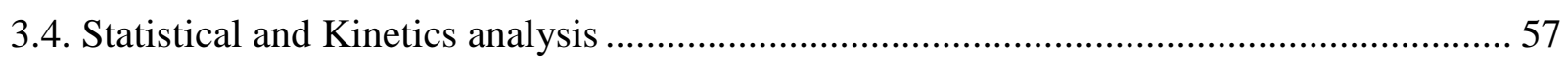

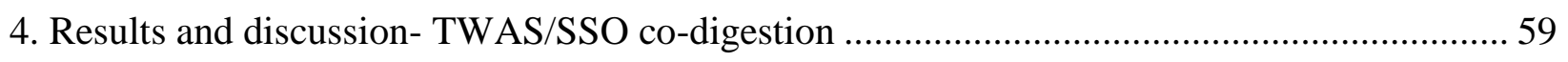

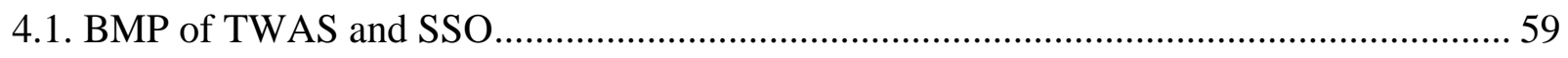

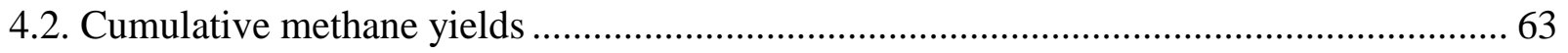

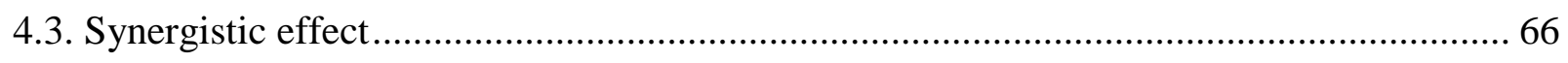

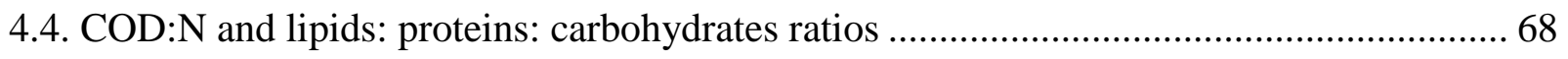

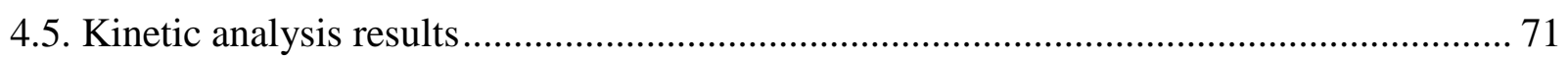

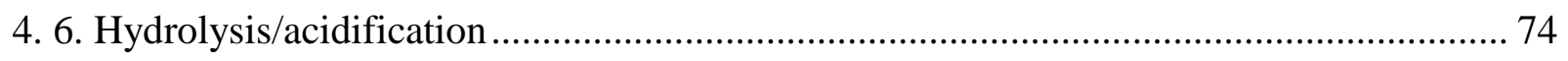

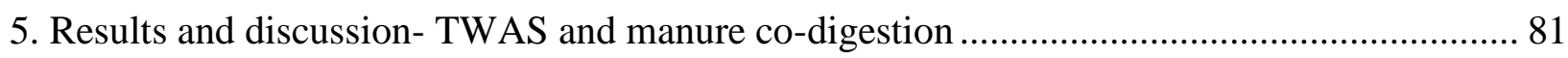

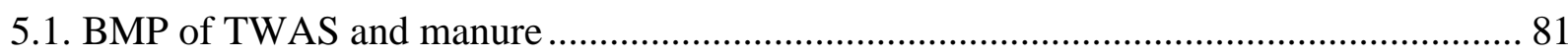

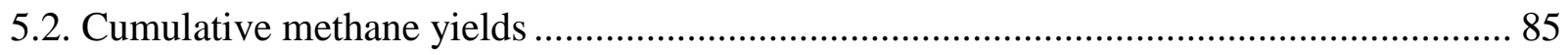

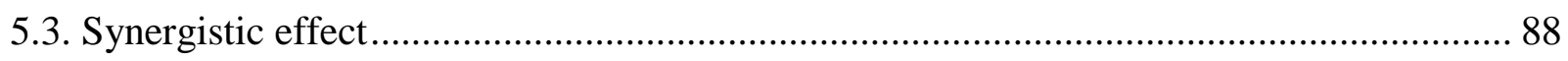

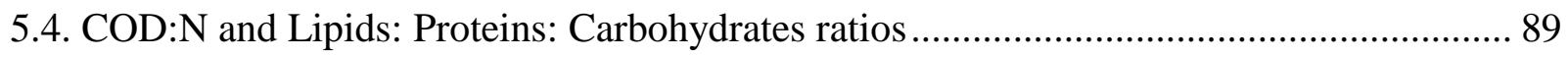

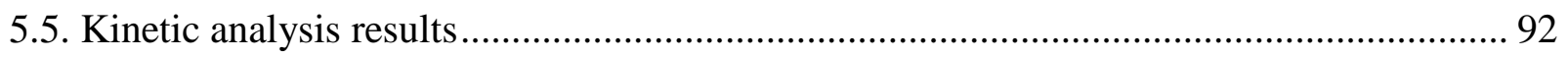

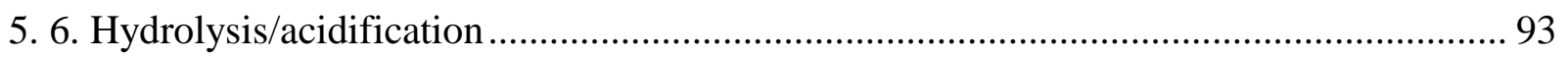

6. Results and discussion- manure and SSO co-digestion ....................................................... 99

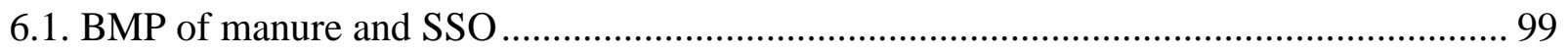

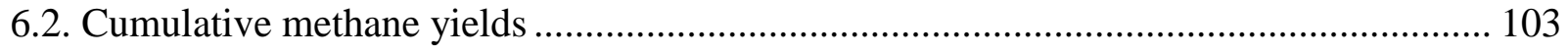

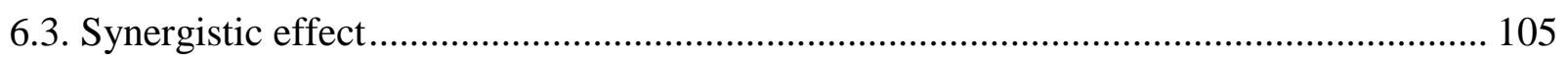

6.4. COD:N and lipids: proteins: carbohydrates ratios ...................................................... 106 


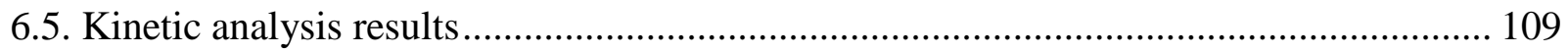

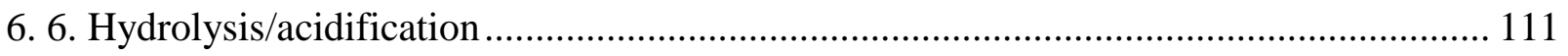

7. Results and discussion- TWAS, manure, SSO co-digestion............................................... 117

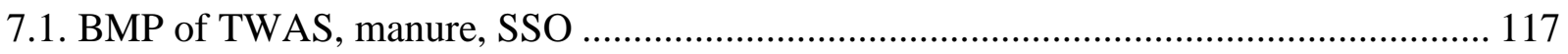

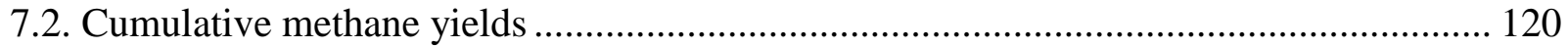

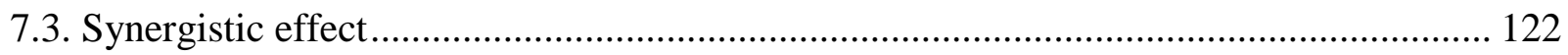

7.4. COD:N and Lipids: Proteins: Carbohydrates ratios ....................................................... 123

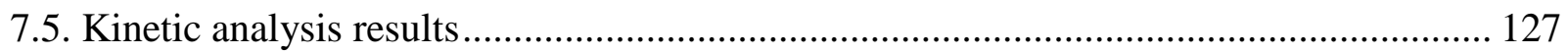

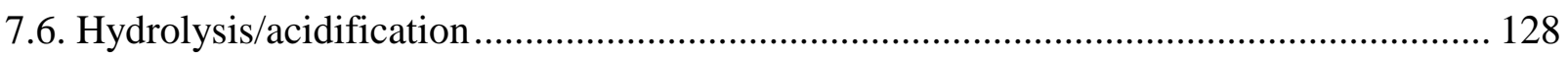

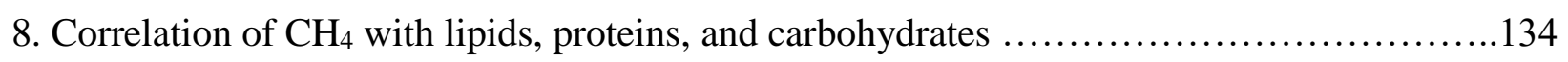

8.1 Correlation of organic compositions and biomethane yield ............................................ 135

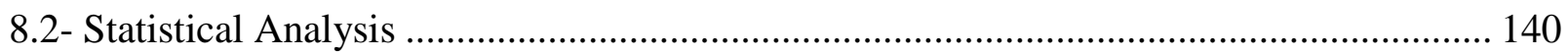

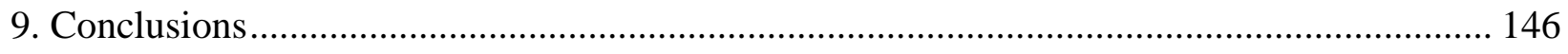

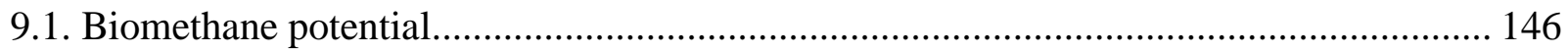

9.1.1. Binary co-digestion of TWAS, manure, and SSO ..................................................... 146

9.1.2. Ternary co-digestion of TWAS, manure and SSO ................................................. 147

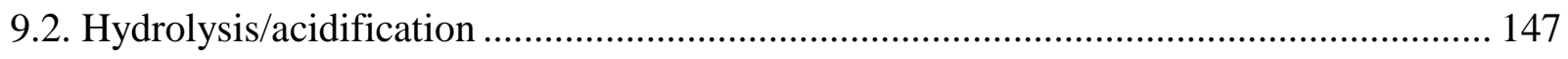

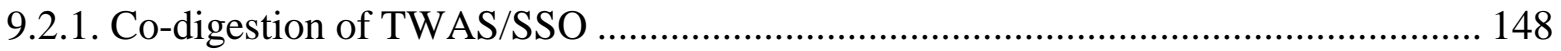

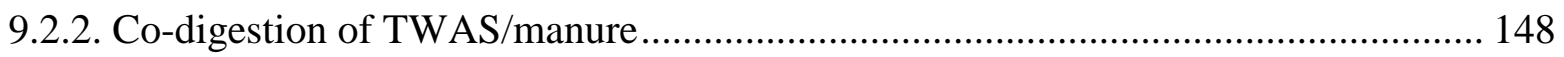

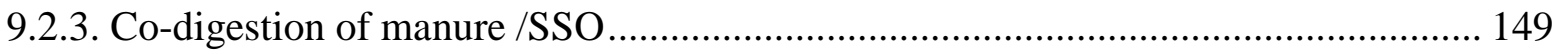

9.2.4. Co-digestion of TWAS/manure /SSO ................................................................... 149

9.3. Correlation of biomethane production with the organic content..................................... 150

9.3.1. COD:N and lipids:proteinscarbohydrates ratios ..................................................... 150

9.3.2. Anaerobic co-digestion model ................................................................................ 151

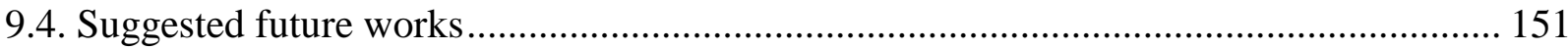




\section{List of Figures}

Figure 2.1. Pathways of anaerobic digestion (Salminen and Rintala, 2002) f.............................. 8

Figure 2.2. Schematic relationship between SRT and degree of digestion (Appels et al., 2008). 17 Figure 2.3. Types of mixing methods for digesters (a) external, pumped recirculation mixing, (b) internal mechanical mixing, and (c) external gas recirculation mixing (Appels et al., 2008)...... 18

Figure 2.4. Co- digestion of multi feedstocks for waste reduction and energy recovery ............. 20 Figure 2.5. Advantages of anaerobic co-digestion systems ................................................... 22 Figure 2.6. Types of digester configuration: (a) Single- stage; (b) Two- stage; and (c) Three-

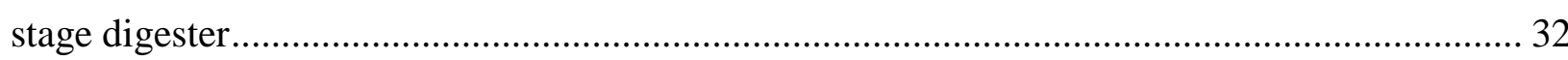

Figure 2.7. Mechanical methods of pretreatment used for AnCoD improvement......................... 35 Figure 3.1. The flow diagram of the Ashbridges Bay Wastewater Treatment Plant (City of

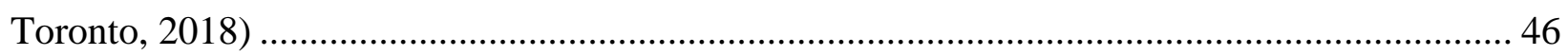

Figure 3.2. Source separated organics processing in Disco Road Facility ................................... 47

Figure 3.3. Experimental set-up for biomethane potential experiment ........................................ 51

Figure 3.4. Experimental set-up for hydrolysis/acidification experiment ................................... 54

Figure 3.5. The spectra and flourometric data for different total lipids concentrations ............... 55

Figure 4.1. Cumulative methane production for different mixing ratios of TWAS and SSO ...... 60

Figure 4.2. Methane production rate $(\mathrm{mL} / \mathrm{d})$ for different mixing ratios of TWAS and SSO ....... 61 Figure 4.3. Maximum methane production rate $(\mathrm{mL} / \mathrm{d})$ for different mixing ratios of TWAS and SSO

Figure 4.4. Methane yields: a) per unit mass TCOD added, b) per unit mass of VSS added and c) per unit volume of substrate added at different mixing ratio of TWAS and SSO ......................... 64

Figure 4.5. Biodegradable fraction of the feedstocks for different mixture ratios of TWAS and SSO

Figure 4.6. Synergetic effect of co-digestion at different mixing ratios of TWAS and SSO ....... 66 Figure 4.7. Main effects polot for $\mathrm{CH} 4$ yield data means in response to feedstock ratios at different lipids:proteins:carbohydrates ratios

Figure 4.8. Matrix effects plot for: a. ultimate $\mathrm{CH}_{4}$ and b. $\mathrm{CH}_{4}$ yield at different COD:N and Lipids: Proteins; Lipids:Carbohydrates and Proteins:Cabohydrates for the same corresponding mixing ratios of TWAS:SSO 70

Figure 4.9. Degree of COD solubilization at different mixing ratios of TWAS and SSO 75 
Figure 4.10. Synergistic effect on solubilization at different mixing ratios of TWAS and SSO.. 75 Figure 4.11. Total VFAs yield at different mixing ratios of TWAS/SSO ............................... 76 Figure 5.1. Cumulative methane production for different mixing ratios of TWAS and manure .81 Figure 5.2. Methane production rate $(\mathrm{mL} / \mathrm{d})$ for different mixing ratios of TWAS and Manure. 82 Figure 5.3. Maximum methane production rate $(\mathrm{mL} / \mathrm{d})$ for different mixing ratios of TWAS and manure.

Figure 5.4. COD mass balance in co-digestion of TWAS and manure for different mixing ratios

Figure 5.5. Methane yields a) per unit mass TCOD added, b) per unit mass of VSS added and c) per volume substrate added at different mixing ratio of TWAS and manure 85

Figure 5.6. Biodegradable fraction of the feedstocks for different mixture ratios of TWAS and manure 86

Figure 5.7. Synergetic effect of co-digestion at different mixing ratios of TWAS and SSO ...... 87

Table 5.2. Ultimate $\mathrm{CH}_{4}$ and yield at different ratios of the substrates, COD:N, and Lipids:Proteins:Carbohydrates 89

Figure 5.8. Main effect plot for $\mathrm{CH}_{4}$ yield data means in response to feedstock and lipids:proteins:carbohydrates ratios at different feedstock mixing ratios in AnCoD of TWAS/manure . 89

Figure 5.9. Matrix plot for: a. ultimate $\mathrm{CH}_{4}$ and b. $\mathrm{CH}_{4}$ yield at different $\mathrm{COD} / \mathrm{N}$ and Lipids:

Proteins, Lipids: Carbohydrates, and Proteins: Carbohydrates ratios....................................... 90

Figure 5.10. Degree of COD solubilization at different mixing ratios of TWAS:Manure .......... 94

Figure 5.11. Synergistic effect on solubilization at different mixing ratios of TWAS:Manure ... 94

Figure 5.12. Total VFAs yield at different mixing ratios of TWAS/Manure ........................... 95

Figure 6.1. Cumulative methane production for different mixing ratios of manure and SSO .... 99 Figure 6.2. Methane production rate $(\mathrm{mL} / \mathrm{d})$ for different mixing ratios of Manure and SSO ... 100 Figure 6.3. Maximum methane production rate $(\mathrm{mL} / \mathrm{d})$ for different mixing ratios manure of and SSO 101

Figure 6.4. COD mass balance in co-digestion of manure and SSO for different mixing ratios 101 Figure 6.5. Methane yields a) per unit mass TCOD added, b) per unit mass of VSS added and c) per volume substrate added at different mixing ratio of TWAS and manure 103 
Figure 6.6. Biodegradable fraction of the feedstocks for different mixture ratios of TWAS and

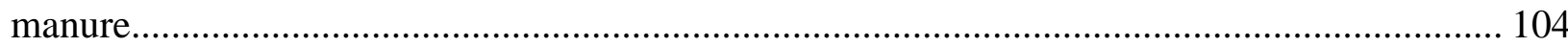

Figure 6.7. Synergetic effect of co-digestion at different mixing ratios of manure and SSO ... 105

Figure 6.8. Main effect plot for $\mathrm{CH}_{4}$ yield data mean in response to feedstock and

lipids:proteins:carbohydrates ratios in AnCoD of manure/SSO ......................................... 107

Figure 6.9. Matrix plot for: a. ultimate $\mathrm{CH}_{4}$ and b. $\mathrm{CH}_{4}$ yield at different COD:N and Lipids:

Proteins, Lipids: Carbohydrates, and Proteins: Carbohydrates Ratios ................................... 108

Figure 6.10. Degree of COD solubilization at different mixing ratios of Manure/SSO ............ 112

Figure 6.11. Synergistic effect on solubilization at different mixing ratios of Manure/SSO ..... 112

Figure 6.12. Total VFAs yield at different mixing ratios of Manure/SSO ............................ 113

Figure 7.1. Cumulative methane production for different mixing ratios of TWAS/manure/SSO

Figure 7.2. Methane production rate $(\mathrm{mL} / \mathrm{d})$ for different mixing ratios of TWAS/Manure/SSO 118

Figure 7.3. Maximum methane production rate $(\mathrm{mL} / \mathrm{d})$ for different mixing ratios TWAS/manure/SSO.

Figure 7.4. COD mass balance in co-digestion of manure and SSO for different mixing ratios 4

Figure 7.5. Methane yields a) per unit mass TCOD added, b) per unit mass of VSS added and c) per volume substrate added at different mixing ratio of TWAS and manure 121

Figure 7.6. Biodegradable fraction of the feedstocks for different mixture ratios of TWAS and manure.

Figure 7.7. Synergetic effect of co-digestion at different mixing ratios of manure and SSO .... 123

Figure

Figure 7.8. Main effect plot for $\mathrm{CH}_{4}$ yield data mean in response to feedstock and

lipids:proteins:carbohydrates ratio in AnCoD of TWAS/manure/SSO

Figure 7.9. Matrix plot for: a. ultimate $\mathrm{CH}_{4}$ and b. $\mathrm{CH}_{4}$ yield at different $\mathrm{COD} / \mathrm{N}$ and Lipids:

Proteins, Lipids: Carbohydrates, and Proteins: Carbohydrates Ratios 126

Figure 7.10. Degree of COD solubilization at different mixing ratios of TWAS/Manure/SSO 130 Figure 7.11. Synergistic effect on solubilization at different mixing ratios of TWAS/Manure/SSO 
Figure 7.12. Total VFAs yield at different mixing ratios of TWAS/Manure/SSO.................. 132

Figure 8.1. Fitted plot of response variable to lipids, Lipids ${ }^{2}$, proteins, and carbohydrate ........ 137

Figure 8.2. Residuals vursus fits plot (residuals versus estimated response) .......................... 139

Figure 8.3. a. Main effect plot for $\mathrm{CH}_{4}$ yield data mean..................................................... 141

Figure 8.3. b. Main effect plot for $\mathrm{CH}_{4}$ yield data mean for the lag phase data mean............... 141

Figure 8.3. c. Main effect plot for $\mathrm{CH}_{4}$ yield data mean for the $\mathrm{CH}_{4}$ maximum rate data mean 142

Figure 8.4. Interval plot of $\mathrm{CH}_{4}$ yield with $95 \%$ confidence interval for the mean................. 143

Figure 8.5. a. Fisher individual $95 \%$ confidence interval differences of means for $\mathrm{CH}_{4}$ yields in

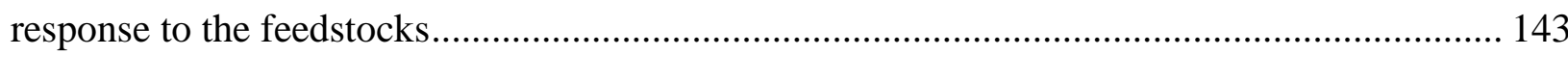

Figure 8.5. b. Fisher individual $95 \%$ confidence interval differences of means for $\mathrm{CH}_{4}$ yields in

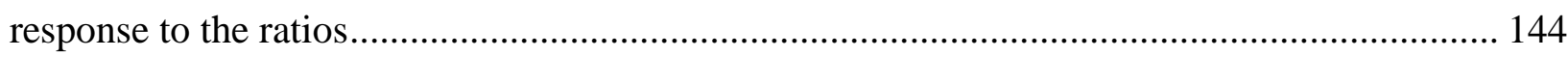




\section{List of Tables}

Table 2.1. Potential feedstocks for co-digestion to balance nutrient with regard to $\mathrm{C} / \mathrm{N}$ ratio

[(Hagos et al., 2017),(R et al., 2017)-(Sosnowski et al., 2003)]

Table 2.2. Microbial consortia diversity in various AnCoD systems for biogas and methane improvement 28

Table 2.3. Comparison of single-stage and two-stage digestion in AnCoD systems 34

Table 2.4. Biological pretreatment for enhanced AnCoD 40

Table 2.5. Hybrid pretreatment for enhanced AnCoD. 42

Table 3.1. Initial Characteristics of the feedstocks and inoculum used in this study 48 Table 3.2 Proportions of digesters' feed for binary co-digestion of TWAS/ SSO, TWAS/manure, and manure/ SSO in BMP experiment. 49

Table 3.3 Proportions of digesters' feed for ternary co-digestion of TWAS, SSO, and manure in BMP experiment 50

Table 3.4 Proportions of digesters' feed for binary co-digestion of TWAS/ SSO, TWAS/manure, and manure/ SSO in hydrolysis/acidification experiment 52 Table 3.5 Proportions of digesters' feed for ternary co-digestion of TWAS, SSO, and manure in in hydrolysis/acidification experiment. 53

Table 4.1. Mean values of the feed characteristics in digesters with different mixing ratios of

TWAS and SSO 58

Table 4.2. Ultimate $\mathrm{CH}_{4}$ at different ratios of the substrates, COD:N, and

Lipids:Proteins:Carbohydrates. 68

Table 4.3. Summary of results of kinetic study using modified Gompertz models..................... 71

Table 4.4. Characteristics of the feedstocks at different mixing ratios of TWAS and SSO ........ 74

Table 4.5. Hydrolysis rate coefficient for COD, lipids, proteins, and carbohydrate content in codigesion of TWAS and SSO at different mixing ratios ................................................... 78

Table 5.1. Characteristics of feed to digesters with different mixing ratios .............................. 80

Table 5.2. Ultimate $\mathrm{CH}_{4}$ and yield at different ratios of the substrates, COD:N, and

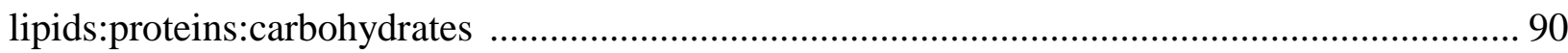

Table 5.3. Summary of results of kinetic study using modified Gompertz model .................... 91

Table 5.4. Characteristics of the feedstocks at different mixing ratios of TWAS and manure .... 93 
Table 5.5. Hydrolysis rate coefficients for COD, lipids, proteins, and carbohydrate content in co-

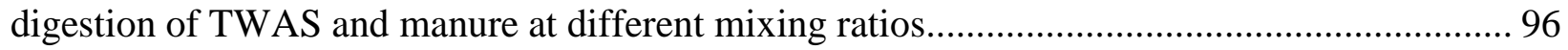
Table 6.1. Characteristics of feed to digesters with different mixing ratios of manure and SSO. 98 Table 6.2. Ultimate $\mathrm{CH}_{4}$ and yield at different ratios of the substrates, COD:N, and

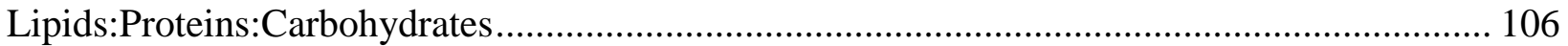

Table 6.3. Summary of results of kinetic study using modified Gompertz model ................... 109 Table 6.4. Characteristics of the feedstocks at different mixing ratios of manure and SSO ..... 111 Table 6.5. Hydrolysis rate coefficients for COD, lipids, proteins, and carbohydrate content in codigestion of SSO and manure at different mixing ratios

Table 7.1. Characteristics of feed to digesters with different mixing ratios of TWAS/ manure/SSO 116

Table 7.2. Ultimate $\mathrm{CH}_{4}$ and yield at different ratios of the substrates, COD:N, and Lipids:Proteins:Carbohydrates.

Table 7.3. Summary of results of kinetic study using modified Gompertz model for

TWAS/manure/SSO co-digestion 127

Table 7.4. Characteristics of the feedstocks at different mixing ratios of TWAS/manure/SSO 129 Table 7.5. Hydrolysis rate coefficients for COD, lipids, proteins, and carbohydrate content in codigestion of TWAS, Manure, and SSO at different mixing ratios 133 Table 8.1.Coefficients of variables in the second-order polynomial model and the results of ANOVA analysis 139

Table 8.2. Experimental set-ups and the corresponding ratios of the feedstock and lipids: proteins: carbohydrates 140 


\section{List of Appendices}

A. Analytical results for AnCoD of TWAS and SSO ...................................................... 152

Table A. 1. Characteristics of raw feedstocks in AnCoD of TWAS and SSO ........................ 152

Table A. 2. Average $\mathrm{CH}_{4}$ production in AnCoD of TWAS and SSO ................................... 153

Table A. 3. Cumulative $\mathrm{CH}_{4}$ production in AnCoD of TWAS and SSO .............................. 154

Table A. 4. Cumulative methane yield per unit mass of COD added in AnCoD of TWAS and

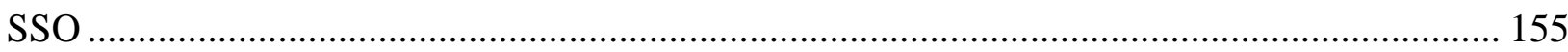

Table A. 5. Cumulative methane yield per unit mass of VSS added in AnCoD of TWAS and SSO 156

Table A. 6. Cumulative methane yield per unit volume of VSS added in AnCoD of TWAS and SSO 157

Figure A.1. Ultimate $\mathrm{CH}_{4}$ production in $\mathrm{AnCoD}$ of TWAS and SSO 158

Table A. 7. Characteristics of raw feedstocks for hydrolysis/acidification in AnCoD of TWAS/SSO 159

Table A. 8. Soluble and particulate COD concentrations (mg/L) over time 160

Figure A.2. Concentration of soluble COD over time for different mixing ratios 161

B. Analytical results for AnCoD of TWAS and manure 162

Table B.1 Characteristics of raw feedstocks in AnCoD of TWAS and manure 162

Table B. 2. Cumulative $\mathrm{CH}_{4}$ production in $\mathrm{AnCoD}$ of TWAS and manure 163

Table B. 3. Cumulative methane yield per unit mass of COD added in AnCoD of TWAS and manure.

Table B. 4. Cumulative methane yield per unit mass of VSS added in AnCoD of TWAS and manure

Table B. 5. Cumulative methane yield per unit volume of substrate added in AnCoD of TWAS and manure. 166

Figure B. 1. Ultimate methane production in AnCoD of TWAS and manure. 167 
Table B. 6. Characteristics of the raw feedstocks for hydrolysis/acidification in AnCoD of

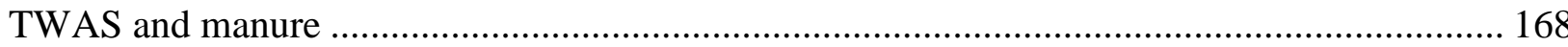

Table B. 7. Measured and theoretical VFAs concentrations over time ................................. 169

Table B. 8. Soluble and particulate COD concentrations over time .................................... 170

Figure B.2. Concentration of soluble COD over time at different mixing ratios of TWAS and

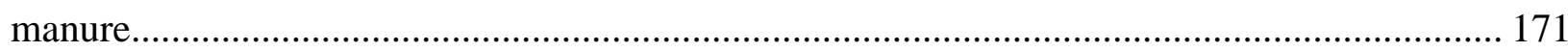

C. Analytical results for AnCoD of manure and SSO ................................................... 172

Table C. 1. Characteristics of raw feedstocks in AnCoD of manure and SSO ........................ 172

Table C. 2. Average $\mathrm{CH}_{4}$ measurements in $\mathrm{AnCoD}$ of manure and $\mathrm{SSO}$............................. 173

Table C. 3. Cumulative $\mathrm{CH}_{4}$ production in $\mathrm{AnCoD}$ of manure and SSO .............................. 174

Table C. 4. Cumulative $\mathrm{CH}_{4}$ production per mass of COD added in AnCoD of manure and SSO

Table C. 5. Cumulative $\mathrm{CH}_{4}$ production per mass of VSS added in AnCoD of manure and SSO

Table C. 6. Cumulative $\mathrm{CH}_{4}$ production per volume of substrate added in AnCoD of manure and $\mathrm{SSO}$

Figure C. 1. Ultimate methane production in AnCoD of TWAS and manure. 178

Table C. 7. Characteristics of the raw feedstocks for hydrolysis/acidification of manure and SSO

Table C. 8. Measured and theoretical VFAs over time in hydrolysis/acidification of manure and SSO 180

Table C. 9. Concentration of soluble and particulate COD over time in hydrolysis/acidification of manure and SSO 181

Figure C.2. Concentration of soluble COD over time at different mixing ratios ..................... 182

D. Analytical results for AnCoD of TWAS, manure and SSO .......................................... 183

Table D. 1. Characteristics of raw feedstocks in AnCoD of TWAS, Manure, and SSO........... 183 
Table D. 2. Average $\mathrm{CH}_{4}$ measurements (mL) in AnCoD of TWAS/Mnaure/SSO (T:TWAS,

M:Manure)

Table D. 3. Cumulative $\mathrm{CH}_{4}$ production (mL) in AnCoD of TWAS/Mnaure/SSO (T:TWAS,

$\mathrm{M}:$ Manure)

Table D. 4. Cumulative $\mathrm{CH}_{4}$ production per mass of COD added in AnCoD of

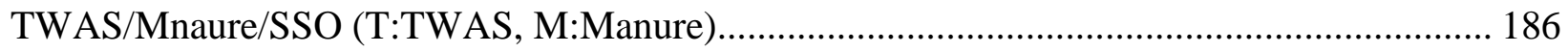

Table D. 5. Cumulative $\mathrm{CH}_{4}$ production per mass of VSS added in AnCoD of

TWAS/Mnaure/SSO (T:TWAS, M:Manure)

Table D. 6. Cumulative $\mathrm{CH}_{4}$ production per volume of substrate added in AnCoD of

TWAS/Mnaure/SSO (T:TWAS, M:Manure) .................................................................. 188

Figure D. 1. Ultimate CH4 production in AnCoD of TWAS/Manure/SSO ........................... 189

Table D. 7. Characteristics of raw feedstocks for hydrolysis/acidification in AnCoD of

TWAS/Manure/SSO

Table D. 8. Measured and theoretical VFAs concentrations (mg/L) over time in

hydrolysis/acidification of TWAS/Manure/SSO

Table D. 9. Soluble and particulate COD concentrations (mg/L) over time in

hydrolysis/acidification of TWAS/Manure/SSO

Figure D.2. Concentration of soluble COD over time at different mixing ratios

References 


\section{List of Nomenclature}

AD

AnCoD

TWAS

SSO

$\mathrm{F}: \mathrm{M}$

COD

TCOD

SCOD

VSS

TSS

VS

TS

VFAs

GC

S Proteins

S Lipids

S Carbohydrates

LCFAs

TN

TSN

$\mathrm{C}: \mathrm{N}$

COD:N
Anaerobic digestion

Anaerobic co-digestion

Thickened waste activated sludge

Source separated organics

Food to microorganism ratio

Chemical Oxygen Demand

Total Chemical Oxygen Demand

Soluble Chemical Oxygen Demand

Volatile suspended solids

Total suspended solids

Volatile solids

Total solids

Volatile fatty aids

Gas chromatograph

Soluble proteins

Soluble lipids

Soluble carbohydrates

Long chain fatty acids

Total nitrogen

Total soluble nitrogen

Carbon to nitrogen ratio

COD to nitrogen ratio 


\section{Chapter 1}

Introduction 


\section{INTRODUCTION}

Waste materials including biowaste are constantly being generated due to inevitable human activities. Different techniques have been employed to manage and reduce the growing amount of biowaste. However, such technologies result in secondary environmental impact. Landfilling can lead to soil and groundwater contamination imposing further actions and cost to remediate the secondary contamination. If not well managed and maintained, incineration will cause air pollution and subsequent environmental and health impact (Elbeshbishy et al., 2017; Feng et al., 2018). Mitigation of climate change and fossil fuel consumption demands a shift to alternative, renewable energy sources (Cornelissen et al., 2012; Kwietniewska and Tys, 2014). As reported by International Energy Outlook, 2011, total world energy consumption will increases from 505 quadrillion British thermal units (Btu) in 2008 to an estimated number of 619 quadrillion Btu in 2020, and it is expected to rise to 770 quadrillion Btu in 2035 which is equivalent to $53 \%$ increase in spam of 27 years (OECD/EIA, 2011). Energy obtained from biomass is regarded as an important future renewable source, as it is capable of providing a continuous power generation and it is also an essential part of the current $\mathrm{CO}_{2}$-mitigation policy (Appels et al., 2011; Kwietniewska and Tys, 2014).

Production of biofuel from biomass, has received increasing attention during recent years. Several treatment processes and technologies have been established to obtain sustainable and affordable biofuel which includes syngas (SNG). SNG is a synthetic gas produced by gasification of a carbon containing fuel that has some energy value. However, production of SNG is narrowly practiced due to its cost (Guo et al., 2015; Schuetzle et al., 2015). Anaerobic digestion (AD) which is widely used for the treatment of wet residual biomass is considered to be one of the most favorable processes for biofuel production from biomass.

In Canada, more than 27 million tons of food waste are disposed yearly. The produced organic waste can lead to serious health and environmental issues. Municipalities have taken different management actions to manage the organic fraction of municipal solid waste (OFMSW). Biological processes for the treatment and/or conversion of OFMSW to value-added products has aroused significant attention due to its financial benefits and less environmental impacts compared to the other waste disposal methods such as landfilling, incineration, gasification, etc. (Luk and Bekmuradov, 2014; Naroznova et al., 2016; Razavi et al., 2019). Organic matters in waste includes

food scraps, yard trimmings, wood waste, paper and cardboard products which normally make up 
around $33 \%$ by weight of the municipal solid waste. Source Separated Organics (SSO) refers to the organic waste which is segregated from other waste materials at the source for separate collection. SSO comprises mostly of food waste which is separated from the residential waste (Kelleher Robins, 2013).The study by Kelleher Environmental have indicated that almost 23\% of waste from non-residential industrial, commercial and institutional (IC\&I) sector is food waste which is generated by institutions and businesses in communities across Canada. The majority of food waste from this sector is created by restaurants, hotels, food processing facilities and hospitals. A considerable amount of biogas through anaerobic digestion process can be produced by food wastes from all of these sources (Government of Canada, 2013; Kelleher Robins, 2013). In areas with vast numbers of large-scale livestock farms, the development of a treatment process for manure is necessary to properly handle the high amount of produced waste. Animal manure provides adequate nutrients for anaerobic digestion to produce biogas as a source of renewable energy. High degradability of manure when flushed and fibrous materials are separated makes its anaerobic digestion a good treatment option to minimize waste above and beyond bioenergy recovery. However, in some farms manure is collected in lagoons or in the open areas which can result in air and water pollution. Manure stockpiling can lead to serious health environmental issues as a result of emissions such as methane, nitrous oxide, ammonia, hydrogen sulfide, volatile organic compounds and particulate matter (Lin et al., 2018; Peter Wright et al., 2013). Co-digestion of manure with additional substrates provided that appropriate mixture ratios are applied can improve digestion process and increase biogas production.

In addition, in Canada more than 660,000 metric tons of dry stabilized biosolids is produced each year. Biosolids are produced during the treatment of wastewater due to the removal of the solids content (sludge) from the liquid effluent. Biosolids management is responsible for almost $50 \%$ of the total operating annual cost of wastewater management. According to Canadian Council of Ministers of the Environment (CCME), a Canada-wide approach for the management of wastewater biosolids was approved on October 11, 2012 which encourages the favorable and thorough management of biosolids. Biosolids are the nutrient-rich, organic materials which makes them a useful resource, containing necessary plant nutrients, and organic matter. They can be recycled as a fertilizer and soil improvement for agricultural utilization. Annually, around 195,000 tons of biosolids is processed by the Ashbridges Bay and Highland Creek wastewater treatment 
plants in City of Toronto. The City's biosoilds management can be classified as the following options (City of Toronto, 2019):

- Land application for agricultural and other purposes

- Pelletization for fertilizer production

- Alkaline stabilization for producing fertilizer, landfill cover, or for the $\mathrm{pH}$ adjustment of acidic soil

- Landfilling

- Incineration

There are some pros and cons associated with any of the above options. However, Anaerobic Digestion (AD) for producing bioenergy from organic waste has shown to be an energy-efficient technology while causing less environmental footprint compared to other technologies such as composting that requires large land and can release uncontrolled odorous volatile organic compounds and pathogens (Bordeleau and Droste, 2011; Lin et al., 2018; Razavi et al., 2019). Nevertheless, in conventional anaerobic digestion of single feedstock some problems such as nutrient imbalance, low biodegradability, toxic substances, etc. can hinder the process causing low production of biogas. Such problems can be modified by simultaneous digestion of two or more feedstocks referred to as anaerobic co-digestion (AnCoD).

\subsection{Research objective}

Anaerobic digestion involves sequential phases comprising hydrolysis, acidogenesis, acetogenesis and methanogenesis. Anaerobic digestion of multi feedstocks has been advantageous compared to conventional anaerobic digestion of single feedstock. Literature has showed contradictory values for the optimum range of the $\mathrm{C}: \mathrm{N}$ or COD:N ratio for optimizing the mixing ratio of the feedstocks in anaerobic co-digestion systems. In addition, hydrolysis of particulate organic matter which is the rate-limiting stage, remains as the least well-defined phase of the process. Therefore, this research focused on the three main following objectives:

1- Study biomethane potential in AnCoD of TWAS, Manure, and SSO. This part of the study included the following purposes: 
Evaluate the influence of the mixing ratio on improving biomethane production in anaerobic co-digestion of TWAS, manure, and SSO.

Investigate the impact of lipids: proteins: carbohydrates on improving biomethane production in anaerobic co-digestion of TWAS, manure, and SSO.

$>$ Compare the impacts of COD:N ratio, lipids:proteins, lipids:carbohydrates, and proteins:carbohydrates on biomethane production in anaerobic co-digestion of TWAS, manure, and SSO.

2- Study the hydrolysis/acidification in AnCoD of TWAS, Manure, and SSO. This part of the present research comprised the following areas:

Monitor the dynamic changes of soluble and particulate COD, lipids, proteins, and carbohydrate content in co-digestion of TWAS, manure, and SSO.

Evaluate the influence of the mixing ratio on hydrolysis/acidification performance in co-digestion of TWAS, manure, and SSO.

Study the hydrolysis kinetics of lipids, proteins, carbohydrates and COD and calculate their hydrolysis rate coefficients for co-digestion of TWAS, manure, and SSO.

3- Investigate the relationship between biomethane production and the lipids, proteins, and carbohydrates contents of the feedstocks aiming to:

Propose an empirical model that explains the relationship between biomethane yield and concentration of lipids, proteins, and carbohydrates at different mixing ratios in anaerobic digestion of manure, TWAS and SSO individually and in their codigestion.

an empirical model that explains the relationship between biomethane yield and concentration of lipids, proteins, and carbohydrates at different mixing ratios in anaerobic digestion of manure, TWAS and SSO individually and in their co-digestion. 


\section{Chapter 2}

\section{Literature review}




\section{LITERATURE REVIEW}

\subsection{Anaerobic digestion}

Biological treatment is an integral part of any wastewater treatment plant that treats wastewater from either municipality or industry which contains soluble organic contaminants or a mix of both source types. There are clear economic advantages of biological systems in terms of capital investment and operating costs when compared to other methods such as chemical oxidation, thermal oxidation, etc. In addition, mostly biological treatment converts toxic contaminants to end products that are less harmful or non-harmful with the help of microorganisms (Mittal, 2011). The two types of biological treatment: aerobic and anaerobic processes are directly related to first the type of microorganisms involved in the degradation of organic compounds in a particular wastewater and second the operating conditions of the bioreactor. Aerobic treatment processes occur in the presence of air and consume those microorganisms also called aerobes, which use molecular/free oxygen to assimilate organic contaminant. They convert them in to carbon dioxide, water and biomass (Eddy, 2003). The anaerobic processes, on other side occur in the absence of air (molecular/free oxygen) by those microorganisms also called anaerobes which do not require air or molecular/free oxygen to assimilate organic contaminants. The end products of organic assimilation in anaerobic treatment contains methane $\left(\mathrm{CH}_{4}\right)$ and carbon dioxide $\left(\mathrm{CO}_{2}\right)$ and biomass.

Anaerobic digestion of municipal wastewater sludge has been widely developed since the early $1900 \mathrm{~s}$ and is the most applied sludge treatment method. Throughout the process about $40 \%$ to $60 \%$ of the organic solids is converted to methane and carbon dioxide. The chemical composition of the produced gas contains 60-65\% methane, 30-35\% carbon dioxide, and small quantities of $\mathrm{H}_{2}, \mathrm{~N}_{2}$, $\mathrm{H}_{2} \mathrm{~S}$, and $\mathrm{H}_{2} \mathrm{O}$. Among these, methane has the most value since it is a hydrocarbon fuel producing $36.5 \mathrm{MJ} / \mathrm{m}^{3}$ in combustion (Lema and Suarez, 2017). The residual organic matter is chemically stable, nearly odorless, and contains considerably reduced levels of pathogens.

Hydrogen production by the use of anaerobic microbial communities also referred to as dark fermentation from organic waste has raised attention due to its ability to produce an environmentally benign energy source, while it stabilizes waste material simultaneously (Sung et al., 2003). Albert Lea facility in Minnesota with 12 million gallons/day of sewage and 4.5 million gallons/day sludge produces $75,000 \mathrm{ft}^{3} /$ day of biogas. Their facility which consists of 4 Capstone 
microturbines, each of them $30 \mathrm{~kW}$, is producing 2,500 $\mathrm{kWh}$ per day electricity at peak production in addition to $28,000 \mathrm{Btu} /$ day of heat.

According to Canadian Biogas Study conducted by Canadian Biogas Association in 2013, biogas production has significant potential for extension and development. All biogas sources excluding energy crops, could meet nearly 3\% of Canada's natural gas demand as biogas contribution is $2,420 \mathrm{Mm}^{3}$ /year of renewable natural gas (RNG) or $1.3 \%$ of its electricity demand where biogas contribution is $810 \mathrm{MW}$. Anaerobic digestion (AD) is a multi-step process during which organic material is converted to biogas and digestate in the absence of oxygen and presence of anaerobic microorganisms. The AD process occurs in four stages including hydrolysis/liquefaction, acidogenesis, acetogenesis, and methanogenesis. The pathways in anaerobic degradation is shown in figure 1.

The first stage of the process is hydrolysis/liquefaction. This stage is very important in AD process since polymers cannot be directly consumed by the fermentative microorganisms. Therefore, hydrolysis makes the substrate available for the following conversion steps. In hydrolysis stage, insoluble complex organic materials are decomposed into their constituents. This allows them transport through microbial cell membrane (Madigan, 2014). Hydrolysis is accomplished due to the function of hydrolytic enzymes. In the first step of hydrolysis, or liquefaction, fermentative bacteria convert the insoluble complex organic compounds like cellulose, into soluble molecules such as sugars, fatty acids, and amino acids.

Hydrolysis/Liquefaction reactions

$$
\begin{aligned}
& \text { Lipids } \rightarrow \text { Fatty Acids } \\
& \text { Polysaccharides } \rightarrow \text { Monosaccharides } \\
& \text { Protein } \rightarrow \text { Amino Acids } \\
& \text { Nucleic Acids } \rightarrow \text { Purines and Pyrimidines }
\end{aligned}
$$

The next stage is acidogenesis/fermentation through which facultative and anaerobic bacteria convert sugars, amino acids and fatty acids to hydrogen, acetate, carbon dioxide, VFAs such as propionic, butyric and acetic acid, ketones, alcohols and lactic acid. Although a simple substrate like glucose can be fermented, various products are created by the diverse bacterial community. 
Conversion of glucose to acetate, ethanol and propionate are shown in the reactions 1, 2 and 3 below respectively (Kangle et al., 2012).

$$
\begin{aligned}
& \mathrm{C}_{6} \mathrm{H}_{12} \mathrm{O}_{6}+2 \mathrm{H}_{2} \mathrm{O} \rightarrow 2 \mathrm{CH}_{3} \mathrm{COOH}+2 \mathrm{CO}_{2}+4 \mathrm{H}_{2} \\
& \mathrm{C}_{6} \mathrm{H}_{12} \mathrm{O}_{6} \rightarrow 2 \mathrm{CH}_{3} \mathrm{CH}_{2} \mathrm{OH}+2 \mathrm{CO}_{2} \\
& \mathrm{C}_{6} \mathrm{H}_{12} \mathrm{O}_{6}+2 \mathrm{H}_{2} \rightarrow 2 \mathrm{CH}_{3} \mathrm{CH}_{2} \mathrm{COOH}+2 \mathrm{H}_{2} \mathrm{O}
\end{aligned}
$$

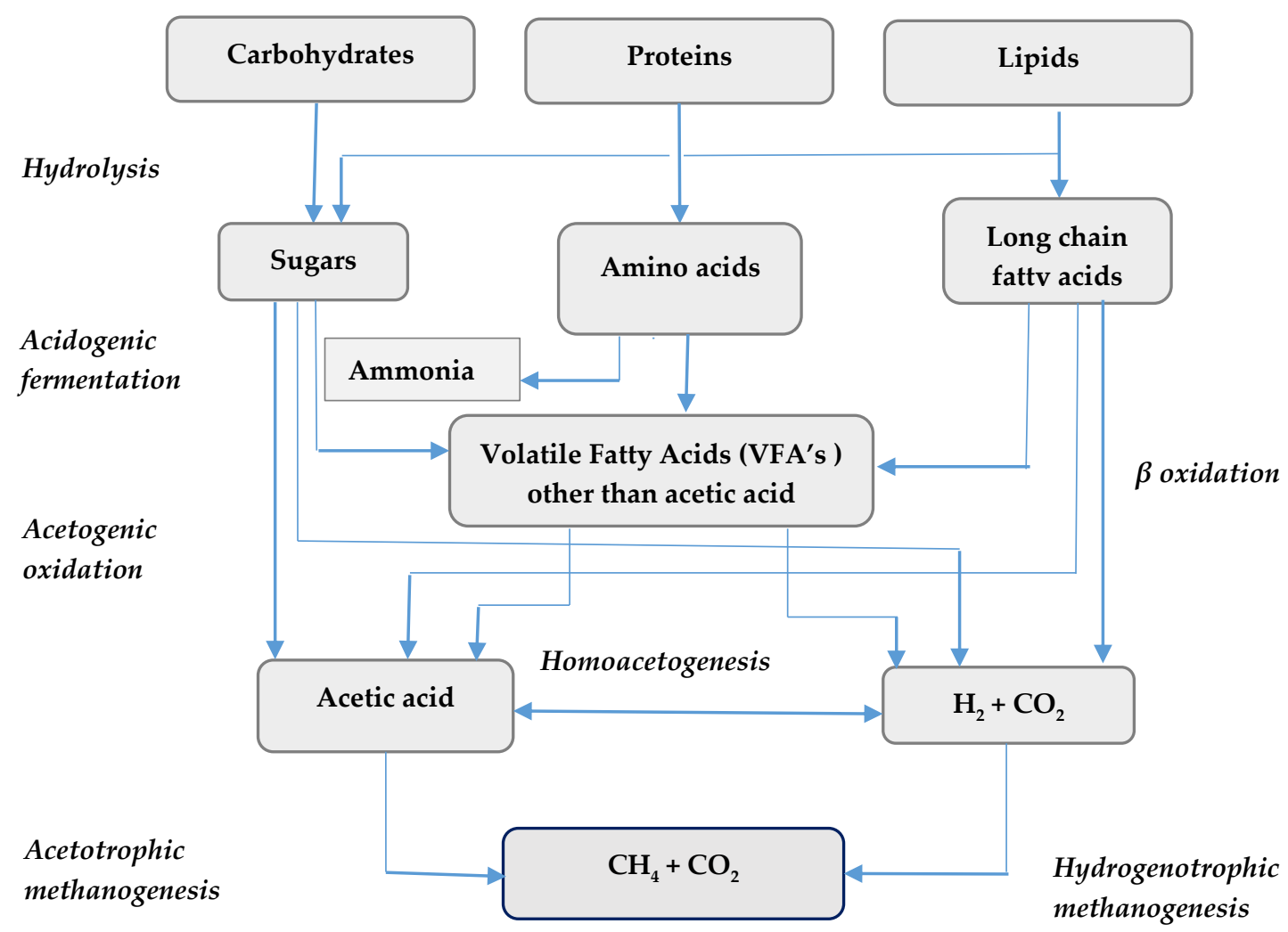

Figure 2.1. Pathways of anaerobic digestion (Salminen and Rintala, 2002)

In equilibrium condition, most of the organic material is converted into substrates such as acetate, hydrogen and carbon dioxide that are readily available for methanogenic microbes. However, a significant portion of about $30 \%$ is converted to short chain fatty acids or alcohols. Degradable organic material is eliminated in this step (Angelidaki et al., 2009a; Kangle et al., 2012). The 
byproduct of amino acids fermentation, ammonia and hydrogen sulfide are released. These compounds can be inhibitory for AD process (Kangle et al., 2012; Salminen and Rintala, 2002).

During the third stage, acetogenesis, certain fermentation products including VFAs with more than two atoms of carbon, alcohols and aromatic fatty acids are converted into acetate and hydrogen these conversions take place via obligate hydrogen producing bacteria (Boe, 2006, Kangle et al., 2012). In this step, the products of the first phase are converted to simple organic acids, carbon dioxide and hydrogen by acetogenic bacteria, also called acid formers. The main acids produced include acetic acid $\left(\mathrm{CH}_{3} \mathrm{COOH}\right)$, propionic acid $\left(\mathrm{CH}_{3} \mathrm{CH}_{2} \mathrm{COOH}\right)$, butyric acid $\left(\mathrm{CH}_{3} \mathrm{CH}_{2} \mathrm{CH}_{2} \mathrm{COOH}\right)$, and ethanol $\left(\mathrm{C}_{2} \mathrm{H}_{5} \mathrm{OH}\right)$. The activities of different microorganisms cause the formation of the products that are created during acetogenesis. These microorganisms include syntrophobacter wolinii, a propionate decomposer and sytrophomonos wolfei, a butyrate decomposer. Also other acid formers include clostridium spp., peptococcus anerobus, lactobacillus, and actinomyces (Kangle et al., 2012; Themelis, 2002). Hydrogen-producing acetogenic bacteria yield acetate, $\mathrm{H}_{2}$ and $\mathrm{CO}_{2}$ from volatile fatty acids and alcohol, whereas, homoacetogenic bacteria produce acetate from $\mathrm{CO}_{2}$ and $\mathrm{H}_{2}$ (Sterling et al., 2001). However, most of the acetate is formed via hydrogen-producing acetogenic bacteria (Angelidaki et al., 2009b). A reaction that occurs during acetogenesis is presented below:

$$
\mathrm{C}_{6} \mathrm{H}_{2} \mathrm{O}_{6} \rightarrow 2 \mathrm{C}_{2} \mathrm{H}_{5} \mathrm{OH}+2 \mathrm{CO}_{2}
$$

The final stage of $\mathrm{AD}$ process is methanogenesis. Various methane-forming bacteria are required in an $\mathrm{AD}$ system. That is because a single species is not able to degrade all the existing substrates. The methanogenic bacteria comprise methanobacterium, methanobacillus, methanococcus, and methanosarcina. Methanogenesis can be classified into two groups. These two groups involves acetate and $\mathrm{H}_{2} / \mathrm{CO}_{2}$ consumers. Methanosarcina spp. and methanothrix spp. Also known as methanosaeta are important in $\mathrm{AD}$ process both as $\mathrm{H}_{2} / \mathrm{CO}_{2}$ and acetate consumers. Almost $70 \%$ of the methane is produced from acetate. The remaining $30 \%$ is produced due to the reduction of carbon dioxide via hydrogen and other electron donors (Smith and Mah, 1966; Varel et al., 1980). With regard to the type of the substrate consumed by the methanogens, methanogenesis can be classified into two main types (Bitton, 2010; Kangle et al., 2012):

- Hydrogenotrophic methanogenesis 
In this type of methanogenesis, hydrogen and carbon dioxide are converted into methane through the following reaction:

$$
\mathrm{CO}_{2}+4 \mathrm{H}_{2} \rightarrow \mathrm{CH}_{4}+2 \mathrm{H}_{2} \mathrm{O}
$$

- Acetotrophic or aceticlastic methanogenesis

This type of methanogenesis involves the formation of methane from the conversion of acetate by the following reaction:

\section{$\mathrm{CH}_{3} \mathrm{COOH} \rightarrow \mathrm{CH}_{4}+\mathrm{CO}_{2}$}

Any substrate that can be converted to methane by anaerobic bacteria is referred to as feedstock. The main components of organic wastes (feedstock) are carbon, oxygen, nitrogen, hydrogen and phosphorus, and microbial cell material of those elements is approximately 50, 20, 12, 8 and $2 \%$ respectively (Gerardi, 2003). Sulfur is also required to synthesize vital proteins in metabolic and anabolic pathways (Madigan, 2014). Feedstocks can be a range of different waste materials from easily degradable wastewater to complex high-solid waste.

AD technologies have shown sufficient adaptability to different feedstocks (Bordoloi et al., 2014). Although $\mathrm{AD}$ is a commercial reality for a range of wastes, anaerobic digestion of single waste may be associated with certain drawbacks such as unbalanced nutrients, rapid acidogenesis, poor buffering capacity, high ammonia nitrogen concentration, inhibition of long chain fatty acids which can inhibit methanogenesis and lead to severe instability and process disruption (Bayr et al., 2014; Silvestre et al., 2014).

The growth rate of anaerobic microorganisms and subsequent biogas production depends highly on the composition of the organic matter in feedstock. The constituents of the feedstock added to the digester are consumed selectively by a range of different microbial consortia. In addition, the existence of nitrogen in the feedstock is necessary for the synthesis of amino acids, proteins and nucleic acids. It is also required for ammonia formation to neutralize VFAs produced during the fermentation process and to maintain neutral $\mathrm{pH}$ condition for cell growth. However, an excess of nitrogen in the feedstocks can result in toxic effects to bacteria by extreme ammonia formation. Therefore, a suitable amount of nitrogen is required to provide sufficient nutrient while avoiding ammonia toxicity (Hagos et al., 2017; P. et al., 2014). 
During anaerobic digestion, a series of complex biological degradation pathways are involved which are influenced by numerous factors. Therefore, a profound understanding of the biochemical activities of anaerobic microorganisms in the AD system is required to support an effective control of the governing factors in anaerobic digestion process (Viotti et al., 2004).

\subsection{Process parameters of anaerobic digestion}

A number of parameters affect the rate of the different steps of the digestion within the anaerobic environment. Overall, two groups of parameters can affect the anaerobic digestion performance including environmental and operational parameters. Environmental factors comprise temperature, $\mathrm{pH}$, alkalinity, waste characteristics such as the amounts of volatile solids (VS) carbon to nitrogen $(\mathrm{C}: \mathrm{N})$ ratio, total solids (TS), nutrients, organic loading rate (OLR), and volatile fatty acids (VFAs). The performance of anaerobic digesters can be reduced by various environmental factors including low $\mathrm{pH}$, accumulation of ammonia and volatile fatty acids (VFAs) which inhibit the activity of methanogenic microorganisms (Heo et al., 2004; Towey, 2013). VS consists of both biodegradable volatile solids (BVS) and the refractory volatile solids (RVS) fractions. BVS fraction of substrate is helpful in better biodegradability of the waste, organic loading rate, $\mathrm{C}: \mathrm{N}$ ratio, and biogas production. Apart from the environmental factors, a number of operational factors including solid retention time (SRT), hydraulic retention time (HRT), digestion mode in terms of single or multistage approaches, digester design being batch or continuous types, and digester mixing also affect the AD performance. These parameters are individually discussed in the following sections.

\subsubsection{Waste composition and volatile solids}

The wastes to be treated by $\mathrm{AD}$ process can be comprised of a biodegradable organic fraction, a combustible and an inert fraction. Waste materials containing high VS and low non- biodegradable material, or refractory volatile solids, are the most suited to AD treatment. Kitchen waste, food waste, and garden waste are biodegradable organic fraction of the waste. The combustible fraction can be slowly degrading lignocellulosic organic compounds such as coarser wood, paper, and cardboard. The lignocellulosic organic content does not readily degrade under anaerobic conditions. Therefore, they are more suitable for waste-to-energy plants. The inert fraction of the waste may contain stones, glass, sand, metal, etc. that can ideally be removed, recycled or used at 
land fill. It is important to remove the inert fraction before digestion, otherwise it causes the increase of digester volume and wear of equipment. The Volatile Solids (VS) of organic wastes are measured as total solids excluding the ash content which is obtained by complete combustion of the feed wastes. The VS contains the Biodegradable Volatile Solids (BVS) fraction and the Refractory Volatile Solids (RVS). Knowing the BVS fraction of substrate can be helpful in better estimation of the biodegradability of the waste, of biogas production, organic loading rate and $\mathrm{C}: \mathrm{N}$ ratio. Lignin is a complex organic compound which is not easily degraded by anaerobic bacteria and forms refractory volatile solids (RVS) in organic matter. Waste materials containing high VS and low non-biodegradable material, or RVS, are the most suited to AD treatment (Elbeshbishy et al., 2017; Kangle et al., 2012).

\subsubsection{Carbon to Nitrogen Ratio (C:N)}

The amount of carbon and nitrogen present in feedstock or $\mathrm{C}: \mathrm{N}$ ratio is a very important parameter of the AD process. A high $\mathrm{C}: \mathrm{N}$ ratio indicates rapid consumption of nitrogen by methanogens and leads to lower gas production. On the contrary, a lower $\mathrm{C}: \mathrm{N}$ ratio results in accumulation of ammonia and exceeding $\mathrm{pH}$ values which is toxic to methanogens. Low $\mathrm{C}: \mathrm{N}$ ratios occur when too much nitrogen is present. On the other side, a high $\mathrm{C}: \mathrm{N}$ ratio leads to deficiency in AD system (Mata-Alvarez et al., 2000a; Poliafico and Murphy, 2007). According to a study (Gerardi, 2003), the optimal gas production can be achieved by feedstock with C:N ratio of 25:1. Other studies reported that the optimum range falls within 25-30 and can be achieved by the co-digestion of different waste streams (Gonzalez-Avila et al., 2011; Monnet, 2003). Some other studies even though reported that the optimum $\mathrm{C}: \mathrm{N}$ ratios in anaerobic digesters are between 20 and 30 while some other studies even reported lower values than 20. Although, a very low C:N ratios occurs when too much nitrogen is present and leads ammonia $\left(\mathrm{NH}_{3}\right)$ to be accumulated which leads to either high pH values or methanogenic inhibition (Salminen et al., 2002, Kangle et al, 2012). On

the contrary, the high $\mathrm{C}: \mathrm{N}$ ratio indicates that nitrogen is rapidly depleted by methanogens and leads to lower gas production. A lower $\mathrm{C}: \mathrm{N}$ ratio results in accumulation of ammonia and exceeding $\mathrm{pH}$ values to over 8.5, which is toxic to methanogens. Mixing materials of high and low $\mathrm{C}: \mathrm{N}$ ratios, such as organic solid waste mixed with animal manure or sewage can help achieve optimum C:N ratios (Poliafico, 2007, Kangle et al, 2012). 


\subsubsection{Nutrients}

Some nutrient elements are needed for the growth of methane-forming bacteria. Particular metals comprising nickel, iron, cobalt, and molybdenum are essential for optimal growth and methane production. Trace metals stimulate methanogenic activity. Some metals including selenium, molybdenum, manganese, aluminum, and boron have been suggested as additional components in media. Addition of metal ions solutions to anaerobic digesters can improve the performance of the AD system. The amounts of 0.002, 0.004, 0.003, $0.02 \mathrm{mg} / \mathrm{g}$ are suggested for iron, cobalt, nickel, and zinc respectively. The requirement for nickel is quite unusual for biological systems and can exclusively characterize methanogenic bacteria. Addition of metal ions solutions to anaerobic digesters can improve the performance of the AD system (Azbar and Speece, 2001; Kangle et al., 2012; Mata-Alvarez et al., 2000a).

\subsubsection{Total solids content (TS)/Organic loading rate (OLR)}

The increase of the total solids (TS) fraction leads a corresponding decrease in the reactor volume. High solids (HS) AD system contain $22 \%$ to $40 \%$ TS, medium solids (MS) system about 15 to $20 \%$ and low solids (LS) AD systems contain less than $10 \%$ of TS. The organic loading rate (OLR) is defined as the organic matter flowing into the digester per time which is expressed as mass of organic matter over digester volume over time (Appels et al., 2008; Kangle et al., 2012). OLR is also defined as measure of the biological conversion capacity of the AD system. Typical values of OLR ranges between 0.5 and $3 \mathrm{~kg} \mathrm{VS} / \mathrm{m}^{3} / \mathrm{d}$. OLR is particularly important parameter in continuous systems. System failures as a result of overloading have been reported by numerous plants (Kangle et al., 2012; Poliafico and Murphy, 2007).

When feeding the system above its sustainable OLR, low biogas yield is obtained. This is caused by the accumulation of the inhibiting substances such as fatty acids in the digester slurry. Any substrate that can be converted to methane by anaerobic bacteria is referred to as feedstock. The main components of feedstock are carbon, oxygen, nitrogen, hydrogen and phosphorus, and microbial cell material of those elements is reported to be approximately 50, 20,12,8 and $2 \%$ respectively. Feedstocks can be a range of different waste materials from easily degradable wastewater to complex high-solid waste (Gerardi, 2003). 


\subsection{5. $\mathrm{pH}$, alkalinity and volatile acids/alkalinity ratio}

There is a different optimum $\mathrm{pH}$ range for each group of micro-organisms. Methanogenic bacteria are very sensitive to $\mathrm{pH}$. The optimum range for them is between 6.5 and 7.2. The fermentative microorganisms are relatively less sensitive and can tolerate a wider range of $\mathrm{pH}$ between 4.0 and 8.5. At low $\mathrm{pH}$, mainly acetic and butyric acids are produced, while at a $\mathrm{pH}$ of around 8.0, acetic and propionic acids are mainly produced. The VFAs produced during AD process result in a $\mathrm{pH}$ reduction. This reduction is normally adjusted by methanogenic bacteria, which produce alkalinity in the form of ammonia, carbon dioxide, and bicarbonate (Appels et al., 2008).

The $\mathrm{pH}$ of the system is controlled by the $\mathrm{CO}_{2}$ in the gas phase and the $\mathrm{HCO}_{3}$-alkalinity of the liquid phase. If the concentration of $\mathrm{CO}_{2}$ remains constant, the addition of $\mathrm{HCO}_{3}$-alkalinity can increase the $\mathrm{pH}$ of digester. In order to maintain a stable and well-buffered digestion process a buffering capacity of 70 meq $\mathrm{CaCO}_{3} / \mathrm{L}$ or a molar ratio of at least $1.4: 1$ of bicarbonate/VFA is required. However, previous studies have shown that particularly the stability of the ratio is very significant and not so much its level.

Except for ammonia, other factors such as sulfide, sodium and potassium, heavy metals, volatile fatty acids, long-chain fatty acids, and hydrogen can also affect the activity of methanogens. Molecular hydrogen is formed throughout different stages of anaerobic digestion. Inhibition can occur due to the lack of balance between the rates of hydrolysis and methanogenesis. A suitable balance between those rates is essential for higher methane production. Rapid methanogenesis is required to prevent accumulation of organic acid lowering $\mathrm{pH}$ to an extent that inhibits methanogenesis (Appels et al., 2008; Pouget et al., 2012).

\subsubsection{Temperature}

The temperature has an important effect on the physicochemical properties of the substrate. Moreover, it is effective on the growth rate and metabolism of micro-organisms and the population dynamics in the reactor. A stable operating temperature is very important to be maintained in the digester, since fluctuations in temperature affect the bacteria particularly the methanogens. Acetotrophic methanogens are one of the most sensitive groups of bacteria to increasing temperatures. The degradation of propionate and butyrate is also sensitive to temperatures above $70^{\circ} \mathrm{C}$. (The propionate or propanoate ion is $\mathrm{C}_{2} \mathrm{H}_{5} \mathrm{COO}-$ the conjugate base of propionic acid- $\mathrm{A}$ propionic or propanoic compound is a small salt or ester of propionic acid. Butyric acid, also 
known under the systematic name butanoic acid, abbreviated BTA, is a carboxylic acid with the structural formula $\mathrm{CH}_{3} \mathrm{CH}_{2} \mathrm{CH}_{2}-\mathrm{COOH}$. Salts and esters of butyric acid are known as butyrates or butanoates) (Appels et al., 2008; Turovskiy and Mathai, 2005).

The temperature has also a substantial effect on the partial pressure of $\mathrm{H}_{2}$ in digesters and therefore, it affects the kinetics of the syntrophic metabolism. Thermodynamic studies show that endergonic reactions under standard conditions, for example the decomposition of propionate into acetate, $\mathrm{CO}_{2}$, and $\mathrm{H}_{2}$, would be more favorable energetically at higher temperature, whereas the exergonic reactions such as hydrogenotrophic methanogenesis are less favored at higher temperatures. Increasing temperature can be favorable for several reasons. It can increase solubility of the organic compounds, enhance chemical and biological reaction rates, and an increase the rate of pathogens' death in thermophilic conditions. Although, the application of high temperatures (thermophilic) has some adverse effects as there will be an increase of the fraction of free ammonia which can be an inhibiting factor for the microorganisms. The increasing pKa of the VFA will make the process more susceptible to inhibition. Therefore, temperature control is a very important concern for thermophilic digestion in comparison with mesophilic digestion. A stable operating temperature is very important to be maintained in the digester, since fluctuations in temperature affect the bacteria particularly the methanogens. Process failure can occur at changes in temperature over $1{ }^{\circ} \mathrm{C} /$ day and changes of more than $0.6{ }^{\circ} \mathrm{C} /$ day in temperature should be avoided Process failure can occur at changes in temperature over $1{ }^{\circ} \mathrm{C} /$ day, and changes of more than $0.6^{\circ} \mathrm{C} /$ day in temperature should be avoided (Appels et al., 2008; Turovskiy and Mathai, 2005).

\subsubsection{Solids and hydraulic retention time}

The average time that the solids spend in a digester is referred to as solids retention time (SRT), and the average time that the liquid sludge is held in the digester is referred to as hydraulic retention time (HRT). SRT and HRT are an important design and operating parameter for all anaerobic processes. Reduction of SRT decreases the extent of the reactions and vice versa. A fraction of the bacterial population is removed each time when the sludge is withdrawn. Therefore, the cell growth must at least compensate the cell removal to maintain steady state and to prevent from process failure. 
The ratio between the solid content of the reactor by the solid flow rate indicates the average SRT. HRT specifies the average time that waste and wastewater are exposed to microorganisms for degradation. In a conventional mixed reactor, the SRT and HRT are equal while in a retained biomass reactor the SRT is usually higher than HRT. Higher SRT can be achieved by increasing the digester volume, increasing the solid content or using a retained biomass reactor. Equation (1.1) shows the calculation for HRT and equation (1.2) shows the calculation for SRT in continues stirred tank reactor (CSTR).

$\mathrm{HRT}=$ Volume of the reactor $(\mathrm{V}) /$ Influent flow rate $=\mathrm{L} / \mathrm{L} / \mathrm{d}=\mathrm{d}$

Eq. 2.1

$\mathrm{SRT}=$ Mass of the biomass in reactor/ mass of the biomass leaving

$=\left(\mathrm{V}^{\times} \mathrm{VSS}\right) /\left(\mathrm{Q}_{\text {out }}{ }^{\times} \mathrm{VSS}\right)=\mathrm{V} / \mathrm{Q}_{\text {out }}=H R T d$

The effect of the retention time on the gas production is mostly studied on laboratory scale. Studies indicated that in a semi-CSTR system retention times shorter than 5 days are insufficient for a stable digestion. VFA concentrations are increasing due to washout of methanogenic bacteria. VFA concentrations are relatively high for 5-8 days SRT. There is an incomplete breakdown of compounds, especially of the lipids for this SRT. It has also been indicated that stable digestion is obtained after 8-10 days. There is low VFA concentrations and the breakdown of lipids starts. According to the studies, the breakdown curve stabilizes at SRT $>10$ days as all sludge compounds are significantly reduced. Figure 2 shows a schematic relationship between SRT and degree of digestion (Appels et al., 2008). 


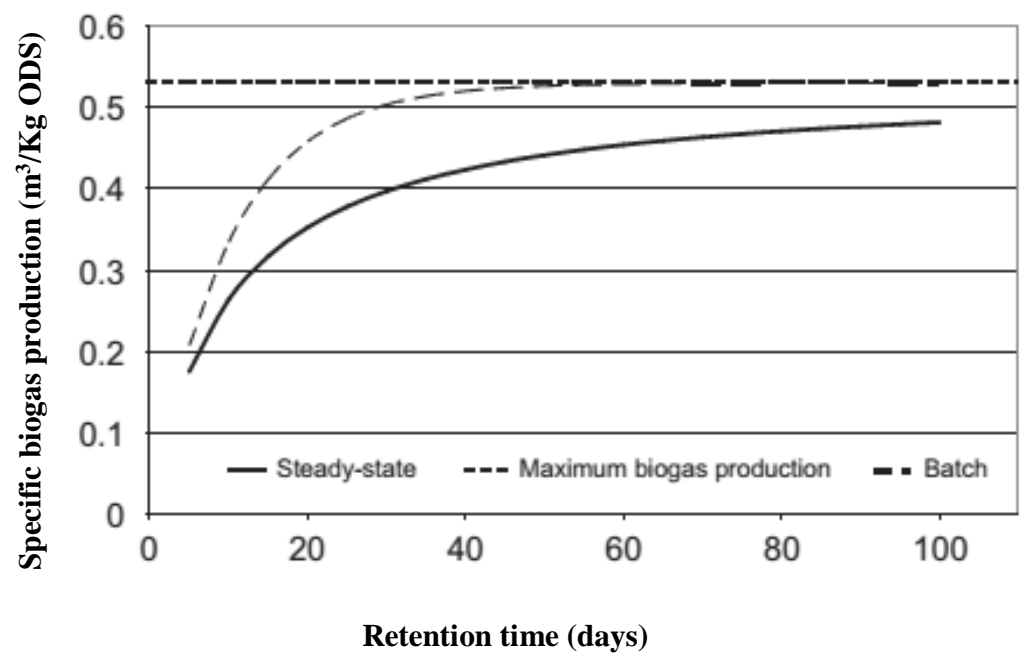

Figure 2.2. Schematic relationship between SRT and degree of digestion (Appels et al., 2008)

\subsubsection{Digester mixing}

To attain an optimum performance for an $\mathrm{AD}$ system, it is essential to maintain a proper mixing. Mixing causes near contact between the feed sludge and active biomass, yielding uniformity of several parameters including temperature, substrate concentration, other chemical, physical and biological aspects all through the digester. Mixing also prevents from the formation of surface scum layers and the sludge deposition on the bottom of the tank (Appels et al., 2008). The rise of gas bubbles and the thermal convection currents caused by the addition of heated sludge, results in some degree of natural mixing in the digestion tank. However, this is not adequate for an optimum performance and auxiliary mixing is required. There are some methods of auxiliary mixing including external pumped recirculation, internal mechanical mixing and internal gas mixing as it is shown in Fig. 3. 
a
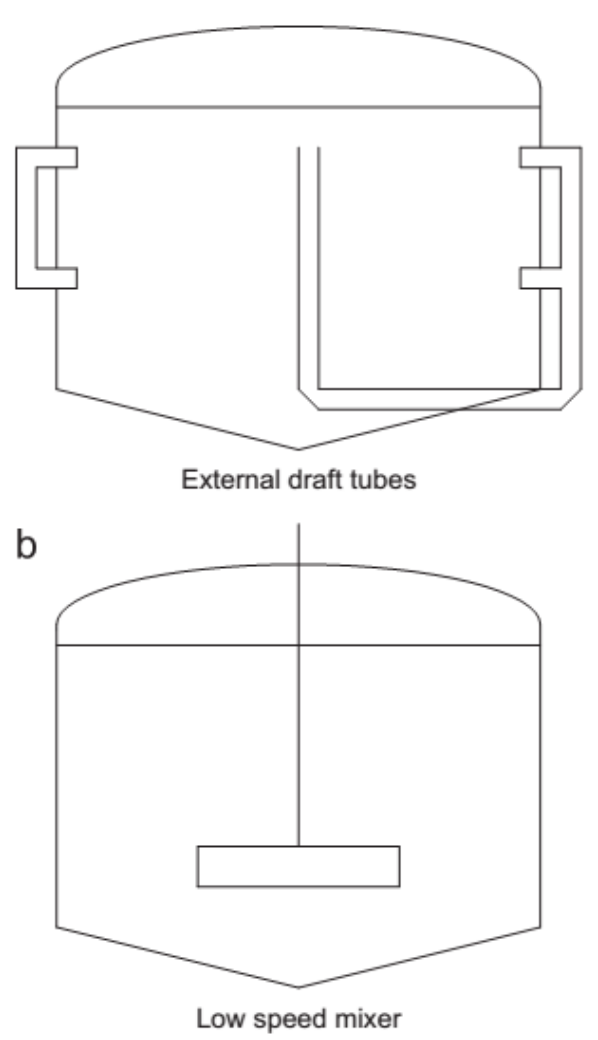

C
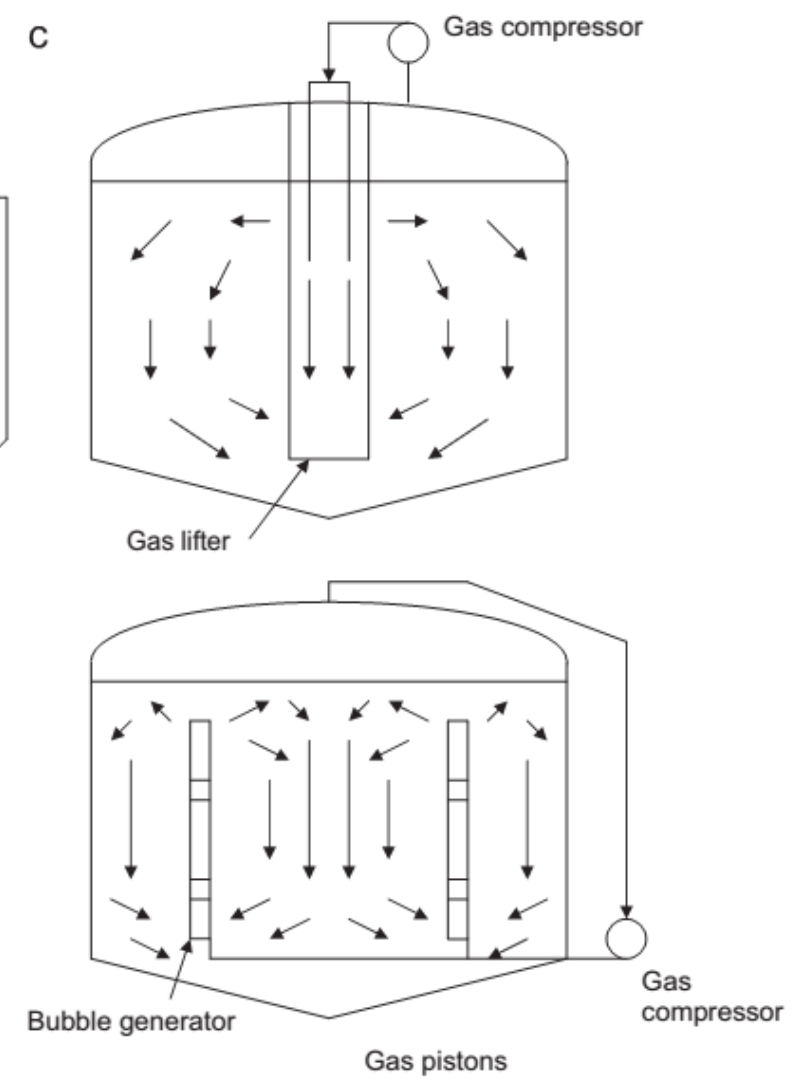

Figure 2.3. Types of mixing methods for digesters (a) external, pumped recirculation mixing, (b) internal mechanical mixing, and (c) external gas recirculation mixing (Appels et al., 2008).

\subsection{Anaerobic Co-Digestion (AnCoD)}

Anaerobic Co-digestion (AnCoD) involves the simultaneous anaerobic digestion of two or more organic waste feedstocks. The anaerobic co-digestion can be referred to as the simultaneous treatment of two or more organic biodegradable waste streams by anaerobic digestion. It provides a proper method of disposal for the organic fraction of solid waste which comes from source or from separate collection systems. This method of treatment, makes it possible to use the existing anaerobic reactors in wastewater treatment plants, with minor modifications and some additional requirements. By combining the treatments of two problematic wastes including for instance organic part of municipal solid waste and paper pulp sludge, higher yield in the production of biogas can be attained. Conventionally, anaerobic digestion was a single substrate and single purpose treatment. Recently, it has been indicated that when an increased variety of substrates 
applied at the same time, more stable AD is achieved. The most common state is when a major amount of a main basic substrate for example manure or sewage sludge is mixed and digested accompanied by minor amounts of a single, or a variety of additional substrate. The usage of cosubstrates usually improves the biogas yields from anaerobic digester due to positive interaction established in the digestion medium and the supply of missing nutrients by the co-substrates (Alvarez et al., 2008, Kangle et al, 2012). Anaerobic co-digestion offers several benefits including: improved nutrient balance and digestion, possible gate fees for waste treatment, additional biogas collection, and additional fertilizer i.e. soil conditioner (Elbeshbishy and Nakhla, 2012; Viotti et al., 2004).

Substrates with high C:N ratio have the poor buffering capacity and produce excessive amounts of VFAs during the fermentation. In contrast, substrates characterized by low $\mathrm{C}: \mathrm{N}$ ratio have high buffer capacity and the increased concentration of ammonia in the fermentation process leads to microbial growth inhibition. Anaerobic co-digestion ( $\mathrm{AnCoD})$, which entails the simultaneous digestion of two or more feedstocks has shown to be beneficial for its economic viability and increasing methane yields (Gelegenis et al., 2007; Karagiannidis and Perkoulidis, 2009; Kwietniewska and Tys, 2014). These problems have made AnCoD of multi feedstock to become a hot research area in the enhancement of conventional $\mathrm{AD}$ technology. The publications on AnCoD significantly increased within the last fifteen years indicating its capability for improving biogas production (Hagos et al., 2017). The main goal of anaerobic co-digestion is to increase biogas mainly biomethane for heat and electricity. As shown in Figure 2, a range of feedstocks can be co-digested at suitable blend ratio to maintain optimum condition required for metabolic activity and improved biogas production for thermal energy and power generation. Anaerobic co-digestion has shown to be a viable option to alleviate the drawbacks of mono-digestion while enhancing economic feasibility of the existing AD plants by increasing methane yields (Bayr et al., 2014; Mata-Alvarez et al., 2014, 2000b; Shah et al., 2015),(Mata-Alvarez et al., 2000a). 


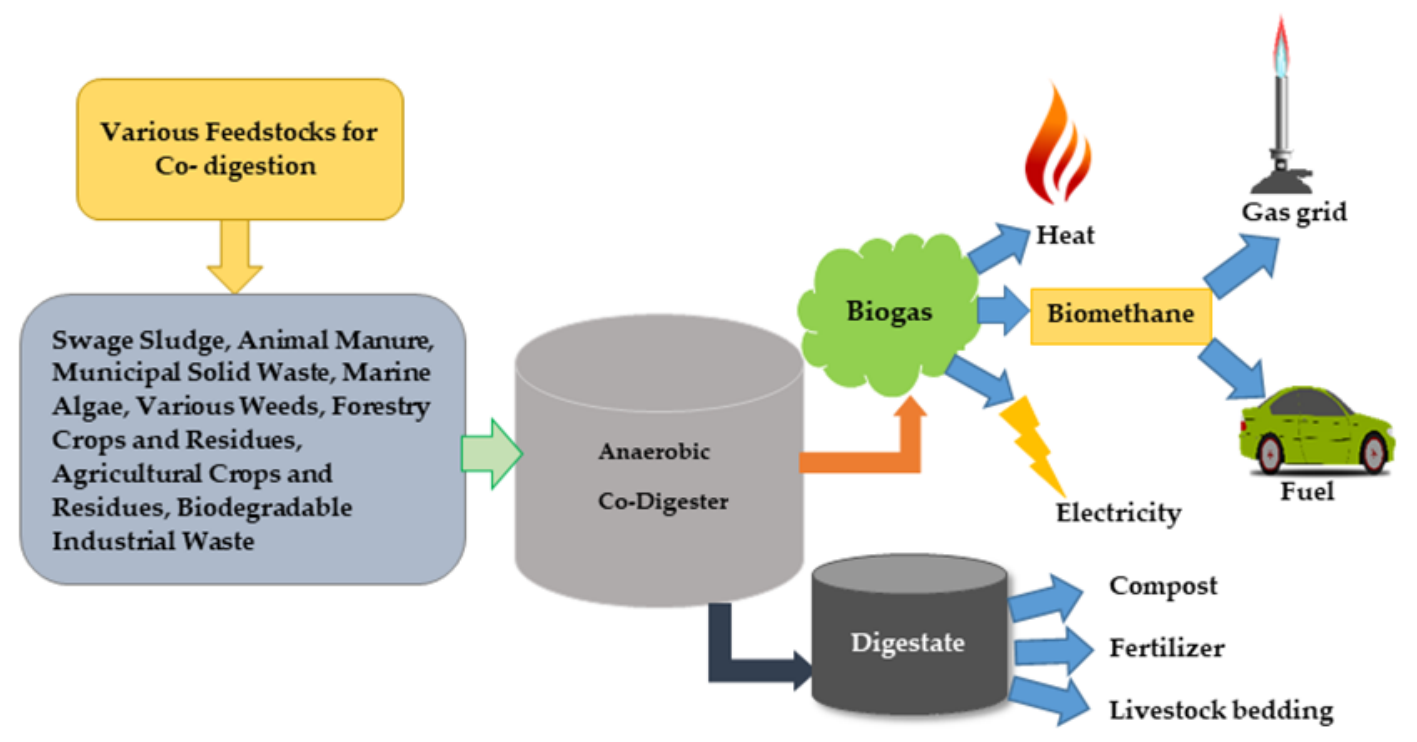

Figure 2.4. Co- digestion of multi feedstocks for waste reduction and energy recovery

Co-digestion can increase biogas production from $25 \%$ to $400 \%$ compared to the mono-digestion of the same substrates. Feedstocks characterized by higher $\mathrm{C}: \mathrm{N}$ ratio $(>50)$ such as rice and wheat straws, corn stalks, seaweed and algae can be co-digested by the feedstocks of lower C:N ratio for instance pig manure, poultry manure, food and kitchen wastes to achieve nutrient balance and to avoid the inhibitions which leads to system instability and reduced biogas production as a result of unsuited C:N ratio (Hagos et al., 2017; R et al., 2017; Sosnowski et al., 2003). Table 2.1 shows the possible feedstocks for co-digestion with regard to $\mathrm{C}: \mathrm{N}$ ratio.

Various advantages of AnCoD systems are presented in Figure 3. Multiple aspects are considered when applying AnCoD. Cost of transporting the co substrate from the generation point to the $\mathrm{AD}$ plant seems to be the primary consideration, the selection of the best co-substrate and blend ratio in order to enhance synergism, dilute disruptive compounds, optimization of methane production and digestate quality, are also important consideration that treatment plants need to evaluate when using the AnCoD (Divya et al., 2015; Mata-Alvarez et al., 2014). Operational and environmental factors as mentioned previously are factors that influence methane yield in anaerobic co-digestion of multi feedstocks (Long et al., 2012). 
Table 2.1. Potential feedstocks for co-digestion to balance nutrient with regard to C/N ratio [(Hagos et al., 2017),(R et al., 2017)-(Sosnowski et al., 2003)]

\begin{tabular}{|c|c|c|c|c|c|}
\hline $\begin{array}{l}\text { Feedstocks with } \max \\
\mathrm{C} / \mathrm{N} \text { ratio }<20\end{array}$ & $\mathrm{C} / \mathrm{N}$ ratio & $\begin{array}{l}\text { Feedstocks with } \max \\
\mathrm{C} / \mathrm{N} \text { ratio } \leq 40\end{array}$ & $\mathrm{C} / \mathrm{N}$ ratio & $\begin{array}{l}\text { Feedstocks with } \mathrm{C} / \mathrm{N} \\
\text { ratio around or }>50\end{array}$ & $\mathrm{C} / \mathrm{N}$ ratio \\
\hline TWAS $^{1}$ & $6-9$ & OFMSW $^{3}$ & 24 & Potatoes & $35-60$ \\
\hline $\mathrm{CSW}^{2}$ & 11 & Cow dung & $16-25$ & Oat straw & $48-50$ \\
\hline Poultry manure & $5-15$ & Horse manure & $20-25$ & Corn stalks/straw & $50-56$ \\
\hline Pig manure & $6-14$ & Kitchen Waste & $25-29$ & Fallen leaves & $50-53$ \\
\hline Goat manure & $10-17$ & Peanut shoots/ hulls & $20-31$ & Rice straw & $51-67$ \\
\hline $\begin{array}{l}\text { Grass/Grass } \\
\text { trimmings }\end{array}$ & $12-16$ & Slaughterhouse waste & $22-37$ & Seaweed & $70-79$ \\
\hline Alfalfa & $12-17$ & Mixed food waste & $15-32$ & Algae & $75-100$ \\
\hline \multirow[t]{3}{*}{ Food Waste } & $3-17$ & Waste cereal & $16-40$ & Sugar cane/ bagasse & $140-150$ \\
\hline & & Sugar beet/ Sugar foliage & $35-40$ & Sawdust & $200-500$ \\
\hline & & Waste cereals & $16-40$ & & \\
\hline
\end{tabular}

${ }^{1}$ Thickened Waste Activated Sludge,

${ }^{2}$ Caned Seafood Waste,

${ }^{3}$ Organic Fraction of Municipal Solid Wastes

However, for the implementation of $\mathrm{AnCoD}$, other than aforementioned factors that govern overall $\mathrm{AD}$ process, additional factors including the selection of co-substrates and their mixing ratio should be taken into consideration. For instance, mixing materials of high and low C:N ratios, such as organic solid waste mixed with animal manure or sewage can help achieve optimum C:N ratios (Akyol et al., 2016). In order to attain an improved co-digestion process, some precautions and suitable procedures are necessary. There may be requirements for supplementary digester equipment depending on the size of the operation, quality of waste, and characteristics of the wastes to be co-digested. Mainly, precautions or supplementary equipment would be required for: homogenization and mixing of co-substrates, delivery of the waste, prevention of excessive foaming and scum layer formation, and removal of sediments from the digester. 


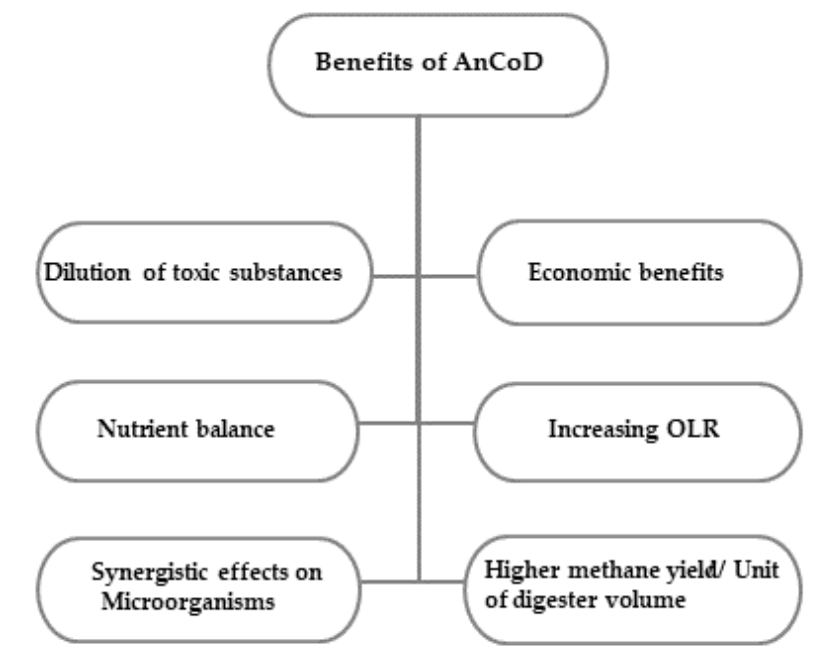

Figure 2.5. Advantages of anaerobic co-digestion systems

Furthermore, proper monitoring parameters should be determined to control and regulate the AnCoD digesters to help maintain an efficient performance when it is under operation. Applying suitable monitoring and control procedures when running the AnCoD process, allows for utilizing the full capacity of the system without overload risks. Monitoring can be performed by measuring indirectly the activity of different groups of organisms for example by measuring the rate of gas production, or the accumulation of intermediates of anaerobic degradation which reflect the existing metabolic status of the active organisms in the system (Gujer and Zehnder, 1983).

Several recommendations in the literature has been proposed specifying what control parameters should be chosen to be measured for this purpose. Some of the more common ones include $\mathrm{pH}$, alkalinity, volatile fatty acids (VFA), gas production rate and the amounts of hydrogen, methane and carbon dioxide in the gas (Ahring et al., 1995; Moletta et al., 1994).

Partial alkalinity (PA) has been considered as a reliable monitoring parameter (Jenkins et al., 1991; Rozzi et al., 1994; Supaphol et al., 2011; Wilcox et al., 1995). The applicability of pH as a process indicator was reported to be intensely dependent on the buffering capacity making it an unreliable monitoring parameter (Ahring et al., 1995). It is expected that the selection and applicability of a specific parameter could not be generalized depending on the individual process configuration and the waste characteristics. For a full-scale municipal system, co-digested excess sludge from the municipal wastewater treatment plant with carbohydrate-rich food processing waste, different parameters were assessed for monitoring and control of the system performance (Björnsson et al., 
2000). Those parameters included the volume of gas produced, $\mathrm{pH}$, VFA, and alkalinity. In addition, gas composition and the degradation of organic matter were also measured at steady state and during process changes. Both full-scale and lab-scale experiments were carried out by that research to evaluate the suitability of those parameters. The digester was run below maximum capacity in order to avoid overload. The only operational limit set for the plant, was that the $\mathrm{pH}$ should not have been below 6.8. So, the $\mathrm{pH}$ was compared with alkalinity, VFA concentration, gas production rate, and the gas composition. Alkalinity was measured as partial alkalinity (PA). OLR changes were monitored both in the full-scale digester and in the lab-scale models (Björnsson et al., 2000).

As indicated by the results of Björnsson et al's study, the load's fluctuations were reflected in the $\mathrm{pH}$, PA, and VFAs concentration. At overload condition, all the three parameters clearly demonstrated the process imbalance. The VFA concentrations proved to be a better indicator for an overload of the microbial system, although alkalinity and $\mathrm{pH}$ showed to be good monitoring parameters as well. The results indicated that gas-phase parameters demonstrated a slow response to load changes. The response of gas production and gas composition was behind and significant change occurred only after severe overload. Prior studies had observed that change in the gas phase parameters only takes place after well-developed imbalance. For that reason, the gas-phase concentration would not always reflect the actual concentration in the liquid, caused by limitations in liquid-to-gas mass transfer (Frigon and Guiot, 1995; Pauss et al., 1990).

At higher OLR, the process showed to be more sensitive to system disturbances. The changes in VFA concentration were not accurately reflected in $\mathrm{pH}$. The increased amounts of VFA were demonstrated in a lower $\mathrm{pH}$, because of the low buffering capacity of the process. Nevertheless, the $\mathrm{pH}$ was not presented as a reliable means of process monitoring, because of possible variation in buffering capacity as a result of variations in substrate composition. Therefore, a process imbalance causing significant accumulation of VFAs, could be unseen by this buffering effect. Therefore, relying on $\mathrm{pH}$ measurements for the process monitoring was not advised and the usage of $\mathrm{pH}$ measurements together with measurements of the PA or VFA was suggested by authors (Björnsson et al., 2000).

Different studies have been carried out to evaluate the impact of various effective factors on AnCoD processes. The main aim of the studies was to assess the influence of those parameters on biogas yield, biogas composition including biomethane or biohydrogen content. However, no 
comprehensive guidelines have been compiled so far to standardize the AnCoD systems. This would be firstly due to the complexity of the process caused by the variety of co-substrates and wastes composition, and secondly because it has not been broadly implemented in full scales.

A study for IEA Bioenergy has presented most important control parameters which regulate anaerobic co-digesters. The control parameters included the overall daily substrate flow $\left(\mathrm{m}^{3} / \mathrm{d}\right.$ or tones/d) and the amount of biogas produced daily $\left(\mathrm{m}^{3} / \mathrm{d}\right)$. Based on the results obtained by this study, for appropriate control of the process, the determination of the $\mathrm{CH}_{4}$ concentration was highly advised. In addition, a periodic calculation of the biogas yield referred to as daily biogas amount divided by daily substrate flow was recommended since it provides the efficiency of the digestion process. The analysis of the ammonia and the volatile fatty acids concentration were also suggested for large-scale operations. In addition, identifying the influences of co-substrates on the digester behavior was recommended. Particularly, detecting the formation of scum layers and bottom sediments was specified. It was suggested to maintain the record of the type and amount of separated contaminations in co-digestion. In the cases that sterilization is also involved, monitoring the type and the amount of waste streams and the treatment conditions such as time and temperature were also considered to be of necessary control parameters. The sampling frequency and methods for analysis required for quality assurance of the end product digestate or compost were also comprehensively defined in that study (Kim et al., 2006).

Although co-digestion of feedstocks such as poultry manure and kitchen waste with low $\mathrm{C}: \mathrm{N}$ ratio with those of higher $\mathrm{C}: \mathrm{N}$ ratio such as agricultural waste including rice and wheat straw is a solution to adjust its ratio at the optimum level, the existence of lignocellulosic material in the agricultural waste cause limitation during $\mathrm{AnCoD}$ as a result of long retention time and low biodegradability (Saha et al., 2011a).

Such problems still may demand for pretreatment techniques in order to speed up the hydrolysis which is the rate-limiting step in the anaerobic digestion process. The main purpose of the pretreatment is to increase the solublization by the breakdown of the complex substrates such as lignin in lingocellulosic feedstocks or tough cell wall in seaweed biomass, in order to accelerate the hydrolysis rate (Elbeshbishy et al., 2011; Giordano, 2012; Noike et al., 1985).

C. Rodriguez et al, 2017 studied the effect of using co-substrate on methane production in codigestion of waste paper (WP) with microalgal biomass (MA). Their study (Rodriguez et al., 2017) was carried out in batch mode and was intended to investigate the influence of the feedstocks 
mixing ratio (WP:MA) as well as feedstock to inoculum (F:I) ratio. They achieved the highest methane yield of $608 \mathrm{ml} \mathrm{CH}_{4} / \mathrm{gVS}$ at the F/I and WP:MA ratios of 0.2 and 50:50 respectively. At this mixing ratio of the feedstocks, the obtained methane yield was more than that of the feedstock's mono-digestion. The maximum $49.58 \%$ increase of the methane yield occurred at the same co-digestion ratio of 50:50 and F:I ratio of 0.4. Their study verified the synergetic effect at the feedstocks mixing ratio of 50:50 and all F:I ratios of 0.2, 0.3, and 0.4.

Pretreatment has proved successful at increasing the methane yield of numerous strains of microalgae in the digestion process. Most species of microalgae reduce the digestion rate due to their tough cell wall consisting of slowly biodegradable material (Uggetti et al., 2017).

With the increasing attention to anaerobic co-digestion, several researches have been allotted for co-digestion of various feedstocks and pre-treatment techniques from mechanical particle size reduction, thermal, chemical and ultrasonic treatment to enzymatic degradation and so on. For instance, mechanical pretreatment with Hollander beater in co-digestion of seaweed biomass with digester sludge increased biogas production by $20 \%$ at the ratio of $2: 3$ of algal pulp to sludge per reactor for $10 \mathrm{~min}$ beating time (Tedesco et al., 2013).

\subsubsection{Microbial Diversity and Synergy in AnCoD}

The selection of sludge inocula plays an important role in the effectiveness of biological anaerobic treatment of organic wastes. The analysis of microbial community dynamics has revealed that various waste streams and environmental factors can affect microbial community dynamics in an anaerobic co-digestion processes (Lin et al., 2012; Supaphol et al., 2011). Reportedly, mesophilic anaerobic co-digestion of mixed wastes allows for a better variety of substrates which in turn supports a wider diversity of bacteria and archaea. More diverse resource input results in more diverse communities and greater metabolic activity (Ike et al., 2010; Supaphol et al., 2011). However, there is limited awareness about the microbial consortia in anaerobic co-digestion process due to the lack of metabolic data on the microorganisms involved in the process. A comprehensive understanding of the microbial community is hindered by limitations of conventional molecular technology approaches that are restricted in terms of detecting sophisticated microbial diversity in the environment. Attempts for the analysis of the 16S rRNA gene sequencing have been carried out as an alternative to conventional culture techniques. This

method is used to identify and compare microorganisms present within a given sample and it is a 
well-established method for studying complex microbial community or environments that are difficult or impossible to study. The method of $16 \mathrm{~S}$ rRNA gene-based fingerprints could provide less biased and higher coverage information and can support many unknown details about the mechanism of microbial response to the digester enhancement. An improved understanding of the function and the metabolic role of microorganisms in the anaerobic co-digestion of various pollutants can be obtained b by the molecular inventories (Rivière et al., 2009; Zhang et al., 2011). Some of the results of these studies are presented in Table 2.2.

Organic matter in $\mathrm{AD}$ process, is decomposed synergistically by a bacterial consortium producing biogas including biomethane (Chandra et al., 2012; Liew et al., 2012). The process involves at least three functional groups of microorganisms that mainly regulate the mutual metabolic interactions under anaerobic conditions. The first microbial community hydrolyzes complex polymeric substances such as lipids, cellulose and protein to fundamental structural building blocks like glucose and amino acids. Subsequently, fermentation of these products to fatty acids, acetate and hydrogen is proceeded by the second community.

Among degradation processes involved in anaerobic digestion, the acidogenesis process has been shown to be the most important step. The third community develops methanogenesis process through which acetate and hydrogen are converted to methane and carbon dioxide. Therefore, microbial communities are vital to a stable and efficient transformation of organic matter to biogas (Zhang et al., 2011). General metabolism of microbial consortia involves extracellular polymeric substances (EPS) of sludge aggregates. It has been indicated that EPS is partly the result of the microbial metabolism that is affected by the microbial community structure and its activity. Growth conditions control the quantity of EPS which in turn affect the anaerobic digestibility and biogas production. It is not yet clear that how different microorganisms contribute to EPS secretion. A comparative study on the pathways of substrates degradation and the by-products of EPS sub-fractions could provide supplementary data on long-term impacts of microbial activity on anaerobic co-digestion reactors (Sheng et al., 2010; Yu et al., 2012). Monitoring qualitative and quantitative changes in a bacterial community structure, allows for the evaluation of the influence of the co-substrate on bacteria contributing to the biogas production.

However, there is not enough literature on this topic. Some attempts have been made to study microbial community structure and its influence on anaerobic co-digestion processes. Such studies as presented in Table 2.2 have been aimed to increase methane production by co-digestion of 
different organic-rich waste streams and they have been mostly developed with a view to the influencing parameters such as mixing ratio, organic loading rate (OLR), and carbon to nitrogen $(\mathrm{C}: \mathrm{N})$ ratio on the population of methanogenic archaea species (Benn and Zitomer, 2018; Gaby et al., 2017; A. J. Li et al., 2011).

It is reported that an even distribution of hydrogenotrophic and acetotrophic methanogen populations in a reactor is indicative of a stable operating condition (Demirel and Scherer, 2008; Kim et al., 2014; Yang et al., 2016). Yang et al. studied the performance of a co-digester for the treatment of sewage sludge with fat, oil and grease (FOG) using a mesophilic semi-continuous reactor and compared it to that of a mono-digester receiving only sewage sludge. It was indicated that the secretion of EPS increased by $40 \%$ in comparison with the mono-digester and that the improvement in co-digestion performance was stimulated due to the release of EPS. The analysis of microbial 16S rRNA gene showed the dynamic change of microbial community through the process. Both bacterial and archaeal community went through a progress with FOG addition, and a large amount of consortia such as Methanosaeta and N09 were involved in the process. As compared to sewer sludge mono-digestion, biogas production and TS removal efficiencies increased up to $35 \%$ and $26 \%$, respectively. It was shown that FOG addition resulted in nutrition balance and regulating microbial composition. Also, metabolic activities were stimulated, and more EPS were obtained with the progressive addition of FOG.

Jihen et al. investigated microbial community's structures in anaerobic co-digestion of dairy wastewater and cattle manure. A maximum volatile solids (VS) reduction of $88.6 \%$ and biogas production of $0.87 \mathrm{~L} / \mathrm{g}$ VS removed were obtained through their research corresponding to the C:N ratio of 24.7 at HRT of 20 days. The bacterial profile analysis showed a large quantity of Uncultured Bacteroidetes, Firmicutes and Synergistetes bacterium.

The Syntrophomonas strains associated with $\mathrm{H}_{2}$-using bacteria comprising Methanospirillum sp., Methanosphaera sp., and Methanobacterium formicicum were observed as well. These syntrophic associations are necessary in anaerobic digestion reactors allowing for maintaining a low hydrogen partial pressure. On the other hand, high concentrations of volatile fatty acids (VFAs) resulted from dairy wastes acidogenesis allowed the growth of Methanosarcina species. It was indicated that high concentrations of VFAs would result preferentially in the growth of the acetoclastic Methanosarcina species (Jihen et al., 2015). 
Table 2.2. Microbial consortia diversity in various AnCoD systems for biogas and methane improvement

\begin{tabular}{|c|c|c|c|c|}
\hline Feedstocks & Microbial Consortia & Digester mode & HRT & $\begin{array}{l}\text { Methane / Biogas } \\
\text { increase \% }\end{array}$ \\
\hline $\begin{array}{l}\text { Fruit vegetable } \\
\text { waste +Food waste } \\
(1: 1)\end{array}$ & $\begin{array}{l}\text { Methanoculleus, } \\
\text { Methanosaeta, } \\
\text { Methanosarcina }\end{array}$ & $\begin{array}{l}\text { CSTR } \\
\text { (mesophilic) }\end{array}$ & $\mathrm{NA}^{1}$ & $0.49 \mathrm{~m}^{3} \mathrm{CH}_{4} / \mathrm{kg} \mathrm{VS}$ \\
\hline \multirow{2}{*}{$\begin{array}{l}\text { Food, fruit, } \\
\text { vegetable + night } \\
\text { soil waste }\end{array}$} & \multirow{2}{*}{$\begin{array}{l}\text { Methanosaeta } \\
\text { (predominant methanogen) } \\
+ \text { hydrogenotrophs }\end{array}$} & \multirow[t]{2}{*}{$\begin{array}{l}\text { Full scale wet fed- } \\
\text { batch }\end{array}$} & $18-20 \mathrm{~d}$ & \multirow[t]{2}{*}{ NA } \\
\hline & & & C:N 8.6 & \\
\hline $\begin{array}{l}\text { Cow manure }+ \\
\text { grass silage }\end{array}$ & $\begin{array}{l}\text { Clostridia, unclassified } \\
\text { Bacteria, Bacteroidets }\end{array}$ & CSTR mesophilic & $20 \mathrm{~d}$ & NA \\
\hline $\begin{array}{l}\text { Cow manure + oat } \\
\text { straw }\end{array}$ & $\begin{array}{l}\text { Clostridia, unclassified } \\
\text { Bacteria, Bacteroidets, } \\
\text { Deltaproteobacteria }\end{array}$ & $\begin{array}{l}\text { CSTR } \\
\text { (mesophilic) }\end{array}$ & $20 \mathrm{~d}$ & NA \\
\hline $\begin{array}{l}\text { Cow manure }+ \\
\text { sugar beet tops }\end{array}$ & $\begin{array}{l}\text { unclassified } \\
\text { Bacteria,Clostridia, } \\
\text { Bacteroidets, Bacilli }\end{array}$ & $\begin{array}{l}\text { CSTR } \\
\text { (mesophilic) }\end{array}$ & $20 \mathrm{~d}$ & NA \\
\hline $\begin{array}{l}\text { STP-OGW + SC- } \\
\text { OFMSW (1:6) }\end{array}$ & $\begin{array}{l}\text { Methanobacterium, } \\
\text { Methanoculleus, } \\
\text { Methanothermobacter } \\
\text { uncultured archaea }\end{array}$ & $\begin{array}{l}\text { Batch } \\
\text { (thermophilic) }\end{array}$ & $14.4 \mathrm{~d}$ & $\begin{array}{l}52 \% \text { biogas and } \\
36 \% \text { methane } \\
\text { increase }\end{array}$ \\
\hline $\begin{array}{l}\text { biodiesel waste } \\
\text { glycerin + } \\
\text { municipal waste } \\
\text { sludge }(1.35: 0.65)\end{array}$ & $\begin{array}{l}\text { Dominated } \\
\text { by:Methanosaeta and } \\
\text { Methanomicrobium }\end{array}$ & $\begin{array}{l}\text { Two-stage CSTR } \\
\text { (mesophilic) }\end{array}$ & $20 \mathrm{~d}$ & $\begin{array}{l}100 \% \text { biogas and } \\
120 \% \text { methane } \\
\text { increase }\end{array}$ \\
\hline $\begin{array}{l}\text { Sewage sludge } \\
\text { +FOG }\end{array}$ & $\begin{array}{l}\text { Dominantly Methanosaeta, } \\
\text { and N09 }\end{array}$ & $\begin{array}{l}\text { Semi-continuous } \\
\text { (mesophilic) }\end{array}$ & $15 \mathrm{~d}$ & $\begin{array}{l}35 \% \text { biogas } \\
\text { increase }\end{array}$ \\
\hline $\begin{array}{l}\text { Dairy wastewater } \\
\text { + Cattle manure }\end{array}$ & $\begin{array}{l}\text { Uncultured Bacteroidetes, } \\
\text { Firmicutes, Synergistetes, } \\
\text { Syntrophomonas strains } \\
\text { Methanosarcina species }\end{array}$ & $\begin{array}{l}\text { ASBR } \\
\text { (mesophilic) }\end{array}$ & $\begin{array}{c}20 \mathrm{~d} \\
(\mathrm{C}: \mathrm{N} 24.7)\end{array}$ & $\begin{array}{l}\text { biogas produced: } \\
0.87 \mathrm{~L} / \mathrm{g} \text { VS } \\
\text { removed }\end{array}$ \\
\hline $\begin{array}{l}\text { Food wastewater } \\
\text { +WAS (3:1) }\end{array}$ & $\begin{array}{l}\text { Dominated by: } \\
\text { Methanothermobacter and } \\
\text { Methanosarcina }\end{array}$ & $\begin{array}{l}\text { CSTR } \\
\text { (thermophilic) }\end{array}$ & $20 \mathrm{~d}$ & $\begin{array}{l}\text { Max biogas: } 316.11 \\
\mathrm{~mL} \mathrm{CH}_{4} / \mathrm{g} \mathrm{COD} \\
\text { removed }\end{array}$ \\
\hline
\end{tabular}

${ }^{1} \overline{\text { Not available }}$

Former studies verify that the AnCoD of cellulosic materials such as grass silage, oat straw, and sugar beet tops to methane to be mediated by bacteria and methanogenic archaea. The polymers which are hydrolyzed into soluble compounds under fermentative condition, are converted to acetate and one-carbon constituents by acidogens and acetogens and these intermediates in turn 
can be transformed directly by methanogenic archaea into methane and carbon dioxide (Nopharatana et al., 1998; Veeken and Hamelers, 1999).

Anaerobic digestion process of cellulosic material including grass silage, oat straw and sugar beet tops, is a multistep process mediated by Bacteria and methanogenic Archaea to produce methane. The process involves the hydrolysis of polymers into soluble compounds under fermentative condition. Subsequently, Acidogenic and acetogenic bacteria convert these soluble intermediates to acetate and mono-carbon compounds. These compounds subsequently can be converted to methane and carbon dioxide by methanogenic Archaea. It was found that in anaerobic digestion of cellulytic feedstocks, significant cellulolytic competences occurs due to the existence of species belonging to the order Clostridiales (Lynd et al., 2002).

Martín-González et al. found that thermophilic conditions was superior to mesophilic conditions for the enhancement of $\mathrm{AnCoD}$ along with the use of sewage treatment plant fat, oil and grease wastes (STP-FOGW) as co-substrates in co-digestion with organic fraction of municipal waste (OFMSW). Monitoring of microbial structure demonstrated that the bacterial profiles clustered in two separate groups, before and after the extended contact with STP-FOGW, whereas, archaeal community structure remained relatively constant throughout the operation. Bacterial population structure showed a dynamic change determined to be due to introducing FOG residues to the reactor.

\subsubsection{Effect of Digester Mode on AnCoD}

In general, anaerobic digesters can be configured as one-stage, two-stage, or multi-stage reactors in which the hydrolysis/acidogenesis and acetogenesis/methanogenesis steps occur in either the same or separated digesters (Figure 4). Separating the digesters makes the process easier to control, and makes it possible to separately optimize the operational and environmental conditions for hydrolysis/acidogenesis and methanogenesis processes in order to enhance the overall reaction rate and biogas yield (Lalman and Bagley, 2002; Nathao et al., 2013).

Fluctuations in organic loading rate, heterogeneity of wastes, and or the presence of excessive inhibitors can lead to instability of the process, and multi-stage systems have shown to be more stable as compared to single-stage ones. Two or multi-stage systems allow for the selection and enrichment of different types of microorganisms in each digester which results in extending the possibility of processing different biomass constituents, improving substrate conversion, enhancing the chemical oxygen demand (COD) reduction, and increasing energy recovery. 
Although, multi-stage digesters are associated with greater construction and maintenance costs, multi-stage digesters provide higher performances as compared to single-stage systems (Azbar and Speece, 2001; Cuetos et al., 2007). Using two-stage digestion, controlled acidogenesis in the first digester, helps maintaining a high soluble feed to the second stage which subsequently enhances the biogas production (Dareioti and Kornaros, 2015).

In the two-stage anaerobic digestion systems, acid fermentation and methanogenesis are separated in two reactors in order to optimize reactor conditions for the different groups of microorganisms. The acidogenic stage is typically operated at a low HRT in the range of 2 to 3 days and a $\mathrm{pH}$ between 5 and 6. While the second stage, methanogenesis, is typically operated with a HRT of 20 to 30 days and a pH between 6 and 8 facilitating the development of slow-growing methanogenic archaea.

Moreover, acidogenesis phase allows for long chain fatty acids (LCFAs) saturation and degradation (Kangle et al., 2012; Kim et al., 2004). Owing to the bent molecular structure of unsaturated LCFAs because of the existence of double bonds, they have a greater cover area of cell wall per molecule as compared to saturated LCFAs. As a result, unsaturated LCFAs have demonstrated stronger inhibitory effects in comparison with saturated LCFAs (Demeyer and Henderickx, 1967; Thies et al., 1994). As such transformation of unsaturated to saturated LCFAs is beneficial. In addition, LCFA saturation would be necessary for the oxidative breakdown of fatty acid molecules and formation of acetic acid (Hanaki et al., 1981; Lalman and Bagley, 2002). The outcome of a study using a two-stage AD system treating a synthetic fat-containing wastewater comprised of glucose and LCFAs mixture revealed that, 19\% of LCFAs were degraded and $12 \%$ of unsaturated LCFAs were saturated in the acidogenic phase (Kim et al., 2004). Acidogenic phase also can convert the unsaturated LCFAs to palmitic acid which reduces the lipid inhibition of methanogenesis in the second stage (Beccari et al., 1998). Food waste, which composes a large portion of OFMSW, contains a substantial amount of organic soluble compounds which can be simply converted to VFA.

Therefore, it can be an ideal substrate for biogas production. Nevertheless, formation of excessive amount of VFA at initial digestion stages can result in a remarkable $\mathrm{pH}$ reduction and subsequently methanogenesis inhibition. Utilization of two-stage anaerobic digestion systems for food waste has shown to be an effective solution for the $\mathrm{pH}$ inhibition of one stage systems (Dinsdale et al., 2000; Kinnunen et al., 2015; Klocke et al., 2008; Y. Li et al., 2011; Shen et al., 2013; Shin et al., 
2010). Most of the studies on anaerobic co-digestion process have aimed to evaluate the digesters performance and optimal operating conditions for a particular type of waste.

Lafitte-Trouque et al. found two-stage thermophilic/mesophilic AnCoD systems to be effective for the co-digestion of sewage sludge and confectionery waste. The system with the second digester operating at a HRT of 12 days provided the best performance in terms of stability, VS reductions, and specific methane yield corresponding to an average $82 \%$ methane in the gas composition. However, a HRT of 8 days in the second stage digester was not able to assimilate high concentrations of volatile acid and low $\mathrm{pH}$ from the first digester. This was related to the insufficient retention time for maintaining a substantial methanogenic population. In a single-stage digester, a HRT of less than 20 days may cause methanogens to be washed out of the digester. Therefore, HRT is one of the important design parameters for the single-phase operations (LafitteTrouqué and Forster, 2000).

The study by Ratanatamskul et al. was conducted in pilot-scale on the development of an energy recovery system using a novel prototype single-stage anaerobic digester. Their system (Ratanatamskul et al., 2015) co-digested food waste with sewage sludge from a high-rise building for on-site biogas production.

The food waste to sewage sludge mixing ratio of $10: 1$ by weight was selected according to the result of lab experiments. Different HRTs of 27, 22, 19 days corresponding to 7.9, 10.8 and 14.0 $\mathrm{kgCOD} / \mathrm{m}^{3} \mathrm{~d}$ OLRs were applied and the optimal methane yield of $76.8 \%$ was achieved by their proposed single-stage reactor when the digester was operated at an HRT of 27 days. Although maximum biogas production occurred at the shortest HRT of 19 days. This indicated that the improvement of methane content of biogas could be attained by adequate operating HRT.

Although, the advantages of two-stage over single-stage digestion systems are addressed in the literature (Bertin et al., 2013; Shen et al., 2013; Yu et al., 2002) there exists a lack of adequate research available in terms of comparing the performances of single, two and or multi-phase codigestion systems. Kim et al. developed a two-stage system comprised of a continuously stirred tank reactor for acidogenesis and a methanogenic up-flow bed reactor for the treatment of a high lipid wastewater from a milk and ice cream factory co-digested with slaughterhouse wastewater. They obtained 1.2 times increase in the COD removal, 1.9 times increase in lipids removal, and 1.4 times increase in the methane production compared to the single phase system (Kim and Shin, 2010). 


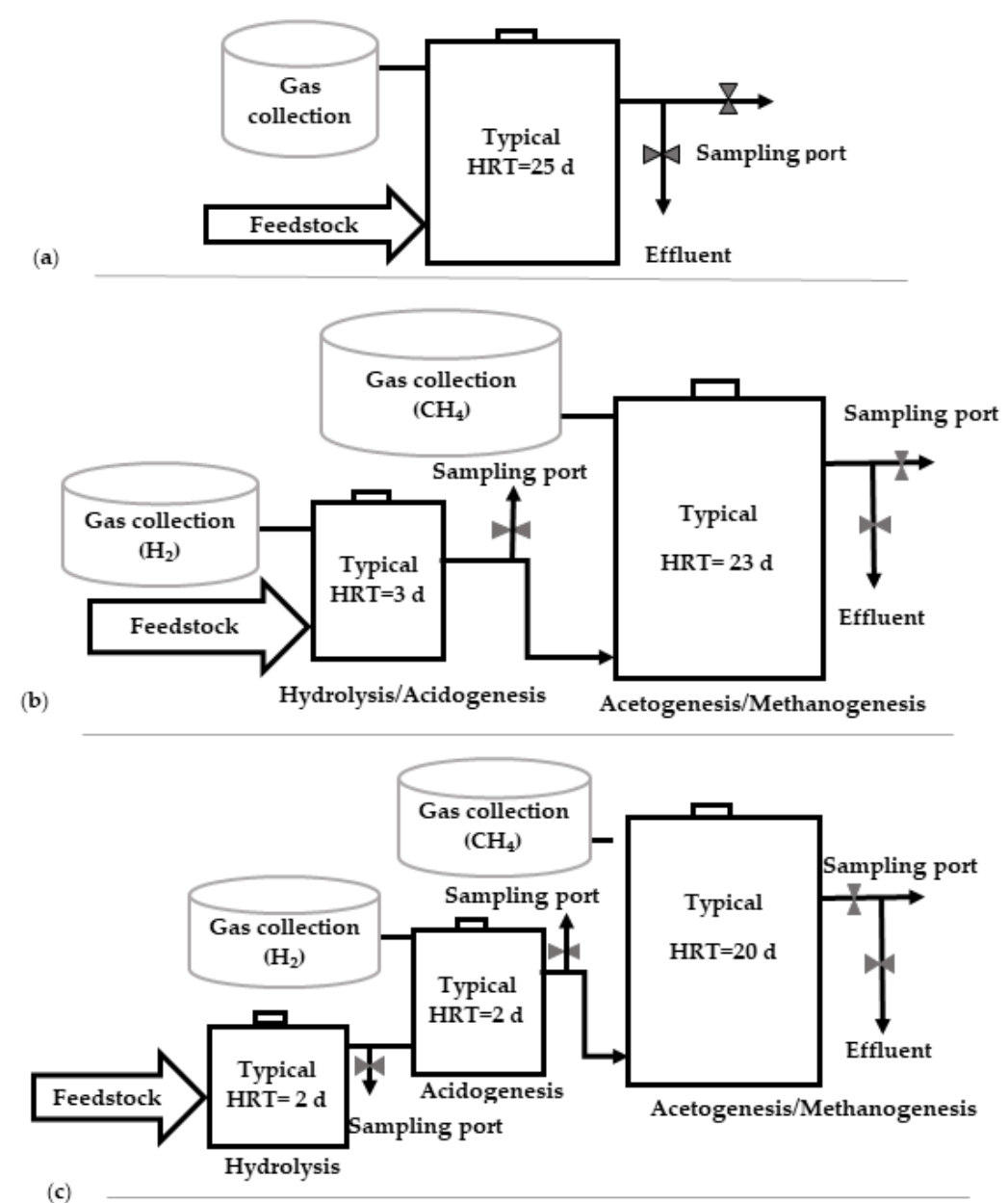

Figure 2.6. Types of digester configuration: (a) Single-stage; (b) Two-stage; and (c) Three-stage digester

Contrary to the result of that research (Kim and Shin, 2010), no significant increase in the overall energy recovery was attained by other study (Schievano et al., 2012) using a two-stage digester co-digesting swine manure and market biowaste in comparison with a single-stage one. Volumetric biogas productions were found to be 0.1 and $0.079 \mathrm{~m}^{3} / \mathrm{L}$ reactor for the single-stage and the twostage systems, respectively. Even though, the average biogas methane content of the two-stage system showed $25 \%$ increase over that of the single-stage digester. The accumulation of undegraded intermediate metabolites such as volatile fatty acids, ketones, amines, amino acids, and phenols was believed to be responsible for the reduced efficiency of the two-stage digester though. It was concluded that although the two-stage system could be capable of a higher bioenergy production, certain incompetent fermentative pathways may lead to formation of recalcitrant and toxic metabolites. 
Hidalgo et al. compared a single-phase with a two-phase reactor for the co-digestion of residues from the used vegetable oil processing industry and pig manure. The maximum methane production of $1.06 \mathrm{~m}^{3} / \mathrm{kg}$ VS removed in the single-stage digestion corresponding to a methane production of $0.69 \mathrm{~m}^{3} \mathrm{CH}_{4} / \mathrm{kg}$ VS removed $\left(65 \% \mathrm{CH}_{4}\right)$ was obtained at the end of first 50-day operational period. The average biogas productions of 0.46 and $0.33 \mathrm{~m}^{3} / \mathrm{kgVS}$ removed were observed for the second and third operational period with methane productions of $0.30(65.5 \%$ $\left.\mathrm{CH}_{4}\right)$ and $0.22\left(66 \% \mathrm{CH}_{4}\right) \mathrm{m}^{3} \mathrm{CH}_{4} / \mathrm{kg} \mathrm{VS}$ removed, respectively (Hidalgo et al., 2014).

The two-phase anaerobic digestion improved VS removal efficiencies and process stability in comparison with the single-phase reactor. Although the single-stage system produced more biogas, a higher methane content in produced biogas was obtained by the two-phase system and the latter was deemed to be more beneficial. Table 2.3 summarizes the results achieved by a number of studies on single and two-stage AnCoD systems.

The results obtained by applying a novel compact three-stage anaerobic digester in co-digestion of food waste and horse manure verified the advantages of the three-phase digester over single and two-stage ones as controls. By using three compartments in the three-stage anaerobic digester, three separated functional zones hydrolysis, acidogenesis and methanogenesis were created. This configuration significantly accelerated the solublization of solid organic matters and the formation of volatile fatty acids leading to an increase of 11 and $23 \%$ in methane yield in the two-stage and three-stage digesters in comparison with the single-stage one respectively. The analysis of $16 \mathrm{~S}$ rDNA showed that different microbial communities comprising hydrolyzing bacteria, acidogenic bacteria and methanogenic archaea were selectively enriched in the three separate reactors of the three-stage digester. It was also indicated that the abundance of the methanogenic archaea was increased by 0.8 and 1.28 times in the two-stage and the three-stage digesters compared to the single stage one correspondingly (Zhang et al., 2017b).

Despite its benefits, AnCoD implementation can be limited owing to long retention time and low biodegradability (Saha et al., 2011b). Therefore, certain techniques are required in order to overcome these obstacles. With the increasing interest in anaerobic co-digestion, a number of researches have been conducted during recent years with the purpose of improving co-digestion of various substrates. Part of those studies has been allotted for pre-treatment techniques from mechanical particle size reduction, thermal and ultrasonic treatment to enzymatic degradation and so on. The main purpose of the pre-treatment methods is to increase the solublization of the 
complex substrates by the breakdown of the complex substrates such as lignin in lingocellulosic feedstocks or tough cell wall in seaweed biomass, in order to accelerate the hydrolysis rate as hydrolysis is the limiting step in the anaerobic digestion process (Eastman and Ferguson, 1981; Esposito et al., 2012; Noike et al., 1985).

Table 2.3. Comparison of single-stage and two-stage digestion in AnCoD systems

\begin{tabular}{|c|c|c|c|c|}
\hline Digester mode & Feedstocks & $\begin{array}{l}\text { Mixing } \\
\text { ratio }\end{array}$ & HRT & Biogas / Methane content \\
\hline $\begin{array}{l}\text { Single- stage (CSTR, } \\
\text { mesophilic) }\end{array}$ & \multirow{2}{*}{$\begin{array}{l}\text { Sewage sludge } \\
+ \text { confectionery } \\
\text { waste }\end{array}$} & \multirow{2}{*}{$\mathrm{NA}^{1}$} & $20 \mathrm{~d}$ & $\begin{array}{l}\text { Methane yield: } 0.36-0.28 \mathrm{~m} 3 / \mathrm{kg} \\
\text { VS applied (76- } 82 \% \text { methane) })^{2}\end{array}$ \\
\hline $\begin{array}{l}\text { Two- stage (CSTR, } \\
\text { thermophilic/mesophilic) }\end{array}$ & & & $12 \mathrm{~d}$ & $\begin{array}{l}\text { Methane yield: } 0.3-0.34 \mathrm{~m} 3 / \mathrm{kg} \\
\text { VS applied }(66-76 \% \text { methane })^{2}\end{array}$ \\
\hline \multirow[t]{2}{*}{ Single stage (plug flow) } & \multirow[t]{2}{*}{$\begin{array}{l}\text { Food waste } \\
\text { +sewage sludge }\end{array}$} & \multirow[t]{2}{*}{$\begin{array}{l}10: 1 \\
\text { (weight) }\end{array}$} & $27 \mathrm{~d}$ & $\begin{array}{l}\text { Biogas production: } \\
1045 \pm 52.81 \mathrm{~L} / \mathrm{d}\end{array}$ \\
\hline & & & $19 \mathrm{~d}$ & $1662.58 \pm 37.32 \mathrm{~L} / \mathrm{d}$ \\
\hline Single- stage (UASB) & \multirow[b]{2}{*}{$\begin{array}{l}\text { Slaughter house } \\
+ \text { milk } \\
\text { wastewater }\end{array}$} & \multirow[b]{2}{*}{ NA } & $2.14 \mathrm{~d}$ & \multirow[b]{2}{*}{$\begin{array}{l}40 \% \text { Methane increase by two- } \\
\text { stage reactor }\end{array}$} \\
\hline Two- stage (CSTR/ UASB) & & & $2.9 \mathrm{~d}$ & \\
\hline $\begin{array}{l}\text { Single- stage (CSTR, } \\
\text { thermophilic) }\end{array}$ & \multirow{2}{*}{$\begin{array}{l}\text { Market biowaste } \\
+ \text { swine manure }\end{array}$} & \multirow{2}{*}{$\begin{array}{l}1: 4 \\
\text { (weight) }\end{array}$} & $25 \mathrm{~d}$ & $0.55 \mathrm{dm} 3 / \mathrm{L}$ digester $\mathrm{d}$ \\
\hline $\begin{array}{l}\text { Two- stage (CSTR, } \\
\text { thermophilic) }\end{array}$ & & & $3 / 22 \mathrm{~d}$ & $0.54 \mathrm{dm} 3 / \mathrm{L}$ digester $\mathrm{d}$ \\
\hline Single- stage (Batch) & \multirow{2}{*}{$\begin{array}{l}\text { Oil processing } \\
\text { wastewater }+ \\
\text { pig manure }\end{array}$} & \multirow{2}{*}{$\begin{array}{l}1: 3 \\
\text { (weight) }\end{array}$} & $20 \mathrm{~d}$ & $\begin{array}{l}\text { Average biogas: } 0.33 \mathrm{~m} 3 / \mathrm{kgVS} \\
\text { removed, }(0.66 \% \text { methane })\end{array}$ \\
\hline Two-stage (Batch) & & & $2 / 18 \mathrm{~d}$ & $\begin{array}{l}\text { Average biogas: } 0.4 \mathrm{~m} 3 / \mathrm{kgVS} \\
\text { removed, }(0.67 \% \text { methane })\end{array}$ \\
\hline Single-stage & \multirow{3}{*}{$\begin{array}{l}\text { Food waste/ } \\
\text { horse manure }\end{array}$} & \multirow{3}{*}{ NA } & $20 \mathrm{~d}$ & $\begin{array}{l}45.4 \text { L cumulative methane } \\
\text { production }\end{array}$ \\
\hline Two-stage & & & $4 / 16 \mathrm{~d}$ & 50.7 L cumulative methane \\
\hline Three-stage & & & $2 / 2 / 16$ & $\begin{array}{l}55.7 \mathrm{~L} \text { cumulative methane } \\
\text { production }\end{array}$ \\
\hline
\end{tabular}

${ }^{1}$ Not Available,

${ }^{2}$ Numbers are mean values after 70 - day period of phase 1 and phase 2 , respectively 


\subsection{Pretreatment for Improving AnCoD}

Pretreatment have been reported to improve the waste stabilization and the methane yield. However, given the additional costs of pretreatment, it mainly requires to be commercially viable (Esposito et al., 2012). Based on the existing literature, the pretreatment methods used for enhancing $\mathrm{AnCoD}$ fall into five main categories including mechanical, thermal/hydrothermal, chemical, biological, and hybrid (combined) pretreatment. As such, the different pretreatment methods that have been used for the enhancement of $\mathrm{AnCoD}$ of multi feedstocks are presented in the following sections.

\subsubsection{Mechanical pretreatment}

Mechanical pretreatment as a mean to improve the AnCoD process has been proposed by a number of researchers. The principal of the mechanical pretreatment technique is to breakdown and/or crushing the substrate particles reducing their particle size. The particle size reduction is proportional to the available specific surface area of the substrate constituents and therefore, it causes a more effective contact between the substrate and the anaerobic microorganisms during the AD process. As a result, the hydrolysis stage (as a known rate-limiting stage) is accelerated and the overall AD process is improved (Esposito et al., 2012).

In general, it has been observed that the smaller the size of the substrate particles is, the higher the methane production rate and yield are in the AD process. In addition to decreasing the particle size, the release of the intracellular components of the substrates has been reported by researchers specifically when the pretreatment was applied on waste activated sludge (WAS). Figure 2.4 illustrates different mechanical pretreatment techniques that have been practiced to enhance the AnCoD process.

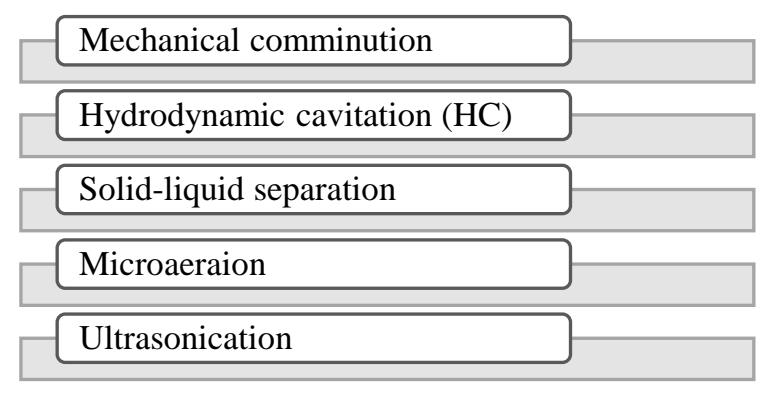

Figure 2.7. Mechanical methods of pretreatment used for AnCoD improvement 


\subsubsection{Thermal pretreatment}

Thermal pretreatment has been commonly applied to both raw and digested substrate to help with dewatering, sludge solublization, viscosity, and pathogen reduction. During thermal pretreatment and as a result of the increase in thermal energy (heat), the structure of the particulate (insoluble) matter changes in a way that it becomes more susceptible to biodegradation (Bougrier et al., 2007). Thermal pretreatment can be applied via conventional heating (conductive heating) or microwave hydrolysis. Edström et al. studied the effect of thermal pretreatment for enhanced co-digestion of animal by-products, food waste from restaurants and food distributors, and sludge from a slaughterhouse wastewater treatment plant. The authors reported a significant increase in the biogas yield from 0.31 to $1.14 \mathrm{~L} / \mathrm{g}$ VS-added when the animal by-product was exposed to conventional heating at a temperature of $70{ }^{\circ} \mathrm{C}$ for a duration of $1 \mathrm{~h}$ before the AnCoD process (Edström et al., 2003). In another study conducted by Paavola et al., thermal pretreatment via conductive heating was applied to a mixture of dairy manure and biowastes. An improvement in methane production at the extent of $14-18 \%$ was achieved compared to the process in the absence of thermal pretreatment (Paavola et al., 2006).

Despite these positive effect of thermal pretreatment on AD performance (including biogas production), the result of a study (Cuetos et al., 2010) indicated a 53\% decrease in methane production in the co-digestion of a mixture of solid slaughterhouse waste and OFMSW that was thermally pretreated at a temperature of $133^{\circ} \mathrm{C}$. The reduction of methane production was due to a foaming problem and accumulation of fats in the reactor that most likely occurred due to the formation of recalcitrant compounds at the elevated temperature of $133{ }^{\circ} \mathrm{C}$, exerting toxic effects on the AD process (Cuetos et al., 2010). Similarly, Guo et al. reported that during the co-digestion of food waste, fruit/vegetable residue, and thermally pretreated dewatered activated sludge, the methanogenesis process was inhibited by the accumulation of VFAs, which reduced the overall biogas production by $6 \%$ (Guo et al., 2014).

\subsubsection{Chemical pretreatment}

Chemical pretreatment can also lead to an improvement in the performance of AnCoD process through enhancement of the rate-limiting hydrolysis stage by facilitating the biodegradation of complex polymers via solubilizing the particulate fraction of the substrate. A variety of chemicals including acids, organic solvents, alkaline, and ionic liquids has shown a positive impact on 
disintegrating the structure of recalcitrant constituents of the substrates such as lignocellulosic compounds (Zhao et al., 2014).

Various types of alkaline compounds comprising $\mathrm{NaOH}, \mathrm{KOH}, \mathrm{Ca}(\mathrm{OH})_{2}$, hydrazine, and anhydrous ammonia can increase the internal surface area of biomass resulting in a decreased degree of polymerization. As a result, the complex structures can be decomposed and the strong bounds between carbohydrate molecules can be disrupted, which results in a higher availability of carbohydrates in the hetero matrix and enhanced reactivity of residual carbohydrates polymers in the biochemical process. Pretreatment by alkaline agents is able to eliminate acetyl and other uronic acid substitutions of hemicellulose that obstructs the accessibility of enzymes to cellulose surface. Alkali pretreatment has shown to be most successful for biomasses with lower lignin contents such as agricultural residues (Anwar et al., 2014; Saini et al., 2015).

Acidic pretreatment including dilute solutions of sulfuric acid, hydro-chloric acid, phosphoric acid, and free nitrous acid can be also employed to disintegrate the substrate structure prior to AnCoD process. Compared to the alkaline pretreatment, the application of concentrated acids is limited due to their corrosive nature and elevated costs. Acidic pretreatment has been effective in the hydrolysis of hemicelluloses to its monomeric units and increasing the bioavailability of cellulose. Acidic pretreatment may also be required to neutralize the hydrolysate providing a favorable environment for microbial activities (Lin et al., 2009). Some of the researches have suggested alkaline pretreatment using an ammonia solution or $\mathrm{NaOH}$ because of their simplicity, ease of operation, and high methane production efficiency (Bali et al., 2015; Guo et al., 2015; Park and Kim, 2012).

Among different substrates utilized in the co-digestion process, the lignocellulosic biomass as a complex mixture of cellulose, hemicelluloses, and lignin was found to be one of the most difficult to digest by compounds as it contains unbalanced carbon to nitrogen ratio as well as recalcitrant lingocellulosic structure. The lignocellulosic biomass is typically found in plant dry matter such as rice straw. Chemical pretreatment of rice straw prior to $\mathrm{AD}$ has been suggested in the literature as an effective way to improve the biodegradability of its constituents (Himmel et al., 2007; Zhao et al., 2014). For instance, the results obtained by Zhao et al. showed that alkali pretreatment using $\mathrm{NaOH}$ in co-digestion of rice straw and municipal waste sludge increased biogas yield by $20 \%$. In comparison, 40 and $45 \%$ increase in the removal rates of cellulose and hemicellulose were also reported, respectively due to the application of chemical pretreatment (Zhao et al., 2014). 
Wei et al. studied the chemical pretreatment using ammonia and $\mathrm{NaOH}$ during AnCoD of corn stover and cattle manure, resulting in improved cumulative biomethane production and solids removal rate. In the co-digestion of cattle manure and ammonia solution-treated corn stover, the required time to reach $80 \%$ of the ultimate methane yield (T80) was found to be $28 \pm 1$ days, which was $20 \%$ shorter than that of the control test with a T80 of $35 \pm 1$ days. Using sodium hydroxide, the T80 was determined to be $22 \pm 1$ days, $37 \%$ shorter as compared to that of the control digester (Wei et al., 2015).

\subsubsection{Biological pretreatment}

Biological pretreatment, which includes the addition of a particular strain, enzymes, or a consortium of microorganisms to the system, offers certain advantages over mechanical, thermal, and chemical pretreatment methods such as low energy requirements, avoiding toxic compounds generation, high yield of desired products, and the capability to target an specific compounds. Biological pretreatment methods such as bioaugmentation (stage 1) can result in the improvement of AD process by breaking complex polymers into simple monomers in hydrolysis stage which leads to increasing the rate of transformation of organic matter to biogas in the second stage. This process has shown to effectively increase both the methane yield and process stability (Song et al., 2014; Yuan et al., 2012; Zhong et al., 2011). Despite many researchers conducted on the application of biological pretreatment for improving conventional mono-digestion in AD systems, limited studies were performed on the application of these methods for enhancing biogas in AnCoD systems.

Table 2.4 summarizes the results of the studies conducted to enhance the AnCoD performance through biological pretreatment. In a study (Wei et al., 2015), biological pretreatment using liquid fraction of digestate (LFD) for AnCoD of cattle manure and corn stover was investigated. LFD obtained from anaerobic digester contains abundant microbes, inorganic substance as well as an organic substance such as amino acids, protein, a sugar that supplies nutrition substance for the process while acting as a microbial agent. As compared to untreated corn stover, pretreatment using LFD increased cumulative biomethane production (CBP) and VS removal rate by 25.40 and $30.12 \%$, respectively. In addition, it reduced T80 and improved buffer capacity of the anaerobic digestion system (Wei et al., 2015). 
Montusiewicz et al. studied the bioaugmentation pretreatment with a commercial product called Arkea ${ }^{\circledR}$ as a method for improving co-digestion of sewage sludge and mature landfill leachate. The co-digestion process was improved due to the enhanced activity of microorganisms involved in bioaugumenting system and their resistance to toxic elements (Montusiewicz, 2014). This was consistent with the results obtained by Duran et al. who used selected strains of Baccillus, Pseudomonas and Actinomycetes species for bioaugmentation (Duran et al., 2006).

In another study by Deng et al., the effect of enzymatic pretreatment on co-digestion of rice straw and soybean straw was evaluated. The research was conducted to investigate the effect of on-site generated cellulase produced by cultivation of Trichoderma reesei RUT C30 on lignocellulose to improve the hydrolysis of cellulose and hemicellulose content of the feedstock. They also investigated the influence of the pretreatment on biogas production via batch experiments. The authors reported more than $300 \%$ increase in the cumulative biogas yield compared to the untreated feedstock. They also achieved $40 \%$ shorter lag time in co-digestion of pretreated feedstock than the pretreated mono-digestion groups verifying a synergistic effect of rice and soybean straws codigestion. This study suggested enzymatic pretreatment using Trichoderma reesei RUT C30 as an effective method for improving biogas production (Deng et al., 2018).

Zhang et al, also investigated the effects of biological co-pretreatment on biogas production in AnCoD of food waste (FW) and waste activated sludge (WAS). They used co-pretreatment of FW and WAS followed by anaerobic co-digestion to improve hydrolysis efficiency. Their method was established based on the fact that a biological solublization process by mixing FW, water and microorganisms was effective as a result of size reduction of substrate particles and increase of solublization. They hypothesized that using a mixture of WAS and FW for biological solublization pretreatment (biological co-pretreatment), would improve the hydrolysis of FW and WAS for the reason that: 1) generation of the alkalis from WAS could buffer VFAs and maintain optimum $\mathrm{pH}$ for hydrolysis stage, and 2) a lower $\mathrm{pH}$ would enhance solublization of WAS through accelerating the hydrolysis of proteins and carbohydrates. Their method of pretreatment included a $2 \mathrm{~L}$ glass reactor with a mechanical stirrer at a mixing speed of $150 \mathrm{rpm}$ as a biological co-pretreatment reactor. They applied a blend ratio of 1:1 (weight) with different co-pretreatment time: $0 \mathrm{~h}, 15 \mathrm{~h}$, $24 \mathrm{~h}$ and $35 \mathrm{~h}$. The pretreated mixtures were used as the feed of the subsequent anaerobic digester. A mixture of fresh FW and WAS with $0 \mathrm{~h}$ co-pretreatment was used as the feed of the subsequent anaerobic digester as control. They achieved $24.6 \%$ higher methane production from co-digestion 
of co-pretreated substrates compared to control substrates without pretreatment. They also observed an increase of $10.1 \%$ in solids reduction under $24 \mathrm{~h}$ optimum pretreatment time (Zhang et al., 2017a).

Table 2.4. Biological pretreatment for enhanced AnCoD

\begin{tabular}{lllll}
\hline $\begin{array}{l}\text { Method of } \\
\text { Pretreatment }\end{array}$ & Feedstock & Digester mode & Mixing ratio & $\begin{array}{l}\text { Methane yield/ } \\
\text { Biogas increase\% }\end{array}$ \\
\hline \hline $\begin{array}{l}\text { Liquid fraction of } \\
\text { digestate }\end{array}$ & $\begin{array}{l}\text { Corn stover+ cattle } \\
\text { manure }\end{array}$ & $\begin{array}{l}\text { Batch } \\
\text { (mesophilic) }\end{array}$ & $3: 1$ (weight) & $\begin{array}{l}\text { 25\% methane } \\
\text { increase }\end{array}$ \\
\hline $\begin{array}{l}\text { Bioaugmentation } \\
\text { by Arkea }{ }^{\circ}\end{array}$ & $\begin{array}{l}\text { sewage sludge }{ }^{*}+ \\
\text { mature landfill }\end{array}$ & $\begin{array}{l}\text { CSTR } \\
\text { (mesophilic, }\end{array}$ & $\begin{array}{l}87: 4.3: 8.7 \\
(\mathrm{v} / \mathrm{v})\end{array}$ & $\begin{array}{l}5-8 \% \text { biogas } \\
\text { decrease }\end{array}$ \\
\hline $\begin{array}{l}\text { Biological } \\
\text { (Enzymatic) }\end{array}$ & Rice straw + & Batch & $1: 1$ TS ratio & $\begin{array}{l}318 \% \text { biogas } \\
\text { increase }\end{array}$ \\
\hline $\begin{array}{l}\text { biological co- } \\
\text { pretreatment }\end{array}$ & Foybean straw & (mesophilic) & & $\begin{array}{l}\text { 24.6\% methane } \\
\text { increase }\end{array}$ \\
\hline \hline
\end{tabular}

\subsubsection{Hybrid pretreatment}

Hybrid pretreatment is a combination two or more methods of mechanical, thermal, chemical, and biological pretreatment techniques. Of all the available hybrid pretreatment methods, thermoalkaline, $\mathrm{NaOH} / \mathrm{H}_{2} \mathrm{O}_{2}$, and Ozone/ $\mathrm{NaOH}$ have received more attention to enhance AnCoD. In a recent study performed by Benn et al. (2018), the usage of thermochemical bioplastic pretreatment was investigated in co-digestion with synthetic primary sludge in batch and continuous mode. The pretreatment experiments were conducted by applying different combinations of temperatures ranging from $35-90^{\circ} \mathrm{C}$ with alkaline conditions in a $\mathrm{pH}$ range from 8 to 12 (Benn and Zitomer, 2018). Percent conversion values for bioplastics to biomethane were calculated as the proportion of BMP value divided by the theoretical maximum methane production value indicated by the bioplastic theoretical oxygen demand loading. According to the authors, the thermos-alkaline pretreatment led to an increase in average BMP values up to over $100 \%$. The batch system codigesting synthetic primary sludge with bioplastic resulted in 80-100\% conversion of bioplastics to biomethane and a $50 \%$ biomethane production increase over the non-pretreated substrate. In comparison with the findings of the batch study, less than $20 \%$ increase in methane production was obtained by continuous co-digestion of pretreated bioplastics with the synthetic primary 
sludge in a CSTR system compared to the system fed with non-pretreated feedstock (Benn and Zitomer, 2018).

Another study conducted by Naran et al. revealed that the cumulative methane yield increased from 116.7 to $177.3 \mathrm{~mL} / \mathrm{g}$ VS added through the co-digestion of thermos-alkaline pretreated food waste from a food waste treatment plant and WAS generated at a municipal treatment compared to the non-pretreated sample (Naran et al., 2016). Similarly, the usage of thermo-alkaline pretreatment method to improve co-digestion of WAS and rice straw was studied and a biogas production of $409 \mathrm{~L} / \mathrm{kgVS}_{\text {added }}$ was obtained under the optimum condition equivalent to a $51 \%$ increase compared to the control test. The authors of that work also reported that the degree of WAS solublization was positively correlated with biogas production and VS removal. It was also observed that, following pretreatment, the cellulose and hemicellulose contents of rice straw decreased remarkably. According to the results of their research, the addition of $\mathrm{NaOH}$ caused $11 \%, 32 \%$, and $22 \%$ reduction in hemicellulose, cellulose, and lignin contents, respectively. The improvement of digestion performance in this study was related to the increased solublization and reduced particle size of the organic matter (Abudi et al., 2016b).

Abudi et al., employed $\mathrm{NaOH} / \mathrm{H}_{2} \mathrm{O}_{2}$ pretreatment on co-digestion of WAS and rice straw. The applied pretreatment resulted in a remarkable reduction in cellulose and hemicellulose contents of rice straw and hence improved the biogas production. $\mathrm{NaOH} / \mathrm{H}_{2} \mathrm{O}_{2}$ pretreatment was able to decrease hemicellulose, cellulose and lignin contents of rice straw by $16 \%, 41 \%$, and $7 \%$, respectively. Pretreatment was more effective in the solublization of hemicellulose content than cellulose and lignin contents of rice straw. Consistent results were obtained by others for single digestion of rice and corn straws using $\mathrm{NaOH} / \mathrm{H}_{2} \mathrm{O}_{2}$ pretreatment (He et al., 2009; Song et al., 2014, 2013).

Rajesh Banu et al. employed ozone/ $\mathrm{NaOH}$ pretreatment for the co-digestion of cow manure and WAS. This resulted in increasing biogas production from 17.9 to $18.8 \mathrm{~L} / \mathrm{d}$. Despite the fact that ozone utilization is considered to be costly, the combination of alkali and ozone not only increased the sludge disintegration efficiency but also saved a considerable amount of energy (Rajesh Banu et al., 2015). The increase in biogas production was most likely due to the particles decomposition resulting from ozone reaction with the organic fraction of sludge and the availability of extra carbon source (Ahn et al., 2002). 
In the study conducted by Ren et al., the effects of hot alkali pretreatment and mixing ratio on anaerobic co-digestion of duckweed and excess sludge were investigated. The result of their study primarily indicated that through co-digestion the delayed stage of gas generation reduced, and a cumulative gas yield of $2963 \mathrm{~mL}$ was obtained which was $11 \%$ higher than the calculated value. The methane content of the produced gas was 57\%, which was $13 \%$ higher than that of the duckweed and $9 \%$ higher than that of the excess sludge single digestion. Additionally, pretreatment of the duckweed in the mixture, improved the methane yield by $8 \%$ (Ren et al., 2018).

In co-digestion of poultry manure with pig manure in a batch system, the effect of a combined thermochemical pretreatment and ammonia stripping on the digester performance was assessed. The result revealed that the optimal blend ratio of poultry manure to pig manure was $24: 76$ on volumetric basis which was corresponding to highest methane production. The combined pretreatment improved the co-digestion system achieving an OLR of $4 \mathrm{~g}$ COD/L $\mathrm{d}$ with a HRT of 20 days (Rodriguez-Verde et al., 2017). The results of these studies are summarized in table 2.5.

Table 2.5. Hybrid pretreatment for enhanced AnCoD

\begin{tabular}{|c|c|c|c|c|}
\hline $\begin{array}{l}\text { Method of } \\
\text { Pretreatment }\end{array}$ & Feedstock & Digester mode & Mixing ratio & $\begin{array}{l}\text { Methane yield/ } \\
\text { Biogas increase \% }\end{array}$ \\
\hline Thermo-alkaline & $\begin{array}{l}\text { Synthetic municipal } \\
\text { primary sludge }+ \\
\text { bioplastics }\end{array}$ & $\begin{array}{l}\text { Batch } \\
\text { (mesophilic) }\end{array}$ & $10: 1(\mathrm{v} / \mathrm{v})$ & $6 \%$ methane increase \\
\hline Thermo-alkaline & WAS + food waste & $\begin{array}{l}\text { Batch } \\
\text { (mesophilic) }\end{array}$ & $7: 3(\mathrm{v} / \mathrm{v})$ & $\begin{array}{l}52 \% \text { methane } \\
\text { increase }\end{array}$ \\
\hline Thermo-alkaline & TWAS+ rice straw & $\begin{array}{l}\text { Batch } \\
\text { (mesophilic) }\end{array}$ & $1: 1(\mathrm{v} / \mathrm{v})$ & $51 \%$ biogas increase \\
\hline $\begin{array}{l}\text { Thermo- } \\
\text { alkaline/ } \mathrm{H}_{2} \mathrm{O}_{2}\end{array}$ & TWAS+ rice straw & $\begin{array}{l}\text { Batch } \\
\text { (mesophilic) }\end{array}$ & $1: 1(\mathrm{v} / \mathrm{v})$ & $56 \%$ biogas increase \\
\hline Ozone/NaOH & $\begin{array}{l}\text { Cow manure +dairy } \\
\text { wastewater }\end{array}$ & HUASB $* * *$ & $3: 1(\mathrm{v} / \mathrm{v})$ & $5 \%$ biogas increase \\
\hline Thermo-alkaline & $\begin{array}{l}\text { duckweed and waste } \\
\text { activated sludge }\end{array}$ & Batch mesophilic & NA & $8 \%$ \\
\hline Thermo-alkaline & $\begin{array}{l}\text { poultry manure and } \\
\text { pig manure }\end{array}$ & $\begin{array}{l}\text { Continuous } \\
\text { mesophilic }\end{array}$ & $24: 76$ & $37 \%$ \\
\hline
\end{tabular}

In summary, due to various advantages that co-digestion offers over conventional mono digestion, this area is attracted by several researchers and studies are still going on to better understand the 
system performance and to investigate different methods for improving the AnCoD systems. Such researches along with studies on control parameters, suitability of different feedstocks and their combinations and optimizing procedures would contribute in further improving this technology. 


\section{Chapter 3}

\section{Materials and methods}




\section{Materials and methods}

\subsection{Feedstocks and Inoculum}

This research was designed to evaluate the effect of mixing ratio of the feedstocks and its relationship with the lipids: proteins: carbohydrates content on anaerobic co-digestion process. The experiment included two sections including BMP assay and hydrolysis/acidification. The BMP was designed to assess the influence of the mixing ratio on biomethane production. Hydrolysis/acidification experiment was carried out to evaluate the effect of mixing ratio on hydrolysis kinetics. Different feedstocks including dairy manure, TWAS and SSO in different combinations were used as digester feedstocks. TWAS (3.8\% TS) and inoculum were collected from Ashbridges Bay Wastewater Treatment Plant Toronto, Ontario. The inoculum was obtained from the effluent of the anaerobic digesters operating at mesophilic condition at a temperature range of $34-38^{\circ} \mathrm{C}$, and receiving approximately $1600 \mathrm{~m}^{3} / \mathrm{d}$ TWAS and $6500 \mathrm{~m}^{3} / \mathrm{d}$ primary sludge. The average organic loading rate and SRT of the anaerobic digesters are $1.1 \mathrm{~kg} \mathrm{VS} / \mathrm{m}^{3}$ and $18 \mathrm{~d}$, respectively.

The Ashbridges Bay Wastewater Treatment Plant is the main wastewater treatment facility among the four treatment plants that service the city of Toronto. After Montreal's Jean-R. Marcotte facility, it is the second largest plant in Canada (Heffez, 2009) The plant treats the wastewater produced by approximately 1.4 million of the city of Toronto's residents and has a capacity of $818,000 \mathrm{~m}^{3} / \mathrm{d}$ (City of Toronto, 2018).

The influent to the treatment plant comes from Mid-Toronto, high level, low level and Lakefront interceptor sewers in addition to Coxwell and Queen Street trunk sewers. Biosolids generated at the plant was approximately 149733 wet tones in 2016 with $28.1 \%$ Total Solid (TS). The influent undergoes treatment processes which comprises preliminary treatment i.e. screening and grit removal, primary treatment, secondary treatment, nutrient removal, disinfection, Waste Activated Sludge (WAS) thickening, anaerobic digestion, biosolids dewatering and biosolids management. The influent Total Suspended Solids (TSS) and Biological Oxygen Demand (BOD) concentrations are $318.6 \mathrm{mg} / \mathrm{L}$ of $244.6 \mathrm{mg} / \mathrm{L}$, respectively (City of Toronto, 2018; Razavi, 2019).

The unit processes of the plant is shown in figure 3.1. The activated sludge system consists of three main stages including aeration tank, settling tank and return activated sludge. In the aeration tank, the atmospheric air is introduced to the primary treated wastewater using air blowers and the biological mass that produces biological flocs is called waste activated sludge (WAS). The 
produced sludge then goes through thickening process using different mechanisms to produce thickened waste activated sludge (TWAS).

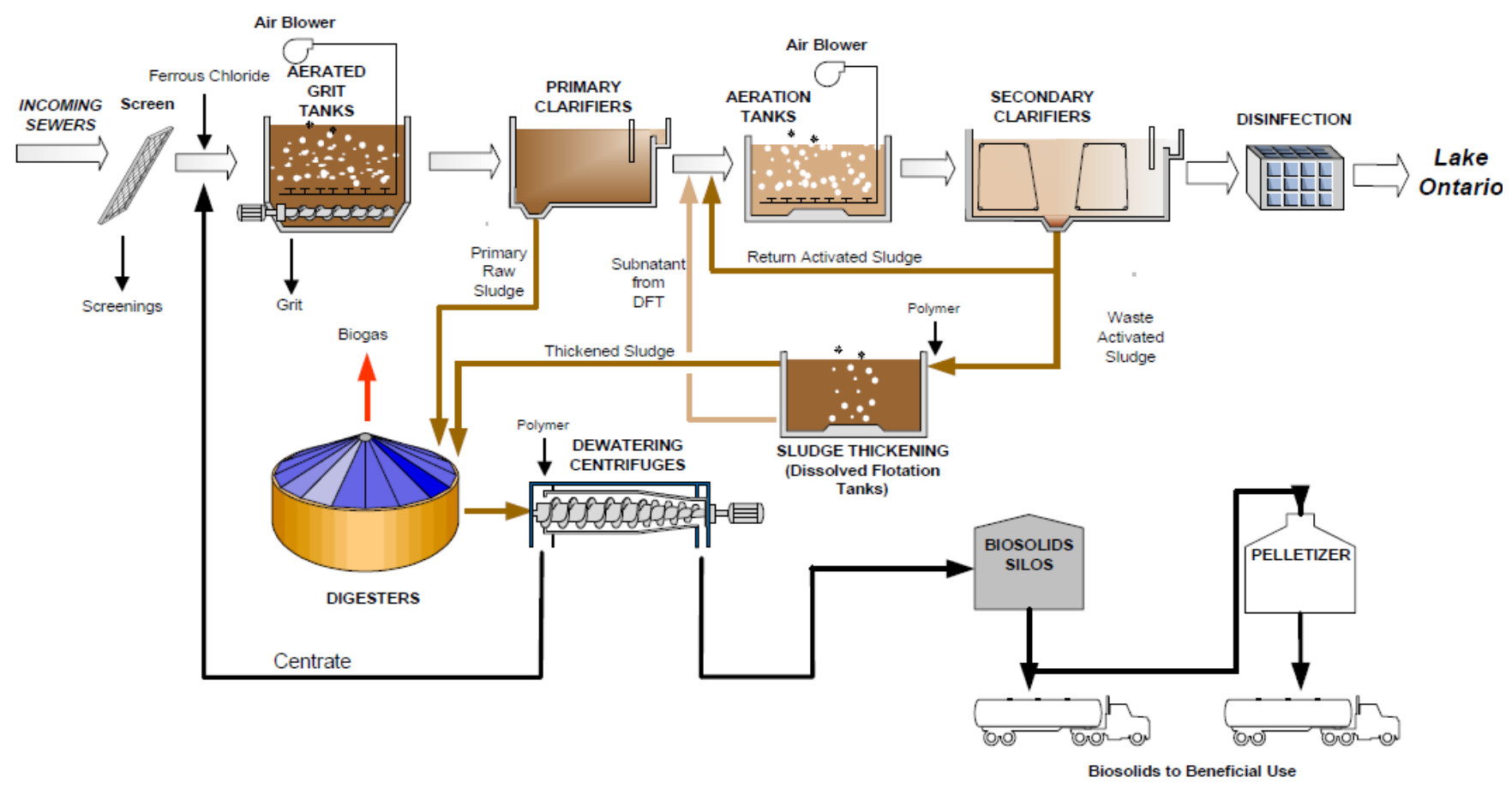

Figure 3.1. The flow diagram of the Ashbridges Bay Wastewater Treatment Plant (City of Toronto, 2018)

SSO was obtained from City of Toronto Disco Road Organics Processing Facility, Toronto, Ontario. The facility is located on 120 Disco Road and on a 1-hectare site and is the first full-scale plant in North America. It has been operated since 2014 for processing the source separated organics by anaerobic digestion. Being one of the municipality's diversion program, it receives almost half of the organics collected in Toronto and is capable of processing up to 75,000 tons of organic waste per year from homes and public buildings. The ultimate capacity of the facility is planned to rise to 130,000 tons in the near future. The acceptable materials to Disco Road facility includes food waste, paper food packaging, pet waste, diapers, houseplants, and biodegradable plastics. The organic processing at the facility is shown in figure 3.2. SSO first is delivered and stored and goes through a visual inspection to remove large unwanted items. At the next stage, the materials undergo the BTA ${ }^{\circledR}$ hydro-mechanical technology through which the organics will convert to a liquid (slurry) pulp. The BTA® consists of screens and hydropulpers for separating 
the unwanted materials including glass shards, plastic bags, metals and sand from the pulp (Razavi, 2019). In this study, the SSO samples were collected after the hydro-mechanical stage and transferred to the lab in the slurry form.
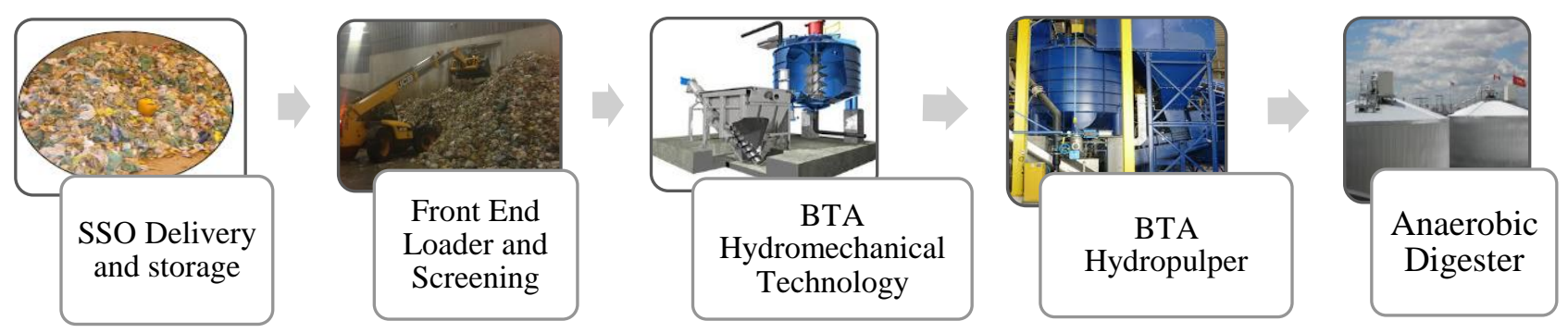

Figure 3.2. Source separated organics processing in Disco Road Facility

Cow manure was collected from a manure pit of a dairy farm located in Newmarket, Ontario. Manure slurry was prepared by addition and homogenization of cow manure with deionized distilled water using a blender followed by a VWR 400 DS bench top homogenizer. The reactors were fed with different combinations of the feedstocks.

The BMP included 3 binary co-digestion experiments using different feedstocks mixtures including different mixing ratios of TWAS/SSO, TWAS/manure, and Manure/SSO. The BMP of TWAS/SSO, TWAS/manure, and Manure/SSO were conducted individually in different periods. It also included a ternary co-digestion using the three feedstocks, TWAS/manure/SSO, at different mixing ratios. Each of the experiments continued until biogas production stopped or was negligible. A series of analysis for characterization of the inoculum, TWAS and SSO and manure was carried out primarily and presented in Table 3.1. Samples were transported and preserved according to Standard Method for Examination of Water and Wastewater (APHA, 2005). Feed of digesters as explained above were mixed at different mixture ratios on a volumetric basis. Batch reactors in working volume of $200 \mathrm{~mL}$ containing inoculum and feedstocks at different mixing ratios were prepared for the BMP essay. TWAS, manure, and SSO alone were also used as control reactor to assess the effect of co-digestion on the efficiency of the system in comparison with the single digestion of the feedstocks.

A series of analysis for characterization of the inoculum, TWAS and SSO was carried out primarily and are presented in table 3.1. The mean values are the average of four measurements on each of 
the parameters for the raw substrates and RSD is the ratio of standard deviation to the mean or relative standard deviation.

Table 3.1. Initial Characteristics of the feedstocks and inoculum used in this study

\begin{tabular}{|c|c|c|c|c|c|c|c|c|c|}
\hline \multirow[t]{2}{*}{ Parameters } & \multirow[t]{2}{*}{ Units } & \multicolumn{2}{|c|}{ SSO } & \multicolumn{2}{|c|}{ Manure } & \multicolumn{2}{|c|}{ TWAS } & \multicolumn{2}{|c|}{ Inoculum } \\
\hline & & MEAN & RSD & MEAN & RSD & MEAN & 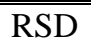 & MEAN & "RSD \\
\hline TCOD & $\mathrm{mg} / \mathrm{L}$ & 110000 & 0.07 & 198833 & 0.03 & 40000 & 0.07 & 16400 & 0.03 \\
\hline SCOD & $\mathrm{mg} / \mathrm{L}$ & 44400 & 0.002 & 10933 & 0.04 & 360 & 0.07 & 362 & 0.05 \\
\hline TSS & $\mathrm{mg} / \mathrm{L}$ & 53833 & 0.12 & 56520 & 0.03 & 31450 & 0.08 & 17033 & 0.02 \\
\hline VSS & $\mathrm{mg} / \mathrm{L}$ & 38478 & 0.095 & 29998 & 0.02 & 25600 & 0.09 & 10900 & 0.02 \\
\hline TS & $\mathrm{mg} / \mathrm{L}$ & 62187 & 0.02 & 73727 & 0.04 & 38810 & 0.12 & 21450 & 0.03 \\
\hline VS & $\mathrm{mg} / \mathrm{L}$ & 43493 & 0.02 & 38647 & 0.02 & 31205 & 0.01 & 13140 & 0.02 \\
\hline Ammonia & $\mathrm{mg} / \mathrm{L}$ & 1738 & 0.003 & 22 & 0.07 & 255 & 0.12 & 1495 & 0.03 \\
\hline pH & - & 5.6 & 0.001 & 6.4 & 0.01 & 6.3 & 0.005 & 7.2 & 0.001 \\
\hline Alkalinity & $\mathrm{mg} \mathrm{CaCO} 3 / \mathrm{L}$ & 7700 & 0.07 & 11133 & 0.05 & 1953 & 0.07 & 3943 & 0.12 \\
\hline TN & $\mathrm{mg} / \mathrm{L}$ & 4167 & 0.18 & 2200 & 0.12 & 2900 & 0.14 & 2025 & 0.10 \\
\hline TSN & $\mathrm{mg} / \mathrm{L}$ & 1793 & 0.02 & 104 & 0.09 & 420 & 0.15 & 696 & 0.16 \\
\hline Total Carbs & $\mathrm{mg} / \mathrm{L}$ & 40360 & 0.09 & 7398 & 0.06 & 1288 & 0.08 & 961 & 0.08 \\
\hline Total Proteins & $\mathrm{mg} / \mathrm{L}$ & 2021 & 0.09 & 5199 & 0.08 & 1459 & 0.18 & 1448 & 0.09 \\
\hline Total Lipids & $\mathrm{mg} / \mathrm{L}$ & 18620 & 0.12 & 7241 & 0.09 & 551 & 0.08 & 1920 & 0.10 \\
\hline
\end{tabular}

\subsection{Experimental design and procedure}

\subsubsection{Co-digestion- BMP assay}

The biochemical methane potential (BMP) assays were conducted according to the procedures described in the literature (Angelidaki et al., 2009a; Moody et al., 2009; Owen et al., 1979). The experiment was initiated by feeding the digesters with different mixing ratios of the feedstocks in triplicates. A 100\% SSO and 100\% TWAS, and 100\% manure as control reactors were assessed in triplicates as well. In this research, a substrate-to-biomass ratio $\left(\mathrm{S}^{0} / \mathrm{X}^{0}\right)$ of $2 \mathrm{~g} \mathrm{COD}$ substrate/g VSS inoculum was kept in all digesters which is within the range that has been suggested by the literature (Elbeshbishy et al., 2012). Substrate to inoculum ratio was selected for all of the batch reactors according to the procedure used in by Nasr et al., 2011 as following:

$\mathrm{S}^{0} / \mathrm{X}^{0}=\frac{\mathrm{g} \mathrm{TCOD}_{\text {substrate }}}{\mathrm{g} \mathrm{VSS}_{\text {inoculum }}}=\frac{\mathrm{V}_{\text {substrate }}{ }^{\times} \text {TCOD }_{\text {substrate }}}{\mathrm{V}_{\text {inoculum }}{ }^{\times} \text {VSS }_{\text {substrate }}}=2$ 
The TCOD of the mixture used in Eq. 3.1 was calculated considering the TCOD of the feedstocks and the mixing ratios. Accordingly, the volumes of the feedstocks were calculated based on the mixing ratios of substrates. Because of the heterogeneous composition of the feedstocks, all of the combinations were prepared in triplicates. The specific amount of the substrates along with the mesophilic inoculum as described above was added to $250 \mathrm{~mL}$ glass bottles. The headspaces in the bottles were flushed with nitrogen gas for 3 minutes at $10 \mathrm{psi}$ and subsequently the bottles were sealed to satisfy the anaerobic conditions. In addition, anaerobic systems require a $\mathrm{pH}$ within the range of 6.5-7.5 according to the literature (Cioabla et al., 2012; Droste, 1997). Therefore, the $\mathrm{pH}$ in each bottle were kept in a range of 7-7.4 using sulfuric acid and sodium hydroxide. The binary co-digestion of TWAS/SSO, TWAS/manure, and manure/SSO were conducted at the mixing ratios as presented in table 3.2 .

Table 3.2 Proportions of digesters'feed for binary co-digestion of TWAS/ SSO, TWAS/manure, and manure/ SSO in BMP experiment

\begin{tabular}{|c|c|c|c|}
\hline Feedstock & TWAS & Manure & SSO \\
\hline \multirow{20}{*}{$\begin{array}{l}\text { Proportion of the } \\
\text { feedstocks in } \\
\text { different runs of } \\
\text { Binary co-digestion } \\
\text { Volume: } 200 \mathrm{~mL}\end{array}$} & 0 & 1 & 0 \\
\hline & 1 & 0 & 0 \\
\hline & 9 & 1 & 0 \\
\hline & 7 & 3 & 0 \\
\hline & 5 & 5 & 0 \\
\hline & 3 & 7 & 0 \\
\hline & 1 & 9 & 0 \\
\hline & 0 & 2 & 4 \\
\hline & 0 & 0 & 1 \\
\hline & 0 & 9 & 1 \\
\hline & 0 & 7 & 3 \\
\hline & 0 & 5 & 5 \\
\hline & 0 & 3 & 7 \\
\hline & 0 & 1 & 9 \\
\hline & 0 & 0 & \\
\hline & 9 & 0 & 1 \\
\hline & 7 & 0 & 3 \\
\hline & 5 & 0 & 5 \\
\hline & 3 & 0 & 7 \\
\hline & 1 & 0 & 9 \\
\hline
\end{tabular}


The ternary co-digestion of TWAS/manure/SSO was carried out at different mixing ratios of TWAS, manure and SSO as presented in Table 3.3. As demonstrated in Table 3.2 and 3.3 reactors containing only manure, only TWAS and only SSO were also used in triplicates as control in each run.

Table 3.3 Proportions of digesters' feed for ternary co-digestion of TWAS, SSO, and manure in BMP experiment

\begin{tabular}{|l|c|c|c|}
\hline \multicolumn{1}{|c|}{ Feedstock } & TWAS & Manure & SSO \\
\hline \multirow{4}{*}{$\begin{array}{l}\text { Proportion of the } \\
\text { feedstocks in }\end{array}$} & 8 & 1 & 1 \\
\cline { 2 - 4 } different runs of \\
\cline { 2 - 4 } ternary co-digestion & 1 & 8 & 1 \\
\cline { 2 - 4 } Volume: 200 mL & 5 & 1 & 2.5 \\
\cline { 2 - 4 } & 2.5 & 5 & 2.5 \\
\cline { 2 - 4 } & 2.5 & 2.5 & 5 \\
\cline { 2 - 4 } & 4 & 4 & 2 \\
\cline { 2 - 4 } & 2 & 2 & 4 \\
\cline { 2 - 4 } & 1 & 4 & 0 \\
\cline { 2 - 4 } & 0 & 1 & 1 \\
\hline
\end{tabular}

The sets of 21 bottles for each of the three binary co-digestion experiments, and the 36 bottles for the ternary co-digestion experiment were placed in the Thermo Scientific MAXQ 4000 shakers and a rotational speed of 150 RPM was applied during the entire process. The incubator temperature was set at $37^{\circ} \mathrm{C}$ to satisfy mesophilic condition for the batch reactors. 


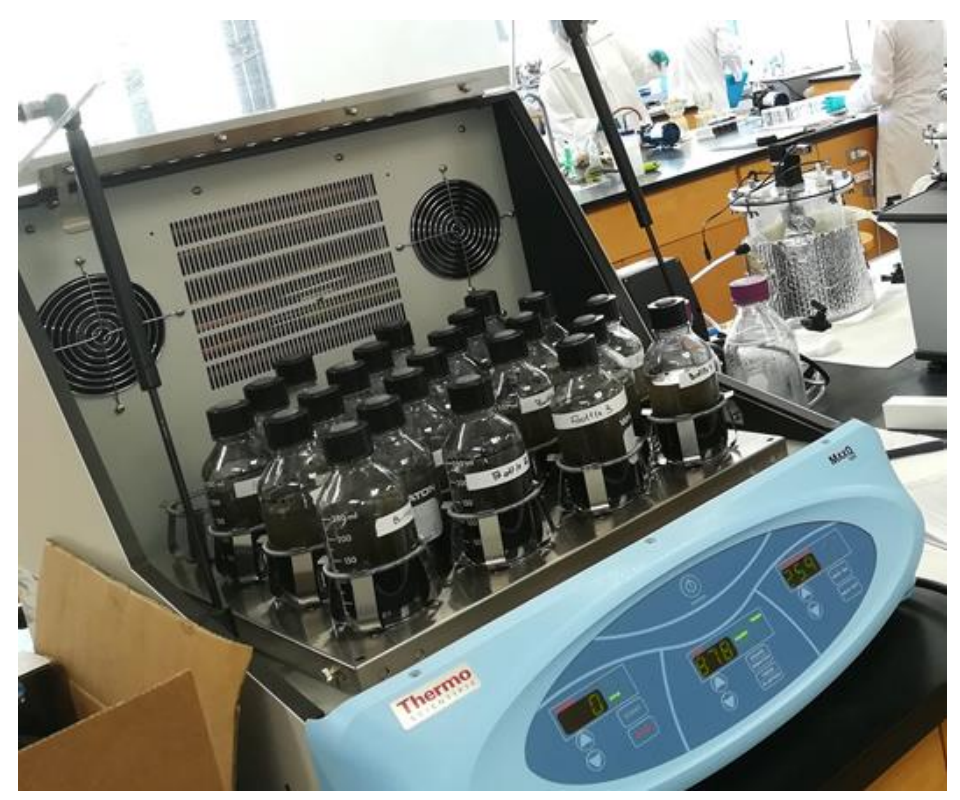

Figure 3.3- Experimental set-up for biomethane potential experiment

\subsubsection{Co-digestion- hydrolysis/acidification experiment}

This experiment was conducted to assess the influence of the mixing ratio and its relationship with the lipids: proteins: carbohydrates in co-digestion on hydrolysis/acidification rate. Similar to BMP experiment, the binary co-digestion of TWAS/ SSO, TWAS/manure, and manure/SSO were conducted at different combinations as presented in Table 3.4. The ternary co-digestion of TWAS/manure/SSO was carried out at the mixing ratios that are presented in Table 3.5. For each of the experiment's rectors containing only TWAS, only manure and only SSO were also used in triplicates as control reactors.

The bottles for each of the three binary co-digestion experiments, and for the ternary co-digestion experiment were placed in the Polyscience WB28 water bathes. Bottles were equipped with a mixer which maintained a rotational speed of 150 RPM the entire process. The temperature in water bathes was set at $37^{\circ} \mathrm{C}$ to satisfy mesophilic condition for the batch reactors.

Consistent with the BMP experiment, the substrate-to-biomass ratio $\left(\mathrm{S}^{0} / \mathrm{X}^{0}\right)$ of to $2 \mathrm{~g}$ COD substrate/g VSS inoculum was kept in all digesters. Feed of digesters as explained above were mixed at different mixture ratios on a volumetric basis. Batch reactors in working volume of $2000 \mathrm{~mL}$ containing inoculum and feedstocks at different mixing ratios were prepared for the BMP essay. In order to deactivate methanogens and to increase the accuracy of the hydrolysis/acidification experiment, 
the inoculum was heated to $70{ }^{\circ} \mathrm{c}$ for $30 \mathrm{~min}$ and $\mathrm{pH}$ was adjusted to a range between 5- 5.5 in all digesters. The experiment continued for a period of three days for each run of the binary codigestions and the ternary co-digestion experiment. Samples were collected with time to evaluate the solublization and hydrolysis rate of the mixtures and to assess the influence of the mixing ratios on them. Consistent with the BMP assay, The TCOD of the mixture in Eq. 3.1 was calculated considering the TCOD of the feedstocks and the mixing ratios. Accordingly, the volumes of the feedstocks were calculated based on the mixing ratios of substrates. Because of the heterogeneous composition of the feedstocks, all of the combinations were prepared in triplicates. The specific amount of the substrates along with the mesophilic inoculum as described above was added to $2500 \mathrm{~mL}$ glass bottles.

Table 3.4 Proportions of digesters' feed for binary co-digestion of TWAS/ SSO, TWAS/manure, and manure/ SSO in hydrolysis/acidification experiment

\begin{tabular}{|c|c|c|c|}
\hline Feedstock & TWAS & Manure & SSO \\
\hline \multirow{20}{*}{$\begin{array}{l}\text { Proportion of the } \\
\text { feedstocks in } \\
\text { different runs of } \\
\text { Binary co-digestion } \\
\text { Volume: } 2000 \mathrm{~mL}\end{array}$} & 0 & 1 & 0 \\
\hline & 1 & 0 & 0 \\
\hline & 9 & 1 & 0 \\
\hline & 7 & 3 & 0 \\
\hline & 5 & 5 & 0 \\
\hline & 3 & 7 & 0 \\
\hline & 1 & 9 & 0 \\
\hline & 0 & 2 & 4 \\
\hline & 0 & 0 & 1 \\
\hline & 0 & 9 & 1 \\
\hline & 0 & 7 & 3 \\
\hline & 0 & 5 & 5 \\
\hline & 0 & 3 & 7 \\
\hline & 0 & 1 & 9 \\
\hline & 0 & 0 & \\
\hline & 9 & 0 & 1 \\
\hline & 7 & 0 & 3 \\
\hline & 5 & 0 & 5 \\
\hline & 3 & 0 & 7 \\
\hline & 1 & 0 & 9 \\
\hline
\end{tabular}


Table 3.5 Proportions of digesters' feed for ternary co-digestion of TWAS, SSO, and manure in in hydrolysis/acidification experiment

\begin{tabular}{|l|c|c|c|}
\hline \multicolumn{1}{|c|}{ Feedstock } & TWAS & Manure & SSO \\
\hline \multirow{4}{*}{$\begin{array}{l}\text { Proportion of the } \\
\text { feedstocks in }\end{array}$} & 8 & 1 & 1 \\
\cline { 2 - 4 } different runs of \\
\cline { 2 - 4 } ternary co-digestion & 1 & 8 & 1 \\
\cline { 2 - 4 } Volume: 200 mL & 5 & 2.5 & 2.5 \\
\cline { 2 - 4 } & 2.5 & 5 & 2.5 \\
\cline { 2 - 4 } & 2.5 & 2.5 & 5 \\
\cline { 2 - 4 } & 4 & 4 & 4 \\
\cline { 2 - 4 } & 2 & 2 & 4 \\
\cline { 2 - 4 } & 1 & 4 & 0 \\
\cline { 2 - 4 } & 0 & 1 & 1 \\
\hline
\end{tabular}

The headspaces in the bottles were flushed with nitrogen gas for 3 minutes at 10 psi and subsequently the bottles were sealed to satisfy the anaerobic conditions. Gas production during the experiment was monitored using water displacement method and displayed real time. The percentage improvements in the soluble contents concentrations (degree of solubilization) (P) values was calculated using Eq 3.2.

$P(\%)=S C_{f}-S C_{i} / P C_{i} * 100 \%$

Eq. 3.2

Where $\mathrm{SC}_{\mathrm{i}}$ and $\mathrm{SC}_{\mathrm{f}}$ are the mass of soluble COD of the digester contents before and after the hydrolysis/acidification phase experiment $(\mathrm{mg})$ and $\mathrm{PC}_{\mathrm{i}}$ is the mass of initial particulate COD in the digesters $(\mathrm{mg})$. 


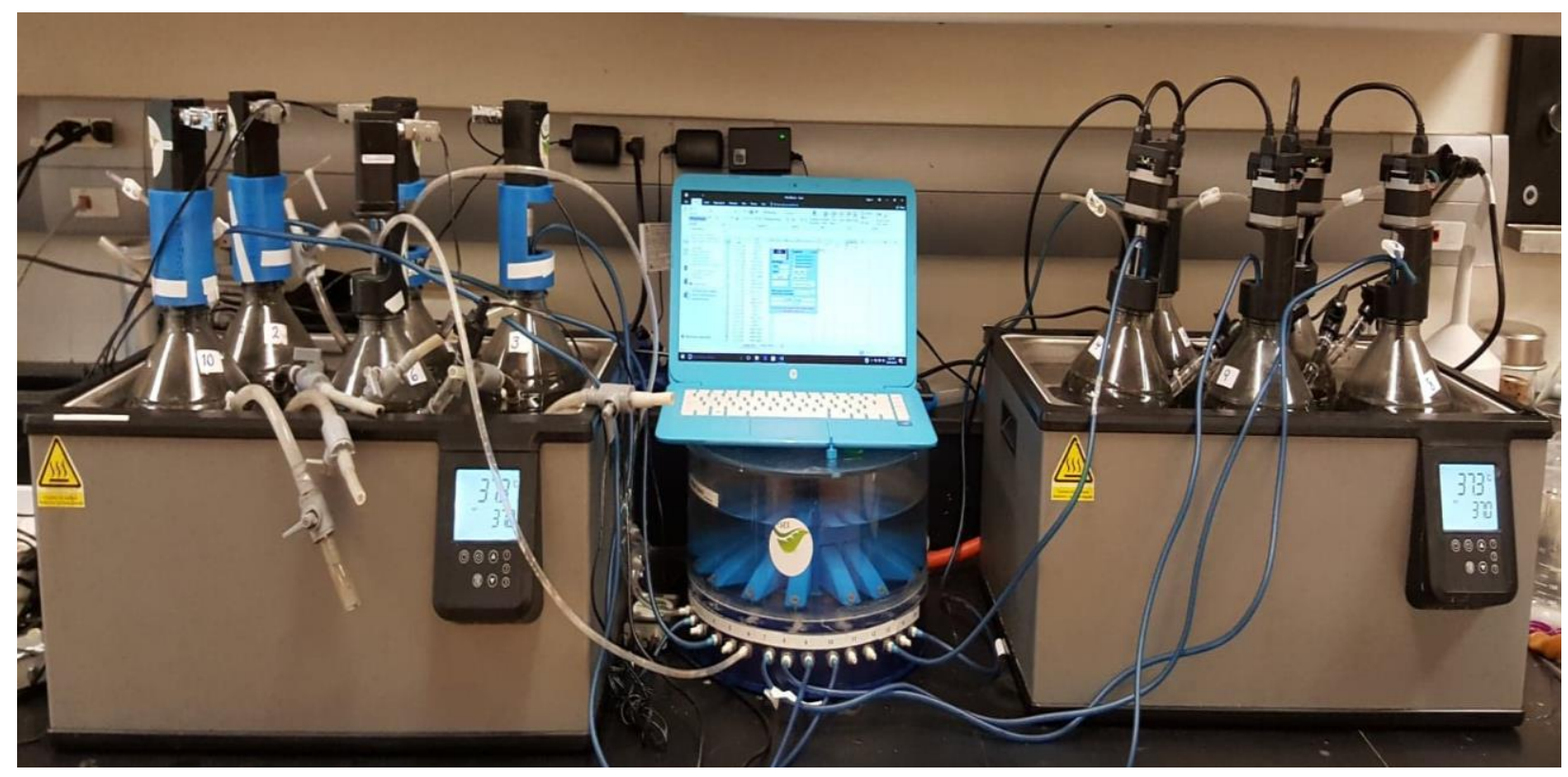

Figure 3.4. Experimental set-up for hydrolysis/acidification experiment

A first-order reaction model was applied using AquaSim 2.0 software to assess the effect of different mixing ratios on biodegradation rates of COD, proteins, lipids, and carbohydrates.

$\mathrm{r}_{\mathrm{su}}=\mathrm{dC} / \mathrm{dt}=-\mathrm{kC}$

Where $\mathrm{C}$ is the concentration $(\mathrm{mg} / \mathrm{L})$ of the parameters (TCOD, proteins, lipids, and carbohydrates) at time $t, k$ is first-order specific biodegradation rate constant $(1 / d)$ and $r_{\text {su }}$ is biodegradation rate (mg/L.d). Eq. 3.4 is derived by integration of Eq. (3.3).

$$
\mathrm{C}_{\mathrm{t}}=\mathrm{C}_{\mathrm{u}} \mathrm{e}^{-\mathrm{kt}}
$$

Where $t, C_{t}$ and $C_{u}$ are time $(d)$, concentration at time $t(\mathrm{mg} / \mathrm{L})$, ultimate particulate parameters (TCOD, proteins, lipids, and carbohydrates) in mg/L, respectively.

\subsection{Analytical analysis}

The analysis of solid contents of the feedstocks including total solids (TS), volatile solids (VS), total suspended solids (TSS) and volatile suspended solids (VSS) of the inoculum, TWAS, and SSO samples were determined according to the Standard Methods procedures (APHA, 2005). Chemical oxygen demand (TCOD) and (SCOD), ammonia, total nitrogen (TN) and total soluble nitrogen (TSN) were measured using a Hach spectrophotometer model 3900. For the measurement 
of the soluble content, samples were prepared by centrifuging at $9000 \mathrm{rpm}$ for $45 \mathrm{~min}$ and then the supernatant was filtered using microfiber filters with a pore size of $0.45 \mu \mathrm{m}$. The absorbance was set at the wavelengths of 600, 560 and $650 \mathrm{~nm}$ for the analysis of COD, ammonia, and alkalinity, respectively.

Total lipids concentration was measured by solvatochromatic method that rely upon a dye or mixture of dyes which change optical properties upon a change in condition of the solvent in which they are dissolved. In this test, there is an increase in fluorescence when there is an increased amount of dissolved lipids that form micelles or other structures. Solvatochromatic analysis was carried out at the fluorescence $405 \mathrm{~nm}$ mode using Vernier SpectroVis spectrometer and Logger Pro software. The data then exported as CSV files for further analysis. Figure 3.5 shows the graph created by Logger Pro at $405 \mathrm{~nm}$ wavelength and the absorbance for different concentrations of total lipids. The peak values of the curves corresponding to the lipids concentration of different standard samples with known concentrations were used to plot the calibration curve and get the curve equation. The measured absorbance values for the actual samples with unknown lipids concentrations were substituted into the calibration equation and was solved for the true value.

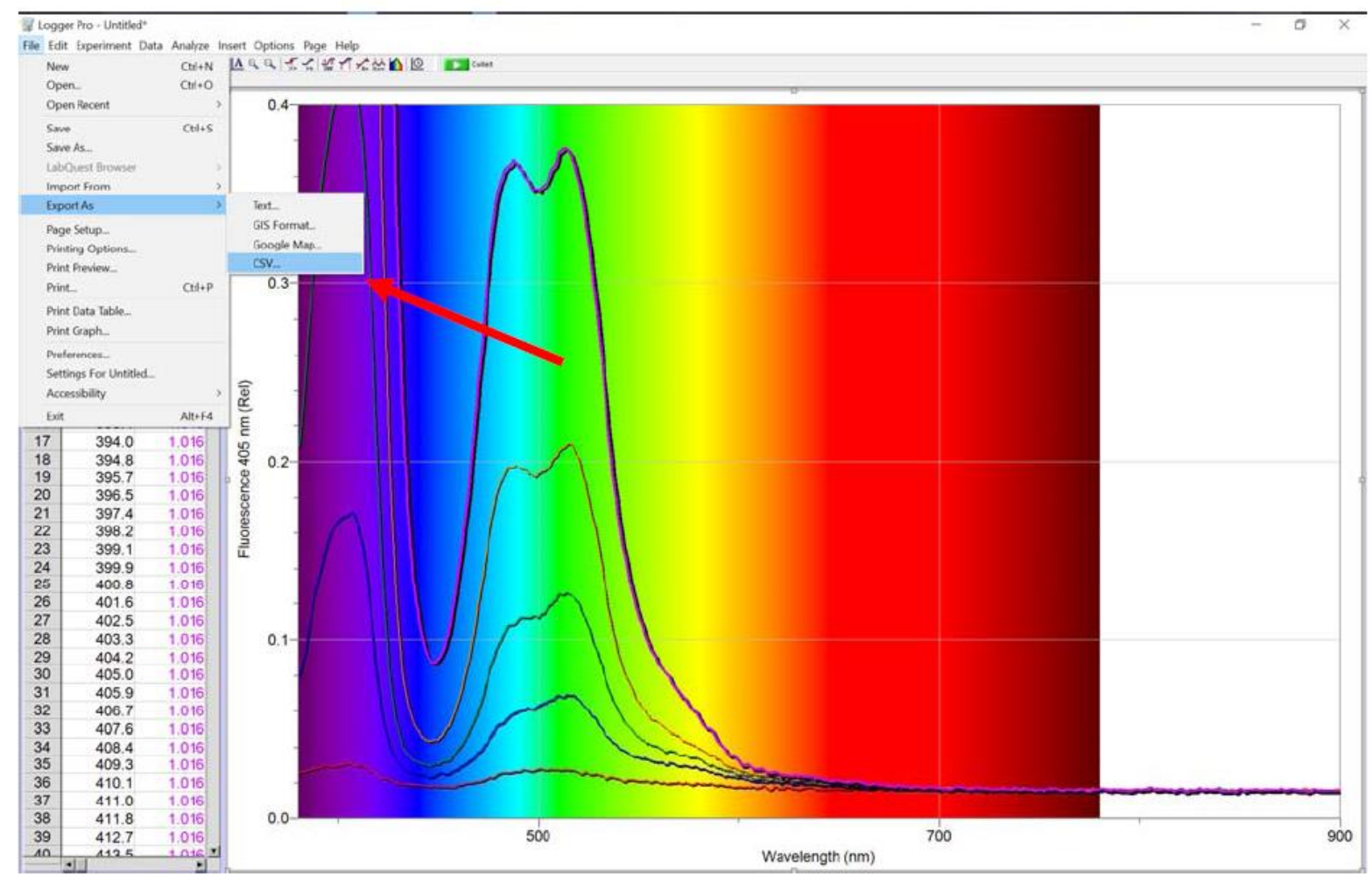

Figure 3.5. The spectra and flourometric data for different total lipids concentrations 
The volume of the produced gas was measured manually using a 100-mL Gastight Luer-Lock glass syringe daily at the beginning of the digestion period. The gas measurement was continued every couple of days later on when the gas production rate slowed down over time. The amount of biomethane in the produced biogas during the anaerobic digestion process was measured using a Thermo Scientific Trace 1310 gas chromatograph (GC). The GC was equipped with a thermal conductivity detector and the temperature of the oven, detector, and filament were set to 80, 100, and $250{ }^{\circ} \mathrm{C}$, respectively. The type of column used was a TG-Bond Msieve 5A model with a $30 \mathrm{~m}$ length and $0.53 \mathrm{~mm}$ diameter.

\subsection{Statistical and Kinetics analysis}

Statistical data including mean and standard deviation were calculated for the data obtained by the experiment. One way ANOVA for the analysis of variance was used to to find statistically significant differences between the group means and the results are presented in chapter 8 .

Gompertz equations provides a wide range of applications in process kinetics of anaerobic digestion and the methane potential studies. In this work, Modified Gompertz model (Elbeshbishy and Nakhla, 2012; Lay et al., 1999) was used to predict the biogas yield and to assess the kinetic parameters and to describe the progress of cumulative methane production through the batch process (eq. 3.52) where $\mathrm{CH}_{4}$ is the cumulative methane production $(\mathrm{mL}), \mathrm{P}$ is the ultimate methane production $(\mathrm{mL}), R_{m}^{e}$ is the maximum methane production rate $(\mathrm{mL} / \mathrm{d}), \lambda$ is the lag phase time (d), $t$ is the digestion time (d).

$\mathrm{CH}_{4}=\mathrm{p} \cdot \exp \left\{-\exp \left[\frac{R_{m}^{e}}{p}(\lambda-\mathrm{t})+1\right]\right\}$ 


\section{Chapter 4}

Results and discussion

\section{TWAS and SSO Co-digestion}




\section{Results and discussion- TWAS/SSO co-digestion}

\subsection{BMP of TWAS and SSO}

This experiment was designed to evaluate the effect of mixing ratio of TWAS with SSO and its relationship with the lipids: proteins: carbohydrates on anaerobic co-digestion process using TWAS and SSO in different combinations as digester feedstocks. Co-digestion of SSO with TWAS was conducted as explained in Chapter 3. The characteristics of the feed in each digester having different mixing ratios of the substrates are summarized in Table 4.1. As presented in Table 4.1, the amount of TCOD of SSO is remarkably higher than that of TWAS. Increasing the fractions of SSO, increased the TCOD of the feed to digesters. The total lipids and total carbohydrates contents of SSO are also significantly more than that of TWAS and therefore, by increasing the proportion of SSO in the co-digesters the concentrations of lipids and carbohydrates increased. $\mathrm{pH}$ was kept at neutral level from 7.0- 7.3 in all the mixtures to satisfy the favorable condition for methanogenesis.

Table 4.1. Mean values of the feed characteristics in digesters with different mixing ratios of TWAS and SSO

\begin{tabular}{|c|c|c|c|c|c|c|c|c|}
\hline & & TWAS Only & SSO Only & $\begin{array}{c}\text { TWAS:SSO } \\
9: 1\end{array}$ & $\begin{array}{c}\text { TWAS:SSO } \\
7: 3\end{array}$ & $\begin{array}{c}\text { TWAS:SSO } \\
1: 1\end{array}$ & $\begin{array}{c}\text { TWAS:SSO } \\
3: 7\end{array}$ & $\begin{array}{c}\text { TWAS:SSO } \\
1: 9\end{array}$ \\
\hline Parameters & Units & Mixture (1) & $\begin{array}{l}\text { Mixture } \\
\text { (2) }\end{array}$ & Mixture (3) & Mixture (4) & Mixture (5) & Mixture (6) & Mixture (7) \\
\hline TCOD & $\mathrm{g} / \mathrm{L}$ & 40 & 110 & 47 & 61 & 75 & 89 & 103 \\
\hline SCOD & $\mathrm{g} / \mathrm{L}$ & 1.4 & 44 & 5.7 & 14 & 23 & 32 & 40 \\
\hline TSS & $\mathrm{g} / \mathrm{L}$ & 32 & 54 & 34 & 38 & 43 & 47 & 52 \\
\hline VSS & $\mathrm{g} / \mathrm{L}$ & 26 & 39 & 27 & 30 & 32 & 35 & 37 \\
\hline TS & $\mathrm{g} / \mathrm{L}$ & 39 & 62 & 41 & 46 & 51 & 55 & 60 \\
\hline VS & $\mathrm{g} / \mathrm{L}$ & 35 & 44 & 35 & 37 & 39 & 41 & 43 \\
\hline Ammonia & $\mathrm{g} / \mathrm{L}$ & 0.3 & 1.1 & 0.3 & 0.5 & 0.7 & 0.9 & 1.0 \\
\hline $\mathrm{pH}$ & & 7.2 & 7.1 & 7.0 & 7.0 & 7.2 & 7.1 & 7.3 \\
\hline Alkalinity & $\mathrm{g} \mathrm{CaCO} 3 / \mathrm{L}$ & 2.0 & 6.1 & 2.4 & 3.2 & 4.0 & 4.8 & 5.7 \\
\hline $\mathrm{TN}$ & $\mathrm{g} / \mathrm{L}$ & 2.9 & 3.3 & 2.9 & 3.0 & 3.1 & 3.2 & 3.2 \\
\hline TSN & $\mathrm{g} / \mathrm{L}$ & 0.4 & 0.9 & 0.5 & 0.6 & 0.7 & 0.8 & 0.9 \\
\hline T-Carbs & $\mathrm{g} / \mathrm{L}$ & 1.1 & 144 & 2.4 & 5.1 & 7.7 & 10.4 & 13.0 \\
\hline T-Proteins & $\mathrm{g} / \mathrm{L}$ & 3.8 & 2.3 & 3.6 & 3.3 & 3.0 & 2.7 & 2.5 \\
\hline T-Lipids & $\mathrm{g} / \mathrm{L}$ & 0.4 & 1.7 & 0.5 & 0.8 & 1.0 & 1.3 & 1.6 \\
\hline
\end{tabular}


As shown in Fig. 4.1, no significant lag-phase occurred in the generation of biogas the reactors. Operation of the digesters continued until no significant biogas was produced. Fig. 4.1 shows the time-course profile of the cumulative biomethane production during the total operation period. As illustrated in the Figure 4.1, no significant lag time was observed for all the digesters. This no sign of significant inhibition would be due to the use of mesophilic inoculum acclimatized to municipal sludge streams similar to the one used in the experiment. The most lag-phase occurred at TWAS:SSO mixing ratios of $1: 1$, and 3:7, and 1:9 which could be due to the existence of particulate matters introduced by SSO to the mixture which could delay the hydrolysis phase and consequently affect methanogenesis.

The amount of biomethane produced by SSO was significantly higher than that of TWAS. Only $542 \mathrm{~mL}$ cumulative methane was produced by TWAS while the amount of cumulative methane obtained by SSO was $1101 \mathrm{~mL}$. This verified the low biodegradability of TWAS compared to SSO and would be due to the composition of TWAS as it mostly consists of proteins and humic substances with some bacterial biomass and carbohydrates. Although proteins, DNA and carbohydrates are anaerobically biodegradable, their biodegradability decreases when they are combined into an organized structure similar to TWAS (Gonzalez et al., 2018; Stuckey and McCarty, 1984). Microbial cells are difficult to break down under anaerobic digestion (Foladori et al., 2015; Wett et al., 2010) and similarly, the presence of humic substances affects enzymatic activity by immobilizing enzymes and as a result, lowers biodegradability (Azman et al., 2015a, 2015b; Fernandes et al., 2015).

Another reason for the low biodegradability of TWAS was investigated by a study, in which low digestibility was attributed to the slow hydrolysis process for the exterior polymeric component of the microbial culture within the sample. Furthermore, the study also found that the ratio of SCOD to TCOD was $34.6 \%$ for untreated TWAS compared to $63.6 \%$ and $68.1 \%$ for thermally and alkaline pretreated samples, respectively. The COD ratio was a clear indicator of the expected biogas production by the TWAS feedstock samples where lower biogas yield was reported for the untreated raw TWAS as compared to pretreated samples (Abudi et al., 2016a). 


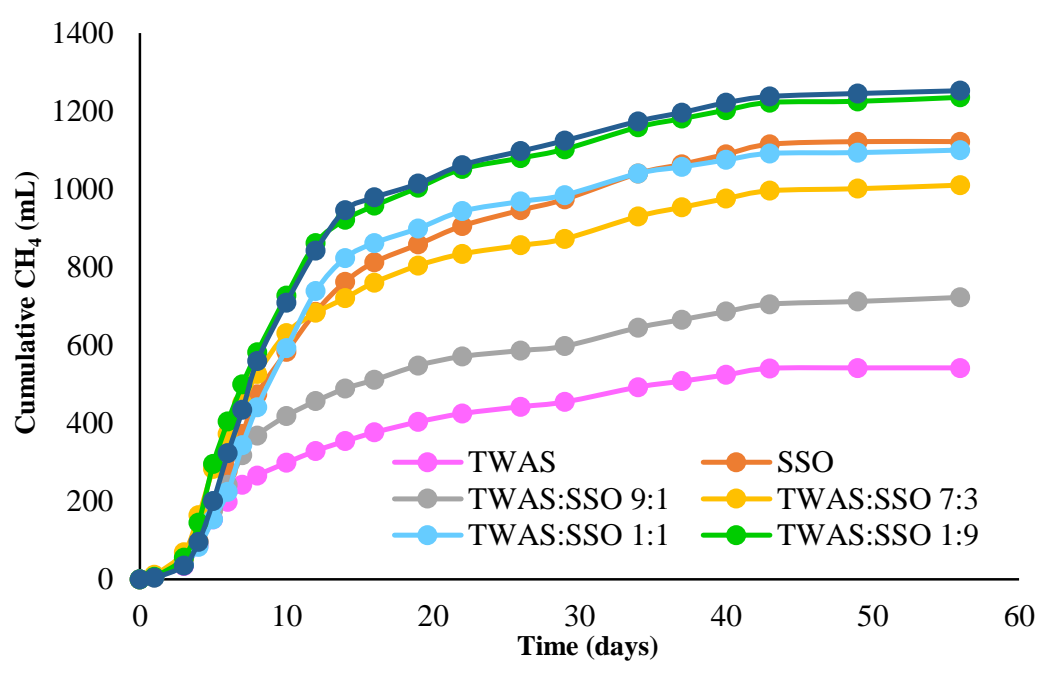

Figure 4.1. Cumulative methane production for different mixing ratios of TWAS and SSO

Addition of SSO to TWAS increased biodegradability and the methane yield compared to TWAS alone. However, as demonstrated in Figure 2, the trend showed an optimal mixing ratio of SSO with TWAS. The TWAS:SSO mixing ratio of 3:7 (V/V) delivered better results compared to TWAS:SSO mixing ratio of 1:9 (V/V) in terms of digestion process and methane enhancement.

Fig 4.2 shows the methane production rate in $\mathrm{mL} \mathrm{CH}_{4} / \mathrm{d}$ through the digestion period. Comparing TWAS and SSO, the methane production rate obtained by SSO was significantly higher than that of TWAS, although the maximum rate for the rectors digesting only TWAS occurred in earlier stage of the digestion process compared to the ones digested only SSO. Similar trend was observed in the digesters containing mixtures of TWAS and SSO so that increasing the fraction of SSO to the co-digesters, caused maximum methane production rate take place later than the co-digesters containing more fraction of TWAS. This could be as a result of abundant particulate matter that affects the hydrolysis rate and prolongs the entire process.

The biomethane data monitoring as presented in Figure 4.2, revealed that the highest portion of the biomethane was produced within the first month. The digesters generated 31-45\%, 65-76\% and $83-90 \%$ of their ultimate biogas productions during first 7, 14 and 30 days of operation, respectively. This would be due to the availability of sufficient nutrient right after the start of the 
operation which increases the metabolic activity of the microorganisms causing rapid conversion of substrate to biogas without inhibition in digesters (A. J. Li et al., 2011; Sung and Dague, 1995). The maximum methane production rate for mono and co-digestions is presented in Figure 4.3. The amount of maximum methane production rate of SSO mono digestion was $109 \mathrm{~mL} \mathrm{CH} / \mathrm{d}$ which was $60 \%$ higher that of TWAS mono digestion corresponding to $68 \mathrm{~mL} \mathrm{CH}_{4} / \mathrm{d}$. Comparing to TWAS mono digestion, increasing the percentage of SSO in the co-digesters containing TWAS:SSO mixtures from the ratio of $1: 9$ ratio to $3: 7$, increased the maximum methane production rate by $25 \%$ and $75 \%$, respectively. Further increasing the percentage of SSO in the co digesters at TWAS:SSO mixing ratios of 1:1, 1:9 and 3:7, significantly increased the maximum methane production rate both compared to TWAS and SSO mono digestion, although the maximum rate values were almost the same for the three mixing ratios.

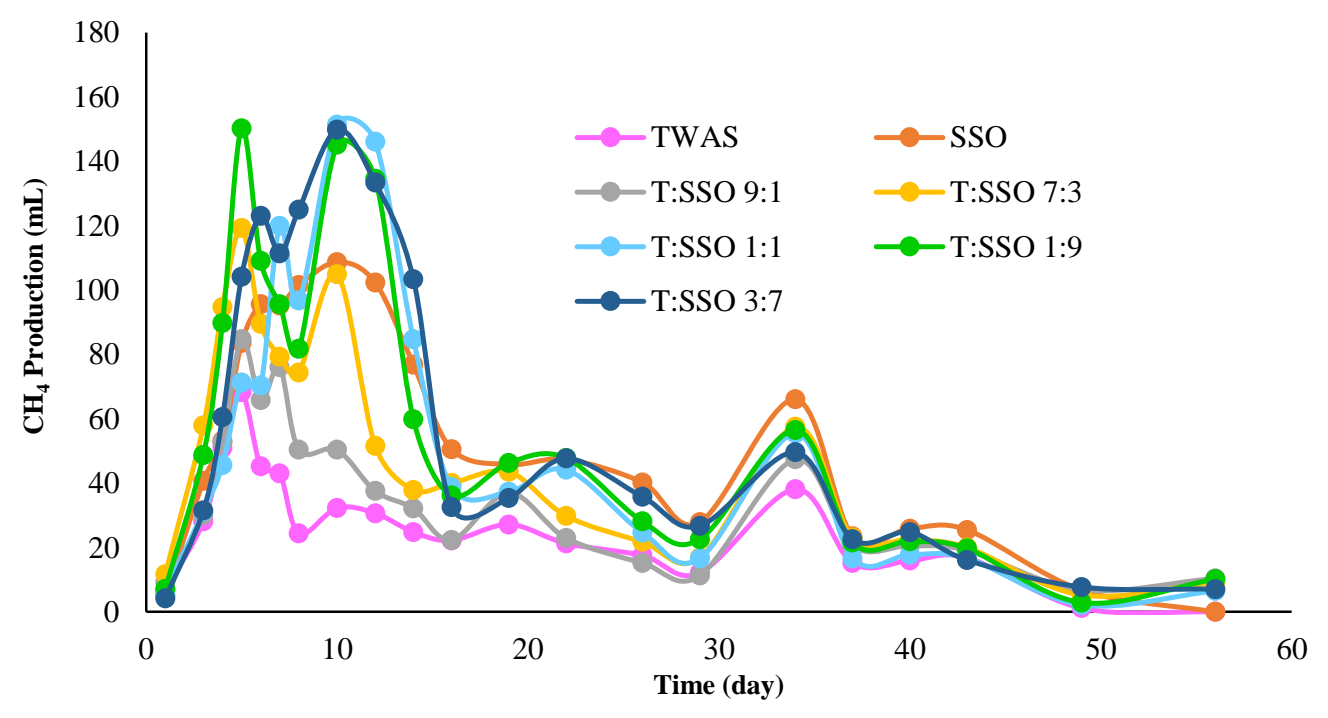

Figure 4.2. Methane production rate $(\mathrm{mL} / \mathrm{d})$ for different mixing ratios of TWAS and SSO

These findings as a matter of fact showed a close agreement to a previously conducted study (Abudi et al., 2016a), where the reported cumulative specific biogas yield (CSBY) for alternating ratios of organic fraction of municipal solid waste (OFMSW) and TWAS resulted in increasing biogas yield. As presented in Figure 4.3, the amount of maximum methane production rate was the highest for the TWAS:SSO mixing ratios of 1:1, 3:7, and 1:9 in a range between 150 to 151 
$\mathrm{mL} / \mathrm{d}$. However, the maximum biomethane production rate for the TWAS:SSO mixing ratio of 3:7 occurred on the day 5 of the operational period while it was observed on the day 10 for the mixing ratios of and 1:1 and 1:9. This would be an evident to the advantage of co-digestion while emphasizing the necessity of a proper mixing ratio of the substrates which fulfills both enhancing the methane production and the process kinetics.

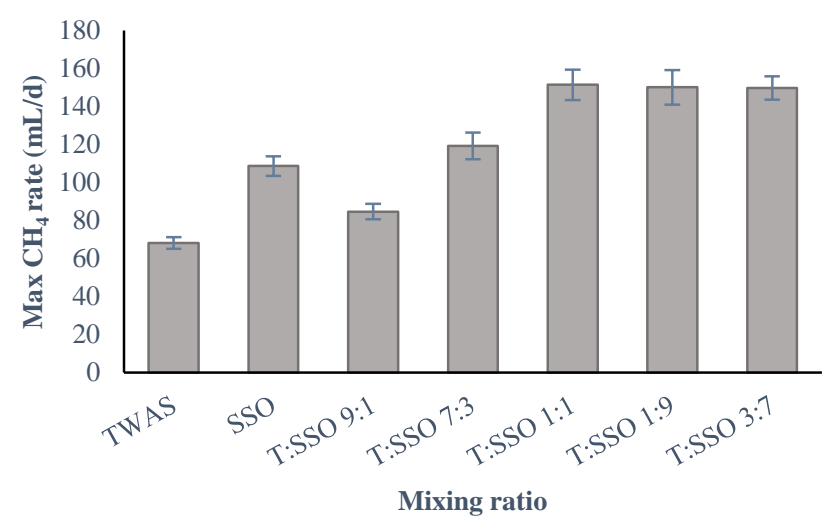

Figure 4.3. Maximum methane production rate $(\mathrm{mL} / \mathrm{d})$ for different mixing ratios of TWAS and SSO

A COD mass balance was conducted for all of the digesters to assess the accuracy of the experiment. The mass balance was carried out with reference to the initial and the final TCOD concentrations of the digester contents, and the theoretical methane production per unit mass of TCOD removed. Comparison between the experimental methane production attained by this research and that of determined by TCOD mass balance, verified a deviation of less than $10 \%$ for all the digesters.

\subsection{Cumulative methane yields}

Cumulative methane yields were calculated and presented in Figures 4.4 a), b) and c. The cumulative methane yield was normalized per substrate unit mass COD added (mLCH4/g TCOD added), per substrate unit mass VSS added ( $\mathrm{mLCH}_{4} / \mathrm{g}$ VSS added) and per unit volume of substrate added ( $\mathrm{mLCH}_{4} / \mathrm{mL}$ substrate added).

Figure 4.4 a) shows the result of cumulative methane yield per mass COD of substrate added for mono and co-digestions. Results showed that $192 \mathrm{mLCH}_{4} / \mathrm{g}$ TCOD added was obtained by TWAS mono digestion while a higher yield corresponding to $308 \mathrm{mLCH}_{4} / \mathrm{g}$ TCOD added was achieved 
by SSO alone. Mixing TWAS with SSO in co-digesters at all ratios excluding TWAS:SSO ratio of 9:1, increased the methane yield in comparison with both TWAS and SSO mono digestion. Even though, the ratio of 9:1 resulted in 27\% increase of the $\mathrm{CH}_{4}$ yield (mL) per mass of TCOD added compared to TWAS alone. The maximum cumulative methane yield was obtained by the 3:7 mixing ratio of TWAS:SSO corresponding to $358 \mathrm{~mL} \mathrm{CH}_{4} / \mathrm{g}$ TCOD added being 85\% and $16 \%$ higher than that of TWAS and SSO alone. It was observed that addition of SSO to co-digesters significantly increased the methane yield compared to mono digestion of TWAS while it did not remarkably increased the yield in comparison with SSO mono digestion.

\section{4. a)}

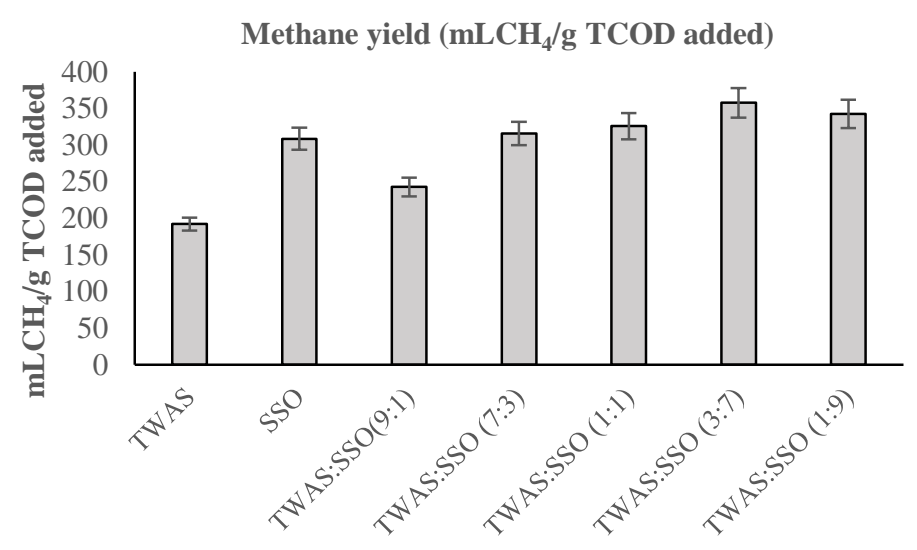

Mixing ratio

4.4. b)

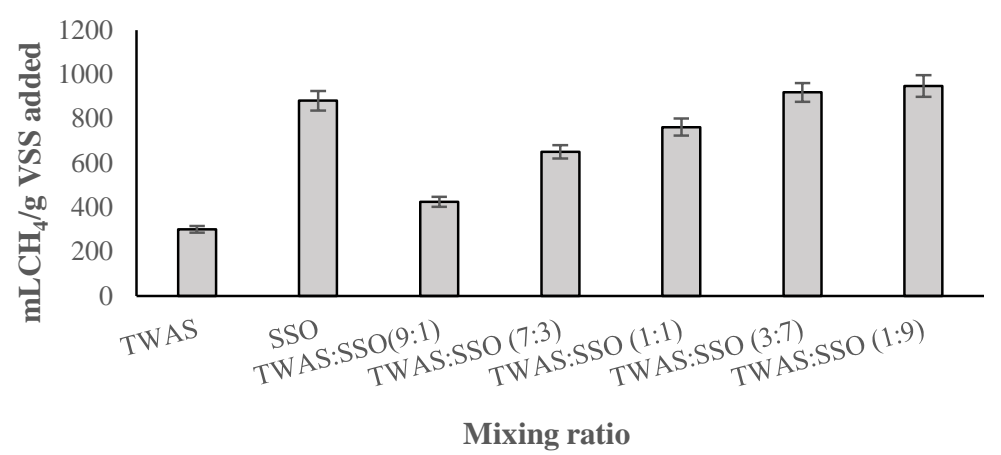


4.4. c)

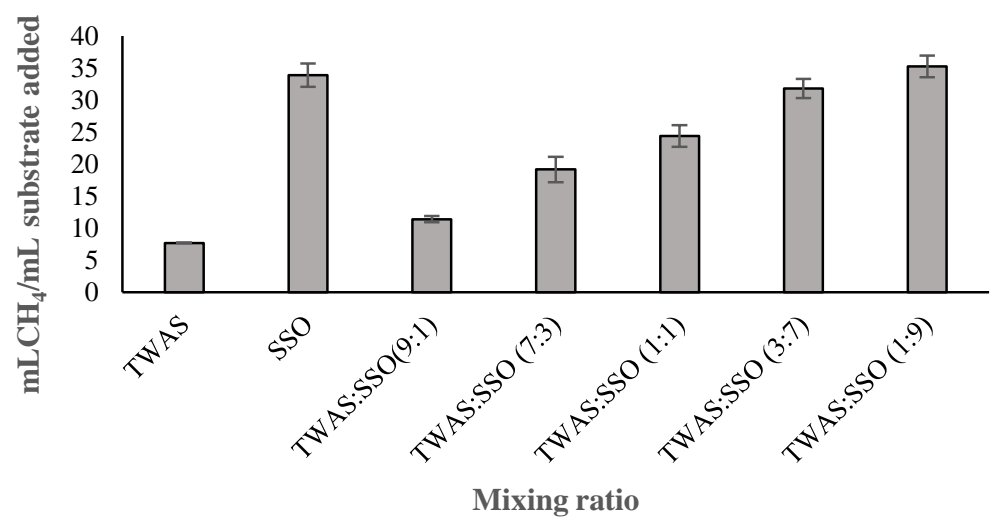

Figure 4.4. Methane yields: a) per unit mass TCOD added, b) per unit mass of VSS added and c) per unit volume of substrate added at different mixing ratio of TWAS and SSO

The yields of cumulative methane in terms of $\mathrm{mLCH}_{4} / \mathrm{g}$ VSS added have been presented in Figure 4.4. b). Similarly, the methane yield per mass of VSS added by SSO mono digestion was significantly higher than that of TWAS. A $300 \mathrm{mLCH}_{4} / \mathrm{g} \mathrm{VSS}$ added and $892 \mathrm{mLCH}_{4} / \mathrm{g}$ VSS added were attained by TWAS and SSO alone, respectively. All co-digesters produced higher methane yield per mass of VSS added compared to TWAS alone. However only the TWAS:SSO mixing ratios of 1:9 and 3:7 resulted in higher methane yields per mass of VSS added in comparison with both TWAS and SSO alone. The maximum methane yield of $975 \mathrm{mLCH}_{4} / \mathrm{g}$ VSS added occurred at the TWAS:SSO mixing ratio of 3:7.

It was revealed that addition of SSO to TWAS significantly increased biomethane production in the rectors co-digesting them compared to the control reactor digesting only TWAS. However, only the TWAS:SSO mixing ratio of 3:7 resulted in the increase of the methane yield compared to both TWAS and SSO mono digesters.

Eq. 4.1 was used for assessing the biodegradable fraction of the feedstocks for each of the rectors and the results are illustrated in Figure 4.5. 
Where $\mathrm{BF}$ is biodegradable fraction, $\mathrm{BM}$ experimental is the measured biomethane by the experiment and $\mathrm{BM}_{\text {theoretical }}$ is the theoretical biomethane production in $\mathrm{mL} \mathrm{CH}_{4}$ per $\mathrm{g}$ TCOD of the substrate in mesophilic condition and standard pressure.

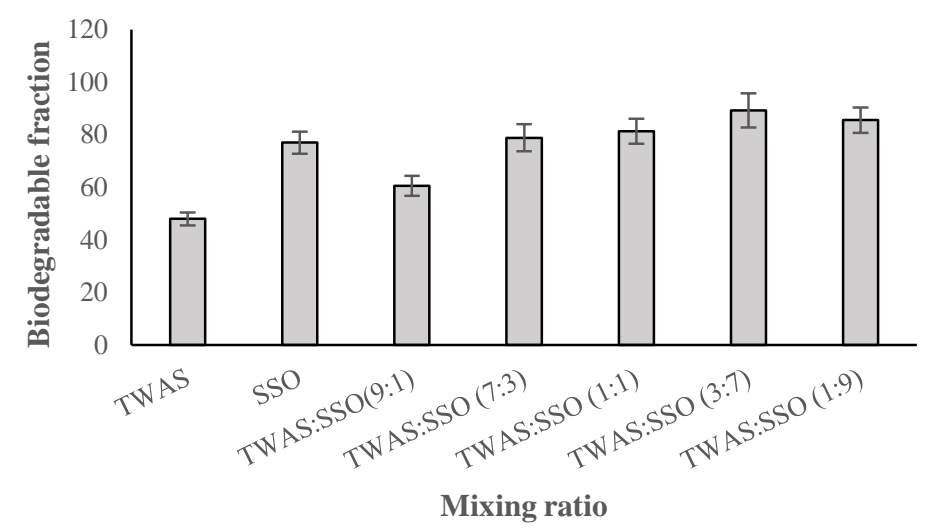

Figure 4.5. Biodegradable fraction of the feedstocks for different mixture ratios of TWAS and SSO

As shown in Figure 4.5, the TWAS:SSO mixing ratio of 3:7 corresponded to the maximum percentage of biodegradable fraction which complies with the maximum methane yield at the same mixing ratio. BF was $48 \%$ and $77 \%$ for TWAS and SSO, respectively. It was verified that addition of SSO as co-substrate increased biodegradability and enhanced the production of methane in the rectors co-digesting SSO with TWAS. All co-digesters had higher BF than TWAS alone. The reason would be the existence of readily biodegradable compounds in SSO that was introduced to the co-digesters. At the mixing ratio of 3:7, the BF was $89 \%$ which was $85 \%$ and $16 \%$ higher than that of TWAS and SSO alone respectively. The trend of BF changes in digesters complies with the methane yields obtained by the experimental results for the corresponding digesters.

\subsection{Synergistic effect}

In anaerobic digestion, production of biomethane develops through a syntrophic metabolism between both communities of methanogens including bacteria and archaea (Viotti et al., 2004). It is evident that both communities of bacteria and archaea are present in AnCoD systems. An 
improvement in the synergy and diverse microbial consortia is obtained when applying codigestion of multiple feedstocks (Zamanzadeh et al., 2017). The synergistic effect of co-digestion can be estimated as an additional methane production $(\mathrm{mL})$ for co-substrates over the weighted average of the methane production of individual substrates (Parra-Orobio et al., 2016). In this research, in order to investigate the synergetic effect of microbial populations on anaerobic codigestion of TWAS and SSO at different mixing ratios, the weighted methane production (MP) of co-substrates were calculated using Eq. (4.2):

$$
\text { Weighted } \mathrm{MP}=\mathrm{MP}_{\mathrm{SSO}} * \mathrm{P}_{\mathrm{SSO}}+\mathrm{MP}_{\mathrm{TWAS}} * \mathrm{P}_{\mathrm{TWAS}}
$$

Where weighted MP is the weighted average of methane production for co-substrates $\left(\mathrm{mLCH}_{4}\right)$; $\mathrm{MP}_{\text {SSO }}$ and $\mathrm{MP}_{\text {TWAS }}$ are the experimental methane production $\left(\mathrm{mLCH}_{4 /} \mathrm{mL}\right.$ substrate added) for SSO and TWAS; and PSSO and PTWAS are the volume $(\mathrm{mL})$ of SSO and TWAS in the substrate's mixture, respectively. When the percentage difference between experimental methane production for the mixtures and the calculated weighted average of methane production was positive, the synergistic effect could be concluded. Figure 4.6 shows the percentage of additional methane production for co-substrates over the weighted average of the methane production of individual substrates. As revealed in Fig 4.6, the maximum synergetic impact was observed in co-digestion of TWAS with SSO at the mixing ratio of 3:7. This is in good agreement with the maximum methane yield that occurred at the same mixing ratio of TWAS and SSO in their co-digestion.

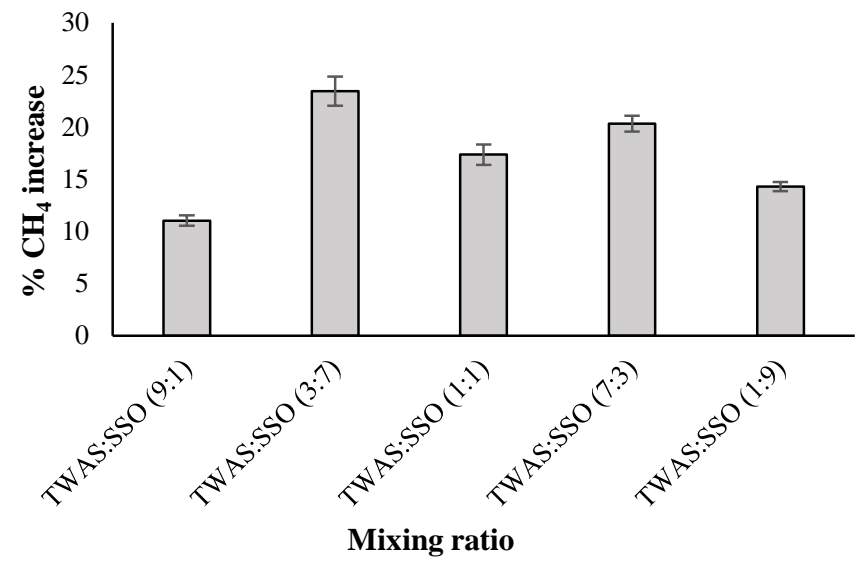

Figure 4.6. Synergetic effect of co-digestion at different mixing ratios of TWAS and SSO 
Although all of the mixings demonstrated the synergetic impact of co-digestion on improving biomethane production, no specific trend for the change of the synergetic effect and methane increase corresponding to the fraction of SSO in the co-digestion mixtures was observed. The percentage of methane increase due to the synergetic impact varied from 11 to 23 . The most percentage of biomethane increase of $23 \%$ as a result of the improved synergy occurred at the mixing ratio of 3:7. This is in good compliance with the results of maximum cumulative methane rate and the maximum methane yield which were achieved at the same mixing ratio. Although introducing fractions of SSO added more amounts of readily biodegradable materials to the codigesters, the more fractions of SSO did not necessarily satisfied the optimum condition for the process improvement.

The improvement of the synergy and biogas yield could be as a result of diverse microbial consortia introduced by applying co-digestion of multiple feedstocks. Nevertheless, enhanced synergy would be dependent on some factors relying on the proper mixing ratios which satisfy the optimal nutrients balance and effective conditions for microbial syntrophy. This could be the reason for the optimum fraction of TWAS and SSO at the mixing ratio of 3:7 in the co-digestion rather than other ratios.

\subsection{COD: $N$ and lipids: proteins: carbohydrates ratios}

The relationship between the COD:N ratios as well as lipids: proteins: carbohydrates ratios at different mixing ratios of TWAS and SSO with methane yield and ultimate methane production is presented in Table 4.2. The table also shows a comparison between the COD:N ratios with lipids: proteins: carbohydrates ratios for the digesters fed with different mixings of TWAS and SSO in volumetric basis. In co-digestion of TWAS and SSO, the COD:N ratio above 20 resulted in higher ultimate methane production, however, variation of the ratios from 28 to 34 caused a reduction of the amount of the produced methane. As shown in the table, the amounts of ultimate methane production were in a range between 542 to $1252 \mathrm{~mL}$ for different mixing ratios. The lowest ultimate methane production of $542 \mathrm{~mL}$ corresponded to a COD:N ratio of 14 and a lipids: proteins: carbohydrates ratio of 1:11:3 which occurred at TWAS:SSO mixing ratio of 1:0 in the reactors digesting TWAS alone. The maximum ultimate $\mathrm{CH}_{4}$ corresponded to COD:N ratio of 28 associated with the lipids: proteins: carbohydrates ratio of 1:2:8 for the reactors co-digesting TWAS and SSO at the mixing ratio of 3:7 (v/v). 
Considering the fact that lipids, proteins and carbohydrates are the main constituents of any organic material, considering the ratios of lipids: proteins: carbohydrates would be a good approach for optimizing the mixing ratios of the substrates. In addition, the optimal C:N or COD:N for some types of the feedstocks have shown to be very different from the generally observed the optimal values.

As presented in Table 4.2, the ratio of COD:N was 14 for TWAS alone while it was higher by 2.4 fold for SSO. The increase of the ratio of COD to $\mathrm{N}$ from 14 to 16, increased ultimate methane from 542 to $723 \mathrm{~mL}$. This increase of methane production could be due to the sufficient amounts of nutrient for microbial activities which led to enhanced biomethane production. On the other hand, an optimal nutrient synergy is required to ensure synergetic interactions of the microbial communities as the increase of COD:N ratio from 28 to 34, decreased the amount of ultimate $\mathrm{CH}_{4}$.

Table 4.2. Ultimate $\mathrm{CH}_{4}$ at different ratios of the substrates, COD:N, and lipids:proteins:carbohydrates

\begin{tabular}{ccccccc}
\hline Digester code & $\begin{array}{c}\text { TWAS: SSO } \\
\text { (V/V) }\end{array}$ & COD:N & $\begin{array}{c}\text { Feedstock } \\
\text { ratios code }\end{array}$ & $\begin{array}{c}\text { Lipids: Proteins: } \\
\text { Carbohydrates }\end{array}$ & $\begin{array}{c}\text { Ultimate } \\
\mathrm{CH}_{4}(\mathrm{~mL})\end{array}$ & $\begin{array}{c}\mathrm{mLCH}_{4} / \mathrm{g} \\
\text { TCOD added }\end{array}$ \\
\hline TWAS Only & $1: 0$ & 14 & AA & $1: 11: 3$ & 542 & 192 \\
SSO Only & $0: 1$ & 34 & BB & $1: 1.3: 8$ & 1122 & 308 \\
$\begin{array}{c}\text { TWAS:SSO } \\
\text { 9:1 }\end{array}$ & $9: 1$ & 16 & A & $1: 7: 5$ & 723 & 243 \\
$\begin{array}{c}\text { TWAS:SSO } \\
\text { 7:3 }\end{array}$ & $7: 3$ & 20 & B & $1: 4: 7$ & 1010 & 316 \\
$\begin{array}{c}\text { TWAS:SSO } \\
1: 1\end{array}$ & $1: 1$ & 24 & $\mathrm{C}$ & $1: 3: 7$ & 1100 & 326 \\
$\begin{array}{c}\text { TWAS:SSO } \\
\text { 3:7 }\end{array}$ & $3: 7$ & 28 & $\mathrm{D}$ & $1: 2: 8$ & 1252 & 358 \\
$\begin{array}{c}\text { TWAS:SSO } \\
1: 9\end{array}$ & $1: 9$ & 32 & $\mathrm{E}$ & $1: 1.5: 8$ & 1235 & 343 \\
\hline \hline
\end{tabular}

The main effect plot for $\mathrm{CH}_{4}$ yield data means in response to the feedstock ratios at different lipids:proteins:carbohydrates ratios is shown in Fig 4.7. As the trend shows, both type of the feedstock and their ratios have significant effect on the methane yield. Each of the feedstock ratios correspond to a different lipids:proteins:carbohydrates ratio. As shown in Figure 4.7, the minimum methane yield corresponds to the mono digestion of TWAS which occurred at the 
lipids:proteins:carbohydrates ratio of 1:11:3. The maximum $\mathrm{CH}_{4}$ yield corresponded to TWAS:SSO ratio of 3:7 corresponding to the lipids: proteins: carbohydrates ratio of 1:2:8.

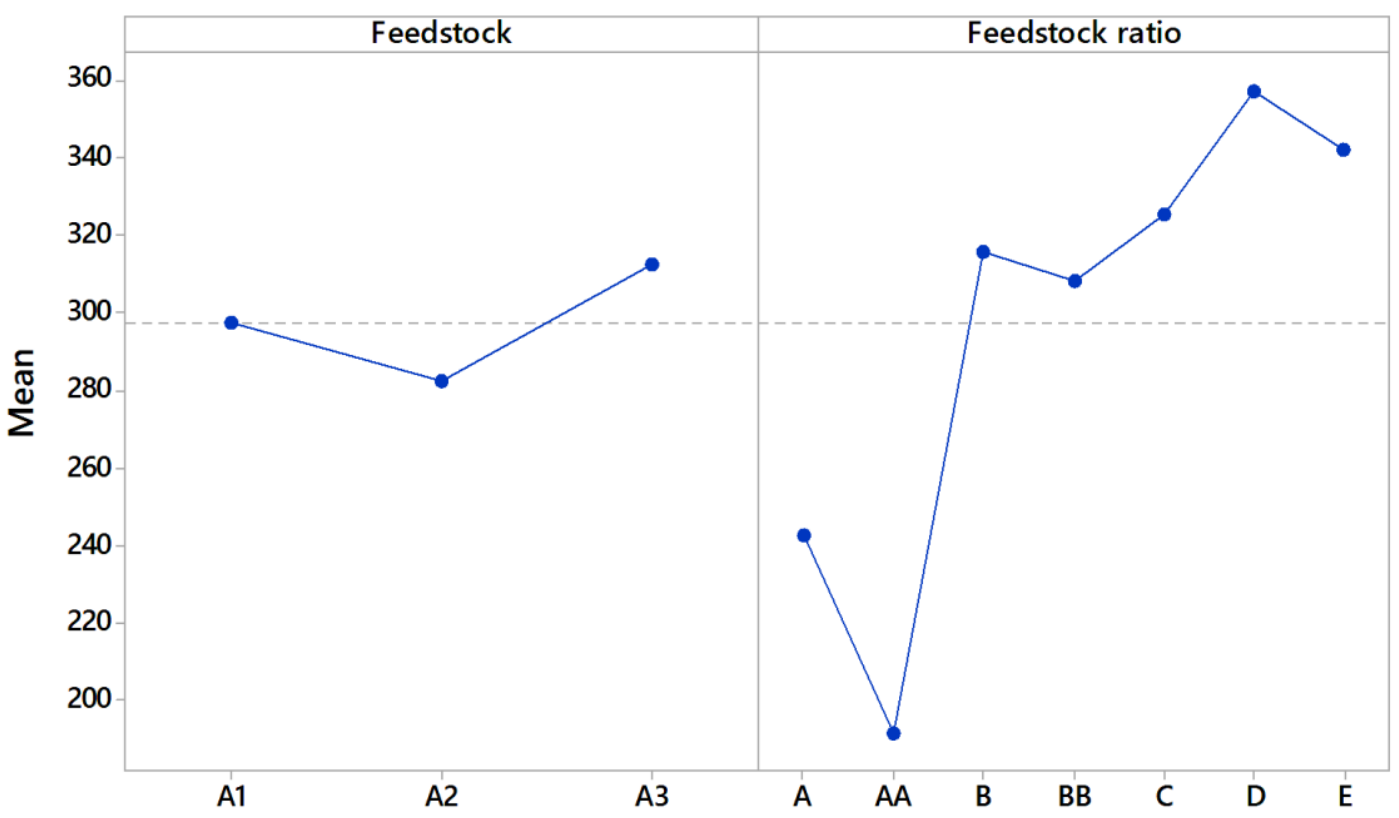

Figure 4.7. Main effects polot for $\mathrm{CH} 4$ yield data means in response to feedstock ratios at different lipids:proteins:carbohydrates ratios

A comparison between COD: $\mathrm{N}$ and the ratios of lipids: proteins, lipids: carbohydrates, and proteins:carbohydrates is presented in Figure 4.8. As illustrated in Figure 4.8 a) and 4.8 b), a same trend for the ultimate methane production in response to both COD:N ratios and lipids: proteins ratios was observed for the corresponding feedstocks mixing ratios. However, the COD:N ratio did not show the same trend as lipids: carbohydrates and proteins: carbohydrates ratios for the same feedstocks mixings, so that the minimum ultimate $\mathrm{CH}_{4}(542 \mathrm{~mL})$ corresponded to the minimum COD:N ratio of 14 while it corresponded to maximum lipids: carbohydrates of 0.32 as well as the maximum proteins: carbohydrates ratio of 3.48. Both lipids to carbohydrates and proteins to carbohydrates ratios showed the same trends at the corresponding feedstocks mixing ratios so that the maximum ultimate methane of $1252 \mathrm{~mL}$ occurred at the minimum lipids: carbohydrates of 0.12 and the minimum proteins: carbohydrates of 0.19 . Similar trends were observed for the $\mathrm{CH}_{4}$ yield ( $\mathrm{mLCH}_{4} / \mathrm{g}$ TCOD added) in response to the COD:N, lipids: proteins, lipids: carbohydrates and proteins: carbohydrates ratios. 


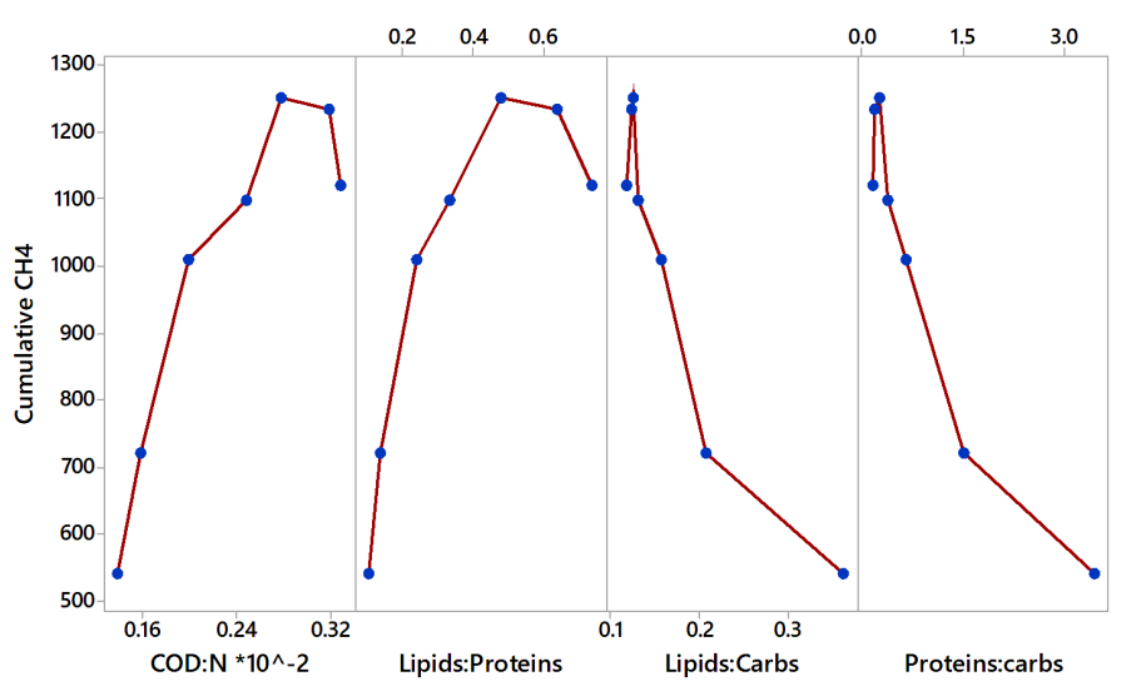

4.8. a

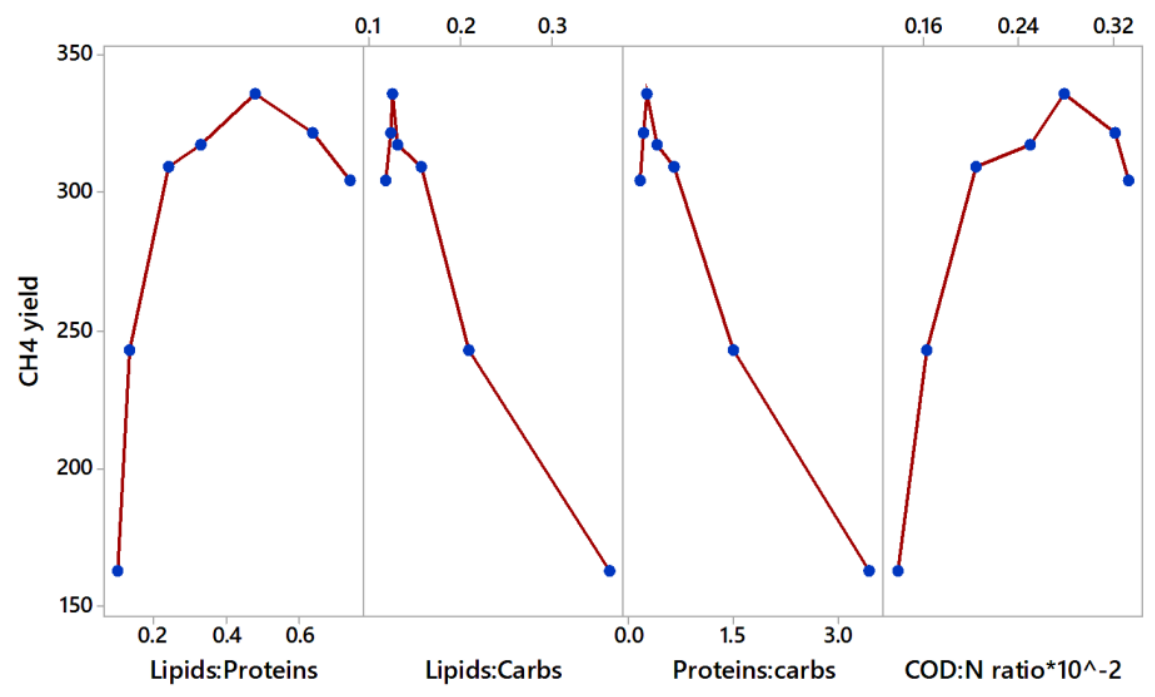

4.8. $\mathrm{b}$

Figure 4.8. Matrix effects plot for: a. ultimate $\mathrm{CH}_{4}$ and $b . \mathrm{CH}_{4}$ yield at different $\mathrm{COD:N}$ and Lipids: Proteins; lipids:carbohydrates and proteins:cabohydrates for the same corresponding mixing ratios of TWAS:SSO

\subsection{Kinetic analysis results}

The modified Gompertz model according to Eq. 3.5 was used to determine the coefficients for cumulated methane production and the results have been presented in Table 4.4. The modified Gompertz model could identify significant parameters related to anaerobic digestion including the maximum methane production rate, maximum methane production and lag phase, which 
underlines the time when the substrate is transformed and its correlation with the methane production phase. The volume data for the controls and for each mixing ratio collected during the experiment were used to apply the modified Gompertz model. The model corresponds to a sigmoid function expressing methane production in the reactor as a function of time (Lay et al., 1999; ParraOrobio et al., 2016).

Applying the collected data for cumulative methane production $\left(\mathrm{CH}_{4}\right)$ per unit substrate in $\mathrm{mL} / \mathrm{g}$ and the digestion time (t) in days to the Gompertz equation, the values of $\mathrm{P}$, representing the maximum methane production per unit substrate $(\mathrm{mL} / \mathrm{g}), R_{m}^{e}$, the maximum methane production rate $(\mathrm{ml} / \mathrm{g} \mathrm{h})$, and $\lambda$, the lag phase time (d) were calculated. The determination coefficient $\left(\mathrm{R}^{2}\right)$ as presented in Table 4 was used as criterion to evaluate the fitted models. The modified Gompertz model for mono- and co-digestions, respectively showed a good fit to the experimental results and the estimated parameters indicated that the co-digestion of SSO with TWAS improved the biogas production rate (Figure 1). The value of $\mathrm{R}^{2}=0.9998$ indicates that the proposed equations can accurately describe the variation of methane yield curves. Typically, S-shaped curves are obtained by the modified Gompertz model which shows a relatively slow upward trend related to the lag phase at the beginning of the curve. Therefore, the lag phase time for all of the TWAS/SSO codigestion mixtures could verify the suitability of the modified Gompertz model in estimated performance of the process.

Table 4.3. Summary of results of kinetic study using modified Gompertz models.

\begin{tabular}{ccccc}
\hline & $\mathrm{P}(\mathrm{mL})$ & $\boldsymbol{R}_{\boldsymbol{m}}^{\boldsymbol{e}}(\mathrm{mL} / \mathrm{d})$ & $\lambda(\mathrm{d})$ & $\mathrm{R}^{2}$ \\
\hline \hline TWAS & 510 & 28 & 0.1 & 0.999 \\
\hline SSO & 1063 & 68 & 2.0 & 0.999 \\
\hline TWAS:SSO 9:1 & 661 & 44 & 1.0 & 0.999 \\
\hline TWAS:SSO 7:3 & 938 & 68 & 1.1 & 0.999 \\
\hline TWAS:SSO 1:1 & 1046 & 84 & 3.1 & 0.999 \\
\hline TWAS:SSO 3:7 & 1182 & 95 & 2.7 & 0.999 \\
\hline TWAS:SSO 1:9 & 1165 & 89 & 1.9 & 0.999 \\
\hline
\end{tabular}


With reference to the adjustment to the Gompertz model, the lag phase varied from 0.1 to 3.1 days for different substrate mixing ratios. The shortest lag phase of $0.1 \mathrm{~d}$ corresponded to TWAS alone digestion. This result would be due to the existence of anaerobic inoculum that are easily assimilated by the microorganisms during this phase and cause rapid acclimatization to the substrate, which can be observed in the methane production. In theory, the inoculum activity, the amount of readily degradable constituent, and the initial $\mathrm{pH}$ of the feedstock affects the AD startup time (Kafle and Chen, 2016).

The results showed that with the increase of the proportion of SSO in TWAS/SSO co-digestion, a longer lag phase time occurred. This trend stopped with further increasing the SSO proportion to the mixing ratio of 1:9. However, the mixing ratio of 3:7 resulted in slightly higher value of $P$ which corresponds to the experimental results that showed the maximum methane production occurred at the mixing ratio of 3:7. Although, a long lag phase time is not favorable as it would increase the residence time and consequently, larger reactor volumes and higher costs during implementation and operation would be required.

The values of $\mathrm{P}$, varied from 509.5 to $1182 \mathrm{~mL}$ corresponding to TWAS alone and TWAS:SSO mixing ratio of 3:7. The trend of changes in the $\mathrm{P}$ values complies with the experimental results as it showed an increasing trend in methane production by increasing the proportion of SSO. Similar to the experimental results, the trend changed from TWAS:SSO mixing ratio of 3:7 to 1:9 so that the ratio of 3:7 corresponded a higher $P$ value for methane production than that $1: 9$. The values obtained for $\mathrm{R}_{\max }^{\mathrm{e}}$ were within a range from 28 to $95 \mathrm{ml} / \mathrm{d}$. The values of $\mathrm{R}_{\max }^{\mathrm{e}}$ and $\mathrm{P}_{\max }$ obtained by Gompertz for the TWAS:SSO ratios of 3:7 were $95 \mathrm{~mL} / \mathrm{d}$ and $1182 \mathrm{~mL}$, respectively.

In co-digestion of TWAS and SSO, the COD:N ratio above 20 resulted in higher ultimate methane production, however, the difference in the ratios from 21 to 26 did not have significant effect on the amounts of the produced methane. Therefore, considering lipids: proteins: carbohydrates ratios would be a good approach for optimizing the mixing ratios of the substrates. A lipids: proteins: carbohydrates ratio of 7:1:15 corresponding to COD:N ratio of 26 , resulted in an ultimate biomethane production of $1252 \mathrm{~mL}$ in TWAS and SSO co-digestion. It was verified that codigestion of TWAS and SSO improved methane production in comparison with conventional single digestion of the feedstocks. It was concluded that cumulative methane production and methane yields varied at different mixing ratios of TWAS and SSO. Co- digestion of TWAS and SSO at the mixing ratio of 3:7 resulted in a biomethane yield of $353 \mathrm{~mL} \mathrm{CH}_{4} / \mathrm{g}$ TCOD added which 
is corresponding to an increase of methane yield by $84 \%$ and $15 \%$ compared to TWAS and SSO alone. A 23\% increase in methane yield was observed as a result of synergetic effect of codigestion at TWAS:SSO mixing ratio of 3:7 (v/v) equivalent to lipids: proteins: carbohydrates ratio of 1:2:8. This study verified the advantage of co-digestion over conventional single digestion of the feedstocks in terms of biomethane improvement and increased microbial synergy resulting higher methane production. Further studies utilizing a range of various feedstocks is required to promote the viability of lipids: proteins: carbohydrates ratio for optimizing the anaerobic digestion process and for optimizing the mixing ratios in anaerobic co-digestion of multiple feedstocks.

\section{6. Hydrolysis/acidification}

This experiment was carried out to evaluate the hydrolysis/acidification stage in anaerobic codigestion of TWAS with SSO. In hydrolysis which is known to be the rate limiting stage in the entire anaerobic digestion process, the large complex organic polymers including lipids, proteins, and carbohydrates break down to simple smaller molecules such as fatty acids, amino acids and sugars. Subsequently, acidogenic microorganisms break down the by-product of hydrolysis to further smaller molecules in acidification step (Gould, 2014). Therefore, this part of the present research was aimed to investigate the degradation of organic compounds in hydrolysis stage through the analysis of the degree of solublization, synergetic effect of co-digestion at different mixing ratios on hydrolysis and liquefaction, volatile fatty acids (VFAs) yield, and kinetics of lipids, proteins, and carbohydrates in co-digestion of TWAS and SSO.

A series of the analysis for the characterization of the feedstocks was primarily carried out in triplicates and the mean values are summarized in Table 4.4. As presented in Table 4.4, the COD concentration of SSO is higher than that of TWAS. Adding SSO to TWAS in the co-digesters, increased the COD concentrations compared to TWAS mono digestion. SSO also contains higher amounts of carbohydrates and lipids than TWAS while the proteins concentration of TWAS is more than SSO by 30\%. The concentrations of total, soluble and particulate COD; total, soluble and particulate proteins; and total, soluble and particulate carbohydrates were monitored over time for calculating their hydrolysis rate coefficients. 
Table 4.4. Characteristics of the feedstocks at different mixing ratios of TWAS and SSO

\begin{tabular}{ccccccccc}
\hline & & TWAS & SSO & T/SSO & T/SSO & T/SSO & T/SSO & T/SSO \\
& & Only & Only & $9: 1$ & $7: 3$ & $1: 1$ & $3: 7$ & $1: 9$ \\
\hline \hline \multirow{2}{*}{ Parameters } & \multirow{2}{*}{ Units } & Mixture & Mixture & Mixture & Mixture & Mixture & Mixture & Mixture \\
& & $(1)$ & $(2)$ & $(3)$ & $(4)$ & $(5)$ & $(6)$ & $(7)$ \\
\hline TCOD & $\mathrm{g} / \mathrm{L}$ & 40 & 110 & 47 & 61 & 75 & 89 & 103 \\
\hline SCOD & $\mathrm{mg} / \mathrm{L}$ & 0.4 & 44.4 & 4.8 & 13.6 & 22.4 & 31.2 & 40.0 \\
\hline TSS & $\mathrm{mg} / \mathrm{L}$ & 31.5 & 53.8 & 33.7 & 38.2 & 42.6 & 47.1 & 51.6 \\
\hline VSS & $\mathrm{mg} / \mathrm{L}$ & 18.5 & 36.0 & 20.2 & 23.7 & 27.2 & 30.8 & 34.3 \\
\hline TS & $\mathrm{mg} / \mathrm{L}$ & 38.8 & 62.2 & 41.1 & 45.8 & 50.5 & 55.2 & 59.8 \\
\hline VS & $\mathrm{mg} / \mathrm{L}$ & 34.5 & 43.5 & 35.4 & 37.2 & 39.0 & 40.8 & 42.6 \\
\hline Ammonia & $\mathrm{mg} / \mathrm{L}$ & 0.3 & 1.1 & 0.3 & 0.5 & 0.7 & 0.9 & 1.0 \\
\hline $\mathrm{pH}$ & - & 5.6 & 5.6 & 5.8 & 5.7 & 5.7 & 5.8 & 5.8 \\
\hline Alkalinity & $\mathrm{mg} \mathrm{CaCO3/L}$ & 2.0 & 6.8 & 2.4 & 3.4 & 4.4 & 5.3 & 6.3 \\
\hline TN & $\mathrm{mg} / \mathrm{L}$ & 2.9 & 4.0 & 3.0 & 3.2 & 3.4 & 3.6 & 3.9 \\
\hline TSN & $\mathrm{mg} / \mathrm{L}$ & 0.4 & 1.1 & 0.5 & 0.6 & 0.7 & 0.9 & 1.0 \\
\hline TotalCarbs & $\mathrm{mg} / \mathrm{L}$ & 0.9 & 13.5 & 2.2 & 4.7 & 7.2 & 9.7 & 12.2 \\
\hline TotalProteins & $\mathrm{mg} / \mathrm{L}$ & 2.8 & 2.1 & 2.7 & 2.6 & 2.4 & 2.3 & 2.2 \\
\hline TotalLipids & $\mathrm{mg} / \mathrm{L}$ & 0.3 & 1.1 & 0.4 & 0.5 & 0.7 & 0.9 & 1.0 \\
\hline \hline
\end{tabular}

The data form total and soluble COD (SCOD) concentrations monitoring with time was used to obtain the degree of COD solublization for each mixture and the results are as follows. The degree of solublization was calculated using Eq. 3.1 and the result is shown in Figure 4.8. The degree of the COD solublization varied from $14 \%$ to $30 \%$. The maximum solublization of COD content was $30 \%$ for TWAS:SSO combination of 1:9 while the minimum value corresponded to the digester containing only TWAS. Except for TWAS:SSO mixing ratio of 9:1, other co-digesters achieved more than $25 \%$ improvement in solublization. The lower degree of solublization in TWAS mono digestion as well as TWAS:SSO mixing ratio of 9:1 with a high portion of TWAS would be due to the existence of slowly biodegradable content which slows down the hydrolysis and liquefaction. SSO contains sufficient amount of carbohydrates which decomposes more rapidly than proteins and lipids. This would be the reason for observing higher degree of solublization for SSO compared to TWAS which contains more proteins than carbohydrates.

However, by calculating the theoretical degree of solublization of each co-digester and comparing them to the ones obtained from the measured values based on the experimental data, it was revealed that co-digestion was effective for improving the solublization due to enhancing microbial synergy. As shown in Figure. 4.10, all co-digesters achieved an improvement in solublization due 
to synergistic effect of the microbial communities from 23 to $44 \%$ corresponding to TWAS:SSO mixing ratios of $3: 7$ and $7: 3$, respectively.

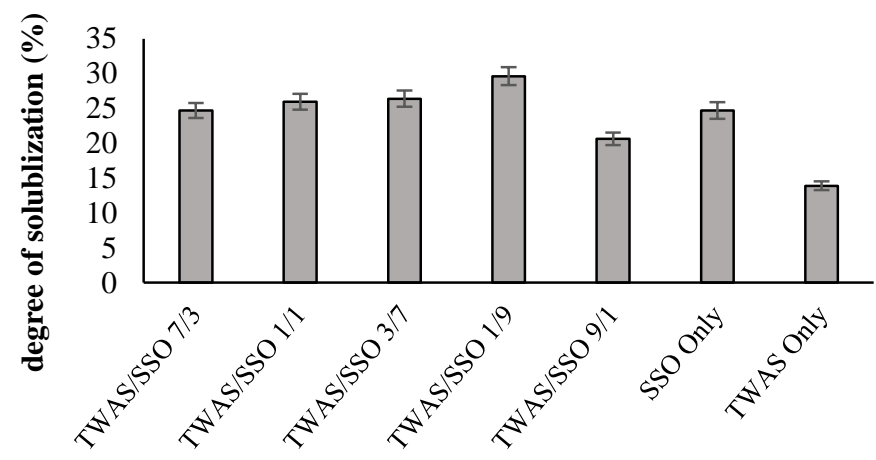

Mixing ratio

Figure 4.9. Degree of COD solublization at different mixing ratios of TWAS and SSO

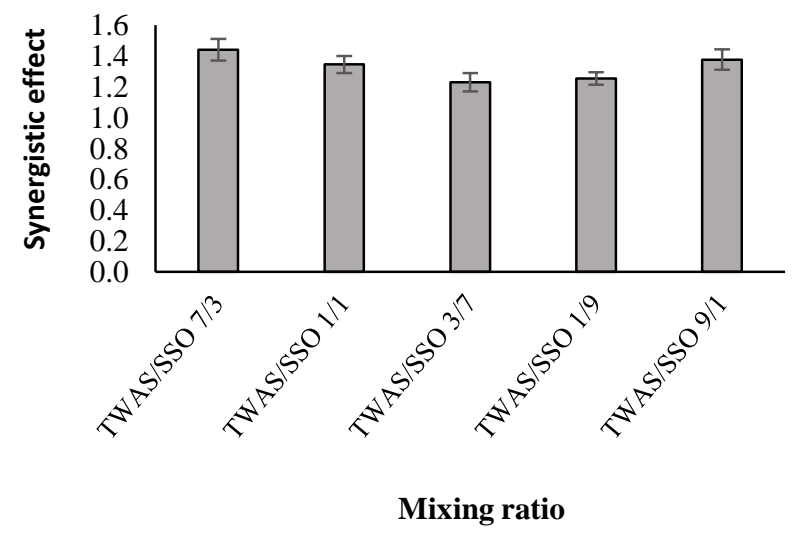

Figure 4.10. Synergistic effect on solublization at different mixing ratios of TWAS and SSO

As discussed earlier, the by-product of hydrolysis is subsequently broken down to smaller molecules by acidogenic bacterial communities. This leads to the formation of volatile fatty acids in acidogenesis. Therefore, monitoring VFAs concentration over time and calculating VFAs yield per mass of VSS added would be a good indicator of acidification progress. The VFAs concentrations monitoring showed an increasing trend during the $72-\mathrm{hr}$ of the hydrolysis/acidification process. The total VFAs concentration was used for calculating the VFAs yield in terms of mass of VFAs produced in mg per mass of VSS added in g. As indicated in Figure 
4.11, the VFAs yield decreased with the addition of TWAS in the digesters. This trend is similar to the trend of COD solublization as demonstrated in Figure 4.11. All of the reactors containing the mix of substrates had higher VFAs yield compared to the reactors containing only TWAS. The VFAs yields were $98 \mathrm{mg}$ VFAs/g VSS added for TWAS mono digestion and $213 \mathrm{mg}$ VFAs/g VSS added for TWAS:SSO ratio of 9:1, respectively. Other digesters resulted in higher amounts of VFAs production from 281 to $328 \mathrm{mg}$ VFAs/g VSS added.

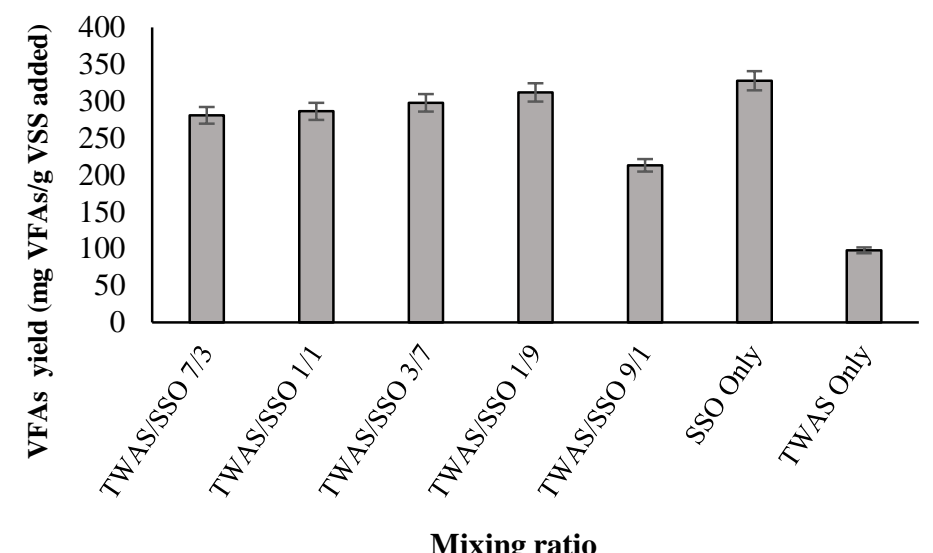

Figure 4.11. Total VFAs yield at different mixing ratios of TWAS/SSO

The analysis of soluble and particulate COD over time during the hydrolysis experimental period, showed an increasing trend in COD solublization and decreasing trend in particulate COD concentrations. Similarly, the analysis of soluble and particulate lipids, proteins, and carbohydrates over time demonstrated an increasing trend in solublization and particulate matter degradation. Although the trend is remarkably slower for lipids and proteins compared to carbohydrates. These results are summarized in the tables that are presented in the appendix. With applying the first order kinetics using equations 3.4., the hydrolysis rate coefficient $\left(\mathrm{K}_{\mathrm{h}}\right)$ was calculated in AquaSim 2.0 for COD, lipids, proteins and carbohydrates based on the particulate matter degradation and the results are summarized in Table 4.5.

As presented in Table 4.4, the hydrolysis rate for COD content of SSO was higher than that of TWAS by 1.8 folds. The $\mathrm{K}_{\mathrm{h}}$ values varied from 0.17 to 0.35 corresponding to TWAS alone and TWAS:SSO mixing ratio of 1:9, respectively. Hydrolysis rate coefficient increased in the reactors containing mixings of TWAS and SSO as compared to the reactors digesting only TWAS. The 
increase of COD hydrolysis rate could be the result of improved microbial synergy and good syntrophic interactions between the hydrolytic and acidogenic microbial communities through the process. The higher kinetic rate coefficient at the mixing ratio of 1:9 is in good agreement with the higher degree of solublization at the same mixing ratio compared to other co-digestion mixtures. Among lipids, proteins, and carbohydrates, lipids showed the lowest hydrolysis rate. In contrast, hydrolysis proceeded more rapidly for carbohydrates compared to lipids and proteins.

The higher hydrolysis rate of carbohydrates would be the result of more rapid biological metabolism of carbohydrates than lipids and proteins. $\mathrm{K}_{\mathrm{h}}$ varied from 0.03 to 0.08 corresponding to hydrolysis of the lipid content of TWAS alone and SSO alone, respectively. All co-digesters had a higher hydrolysis rate of the lipid content in comparison with the digesters containing only TWAS.

As shown in Table 4.5, the hydrolysis rate of proteins content of the digesters was higher than that of lipids content, however it was still lower compared to the hydrolysis rate of carbohydrates content of the feedstocks. $\mathrm{K}_{\mathrm{h}}$ for the proteins content was in a range between 0.19 to 0.34 corresponding to TWAS alone and TWAS:SSO mixings of 1:9, respectively. All of the reactors co-digesting TWAS with SSO demonstrated bigger hydrolysis rate coefficient than that of the TWAS mono digesters. The hydrolysis rate coefficient of the carbohydrates content was within a range between 0.32 to 0.68 corresponding to TWAS alone and the co-digestion of TWAS with SSO at the mixing ratio of 1:9. Contrary to expectation, the $\mathrm{K}_{\mathrm{h}}$ values of carbohydrates did not increase with the addition of SSO portion in the mixtures.

The variation of hydrolysis rate for the carbohydrates content did not show the same trend as the lipids and proteins contents. This verifies that the hydrolysis of the lipids, proteins and carbohydrates of the feedstocks occurs independently during the hydrolysis/acidification stage. It was observed that co-digestion had an effect on the hydrolysis rate by mostly improving the hydrolysis of proteins and carbohydrates content rather than lipids. 
Table 4.5. Hydrolysis rate coefficient for COD, lipids, proteins, and carbohydrate content in co-digesion of TWAS and SSO at different mixing ratios

\begin{tabular}{|c|c|c|c|c|c|c|c|}
\hline \multirow[t]{2}{*}{$\mathbf{K}_{\mathbf{h}}$} & TWAS:SSO & TWAS:SSO & TWAS:SSO & TWAS:SSO & TWAS:SSO & \multirow[t]{2}{*}{ TWAS } & \multirow[t]{2}{*}{ SSO } \\
\hline & $7: 3$ & $5: 5$ & $3: 7$ & & & & \\
\hline $\mathrm{Kh}_{\mathrm{COD}}$ & 0.30 & 0.32 & 0.32 & 0.25 & 0.35 & 0.17 & 0.30 \\
\hline Kh Lipids & 0.06 & 0.07 & 0.07 & 0.03 & 0.05 & 0.03 & 0.08 \\
\hline $\mathrm{Kh}$ Proteins & 0.28 & 0.27 & 0.33 & 0.25 & 0.34 & 0.19 & 0.28 \\
\hline $\mathrm{K}_{\mathrm{h} \text { Carbohydrates }}$ & 0.56 & 0.57 & 0.54 & 0.46 & 0.68 & 0.32 & 0.55 \\
\hline
\end{tabular}




\section{Chapter 5}

Results and discussion

\section{TWAS and Manure Co- digestion}




\section{Results and discussion- TWAS and manure co-digestion}

\subsection{BMP of TWAS and manure}

This experiment was designed to investigate the effect of mixing ratio of TWAS with manure and its correlation with the lipids: proteins: carbohydrates ratios on anaerobic co-digestion of TWAS and manure. The characteristics of the feed in each digester having different mixing ratios of the substrates are summarized in table 5.1. The COD concentration of manure slurry was significantly higher than that of TWAS. The average TCOD of manure was $122 \mathrm{~g} / \mathrm{L}$ while it was $45 \mathrm{~g} / \mathrm{L}$ for TWAS. Manure also contained significantly higher amount of total carbohydrates (27 g/L), proteins $(5.1 \mathrm{~g} / \mathrm{L})$, and lipids $(1.4 \mathrm{~g} / \mathrm{L})$ concentrations compared to TWAS. Mixtures of TWAS and manure as described in chapter 3 were prepared in different combinations and used as digester feedstocks. Addition of manure to TWAS increased the concentration of carbohydrates in codigesters by a large extent as compared to TWAS mono digesters. It also increased lipids and proteins content of the co-digesters in comparison with the digester containing TWAS alone.

Table 5.1. Characteristics of feed to digesters with different mixing ratios

\begin{tabular}{|c|c|c|c|c|c|c|c|c|}
\hline & & TWAS & Manure & $\mathrm{T}^{*}: \mathrm{M}^{* *} 9: 1$ & $\mathrm{~T}: \mathrm{M}$ 7:3 & $\mathrm{T}: \mathrm{M} 1: 1$ & T:M 3:7 & T:M 1:9 \\
\hline Parameters & Units & $\begin{array}{c}\text { Mixture } \\
\text { (1) }\end{array}$ & $\begin{array}{c}\text { Mixture } \\
\text { (2) }\end{array}$ & Mixture (3) & $\begin{array}{c}\text { Mixture } \\
\text { (4) }\end{array}$ & $\begin{array}{c}\text { Mixture } \\
\text { (5) }\end{array}$ & $\begin{array}{c}\text { Mixture } \\
\text { (6) }\end{array}$ & $\begin{array}{c}\text { Mixture } \\
\text { (7) }\end{array}$ \\
\hline TCOD & $\mathrm{g} / \mathrm{L}$ & 45 & 122 & 53 & 68 & 84 & 99 & 115 \\
\hline SCOD & $\mathrm{g} / \mathrm{L}$ & 2.0 & 8.9 & 2.7 & 4.1 & 5.5 & 6.7 & 8.2 \\
\hline TSS & $\mathrm{g} / \mathrm{L}$ & 36 & 80 & 40 & 49 & 58 & 67 & 75 \\
\hline VSS & $\mathrm{g} / \mathrm{L}$ & 27 & 77 & 38 & 42 & 52 & 62 & 72 \\
\hline $\mathrm{TS}$ & $\mathrm{g} / \mathrm{L}$ & 38 & 99 & 44 & 56 & 68 & 80 & 93 \\
\hline VS & $\mathrm{g} / \mathrm{L}$ & 28 & 86 & 33 & 45 & 57 & 68 & 80 \\
\hline Ammonia & $\mathrm{g} / \mathrm{L}$ & 0.32 & 0.02 & 0.29 & 0.23 & 0.17 & 0.11 & 0.05 \\
\hline $\mathrm{pH}$ & - & 7.1 & 7.0 & 6.9 & 7.0 & 7.1 & 7.2 & 7.4 \\
\hline Alkalinity & $\mathrm{g} \mathrm{CaCO} 3 / \mathrm{L}$ & 4.8 & 5.1 & 4.8 & 4.9 & 4.9 & 5.0 & 5.1 \\
\hline $\mathrm{TN}$ & $\mathrm{g} / \mathrm{L}$ & 2.9 & 2.2 & 2.8 & 2.7 & 2.5 & 2.4 & 2.3 \\
\hline TSN & $\mathrm{g} / \mathrm{L}$ & 0.4 & 0.1 & 0.4 & 0.3 & 0.3 & 0.2 & 0.1 \\
\hline T-Carbs & $\mathrm{g} / \mathrm{L}$ & 1.3 & 27.1 & 3.9 & 9.1 & 14.2 & 19.4 & 24.5 \\
\hline T-Proteins & $\mathrm{g} / \mathrm{L}$ & 3.9 & 5.1 & 4.1 & 4.3 & 4.5 & 4.8 & 5.0 \\
\hline T-Lipids & $\mathrm{g} / \mathrm{L}$ & 0.5 & 1.4 & 0.6 & 0.8 & 1.0 & 1.1 & 1.3 \\
\hline
\end{tabular}


The range of carbohydrates concentrations were within 3.9 to $24 \mathrm{~g} / \mathrm{L}$. Total proteins concentrations were 3.9 and 5.1 for TWAS and manure respectively and varied from 4.1 to $5 \mathrm{~g} / \mathrm{L}$ in the codigesters. The total lipids concentration of manure was $1.4 \mathrm{~g} / \mathrm{L}$ and it was higher by 2.8 fold than TWAS. Adding manure to TWAS increased the lipids content. In the reactors containing the mixtures of the TWAS and manure, lipids concentrations varied from 0.6 to $1.3 \mathrm{~g} / \mathrm{L}$.

Operation of the digesters continued until no significant biogas was produced. Fig. 5.1 shows the profile of the cumulative biomethane production versus time during the total digestion period. TWAS resulted in minimum cumulative methane production of $590 \mathrm{ml}$ while manure alone produced $838 \mathrm{~mL}$ of cumulative methane during the operation period.

As shown in figure 5.1, the maximum cumulative methane production was obtained by codigestion of TWAS:manure at the mixing ratio of 3:7 corresponding to the lipids: proteins: carbohydrates ratio of 1:4:17. Compared to mono digestion of manure, co-digestion at the mixing ratio of 9:1 did not increase methane production, although higher cumulative methane was obtained at that ratio in comparison with TWAS mono digestion. In fact TWAS alone with lipids: proteins: carbohydrates ratio of 1:7:2.5, produced the least amount of methane. Other co-digesters produced higher methane than that of TWAS and manure single digestion.

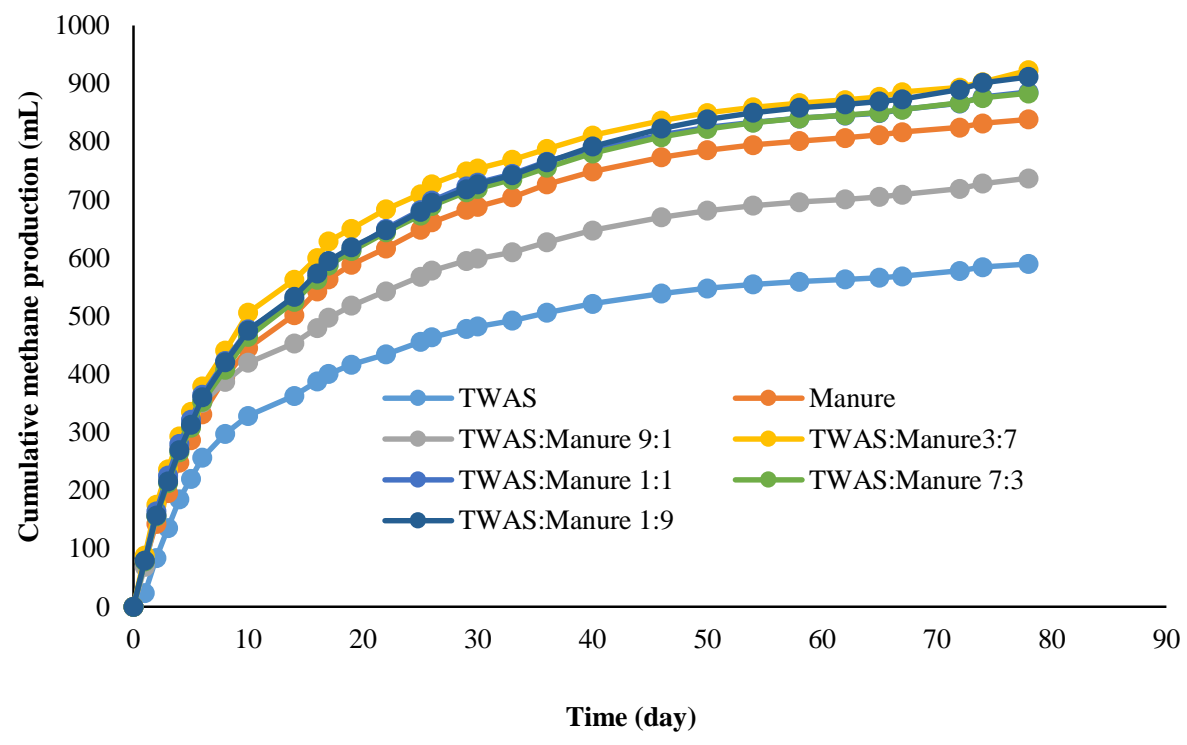

Figure 5.1. Cumulative methane production for different mixing ratios of TWAS and manure 
As illustrated in Figure 5.2, for all of the digesters, the maximum methane rate occurred in the first week of the operation period. The amount of maximum methane production rate of the digesters differed from each other, although a similar trend was observed for all of the co-digestion mixtures. The reactors generated $47 \%$ to $52 \%$ of their ultimate methane production at the first week of the digestion period and 59\% to $62 \%$ in two weeks operation. Compared to TWAS, manure alone resulted in higher methane rate. Addition of manure to TWAS increased partially the maximum methane production rate so that all of the co-digesters demonstrated slightly higher maximum methane rate compared to TWAS and manure mono digesters except for the reactor containing the TWAS and manure at the mixing ratios of 7:3 and 1:9 which had almost the same maximum methane rate as of manure alone. In comparison with TWAS alone, the maximum methane rate in co-digestion reactors were $30 \%$ to $47 \%$ higher.

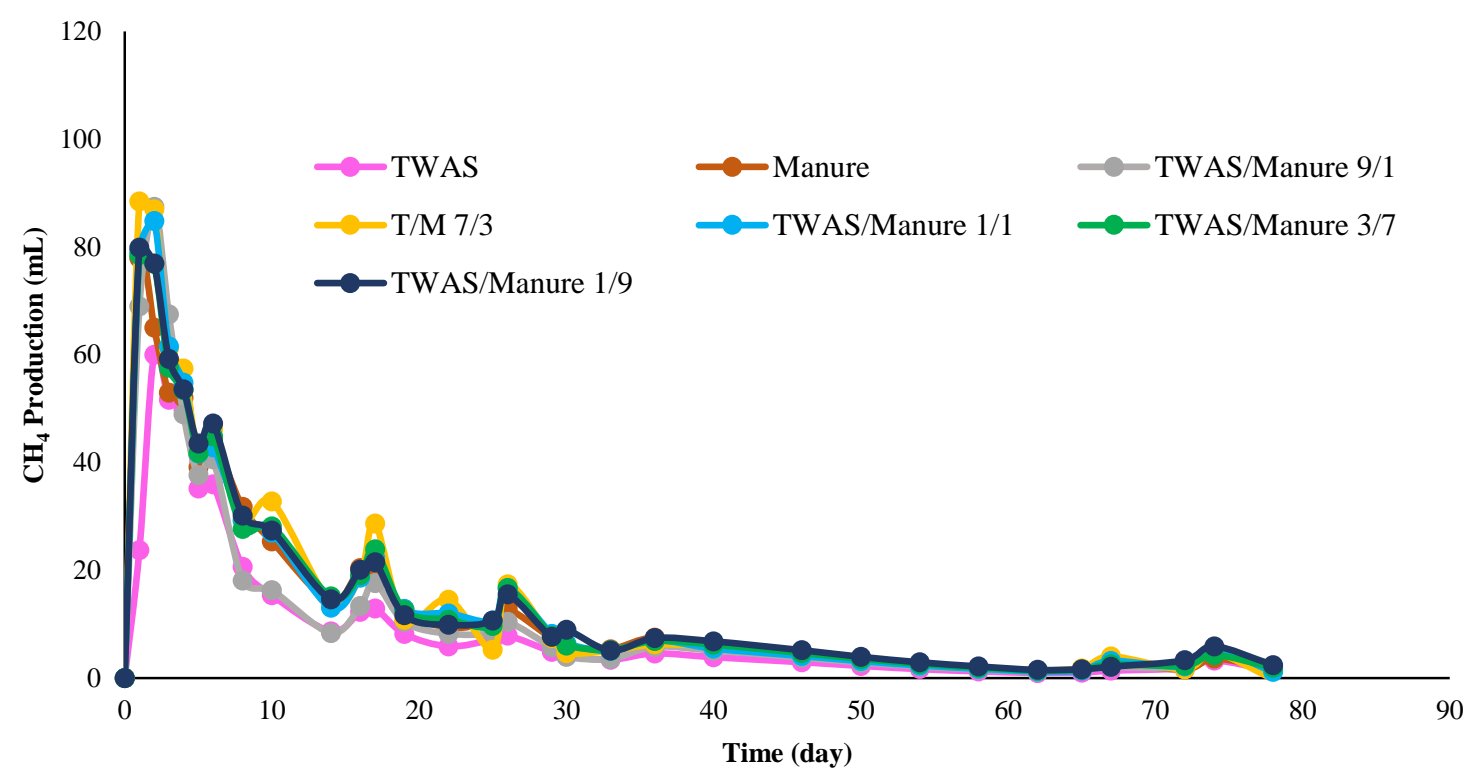

Figure 5.2. Methane production rate $(\mathrm{mL} / \mathrm{d})$ for different mixing ratios of TWAS and Manure

The maximum $\mathrm{CH}_{4}$ production rate at different mixing ratios is presented in Figure 5.3. As shown in Figure 5.3, the maximum rate was $100 \mathrm{~mL} /$ day that occurred at the first day of the process for TWAS/manure mixing ratio of 1:1 corresponding to the lipids: proteins: carbohydrates ratio of 1:25:78 and the minimum rate corresponded to TWAS mono digestion at the lipids: proteins: carbohydrates ratio of 1:7:2.5. 


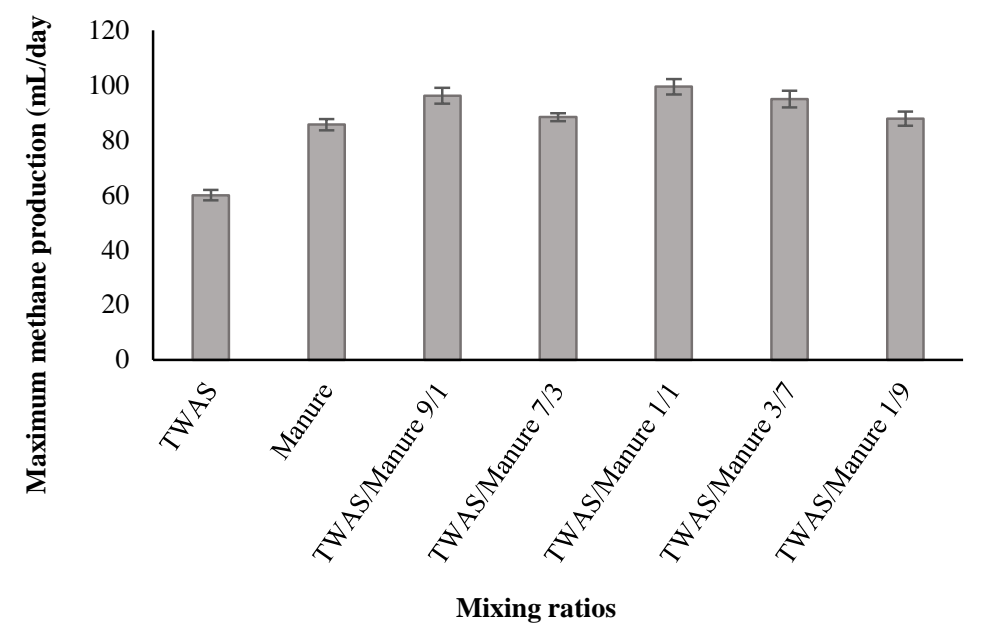

Figure 5.3. Maximum methane production rate $(\mathrm{mL} / \mathrm{d})$ for different mixing ratios of TWAS and manure

During the anaerobic process COD is only rearranged meaning that all COD that enters the system converts eventually to the end products methane minus the COD that incorporates in new microbial mass. Therefore, COD is generally taken as a useful control tool for the operation of anaerobic systems (Henze et al., 2015). A COD mass balance was conducted for all of the digesters to assess the accuracy of the experiment. The mass balance was carried out with reference to the initial and the final TCOD concentrations of the digester contents, and the theoretical methane production per unit mass of TCOD removed. Comparison between the experimental methane production attained by this research and that of determined by TCOD mass balance, as presented in figure 5.4 verified a deviation of less than $10 \%$ for all the digesters. The COD balance varied from $90 \%$ to $98 \%$ in all mono digestion and co-digestion reactors.

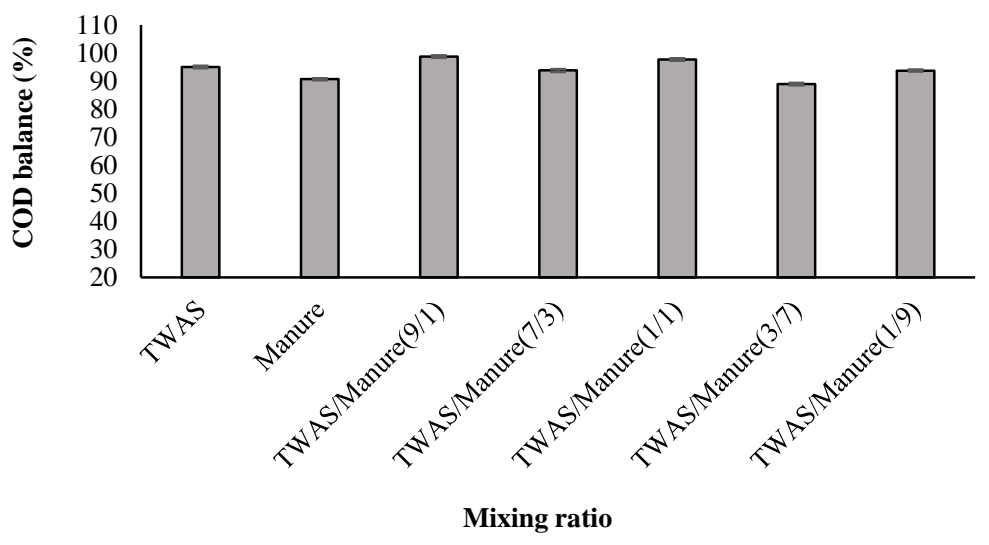

Figure 5.4. COD mass balance in co-digestion of TWAS and manure for different mixing ratios 


\subsection{Cumulative methane yields}

Cumulative methane yields including $\mathrm{mLCH}_{4} / \mathrm{g}$ TCOD added, $\mathrm{mLCH}_{4} / \mathrm{g}$ VSS added, and $\mathrm{mLCH}_{4} / \mathrm{mL}$ substrate added are presented in Figure 5.5. Figure 5.5 a. shows cumulative methane yield per mass COD of substrate added for mono and co-digestions. Manure produced a higher amount of biomethane compared to TWAS. The methane yield was $196 \mathrm{mLCH}_{4} / \mathrm{g}$ TCOD added and $298 \mathrm{mLCH}_{4} / \mathrm{g}$ TCOD added for TWAS and manure mono digestion, respectively. The addition of manure to TWAS, only increased the methane yield by $15 \%$ in co-digesters compared to digestion of manure alone. Even though, it increased the methane yield by $65 \%$ in comparison with mono digestion of TWAS. Results showed that 320 and $324 \mathrm{mLCH}_{4} / \mathrm{g}$ TCOD added was obtained by co-digestion at the mixing ratios of 1:9 and 3:7. Mixing TWAS with manure in codigesters except for TWAS:manure ratio of 9:1 and 7:3 enhanced the methane yield in comparison with both TWAS and manure mono digestion. Although, the ratios of 9:1 and 3:7, increased the methane yield per unit mass of COD added by $40 \%$ and $59 \%$ compared to TWAS alone. The maximum cumulative methane yield of $324 \mathrm{~mL} \mathrm{CH}_{4} / \mathrm{g}$ TCOD added at TWAS/SSO ratio of 3:7 corresponded to 1:4:17 lipids: proteins: carbohydrates ratio.

The methane yield per volume of substrate added in co-digesters were lower than that of manure alone. However, all of the co-digesters yielded higher methane per volume substrate added in comparison with TWAS mono digestion. Only $4 \mathrm{~mL}$ methane per unit volume of substrate added in single digestion of TWAS was obtained.

As shown in figure 5.5. b, cumulative methane yield in terms of $\mathrm{mLCH}_{4} / \mathrm{g}$ VSS added significantly increased compared to TWAS alone due to addition of manure as co-substrate. Although the yield $\left(\mathrm{mLCH}_{4} / \mathrm{g}\right.$ VSS added) only increased by $12 \%$ compared to manure alone. Co-digestion of manure with TWAS significantly increased the methane yield per unit mass of VSS added compared to TWAS alone. A $136 \mathrm{mLCH}_{4} / \mathrm{g}$ VSS added and $401 \mathrm{mLCH}_{4} / \mathrm{g}$ VSS added were attained by TWAS and manure alone, respectively. The amount of methane yield per unit mass of VSS added in codigesters varied from 206 to $448 \mathrm{mLCH}_{4} / \mathrm{g}$ VSS added. The maximum $\mathrm{CH}_{4}$ yield per unit mass of VSS added corresponded to lipids: proteins: carbohydrates ratio of 1:4:17 at TWAS:manure mixing ratio of $3: 7(\mathrm{v} / \mathrm{v})$. 


\section{5. a)}

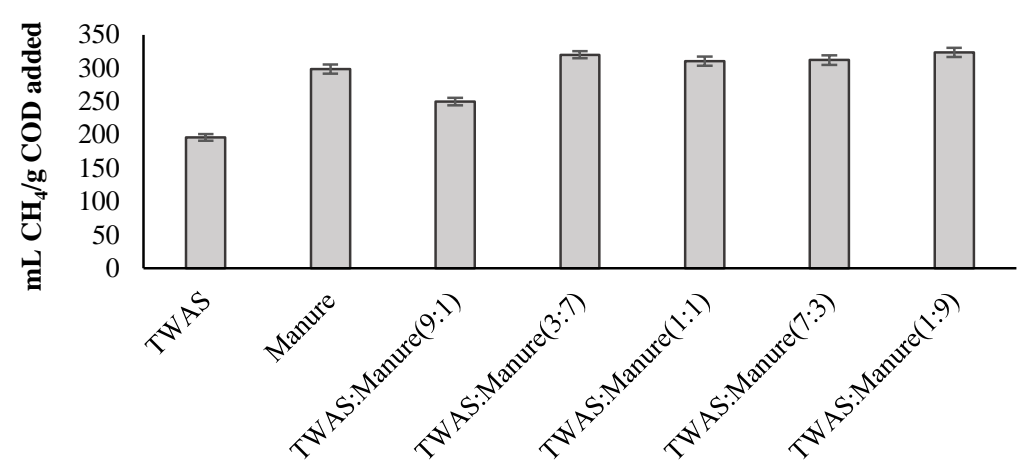

Mixing ratios

\section{5. b)}

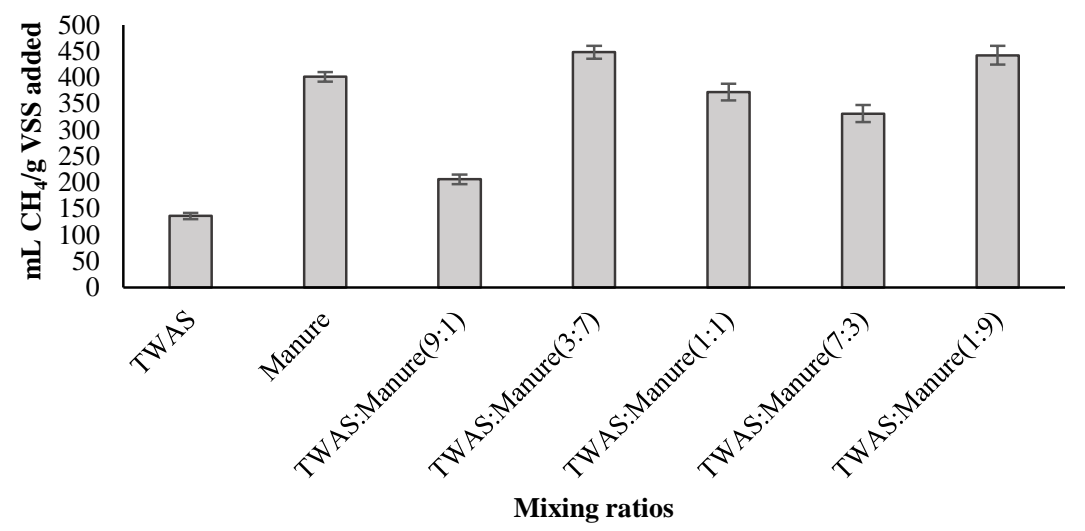

\section{5. c)}

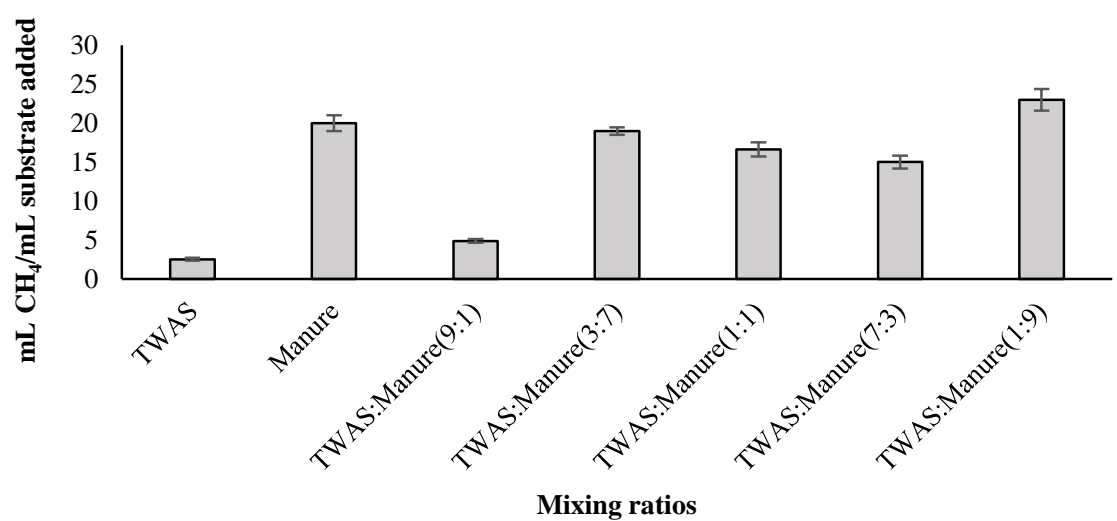

Figure 5.5. Methane yields a) per unit mass TCOD added, $b$ ) per unit mass of VSS added and c) per volume substrate added at different mixing ratio of TWAS and manure 
Assessing biodegradable fraction of the feedstocks with reference to Eq. 4.1, confirmed a 53\% more biodegradable fraction for manure than TWAS. This verifies the higher amount of biomethane obtained by manure compared to TWAS. Co- digestion enhanced biodegradability by $8 \%$ and $65 \%$ in comparison with the control reactors digesting only manure and TWAS, respectively.

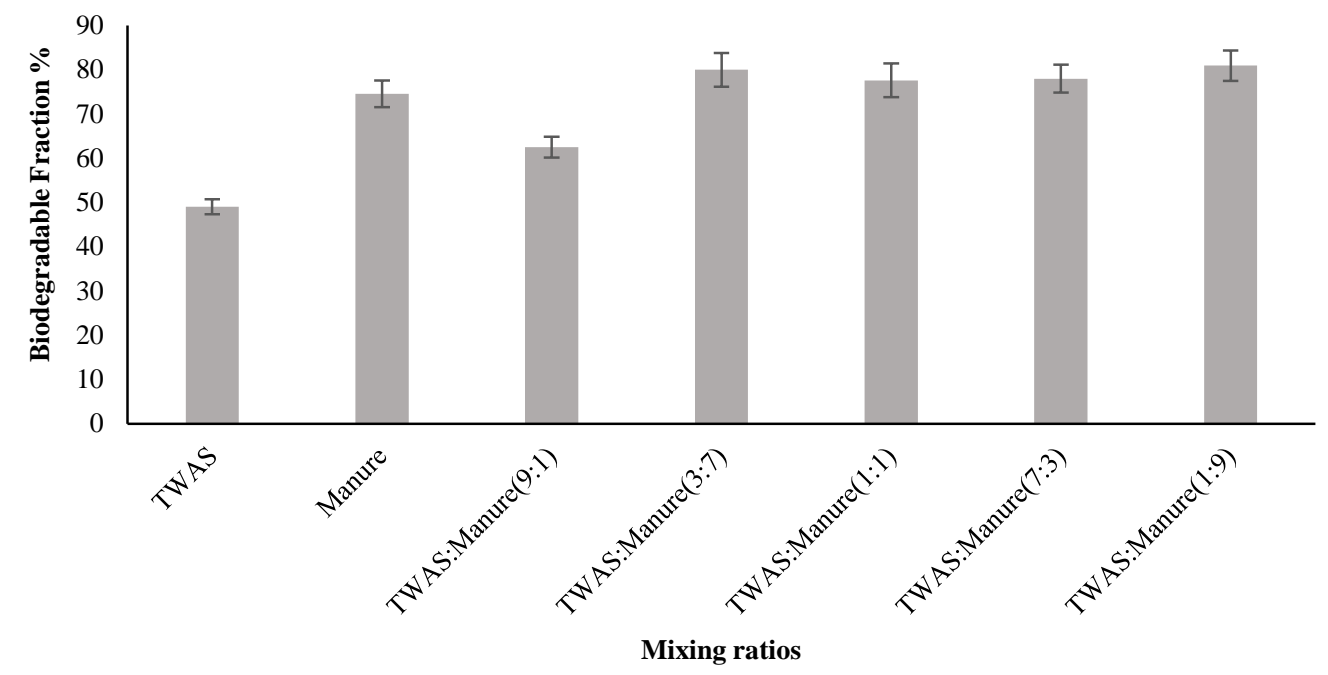

Figure 5.6. Biodegradable fraction of the feedstocks for different mixture ratios of TWAS and manure

As shown in Figure 5.6, biodegradable fraction was 49\% for TWAS while it was $75 \%$ for manure. The mixing ratios of $3: 7$ and 1:9 had the highest biodegradable fraction of almost $80 \%$ in TWAS/manure co-digestion. It was verified that addition of manure as co-substrate increased biodegradability and enhanced the production of methane in the rectors co-digesting manure with TWAS. All co-digesters had higher biodegradable fraction than TWAS alone. The reason would be the existence of readily biodegradable compounds in manure that was introduced to the codigesters. At the mixing ratio of 3:7, the biodegradable fraction was almost $10 \%$ and $0.53 \%$ higher than that of manure and TWAS alone. The trend of biodegradable fraction changes in digesters complies with the methane yields obtained by the experimental results for the corresponding digesters. 


\subsection{Synergistic effect}

Synergistic effect was evaluated with reference to Eq. 4.2 as described in chapter 4. Figure 5.7 shows the percentage of additional methane yield for co-substrates that was measured by the experiment, over the weighted average of the methane production of individual substrates per unit volume of substrate added. As shown in Fig 5.7, the synergistic effect varied from 10 to $24 \%$ in co-digesters. The maximum synergetic impact was observed in co-digestion of TWAS/manure at the mixing ratio of 3:7. This is in compliance with the maximum methane yield that was obtained at the same mixing ratio of TWAS and manure in the reactors co-digesting them. In co-digestion of TWAS and manure, $24 \%$ improvement was achieved due to synergistic effect at the mixing ratios of 3:7.

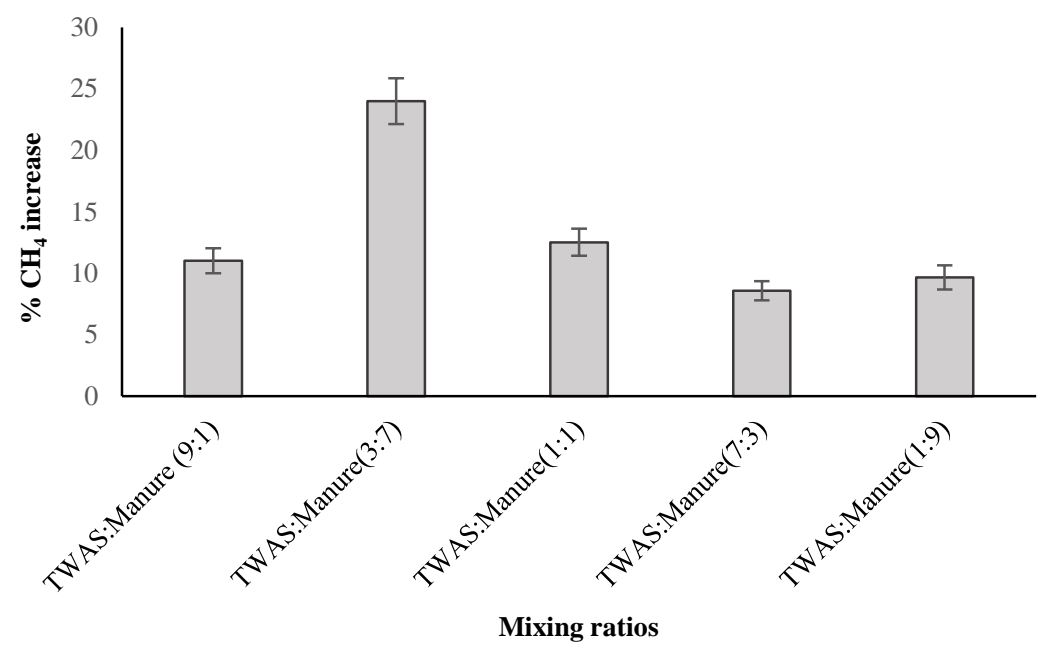

Figure 5.7. Synergetic effect of co-digestion at different mixing ratios of TWAS and SSO

Although all of the mixings demonstrated the synergetic impact of co-digestion on improving biomethane production, no specific trend for the change of the synergetic effect and methane increase corresponding to the fraction of manure in the co-digestion mixtures was observed. Similar to co-digestion of TWAS/SSO, adding fractions of manure as co-substrate enhanced the amounts of readily biodegradable materials to the co-digesters. The ratios with more fraction of carbohydrates produced more methane as the biodegradation of carbohydrate occurs more rapidly than lipids and proteins content. However, increasing the fractions of manure did not essentially resulted in the process improvement. 
The improvement of the synergy and biogas yield in TWAS/manure co-digestion could be due to the abundance of methanogenic populations and diversity of archaeal communities present in manure. Nevertheless, improved synergy would also depend on the optimal nutrients balance and effective conditions for microbial growth. This could explain the optimum fraction of TWAS and manure at the mixing ratio of 3:7 in the co-digestion rather than other ratios.

\subsection{COD:N and Lipids: Proteins: Carbohydrates ratios}

Table 5.2 presents the ultimate methane production and the methane yield per unit mass of COD added, the COD:N and lipids: proteins: carbohydrates ratios of the digesters. Table 5.2 provides a comparison between the COD: $\mathrm{N}$ ratios and the lipids: proteins: carbohydrates ratios of the digesters fed with different mixings of TWAS and manure. In co-digestion of TWAS and manure, the COD: $\mathrm{N}$ ratios between 33 and 56 resulted in higher ultimate methane production, however, the ratios below 20 resulted in lower methane yields. The ultimate methane production and methane yield ranged between 590 to $1069 \mathrm{~mL}$ and 196 to $324 \mathrm{~mL} / \mathrm{g}$ TCOD added for different mixing ratios, respectively.

As presented in Table 5.2, the ratios of COD:N and lipids: proteins: carbohydrates were 16 and 1:7:2.5 for TWAS alone while they were 56 and 1:4:20 for manure, respectively. The methane yield obtained by manure was $52 \%$ more than that of TWAS. The lowest ultimate methane production and methane yield of $590 \mathrm{~mL}$ and $196 \mathrm{~mL} / \mathrm{g}$ TCOD added, corresponded to the TWAS/manure mixing ratio of 1:0 in the control reactors digesting only TWAS. The maximum ultimate $\mathrm{CH}_{4}$ and $\mathrm{CH}_{4}$ yield corresponded to the COD:N ratio of 41 and the lipids: proteins: carbohydrates ratio of 1:4:17 for the reactors co-digesting TWAS and manure at the mixing ratio of $3: 7(\mathrm{v} / \mathrm{v})$.

Figure 5.8. shows the main effect plot for $\mathrm{CH}_{4}$ yield data means in response to feedstock and lipids:proteins:carbohydrates ratios at different feedstock mixing ratios in co-digestion of TWAS/manure. As illustrated in Figure 5.8, both feedstock and lipids:proteins:carbohydrates ratios has significant effect on the methane yield. The minimum methane yield corresponded to TWAS alone with lipids:proteins:carbohydrate ratio of 1:7:2.5 while the maximum yield occurred at the mixing ratio of 3:7 and lipids:proteins:carbohydrate ratio of 1:4:17 in co-digestion of TWAS with SSO. With reference to the results of the ANOVA test, the both COD:N and Lipids: Proteins: 
Carbohydrates ratios had statistically significant effects on the ultimate methane production $(\mathrm{P}<$ $0.05)$.

Table 5.2. Ultimate $\mathrm{CH}_{4}$ and yield at different ratios of the substrates, $\mathrm{COD}: \mathrm{N}$, and lipids:proteins:carbohydrates

\begin{tabular}{ccccccc}
\hline Digester code & $\begin{array}{c}\text { TWAS: } \\
\text { Manure (V/V) }\end{array}$ & COD:N & $\begin{array}{c}\text { Feedstock } \\
\text { ratios } \\
\text { code }\end{array}$ & $\begin{array}{c}\text { Lipids: } \\
\text { Proteins: } \\
\text { Carbohydrates }\end{array}$ & $\begin{array}{c}\text { Ultimate } \\
\text { CH}_{4}(\mathbf{m L})\end{array}$ & $\begin{array}{c}\text { mLCH4/g } \\
\text { TCOD } \\
\text { added }\end{array}$ \\
\hline \hline TWAS Only & $1: 0$ & 16 & $\mathrm{AA}$ & $1: 7: 2.5$ & 590 & 196 \\
$\begin{array}{c}\text { Manure Only } \\
\text { TWAS/Manure } \\
\text { 9/1 }\end{array}$ & $0: 1$ & 56 & $\mathrm{CC}$ & $1: 4: 20$ & 922 & 298 \\
$\begin{array}{c}\text { TWAS/Manure } \\
\text { 7/3 }\end{array}$ & $9: 1$ & 19 & $\mathrm{~A}$ & $1: 7: 6$ & 811 & 250 \\
$\begin{array}{c}\text { TWAS/Manure } \\
\text { 1/1 }\end{array}$ & $1: 1$ & 26 & $\mathrm{~B}$ & $1: 5.5: 12$ & 902 & 320 \\
$\begin{array}{c}\text { TWAS/Manure } \\
\text { 3/7 }\end{array}$ & $3: 7$ & 33 & $\mathrm{C}$ & $1: 25: 78$ & 1015 & 310 \\
$\begin{array}{c}\text { TWAS/Manure } \\
\text { 1/9 }\end{array}$ & $1: 9$ & 41 & $\mathrm{D}$ & $1: 4: 17$ & 1069 & 324 \\
\hline \hline
\end{tabular}

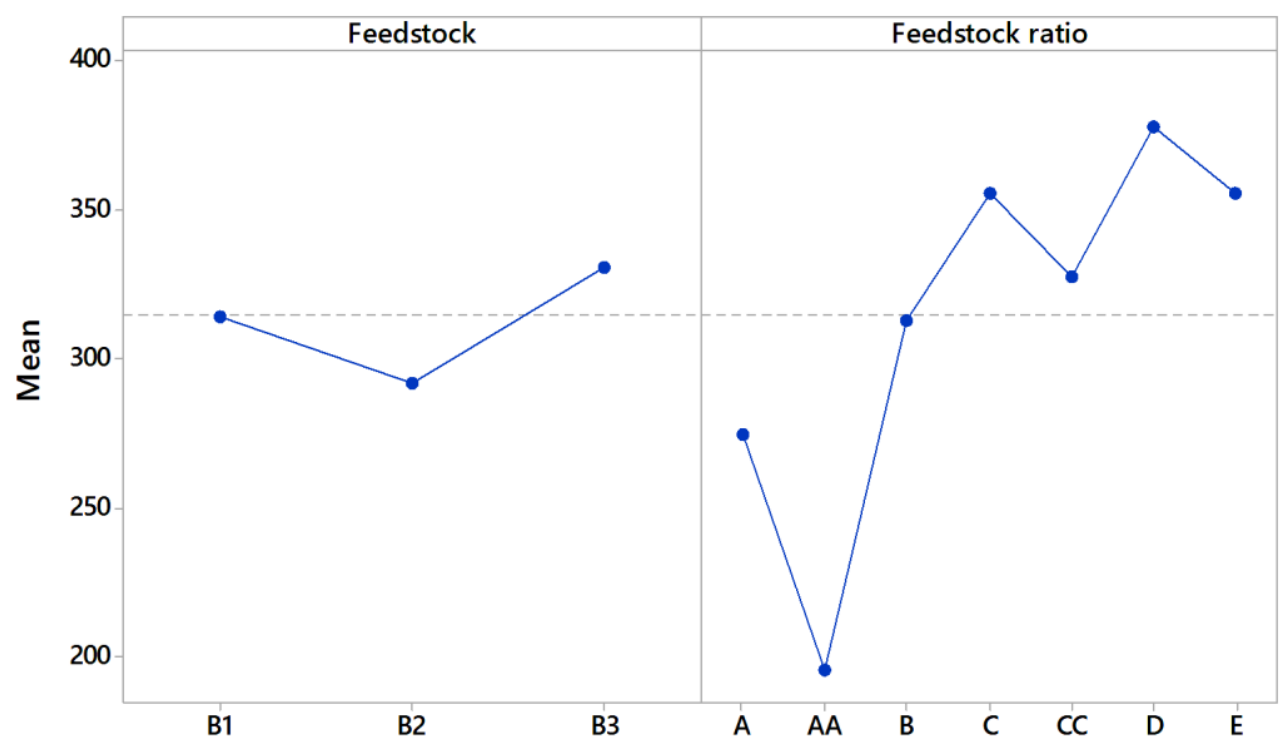

Figure 5.8. Main effect plot for $\mathrm{CH}_{4}$ yield data means in response to feedstock and lipids:proteins:carbohydrates ratios at different feedstock mixing ratios in AnCoD of TWAS/manure 
Figures 5.9. a and 5.9. $\mathrm{b}$ show the variation of ultimate methane and the methane yield versus COD:N ratio, lipids:proteins ratio, and proteins:carbohydrates ratios. As illustrated in Figure 5.9, different trends for the variations of methane yield and the ultimate methane versus COD:N, lipids: proteins, lipids: carbohydrates, and proteins: carbohydrates ratios were observed. For the corresponding feedstocks mixing ratios, a similar trend for the variations of methane versus COD: $N$ and lipids: proteins ratios was observed. However, the COD:N ratio did not show the same trend as lipids: carbohydrates and proteins: carbohydrates ratios for the same feedstocks mixings.

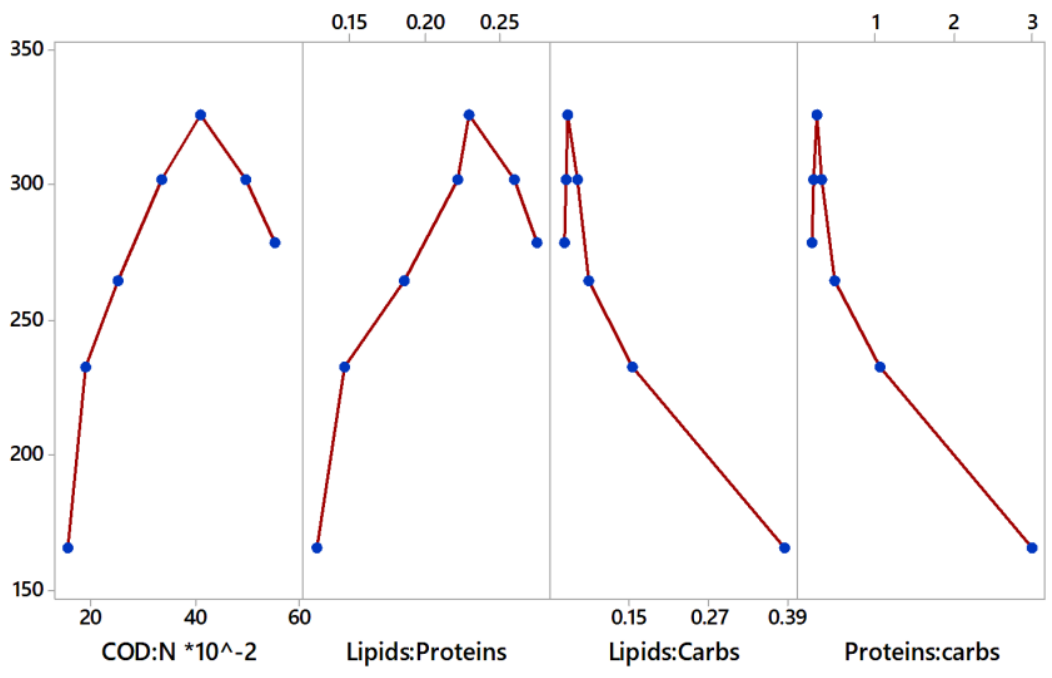

5.9. a

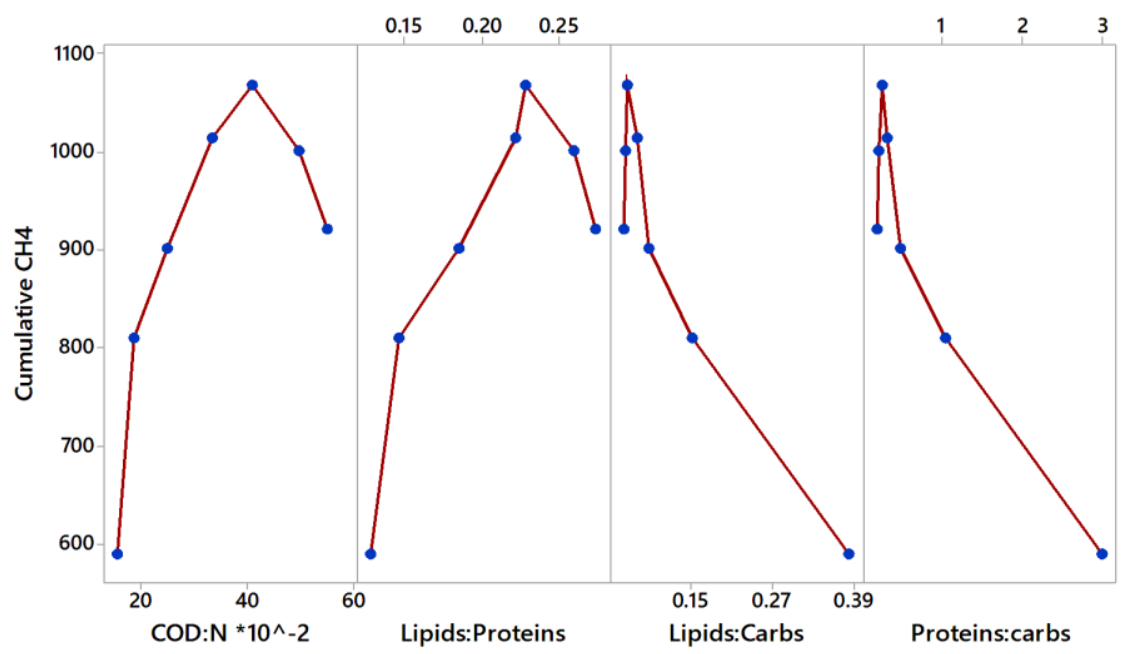

5.9 b.

Figure 5.10. Matrix plot for: a. ultimate $\mathrm{CH}_{4}$ and $b . \mathrm{CH}_{4}$ yield at different $\mathrm{COD} / \mathrm{N}$ and lipids: proteins, lipids: carbohydrates, and proteins: carbohydrates ratios 


\subsection{Kinetic analysis results}

Table 5.3 presents the results of analyzing biomethane production according to the modified Gompertz model using Eq. 3.5. The data collected by the experiment from the control reactors and from each co-digester was applied to the model. The experimental data for cumulative methane production $\left(\mathrm{CH}_{4}\right)$ per unit substrate in $\mathrm{mL} / \mathrm{g}$ and the digestion time $(\mathrm{t})$ in days were applied to the Gompertz equation to calculate the values of $\mathrm{P}$, representing the maximum methane production per unit substrate $(\mathrm{mL} / \mathrm{g}), R_{m}^{e}$, the maximum methane production rate $(\mathrm{mL} / \mathrm{g} \mathrm{h})$, and $\lambda$, the lag phase time (d). The modified Gompertz model for mono- and co-digestions of TWAS and manure, showed a good fit to the experimental results with less than 5\% diversion from the experimental values. The estimated parameters indicated that the co-digestion of manure with TWAS enhanced the biogas production rate. Such performance $\left(R^{2}=0.9998\right)$ shows that the proposed equations can accurately describe the variation of methane yield curves.

Table 5.3. Summary of results of kinetic study using modified Gompertz model

\begin{tabular}{ccccc}
\hline & $\mathbf{P}(\mathbf{m L})$ & $\boldsymbol{R}_{\boldsymbol{m}}^{\boldsymbol{e}}(\mathbf{m L} / \mathbf{d})$ & $\lambda(\mathbf{d})$ & $\mathbf{R}^{2}$ \\
\hline \hline TWAS & 554 & 21 & 0.09 & 0.999 \\
\hline Manure & 880 & 31 & 0.38 & 0.999 \\
\hline TWAS/Manure 9/1 & 763 & 27 & 0.06 & 0.999 \\
\hline TWAS/Manure 7/3 & 861 & 32 & 0.04 & 0.999 \\
\hline TWAS/Manure 5/5 & 969 & 36 & 0.04 & 0.999 \\
\hline TWAS/Manure 3/7 & 1016 & 35 & 0.04 & 0.999 \\
\hline TWAS/Manure 1/9 & 947 & 31 & 0.05 & 0.999 \\
\hline
\end{tabular}

With reference to the adjustment to the nonlinear regression Gompertz model, the lag phase varied from 0.04 to 0.06 days for the co-digesters with different substrate mixing ratios. By increasing the proportion of manure higher values of $\mathrm{P}$ was estimated. The mixing ratio of 7:3 and 1:9 corresponded to the higher $\mathrm{P}$ values. These findings were in good agreement with the data collected from the experiment. As verified by the experiment, a longer lag phase was observed in codigestion of TWAS:manure compared to co-digestion of TWAS:SSO. As mentioned before, a long lag phase time is unfavorable as it demands for a higher residence time and consequently, larger 
reactor volumes which increases the operational costs of the anaerobic system. The values of $\mathrm{P}$, varied from 554 to $1016 \mathrm{~mL}$ corresponding to TWAS alone and TWAS:manure mixing ratio of 3:7. The trend of changes in the $\mathrm{P}$ values complies with the experimental data as it showed an increasing trend in methane production by increasing the proportion of manure. The values of $\mathrm{R}_{\max }^{\mathrm{e}}$ were within a range from 21.3 to $36.2 \mathrm{~mL} / \mathrm{d}$. The values of $\mathrm{R}_{\max }^{\mathrm{e}}$ and $\mathrm{P}_{\max }$ obtained by Gompertz for the TWAS/manure ratios of 3:7, were $35.1 \mathrm{~mL} / \mathrm{d}$ and $1016 \mathrm{~mL}$, respectively.

\section{6. Hydrolysis/acidification}

This experiment was conducted to investigate the hydrolysis/acidification phase in anaerobic codigestion of TWAS and manure. The degradation of organic compounds in hydrolysis stage was evaluated using a series of analysis such as degree of solublization, synergetic effect of codigestion at different mixing ratios on hydrolysis and liquefaction, volatile fatty acids (VFAs) yield, and hydrolysis kinetics of lipids, proteins, and carbohydrates .

Characterization of the feedstocks was initially carried out in triplicates and the mean values are summarized in table 5.4. As presented in the table, the amount of COD concentration is remarkably higher in manure than TWAS. Adding manure to TWAS in the co-digesters, increased the COD concentrations compared to the reactor digesting only TWAS. Manure also contains higher amounts of carbohydrates, lipids, and proteins than TWAS. The concentrations of total, soluble and particulate COD; total, soluble and particulate proteins; and total, soluble and particulate carbohydrates were monitored over time to obtain their hydrolysis rate coefficients.

The data form total and soluble COD (SCOD) concentrations monitoring over time during a 72- $\mathrm{h}$ experimental period was used to obtain the degree of COD solublization for each mixture. The degree of solublization was calculated using Eq. 3.1 and the result is summarized in Figure 5.10. The degree of the COD solublization varied from $21 \%$ to $34 \%$. The maximum solublization $34 \%$ occurred at TWAS/manure combination of 3:7 while the minimum value corresponded to the digesters containing only TWAS. Except for TWAS/manure mixing ratio of 9:1, other co-digesters demonstrated an increase of solublization compared to both TWAS and manure alone. A $31 \%$ and $62 \%$ improvement was achieved by TWAS/Manure co-digestion at the mixing ratio of 3:7 compared to manure and TWAS single digestion, respectively. Manure contains sufficient amount of rapidly biodegradable materials than TWAS. The lower degree of solublization in TWAS mono digestion as well as TWAS:manure mixing ratio of 9:1 with a high portion of TWAS could be due 
to the existence of slowly biodegradable materials which slows down the hydrolysis and liquefaction process and decreases the degree of solublization.

Table 5.4. Characteristics of the feedstocks at different mixing ratios of TWAS and manure

\begin{tabular}{|c|c|c|c|c|c|c|c|c|}
\hline & & TWAS & Manure & $\begin{array}{c}\mathrm{T}^{*}: \mathrm{M}^{* *} \\
9: 1\end{array}$ & $\mathrm{~T}: \mathrm{M}$ 7:3 & $\mathrm{T}: \mathrm{M} \mathrm{1:1}$ & $\mathrm{T}: \mathrm{M} 3: 7$ & $\mathrm{~T}: \mathrm{M} 1: 9$ \\
\hline Parameters & Units & $\begin{array}{c}\text { Mixture } \\
(1)\end{array}$ & $\begin{array}{c}\text { Mixture } \\
\text { (2) }\end{array}$ & $\begin{array}{c}\text { Mixture } \\
(3)\end{array}$ & $\begin{array}{c}\text { Mixture } \\
(4)\end{array}$ & $\begin{array}{c}\text { Mixture } \\
(5)\end{array}$ & $\begin{array}{c}\text { Mixture } \\
(6)\end{array}$ & Mixture (7) \\
\hline TCOD & $\mathrm{g} / \mathrm{L}$ & 41 & 110 & 48 & 62 & 76 & 90 & 104 \\
\hline SCOD & $\mathrm{g} / \mathrm{L}$ & 2.2 & 6.6 & 2.6 & 3.5 & 4.4 & 5.2 & 6.1 \\
\hline TSS & $\mathrm{g} / \mathrm{L}$ & 37.3 & 52.6 & 38.8 & 41.9 & 44.9 & 48.0 & 51.1 \\
\hline VSS & $\mathrm{g} / \mathrm{L}$ & 26.5 & 42.8 & 28.1 & 31.4 & 34.7 & 37.9 & 41.2 \\
\hline $\mathrm{TS}$ & $\mathrm{g} / \mathrm{L}$ & 39.2 & 68.0 & 42.1 & 47.9 & 53.6 & 59.4 & 65.2 \\
\hline VS & $\mathrm{g} / \mathrm{L}$ & 28.6 & 58.5 & 31.6 & 37.5 & 43.5 & 49.5 & 55.5 \\
\hline Ammonia & $g / L$ & 0.3 & 0.0 & 0.3 & 0.2 & 0.2 & 0.1 & 0.0 \\
\hline $\mathrm{pH}$ & - & 5.8 & 5.8 & 5.6 & 5.7 & 5.8 & 5.8 & 5.8 \\
\hline Alkalinity & $\mathrm{g} \mathrm{CaCO}_{3} / \mathrm{L}$ & 5.0 & 7.7 & 5.2 & 5.8 & 6.3 & 6.9 & 7.4 \\
\hline $\mathrm{TN}$ & $\mathrm{g} / \mathrm{L}$ & 3.0 & 1.4 & 2.8 & 2.5 & 2.2 & 1.9 & 1.6 \\
\hline $\mathrm{TSN}$ & $\mathrm{g} / \mathrm{L}$ & 0.4 & 0.1 & 0.4 & 0.3 & 0.2 & 0.2 & 0.1 \\
\hline T-Carbs & $\mathrm{g} / \mathrm{L}$ & 1.3 & 27.1 & 3.9 & 9.1 & 14.2 & 19.4 & 24.5 \\
\hline T-Proteins & $\mathrm{g} / \mathrm{L}$ & 4.0 & 5.1 & 4.1 & 4.3 & 4.6 & 4.8 & 5.0 \\
\hline T-Lipids & $\mathrm{g} / \mathrm{L}$ & 0.5 & 1.4 & 0.6 & 0.8 & 0.9 & 1.1 & 1.3 \\
\hline
\end{tabular}

${ }^{*}$ T: TWAS

** M: Manure

Manure contains a large portion of carbohydrates which decomposes more rapidly than proteins and lipids. This would lead to a higher degree of solublization of manure than TWAS which contains more proteins than carbohydrates.

Comparing the theoretical degree of solublization of each co-digester to the ones obtained from the experimental data, showed that co-digestion improved solublization due to enhancing microbial synergy. As shown in Figure. 5.11, all co-digesters demonstrated an improvement in solublization due to synergistic effect of the microbial communities from 11 to $38 \%$ corresponding to TWAS:manure mixing ratios of 1:1 and 3:7, respectively. 


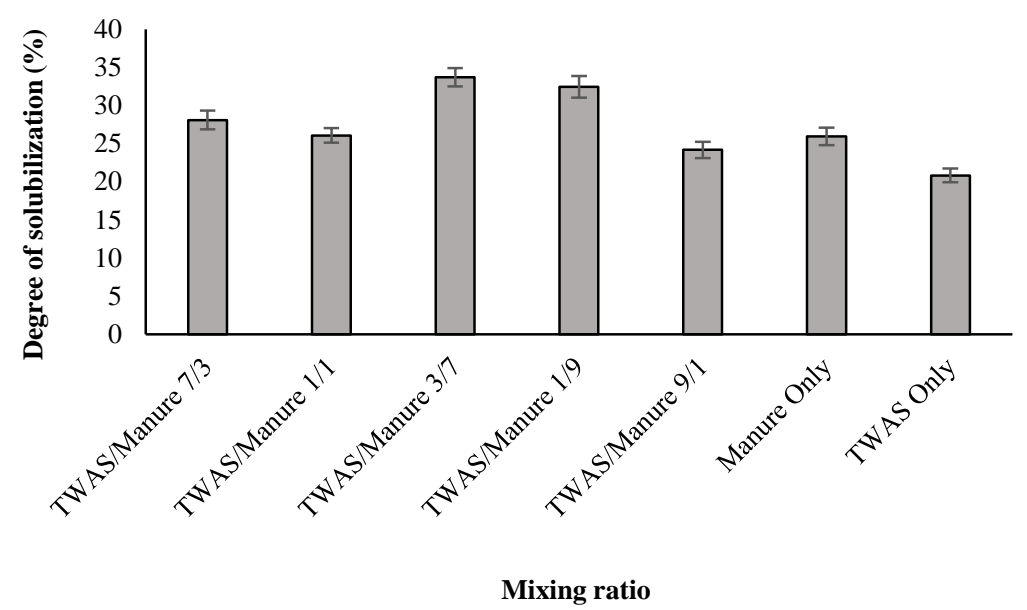

Figure 5.10. Degree of COD solublization at different mixing ratios of TWAS:Manure

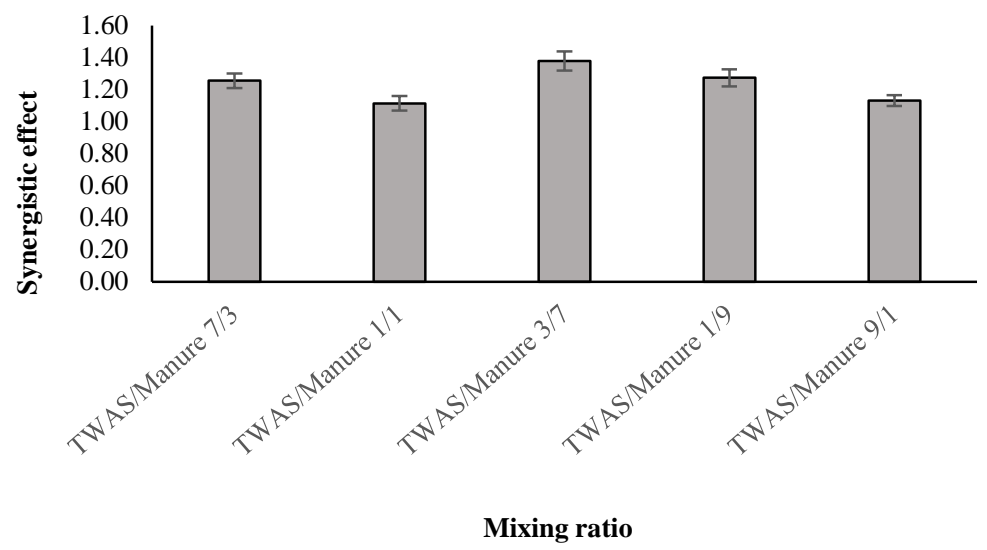

Figure 5.11. Synergistic effect on solublization at different mixing ratios of TWAS:Manure

The VFAs concentrations monitoring showed an increasing trend over the 72-hr of the hydrolysis/acidification experimental period. The total VFAs yield is presented in terms of mass of VFAs produced in mg per mass of VSS added in g. As indicated in Figure 5.12, manure alone had a significantly higher VFAs yields than TWAS alone. The VFAs yield in the co-digester was correlated to the mixing ratios of the feedstocks. The trend of VFAs yield did not conform to the trend of COD solublization of the corresponding mixing ratios (Figure 5.10). Therefore, hydrolysis and liquefaction showed a different trend from acidification. All of the reactors containing the mix of substrates had higher VFAs yield compared to the reactors containing only TWAS. The VFAs yields were $95 \mathrm{mg}$ VFAs/g VSS added and $260 \mathrm{mg}$ VFAs/g VSS added for TWAS and manure 
mono digestions, respectively. A VFAs yield of $307 \mathrm{mg}$ VFAs/g VSS added was achieved for TWAS:manure ratio of 3:7.

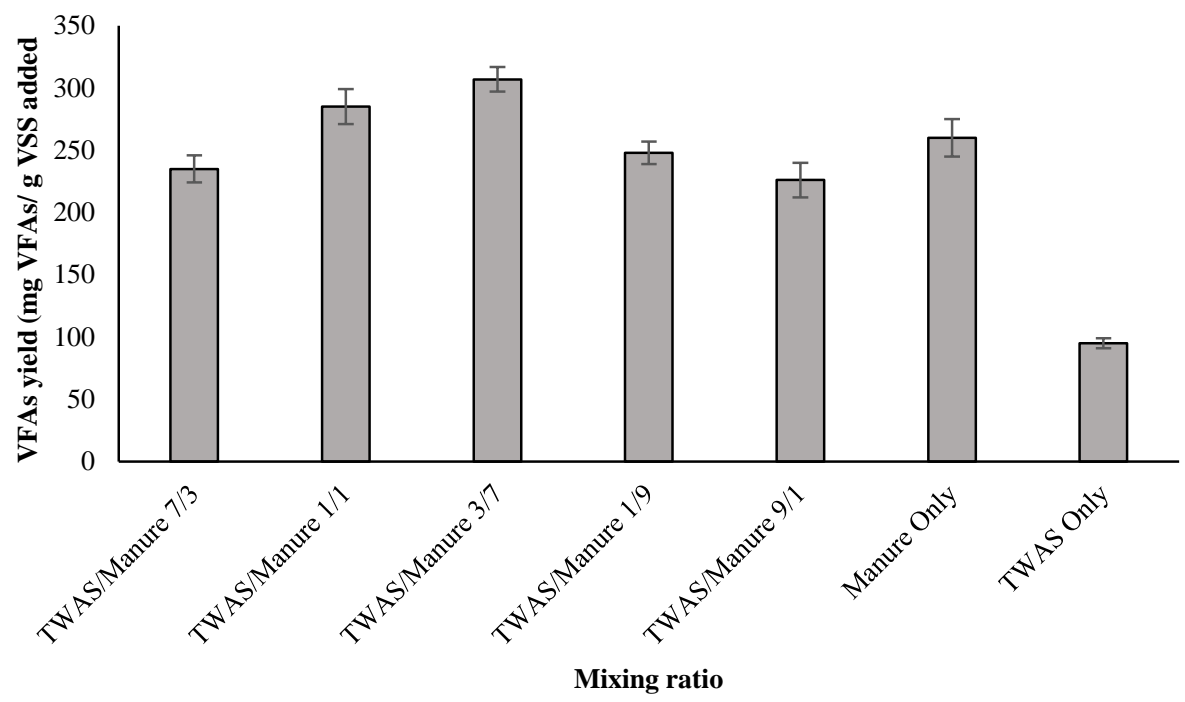

Figure 5.12. Total VFAs yield at different mixing ratios of TWAS/Manure

Monitoring COD over time, showed an increasing trend in solublization of COD and decreasing particulate COD concentrations. In addition, the analysis of soluble and particulate lipids, proteins, and carbohydrates over time showed an increasing trend in solublization and particulate matter degradation. For lipids and proteins, the hydrolysis rate was slower than carbohydrate. These results are summarized in the tables in the appendix. The hydrolysis rate coefficient $\left(\mathrm{K}_{\mathrm{h}}\right)$ was calculated by applying first order kinetics using AquaSim 2.0 for COD, lipids, proteins and carbohydrates based on the particulate degradation and results are summarized in Table 5.5.

As presented in Table 5.5, the hydrolysis rate for COD content of TWAS was higher than that of manure by $35 \%$. The $\mathrm{K}_{\mathrm{h}}$ values in the co-digesters varied from 0.21 to 0.33 corresponding to TWAS and manure mixing ratios of 9:1 and 3:7, respectively. The maximum hydrolysis rate coefficient corresponded to the reactors containing mixings of TWAS and manure at the ratio of $3: 7$.

Lipids showed the lowest hydrolysis rate compared to proteins, and carbohydrates. On the contrary, the most rapid hydrolysis rate was observed for carbohydrates as a result of more rapid biological metabolism of carbohydrates than lipids and proteins. $\mathrm{K}_{\mathrm{h}}$ varied from 0.4 to 0.09 in the 
reactors co-digesting TWAS with manure. The maximum hydrolysis rate coefficient of the lipids contents also corresponded to TWAS:manure mixing of 7:3. TWAS alone has the minimum $\mathrm{K}_{\mathrm{h}}$ for the lipids. TWAS alone and manure alone had $\mathrm{K}_{\mathrm{h}}$ values of 0.03 and 0.07 , respectively. As presented in Table 5.5, the hydrolysis rate of proteins was slightly higher than that of lipids content of the digesters, although it was still lower than the hydrolysis rate of carbohydrates. $\mathrm{K}_{\mathrm{h}}$ for the proteins content of the feedstocks was within a range between 0.22 to 0.27 corresponding to TWAS:manure mixings of $1: 9$ and 3:7, respectively. The hydrolysis rate coefficient of the carbohydrates varied from 0.38 to 0.59 corresponding to the digestion of TWAS:manure with the mixing ratios of 9:1 and 3:7, respectively. The carbohydrates content of the manure showed more rapid biodegradability than TWAS. The hydrolysis rate variation of lipids did not show the same trend as the proteins and carbohydrates of the feedstocks. This revealed that the hydrolysis of the lipids, proteins and carbohydrates of the feedstocks developed independently during the hydrolysis/acidification stage. This independent hydrolysis of the lipids, proteins, and carbohydrates was observed in co-digestion of TWAS and SSO as well.

Table 5.5. Hydrolysis rate coefficients for COD, lipids, proteins, and carbohydrate content in co-digestion of TWAS and manure at different mixing ratios

\begin{tabular}{lccccccc}
\hline $\mathbf{K}_{\mathbf{h}}$ & $\mathrm{T} / \mathrm{M} \mathrm{7/3}$ & $\mathrm{T} / \mathrm{M} 5 / 5$ & $\mathrm{~T} / \mathrm{M} 3 / 7$ & $\mathrm{~T} / \mathrm{M}$ 1/9 & $\mathrm{T} / \mathrm{M}$ 9/1 & TWAS & Manure \\
\hline \hline Kh $\mathrm{COD}$ & 0.26 & 0.24 & 0.33 & 0.28 & 0.21 & 0.17 & 0.23 \\
\hline $\mathrm{Kh}_{\text {Lipids }}$ & 0.04 & 0.05 & 0.09 & 0.07 & 0.04 & 0.03 & 0.07 \\
\hline $\mathrm{Kh}_{\text {Proteins }}$ & 0.23 & 0.22 & 0.27 & 0.25 & 0.22 & 0.19 & 0.21 \\
\hline $\mathrm{K}_{\mathrm{h} \text { Carbohydrates }}$ & 0.49 & 0.44 & 0.59 & 0.48 & 0.38 & 0.32 & 0.43 \\
\hline
\end{tabular}

${ }^{*} \mathrm{~T}$ : TWAS

${ }^{* *} \mathrm{M}$ : Manure 


\section{Chapter 6}

\section{Results and discussion}

\section{Manure and SSO Co- digestion}




\section{Results and discussion- manure and SSO co-digestion}

\subsection{BMP of manure and SSO}

In this experiment co-digestion of manure and SSO was investigated. Manure slurry was prepared as discussed in chapter 3 and was fed to the reactors in different combinations with SSO. The influence of the feedstocks mixing ratios and their correlation with the lipids: proteins: carbohydrates ratios on biomethane production in anaerobic co-digestion of manure and SSO was evaluated. The characteristics of the feed in each digester containing different mixing ratios of the substrates are summarized in table 6.1. The values are the average of each parameter that was measured in triplicate. As presented in Table 6.1, both manure and SSO have high amount of COD concentrations and as a result, the amount of COD in the digesters are high and exceed $100 \mathrm{~g} / \mathrm{L}$. Both VSS and COD values did not vary significantly (less than $8 \%$ ) in the digesters. The amount of carbohydrates and proteins of manure is significantly higher than that of SSO. Therefore, addition of manure increased the carbohydrates and proteins content of the co-digesters compared to the rectors digesting only SSO.

Table 6.1. Characteristics of feed to digesters with different mixing ratios of manure and SSO

\begin{tabular}{ccccccccc}
\hline & & Manure & \multirow{2}{*}{ SSO Only } & M/SSO & M/SSO & M/SSO & M/SSO & \multicolumn{2}{c}{ M/SSO } \\
& & Only & & $9 / 1$ & $7 / 3$ & $5 / 5$ & $3 / 7$ & $1 / 9$ \\
\hline \hline \multirow{2}{*}{ Parameters } & Units & Mixture & Mixture & Mixture & Mixture & Mixture & Mixture & Mixture \\
& & $(1)$ & $(2)$ & $(3)$ & $(4)$ & $(5)$ & $(6)$ & $(7)$ \\
TCOD & $\mathrm{g} / \mathrm{L}$ & 105 & 115 & 106 & 108 & 110 & 112 & 114 \\
SCOD & $\mathrm{g} / \mathrm{L}$ & 44 & 43 & 44 & 44 & 44 & 43 & 43 \\
TSS & $\mathrm{g} / \mathrm{L}$ & 54 & 62 & 55 & 57 & 58 & 60 & 61 \\
VSS & $\mathrm{g} / \mathrm{L}$ & 46.4 & 45.6 & 46.3 & 46.2 & 46.0 & 45.8 & 45.7 \\
$\mathrm{TS}$ & $\mathrm{g} / \mathrm{L}$ & 70 & 68 & 70 & 69 & 69 & 69 & 68 \\
VS & $\mathrm{g} / \mathrm{L}$ & 59 & 49 & 58 & 56 & 54 & 52 & 50 \\
Ammonia & $\mathrm{g} / \mathrm{L}$ & 0.02 & 1.07 & 0.13 & 0.3 & 0.5 & 0.8 & 1.0 \\
pH & - & 6.6 & 5.8 & 6.9 & 7.0 & 7.2 & 7.1 & 7.0 \\
Alkalinity & $\mathrm{g}$ CaCO3/L & 4.9 & 6.2 & 5.0 & 5.3 & 5.6 & 5.8 & 6.1 \\
TN & $\mathrm{g} / \mathrm{L}$ & 2.5 & 3.5 & 2.6 & 2.8 & 3.0 & 3.2 & 3.4 \\
TSN & $\mathrm{g} / \mathrm{L}$ & 0.1 & 1.0 & 0.2 & 0.4 & 0.6 & 0.8 & 1.0 \\
T-Carbs & $\mathrm{g} / \mathrm{L}$ & 29 & 13 & 27 & 24 & 21 & 18 & 15 \\
T-Proteins & $\mathrm{g} / \mathrm{L}$ & 5.9 & 2.1 & 5.5 & 4.8 & 4.0 & 3.2 & 2.5 \\
T-Lipids & $\mathrm{g} / \mathrm{L}$ & 1.4 & 1.1 & 1.4 & 1.3 & 1.3 & 1.2 & 1.1 \\
\hline${ }^{*}$ M: Manure & & & & & & & &
\end{tabular}


Carbohydrates concentrations were within a range between 13 to $29 \mathrm{~g} / \mathrm{L}$ in the rectors. Total proteins concentrations of manure and SSO were 2.1 and 5.9, respectively and varied from 2.5 to $5.5 \mathrm{~g} / \mathrm{L}$ in the co-digesters. The total lipids concentrations varied from 1.1 to $1.4 \mathrm{~g} / \mathrm{L}$ in the digesters including the controls. Adding manure slightly increased the lipids content in the reactors containing the combination of manure and SSO.

Operation of the digesters proceeded until no significant amount of biogas was generated. Fig. 6.1 shows the profile of the cumulative biomethane production versus time during the digestion period of manure with SSO including the controls. The cumulative methane production generated by SSO was higher than that of manure in the control reactors. All co-digesters produced more biomethane than the control reactors containing only manure and only SSO.

SSO alone produced $15 \%$ more methane than manure alone. The amount of ultimate $\mathrm{CH}_{4}$ obtained by single digestion of SSO and manure was 1063 and $919 \mathrm{~mL}$, corresponding to the lipids: proteins: carbohydrates ratios of 1:2:12 and 1:4.2:21, respectively. As shown in figure 6.1, the maximum cumulative methane production of $1186 \mathrm{~mL}$ corresponded to the manure:SSO mixing ratio of 7:3 and lipids: proteins: carbohydrates ratio of 1:3.5:18.5.

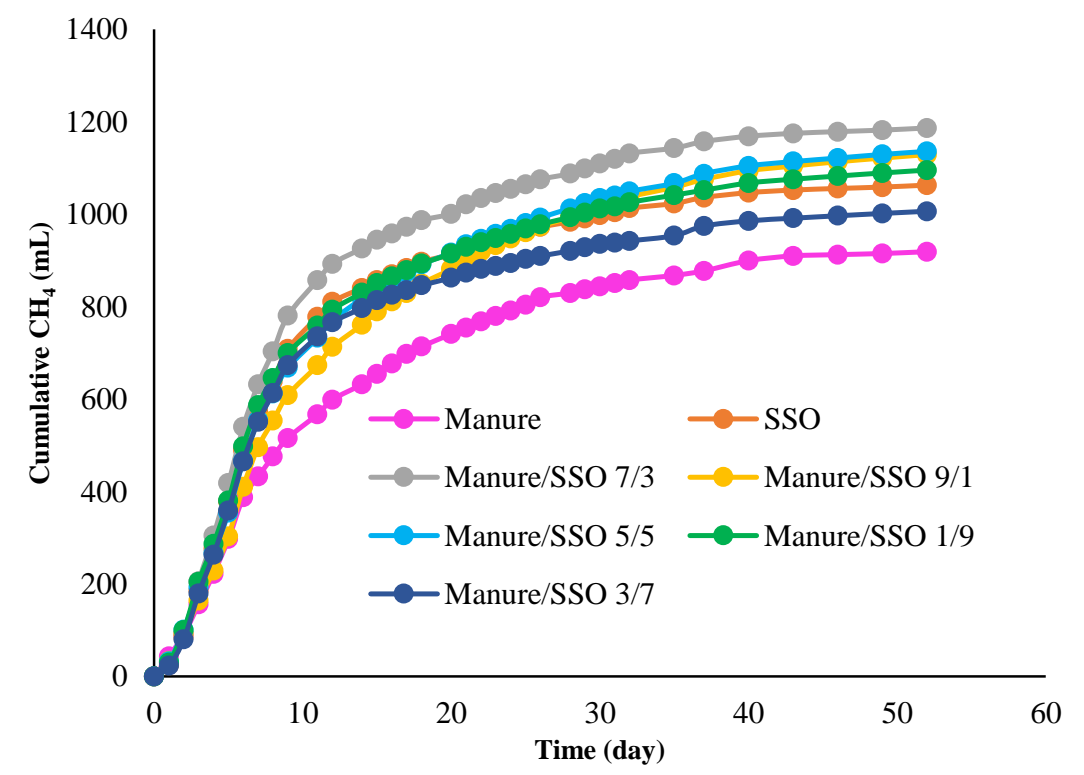

Figure 6.1. Cumulative methane production for different mixing ratios of manure and SSO

Figure 6.2, shows the methane rate in $\mathrm{mL} /$ day for all of the feedstocks combinations including the control reactors. For all of the digesters, the maximum methane rate occurred in the first week of 
the operation period. The maximum methane production rate of the digesters containing the mixings of manure and SSO was higher compared to the control reactors. The reactors generated $44 \%$ to $55 \%$ of their ultimate methane production at the first week of the digestion period and $69 \%$ to $79 \%$ of it in two weeks of operation. Compared to SSO alone, a higher $\mathrm{CH}_{4}$ rate was observed for single digestion of manure. Addition of manure to SSO increased the maximum methane production rate so that all of the co-digester demonstrated a higher maximum methane rate compared to the control rectors. The manure/SSO mixing ratio of $7: 3$ achieved the highest maximum biomethane rate in comparison with other combinations and the lowest value for the

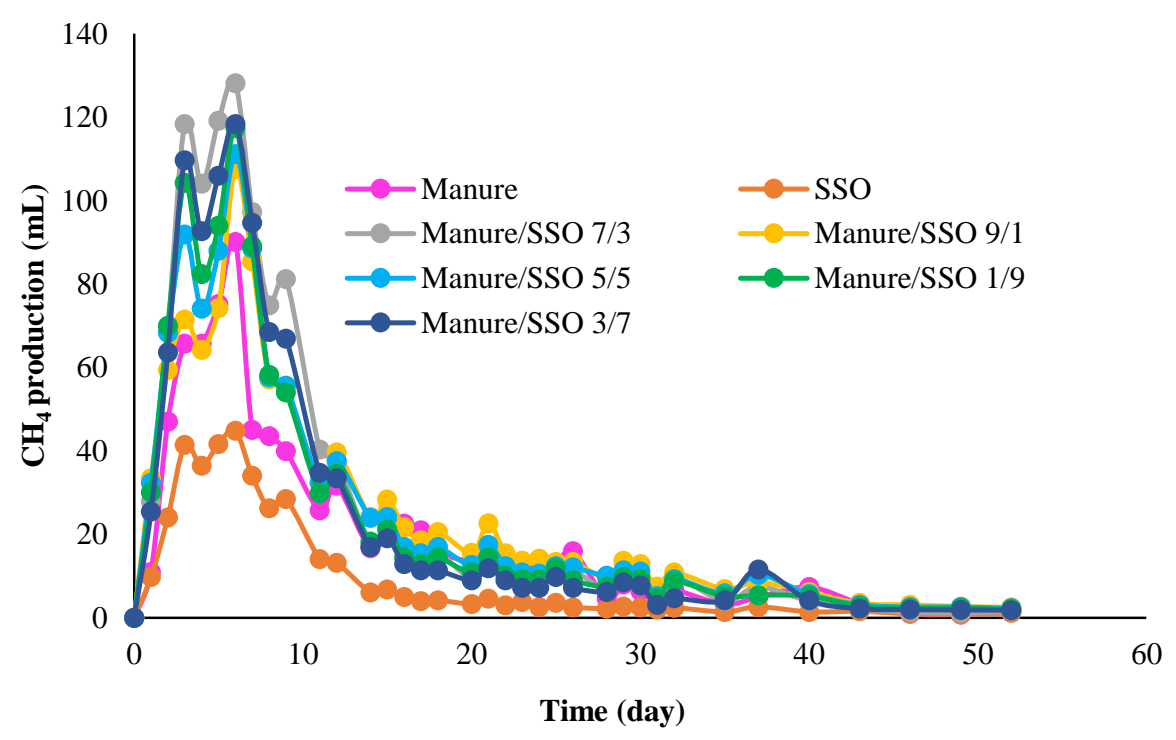

Figure 6.2. Methane production rate $(\mathrm{mL} / \mathrm{d})$ for different mixing ratios of Manure and SSO

maximum biomethane rate corresponded to single digestion of $\mathrm{SSO}$. The maximum $\mathrm{CH}_{4}$ production rate at different mixing ratios is shown in Figure 6.3. As presented in the figure, the most maximum rate was $128 \mathrm{~mL} /$ day corresponding to the mixing ratio of $7: 3$. The lowest value of $45 \mathrm{~mL} /$ day corresponded to single digestion of SSO. Manure alone had the maximum $\mathrm{CH}_{4}$ rate of $90 \mathrm{~mL} /$ day which it was higher than that of SSO by 2 fold. The maximum $\mathrm{CH}_{4}$ rate varied from 108 to $128 \mathrm{~mL} /$ day for the digesters containing combinations of manure and SSO. 


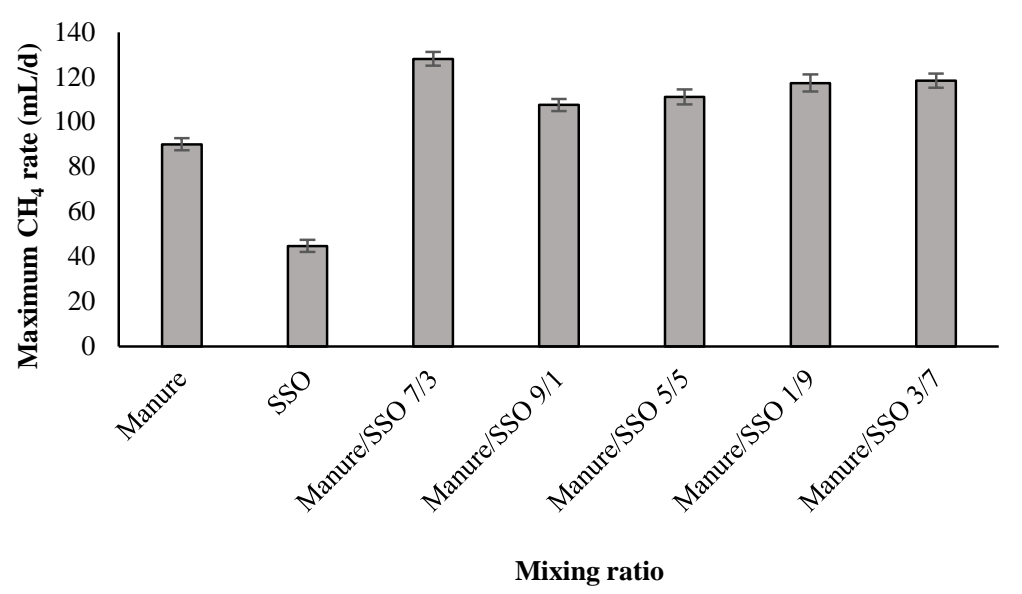

Figure 6.3. Maximum methane production rate $(\mathrm{mL} / \mathrm{d})$ for different mixing ratios manure of and SSO

The COD mass balance was conducted for all of the digesters with reference to the initial and the final TCOD concentrations of the digester contents, and the theoretical methane production per unit mass of TCOD removed. Comparison between the experimental methane production data and that of obtained by TCOD mass balance, showed a deviation of less than $8 \%$ for all the digesters. As shown in Figure 6.4, the COD balance varied from almost 90\% to $97 \%$ in all mono and codigesters.

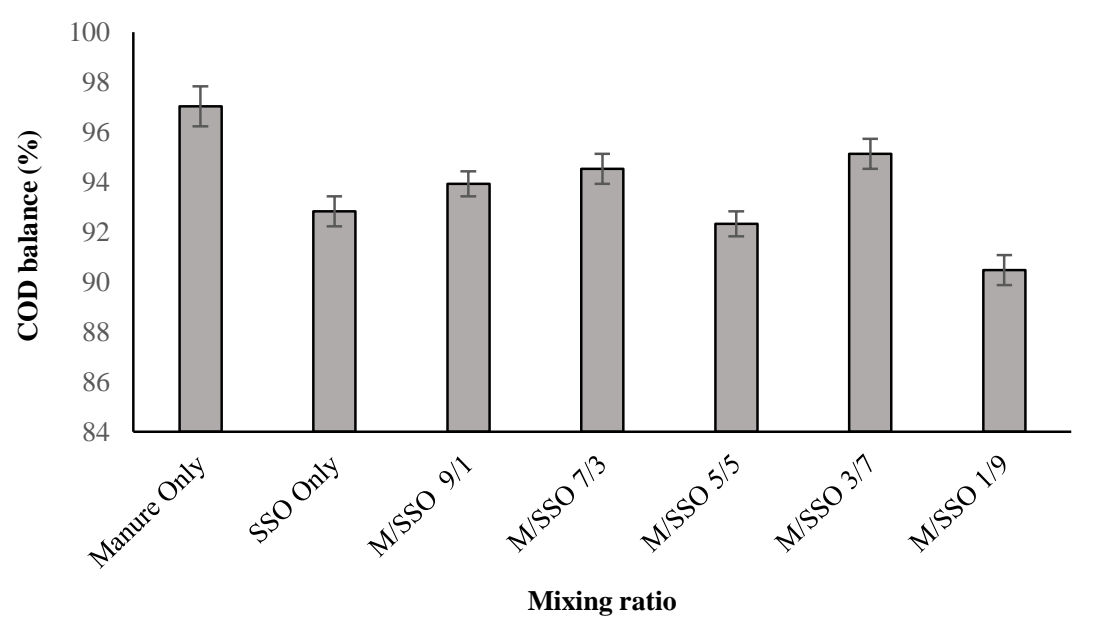

Figure 6.4. COD mass balance in co-digestion of manure and SSO for different mixing ratios 


\subsection{Cumulative methane yields}

Cumulative methane yields including $\mathrm{mLCH}_{4} / \mathrm{g}$ TCOD added, $\mathrm{mLCH}_{4} / \mathrm{g}$ VSS added, and $\mathrm{mLCH}_{4} / \mathrm{mL}$ substrate added are presented in Figure 6.5. It was observed that cumulative methane yield per mass COD of substrate added increased in co-digesters in comparison with the control reactors. As shown in Figure 6.5. a), SSO and manure alone produced $303 \mathrm{mLCH}_{4} / \mathrm{g}$ TCOD added and $287 \mathrm{mLCH}_{4} / \mathrm{g}$ TCOD added, respectively. The addition of manure with SSO, increased the methane yield in the co-digesters. The amounts of the biomethane yields were within a range between 316 and $362 \mathrm{mLCH}_{4} / \mathrm{g}$ TCOD added in the co-digesters. The highest yield of 362 $\mathrm{mLCH}_{4} / \mathrm{g}$ TCOD added occurred at the manure/SSO mixing ratio of 7:3. $\mathrm{CH}_{4}$ yield increased by $26 \%$ and $20 \%$ compared to single digestion of manure and SSO, respectively.

Figure 6.5. b), shows the methane yields in $\mathrm{mLCH}_{4} / \mathrm{g}$ VSS added. Manure produced $654 \mathrm{mLCH}_{4} / \mathrm{g}$ VSS added. The yield was higher for SSO corresponding to $793 \mathrm{mLCH}_{4} / \mathrm{g}$ VSS added. The highest yield occurred at the manure/SSO mixing ratio of 7:3 corresponding to $876 \mathrm{mLCH}_{4} / \mathrm{g}$ VSS added. The $\mathrm{CH}_{4}$ yield improved by $30 \%$ compared to the digestion of manure alone. All of the reactors with mixings of manure and SSO resulted in higher amounts of methane yields per unit mass of VSS added. The methane yields of from 819 to $847 \mathrm{mLCH}_{4} / \mathrm{g}$ VSS added were obtained in codigestion of manure and SSO at different mixing ratios.

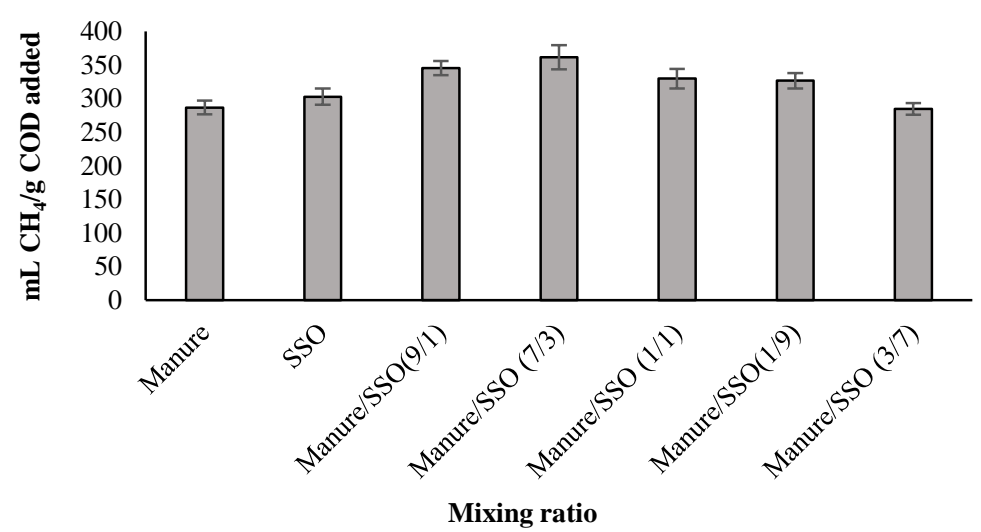

6.5. a)

Mixing ratio 


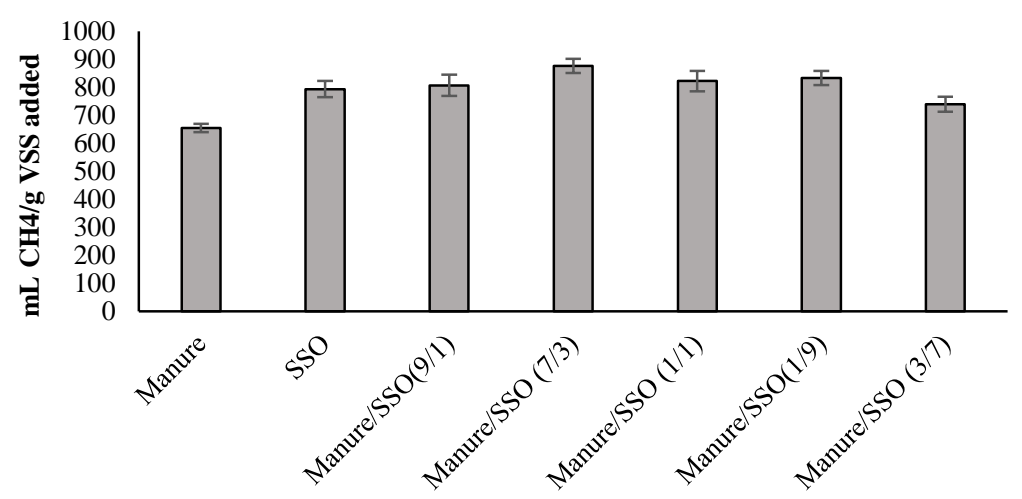

6.5. b)

Mixing ratio

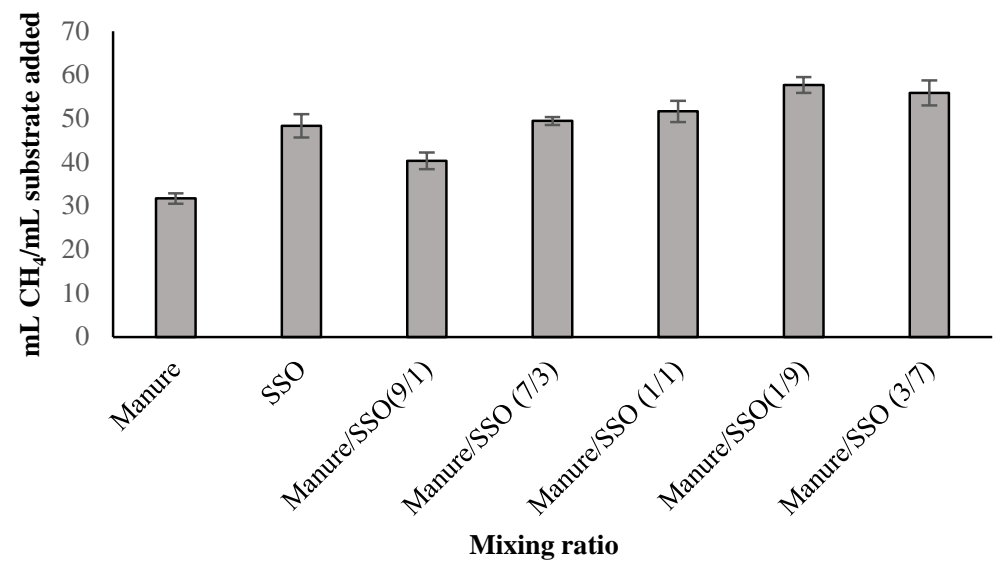

Figure 6.5. Methane yields a) per unit mass TCOD added, $b$ ) per unit mass of VSS added and c) per volume substrate added at different mixing ratio of TWAS and manure

Figure 6.5. c), shows the methane yield per unit volume of substrate added in $\mathrm{mLCH}_{4} / \mathrm{mL}$ substrate added. Manure and SSO individually produced $32 \mathrm{mLCH}_{4} / \mathrm{mL}$ substrate added and $48 \mathrm{mLCH}_{4} /$ $\mathrm{mL}$ substrate added, respectively. The reactors with the combinations of manure and SSO resulted in methane yields ranging from $40 \mathrm{mLCH}_{4} / \mathrm{mL}$ substrate added to $58 \mathrm{mLCH}_{4} / \mathrm{mL}$ substrate added. The most values of the $\mathrm{CH}_{4}$ yield in terms of unit volume of substrate added corresponded to manure/SSO co-digestion at the mixing ratios of 1:9 and 3:7.

Biodegradable fraction of the feedstocks was obtained using the Eq. 4.1 and the result is summarized in Figure 6.6. Manure and SSO individually had biodegradable fractions of $72 \%$ and $76 \%$, respectively. Co- digestion increased biodegradable fraction of the feedstocks by $20 \%$ and $26 \%$ in comparison with the control reactors digesting only SSO and manure, respectively. The 
most percentage of biodegradable fraction occurred at the reactor co-digesting manure with SSO at the mixing ratio of $7: 3$. This in good compliance with the maximum methane production at the same mixing ratio.

As shown in Figure 6.6, the trend of biodegradable fraction variations in the digesters conforms to the trend of the methane yields obtained by the experimental results for the corresponding digesters. It was verified that addition of manure to SSO as co-substrate increased biodegradability and enhanced methane production in the rectors co-digesting manure and SSO. The reason would be the existence of abundant of methanogenic populations in manure that was introduced to the co-digesters and enhanced degradation of organic matters.

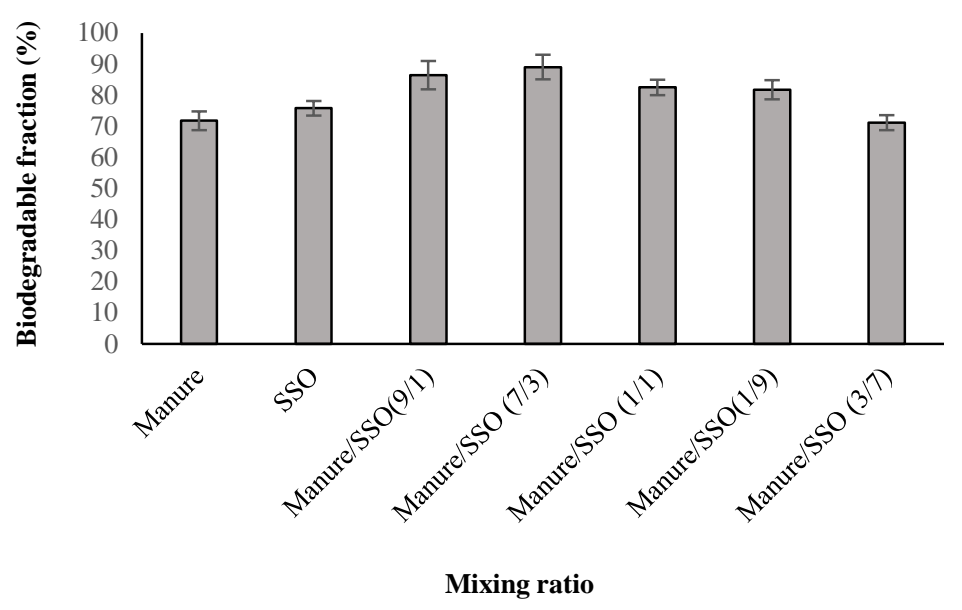

Figure 6.6. Biodegradable fraction of the feedstocks for different mixture ratios of TWAS and manure

\subsection{Synergistic effect}

Synergistic effect was assessed using Eq. 4.2 as presented in chapter 4 and the result is summarized in Figure 6.7. Synergistic effect represents the percentage of additional methane yield for cosubstrates that was measured by the experiment, over the weighted average of the methane yield of individual substrates per unit volume of substrate added. As demonstrated in Fig 6.7, the most synergetic impact corresponds to the co-digestion of manure/SSO at the mixing ratio of 7:3. In codigestion of manure with $\mathrm{SSO}$, the increase of $\mathrm{CH}_{4}$ yield due to synergistic effect ranged from $22 \%$ to $36 \%$ corresponding to the reactor co-digesting manure and SSO at the mixing ratio of 9:1 and 7:3, respectively. It was revealed that only adding the fraction of manure in co-digesters, did not lead to increasing synergy. Although increasing the fraction of manure would introduce more 
populations of methanogenic archaea and bacteria, a balance between the microbial populations and nutrient is necessary for the effective microbial growth and enhanced methanogenesis.

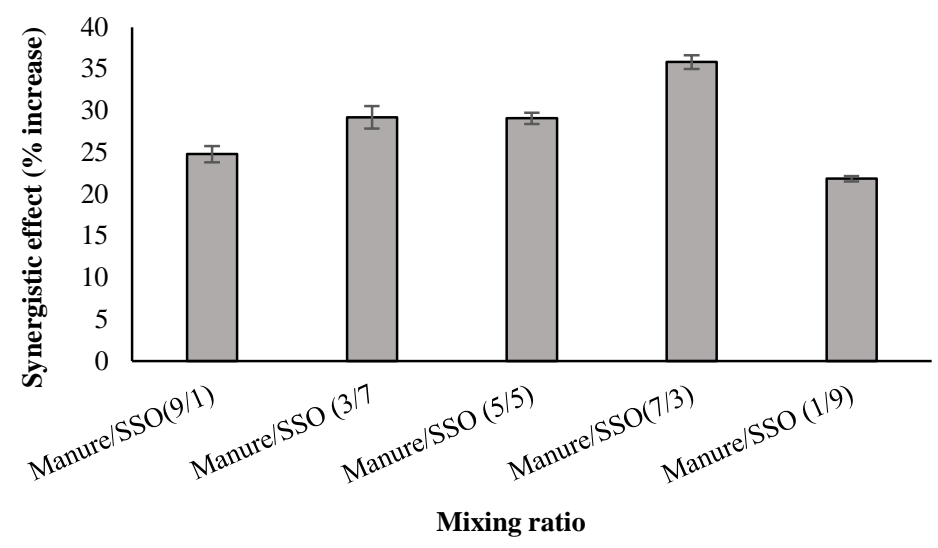

Figure 6.7. Synergetic effect of co-digestion at different mixing ratios of manure and SSO

\subsection{COD: $\mathrm{N}$ and lipids: proteins: carbohydrates ratios}

Table 6.2 presents the COD:N ratios, lipids: proteins: carbohydrates ratios, the ultimate methane production, and the methane yield per unit mass of COD added of the digesters with different mixing ratios. The COD:N ratios were 33 and 42 corresponding to SSO and manure alone, respectively. The values of COD:N varied from 34 to 41 in the co-digesters. The ultimate methane production and methane yield ranged from 919 to $1186 \mathrm{~mL}$, and 287 to $363 \mathrm{~mL} / \mathrm{g}$ TCOD added for different mixing ratios, respectively.

As shown in Table 6.2 The minimum ultimate methane and methane yield occurred at mono digestion of manure corresponding to the COD: $\mathrm{N}$ ratio of 42 and lipids: proteins: carbohydrates ratio of 1:4.2:21. However, the maximum ultimate methane and methane yield occurred at the mixing ratio of 7:3 corresponding to the COD:N ratio of 41 and lipids: proteins: carbohydrates ratio of 1:3.5:18.5. SSO alone with a lipids: proteins: carbohydrates ratio of 1:2:12 produced 15\% more ultimate methane than manure alone with the lipids: proteins: carbohydrates ratio of 1:4.2:21. Although, it only resulted in 6\% more methane yield per unit mass of COD added than manure. On the other side, the 1:4.2:21 lipids: proteins: carbohydrates ratios for manure alone and 1:4:20 for manure/SSO co-digestion at the mixing ratio of 9:1 had only a minor variation while the ultimate methane and the methane yield were $23 \%$ and $20 \%$ higher for the latter. 
Table 6.2. Ultimate $\mathrm{CH}_{4}$ and yield at different ratios of the substrates, $\mathrm{COD}: \mathrm{N}$, and Lipids:Proteins:Carbohydrates

\begin{tabular}{ccccccc}
\hline Digester code & $\begin{array}{c}\text { TWAS: Manure } \\
\text { (V/V) }\end{array}$ & COD:N & $\begin{array}{c}\text { Feedstock } \\
\text { ratio codes }\end{array}$ & $\begin{array}{c}\text { Lipids: Proteins: } \\
\text { Carbohydrates }\end{array}$ & $\begin{array}{c}\text { Ultimate } \\
\mathrm{CH}_{4}(\mathrm{~mL})\end{array}$ & $\begin{array}{c}\mathrm{mLCH} / \mathrm{g} \\
\text { TCOD added }\end{array}$ \\
\hline \hline Manure & $1: 0$ & 42 & $\mathrm{CC}$ & $1: 4.2: 21$ & 919 & 287 \\
SSO & $0: 1$ & 33 & $\mathrm{BB}$ & $1: 2: 12$ & 1063 & 303 \\
$\begin{array}{c}\text { Manure/SSO } \\
7: 3\end{array}$ & $7: 3$ & 41 & $\mathrm{~A}$ & $1: 3.5: 18.5$ & 1186 & 363 \\
$\begin{array}{c}\text { Manure/SSO } \\
\text { 9:1 }\end{array}$ & $9: 1$ & 39 & $\mathrm{~B}$ & $1: 4: 20$ & 1129 & 344 \\
$\begin{array}{c}\text { Manure/SSO } \\
5: 5\end{array}$ & $1: 1$ & 37 & $\mathrm{C}$ & $1: 3: 17$ & 1136 & 330 \\
$\begin{array}{c}\text { Manure/SSO } \\
\text { 3:7 }\end{array}$ & $3: 7$ & 35 & $\mathrm{D}$ & $1: 2.7: 15$ & 1095 & 327 \\
$\begin{array}{c}\text { Manure/SSO } \\
1: 9\end{array}$ & $1: 9$ & 34 & $\mathrm{E}$ & $1: 2: 13$ & 1115 & 316 \\
\hline \hline
\end{tabular}

Manure alone with lipids:proteins:carbohydrates ratio of 1:4.2:21 corresponded the minimum methane yield while the maximum yield occurred at manure:SSO mixing ratio of 7:3 and lipids:proteins:carbohydrates ratio of 1:3.5:18.5. This increase of the methane would be the result of microbial population diversity introduced by manure to the co-digesters and the synergetic impact of co-digestion rather than the ratio of lipids: proteins: carbohydrates.

Figure 6.8. shows the main effect plot for $\mathrm{CH}_{4}$ yield data mean in response to feedstock and lipids:proteins:carbohydrates ratios in $\mathrm{AnCoD}$ of manure/SSO. As shown in figure 6.8, the different lipids:proteins:carbohydrates ratios for the different mixing ratios of the feedstocks have significant effect on the methane yield. 


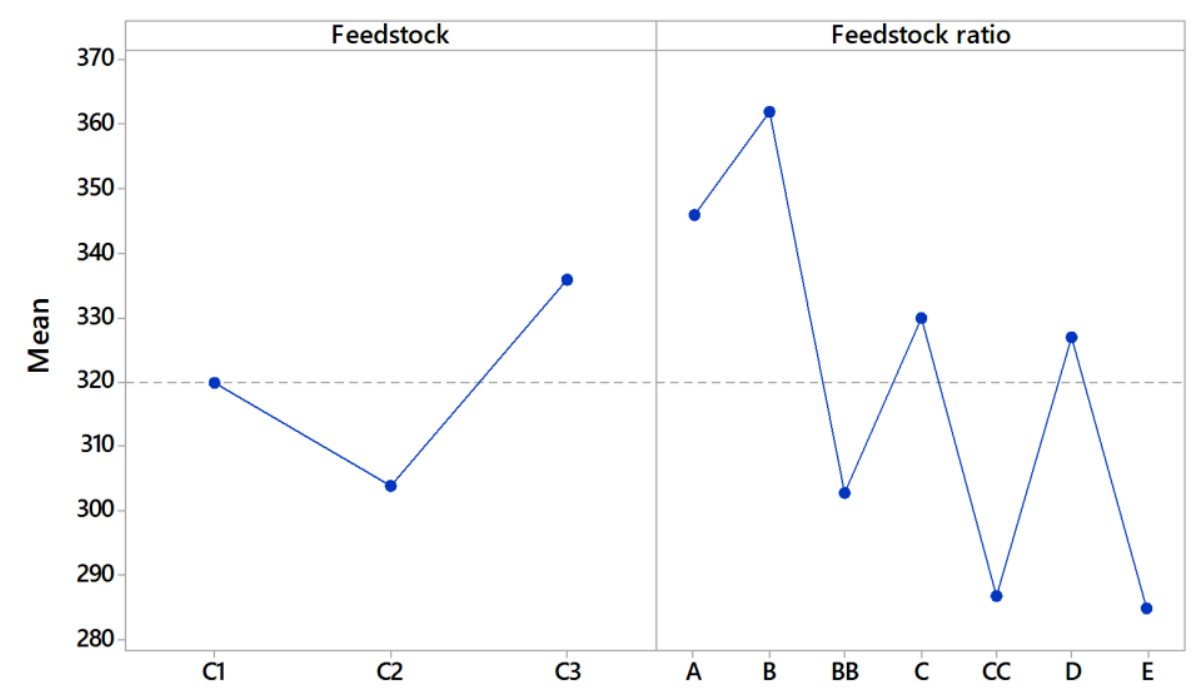

Figure 6.8. Main effect plot for $\mathrm{CH}_{4}$ yield data mean in response to feedstock and lipids:proteins:carbohydrates ratios in AnCoD of manure/SSO

Figure 6.9 shows the matrix plot for the variations of the methane yield and the ultimate methane versus COD:N ratio, proteins: lipids, carbohydrates: lipids, and carbohydrates: proteins ratios. As illustrated in Figure 6.9. a, the trend of variations of the methane yields versus COD:N and versus proteins: carbohydrates were similar. lipids: proteins and lipids: carbohydrates also showed a similar trend but for both of them the trend was the mirror image of COD:N and proteins: carbohydrates. The ultimate methane as illustrated in Figure 6.9. $\mathrm{b}$ also demonstrated a similar response to those ratios. These observations were contrary to the results of TWAS:SSO and TWAS/Manure co-digestion. The reason would be the minor variations of proteins: lipids ratios at the different combinations of manure and SSO. As mentioned earlier, in co-digestion of manure/SSO the methane yield in the co-digesters could more depend on the microbial diversity than the ratio of the lipids: proteins: carbohydrates. 

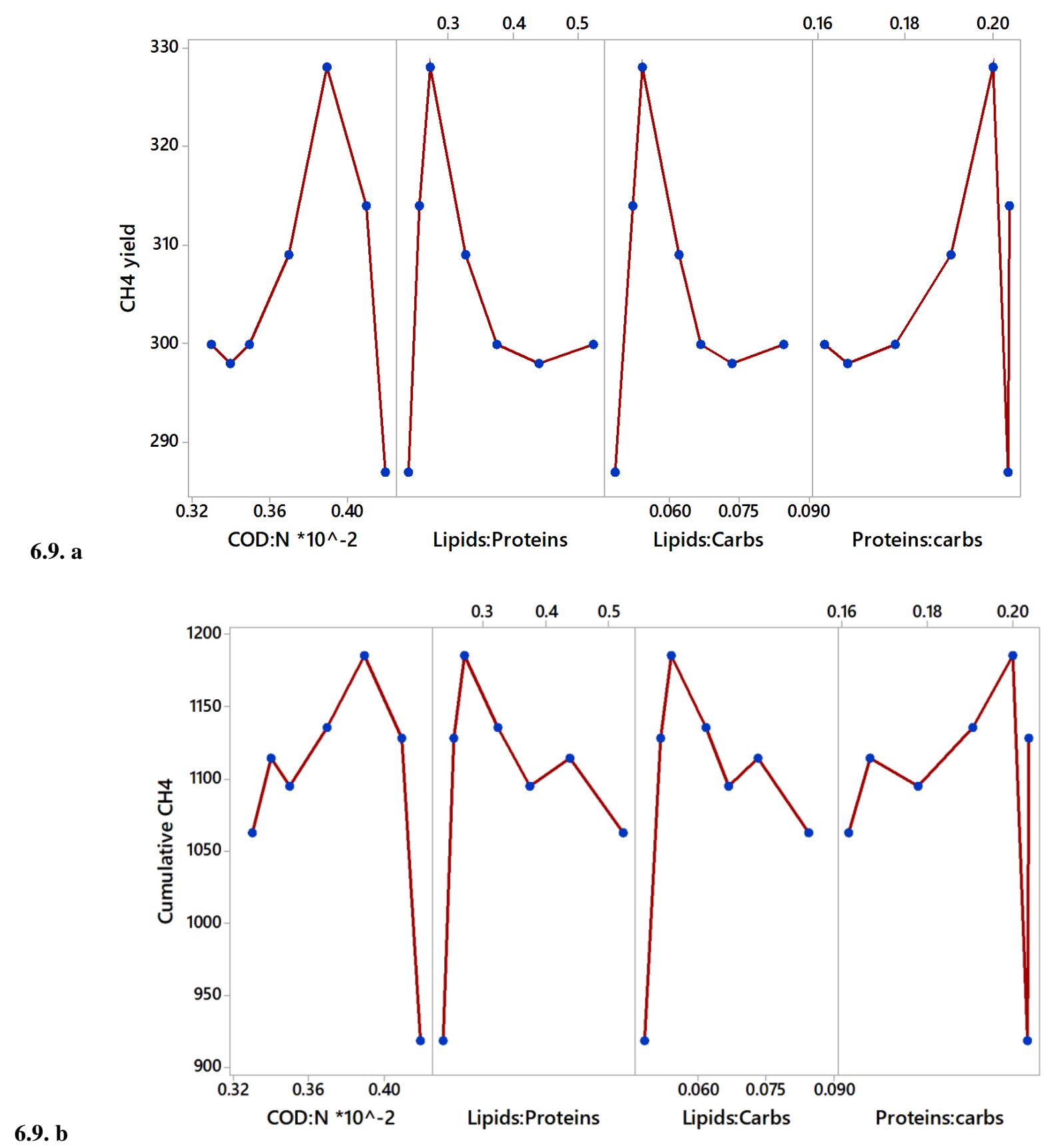

Figure 6.10. Matrix plot for: a. ultimate $\mathrm{CH}_{4}$ and b. $\mathrm{CH}_{4}$ yield at different COD:N and Lipids: Proteins, Lipids:

Carbohydrates, and Proteins: Carbohydrates Ratios

\subsection{Kinetic analysis results}

The results of kinetic study by modified Gompertz model using Eq. 3.5 is summarized in Table 6.3. The model was applied to the experimental data from mono and co-digestion of manure and 
SSO. The values of $\mathrm{P}$ for the maximum methane production per unit substrate $(\mathrm{mL} / \mathrm{g}), R_{m}^{e}$, the maximum methane production rate $(\mathrm{mL} / \mathrm{g} \mathrm{h})$, and $\lambda$, the lag phase time (d) were calculated and the results values are summarized in Table 6.3. The modified Gompertz model for mono- and codigestions of manure and SSO, rather showed a good fit to the experimental results with less than $10 \%$ diversion from the measured values. The estimated values and their correlation with the mixing ratio of the feedstock were in good compliance with the data obtained by the experiment. An increasing in $\mathrm{P}$ values was observed in co-digestion of manure with SSO which conformed to the experimental results.

Table 6.3. Summary of results of kinetic study using modified Gompertz model

\begin{tabular}{ccccc}
\hline & $\mathbf{P}(\mathbf{m L})$ & $\boldsymbol{R}_{\boldsymbol{m}}^{\boldsymbol{e}}\left(\frac{\mathbf{m L}}{\mathbf{D}}\right)$ & $\lambda(\mathbf{d})$ & $\mathbf{R}^{2}$ \\
\hline \hline Manure & 875 & 48 & 0.4 & 0.999 \\
\hline SSO & 998 & 83 & 1.6 & 0.999 \\
\hline Manure/SSO 7/3 & 1112 & 89 & 0.9 & 0.999 \\
\hline Manure/SSO 9/1 & 1065 & 57 & 0.7 & 0.999 \\
\hline Manure/SSO 5/5 & 1060 & 66 & 0.9 & 0.999 \\
\hline Manure/SSO 1/9 & 1018 & 76 & 1.1 & 0.999 \\
\hline Manure/SSO 3/7 & 936 & 81 & 0.8 & 0.999 \\
\hline
\end{tabular}

The trend of $\mathrm{P}$ variations complied with the experimental data as it showed the same trend in response to the corresponding mixing ratios. For instance, the highest $\mathrm{P}$ value of $1112 \mathrm{~mL}$ corresponded to the mixing ratio of 7:3. The lag phase varied from 0.01 to 0.7 days for different substrate mixing ratios. The lag phase time was quite short and less than 1 day for all the digesters. A short lag phase is advantageous as it does not demand for a long residence time and therefore, it does not require a large reactor volume which reduces the operational costs of the system. The values of $\mathrm{R}_{\max }^{\mathrm{e}}$ ranged from 48 to $89 \mathrm{~mL} / \mathrm{d}$ corresponding to manure mono digestion and manure/SSO mixing ratio of 7:3, respectively. 


\section{6. Hydrolysis/acidification}

This experiment was carried out for evaluating the hydrolysis/acidification phase in anaerobic codigestion of manure and SSO. A series of analysis such as degree of solublization, synergetic effect of co-digestion at different mixing ratios on hydrolysis and liquefaction, volatile fatty acids (VFAs) yield, and hydrolysis kinetics of lipids, proteins, and carbohydrates in co-digestion of manure and SSO was carried out and the results are summarized below.

Initially, characterization of the feedstocks in triplicates was conducted and the mean values are presented in Table 6.4. As shown in Table 6.4, both manure and SSO have high amount of COD concentration which is above $100 \mathrm{~g} / \mathrm{L}$. Therefore, COD concentrations of the feed for all of the reactors containing the combinations of manure and SSO were above $100 \mathrm{~g} / \mathrm{L}$. Manure contains high amount of carbohydrates and proteins. Adding manure to SSO increased carbohydrates and proteins concentrations in the co-digesters compared to the reactor digesting SSO alone. In order to obtain the hydrolysis rate coefficients of COD, lipids, proteins, and manure, the concentrations of total, soluble and particulate COD; total, soluble and particulate proteins; and total, soluble and particulate carbohydrates were monitored over time.

The data form total and soluble COD (SCOD) concentrations monitoring in all the digesters over time during a 72-h hydrolysis/acidification period was used to calculate the degree of COD solublization for each digester. As explained earlier, the degree of solublization was calculated using Eq. 3.1 and the result is summarized in Figure 6.10.

As illustrated in figure 6.10, the degree of the COD solublization ranged from $24 \%$ to $35 \%$. The most solublization of the COD content was $35 \%$ which corresponded to manure:SSO combination of 3:7 and 1:9. The COD solublization in mono digestion of manure and SSO were $24 \%$ and $28 \%$, respectively. The reactors containing only manure had the lowest degree of solublization. Manure resulted in a relatively lower solublization than SSO which could be due to the presence of some recalcitrant contents such as fibers and cellulosic compounds in manure that delay its hydrolysis and liquefaction. 
Table 6.4. Characteristics of the feedstocks at different mixing ratios of manure and SSO

\begin{tabular}{ccccccccc}
\hline & & Manure & SSO & M $/$ SSO & M/SSO & M/SSO & M/SSO & M/SSO \\
& & & & $9 / 1$ & $7 / 3$ & $5 / 5$ & $3 / 7$ & $1 / 9$ \\
\hline \hline Parameters & Units & Mixture & Mixture & Mixture & Mixture & Mixture & Mixture & Mixture \\
& & $(1)$ & $(2)$ & $(3)$ & $(4)$ & $(5)$ & $(6)$ & $(7)$ \\
TCOD & & Ave. & & & & & & \\
SCOD & $\mathrm{g} / \mathrm{L}$ & 101 & 115 & 103 & 105 & 108 & 111 & 114 \\
TSS & $\mathrm{g} / \mathrm{L}$ & 55 & 47 & 54 & 52 & 51 & 49 & 48 \\
VSS & $\mathrm{g} / \mathrm{L}$ & 32.1 & 39.9 & 32.9 & 34.5 & 36.0 & 37.6 & 39.2 \\
TS & $\mathrm{g} / \mathrm{L}$ & 69 & 78 & 69 & 71 & 73 & 75 & 77 \\
VS & $\mathrm{g} / \mathrm{L}$ & 59 & 64 & 59 & 60 & 61 & 62 & 63 \\
Ammonia & $\mathrm{g} / \mathrm{L}$ & 0.02 & 1.40 & 0.16 & 0.4 & 0.7 & 1.0 & 1.3 \\
pH & & 5.6 & 5.8 & 5.9 & 5.7 & 5.7 & 5.6 & 5.8 \\
Alkalinity & $\mathrm{g}$ CaCO3/L & 7.2 & 6.7 & 7.2 & 7.1 & 7.0 & 6.9 & 6.8 \\
TN & $\mathrm{g} / \mathrm{L}$ & 1.7 & 4.2 & 2.0 & 2.5 & 3.0 & 3.5 & 4.0 \\
TSN & $\mathrm{g} / \mathrm{L}$ & 0.1 & 1.1 & 0.2 & 0.4 & 0.6 & 0.8 & 1.0 \\
T-Carbs & $\mathrm{g} / \mathrm{L}$ & 26.8 & 14 & 25.5 & 23.0 & 20.5 & 17.9 & 15.4 \\
T-Proteins & $\mathrm{g} / \mathrm{L}$ & 5.4 & 2 & 5.1 & 4.4 & 3.8 & 3.1 & 2.5 \\
T-Lipids & $\mathrm{g} / \mathrm{L}$ & 1.7 & 1.5 & 1.7 & 1.6 & 1.6 & 1.5 & 1.5 \\
\hline M: Manure & & & & & & & &
\end{tabular}

It was observed that co-digestion increased solublization as all of the reactors containing the mixings of manure and SSO achieved a higher degree of solublization than the control reactors.

A $46 \%$ and $25 \%$ improvement in solublization was achieved in the co-digestion of manure and SSO in comparison with the control reactors digesting only manure and only SSO. Although SSO demonstrated more solublization than manure, no correlation between the portion of SSO and the degree of solublization at different mixing ratio was observed. This could verify that a proper mixing ratio is requires to enhance the microbial synergy for the process improvement.

The synergistic effect on the solublization of the feedstocks was evaluated by comparing the theoretical degree of solublization of each co-digester to the measured data from the experiment. The results indicated that co-digestion improved solublization by improving microbial synergy. As shown in Figure 6.11, all co-digesters achieved an improvement in solublization due to the synergistic effect of the microbial communities. The synergistic effect varied from 18 to $34 \%$ corresponding to manure/SSO mixing ratios of 5:5 and 9:1, respectively. 


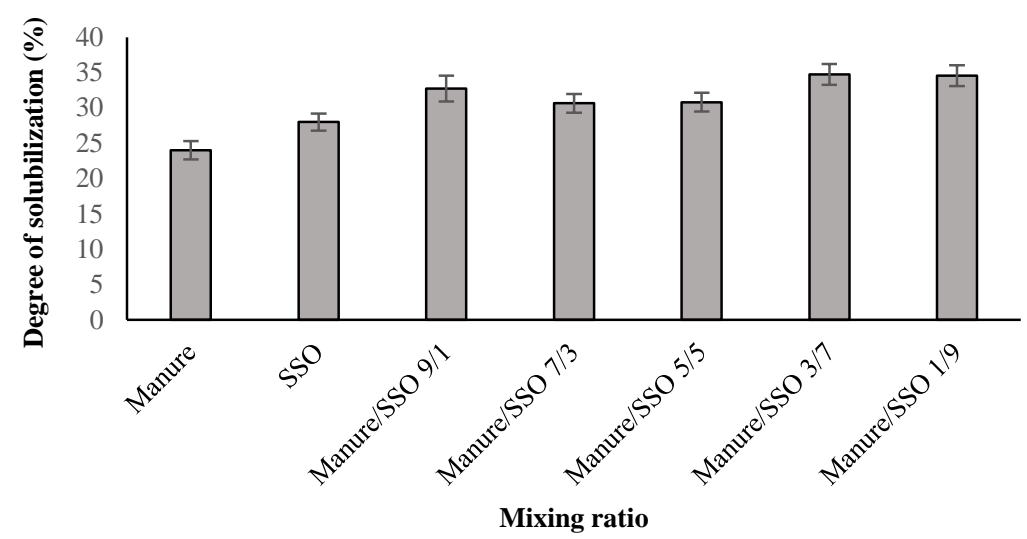

Figure 6.10. Degree of COD solublization at different mixing ratios of Manure/SSO

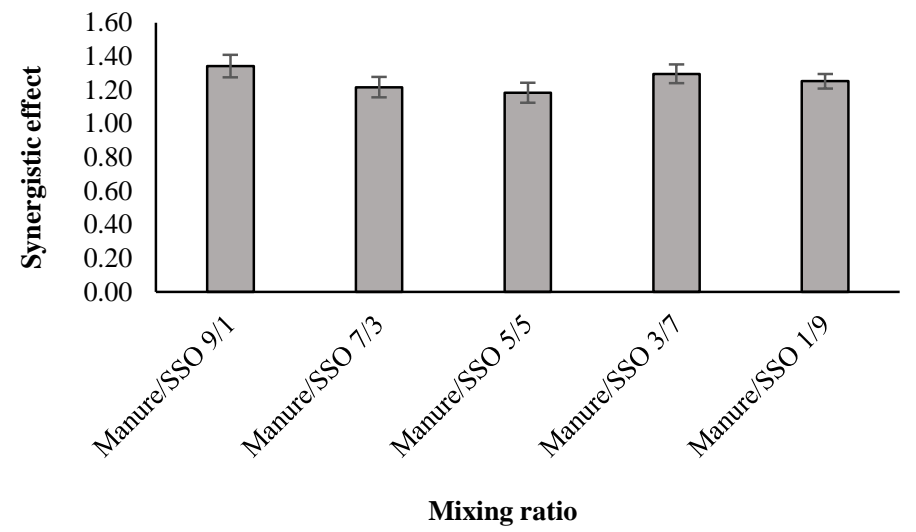

Figure 6.11. Synergistic effect on solublization at different mixing ratios of Manure/SSO

The monitoring of VFAs concentrations over time showed an increasing trend during the 72-hr of the hydrolysis/acidification process. The total VFAs yields were calculated in terms of mass of produced VFAs per mass of VSS added (mg VFAs/g VSS added). As illustrated in Figure 6.12, SSO alone had more VFAs yields than manure alone. The VFAs yield in the co-digester was correlated to the mixing ratios of the feedstocks so that the VFAs yield increased by increasing the fraction of SSO in the co-digesters. The trend of VFAs yield did not comply with the trend of COD solublization of the corresponding mixing ratios (Figure 6.10). Hence, hydrolysis/liquefaction occurred independently of acidification and followed a different trend from acidification. All of the reactors containing the mix of manure and SSO had higher VFAs yield than the control reactors. The VFAs yields were $231 \mathrm{mg}$ VFAs/g VSS added and $325 \mathrm{mg}$ VFAs/g VSS added for the manure and SSO mono digestions, respectively. The maximum VFAs yield of $400 \mathrm{mg}$ VFAs/g VSS added was achieved by manure/SSO ratio of 1:9. 


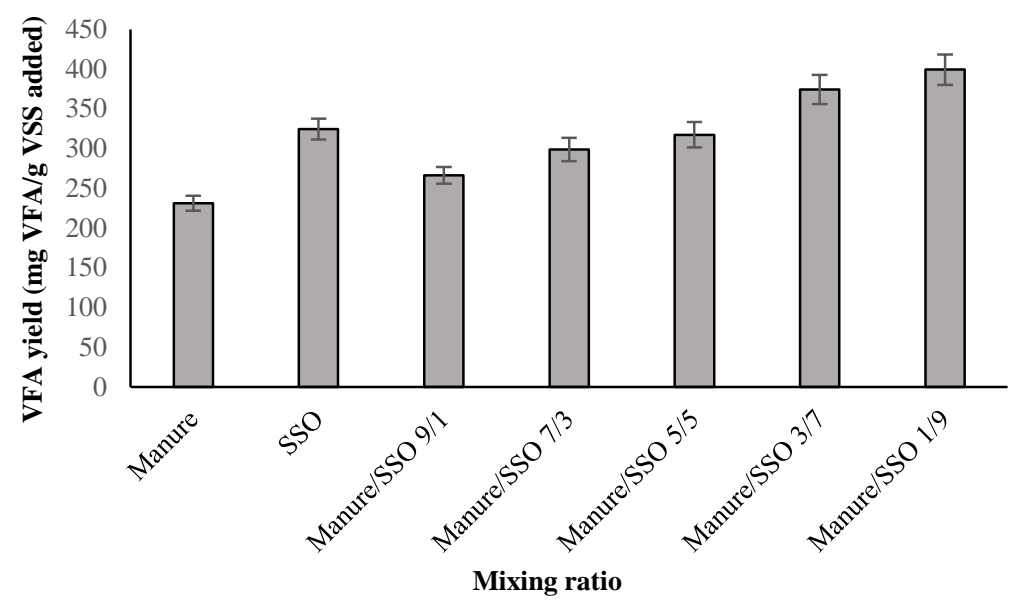

Figure 6.12. Total VFAs yield at different mixing ratios of Manure/SSO

Monitoring soluble and particulate COD over time, indicated an increasing trend in solublization of COD and particulate COD degradation. Furthermore, the monitoring of soluble and particulate lipids, proteins, and carbohydrates over time also demonstrated an increasing trend in solublization and particulate matter degradation. Similar to the previous experiments, in codigestion of manure and SSO, the hydrolysis and solublization rates of the lipids and proteins, were slower than carbohydrate. These results are summarized in the tables presented in the appendix. The hydrolysis rate coefficient $\left(\mathrm{K}_{\mathrm{h}}\right)$ based on first order kinetics (equations 3.3 and 3.4) was calculated using AquaSim 2.0 for COD, lipids, proteins and carbohydrates based on the particulate degradation and results are summarized in Table 6.5.

In manure and SSO co-digestion, hydrolysis did not show the same trend as methanogenesis. The hydrolysis kinetics showed a higher rate in the manure:SSO mixing ratio of 3:7 while the most methane yield occurred at the mixing ratio of 7:3 with more fraction of manure. This would be due to the more microbial population of methanogens which was introduced by more fraction of manure in the co-digester. Similar to the co-digestion of TWAS with SSO and TWAS with manure, the improvement of the hydrolysis in co-digestion of manure with SSO resulted from the improvement of proteins and carbohydrates hydrolysis than that of lipids contents of the feedstocks. Unlike TWAS/manure and TWAS/SSO co-digestion, the fluctuations of hydrolysis rate coefficient of lipids and carbohydrates at different feedstocks mixing ratios showed similar 
trend in manure/SSO co-digestion. Nevertheless, in co-digestion of manure with SSO the variations of $\mathrm{K}_{\mathrm{h}}$ for proteins demonstrated a different trend from that of lipids and carbohydrates. The maximum hydrolysis rate of proteins corresponded to the manure:SSO mixing ratio of 1:9. However, the maximum $\mathrm{K}_{\mathrm{h}}$ values for lipids and carbohydrates were 0.11 and 0.68 corresponding to the mixing ratio of $3: 7$.

Table 6.5. Hydrolysis rate coefficients for COD, lipids, proteins, and carbohydrate content in co-digestion of SSO and manure at different mixing ratios

\begin{tabular}{lccccccc}
\hline \multicolumn{1}{c}{$\mathbf{K}_{\mathbf{h}}$} & $\mathrm{M}^{*} / \mathrm{SSO} 1 / 9$ & $\mathrm{M} / \mathrm{SSO} 3 / 7$ & $\mathrm{M} / \mathrm{SSO} 5 / 5$ & $\mathrm{M} / \mathrm{SSO} 7 / 3$ & $\mathrm{M} / \mathrm{SSO}$ 9/1 & Manure & SSO \\
\hline \hline $\mathrm{K}_{\mathrm{h} \text { COD }}$ & 0.37 & 0.38 & 0.31 & 0.32 & 0.35 & 0.23 & 0.30 \\
\hline $\mathrm{K}_{\mathrm{h} \text { Lipids }}$ & 0.09 & 0.11 & 0.08 & 0.07 & 0.08 & 0.07 & 0.08 \\
\hline $\mathrm{K}_{\mathrm{h} \text { Proteins }}$ & 0.35 & 0.33 & 0.25 & 0.29 & 0.32 & 0.21 & 0.28 \\
\hline $\mathrm{K}_{\mathrm{h} \text { Carbohydrates }}$ & 0.63 & 0.68 & 0.57 & 0.55 & 0.61 & 0.43 & 0.55
\end{tabular}

${ }^{*} \mathrm{M}$ : Manure 


\section{Chapter 7}

Results and discussion

$$
\begin{gathered}
\text { TWAS, Manure, SSO Co- } \\
\text { digestion }
\end{gathered}
$$




\section{Results and discussion- TWAS, manure, SSO co-digestion 7.1. BMP of TWAS, manure, SSO}

This experiment was conducted on ternary co-digestion of TWAS, manure, and SSO. Manure slurry was prepared as discussed in chapter 3 and was fed to the reactors in different combinations with SSO and TWAS in triplicates. Control reactors containing TWAS, manure, and SSO individually were also used in triplicates. The influence of feedstocks mixing ratios and their correlation with the lipids: proteins: carbohydrates ratios on biomethane production in anaerobic co-digestion of TWAS/manure/SSO was evaluated. The characteristics of the feed in each digester with different mixing ratios of the substrates for the average of three measurement of each parameter are summarized in Table 7.1.

As presented in Table 7.1, both manure and SSO have high amount of COD concentrations compared to TWAS. The COD concentrations of the feedstocks were 100, 109, and $101 \mathrm{~g} / \mathrm{L}$ for the digesters fed with manure, SSO and mixture of TWAS/manure/SSO at 1:1:8 ratio, respectively. Other reactors which contained the combinations of TWAS, manure, and SSO had a COD concentration ranging from 53 to $95 \mathrm{~g} / \mathrm{L}$. VSS concentrations were 26.5, 45.4, and 47.0 for TWAS, manure, and SSO respectively and ranged from 30.4 to 44.8 for the reactors containing the combinations of them. The amount of carbohydrates and lipids of manure and SSO were significantly more than that of TWAS. Therefore, addition of manure and SSO to TWAS increased the carbohydrates and lipids content of the co-digesters compared to the rectors digesting only TWAS.

Carbohydrates concentrations were within a range between 5.4 to $23.8 \mathrm{~g} / \mathrm{L}$ in the co-digesters. Total proteins concentrations of TWAS, manure, and SSO were 3.9, 5.8, and 2.4 respectively and varied from 2.9 to $4.5 \mathrm{~g} / \mathrm{L}$ in the co-digesters. The total lipids concentrations of TWAS was 0.4 $\mathrm{g} / \mathrm{L}$ while manure and SSO had lipids concentrations of 1.4 and $1.5 \mathrm{~g} / \mathrm{L}$ respectively. Adding manure and SSO increased the lipids content in the reactors containing the combination of TWAS, manure, and SSO compared to the reactors containing only TWAS. The total lipids concentrations varied from 0.6 to $1.3 \mathrm{~g} / \mathrm{L}$ in the co-digesters containing the mix of the three feedstocks. $\mathrm{pH}$ was around neutral point varying from 7 to 7.2 for all of the reactors. 
Table 7.1. Characteristics of feed to digesters with different mixing ratios of TWAS/ manure/SSO

\begin{tabular}{|c|c|c|c|c|c|c|c|c|c|c|c|c|c|}
\hline & & TWAS & Manure & SSO & $\begin{array}{c}\mathrm{T}^{*}: \mathrm{M}^{* *}: \mathrm{SSO} \\
8: 1: 1\end{array}$ & $\begin{array}{c}\text { T:M:SSO } \\
1: 8: 1\end{array}$ & $\begin{array}{c}\text { T/M/SSO } \\
1: 1: 8\end{array}$ & $\begin{array}{l}\text { T/M/SSO } \\
5: 2.5: 2.5\end{array}$ & $\begin{array}{l}\text { T/M/SSO } \\
2.5: 5: 2.5\end{array}$ & $\begin{array}{l}\text { T/M/SSO } \\
2.5: 2.5: 5\end{array}$ & $\begin{array}{c}\text { T/M/SSO } \\
4: 4: 2\end{array}$ & $\begin{array}{c}\text { T/M/SSO } \\
2: 4: 4\end{array}$ & $\begin{array}{c}\text { T/M/SSO } \\
4: 2: 4\end{array}$ \\
\hline Parameters & Units & $\begin{array}{c}\text { Mixture } \\
\text { (1) }\end{array}$ & $\begin{array}{c}\text { Mixture } \\
\text { (2) }\end{array}$ & $\begin{array}{c}\text { Mixture } \\
\text { (3) }\end{array}$ & Mixture (4) & $\begin{array}{c}\text { Mixture } \\
(5)\end{array}$ & $\begin{array}{c}\text { Mixture } \\
\text { (6) }\end{array}$ & $\begin{array}{c}\text { Mixture } \\
(7)\end{array}$ & $\begin{array}{c}\text { Mixture } \\
(8)\end{array}$ & $\begin{array}{c}\text { Mixture } \\
(9)\end{array}$ & $\begin{array}{c}\text { Mixture } \\
(10)\end{array}$ & $\begin{array}{c}\text { Mixture } \\
\text { (11) }\end{array}$ & $\begin{array}{c}\text { Mixture } \\
\text { (12) }\end{array}$ \\
\hline TCOD & $\mathrm{g} / \mathrm{L}$ & 40 & 100 & 109 & 53 & 95 & 101 & 72 & 87 & 89 & 78 & 71 & 79 \\
\hline SCOD & $\mathrm{g} / \mathrm{L}$ & 1.4 & 42 & 41 & 9 & 38 & 37 & 21 & 32 & 31 & 26 & 25 & 25 \\
\hline TSS & $\mathrm{g} / \mathrm{L}$ & 31 & 52 & 62 & 37 & 51 & 58 & 44 & 50 & 52 & 46 & 42 & 48 \\
\hline VSS & $\mathrm{g} / \mathrm{L}$ & 26.5 & 45.4 & 47.0 & 30.4 & 43.7 & 44.8 & 36.3 & 41.1 & 41.5 & 38.2 & 33.2 & 38.5 \\
\hline $\mathrm{TS}$ & $\mathrm{g} / \mathrm{L}$ & 38.9 & 67.8 & 67.0 & 44.6 & 64.8 & 64.3 & 53.2 & 60.4 & 60.2 & 56.1 & 48.1 & 55.9 \\
\hline VS & $\mathrm{g} / \mathrm{L}$ & 35.2 & 55.6 & 49.6 & 38.6 & 52.9 & 48.7 & 43.9 & 49.0 & 47.5 & 46.2 & 38.0 & 45.0 \\
\hline $\mathrm{pH}$ & - & 7.0 & 7.0 & 7.2 & 7.1 & 7.0 & 7.0 & 7.1 & 7.1 & 7.1 & 7.0 & 7.0 & 7.1 \\
\hline Alkalinity & $\begin{array}{c}\mathrm{g} \\
\mathrm{CaCO}_{3} / \mathrm{L}\end{array}$ & 1.9 & 5.2 & 6.2 & 2.7 & 5.0 & 5.7 & 3.8 & 4.6 & 4.9 & 4.1 & 3.9 & 4.3 \\
\hline $\mathrm{TN}$ & $\mathrm{g} / \mathrm{L}$ & 2.8 & 2.1 & 4.0 & 2.8 & 2.4 & 3.7 & 2.9 & 2.7 & 3.2 & 2.7 & 2.6 & 3.1 \\
\hline TSN & $\mathrm{g} / \mathrm{L}$ & 0.4 & 0.1 & 1.0 & 0.4 & 0.2 & 0.8 & 0.5 & 0.4 & 0.6 & 0.4 & 0.5 & 0.6 \\
\hline Total carbs & $\mathrm{g} / \mathrm{L}$ & 1.5 & 27.8 & 14.1 & 5.4 & 23.8 & 14.2 & 11.3 & 17.8 & 14.4 & 14.6 & 11.5 & 11.8 \\
\hline $\begin{array}{c}\text { Total } \\
\text { proteins }\end{array}$ & $\mathrm{g} / \mathrm{L}$ & 3.9 & 5.8 & 2.4 & 3.9 & 5.2 & 2.9 & 4.0 & 4.5 & 3.6 & 4.3 & 2.9 & 3.7 \\
\hline Total lipids & $\mathrm{g} / \mathrm{L}$ & 0.4 & 1.4 & 1.5 & 0.6 & 1.3 & 1.4 & 0.9 & 1.2 & 1.2 & 1.0 & 0.9 & 1.0 \\
\hline
\end{tabular}

${ }^{*}$ T: TWAS

** M: Manure 
Operation of the digesters carried on until no significant amount of biogas was produced. Fig. 7.1 shows the profile of the cumulative biomethane production versus time during the co-digestion of TWAS, manure and SSO including the control rectors. SSO alone produced more cumulative methane than TWAS in the control reactors. The amount of methane produced by SSO alone was $13 \%$ more methane than manure alone.

All co-digesters produced more biomethane than the control reactors containing only TWAS. The amounts of ultimate $\mathrm{CH}_{4}$ obtained by single digestion of SSO and manure were higher than that of TWAS alone by 3.2 and 2.9 fold. As illustrated in figure 7.1, all of the co-digesters generated higher amounts of $\mathrm{CH}_{4}$ than the control reactors containing only manure. The reactors with the mix of the three feedstocks at the ratios of $8: 1: 1$ and 5:2.5:2.5 produced more methane than TWAS alone however, they did not show any improvement in comparison with single digestion of manure and SSO. Other combinations produced more methane than TWAS and SSO alone, although only the three of them with the mixing ratios of 2.5:2.5:5, 4:2:2, and 4:2:4 resulted in higher cumulative $\mathrm{CH}_{4}$ production comparing to all of the control reactors. The maximum cumulative methane production of $1424 \mathrm{~mL}$ corresponded to the TWAS/manure/SSO mixing ratio of 2:4:4 and lipids: proteins: carbohydrates ratio of $1: 3: 12$.

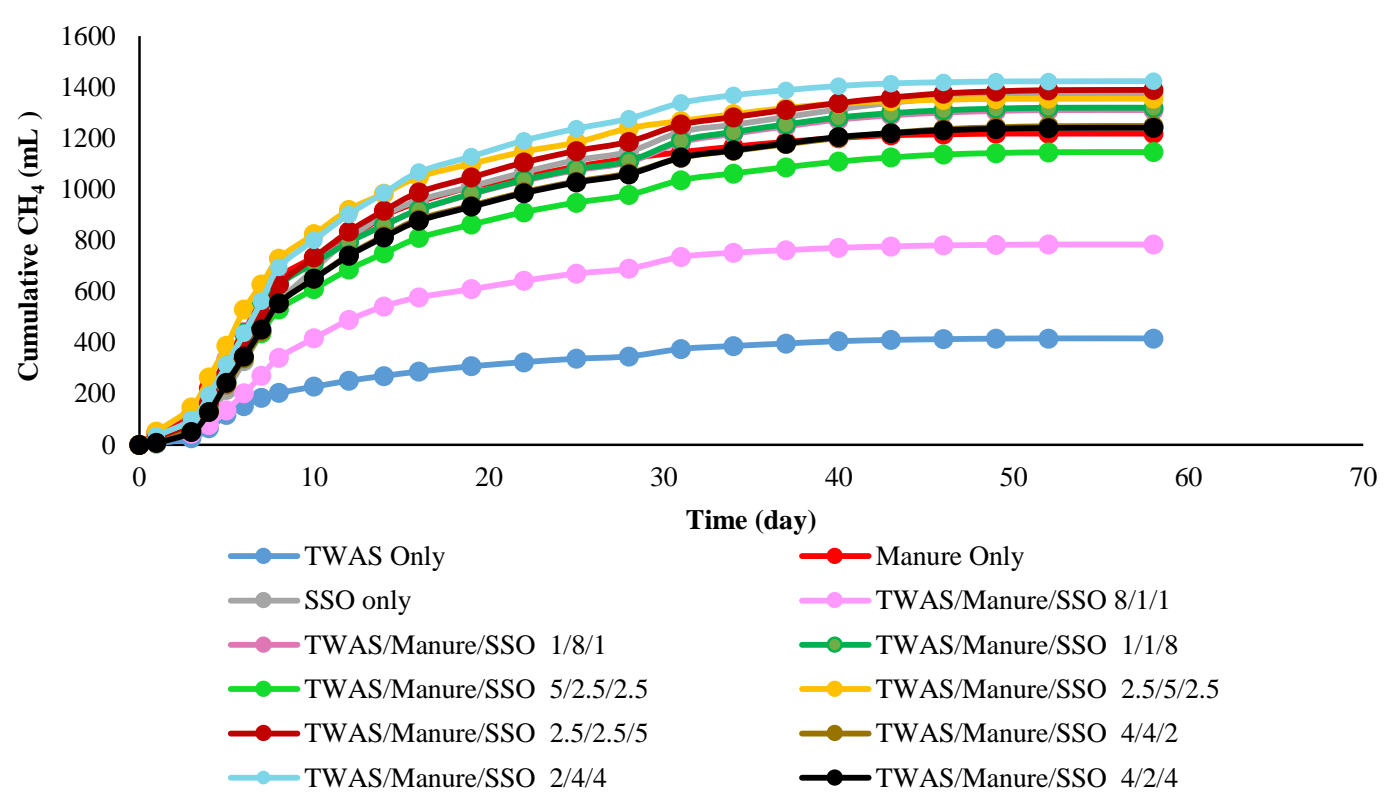

Figure 7.1. Cumulative methane production for different mixing ratios of TWAS/manure/SSO 
Figure 7.2, illustrates the methane rate in $\mathrm{mL} /$ day for all of the feedstocks combinations including the control reactors. The lowest maximum $\mathrm{CH}_{4}$ rate corresponded to TWAS mono digestion. Among control reactors, manure had the highest maximum $\mathrm{CH}_{4}$ production rate. All of the digesters, reached their maximum methane rate in less than 10 days of the process operation. The digesters containing the mixings of TWAS/manure/SSO at the ratio of 1:8:1 achieved the highest maximum methane production rate at the first week of the operational period. The digesters produced $32 \%$ to $47 \%$ of their ultimate $\mathrm{CH}_{4}$ production during the first week and $65 \%$ to $73 \%$ of it during the two weeks of the digestion period.

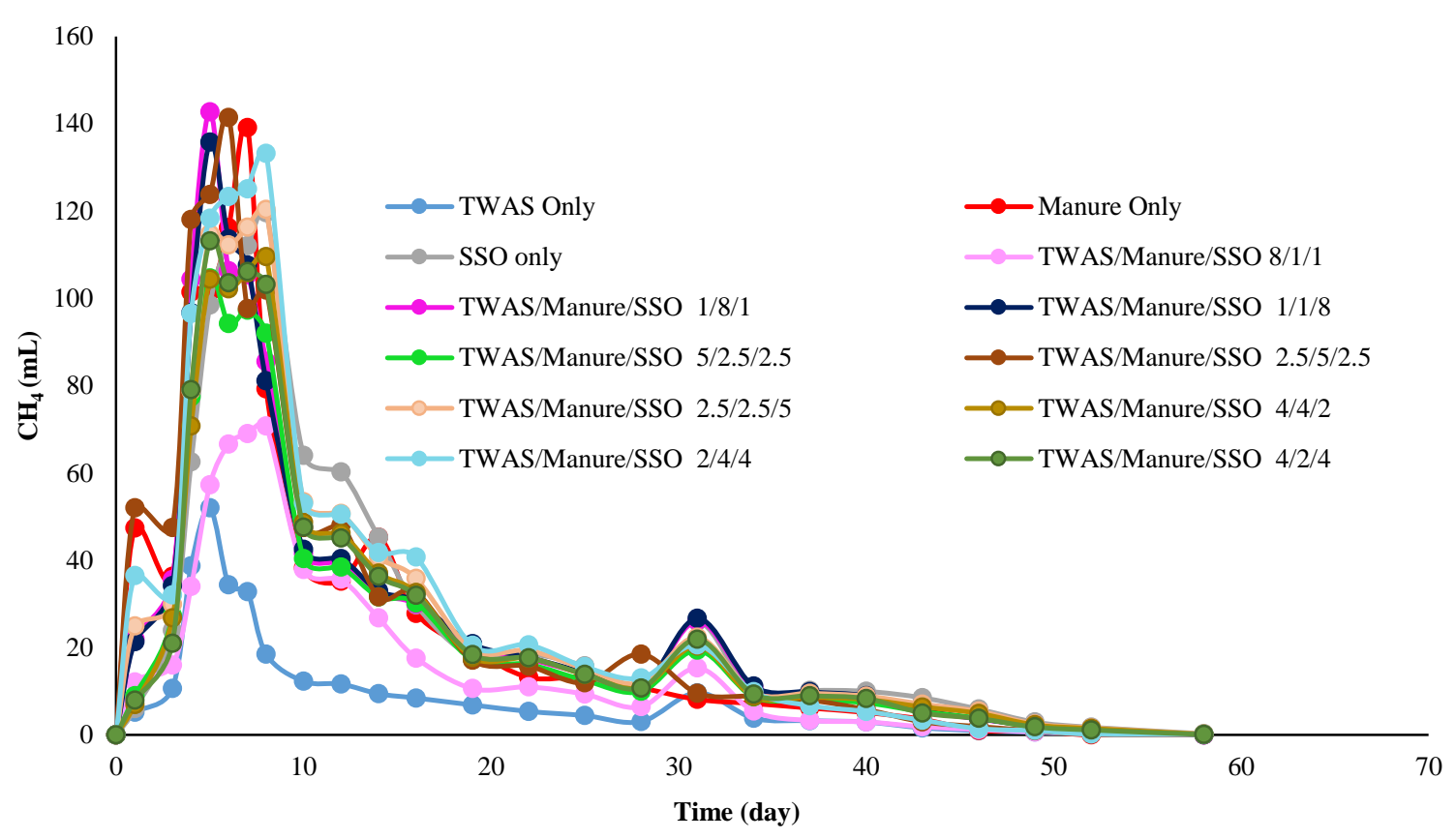

Figure 7.2. Methane production rate $(\mathrm{mL} / \mathrm{d})$ for different mixing ratios of TWAS/Manure/SSO

The maximum $\mathrm{CH}_{4}$ production rates at different mixing ratios including the controls are shown in Figure 7.3. The lowest maximum rate of $52 \mathrm{~mL} /$ day corresponded to single digestion of TWAS. The maximum $\mathrm{CH}_{4}$ rates of manure and SSO alone were $139 \mathrm{~mL} /$ day and $120 \mathrm{~mL} / \mathrm{day}$, respectively. The maximum $\mathrm{CH}_{4}$ rates of the co-digesters including the mix of the three feedstocks varied from 71 to $143 \mathrm{~mL} / \mathrm{day}$. As presented in the figure, the highest maximum rate of $143 \mathrm{~mL} /$ day corresponded to the TWAS/manure/SSO mixing ratio of 1:8:1. 


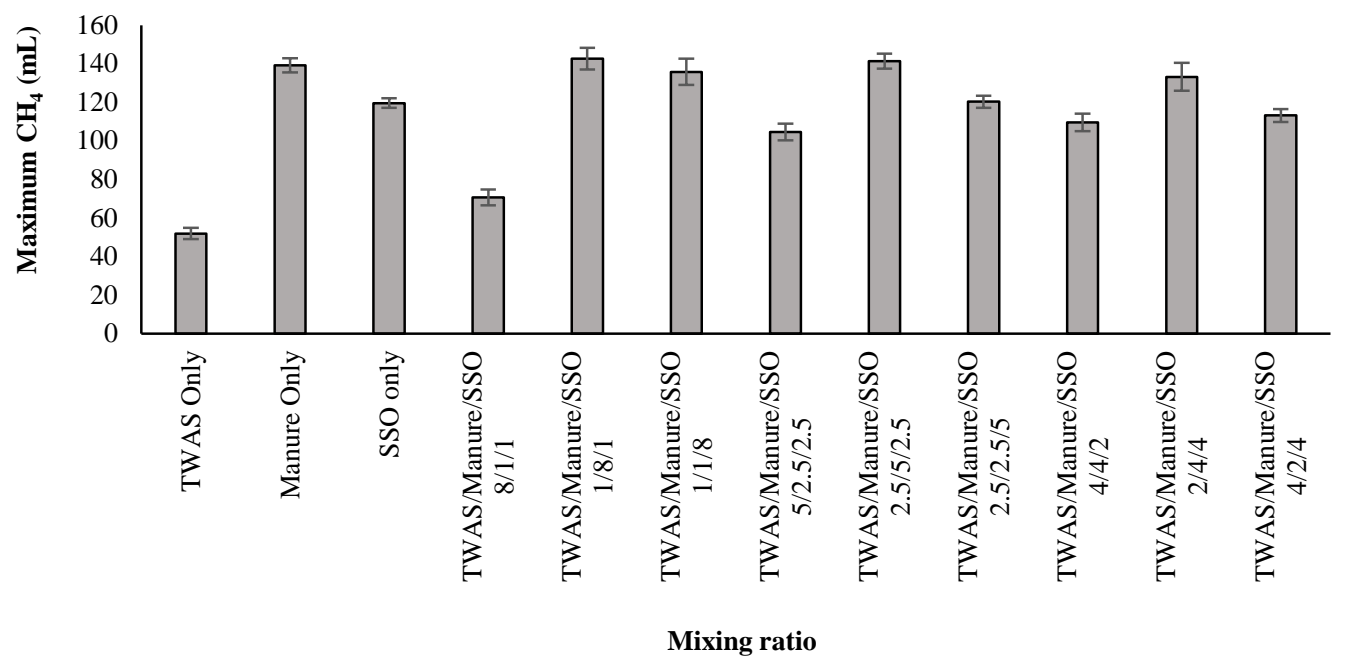

Figure 7.3. Maximum methane production rate $(\mathrm{mL} / \mathrm{d})$ for different mixing ratios TWAS/manure/SSO

The COD mass balance was conducted for all of the digesters and the result is summarized in Figure 7.4. The mass balance was calculated considering the initial and the final TCOD concentrations of the reactors' contents, and the theoretical methane production per unit mass of TCOD removed. Comparing the experimental methane production data with the ones obtained by the calculations, showed a deviation of less than $5 \%$ for all of the digesters.

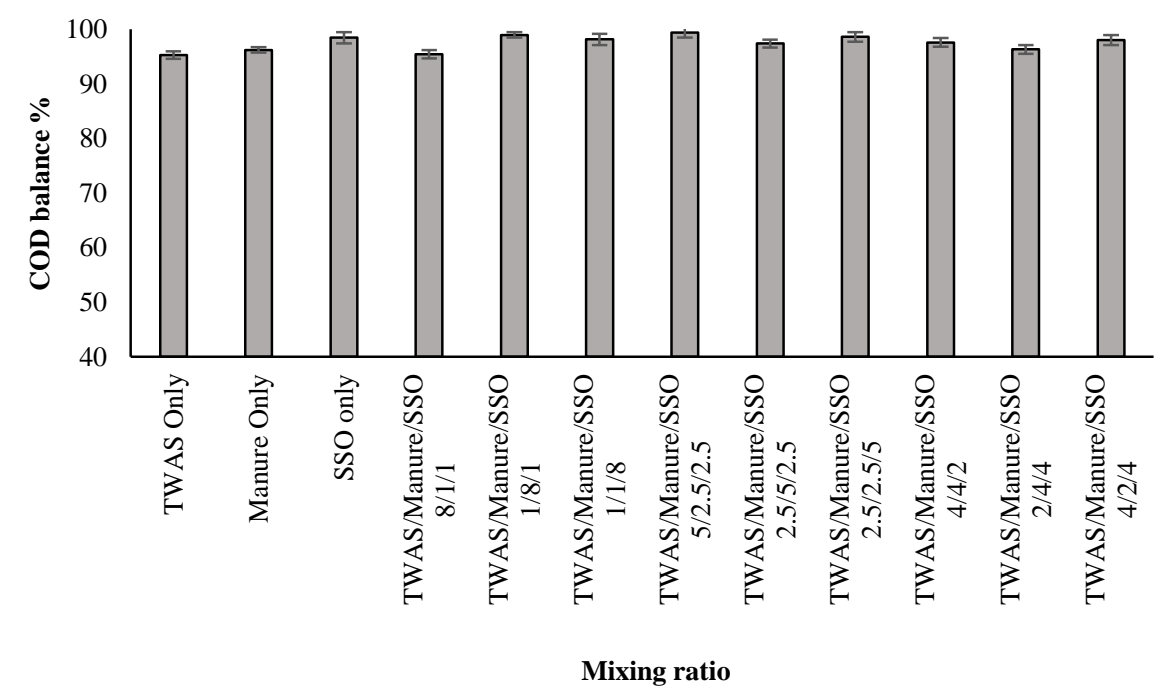

Figure 7.4. COD mass balance in co-digestion of manure and SSO for different mixing ratios 4 


\subsection{Cumulative methane yields}

Figure 7.5 shows the cumulative methane yields of the digesters including $\mathrm{mLCH}_{4} / \mathrm{g}$ TCOD added, $\mathrm{mLCH}_{4} / \mathrm{g}$ VSS added, and $\mathrm{mLCH}_{4} / \mathrm{mL}$ substrate added. The cumulative methane yield per mass COD of substrate added were 134, 299, and $332 \mathrm{mLCH}_{4} / \mathrm{g}$ TCOD added for the control reactors digesting only TWAS, manure, and SSO, respectively. As shown in Figure 7.5. a), the minimum and the maximum yield corresponded to mono digestion of TWAS and TWAS/manure/SSO mixing ratio of 2:4:4, respectively. The biomethane yields ranged from 228 to $356 \mathrm{mLCH}_{4} / \mathrm{g}$ TCOD added in the co-digesters. $\mathrm{CH}_{4}$ yield increased by $165 \%, 19 \%$ and $7 \%$ compared to single digestion of TWAS, manure, and SSO, respectively.

The methane yields in $\mathrm{mLCH}_{4} / \mathrm{g}$ VSS added are presented in Figure 7.5. b). The $\mathrm{CH}_{4}$ yields of 202, 659, and $766 \mathrm{mLCH}_{4} / \mathrm{g}$ VSS added corresponded TWAS, manure, and SSO for the control reactors, respectively. The $\mathrm{CH}_{4}$ yield varied from 395 to $982 \mathrm{mLCH}_{4} / \mathrm{g}$ VSS added in the reactors containing the combinations of the three feedstocks. The maximum $\mathrm{CH}_{4}$ yield of $395 \mathrm{mLCH}_{4} / \mathrm{g}$ VSS added was achieved by the co-digestion of TWAS/manure/SSO at the mixing ratio of 2:4:4. Figure 7.5. c), illustrates the methane yield per unit volume of substrate added. TWAS, Manure and SSO individually resulted in 5.3, 30 and $36 \mathrm{mLCH}_{4} / \mathrm{mL}$ substrate added, respectively. The reactors with the combinations of manure and SSO resulted in methane yields ranging from 12 $\mathrm{mLCH}_{4} / \mathrm{mL}$ substrate added to $32.6 \mathrm{mLCH}_{4} / \mathrm{mL}$ substrate added. The most $\mathrm{CH}_{4}$ yield per unit volume of substrate added corresponded to single digestion of SSO and TWAS/manure/SSO codigestion at the mixing ratios of 2:4:4.

Biodegradable fraction as mentioned earlier was calculated using the Eq. 4.1 and the result is summarized in Figure 7.6. The biodegradable fraction of the digesters ranged from 33\% to 89\%. TWAS, manure and SSO alone, corresponded to biodegradable fractions of 33\%, $75 \%$ and $83 \%$, respectively. The most percentage of biodegradable fraction occurred at the reactor co-digesting TWAS, manure and SSO at the mixing ratio of 2:4:4. This in good compliance with the maximum methane production that occurred at the same mixing ratio. The trend of variation in the methane

yields also conforms to the trend that was observed for the variations of biodegradable fractions at the different mixing ratios. All of the co-digesters demonstrated a more biodegradable fraction than TWAS alone, nevertheless not all of the combinations resulted in more biodegradable fraction than manure and SSO alone. 
7.5. a)

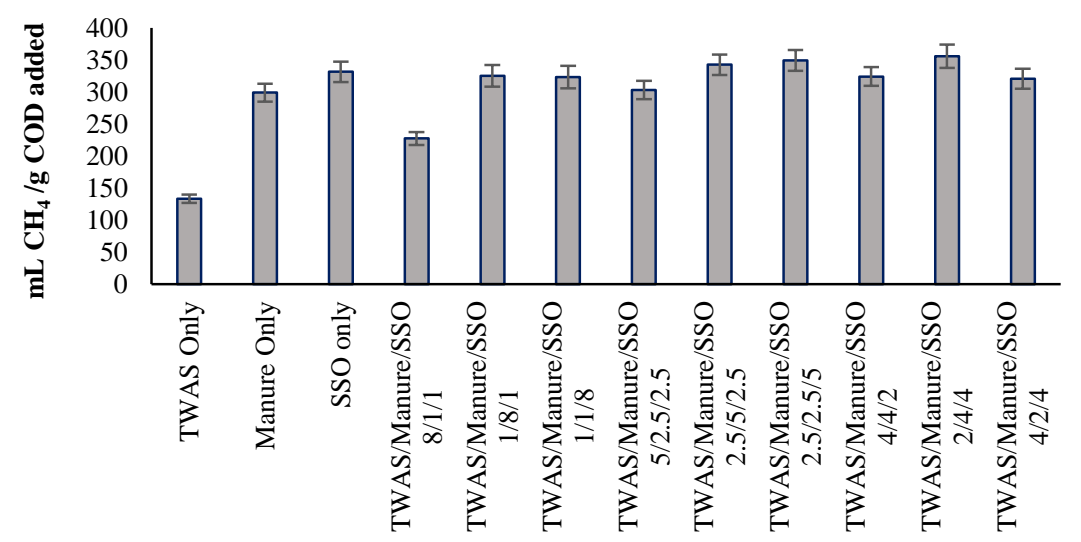

7.5. b)

Mixing ratios

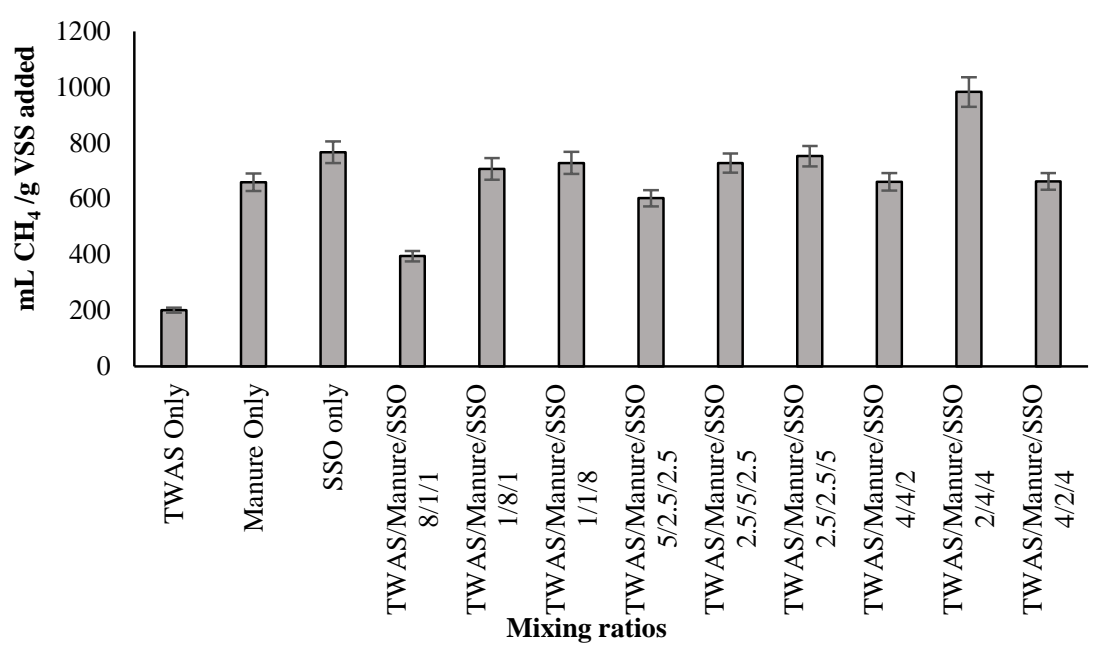

7.5. c)

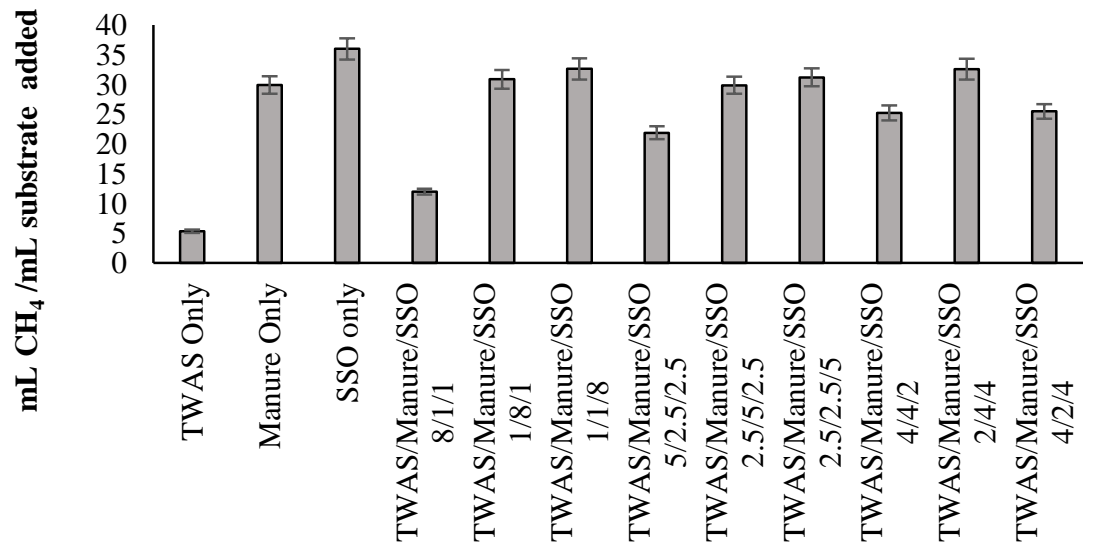

\section{Mixing ratios}

Figure 7.5. Methane yields a) per unit mass TCOD added, $b$ ) per unit mass of VSS added and c) per volume substrate added at different mixing ratio of TWAS and manure 
The reason would be that the balance of the nutrient with the microbial communities was not necessarily ideal for methanogenic populations in the reactors containing the mix of the three feedstocks at all of the mixing ratios.

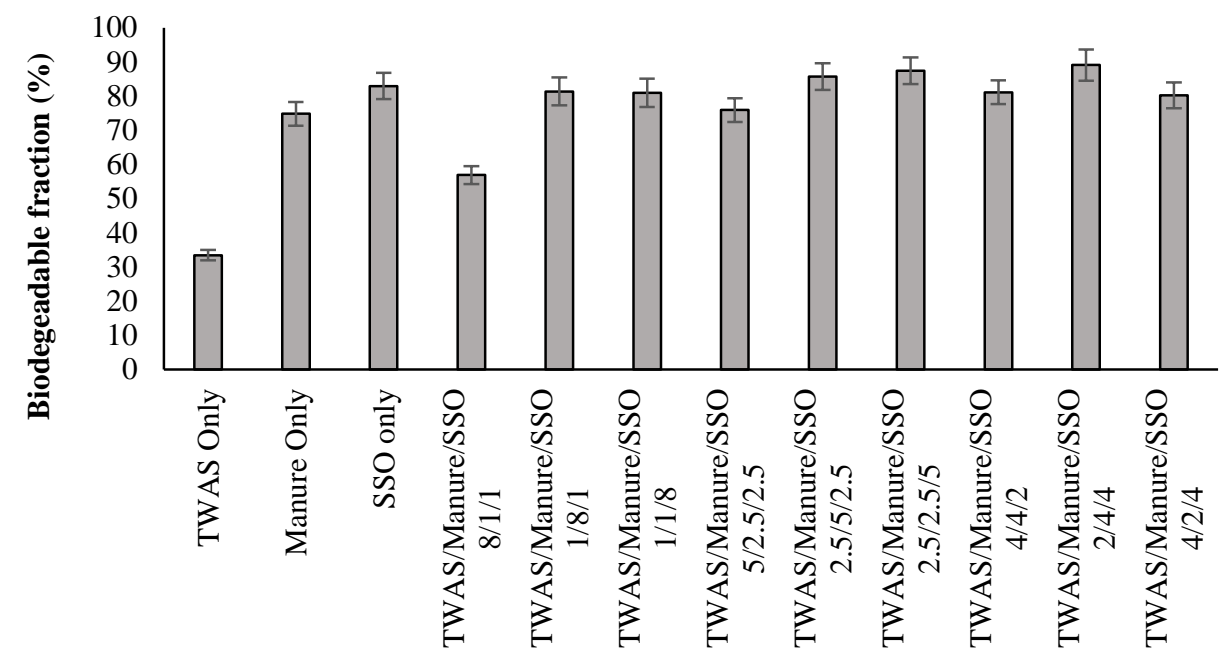

Mixing ratios

Figure 6.6. Biodegradable fraction of the feedstocks for different mixture ratios of TWAS and manure

\subsection{Synergistic effect}

Synergistic effect was obtained by calculating the percentage of additional methane yield achieved in co-digestion. This was done by dividing the measured yields, over the weighted average of the methane yield of individual substrates per unit volume of substrate added. Figure 7.7 shows the percentage improvement of biomethane production due to the synergistic impact. As demonstrated in Fig 7.7, in the ternary co-digestion of TWAS/manure/SSO, the most synergetic impact corresponds to the mixing ratio of 2:4:4. No significant improvement due to synergy was observed at the mixing ratio of 1:1:8. The maximum synergistic effect that was achieved by this experiment was $19 \%$ which was slightly lower than the result obtained by some of digesters in the binary codigestion. 


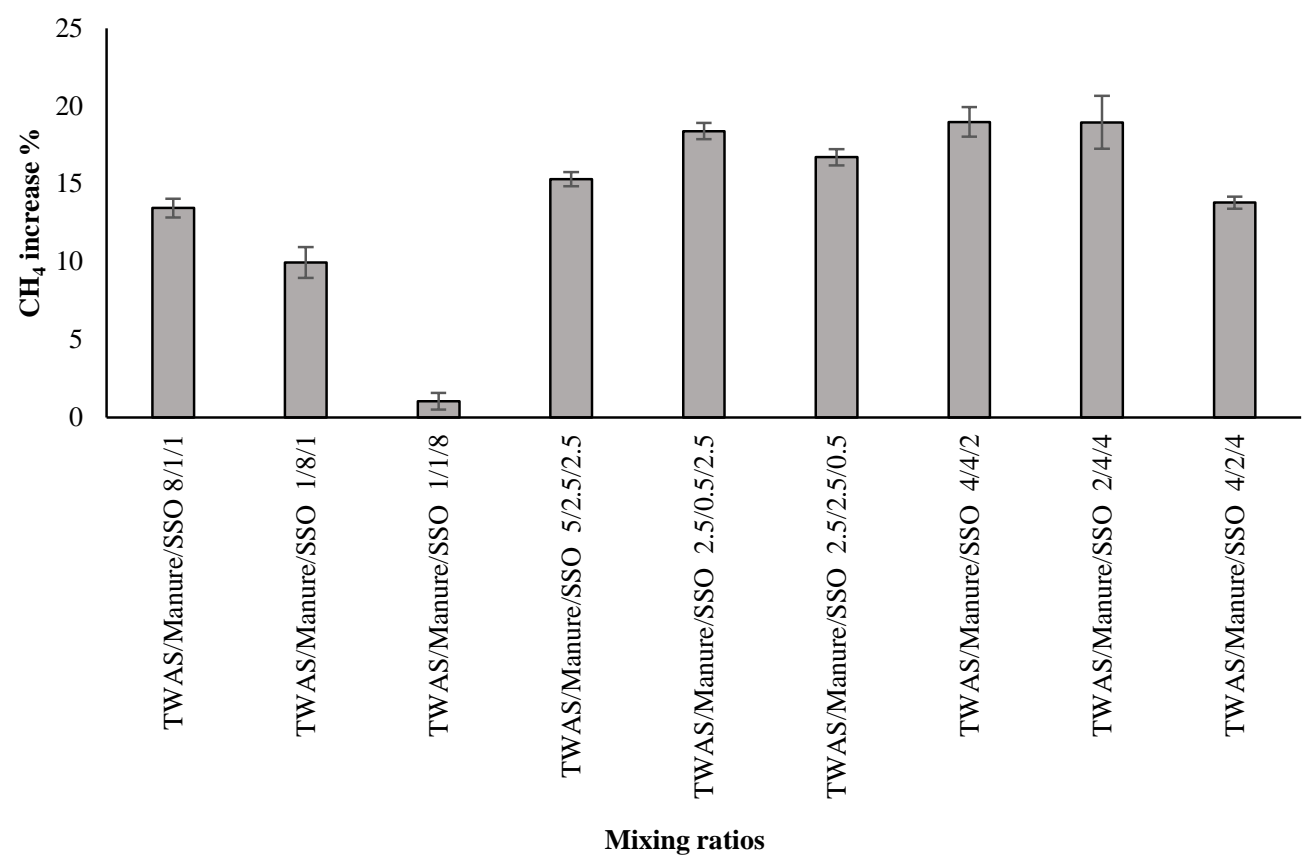

Figure 7.7. Synergetic effect of co-digestion at different mixing ratios of manure and SSO

\subsection{COD:N and Lipids: Proteins: Carbohydrates ratios}

The values of COD: $\mathrm{N}$ ratios, lipids: proteins: carbohydrates ratios, the ultimate methane production and the methane yield per unit mass of COD added at different mixing ratios are presented in Table 7.2. The COD:N ratios of TWAS, manure and SSO were 15, 47, and 27 respectively. For the co-digesters, COD:N varied from 19 to 40 . The lipids: proteins: carbohydrates ratios were 1:10:4, 1:4:20, and 1:1.6:9 for TWAS, manure, and SSO respectively. Among them SSO had the most ultimate methane production and methane yield corresponding to $1373 \mathrm{~mL}$ and $332 \mathrm{~mL} \mathrm{CH}_{4} / \mathrm{g}$ COD added. As shown in Table 7.2, the minimum ultimate methane production and the methane yield occurred at TWAS mono digestion corresponding to the COD:N ratio of 15 and lipids: proteins: carbohydrates ratio of 1:10:4. On the other side, the maximum ultimate methane production and yields occurred at the mixing ratios of 2:4:4 corresponding to the COD:N ratio of 28 and lipids: proteins: carbohydrates ratio of 1:3:12. 
Table 7.2. Ultimate $\mathrm{CH}_{4}$ and yield at different ratios of the substrates, $\mathrm{COD}: \mathrm{N}$, and lipids:proteins:carbohydrates

\begin{tabular}{|c|c|c|c|c|c|c|}
\hline $\begin{array}{l}\text { Digester } \\
\text { code }\end{array}$ & $\begin{array}{c}\text { TWAS: Manure: SSO } \\
(\mathrm{V} / \mathrm{V})\end{array}$ & COD:N & $\begin{array}{l}\text { Feedstock } \\
\text { ratio codes }\end{array}$ & $\begin{array}{l}\text { Lipids: Proteins: } \\
\text { Carbohydrates }\end{array}$ & $\begin{array}{c}\text { Ultimate } \\
\mathrm{CH}_{4} \\
(\mathrm{~mL})\end{array}$ & $\begin{array}{c}\mathrm{mLCH}_{4} / \mathrm{g} \\
\text { TCOD } \\
\text { added }\end{array}$ \\
\hline $\begin{array}{l}\text { TWAS } \\
\text { Only }\end{array}$ & 1:0:0 & 15 & AA & $1: 10: 4$ & 417 & 134 \\
\hline $\begin{array}{l}\text { Manure } \\
\text { Only }\end{array}$ & $0: 1: 0$ & 47 & $\mathrm{CC}$ & $1: 4: 20$ & 1218 & 299 \\
\hline SSO only & 0:0:1 & 27 & BB & 1:1.6:9 & 1373 & 332 \\
\hline $\begin{array}{c}\mathrm{T} / \mathrm{M} / \mathrm{SSO} \\
8 / 1 / 1\end{array}$ & $8: 1: 1$ & 19 & $\mathrm{~F}$ & $1: 6.5: 9$ & 784 & 228 \\
\hline $\begin{array}{c}\mathrm{T} / \mathrm{M} / \mathrm{SSO} \\
1 / 8 / 1\end{array}$ & $1: 8: 1$ & 40 & G & $1: 4: 19$ & 1311 & 325 \\
\hline $\begin{array}{c}\mathrm{T} / \mathrm{M} / \mathrm{SSO} \\
1 / 1 / 8\end{array}$ & $1: 1: 8$ & 28 & $\mathrm{H}$ & $1: 2: 10$ & 1320 & 324 \\
\hline $\begin{array}{l}\mathrm{T} / \mathrm{M} / \mathrm{SSO} \\
5 / 2.5 / 2.5\end{array}$ & $5: 2.5: 2.5$ & 25 & I & $1: 4: 12$ & 1146 & 304 \\
\hline $\begin{array}{l}\mathrm{T} / \mathrm{M} / \mathrm{SSO} \\
2.5 / 5 / 2.5\end{array}$ & $2.5: 5: 2.5$ & 32 & $\mathrm{~J}$ & $1: 3.8: 15.5$ & 1355 & 343 \\
\hline $\begin{array}{l}\mathrm{T} / \mathrm{M} / \mathrm{SSO} \\
2.5 / 2.5 / 5\end{array}$ & $2.5: 2.5: 5$ & 28 & $\mathrm{~K}$ & $1: 3: 12$ & 1390 & 350 \\
\hline $\begin{array}{c}\mathrm{T} / \mathrm{M} / \mathrm{SSO} \\
4 / 4 / 2\end{array}$ & $4: 4: 2$ & 28 & $\mathrm{~L}$ & $1: 4: 15$ & 1248 & 324 \\
\hline $\begin{array}{c}\mathrm{T} / \mathrm{M} / \mathrm{SSO} \\
2 / 4 / 4\end{array}$ & $2: 4: 4$ & 28 & M & $1: 3: 12$ & 1424 & 356 \\
\hline $\begin{array}{c}\mathrm{T} / \mathrm{M} / \mathrm{SSO} \\
4 / 2 / 4\end{array}$ & $4: 2: 4$ & 26 & $\mathrm{~N}$ & $1: 3.7: 11.8$ & 1241 & 321 \\
\hline
\end{tabular}

Figure 7.8. illustrates the main effect plot for $\mathrm{CH}_{4}$ yield data mean in response to feedstock and lipids:proteins:carbohydrates ratios in AnCoD of TWAS/manure/SSO. As shown in Figure 7.8, feedstocks and lipids:proteins:carbohydrates ratios have significant effect on $\mathrm{CH}_{4}$ yield. TWAS only with lipids:proteins:carbohydrates ratio of 1:10:4 has the minimum methane yield. TWAS/manure/SSO co-digestion at the mixing ratio of 2:4:4 and lipids:proteins:carbohydrates ratio of 1:3:12 corresponded to the maximum methane yield.

The matrix plot in Figure 7.9 shows the relationship of COD:N, proteins: lipids, carbohydrates: lipids, and carbohydrates: proteins ratios with the ultimate methane production $(\mathrm{mL})$ and with the 
yield (mg CH $4 / \mathrm{g}$ TCOD added). The responses of the ultimate methane production and the methane yield to the different ratios is illustrated in figures 7.9. a) and 7.9. b), respectively.

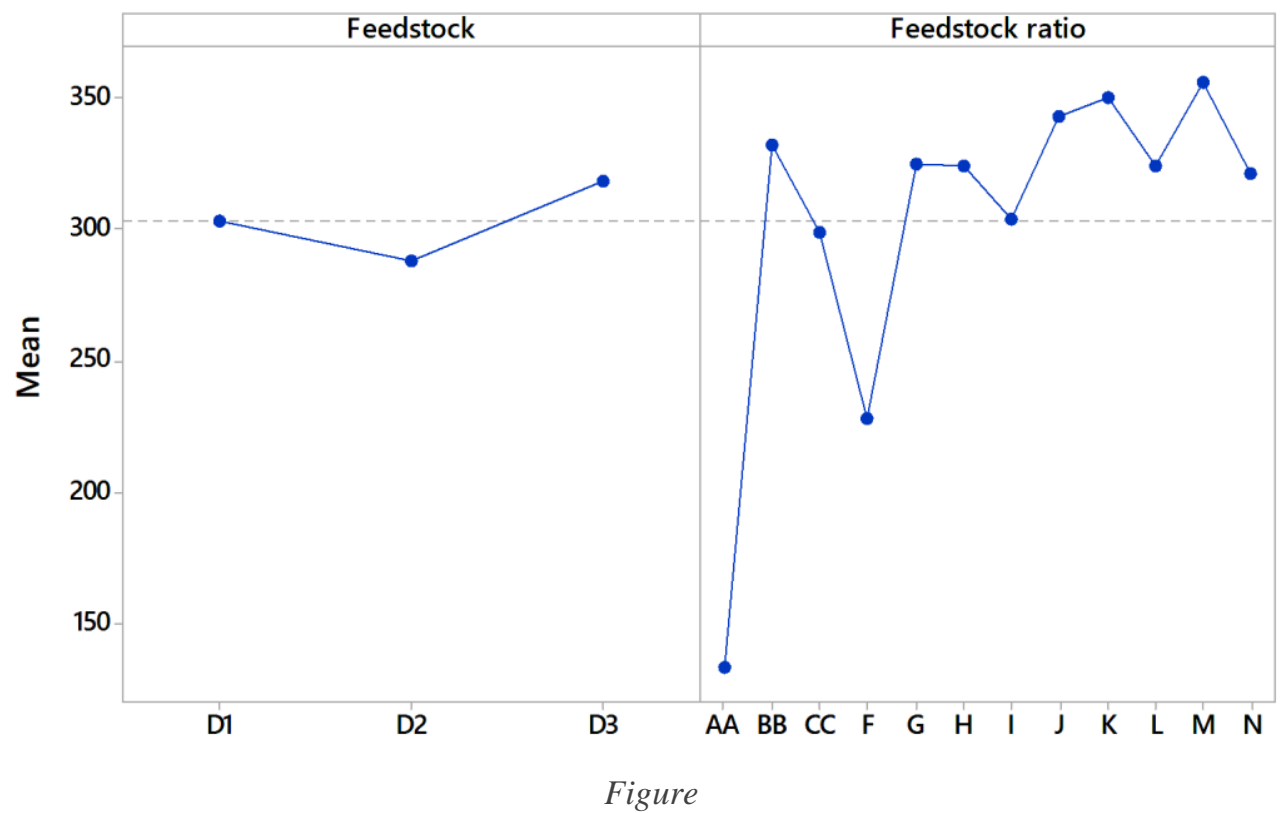

Figure 7.8. Main effect plot for $\mathrm{CH}_{4}$ yield data mean in response to feedstock and lipids:proteins:carbohydrates ratio in AnCoD of TWAS/manure/SSO

As shown in Figures 7.9. a) and 7.9. b) the trends of variations of methane versus COD:N, and lipids: proteins were similar. However, lipids: carbohydrates and proteins: carbohydrates did not conform to COD:N ratio. As illustrated in the Figures 7.8. a), the minimum ultimate methane production corresponds to the minimum COD:N ratio and the minimum lipids: proteins ratio while it corresponded to the maximum lipids: carbohydrates and maximum protein: carbohydrates ratios. The trend of the ultimate methane production variations in response to the COD:N and to the lipids: proteins ratios relatively conform to each other excluding some of the ratios. The ultimate methane production was higher at the COD:N ratios between 26 and 47 and at the lipids: proteins ratios between 0.23 and 0.62 . On the contrary, the increase of the lipids: carbohydrates and proteins: carbohydrates ratios, reduced the ultimate methane production. The most ultimate methane production occurred at the minimum lipids: carbohydrates and proteins: carbohydrates ratios of 0.05 and 0.17 , respectively. In contrast the minimum ultimate methane production corresponded to the maximum lipids: carbohydrates and proteins: carbohydrates ratios of 0.26 and 2.55 , respectively. The lipids: carbohydrates ratios above 0.1 and proteins: carbohydrates ratios above 
0.3 resulted in significant decrease in the ultimate methane production. Similar trend was observed for the methane yield in response to the COD:N, lipids: proteins and lipids: carbohydrates, and proteins: carbohydrates ratios.

7.9. a)

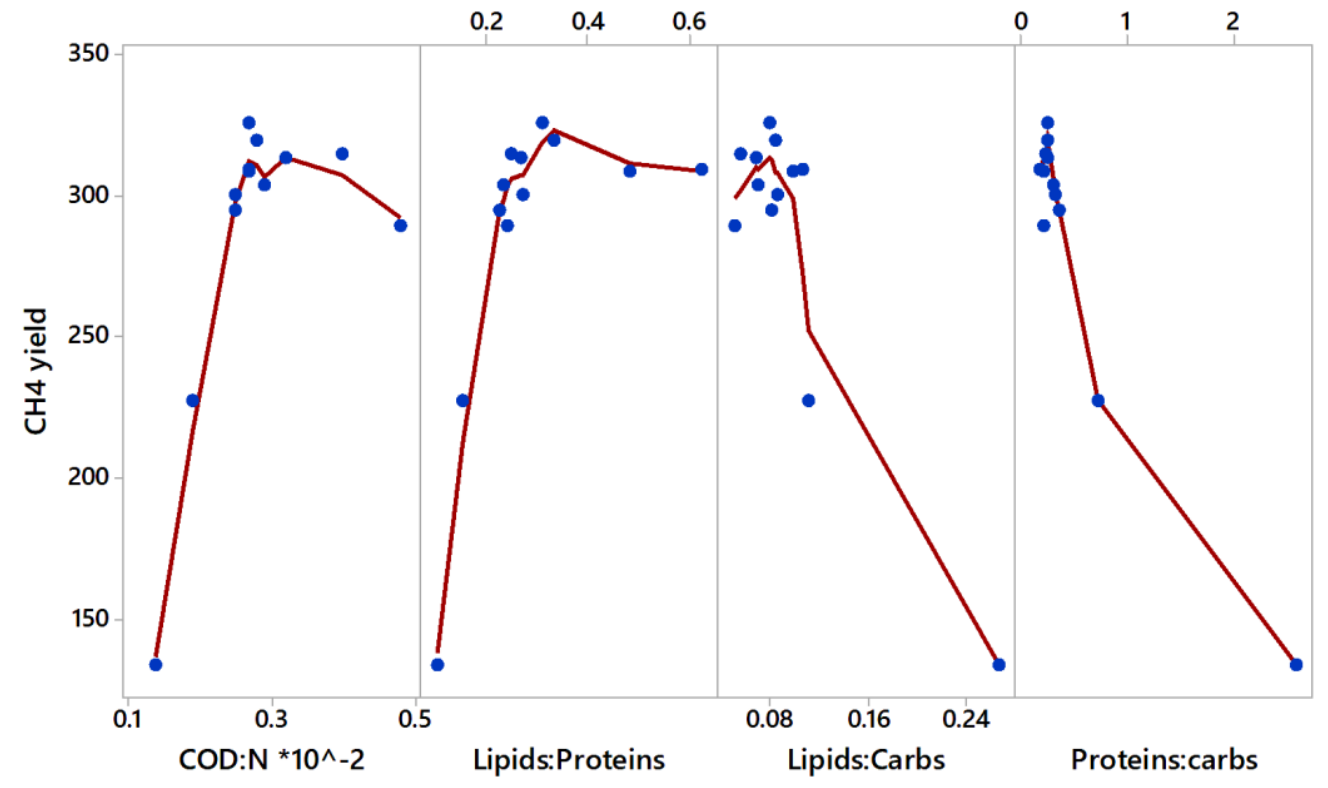

7.9. b)

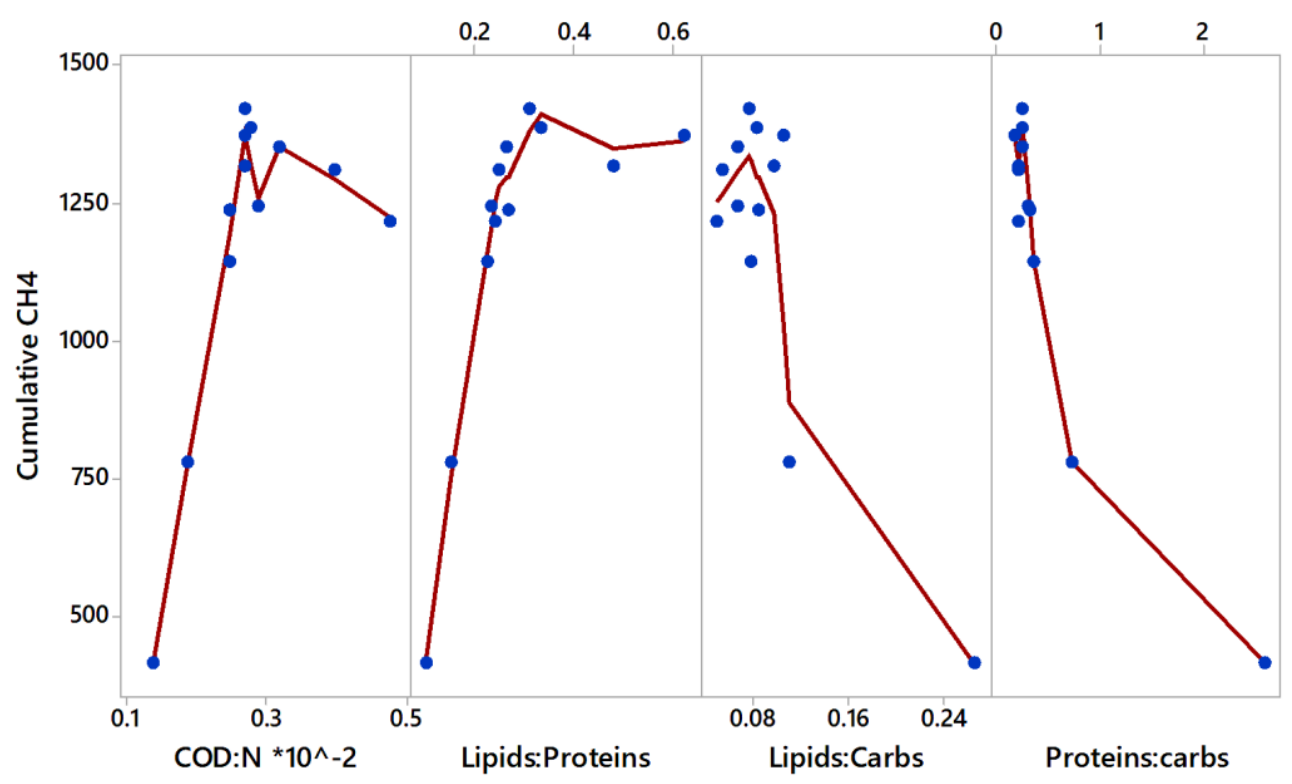

Figure 7.9. Matrix plot for: a. ultimate $\mathrm{CH}_{4}$ and b. $\mathrm{CH}_{4}$ yield at different $\mathrm{COD} / \mathrm{N}$ and Lipids: Proteins, Lipids:

Carbohydrates, and Proteins: Carbohydrates Ratios 


\subsection{Kinetic analysis results}

The results of kinetic by modified Gompertz model using Eq. 3.5 is summarized in Table 7.3. The model was applied to the experimental data from mono and co-digestion of manure and SSO. The values of $\mathrm{P}$ for the maximum methane production $(\mathrm{mL}), R_{m}^{e}$, the maximum methane production rate (mL/g day), and $\lambda$, the lag phase time $(\mathrm{d})$ were calculated and the estimated values are presented in Table 7.3. The modified Gompertz model for mono- and co-digestions of TWAS, manure and SSO, showed a good fit to the experimental results with less than $10 \%$ diversion from the measured values. The estimated values and their correlation with the mixing ratio of the feedstock showed compliance with the data obtained by the experiment. The trend of $\mathrm{P}$ variations showed the same trend as the trend observed by the experimental data in response to the corresponding mixing ratios.

Table 7.3. Summary of results of kinetic study using modified Gompertz model for TWAS/manure/SSO co-digestion

\begin{tabular}{|c|c|c|c|c|}
\hline Digester codes & $\mathbf{P}(\mathbf{m L})$ & $R_{m}^{e}(\mathrm{~mL} / \mathrm{d})$ & $\lambda(\mathbf{d})$ & $\mathbf{R}^{2}$ \\
\hline TWAS Only & 400 & 20.3 & 0.02 & 0.999 \\
\hline Manure Only & 1180 & 78.4 & 0.7 & 0.999 \\
\hline SSO only & 1307 & 73.5 & 1.76 & 0.999 \\
\hline $\mathrm{T}^{*}: \mathrm{M}^{* *}: \mathrm{SSO} 8: 1: 1$ & 760 & 46.6 & 1.7 & 0.999 \\
\hline $\mathrm{T}: \mathrm{M}: S S O$ 1:8:1 & 1257 & 66.9 & 0.2 & 0.999 \\
\hline $\mathrm{T}: \mathrm{M}: \mathrm{SSO} 1: 1: 8$ & 1267 & 66.1 & 0.2 & 0.999 \\
\hline $\mathrm{T}: \mathrm{M}: S S O$ 5:2.5:2.5 & 1099 & 60.7 & 0.8 & 0.999 \\
\hline T:M:SSO 2.5:5:2.5 & 1308 & 84.9 & 0.5 & 0.999 \\
\hline $\mathrm{T}: \mathrm{M}: S S O 2.5: 2.5: 5$ & 1330 & 74.4 & 1.0 & 0.999 \\
\hline T:M:SSO 4:4:2 & 1193 & 67.2 & 1.2 & 0.999 \\
\hline $\mathrm{T}: \mathrm{M}: \mathrm{SSO} 2: 4: 4$ & 1382 & 84.6 & 1.1 & 0.999 \\
\hline $\mathrm{T}: \mathrm{M}: \mathrm{SSO} 4: 2: 4$ & 1191 & 66.8 & 1.2 & 0.999 \\
\hline
\end{tabular}


The Maximum $\mathrm{P}$ value for the predicted cumulative methane production was $1382 \mathrm{~mL}$ and corresponded to the mixing ratio of 2:4:4. The lag phase varied from 0.0 to 1.7 days for different substrate mixing ratios. The lag phase time was less than 1 day for half of the digesters. The other half had a lag phase time from 1 to 1.7 days. The values of $\mathrm{R}_{\max }^{\mathrm{e}}$ ranged from 20 to $84.9 \mathrm{~mL} / \mathrm{g} \mathrm{d}$ corresponding to TWAS mono digestion and TWAS/manure/SSO mixing ratio of 2:4:4, respectively.

\subsection{Hydrolysis/acidification}

This experiment was carried out for evaluating the hydrolysis/acidification phase in anaerobic codigestion of TWAS, manure, and SSO. The analysis of degree of solublization, synergistic effect of co-digestion at different mixing ratios, volatile fatty acids (VFAs) yield, and hydrolysis kinetics of lipids, proteins, and carbohydrates was conducted by monitoring the soluble and particulate contents during a 72-hr period. Initially, characterization of the feedstocks in triplicates was carried out and the mean values are presented in Table 7.4. As shown in the table, both manure and SSO have higher amount of COD concentration than TWAS. Manure contains high amount of carbohydrates and proteins. The lipids and carbohydrates content of manure and SSO are also remarkably higher than that of TWAS. $\mathrm{pH}$ values of the digesters were adjusted to satisfy the required condition for hydrolysis/acidification and ranged from 5.6 to 5.8 in this experiment.

The data form total and soluble COD (SCOD) concentrations monitoring in all of the digesters over time during a 72-h hydrolysis/acidification period was used to obtain the degree of COD solublization for each digester. As explained earlier, the degree of solublization was calculated using Eq. 3.1 and the result is summarized in Figure 7.10.

As illustrated in figure 7.10, the degree of the COD solublization varied from $15 \%$ to $30 \%$. The most solublization of the COD content of $30 \%$ corresponded to TWAS/manure/SSO combinations of 1:8:1 and 2:4:4. For the control reactors, the COD solublization in mono digestion of TWAS, manure and SSO were $15 \%$ and $23 \%$, and $26 \%$ respectively. The reactors containing only TWAS had the lowest degree of solublization. Similar to the results of previous experiments, manure resulted in a relatively lower solublization than SSO which would be due to the presence of some recalcitrant contents such as fibers and cellulosic compounds that delay hydrolysis and liquefaction. 
Table 7.4. Characteristics of the feedstocks at different mixing ratios of TWAS/manure/SSO

\begin{tabular}{|c|c|c|c|c|c|c|c|c|c|c|c|c|c|}
\hline & & TWAS & Manure & SSO & $\begin{array}{c}\mathrm{T}^{*}: \mathrm{M}^{* *}: \mathrm{SSO} \\
8: 1: 1\end{array}$ & $\begin{array}{c}\text { T:M:SSO } \\
1: 8: 1\end{array}$ & $\begin{array}{c}\text { T:M:SSO } \\
1: 1: 8\end{array}$ & $\begin{array}{l}\text { T:M:SSO } \\
5: 2.5: 2.5\end{array}$ & $\begin{array}{l}\text { T:M:SSO } \\
2.5: 5: 2.5\end{array}$ & $\begin{array}{l}\text { T:M:SSO } \\
2.5: 2.5: 5\end{array}$ & $\begin{array}{c}\text { T:M:SSO } \\
4: 4: 2\end{array}$ & $\begin{array}{c}\text { T:M:SSO } \\
2: 4: 4\end{array}$ & $\begin{array}{c}\text { T:M:SSO } \\
4: 2: 4\end{array}$ \\
\hline Parameters & Units & $\begin{array}{c}\text { Mixture } \\
\text { (1) }\end{array}$ & $\begin{array}{c}\text { Mixture } \\
\text { (2) }\end{array}$ & $\begin{array}{c}\text { Mixture } \\
\text { (3) }\end{array}$ & Mixture (4) & $\begin{array}{c}\text { Mixture } \\
(5)\end{array}$ & $\begin{array}{c}\text { Mixture } \\
(6)\end{array}$ & $\begin{array}{c}\text { Mixture } \\
\text { (7) }\end{array}$ & $\begin{array}{c}\text { Mixture } \\
(8)\end{array}$ & $\begin{array}{c}\text { Mixture } \\
(9)\end{array}$ & $\begin{array}{c}\text { Mixture } \\
(10)\end{array}$ & $\begin{array}{c}\text { Mixture } \\
\text { (11) }\end{array}$ & $\begin{array}{c}\text { Mixture } \\
(12)\end{array}$ \\
\hline TCOD & $\mathrm{g} / \mathrm{L}$ & 37 & 100 & 102 & 50 & 94 & 95 & 69 & 85 & 85 & 75 & 68 & 76 \\
\hline SCOD & $\mathrm{g} / \mathrm{L}$ & 1.9 & 10 & 37 & 6 & 12 & 31 & 13 & 15 & 22 & 12 & 17 & 17.7 \\
\hline TSS & $\mathrm{g} / \mathrm{L}$ & 29 & 54 & 50 & 34 & 51 & 48 & 40 & 47 & 46 & 43 & 36 & 42 \\
\hline VSS & $\mathrm{g} / \mathrm{L}$ & 23 & 45 & 44 & 27 & 43 & 42 & 34 & 39 & 39 & 36 & 31 & 36 \\
\hline $\mathrm{TS}$ & $\mathrm{g} / \mathrm{L}$ & 36 & 68 & 77 & 43 & 65 & 72 & 54 & 62 & 64 & 57 & 51 & 59 \\
\hline VS & $\mathrm{g} / \mathrm{L}$ & 32 & 58 & 63 & 37 & 56 & 59 & 46 & 53 & 54 & 48 & 43 & 49 \\
\hline Ammonia & $\mathrm{g} / \mathrm{L}$ & 0.2 & 0.0 & 1.3 & 0.3 & 0.2 & 1.1 & 0.5 & 0.4 & 0.7 & 0.4 & 0.6 & 0.6 \\
\hline $\mathrm{pH}$ & - & 5.6 & 5.7 & 5.8 & 5.6 & 5.7 & 5.8 & 5.7 & 5.7 & 5.7 & 5.7 & 5.8 & 5.7 \\
\hline Alkalinity & $\mathrm{g} \mathrm{CaCO} 3 / \mathrm{L}$ & 1.8 & 7.0 & 6.4 & 2.8 & 6.4 & 6.0 & 4.2 & 5.5 & 5.4 & 4.8 & 4.3 & 4.7 \\
\hline TN & $\mathrm{g} / \mathrm{L}$ & 2.7 & 1.7 & 4.1 & 2.7 & 2.0 & 3.7 & 2.8 & 2.5 & 3.1 & 2.6 & 2.5 & 3.0 \\
\hline TSN & $\mathrm{g} / \mathrm{L}$ & 0.4 & 0.1 & 1.1 & 0.4 & 0.2 & 0.9 & 0.5 & 0.4 & 0.7 & 0.4 & 0.5 & 0.6 \\
\hline T-Carbs & $\mathrm{g} / \mathrm{L}$ & 0.8 & 26.0 & 13.6 & 4.6 & 22.2 & 13.5 & 10.3 & 16.6 & 13.5 & 13.4 & 10.8 & 11.0 \\
\hline T-Proteins & $\mathrm{g} / \mathrm{L}$ & 2.5 & 5.1 & 2.1 & 2.8 & 4.6 & 2.4 & 3.1 & 3.7 & 3.0 & 3.5 & 2.4 & 2.9 \\
\hline T-Lipids & $\mathrm{g} / \mathrm{L}$ & 0.3 & 1.7 & 1.4 & 0.5 & 1.5 & 1.3 & 0.9 & 1.2 & 1.2 & 1.0 & 0.9 & 1.0 \\
\hline
\end{tabular}

*T: TWAS

${ }^{* *} \mathrm{M}$ : Manure 


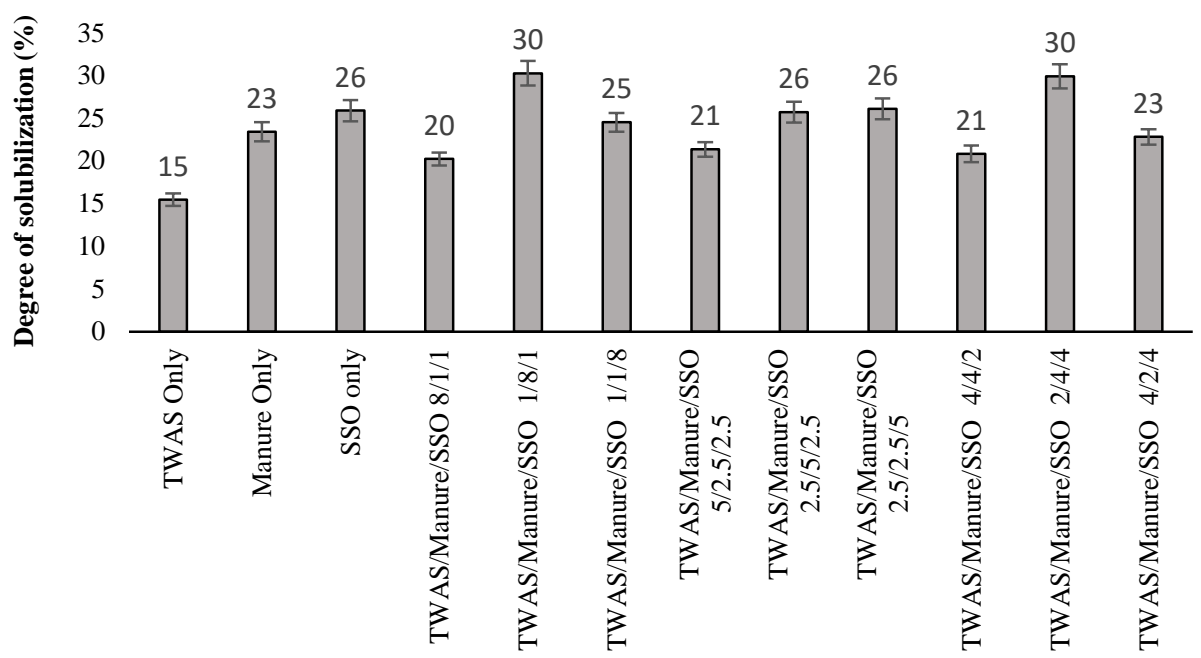

Mixing ratio

Figure 7.10. Degree of COD solublization at different mixing ratios of TWAS/Manure/SSO

It was observed that all of the reactors containing the mixings of TWAS, manure and SSO achieved a higher degree of solublization than the control reactors containing only TWAS. Solublization increased by $100 \%, 30 \%$ and $15 \%$ in the co-digestion of TWAS/manure/SSO in comparison with the control reactors digesting only TWAS, manure and SSO, respectively. Although SSO demonstrated more solublization than manure, no correlation between the portion of SSO and the degree of solublization at different mixing ratio was observed. This could verify that a proper mixing ratio is required to enhance the microbial synergy of the hydrolytic and acidogenic microbial communities for the process improvement.

The synergistic effect on the solublization of the feedstocks was evaluated by comparing the theoretical degree of solublization of each co-digester to the measured data from the experiment and the result is summarized in Figure 7.11. Although the improvement of synergy was not significant at some combinations, most of the co-digesters resulted in an increase of solublization due to the synergistic effect of the microbial communities. The synergistic effect varied from $2 \%$ to $35 \%$ in hydrolysis/acidification of TWAS/manure/SSO at different mixing ratios. 


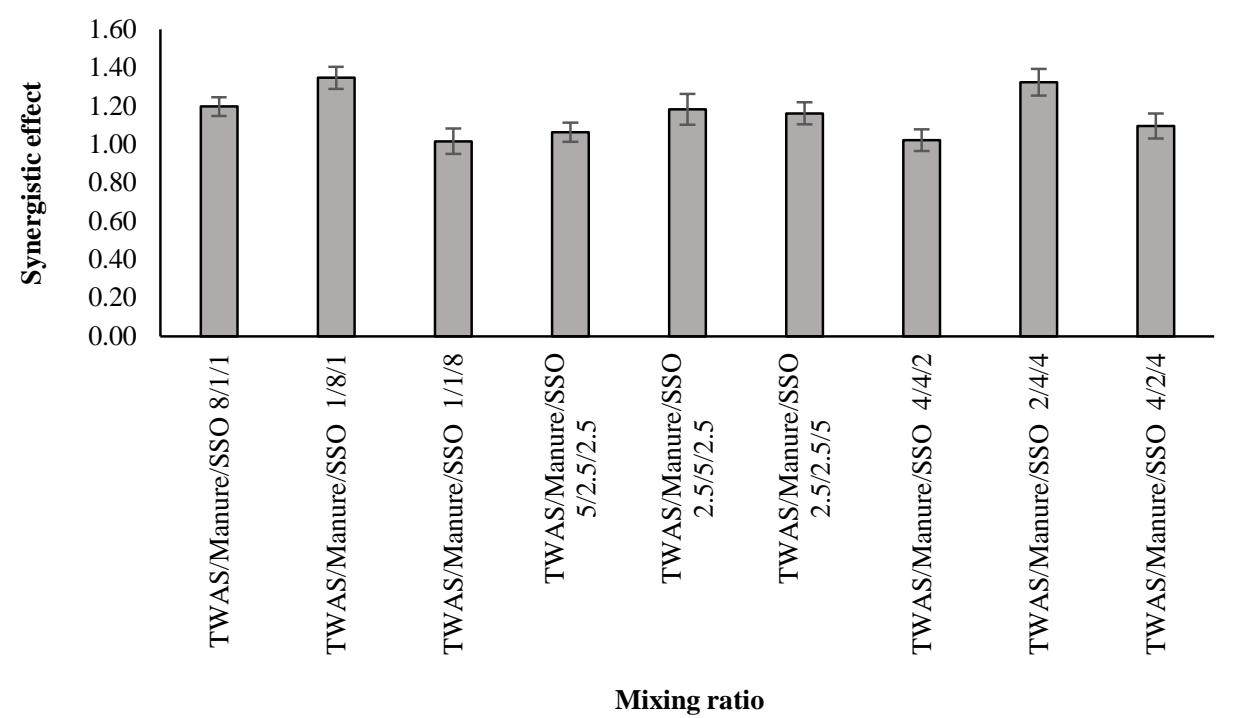

Figure 7.11. Synergistic effect on solublization at different mixing ratios of TWAS/Manure/SSO

The monitoring of VFAs concentrations over time indicated an increasing trend during the 72-hr of the hydrolysis/acidification period. The total VFAs yields were calculated in terms of mass of produced VFAs per mass of VSS added (mg VFAs/g VSS added). As illustrated in Figure 7.12, SSO alone had more VFAs yields than manure alone. Both manure and SSO had a higher VFAs yield than TWAS. The VFAs yield in the co-digester was correlated to the mixing ratios of the feedstocks so that the VFAs yield increased by increasing the fractions of manure and SSO in the co-digesters. Similar to the result of the previous experiments, the trend of VFAs yield did not comply with the trend of COD solublization of the corresponding mixing ratios (Figure 7.10). Therefore, it was assumed that hydrolysis/liquefaction would occur independently of acidification. All of the reactors containing the mix of manure and SSO had higher VFAs yield than the control reactors containing TWAS alone. The VFAs yields were $231 \mathrm{mg}$ VFAs/g VSS added and $325 \mathrm{mg}$ VFAs/g VSS added for the manure and SSO mono digestions, respectively. The maximum VFAs yield of $400 \mathrm{mg}$ VFAs/g VSS added was achieved by manure:SSO ratio of 1:9. 


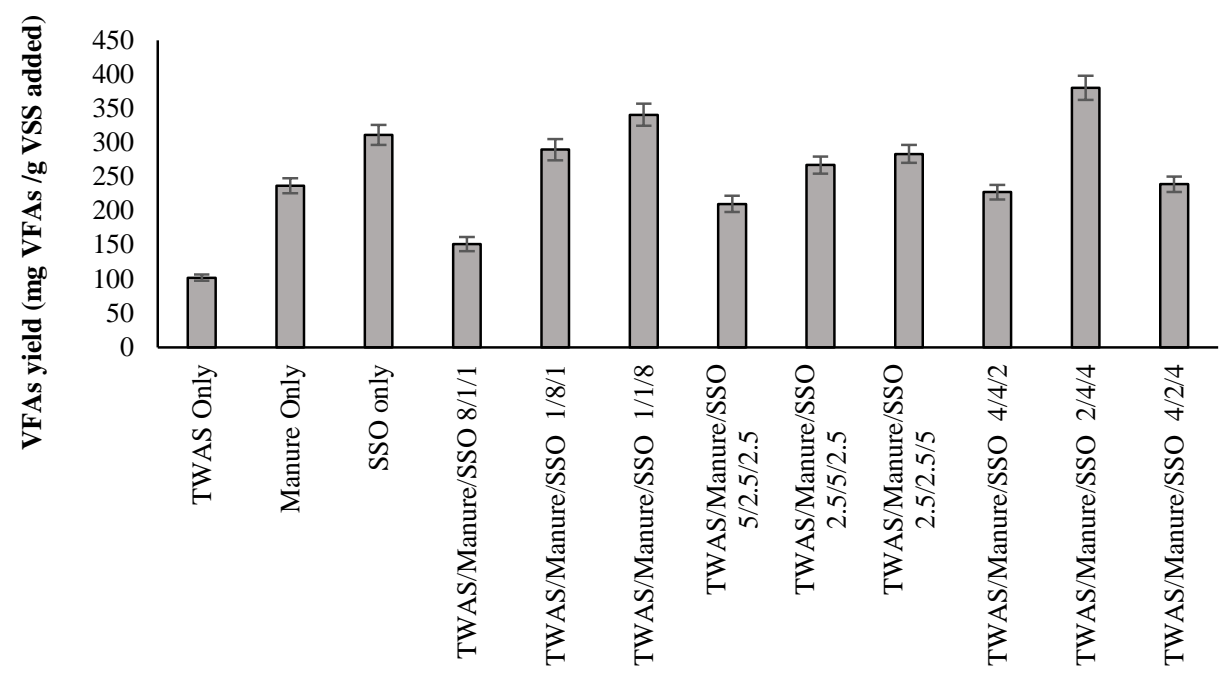

Mixing ratio

Figure 7.12. Total VFAs yield at different mixing ratios of TWAS/Manure/SSO

In co-digestion of TWAS/manure/SSO, by monitoring soluble and particulate COD over time, an increasing trend in solublization of COD and decreasing particulate COD concentrations was observed. Furthermore, monitoring soluble and particulate lipids, proteins, and carbohydrates over time also showed an increasing trend in solublization and particulate matter degradation of the feedstocks. Similar to the previous experiments, the hydrolysis rates of the lipids and proteins, were slower than carbohydrate. These results are summarized in the tables presented in the appendix. The hydrolysis rate coefficient $\left(\mathrm{K}_{\mathrm{h}}\right)$ was calculated according to first order kinetic (equations 3.3 and 3.4) for COD, lipids, proteins and carbohydrates based on the particulate degradation and results are summarized in Table 7.5.

Although the first order kinetics was compatible with the trend of particulate matters degradation, the hydrolysis kinetics coefficient did not show the same trend as solublization and acidification as well as methanogenesis. Hydrolysis rate coefficients of the co-digesters increased in the codigesters although, it did not change significantly in all of them compared to the single digestion of SSO. In some co-digesters the $\mathrm{K}_{\mathrm{h}}$ values were even lower than that of SSO alone. The maximum VFAs yield corresponded to TWAS:manure:SSO 2:4:4 and 1:1:8. However, the Kh for the mixing ratio of $1: 8: 1$ was higher than that of $1: 1: 8$. The maximum solublization corresponded to 
TWAS:manure:SSO mixing ratios of 2:4:4 and 1:8:1. However, the maximum methane yield occurred at the mixing ratios of 2:4:4 and 2.5:2.5:5 in the reactors co-digesting TWAS, manure, and SSO. Only the mixing ratio of 2:4:4 resulted in improving both methanogenesis and hydrolysis which would be due to the balance of the nutrients that favors both methanogenic and hydrolytic microbial consortia.

Table 7.5. Hydrolysis rate coefficients for COD, lipids, proteins, and carbohydrate content in co-digestion of TWAS,

Manure, and SSO at different mixing ratios

\begin{tabular}{|c|c|c|c|c|c|c|c|c|c|c|c|c|}
\hline$K$ & & & & $\mathrm{~T} / \mathrm{M} / \mathrm{SSO}$ & $\mathrm{T} / \mathrm{M} / \mathrm{SSO}$ & $\mathrm{T} / \mathrm{M} / \mathrm{SSO}$ & $\mathrm{T} / \mathrm{M} / \mathrm{SSO}$ & $\mathrm{T} / \mathrm{M} / \mathrm{SSO}$ & $\mathrm{T} / \mathrm{M} / \mathrm{SSO}$ & $\mathrm{T} / \mathrm{M} / \mathrm{SSO}$ & $\mathrm{T} / \mathrm{M} / \mathrm{SSO}$ & $\mathrm{T} / \mathrm{M} / \mathrm{SSO}$ \\
\hline $\mathbf{N h}$ & TWAS & Manure & SSO & $8 / 1 / 1$ & $1 / 8 / 1$ & $1 / 1 / 8$ & $5 / 2.5 / 2.5$ & $2.5 / 5 / 2.5$ & $2.5 / 2.5 / 5$ & $4 / 4 / 2$ & $2 / 4 / 4$ & $4 / 2 / 4$ \\
\hline $\mathrm{Kh}_{\mathrm{COD}}$ & 0.17 & 0.23 & 0.3 & 0.21 & 0.37 & 0.27 & 0.24 & 0.31 & 0.3 & 0.24 & 0.39 & 0.26 \\
\hline Kh Lipids & 0.03 & 0.07 & 0.08 & 0.05 & 0.12 & 0.06 & 0.05 & 0.09 & 0.08 & 0.09 & 0.14 & 0.05 \\
\hline $\mathrm{Kh}$ Proteins & 0.19 & 0.21 & 0.28 & 0.23 & 0.31 & 0.24 & 0.2 & 0.23 & 0.24 & 0.21 & 0.31 & 0.21 \\
\hline $\mathrm{K}_{\mathrm{h} \text { Carbs }}$ & 0.32 & 0.43 & 0.55 & 0.36 & 0.66 & 0.51 & 0.46 & 0.63 & 0.58 & 0.26 & 0.69 & 0.52 \\
\hline
\end{tabular}




\section{Chapter 8}

\section{Correlation of $\mathrm{CH}_{4}$}

\section{with lipids, proteins,}

and carbohydrates 


\subsection{Correlation of organic compositions and biomethane yield}

Lipids, proteins and carbohydrates make up the principal constituents of organic waste. Currently, there is limited information on how the three main components of organics impacts the AD performance. Therefore, assessing the correlation between the performance of anaerobic digestion system and the three main organic composition is required in order to enhance the efficiency of the AD process and to provide a tool for the prediction of the system performance.

In addition, in designing a co-digestion system this would provide a tool to optimize the system based on lipids, proteins, and carbohydrates fractions along with $\mathrm{C}: \mathrm{N}$ ratio as they are both important design parameters. In this work the influence of lipids, proteins and carbohydrates content on the anaerobic digestion of TWAS, SSO and manure was investigated. The feedstocks and their mixtures with different ratios as presented in Table 3.2- Table 3.5, were analysed to determine their total lipids, proteins, and carbohydrates contents. The results of biogas monitoring and the methane in biogas using GC from the sets of BMP essay were used to determine an empirical model based on the relationship between lipids, proteins, carbohydrates components and the system efficiency in terms of methane production and yield. The functional relationship between responses $\left(\mathrm{CH}_{4}\right)$ and the factors $(\mathrm{Lp}, \mathrm{Pr}$, and $\mathrm{Cr}$ ) were described by estimating the coefficients of the second-order polynomial model based on the experimental data according to Eq. 8.1 where $\mathrm{B}_{0}$ is a constant, $\mathrm{B}_{1}, \mathrm{~B}_{3}$, and $\mathrm{B}_{4}$ are linear coefficients and $\mathrm{B}_{2}$ is quadratic coefficient. The empirical model was used to predict the methane yield ( $\left.\mathrm{mLCH}_{4} / \mathrm{gCOD}_{\text {added }}\right)$ in terms of lipids, proteins, and carbohydrates components and the results are depicted by fit plots and residual plots as presented in figures 8.1 and 8.2.

$\mathrm{CH}_{4}=\mathrm{B}_{0}+\mathrm{B}_{1} \mathrm{Lp}+\mathrm{B}_{2} \mathrm{Lp}^{2}+\mathrm{B}_{3} \mathrm{Pr}+\mathrm{B}_{4} \mathrm{Cr}$

Each of the fit plots as shown in Figure 8.1 contains adequate number of observations throughout the entire range of the predictor values. Although for the lipids a few points on the top left corner of the plot seems to be outlier, the multiple $\mathrm{R}$ value of 0.7 indicates the model properly fits in the data. 
8.1. a)

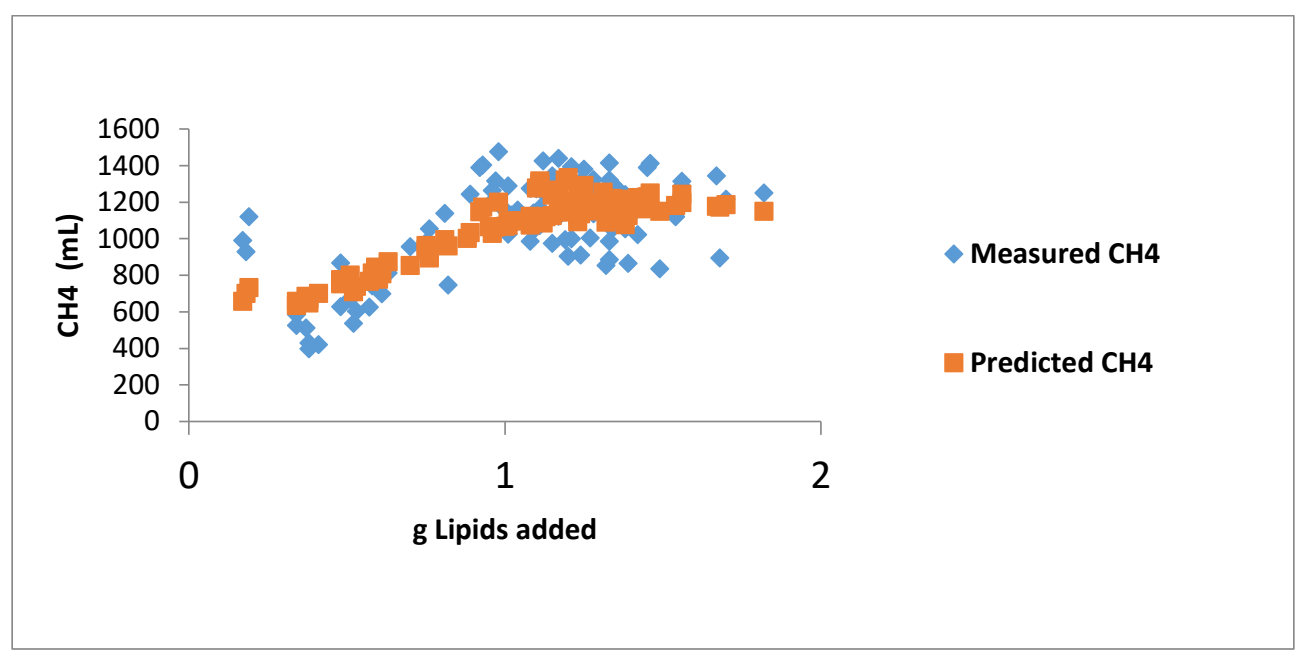

8.1.b)

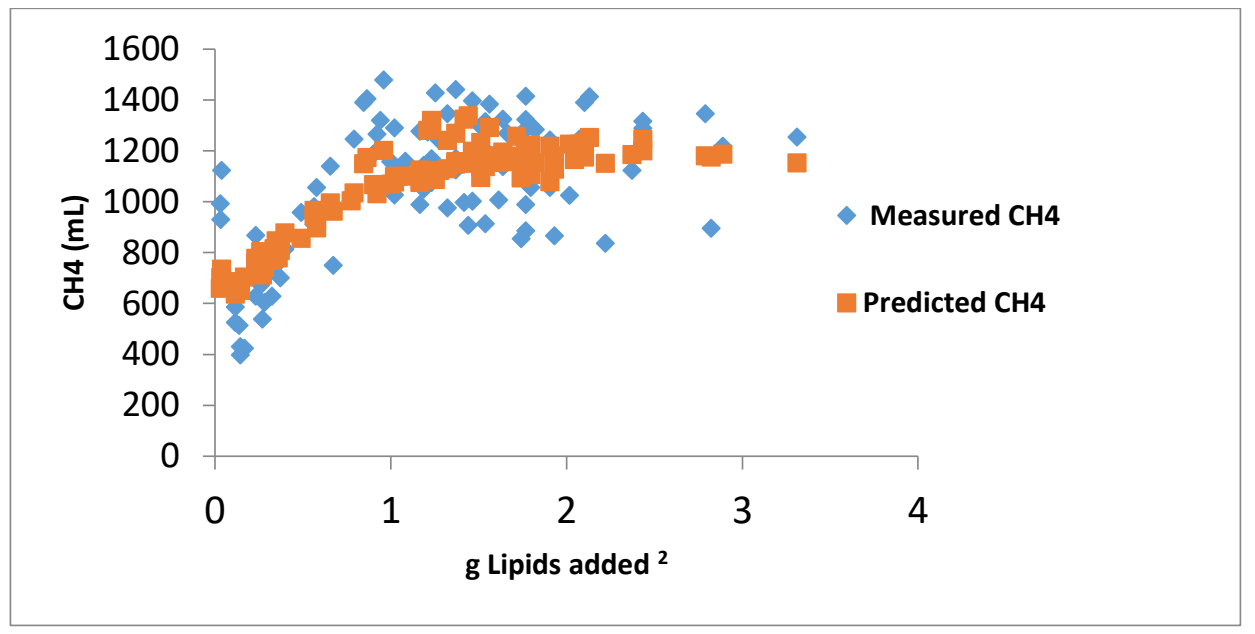


8.1.c)

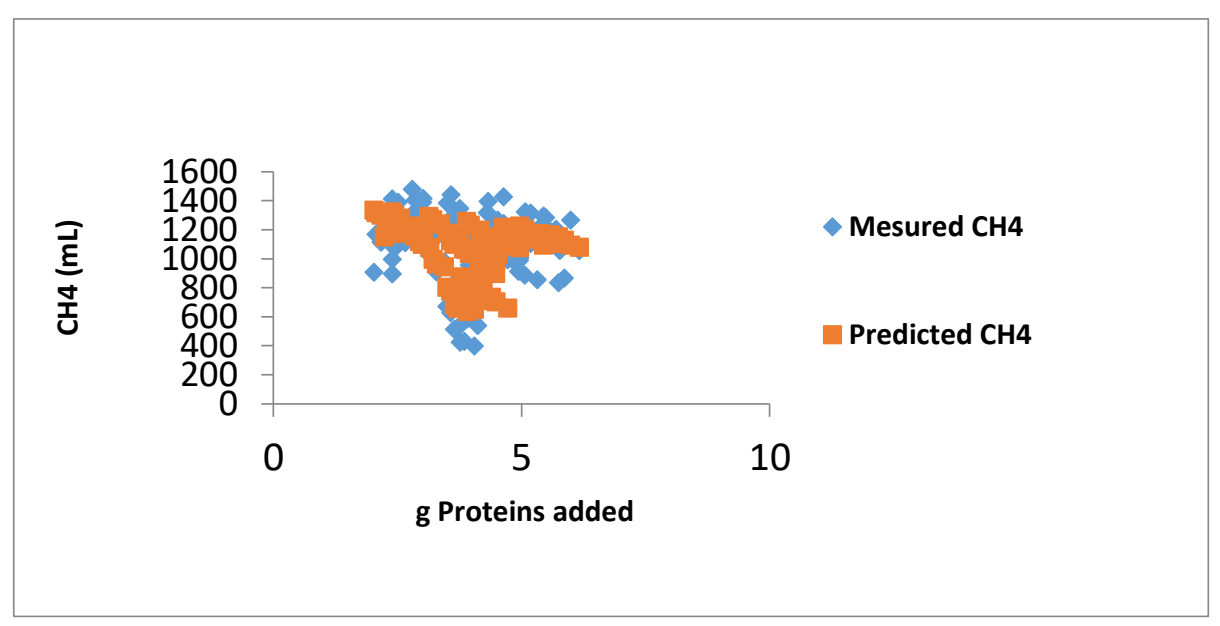

8.1. d)

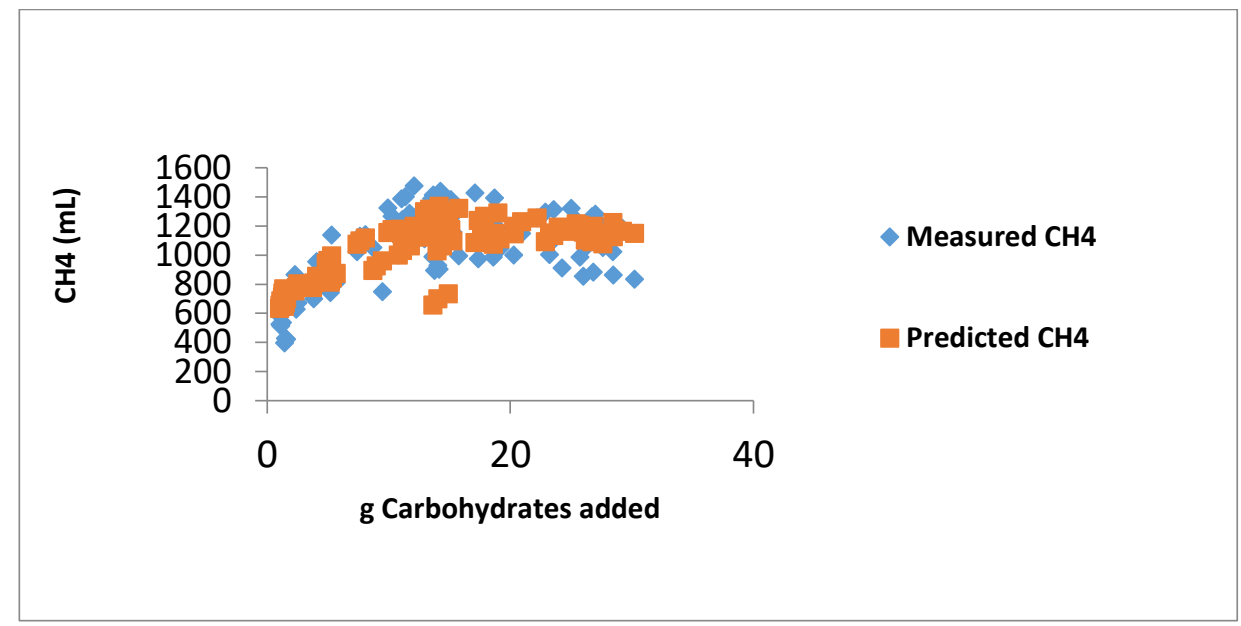

Figure 8.1. Fitted plot of response variable to lipids, Lipids², proteins, and carbohydrate

The residual plot was used to determine whether the model adequately meets the assumptions of the model. As the patterns in the points show, the points on the residual plots are randomly on both side of 0 which indicate that the model meets the model assumptions. 
8.2. a)

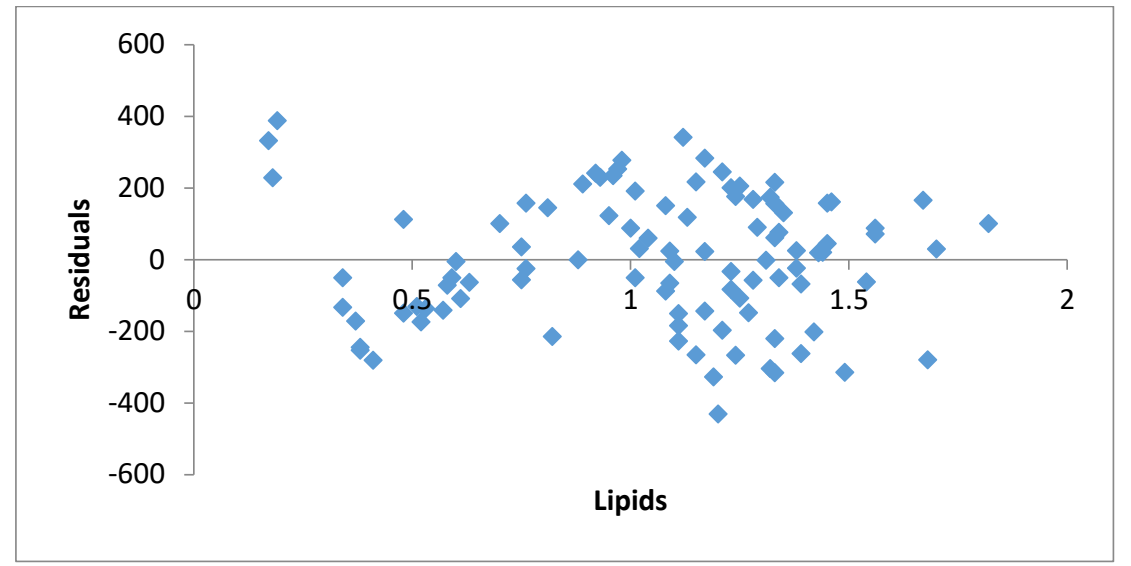

8.2. b)

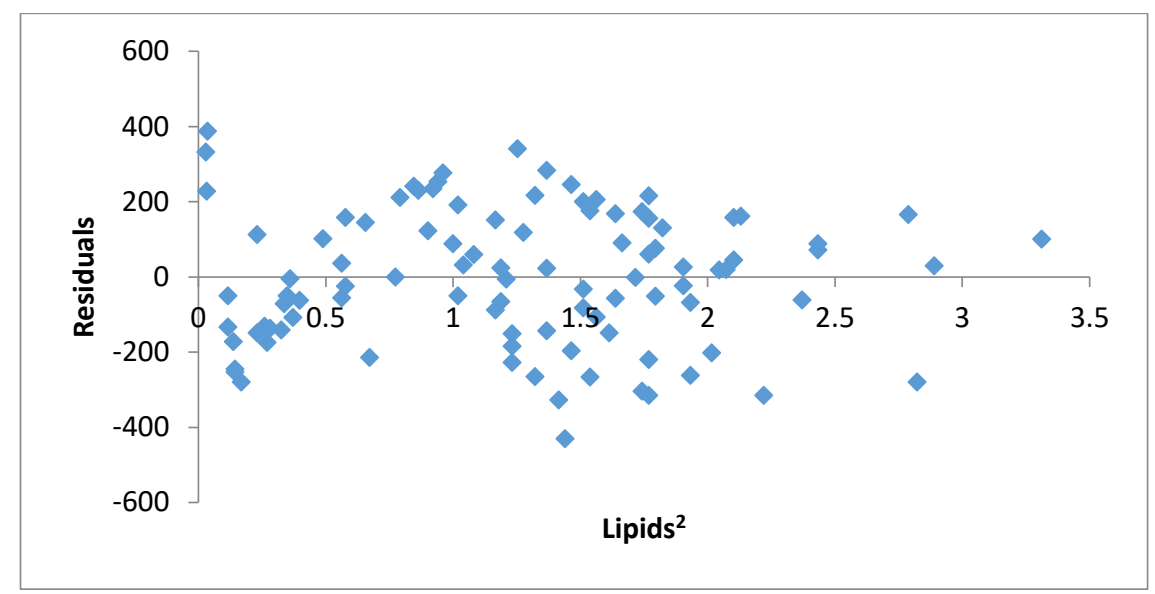

8.2. c)

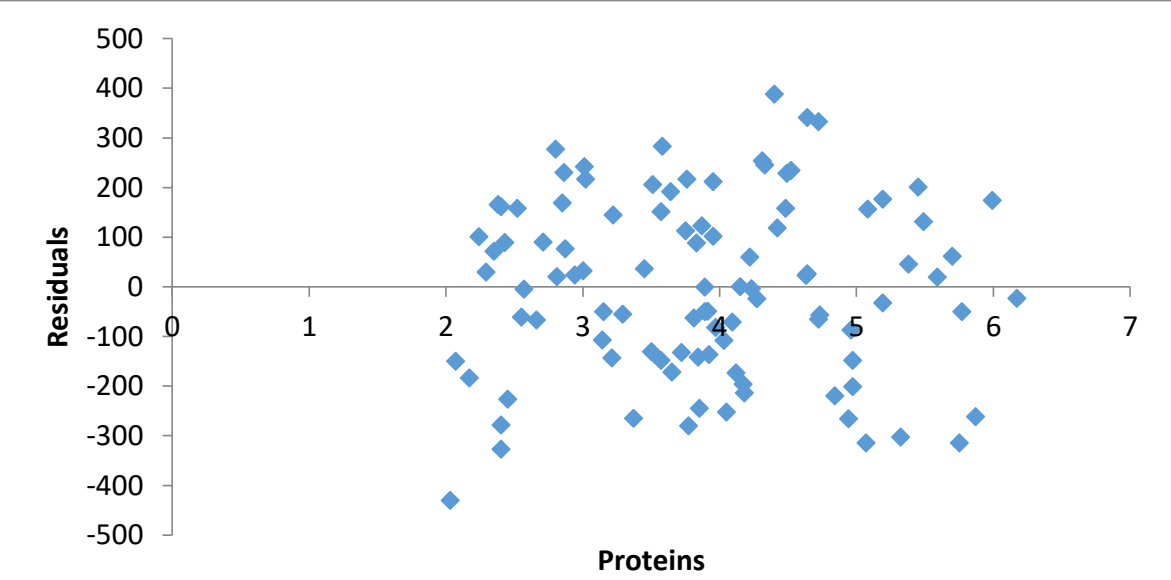


8.2.d.)

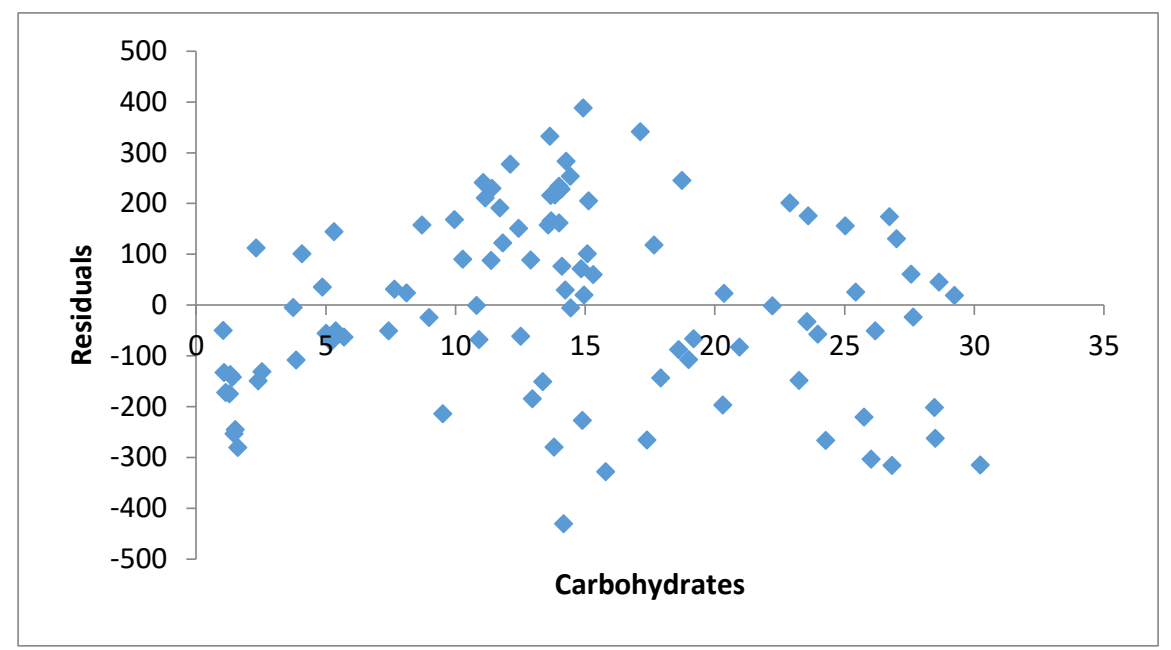

Figure 8.2. Residuals versus fits plot (residuals versus estimated response)

Table 8.1 includes the intercept, linear and quadratic coefficients of the Eq. 8.1 and summarizes some of the results of ANOVA analysis. The P-values $<0.05$ as shown in the table verifies that the correlation between the response and the variables is statistically significant. The findings suggest that the amount of lipids in substrate is directly proportional to the methane yield and its impact is significantly more than carbohydrates. While the lower fraction of proteins than lipids and carbohydrates increase the methane yield. Y. Li et al, 2017 also used a second-order polynomial model as a quick estimation of AD parameters, however their model only included proteins and lipids as variables. Their findings though verified that the more fraction of lipids significantly increases the methane production compared to proteins.

Table 8.1.Coefficients of variables in the second-order polynomial model and the results of ANOVA analysis

\begin{tabular}{lccccccc}
\hline & & & & Upper & Lower & Upper \\
& Coefficients & t Stat & P-value & Lower 95\% & $\mathbf{9 5 \%}$ & $\mathbf{9 5 . 0 \%}$ & $\mathbf{9 5 . 0 \%}$ \\
\hline Intercept & 825.279203 & 5.12631511 & $1.576 \mathrm{E}-06$ & 505.632211 & 1144.926 & 505.6322 & 1144.926 \\
Lipids & 862.890011 & 3.63241266 & 0.0004573 & 391.223242 & 1334.557 & 391.2232 & 1334.557 \\
Lipids $^{2}$ & -380.17074 & -3.2738253 & 0.0014856 & -610.738303 & -149.603 & -610.738 & -149.603 \\
Proteins & -117.12258 & -3.7852707 & 0.0002703 & -178.557985 & -55.6872 & -178.558 & -55.6872 \\
Carbs & 18.4329527 & 3.36910525 & 0.0010949 & 7.56981336 & 29.29609 & 7.569813 & 29.29609 \\
\hline
\end{tabular}




\section{2- Statistical Analysis}

Table 8.2 shows the experimental set-ups and the corresponding feedstock ratios and lipids: proteins: carbohydrates ratios. ANOVA was carried out for the $\mathrm{CH}_{4}$ yields in response to the feedstock ratios and lipids: proteins: carbohydrates ratios. The main effect plot for $\mathrm{CH}_{4}$ yield data mean is presented in Figure 8.3. The main effect plot shows the mean response of each level factors connected by the line. The steeper slope in the line explains the greater scale of the main effect. An effect is the variation in the methane yield when the factors i.e. the feedstock and lipids: proteins: carbohydrates ratios change from one level to another. The $\mathrm{P}$ value from the ANOVA results showed that the feedstock and lipids: proteins: carbohydrates ratios have significant effects on the $\mathrm{CH}_{4}$ yield.

Table 8.2. Experimental set-ups and the corresponding ratios of the feedstock and lipids: proteins: carbohydrates

\begin{tabular}{llcc}
\hline Experiment code & Feedstock ratios & Feedstock ratios code & Lipids: Proteins: Carbs \\
\hline A & TWAS Only & AA & $1: 11: 3$ \\
\hline A & SSO Only & BB & $1: 1.3: 8$ \\
\hline A & TWAS/SSO 9/1 & A & $1: 7: 5$ \\
\hline A & TWAS /SSO 7/3 & B & $1: 4: 7$ \\
\hline A & TWAS /SSO 1/1 & C & $1: 3: 7$ \\
\hline A & TWAS /SSO 3/7 & D & $1: 2: 8$ \\
\hline A & TWAS /SSO 1/9 & E & $1: 1.5: 8$ \\
\hline B & TWAS & AA & $1: 7: 2.5$ \\
\hline B & CC & $1: 4: 20$ \\
\hline B & Manure & A & $1: 7: 6$ \\
\hline B & TWAS / Manure 9/1 & B & $1: 5: 12$ \\
\hline B & TWAS / Manure 7/3 & C & $1: 4: 17$ \\
\hline B & TWAS / Manure 1/1 & D & $1: 4: 19$ \\
\hline B & TWAS / Manure 3/7 & E & $1: 4.2: 21$ \\
\hline C & TWAS / Manure 1/9 & CC & $1: 2: 12$ \\
\hline C & Manure & BB & $1: 3.5: 18.5$ \\
\hline C & SSO & A & $1: 4: 20$ \\
\hline C & Manure/SSO 9/1 & B & $1: 3: 17$ \\
\hline C & Manure /SSO 7/3 & D & $1: 2.7: 15$ \\
\hline C & Manure /SSO 5/5 & & \\
\hline
\end{tabular}




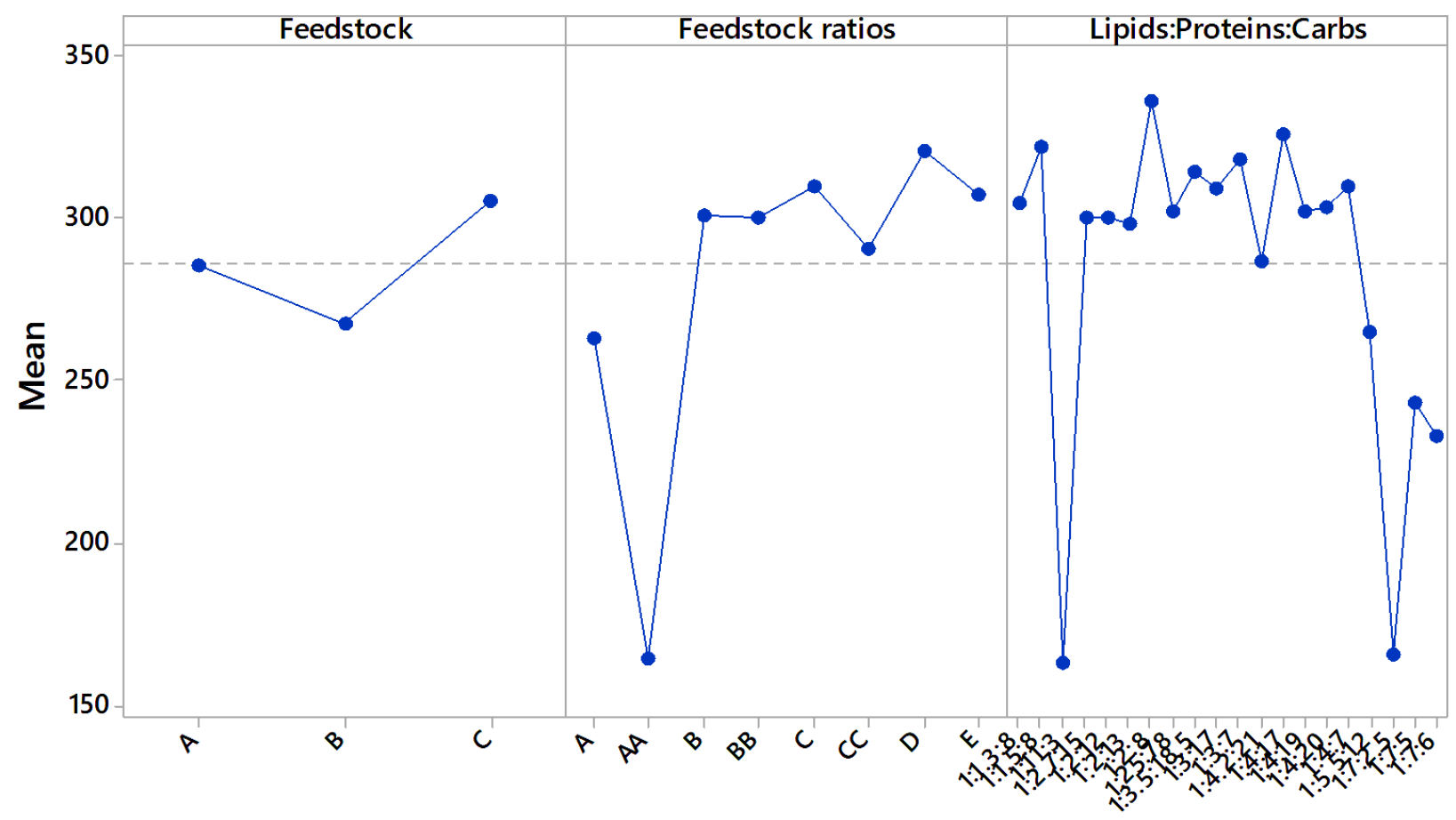

Figure 8.3. a. Main effect plot for $\mathrm{CH}_{4}$ yield data mean

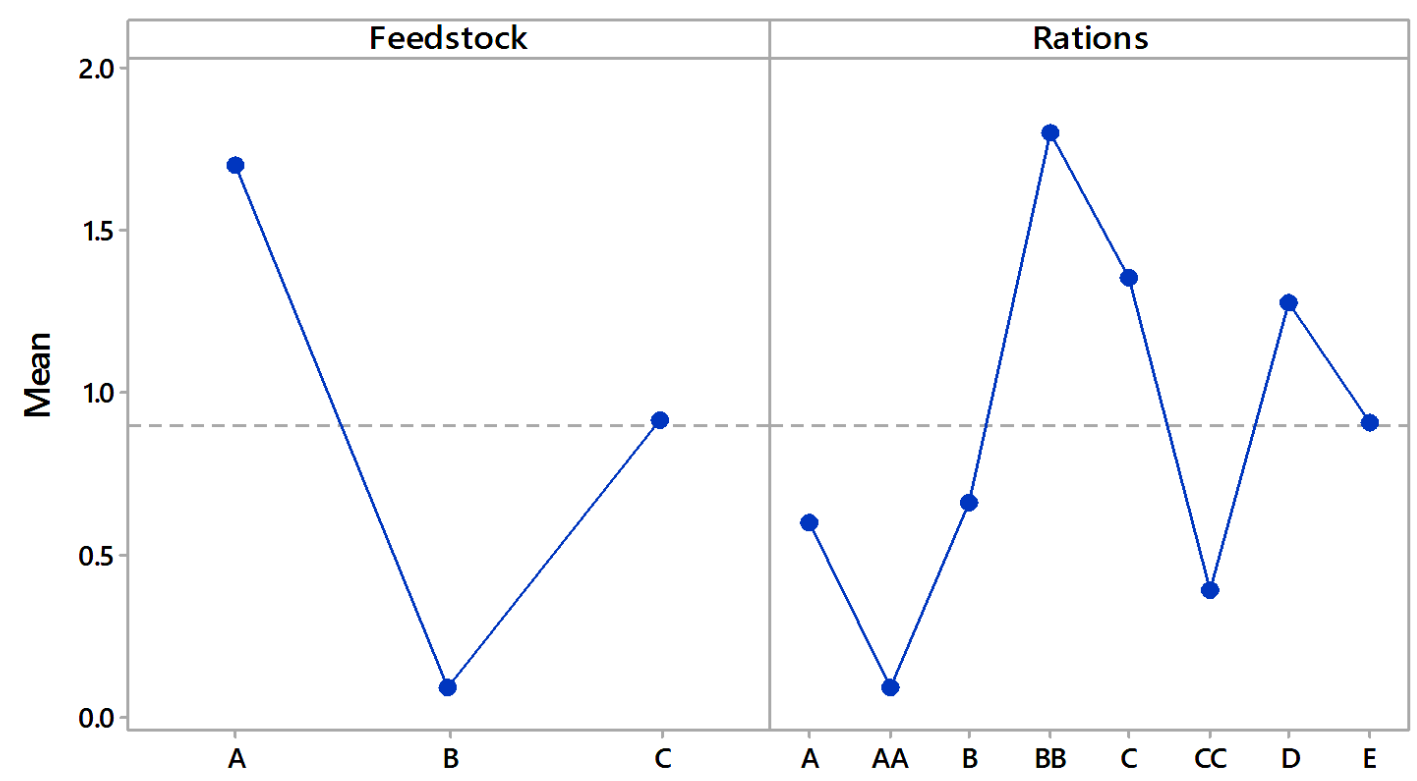

Figure 8.3. b. Main effect plot for $\mathrm{CH}_{4}$ yield data mean for the lag phase data mean 


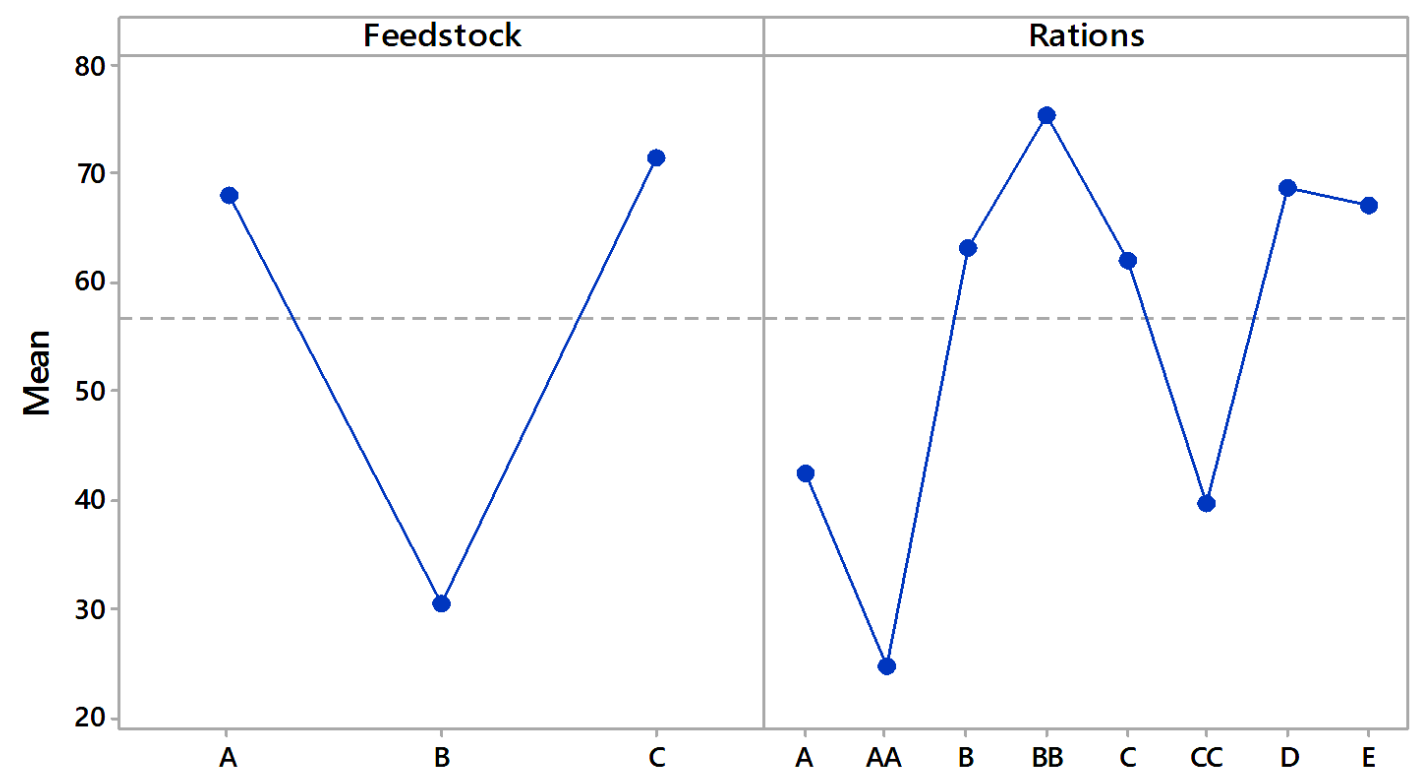

Figure 8.3. c. Main effect plot for $\mathrm{CH}_{4}$ yield data mean for the $\mathrm{CH}_{4}$ maximum rate data mean

The interval plot of $\mathrm{CH}_{4}$ yield with 95\% confidence interval for the mean using individual standard deviations to calculate the intervals is shown in Figure 8.4. The width of the interval plot represents the extent of variation in the data. A small interval would indicate more consistent data and less variation. As shown in Figure 8.4 the variation is more for some ratios than the other ones. The variation in the methane yield for the feedstock ratios of 1:9 and 7:3 were more than the other feedstock mixing ratios. The data was quite consistent with that of SSO alone. As shown in Figure 8.5.a, all of the intervals include 0 which means that the corresponding $\mathrm{CH}_{4}$ yield data means are not significantly different in response to the feedstocks. However, according to Figure 8.5.b., for some of the feedstock mixing ratios, the corresponding means are significantly different. 


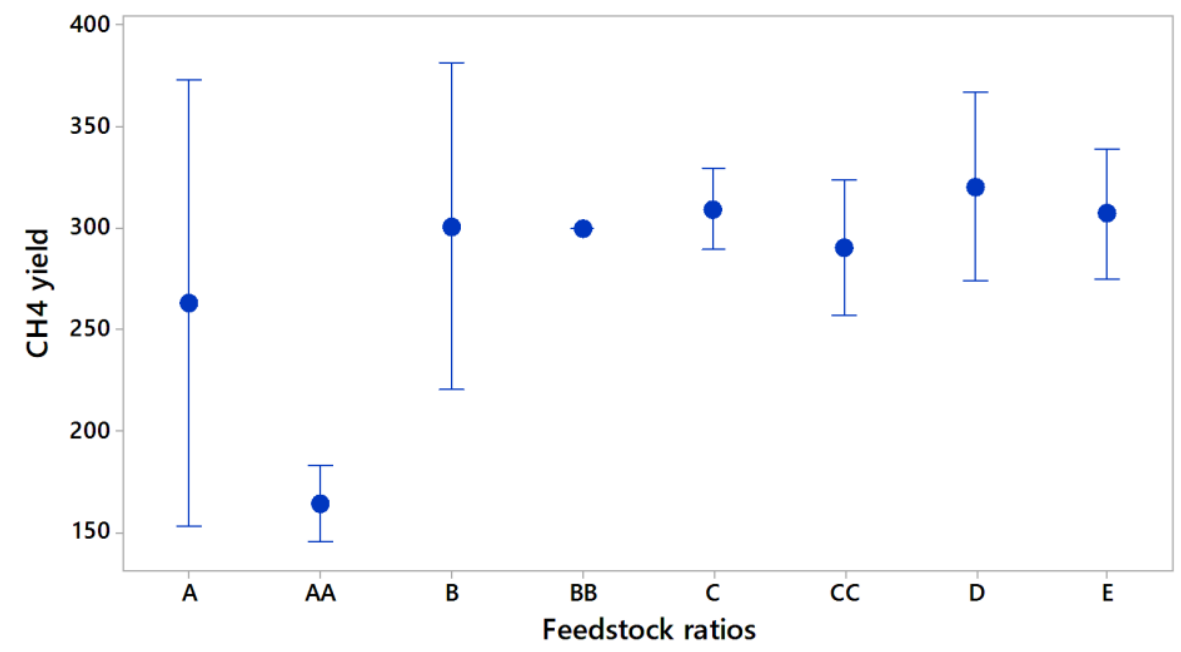

Figure 8.4. Interval plot of $\mathrm{CH}_{4}$ yield with $95 \%$ confidence interval for the mean

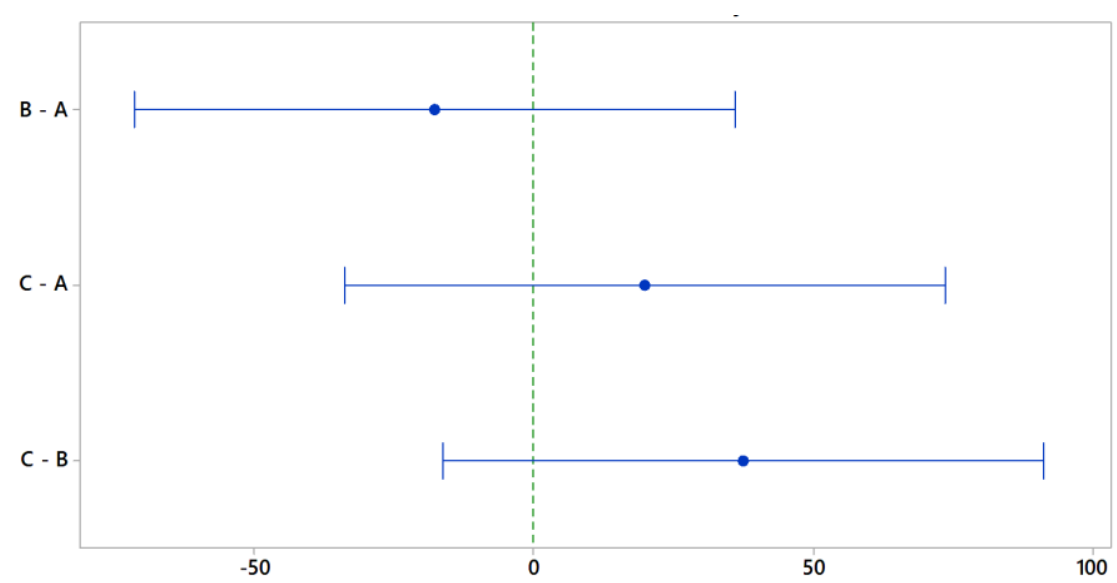

Figure 8.5. a. Fisher individual 95\% confidence interval differences of means for $\mathrm{CH}_{4}$ yields in response to the feedstocks 


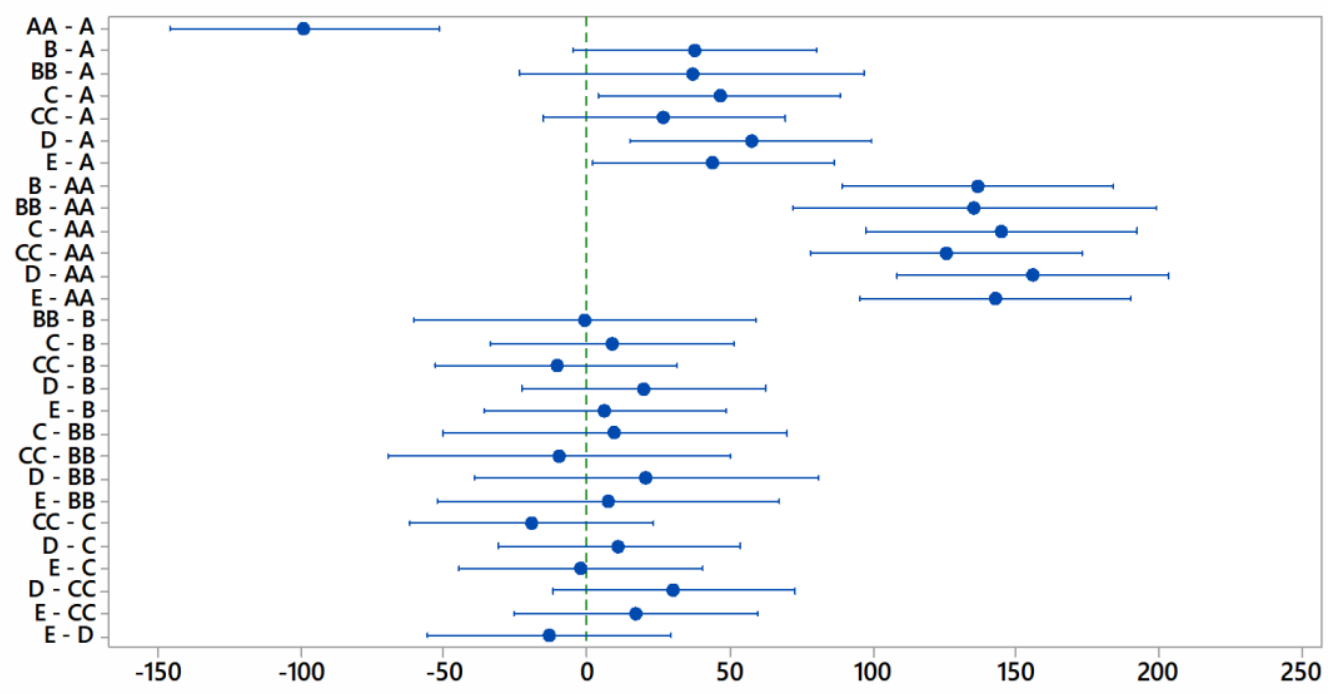

Figure 8.5. b. Fisher individual $95 \%$ confidence interval differences of means for $\mathrm{CH}_{4}$ yields in response to the ratios 


\section{Chapter 9}

Conclusions 


\section{Conclusions}

\subsection{Biomethane potential}

A series of binary and ternary anaerobic co-digestion was designed to evaluate the biomethane potential and to investigate the correlation between the biomethane production and the mixing ratios as well as lipids: proteins: carbohydrates ratios of the feedstock. Gompertz model was used to assess the compatibility of the model to the data obtained by the experiment. A first order kinetic model was applied using AquaSim 2.0 to assess the hydrolysis kinetics and its influence on the process. A simple empirical model was derived based on the correlation of biomethane production to the lipids, proteins and carbohydrates content of the feedstocks which included TWAS, manure and SSO.

\subsubsection{Binary co-digestion of TWAS, manure, and SSO}

$>$ The three sets of the binary co-digestion primarily verified the advantages of co-digestion over mono-digestion of TWAS, manure and SSO.

$>$ Among the three binary co-digestion including TWAS/SSO, TWAS/manure, and Manure/SSO, the most amount of the methane yield was achieved by co-digestion of Manure/SSO at the mixing ratio of 7:3 corresponding to $363 \mathrm{~mL} \mathrm{CH}_{4} / \mathrm{g}$ COD added.

$>$ The modified Gompertz model showed a good fit to the experimental data by a diversion ranging from $4 \%$ to $6 \%$.

$>$ The optimum ratios of the feedstocks as well as the lipids: proteins: carbohydrates varied by the different feedstocks.

$>$ In co-digestion of TWAS/SSO the maximum ultimate $\mathrm{CH}_{4}$ of $1252 \mathrm{~mL}^{2}$ and $\mathrm{CH}_{4}$ yield of $357 \mathrm{~mL} \mathrm{CH}_{4} / \mathrm{g} \mathrm{COD}$ added corresponded to the mixing ratio of 3:7 and lipids: proteins: carbohydrates ratio of $1: 2: 8$.

TWAS/manure co-digestion resulted in $1069 \mathrm{~mL}$ ultimate $\mathrm{CH}_{4}$ production and $324 \mathrm{~mL}$ $\mathrm{CH}_{4} / \mathrm{g}$ COD added biomethane yield at the mixing ratio of 3:7 and lipids: proteins: carbohydrates ratio of $1: 4: 17$.

$>$ For Manure/SSO co-digestion an ultimate $\mathrm{CH}_{4}$ of $1186 \mathrm{~mL}$ and a $\mathrm{CH}_{4}$ yield of $363 \mathrm{~mL}$ $\mathrm{CH}_{4} / \mathrm{g} \mathrm{COD}$ added was achieved corresponding to the mixing ratio of $7: 3$ and lipids: proteins: carbohydrate ratio of $1: 3.5: 18.5$. 


\subsubsection{Ternary co-digestion of TWAS, manure and SSO}

$>$ Among co-digestion of the three feedstocks at different ternary combinations, the most ultimate methane production and yield occurred at TWAS/manure/SSO mixing ratio of 2:4:4 and lipids: proteins: carbohydrate ratio of $1: 3: 12$.

$>$ The maximum methane production and yield in co-digestion of TWAS/manure/SSO were $1424 \mathrm{~mL} 356 \mathrm{~mL} \mathrm{CH}_{4} / \mathrm{g}$ COD added, respectively.

$>$ The modified Gompertz model showed a good fit to the results obtained by the experiment with less than $5 \%$ diversion.

$>$ A higher maximum ultimate methane and methane yield was achieved by ternary codigestion of TWAS/manure and SSO, however at some ratios the ternary co-digestion did not produce higher methane than some of the combinations in the binary co-digestion.

$>$ Synergistic effect did not demonstrate further improvement in the ternary co-digestion of TWAS, manure, and SSO compared to their combinations in the binary co-digestion.

$>$ The most synergistic effect in co-digestion of TWAS/manure/SSO was 19\% while the maximum synergistic effect was $36 \%$ in manure/SSO co-digestion.

\subsection{Hydrolysis/acidification}

Along with the biomethane potential assay, a series of hydrolysis/ acidification of the binary and ternary combinations of the feedstocks was designed to evaluate the hydrolysis and acidification process in co-digestion of TWAS, manure, and SSO. The binary and ternary anaerobic codigestion were set up with the same combination as the BMP experiments for investigating the correlation between the solublization, VFAs yield, and hydrolysis rate, with the mixing ratios as well as lipids: proteins: carbohydrates ratios of the feedstock. First-order kinetic model was used to assess the compatibility of the model to the data obtained by the experiment. The model was used to obtain the kinetic rare coefficients of COD, lipids, proteins and carbohydrates of the feedstocks at different mixing ratios. 


\subsubsection{Co-digestion of TWAS/SSO}

An improvement of hydrolysis/acidification was observed in co-digestion of the feedstocks at different mixing ratios

$>$ In TWAS/SSO co-digestion the maximum degree of solublization was $30 \%$ at the mixing ratio of 1:9.

$>$ The synergistic effect was the highest at TWAS/SSO mixing ratio of 7:3 corresponding to $44 \%$ improvement as a result of using co-substrate and improving synergy.

$>$ A maximum VFAs yield of $312 \mathrm{mg} / \mathrm{g}$ VSS added was attained by TWAS/SSO codigestion at the mixing ratio of 1:9.

$>$ The hydrolysis kinetic rate coefficient was compatible to the first order kinetic model with a diversion less than $10 \%$.

$>$ The maximum hydrolysis rate coefficient of COD, proteins, and carbohydrates in codigestion of TWAS/SSO were $0.35,0.07,0.34$ and $0.68 \mathrm{~d}^{-1}$, corresponding to the mixing ratios of 1:9, 3:7, 1:9, respectively.

$>$ The maximum hydrolysis rate coefficient of lipids was 0.07 which corresponded to the TWAS:SSO mixing ratios of 5:5 and 3:7.

\subsubsection{Co-digestion of TWAS/manure}

The percentage improvement of hydrolysis/acidification varied by different mixing ratios of TWAS and manure.

$>$ In co-digestion of TWAS/manure, the maximum degree of solublization was $34 \%$ corresponding to the TWAS/manure mixing ratio of 3:7.

$>$ A synergistic effect of $38 \%$ was achieved by TWAS/manure co-digestion at the mixing ratio of $3: 7$.

$>$ The maximum VFAs yield of $507 \mathrm{mg} / \mathrm{g}$ VSS added was achieved by TWAS/manure mixing ratio of 3:7.

$>$ The hydrolysis kinetic rate coefficient was compatible to the first order kinetic model with a diversion less than $10 \%$. 
The maximum hydrolysis rate coefficient of COD, lipids, proteins, and carbohydrates in co-digestion of TWAS/manure were $0.33,0.09,0.27$, and $0.59 \mathrm{~d}^{-1}$, corresponding to the mixing ratio of $3: 7$.

\subsubsection{Co-digestion of manure /SSO}

The hydrolysis/acidification improved to different extents in response to the different mixing ratios in co-digestion of TWAS and manure.

$>$ The most degree of solublization was $35 \%$ corresponding to manure/SSO mixing ratios of $3: 7$ and $1: 9$.

$>$ The highest synergistic effect of $34 \%$ in co-digestion of manure and SSO occurred at the mixing ratio of $9: 1$.

$>$ In manure/SSO co-digestion, the maximum VFAs yield of $400 \mathrm{mg} / \mathrm{g}$ VSS added was achieved at the mixing ratio of 1:9.

$>$ The hydrolysis kinetic rate coefficient was compatible to the first order kinetic model with a diversion less than $10 \%$

$>$ The maximum hydrolysis rate coefficient of COD, lipids, proteins, and carbohydrates in co-digestion of manure/SSO were $0.38,0.11,0.33$, and $0.68 \mathrm{~d}-1$, corresponding to the mixing ratio of $3: 7$.

\subsubsection{Co-digestion of TWAS/manure /SSO}

$>$ In the ternary co-digestion of TWAS, manure, and SSO the maximum solublization was $30 \%$ corresponding to TWAS/manure/SSO mixing ratio of 2:4:4 and 1:8:1.

$>$ The maximum synergistic effect of $35 \%$ corresponded to TWAS/manure/SSO mixing ratio of $1: 8: 1$.

$>$ In co-digestion of TWAS/manure/SSO the highest VFAs yield of $380 \mathrm{mg}$ VFAs/g VSS added was achieved at the mixing ratio of $2: 4: 4$.

$>$ The hydrolysis kinetic rate coefficient was compatible to the first order kinetic model with a diversion less than $10 \%$

$>$ The hydrolysis rate coefficient of COD were within the range of 0.21 to 0.39 for all of the co-digesters. 
$>$ The maximum hydrolysis rate coefficients of COD, lipids, and carbohydrates in codigestion of TWAS/manure/SSO were $0.39,0.14$, and $0.69 \mathrm{~d}^{-1}$ corresponding to the mixing ratio of $2: 4: 4$.

$>$ In co-digestion of TWAS/manure/SSO, the maximum hydrolysis rate coefficient for the proteins content was 0.23 which corresponded to the mixing ratios of $8: 1: 1$ and $2.5: 5: 2.5$.

\subsection{Correlation of biomethane production with the organic content}

\subsubsection{COD: $N$ and lipids:proteinscarbohydrates ratios}

$>$ In TWAS/SSO co-digestion the maximum ultimate $\mathrm{CH}_{4}$ corresponded to COD:N ratio of 28 corresponding to the lipids: proteins: carbohydrates ratio of $1: 2: 8$. The trend of variations of the methane yield versus COD: $\mathrm{N}$ was similar to the trend that was observed for its variation vursus lipids:proteins. The changes of methane yield versus lipids:carbohydrates and proteins:carbohydrates showed a similar trend while it did not comply with that of COD: $\mathrm{N}$ ratio.

$>$ In TWAS/manure co-digestion, the maximum $\mathrm{CH}_{4}$ yield occurred at COD:N ratio of 41 corresponding to the lipids: proteins: carbohydrates ratio of 1:4:17. Similar to the codigestion of TWAS/SSO, in TWAS/manure co-digestion the trend of variations of the methane yield versus COD: $\mathrm{N}$ was similar to the trend that was observed for its variation versus lipids:proteins. The changes of methane yield versus lipids:carbohydrates and proteins:carbohydrates did not comply with that of COD:N ratio although they both showed a simiar trend.

$>$ In Manure/SSO co-digestion, the maximum methane yield corresponded to the COD:N ratio of 41 and lipids: proteins: carbohydrates ratio of 1:3.5:18.5. The variations of methane yield versus COD:N and lipids: proteins did not show a similar trend. Although, in terms of the changes of the $\mathrm{CH}_{4}$ yield, both lipids: carbohydrates and proteins: carbohydrates ratios demonstrated similar trend.

In TWAS/manure/SSO co-digestion the maximum methane yield corresponded to COD:N tatio of 28 and the lipids:proteins:carbohydrates ratio of 1:3:12. The trend of variations of the methane yield vursus COD:N was similar to the trend that was observed for its variation versus lipids:proteins. The changes of methane yield vursus lipids:carbohydrates and 
proteins:carbohydrates showed a similar trend while it did not comply with that of COD:N. TWAS/manure/SSO co-digestion.

\subsubsection{Anaerobic co-digestion model}

An empirical model would be a useful approach to predict the methane yield $\left(\mathrm{mLCH}_{4} / \mathrm{gCOD}_{\text {added }}\right)$ based on the lipids, proteins, and carbohydrates components of the feedstock in anaerobic codigestion of multi substrate. As the three main components of any type of feedstocks in biowaste are lipids, proteins, and carbohydrates, an empirical model was presented that correlates the $\mathrm{CH}_{4}$ yields with lipids, proteins and carbohydrates content of the feedstock. The functional relationship between responses which was $\mathrm{CH}_{4}$ yield and the factors including lipids, proteins and carbohydrates (Lp, Pr, and Cr) were described by estimating the coefficients of the second-order polynomial model based on the experimental data. The fit plots together with the residual plots for lipids, proteins and carbohydrates showed that the model adequately fits to the data and meet the model assumption.

\subsection{Suggested future works}

Validating the batch study results for AnCoD of Manure/SSO and TWAS/Manure/SSO at 7:3 and 2:4:4 mixing ratios in CSTR mode

$>$ Investigating the validity of the proposed empirical model for a variety of the feedstocks including the industrial wastes

$>$ Investigating the validity of the proposed empirical model using CSTR mode.

$>$ Implementing a cost-benefit analysis for applying AnCoD of Manure/SSO and TWAS/Manure/SSO for GTA versus individual AD of manure and SSO.

Analyzing carbon footprint reduction by AnCoD of manure with TWAS as compared to conventional $\mathrm{AD}$ and composting 


\section{Appendices}

\section{A. Analytical results for AnCoD of TWAS and SSO}

Table A. 1. Characteristics of raw feedstocks in AnCoD of TWAS and SSO

\begin{tabular}{|c|c|c|c|c|c|c|c|}
\hline \multirow{2}{*}{ Parameters } & \multirow{2}{*}{ Units } & \multicolumn{2}{|c|}{ SSO } & \multicolumn{2}{|c|}{ TWAS } & \multicolumn{2}{|c|}{ Seed } \\
\hline & & MEAN & SD & MEAN & SD & MEAN & $\mathrm{SD}$ \\
\hline TCOD & $\mathrm{mg} / \mathrm{L}$ & 110000 & 7795 & 40000 & 2053 & 16400 & 444 \\
\hline SCOD & $\mathrm{mg} / \mathrm{L}$ & 44400 & 100 & 1360 & 26 & 762 & 19 \\
\hline TSS & $\mathrm{mg} / \mathrm{L}$ & 53833 & 6252 & 31450 & 1006 & 15033 & 400 \\
\hline VSS & $\mathrm{mg} / \mathrm{L}$ & 38478 & 3649 & 25600 & 2216 & 10900 & 200 \\
\hline TS & $\mathrm{mg} / \mathrm{L}$ & 62187 & 1025 & 38810 & 1859 & 17450 & 582 \\
\hline VS & $\mathrm{mg} / \mathrm{L}$ & 43493 & 761 & 34477 & 1027 & 13140 & 328 \\
\hline Ammonia & $\mathrm{mg} / \mathrm{L}$ & 1138 & 6 & 255 & 9 & 795 & 40 \\
\hline $\mathrm{pH}$ & $\begin{array}{c}- \\
\mathrm{mg}\end{array}$ & 5.6 & 0.2 & 6.3 & 0.2 & 7.2 & 0.2 \\
\hline Alkalinity & $\mathrm{CaCO} 3 / \mathrm{L}$ & 6080 & 522 & 1953 & 148 & 4943 & 465 \\
\hline $\mathrm{TN}$ & $\mathrm{mg} / \mathrm{L}$ & 3267 & 751 & 2900 & 400 & 1425 & 203 \\
\hline TSN & $\mathrm{mg} / \mathrm{L}$ & 910 & 38 & 420 & 64 & 696 & 112 \\
\hline Total Carbs & $\mathrm{mg} / \mathrm{L}$ & 14360 & 3580 & 1080 & 112 & 549 & 82 \\
\hline Total Proteins & $\mathrm{mg} / \mathrm{L}$ & 2308 & 198 & 3759 & 298 & 1648 & 56 \\
\hline Total Lipids & $\mathrm{mg} / \mathrm{L}$ & 1731 & 2420 & 351 & 27 & 163 & 52 \\
\hline
\end{tabular}


Table A. 2. Average $\mathrm{CH}_{4}$ production in AnCoD of TWAS and SSO

\begin{tabular}{|c|c|c|c|c|c|c|c|c|c|c|c|c|c|}
\hline \multicolumn{14}{|c|}{ Average $\mathrm{CH}_{4}$ Measurement $(\mathrm{mL})$} \\
\hline $\begin{array}{l}\text { Time } \\
\text { (day) }\end{array}$ & TWAS & SD & SSO & SD & $\begin{array}{c}\text { T/SSO } \\
9 / 1\end{array}$ & SD & $\begin{array}{c}\text { T/SSO } \\
7 / 3\end{array}$ & SD & $\begin{array}{c}\text { T/SSO } \\
1 / 1\end{array}$ & SD & $\begin{array}{c}\mathrm{T} / \mathrm{SSO} \\
1 / 9\end{array}$ & SD & $\begin{array}{c}\mathrm{T} / \mathrm{SSO} \\
3 / 7\end{array}$ \\
\hline 1 & 6 & 0.3 & 3 & 0.1 & 6 & 0.3 & 7 & 0.3 & 3 & 0.1 & 4 & 0.1 & 3 \\
\hline 3 & 24 & 1.2 & 20 & 1.0 & 20 & 1.0 & 33 & 1.7 & 17 & 0.7 & 27 & 1.0 & 20 \\
\hline 4 & 43 & 2.3 & 27 & 1.3 & 35 & 1.7 & 54 & 2.8 & 25 & 1.1 & 49 & 1.9 & 38 \\
\hline 5 & 58 & 3.0 & 42 & 2.0 & 57 & 2.8 & 68 & 3.5 & 39 & 1.7 & 82 & 3.1 & 65 \\
\hline 6 & 39 & 2.0 & 48 & 2.3 & 44 & 2.2 & 51 & 2.6 & 39 & 1.6 & 60 & 2.3 & 76 \\
\hline 7 & 37 & 1.9 & 48 & 2.3 & 51 & 2.5 & 45 & 2.3 & 66 & 2.8 & 52 & 2.0 & 69 \\
\hline 8 & 21 & 1.1 & 51 & 2.5 & 34 & 1.7 & 42 & 2.2 & 53 & 2.2 & 45 & 1.7 & 78 \\
\hline 10 & 28 & 1.4 & 55 & 2.7 & 34 & 1.7 & 60 & 3.0 & 84 & 3.5 & 80 & 3.0 & 93 \\
\hline 12 & 26 & 1.4 & 51 & 2.5 & 25 & 1.2 & 29 & 1.5 & 81 & 3.4 & 74 & 2.8 & 83 \\
\hline 14 & 21 & 1.1 & 39 & 1.9 & 22 & 1.1 & 22 & 1.1 & 47 & 2.0 & 33 & 1.2 & 64 \\
\hline 16 & 19 & 1.0 & 25 & 1.2 & 15 & 0.7 & 23 & 1.2 & 21 & 0.9 & 20 & 0.8 & 20 \\
\hline 19 & 23 & 1.2 & 23 & 1.1 & 25 & 1.2 & 25 & 1.3 & 21 & 0.9 & 25 & 1.0 & 22 \\
\hline 22 & 18 & 0.9 & 24 & 1.2 & 15 & 0.8 & 17 & 0.9 & 24 & 1.0 & 26 & 1.0 & 30 \\
\hline 26 & 15 & 0.8 & 20 & 1.0 & 10 & 0.5 & 12 & 0.6 & 14 & 0.6 & 15 & 0.6 & 22 \\
\hline 29 & 10 & 0.5 & 14 & 0.7 & 8 & 0.4 & 10 & 0.5 & 9 & 0.4 & 12 & 0.5 & 17 \\
\hline 34 & 33 & 1.7 & 33 & 1.6 & 32 & 1.6 & 33 & 1.7 & 31 & 1.3 & 31 & 1.2 & 31 \\
\hline 37 & 13 & 0.7 & 12 & 0.6 & 14 & 0.7 & 13 & 0.7 & 9 & 0.4 & 12 & 0.4 & 14 \\
\hline 40 & 14 & 0.7 & 13 & 0.6 & 14 & 0.7 & 13 & 0.7 & 10 & 0.4 & 12 & 0.5 & 15 \\
\hline 43 & 14 & 0.7 & 13 & 0.6 & 13 & 0.6 & 11 & 0.6 & 9 & 0.4 & 11 & 0.4 & 10 \\
\hline 49 & 1 & 0.1 & 3 & 0.2 & 5 & 0.2 & 3 & 0.2 & 1 & 0.1 & 2 & 0.1 & 5 \\
\hline 56 & 0 & 0.0 & 0 & 0.0 & 7 & 0.3 & 5 & 0.3 & 4 & 0.2 & 6 & 0.2 & 4 \\
\hline
\end{tabular}


Table A. 3. Cumulative $\mathrm{CH}_{4}$ production in AnCoD of TWAS and SSO

\begin{tabular}{|c|c|c|c|c|c|c|c|c|c|c|c|c|c|c|}
\hline \multicolumn{15}{|c|}{ Cumulative $\mathrm{CH}_{4}$ Production (mL) } \\
\hline $\begin{array}{l}\text { Time } \\
\text { (day) }\end{array}$ & TWAS & SD & SSO & SD & $\begin{array}{c}\mathrm{T} / \mathrm{SSO} \\
9 / 1\end{array}$ & SD & $\begin{array}{c}\text { T/SSO } \\
7 / 3\end{array}$ & SD & $\begin{array}{c}\mathrm{T} / \mathrm{SSO} \\
1 / 1\end{array}$ & SD & $\begin{array}{c}\text { T/SSO } \\
1 / 9\end{array}$ & SD & $\begin{array}{l}\text { T/SSO } \\
3 / 7\end{array}$ & SD \\
\hline 0 & 0 & 0 & 0 & 0 & 0 & 0 & 0 & 0 & 0 & 0 & 0 & 0 & 0 & 0.0 \\
\hline 1 & 7 & 0 & 5 & 0 & 9 & 0 & 12 & 1 & 6 & 0 & 7 & 0 & 4 & 0.2 \\
\hline 3 & 35 & 2 & 46 & 2 & 39 & 2 & 70 & 3 & 37 & 2 & 56 & 3 & 36 & 1.4 \\
\hline 4 & 86 & 4 & 99 & 5 & 92 & 5 & 164 & 8 & 83 & 4 & 146 & 8 & 96 & 3.8 \\
\hline 5 & 154 & 8 & 182 & 8 & 177 & 8 & 284 & 14 & 154 & 8 & 296 & 16 & 200 & 8.0 \\
\hline 6 & 199 & 10 & 278 & 13 & 243 & 13 & 373 & 18 & 225 & 11 & 405 & 22 & 323 & 12.9 \\
\hline 7 & 242 & 12 & 373 & 17 & 319 & 17 & 452 & 22 & 345 & 18 & 500 & 27 & 435 & 17.4 \\
\hline 8 & 267 & 13 & 475 & 22 & 369 & 22 & 527 & 26 & 441 & 23 & 582 & 31 & 560 & 22.3 \\
\hline 10 & 299 & 15 & 583 & 27 & 419 & 27 & 631 & 31 & 593 & 30 & 727 & 39 & 710 & 28.3 \\
\hline 12 & 330 & 16 & 685 & 31 & 457 & 31 & 683 & 34 & 739 & 38 & 862 & 46 & 843 & 34.5 \\
\hline 14 & 354 & 17 & 762 & 35 & 489 & 35 & 721 & 35 & 823 & 42 & 922 & 49 & 946 & 37.8 \\
\hline 16 & 377 & 18 & 813 & 37 & 511 & 37 & 761 & 37 & 862 & 44 & 958 & 51 & 979 & 39.1 \\
\hline 19 & 404 & 20 & 859 & 39 & 548 & 39 & 804 & 40 & 899 & 46 & 1004 & 54 & 1014 & 40.5 \\
\hline 22 & 425 & 21 & 906 & 42 & 571 & 42 & 834 & 41 & 944 & 48 & 1052 & 56 & 1062 & 42.4 \\
\hline 26 & 443 & 22 & 946 & 43 & 586 & 43 & 856 & 42 & 968 & 49 & 1080 & 58 & 1098 & 43.8 \\
\hline 29 & 455 & 22 & 974 & 45 & 598 & 45 & 873 & 43 & 985 & 50 & 1102 & 59 & 1124 & 44.9 \\
\hline 34 & 493 & 24 & 1040 & 48 & 645 & 48 & 930 & 46 & 1040 & 53 & 1159 & 62 & 1174 & 46.8 \\
\hline 37 & 508 & 25 & 1064 & 49 & 666 & 49 & 953 & 47 & 1057 & 54 & 1180 & 63 & 1196 & 47.7 \\
\hline 40 & 524 & 26 & 1089 & 50 & 686 & 50 & 976 & 48 & 1075 & 55 & 1202 & 64 & 1221 & 48.7 \\
\hline 43 & 541 & 26 & 1115 & 51 & 705 & 51 & 996 & 45 & 1091 & 56 & 1222 & 65 & 1237 & 45.4 \\
\hline 49 & 542 & 26 & 1122 & 48 & 712 & 49 & 1001 & 49 & 1094 & 55 & 1225 & 62 & 1245 & 49.7 \\
\hline 56 & 542 & 26 & 1122 & 47 & 723 & 50 & 1010 & 50 & 1100 & 57 & 1235 & 66 & 1252 & 50.0 \\
\hline
\end{tabular}


Table A. 4. Cumulative methane yield per unit mass of COD added in AnCoD of TWAS and SSO

\begin{tabular}{|c|c|c|c|c|c|c|c|c|c|c|c|c|c|c|}
\hline \multicolumn{15}{|c|}{ Cumulative $\mathrm{CH}_{4}$ yield (mLCH $4 / \mathrm{COD}$ added) } \\
\hline $\begin{array}{l}\text { Time } \\
\text { (day) }\end{array}$ & TWAS & SD & SSO & SD & $\begin{array}{l}\mathrm{T} / \mathrm{SSO} \\
9 / 1\end{array}$ & SD & $\begin{array}{l}\mathrm{T} / \mathrm{SSO} \\
7 / 3\end{array}$ & SD & $\begin{array}{l}\text { T/SSO } \\
1 / 1\end{array}$ & SD & $\begin{array}{l}\mathrm{T} / \mathrm{SSO} \\
3 / 7\end{array}$ & SD & $\begin{array}{l}\mathrm{T} / \mathrm{SSO} \\
1 / 9\end{array}$ & SD \\
\hline 0 & 0 & 0 & 0 & 0 & 0 & 0 & 0 & 0 & 0 & 0 & 0 & 0 & 0 & 0 \\
\hline 1 & 2 & 0.1 & 1 & 0.1 & 3 & 0.2 & 4 & 0.2 & 2 & 0.1 & 2 & 0.1 & 1 & 0.1 \\
\hline 3 & 12 & 0.6 & 13 & 0.7 & 13 & 0.6 & 22 & 1.1 & 11 & 0.5 & 16 & 0.9 & 10 & 0.4 \\
\hline 4 & 30 & 1.6 & 27 & 1.5 & 31 & 1.5 & 51 & 2.5 & 25 & 1.1 & 42 & 2.3 & 27 & 1.2 \\
\hline 5 & 55 & 2.8 & 50 & 2.7 & 59 & 2.9 & 88 & 4.3 & 46 & 2.1 & 84 & 4.6 & 56 & 2.4 \\
\hline 6 & 71 & 3.7 & 76 & 4.1 & 81 & 4.0 & 116 & 5.7 & 66 & 3.0 & 116 & 6.4 & 90 & 3.9 \\
\hline 7 & 86 & 4.5 & 103 & 5.5 & 107 & 5.2 & 141 & 6.9 & 102 & 4.6 & 143 & 7.9 & 121 & 5.2 \\
\hline 8 & 94 & 4.9 & 130 & 7.0 & 124 & 6.1 & 164 & 8.0 & 131 & 5.9 & 166 & 9.1 & 156 & 6.7 \\
\hline 10 & 106 & 5.5 & 160 & 8.7 & 141 & 6.9 & 197 & 9.6 & 175 & 7.9 & 208 & 11.4 & 197 & 8.5 \\
\hline 12 & 117 & 6.1 & 188 & 10.2 & 153 & 7.5 & 213 & 10.4 & 219 & 9.8 & 246 & 13.5 & 234 & 10.1 \\
\hline 14 & 126 & 6.5 & 209 & 11.3 & 164 & 8.0 & 224 & 11.0 & 244 & 11.0 & 263 & 14.5 & 263 & 10.5 \\
\hline 16 & 133 & 6.9 & 223 & 12.1 & 172 & 8.4 & 237 & 11.6 & 255 & 11.5 & 273 & 15.0 & 272 & 11.7 \\
\hline 19 & 143 & 7.4 & 236 & 12.7 & 184 & 9.0 & 250 & 12.3 & 266 & 12.0 & 287 & 15.8 & 282 & 12.1 \\
\hline 22 & 151 & 7.8 & 249 & 13.4 & 192 & 9.4 & 260 & 12.7 & 279 & 12.6 & 300 & 16.5 & 295 & 11.3 \\
\hline 26 & 157 & 8.2 & 260 & 13.8 & 197 & 9.6 & 266 & 13.1 & 287 & 12.9 & 308 & 17.0 & 305 & 13.1 \\
\hline 29 & 161 & 8.4 & 268 & 14.5 & 201 & 9.8 & 272 & 13.3 & 292 & 13.1 & 315 & 17.3 & 312 & 13.7 \\
\hline 34 & 175 & 9.1 & 286 & 15.4 & 217 & 10.6 & 290 & 14.2 & 308 & 13.9 & 331 & 18.2 & 326 & 14.0 \\
\hline 37 & 180 & 9.4 & 292 & 15.8 & 223 & 11.0 & 297 & 14.5 & 313 & 14.1 & 337 & 18.5 & 332 & 14.3 \\
\hline 40 & 186 & 9.7 & 299 & 14.8 & 230 & 10.3 & 304 & 14.9 & 318 & 12.7 & 343 & 18.9 & 339 & 13.5 \\
\hline 43 & 192 & 10.2 & 306 & 16.5 & 237 & 12.2 & 310 & 16.1 & 323 & 14.5 & 349 & 17.2 & 344 & 14.8 \\
\hline 49 & 192 & 9.8 & 308 & 15.5 & 239 & 11.7 & 312 & 15.3 & 324 & 13.2 & 350 & 16.3 & 346 & 15.2 \\
\hline 56 & 192 & 9.5 & 308 & 16.2 & 243 & 10.6 & 315 & 14.4 & 326 & 14.7 & 353 & 15.8 & 348 & 14.4 \\
\hline
\end{tabular}


Table A. 5. Cumulative methane yield per unit mass of VSS added in AnCoD of TWAS and SSO

\begin{tabular}{|c|c|c|c|c|c|c|c|c|c|c|c|c|c|c|}
\hline \multicolumn{15}{|c|}{ Cumulative $\mathrm{CH}_{4}$ yield (mLCH$/$ VSS added) } \\
\hline $\begin{array}{l}\text { Time } \\
\text { (day) }\end{array}$ & TWAS & SD & SSO & SD & $\begin{array}{l}\mathrm{T} / \mathrm{SSO} \\
9 / 1\end{array}$ & SD & $\begin{array}{l}\mathrm{T} / \mathrm{SSO} \\
7 / 3\end{array}$ & SD & $\begin{array}{l}\mathrm{T} / \mathrm{SSO} \\
1 / 1\end{array}$ & SD & $\begin{array}{l}\text { T/SSO } \\
3 / 7\end{array}$ & SD & $\begin{array}{l}\mathrm{T} / \mathrm{SSO} \\
1 / 9\end{array}$ & SD \\
\hline 0 & 0 & 0 & 0 & 0 & 0 & 0 & 0 & 0 & 0 & 0 & 0 & 0 & 0 & 0 \\
\hline 1 & 4 & 0.2 & 1 & 0.0 & 5 & 0.3 & 8 & 0.4 & 4 & 0.2 & 5 & 0.3 & 3 & 0.2 \\
\hline 3 & 19 & 0.9 & 10 & 0.4 & 23 & 1.2 & 45 & 2.2 & 26 & 1.3 & 41 & 2.0 & 28 & 1.3 \\
\hline 4 & 47 & 2.3 & 21 & 0.8 & 54 & 2.8 & 106 & 5.2 & 58 & 2.8 & 107 & 5.2 & 74 & 3.6 \\
\hline 5 & 85 & 4.1 & 39 & 1.6 & 104 & 5.4 & 183 & 9.0 & 107 & 5.2 & 217 & 10.6 & 154 & 7.6 \\
\hline 6 & 110 & 5.4 & 60 & 2.4 & 142 & 7.4 & 240 & 11.9 & 156 & 7.6 & 297 & 14.6 & 249 & 12.2 \\
\hline 7 & 134 & 6.5 & 81 & 3.2 & 187 & 9.7 & 291 & 14.4 & 239 & 11.6 & 367 & 18.0 & 335 & 16.4 \\
\hline 8 & 148 & 7.2 & 102 & 4.1 & 216 & 11.3 & 339 & 16.8 & 306 & 14.9 & 427 & 20.9 & 431 & 21.1 \\
\hline 10 & 165 & 8.0 & 126 & 5.0 & 246 & 12.8 & 407 & 20.1 & 411 & 20.0 & 534 & 26.2 & 546 & 26.8 \\
\hline 12 & 182 & 8.9 & 148 & 5.9 & 268 & 14.0 & 440 & 21.8 & 512 & 24.9 & 633 & 31.0 & 649 & 31.8 \\
\hline 14 & 196 & 9.5 & 165 & 6.6 & 287 & 15.0 & 465 & 23.0 & 571 & 27.8 & 677 & 33.2 & 728 & 35.7 \\
\hline 16 & 208 & 10.1 & 175 & 7.0 & 300 & 15.6 & 490 & 24.3 & 597 & 29.1 & 703 & 34.5 & 753 & 36.9 \\
\hline 19 & 224 & 10.9 & 185 & 7.4 & 322 & 16.8 & 518 & 25.7 & 623 & 30.4 & 737 & 36.1 & 781 & 38.2 \\
\hline 22 & 235 & 11.4 & 196 & 7.8 & 335 & 17.5 & 538 & 26.6 & 654 & 31.8 & 772 & 37.8 & 817 & 40.0 \\
\hline 26 & 245 & 11.9 & 204 & 8.1 & 344 & 17.9 & 552 & 26.5 & 671 & 32.7 & 793 & 38.8 & 845 & 41.4 \\
\hline 29 & 252 & 12.2 & 210 & 8.4 & 351 & 18.3 & 563 & 27.8 & 683 & 33.2 & 809 & 39.7 & 865 & 42.4 \\
\hline 34 & 273 & 13.3 & 225 & 8.9 & 379 & 19.7 & 600 & 29.7 & 721 & 35.1 & 851 & 41.7 & 903 & 44.3 \\
\hline 37 & 281 & 13.7 & 230 & 9.1 & 391 & 20.4 & 615 & 30.4 & 732 & 35.7 & 867 & 42.5 & 921 & 45.1 \\
\hline 40 & 290 & 15.4 & 235 & 9.4 & 403 & 21.0 & 629 & 32.3 & 745 & 36.3 & 883 & 43.3 & 940 & 46.1 \\
\hline 43 & 299 & 14.6 & 241 & 10.0 & 414 & 20.2 & 642 & 31.8 & 756 & 36.8 & 897 & 43.2 & 952 & 46.7 \\
\hline 49 & 300 & 13.8 & 242 & 9.9 & 418 & 19.8 & 645 & 30.5 & 758 & 35.4 & 899 & 45.4 & 958 & 45.1 \\
\hline 56 & 300 & 15.1 & 242 & 9.2 & 424 & 21.6 & 651 & 32.2 & 762 & 37.1 & 907 & 44.4 & 964 & 47.2 \\
\hline
\end{tabular}


Table A. 6. Cumulative methane yield per unit volume of VSS added in AnCoD of TWAS and SSO

\begin{tabular}{|c|c|c|c|c|c|c|c|c|c|c|c|c|c|c|}
\hline \multicolumn{15}{|c|}{ Cumulative $\mathrm{CH}_{4}$ yield (mLCH $4 / \mathrm{mL}$ substrate added) } \\
\hline $\begin{array}{l}\text { Time } \\
\text { (day) }\end{array}$ & TWAS & SD & SSO & SD & $\begin{array}{l}\mathrm{T} / \mathrm{SSO} \\
9 / 1\end{array}$ & SD & $\begin{array}{l}\text { T/SSO } \\
7 / 3\end{array}$ & SD & $\begin{array}{l}\mathrm{T} / \mathrm{SSO} \\
1 / 1\end{array}$ & SD & $\begin{array}{l}\text { T/SSO } \\
3 / 7\end{array}$ & SD & $\begin{array}{l}\mathrm{T} / \mathrm{SSO} \\
1 / 9\end{array}$ & SD \\
\hline 0 & 0.00 & 0.00 & 0.00 & 0.00 & 0.00 & 0.00 & 0.00 & 0.00 & 0.00 & 0.00 & 0.00 & 0.00 & 0.00 & 0.00 \\
\hline 1 & 0.10 & 0.00 & 0.15 & 0.01 & 0.14 & 0.01 & 0.22 & 0.01 & 0.14 & 0.01 & 0.18 & 0.01 & 0.12 & 0.00 \\
\hline 3 & 0.49 & 0.02 & 1.38 & 0.07 & 0.62 & 0.03 & 1.32 & 0.06 & 0.83 & 0.04 & 1.42 & 0.07 & 1.02 & 0.04 \\
\hline 4 & 1.22 & 0.05 & 2.99 & 0.14 & 1.45 & 0.08 & 3.12 & 0.15 & 1.84 & 0.09 & 3.70 & 0.18 & 2.76 & 0.10 \\
\hline 5 & 2.18 & 0.09 & 5.51 & 0.27 & 2.79 & 0.15 & 5.38 & 0.26 & 3.43 & 0.17 & 7.52 & 0.36 & 5.74 & 0.22 \\
\hline 6 & 2.82 & 0.12 & 8.40 & 0.41 & 3.83 & 0.21 & 7.08 & 0.34 & 4.99 & 0.25 & 10.29 & 0.49 & 9.26 & 0.35 \\
\hline 7 & 3.43 & 0.14 & 11.28 & 0.55 & 5.03 & 0.27 & 8.59 & 0.41 & 7.65 & 0.39 & 12.72 & 0.61 & 12.45 & 0.47 \\
\hline 8 & 3.78 & 0.16 & 14.34 & 0.70 & 5.82 & 0.32 & 10.00 & 0.48 & 9.80 & 0.50 & 14.79 & 0.71 & 16.02 & 0.61 \\
\hline 10 & 4.24 & 0.18 & 17.63 & 0.85 & 6.62 & 0.36 & 11.99 & 0.58 & 13.16 & 0.67 & 18.48 & 0.88 & 20.31 & 0.77 \\
\hline 12 & & 0.20 & 20 & 1.00 & & 0.39 & 12.97 & 0.62 & 16.40 & 0.84 & 21.90 & 1.05 & 24.12 & 92 \\
\hline 14 & 5.02 & 0.21 & 23.04 & 1.12 & 7.72 & 0.42 & 13.69 & 0.66 & 18.28 & 0.93 & 23.42 & 1.12 & 27.08 & 1.03 \\
\hline 16 & 5.34 & 0.22 & 24.57 & 1.19 & 8.07 & 0.44 & 14.45 & 0.69 & 19.14 & 0.98 & 24.34 & 1.16 & 28.01 & 1.06 \\
\hline 19 & 5.72 & 0.24 & 25.95 & 1.26 & 8.65 & 0.47 & 15.27 & 0.73 & 19.97 & 1.02 & 25.51 & 1.22 & 29.03 & 1.10 \\
\hline 22 & 6.02 & 0.25 & 27.39 & 1.33 & 9.01 & 0.49 & 15.84 & 0.76 & 20.95 & 1.07 & 26.73 & 1.28 & 30.39 & 1.15 \\
\hline 26 & 6.27 & 0.26 & 28.60 & 1.39 & 9.25 & 0.51 & 16.25 & 0.78 & 21.50 & 1.10 & 27.44 & 1.31 & 31.42 & 1.19 \\
\hline 29 & 6.45 & 0.27 & 29.44 & 1.43 & 9.43 & 0.51 & 16.57 & 0.80 & 21.87 & 1.12 & 28.02 & 1.34 & 32.18 & 1.22 \\
\hline 34 & 6.99 & 0.29 & 31.44 & 1.52 & 10.18 & 0.56 & 17.67 & 0.85 & 23.10 & 1.18 & 29.45 & 1.41 & 33.60 & 1.28 \\
\hline 37 & 7.20 & 0.30 & 32.15 & 1.56 & 10.50 & 0.57 & 18.11 & 0.87 & 23.47 & 1.20 & 30.00 & 1.48 & 34.24 & 1.30 \\
\hline 40 & 7.43 & 0.31 & 32.93 & 1.60 & 10.83 & 0.59 & 18.53 & 0.89 & 23.86 & 1.22 & 30.56 & 1.46 & 34.95 & 1.33 \\
\hline 43 & 7.67 & 0.29 & 33.70 & 1.63 & 11.13 & 0.61 & 18.91 & 0.78 & 24.23 & 1.24 & 31.06 & 1.48 & 35.42 & 1.45 \\
\hline 49 & 7.68 & 0.22 & 33.91 & 1.56 & 11.24 & 0.52 & 19.01 & 0.88 & 24.28 & 1.35 & 31.13 & 1.39 & 35.64 & 1.25 \\
\hline 56 & 7.68 & 0.32 & 33.91 & 1.70 & 11.40 & 0.65 & 19.18 & 0.92 & 24.42 & 1.25 & 31.39 & 1.50 & 35.84 & 1.36 \\
\hline
\end{tabular}




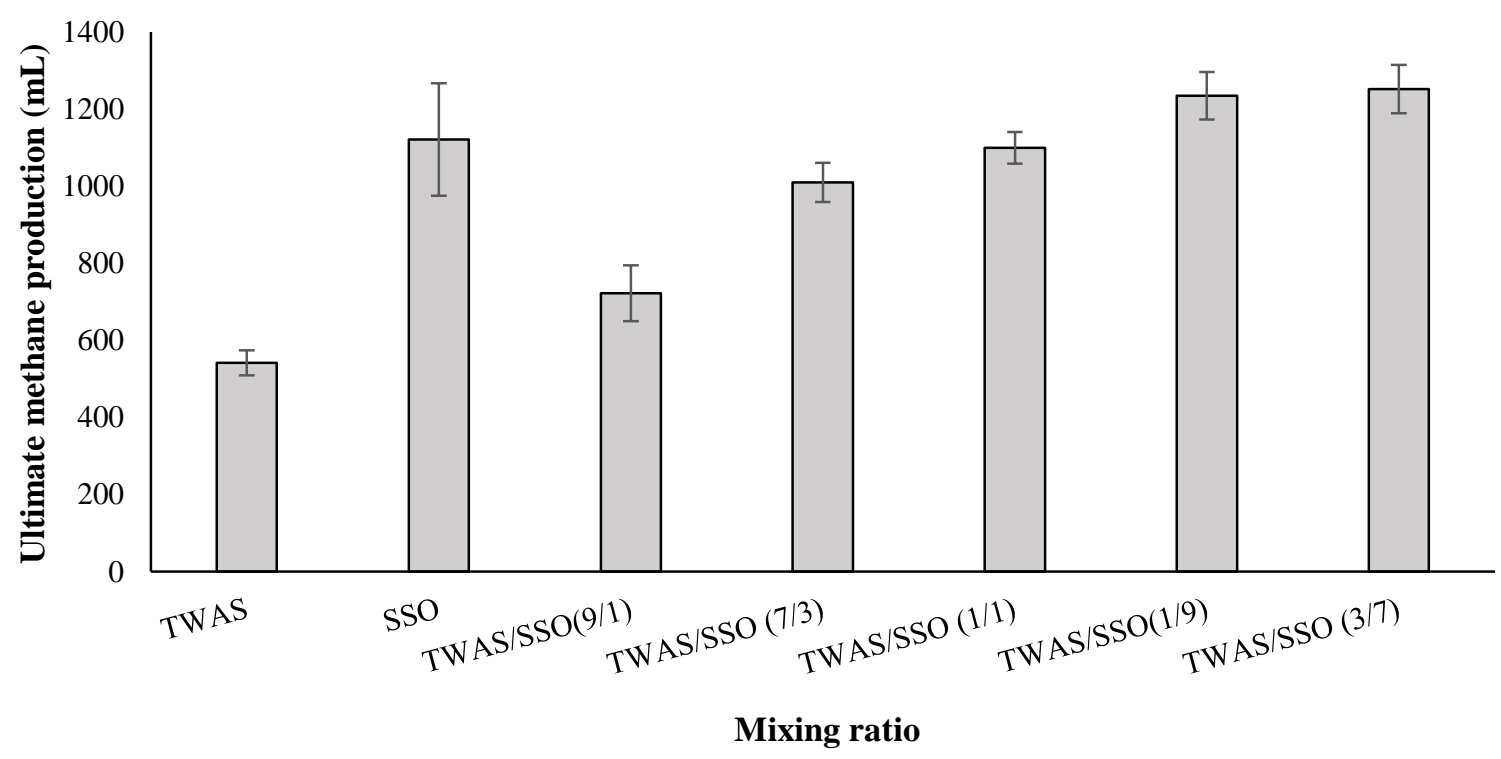

Figure A.1. Ultimate $\mathrm{CH}_{4}$ production in $\mathrm{AnCoD}$ of TWAS and SSO 
Table A. 7. Characteristics of raw feedstocks for hydrolysis/acidification in AnCoD of TWAS/SSO

\begin{tabular}{cccccccc}
\hline \multirow{2}{*}{ Parameters } & \multirow{2}{*}{ Units } & \multicolumn{2}{c}{ SSO } & \multicolumn{2}{c}{ TWAS } & \multicolumn{2}{c}{ Seed } \\
& & MEAN & SD & MEAN & SD & MEAN & SD \\
\hline TCOD & $\mathrm{mg} / \mathrm{L}$ & 110000 & 7795 & 40000 & 2853 & 16400 & 444 \\
SCOD & $\mathrm{mg} / \mathrm{L}$ & 44400 & 100 & 2360 & 26 & 960 & 19 \\
TSS & $\mathrm{mg} / \mathrm{L}$ & 53833 & 6252 & 31450 & 1006 & 17033 & 400 \\
VSS & $\mathrm{mg} / \mathrm{L}$ & 36030 & 3649 & 18460 & 2216 & 10900 & 200 \\
TS & $\mathrm{mg} / \mathrm{L}$ & 62187 & 1025 & 38810 & 6859 & 21450 & 582 \\
VS & $\mathrm{mg} / \mathrm{L}$ & 43493 & 761 & 34477 & 27 & 13140 & 328 \\
Ammonia & $\mathrm{mg} / \mathrm{L}$ & 1138 & 6 & 255 & 30 & 1495 & 40 \\
$\mathrm{pH}$ & - & 5.6 & 0.006 & 6.3 & 0.03 & 7.2 & 0.1 \\
Alkalinity & $\mathrm{mg} \mathrm{CaCO3/L}$ & 6800 & 522 & 1953 & 148 & 3943 & 465 \\
TN & $\mathrm{mg} / \mathrm{L}$ & 3967 & 751 & 2900 & 400 & 2025 & 203 \\
TSN & $\mathrm{mg} / \mathrm{L}$ & 1055 & 38 & 420 & 64 & 696 & 112 \\
Total Carbs & $\mathrm{mg} / \mathrm{L}$ & 13495 & 3580 & 923 & 112 & 545 & 82 \\
Total Proteins & $\mathrm{mg} / \mathrm{L}$ & 2087 & 198 & 2769 & 298 & 1635 & 56 \\
Total Lipids & $\mathrm{mg} / \mathrm{L}$ & 1103 & 2420 & 289 & 27 & 164 & 52 \\
\hline
\end{tabular}


Table A. 8. Soluble and particulate COD concentrations (mg/L) over time

\begin{tabular}{|c|c|c|c|c|c|c|c|}
\hline \multicolumn{8}{|c|}{ Soluble COD concentrations $(\mathrm{mg} / \mathrm{L}$ ) over time } \\
\hline Time & $\mathrm{T} / \mathrm{SSO} 7 / 3$ & T/SSO $1 / 1$ & T/SSO 3/7 & T/SSO $1 / 9$ & $\mathrm{~T} / \mathrm{SSO}$ 9/1 & SSO Only & TWAS Only \\
\hline 0 & 4590 & 5527 & 6525 & 7318 & 2281 & 7630 & 1905 \\
\hline 2 & 4698 & 6087 & 6959 & 7857 & 2529 & 8123 & 2254 \\
\hline 4 & 5036 & 6527 & 7233 & 8216 & 2727 & 8416 & 2377 \\
\hline 6 & 5284 & 6767 & 7557 & 8655 & 3345 & 8689 & 2524 \\
\hline 8 & 5802 & 7017 & 8011 & 8994 & 3503 & 9082 & 2548 \\
\hline 10 & 5990 & 7487 & 8275 & 9353 & 3961 & 9355 & 2597 \\
\hline 12 & 6198 & 7787 & 8599 & 9712 & 4209 & 9858 & 2769 \\
\hline 24 & 6616 & 8047 & 8933 & 10221 & 4397 & 10261 & 2988 \\
\hline 48 & 7534 & 8427 & 9337 & 10540 & 4715 & 11094 & 3087 \\
\hline 72 & 7732 & 9067 & 9981 & 11079 & 5435 & 10817 & 3120 \\
\hline \multicolumn{8}{|c|}{ Particulate COD concentrations $(\mathrm{mg} / \mathrm{L})$ over time } \\
\hline Time & $\mathrm{T} / \mathrm{SSO} 7 / 3$ & T/SSO 1/1 & T/SSO 3/7 & T/SSO $1 / 9$ & T/SSO 9/1 & SSO Only & TWAS Only \\
\hline 0 & 24029 & 24473 & 24182 & 24237 & 23558 & 13979 & 23635 \\
\hline 2 & 23817 & 24256 & 23905 & 24105 & 23416 & 13717 & 22604 \\
\hline 4 & 23681 & 23968 & 23495 & 23987 & 23203 & 13527 & 22518 \\
\hline 6 & 23432 & 22832 & 22843 & 23602 & 22825 & 13256 & 22418 \\
\hline 8 & 22948 & 22683 & 22747 & 23360 & 22635 & 12967 & 22325 \\
\hline 10 & 22629 & 22410 & 22684 & 23020 & 22438 & 12392 & 22223 \\
\hline 12 & 22379 & 22112 & 21558 & 22844 & 22289 & 12228 & 22125 \\
\hline 24 & 21256 & 21110 & 21222 & 21163 & 20870 & 11287 & 21897 \\
\hline 48 & 20684 & 20371 & 21021 & 20815 & 20376 & 10951 & 21581 \\
\hline 72 & 20484 & 20233 & 20574 & 20471 & 20154 & 10623 & 21508 \\
\hline
\end{tabular}




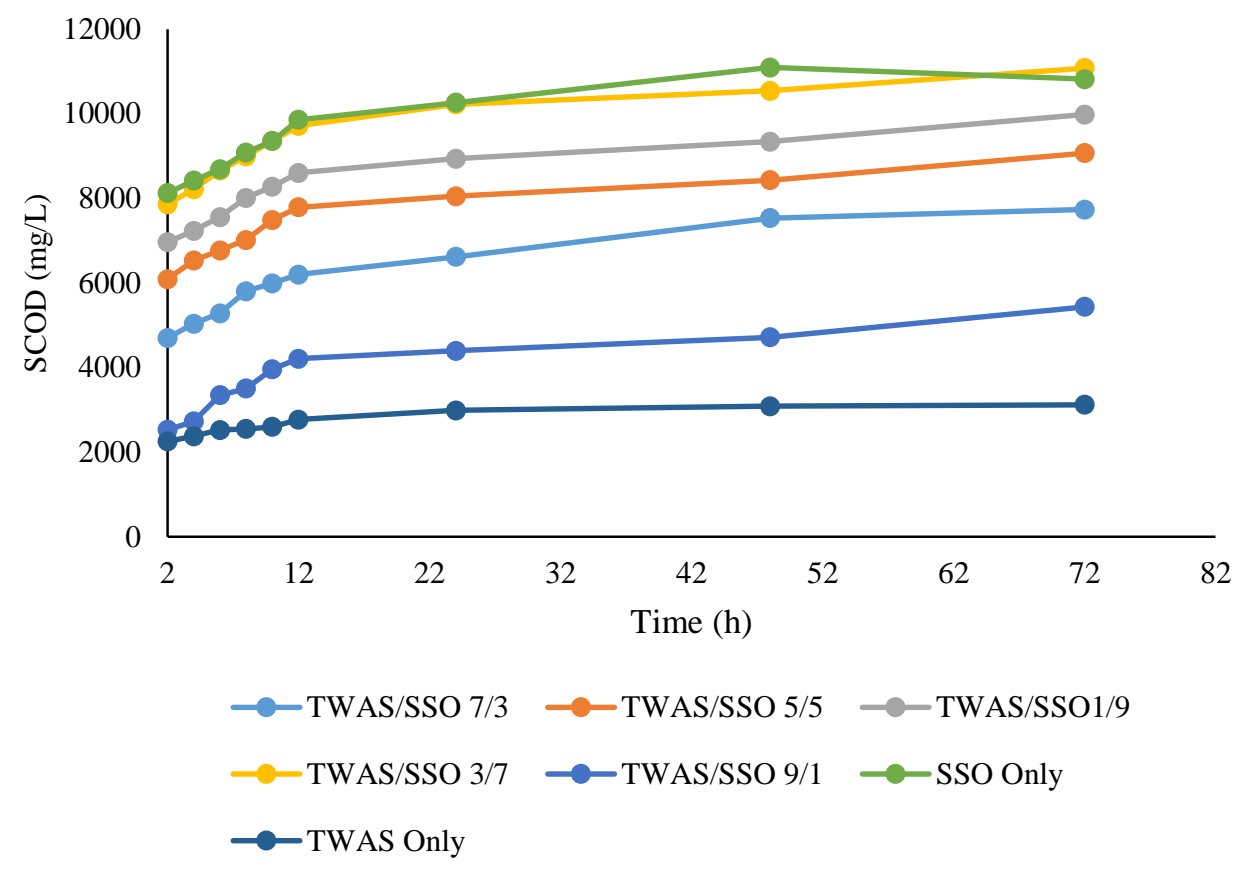

Figure A.2. Concentration of soluble COD over time for different mixing ratios 


\section{B. Analytical results for AnCoD of TWAS and manure}

Table B.1 Characteristics of raw feedstocks in AnCoD of TWAS and manure

\begin{tabular}{cccccccc}
\hline \multirow{2}{*}{ Parameters } & \multirow{2}{*}{ Units } & \multicolumn{2}{c}{ Manure } & \multicolumn{2}{c}{ TWAS } & \multicolumn{2}{c}{ Inoculum } \\
& & MEAN & SD & MEAN & SD & MEAN & SD \\
\hline TCOD & $\mathrm{mg} / \mathrm{L}$ & 122833 & 19553 & 45600 & 500 & 17167 & 306 \\
SCOD & $\mathrm{mg} / \mathrm{L}$ & 8933 & 503 & 2020 & 265 & 1250 & 173 \\
TSS & $\mathrm{mg} / \mathrm{L}$ & 79920 & 19060 & 36150 & 538 & 15580 & 459 \\
VSS & $\mathrm{mg} / \mathrm{L}$ & 76998 & 1789 & 26710 & 349 & 10313 & 284 \\
TS & $\mathrm{mg} / \mathrm{L}$ & 98727 & 1035 & 38053 & 1832 & 16590 & 302 \\
VS & $\mathrm{mg} / \mathrm{L}$ & 85647 & 1123 & 27730 & 1092 & 10180 & 286 \\
Ammonia & $\mathrm{mg} / \mathrm{L}$ & 22 & 3 & 317 & 20 & 497 & 68 \\
$\mathrm{pH}$ & - & 6.4 & 0.1 & 6.8 & 0.1 & 7 & 0.1 \\
Alkalinity & $\mathrm{mg} \mathrm{CaCO3/L}$ & 5133 & 902 & 4820 & 452 & 5517 & 306 \\
Total N & $\mathrm{mg} / \mathrm{L}$ & 2200 & 100 & 2883 & 278 & 2050 & 278 \\
Total Soluble N & $\mathrm{mg} / \mathrm{L}$ & 104 & 15 & 410 & 22 & 747 & 15 \\
Total Carbs & $\mathrm{mg} / \mathrm{L}$ & 27100 & 958 & 1322 & 104 & 508 & 23 \\
Total Proteins & $\mathrm{mg} / \mathrm{L}$ & 5115 & 244 & 3958 & 276 & 1498 & 50 \\
Total Lipids & $\mathrm{mg} / \mathrm{L}$ & 1355 & 54 & 536 & 44 & 205 & 8 \\
\hline
\end{tabular}


Table B. 2. Cumulative $\mathrm{CH}_{4}$ production in AnCoD of TWAS and manure

\begin{tabular}{|c|c|c|c|c|c|c|c|c|c|c|c|c|c|c|}
\hline \multicolumn{15}{|c|}{ Cumulative methane production $(\mathrm{mL})$} \\
\hline $\begin{array}{l}\text { time } \\
\text { (day) }\end{array}$ & TWAS & SD & Manure & SD & $\begin{array}{c}\mathrm{T} / \mathrm{M} \\
9 / 1\end{array}$ & SD & $\begin{array}{c}\mathrm{T} / \mathrm{M} \\
7 / 3\end{array}$ & SD & $\begin{array}{c}\mathrm{T} / \mathrm{M} \\
1 / 1\end{array}$ & SD & $\begin{array}{c}\mathrm{T} / \mathrm{M} \\
3 / 7\end{array}$ & SD & $\begin{array}{c}\mathrm{T} / \mathrm{M} \\
1 / 9\end{array}$ & SD \\
\hline 0 & 0 & 0 & 0 & 0 & 0 & 0 & 0 & 0 & 0 & 0 & 0 & 0 & 0 & 0 \\
\hline 1 & 24 & 1 & 86 & 5 & 76 & 3 & 88 & 5 & 100 & 5 & 95 & 5 & 88 & 4.39 \\
\hline 2 & 84 & 4 & 157 & 9 & 172 & 7 & 175 & 9 & 197 & 7 & 188 & 9 & 172 & 8.62 \\
\hline 3 & 135 & 7 & 215 & 12 & 246 & 11 & 237 & 12 & 266 & 12 & 258 & 13 & 238 & 11.9 \\
\hline 4 & 185 & 9 & 273 & 15 & 300 & 13 & 294 & 15 & 331 & 15 & 322 & 16 & 297 & 14.8 \\
\hline 5 & 220 & 11 & 316 & 17 & 341 & 15 & 336 & 17 & 378 & 17 & 373 & 18 & 344 & 17.2 \\
\hline 6 & 256 & 12 & 364 & 20 & 386 & 17 & 382 & 20 & 430 & 20 & 427 & 21 & 396 & 19.8 \\
\hline 8 & 298 & 14 & 434 & 24 & 426 & 19 & 441 & 23 & 496 & 23 & 494 & 24 & 463 & 23.1 \\
\hline 10 & 328 & 16 & 490 & 27 & 462 & 20 & 506 & 26 & 570 & 26 & 562 & 28 & 523 & 26.1 \\
\hline 14 & 363 & 18 & 552 & 30 & 498 & 22 & 562 & 29 & 633 & 29 & 635 & 31 & 587 & 29.4 \\
\hline 16 & 388 & 19 & 597 & 33 & 528 & 23 & 600 & 31 & 675 & 31 & 681 & 33 & 631 & 31.6 \\
\hline 17 & 400 & 19 & 620 & 34 & 547 & 24 & 629 & 33 & 708 & 33 & 710 & 35 & 655 & 32.7 \\
\hline 19 & 417 & 20 & 648 & 36 & 570 & 25 & 650 & 34 & 732 & 34 & 740 & 36 & 680 & 34 \\
\hline 22 & 434 & 21 & 678 & 37 & 597 & 26 & 694 & 36 & 781 & 36 & 780 & 38 & 713 & 35.6 \\
\hline 25 & 456 & 22 & 713 & 39 & 625 & 27 & 710 & 37 & 799 & 37 & 815 & 40 & 748 & 37.4 \\
\hline 26 & 464 & 22 & 728 & 40 & 636 & 28 & 727 & 38 & 818 & 38 & 835 & 41 & 765 & 38.2 \\
\hline 29 & 478 & 23 & 752 & 41 & 655 & 28 & 749 & 39 & 843 & 39 & 863 & 42 & 790 & 39.5 \\
\hline 30 & 482 & 23 & 757 & 42 & 659 & 29 & 754 & 39 & 848 & 39 & 870 & 43 & 800 & 39 \\
\hline 33 & 492 & 24 & 775 & 43 & 671 & 29 & 769 & 40 & 865 & 40 & 889 & 44 & 817 & 40.8 \\
\hline 36 & 506 & 24 & 799 & 44 & 690 & 30 & 788 & 41 & 886 & 41 & 914 & 45 & 841 & 42.1 \\
\hline 40 & 521 & 25 & 824 & 45 & 712 & 31 & 811 & 42 & 912 & 42 & 944 & 46 & 871 & 43.5 \\
\hline 46 & 539 & 26 & 851 & 47 & 737 & 32 & 836 & 43 & 941 & 43 & 978 & 48 & 905 & 45.2 \\
\hline 50 & 548 & 27 & 864 & 48 & 750 & 33 & 850 & 44 & 956 & 44 & 995 & 49 & 922 & 46.1 \\
\hline 54 & 555 & 27 & 874 & 48 & 759 & 33 & 859 & 45 & 967 & 42 & 1007 & 49 & 935 & 46.7 \\
\hline 58 & 559 & 27 & 881 & 48 & 766 & 33 & 866 & 42 & 975 & 45 & 1017 & 48 & 944 & 47.2 \\
\hline 62 & 563 & 27 & 887 & 49 & 771 & 34 & 872 & 44 & 981 & 41 & 1023 & 51 & 951 & 45 \\
\hline 65 & 566 & 27 & 893 & 49 & 776 & 34 & 877 & 46 & 987 & 43 & 1029 & 50 & 956 & 47.8 \\
\hline 67 & 569 & 28 & 898 & 49 & 780 & 34 & 885 & 45 & 996 & 44 & 1035 & 52 & 961 & 42 \\
\hline 72 & 578 & 28 & 907 & 50 & 791 & 34 & 893 & 46 & 1005 & 42 & 1049 & 48 & 979 & 48.9 \\
\hline 74 & 584 & 28 & 915 & 50 & 801 & 33 & 903 & 47 & 1016 & 45 & 1059 & 49 & 992 & 43 \\
\hline 78 & 590 & 29 & 922 & 51 & 811 & 35 & 902 & 43 & 1015 & 47 & 1069 & 51 & 1002 & 50.1 \\
\hline
\end{tabular}


Table B. 3. Cumulative methane yield per unit mass of COD added in AnCoD of TWAS and manure

\begin{tabular}{|c|c|c|c|c|c|c|c|c|c|c|c|c|c|c|}
\hline \multicolumn{15}{|c|}{ Cumulative methane yield ( $\mathrm{mL} \mathrm{CH}_{4} / \mathrm{g}$ COD added) } \\
\hline $\begin{array}{l}\text { time } \\
\text { (day) }\end{array}$ & TWAS & SD & Manure & SD & $\begin{array}{c}\mathrm{T} / \mathrm{M} \\
9 / 1\end{array}$ & $\mathrm{SD}$ & $\begin{array}{c}\mathrm{T} / \mathrm{M} \\
7 / 3\end{array}$ & SD & $\begin{array}{c}\mathrm{T} / \mathrm{M} \\
1 / 1\end{array}$ & SD & $\begin{array}{c}\mathrm{T} / \mathrm{M} \\
3 / 7\end{array}$ & SD & $\begin{array}{c}\text { T/M } \\
1 / 9\end{array}$ & SD \\
\hline 0 & 0 & 0 & 0 & 0 & 0 & 0 & 0 & 0 & 0 & 0 & 0 & 0 & 0 & 0 \\
\hline 1 & 8 & 0.3 & 30 & 1.6 & 26 & 1.2 & 31 & 1.4 & 35 & 1.3 & 34 & 1.8 & 34 & 1.2 \\
\hline 2 & 28 & 1.2 & 56 & 2.9 & 58 & 2.7 & 61 & 2.9 & 69 & 2.6 & 66 & 3.7 & 67 & 2.5 \\
\hline 3 & 45 & 1.9 & 77 & 4.0 & 84 & 3.8 & 82 & 3.9 & 93 & 3.5 & 91 & 5.0 & 91 & 3.4 \\
\hline 4 & 62 & 2.7 & 97 & 5.0 & 102 & 4.7 & 102 & 4.8 & 116 & 4.4 & 114 & 6.3 & 114 & 4.2 \\
\hline 5 & 73 & 3.2 & 112 & 5.8 & 116 & 5.3 & 116 & 5.5 & 133 & 5.0 & 132 & 7.2 & 132 & 4.9 \\
\hline 6 & 85 & 3.7 & 130 & 6.7 & 131 & 6.0 & 132 & 6.2 & 151 & 5.7 & 151 & 8.3 & 152 & 5.6 \\
\hline 8 & 99 & 4.3 & 154 & 8.0 & 144 & 6.6 & 153 & 7.2 & 174 & 6.6 & 175 & 9.6 & 175 & 6.5 \\
\hline 10 & 109 & 4.7 & 174 & 9.1 & 157 & 7.2 & 175 & 8.2 & 200 & 7.6 & 199 & 10.9 & 200 & 7.4 \\
\hline 14 & 121 & 5.2 & 196 & 10.2 & 169 & 7.8 & 195 & 9.2 & 222 & 8.4 & 224 & 12.3 & 225 & 8.3 \\
\hline 16 & 129 & 5.5 & 212 & 11.0 & 179 & 8.2 & 208 & 9.8 & 237 & 9.0 & 241 & 13.2 & 242 & 8.9 \\
\hline 17 & 133 & 5.7 & 221 & 11.5 & 186 & 8.5 & 218 & 10.2 & 248 & 9.4 & 251 & 13.8 & 252 & 9.3 \\
\hline 19 & 139 & 6.0 & 230 & 12.0 & 193 & 8.9 & 225 & 10.6 & 257 & 9.8 & 262 & 14.4 & 263 & 9.7 \\
\hline 22 & 145 & 6.2 & 241 & 12.6 & 202 & 9.3 & 241 & 11.3 & 274 & 10.4 & 275 & 15.2 & 277 & 10.2 \\
\hline 25 & 152 & 6.5 & 254 & 13.2 & 212 & 9.7 & 246 & 11.6 & 280 & 10.6 & 288 & 15.8 & 289 & 10.7 \\
\hline 26 & 154 & 6.6 & 259 & 13.5 & 216 & 9.9 & 252 & 11.8 & 287 & 10.9 & 295 & 16.2 & 296 & 11.0 \\
\hline 29 & 159 & 6.8 & 267 & 13.9 & 222 & 10.2 & 260 & 12.2 & 296 & 11.2 & 305 & 16.8 & 306 & 11.3 \\
\hline 30 & 160 & 6.9 & 269 & 14.0 & 224 & 10.3 & 261 & 12.3 & 298 & 10.2 & 307 & 16.9 & 309 & 11.4 \\
\hline 33 & 164 & 7.0 & 276 & 14.3 & 227 & 10.5 & 267 & 12.5 & 303 & 11.5 & 314 & 17.3 & 316 & 11.7 \\
\hline 36 & 168 & 7.2 & 284 & 14.8 & 234 & 10.8 & 273 & 12.8 & 311 & 11.8 & 323 & 17.8 & 325 & 13.3 \\
\hline 40 & 173 & 7.5 & 293 & 15.2 & 242 & 11.1 & 281 & 13.2 & 320 & 12.2 & 334 & 18.3 & 335 & 12.4 \\
\hline 46 & 179 & 7.7 & 303 & 15.7 & 250 & 11.5 & 290 & 13.6 & 330 & 12.5 & 345 & 17.6 & 347 & 12.8 \\
\hline 50 & 182 & 7.8 & 307 & 16.0 & 254 & 11.7 & 294 & 13.8 & 335 & 10.7 & 351 & 19.3 & 353 & 14.1 \\
\hline 54 & 184 & 7.9 & 311 & 16.2 & 257 & 11.8 & 298 & 14.0 & 339 & 12.9 & 356 & 17.5 & 358 & 13.2 \\
\hline 58 & 186 & 8.0 & 314 & 16.3 & 260 & 11.9 & 300 & 13.5 & 342 & 13.5 & 359 & 19.8 & 361 & 14.2 \\
\hline 62 & 187 & 7.5 & 316 & 15.7 & 261 & 12.0 & 302 & 14.2 & 344 & 12.9 & 362 & 19.3 & 363 & 13.4 \\
\hline 65 & 188 & 8.1 & 318 & 16.5 & 263 & 11.8 & 304 & 14.3 & 346 & 13.2 & 364 & 20.0 & 365 & 12.8 \\
\hline 67 & 189 & 7.9 & 320 & 15.5 & 265 & 12.2 & 307 & 13.8 & 349 & 14.1 & 366 & 19.2 & 368 & 14.5 \\
\hline 72 & 192 & 8.3 & 323 & 16.8 & 268 & 10.9 & 310 & 14.6 & 353 & 12.8 & 371 & 20.4 & 372 & 11.7 \\
\hline 74 & 194 & 7.6 & 326 & 15.3 & 272 & 11.5 & 313 & 13.5 & 356 & 13.5 & 374 & 18.9 & 376 & 13.9 \\
\hline 78 & 196 & 8.4 & 328 & 17.1 & 275 & 12.6 & 313 & 14.7 & 356 & 11.6 & 378 & 20.8 & 379 & 14.0 \\
\hline
\end{tabular}


Table B. 4. Cumulative methane yield per unit mass of VSS added in AnCoD of TWAS and manure

\begin{tabular}{|c|c|c|c|c|c|c|c|c|c|c|c|c|c|c|}
\hline \multicolumn{15}{|c|}{ Cumulative methane yield (mL CH4/g VSS added) } \\
\hline $\begin{array}{l}\text { time } \\
\text { (day) }\end{array}$ & TWAS & SD & Manure & $\mathrm{SD}$ & $\begin{array}{c}\mathrm{T} / \mathrm{M} \\
9 / 1\end{array}$ & $\mathrm{SD}$ & $\begin{array}{c}\mathrm{T} / \mathrm{M} \\
7 / 3\end{array}$ & SD & $\begin{array}{c}\mathrm{T} / \mathrm{M} \\
1 / 1\end{array}$ & SD & $\begin{array}{c}\mathrm{T} / \mathrm{M} \\
3 / 7\end{array}$ & $\mathrm{SD}$ & $\begin{array}{c}\mathrm{T} / \mathrm{M} \\
1 / 9\end{array}$ & SD \\
\hline 0 & 0 & 0 & 0 & 0 & 0 & 0 & 0 & 0 & 0 & 0 & 0 & 0 & 0 & 0 \\
\hline 1 & 16 & 0.7 & 127 & 5.8 & 62 & 2.4 & 92 & 4.2 & 119 & 5.1 & 136 & 4.8 & 117 & 4.208 \\
\hline 2 & 58 & 2.6 & 233 & 10.7 & 142 & 5.5 & 182 & 8.4 & 237 & 10.2 & 270 & 9.4 & 229 & 8.258 \\
\hline 3 & 94 & 4.2 & 320 & 14.7 & 203 & 7.9 & 245 & 11.3 & 319 & 13.7 & 370 & 13.0 & 316 & 11.38 \\
\hline 4 & 128 & 5.8 & 404 & 18.6 & 247 & 9.6 & 304 & 14.0 & 397 & 17.1 & 463 & 16.2 & 394 & 14.2 \\
\hline 5 & 153 & 6.9 & 468 & 21.5 & 281 & 11.0 & 348 & 16.0 & 453 & 19.5 & 536 & 18.7 & 458 & 16.49 \\
\hline 6 & 178 & 8.0 & 540 & 24.9 & 318 & 12.4 & 396 & 18.2 & 516 & 22.2 & 613 & 21.5 & 527 & 18.98 \\
\hline 8 & 206 & 9.3 & 644 & 29.6 & 351 & 13.7 & 456 & 21.0 & 595 & 25.6 & 710 & 24.8 & 615 & 22.15 \\
\hline 10 & 227 & 10.2 & 727 & 33.4 & 380 & 14.8 & 524 & 24.1 & 683 & 29.4 & 807 & 28.3 & 695 & 25.03 \\
\hline 14 & 251 & 11.3 & 819 & 37.7 & 410 & 15.6 & 582 & 26.8 & 759 & 32.6 & 912 & 31.9 & 781 & 28.11 \\
\hline 16 & 268 & 12.1 & 885 & 40.7 & 435 & 16.9 & 621 & 28.6 & 810 & 34.8 & 979 & 34.3 & 839 & 30.22 \\
\hline 17 & 277 & 12.5 & 919 & 42.3 & 451 & 17.6 & 651 & 29.9 & 849 & 36.5 & 1020 & 35.7 & 871 & 31.35 \\
\hline 19 & 289 & 13.0 & 961 & 44.2 & 469 & 18.3 & 673 & 31.0 & 878 & 37.7 & 1064 & 37.2 & 905 & 32.58 \\
\hline 22 & 301 & 13.5 & 1006 & 45.3 & 492 & 19.2 & 719 & 33.1 & 937 & 40.3 & 1120 & 39.2 & 948 & 34.13 \\
\hline 25 & 316 & 14.2 & 1058 & 48.7 & 514 & 20.1 & 735 & 33.8 & 958 & 39.1 & 1170 & 41.0 & 995 & 35.81 \\
\hline 26 & 321 & 14.5 & 1079 & 46.7 & 524 & 18.7 & 753 & 34.6 & 981 & 42.2 & 1199 & 42.0 & 1017 & 36.63 \\
\hline 29 & 331 & 14.9 & 1115 & 51.3 & 539 & 21.0 & 776 & 35.7 & 1011 & 43.5 & 1240 & 43.4 & 1051 & 39.2 \\
\hline 30 & 334 & 13.8 & 1123 & 49.5 & 543 & 22.4 & 781 & 34.2 & 1017 & 42.8 & 1250 & 43.8 & 1064 & 38.3 \\
\hline 33 & 341 & 15.4 & 1149 & 52.9 & 552 & 21.5 & 796 & 36.6 & 1038 & 44.6 & 1277 & 44.7 & 1086 & 39.1 \\
\hline 36 & 351 & 15.8 & 1186 & 54.5 & 568 & 20.3 & 815 & 37.5 & 1063 & 45.7 & 1313 & 46.0 & 1119 & 38.6 \\
\hline 40 & 361 & 16.3 & 1222 & 50.6 & 586 & 22.9 & 840 & 38.6 & 1094 & 47.1 & 1356 & 44.8 & 1158 & 41.7 \\
\hline 46 & 373 & 14.5 & 1261 & 58.0 & 607 & 23.7 & 866 & 39.8 & 1129 & 48.5 & 1404 & 49.2 & 1204 & 43.33 \\
\hline 50 & 380 & 17.1 & 1281 & 54.5 & 617 & 24.1 & 880 & 40.5 & 1147 & 45.2 & 1429 & 50.0 & 1226 & 44.15 \\
\hline 54 & 384 & 16.9 & 1296 & 59.6 & 625 & 23.8 & 890 & 39.3 & 1160 & 49.9 & 1447 & 48.7 & 1243 & 44.76 \\
\hline 58 & 387 & 15.2 & 1307 & 58.3 & 631 & 24.6 & 897 & 41.3 & 1169 & 50.3 & 1461 & 51.1 & 1256 & 46.3 \\
\hline 62 & 390 & 17.6 & 1316 & 60.5 & 635 & 25.2 & 903 & 41.5 & 1177 & 48.6 & 1470 & 52.6 & 1265 & 45.52 \\
\hline 65 & 392 & 16.8 & 1324 & 56.4 & 639 & 24.9 & 908 & 39.7 & 1184 & 50.9 & 1478 & 49.8 & 1272 & 45.78 \\
\hline 67 & 394 & 17.7 & 1332 & 61.3 & 642 & 23.5 & 917 & 40.9 & 1195 & 49.7 & 1487 & 51.3 & 1278 & 46 \\
\hline 72 & 400 & 16.7 & 1345 & 59.2 & 651 & 25.4 & 925 & 42.6 & 1206 & 51.8 & 1507 & 52.7 & 1302 & 45.2 \\
\hline 74 & 405 & 17.5 & 1357 & 58.1 & 660 & 24.8 & 935 & 43.0 & 1218 & 47.4 & 1522 & 50.2 & 1319 & 47.48 \\
\hline 78 & 408 & 18.4 & 1368 & 60.8 & 667 & 26.7 & 934 & 41.5 & 1217 & 50.2 & 1535 & 53.7 & 1333 & 47.98 \\
\hline
\end{tabular}


Table B. 5. Cumulative methane yield per unit volume of substrate added in AnCoD of TWAS and manure

\begin{tabular}{|c|c|c|c|c|c|c|c|c|c|c|c|c|c|c|}
\hline \multicolumn{15}{|c|}{ Cumulative methane yield ( $\mathrm{mL} \mathrm{CH}_{4} / \mathrm{mL}$ substrate added) } \\
\hline $\begin{array}{l}\text { time } \\
\text { (day) }\end{array}$ & TWAS & & Manure & & $\begin{array}{c}\mathrm{T} / \mathrm{M} \\
9 / 1\end{array}$ & & $\begin{array}{c}\mathrm{T} / \mathrm{M} \\
7 / 3\end{array}$ & & $\begin{array}{c}\mathrm{T} / \mathrm{M} \\
1 / 1\end{array}$ & & $\begin{array}{c}\mathrm{T} / \mathrm{M} \\
3 / 7\end{array}$ & & $\begin{array}{c}\mathrm{T} / \mathrm{M} \\
1 / 9\end{array}$ & \\
\hline 0 & 0 & 0 & 0 & 0 & 0 & 0 & 0 & 0.00 & 0 & 0.00 & 0 & 0.00 & 0 & 0.00 \\
\hline 1 & 0.3 & 0.01 & 2.2 & 0.10 & 0.7 & 0.03 & 1.1 & 0.04 & 1.4 & 0.08 & 1.8 & 0.10 & 2.0 & 0.10 \\
\hline 2 & 1.0 & 0.04 & 4.0 & 0.18 & 1.6 & 0.10 & 2.2 & 0.08 & 2.9 & 0.16 & 3.5 & 0.19 & 4.0 & 0.19 \\
\hline 3 & 1.6 & 0.06 & 5.5 & 0.24 & 2.2 & 0.09 & 3.0 & 0.11 & 3.9 & 0.21 & 4.8 & 0.26 & 5.5 & 0.26 \\
\hline 4 & 2.2 & 0.08 & 7.0 & 0.31 & 2.7 & 0.11 & 3.7 & 0.14 & 4.8 & 0.26 & 6.0 & 0.33 & 6.9 & 0.32 \\
\hline 5 & 2.6 & 0.09 & 8.1 & 0.36 & 3.1 & 0.12 & 4.2 & 0.16 & 5.5 & 0.30 & 6.9 & 0.38 & 8.0 & 0.38 \\
\hline 6 & 3.1 & 0.11 & 9.3 & 0.41 & 3.5 & 0.14 & 4.8 & 0.18 & 6.2 & 0.34 & 7.9 & 0.43 & 9.2 & 0.43 \\
\hline 8 & 3.5 & 0.13 & 11.1 & 0.49 & 3.9 & 0.13 & 5.5 & 0.21 & 7.2 & 0.40 & 9.1 & 0.50 & 10.8 & 0.51 \\
\hline 10 & 3.9 & 0.14 & 12.6 & 0.55 & 4.2 & 0.16 & 6.3 & 0.24 & 8.3 & 0.45 & 10.4 & 0.57 & 12.2 & 0.57 \\
\hline 14 & 4.3 & 0.16 & 14.2 & 0.62 & 4.5 & 0.18 & 7.0 & 0.27 & 9.2 & 0.50 & 11.8 & 0.65 & 13.7 & 0.64 \\
\hline 16 & 4.6 & 0.17 & 15.3 & 0.67 & 4.8 & 0.19 & 7.5 & 0.29 & 9.8 & 0.54 & 12.6 & 0.69 & 14.7 & 0.69 \\
\hline 17 & 4.8 & 0.15 & 15.9 & 0.70 & 5.0 & 0.15 & 7.9 & 0.30 & 10.3 & 0.56 & 13.1 & 0.72 & 15.2 & 0.72 \\
\hline 19 & 5.0 & 0.18 & 16.6 & 0.73 & 5.2 & 0.20 & 8.1 & 0.31 & 10.6 & 0.58 & 13.7 & 0.75 & 15.8 & 0.74 \\
\hline 22 & 5.2 & 0.19 & 17.4 & 0.77 & 5.4 & 0.21 & 8.7 & 0.33 & 11.3 & 0.62 & 14.4 & 0.79 & 16.6 & 0.78 \\
\hline 25 & 5.4 & 0.20 & 18.3 & 0.80 & 5.7 & 0.22 & 8.9 & 0.34 & 11.6 & 0.64 & 15.1 & 0.83 & 17.4 & 0.82 \\
\hline 26 & 5.5 & 0.19 & 18.7 & 0.82 & 5.8 & 0.23 & 9.1 & 0.35 & 11.9 & 0.65 & 15.5 & 0.85 & 17.8 & 0.84 \\
\hline 29 & 5.7 & 0.20 & 19.3 & 0.85 & 6.0 & 0.23 & 9.4 & 0.36 & 12.2 & 0.67 & 16.0 & 0.88 & 18.4 & 0.86 \\
\hline 30 & 5.7 & 0.21 & 19.4 & 0.85 & 6.0 & 0.27 & 9.4 & 0.36 & 12.3 & 0.68 & 16.1 & 0.89 & 18.6 & 0.87 \\
\hline 33 & 5.9 & 0.21 & 19.9 & 0.87 & 6.1 & 0.24 & 9.6 & 0.41 & 12.5 & 0.69 & 16.5 & 0.91 & 19.0 & 0.89 \\
\hline 36 & 6.0 & 0.20 & 20.5 & 0.90 & 6.3 & 0.20 & 9.8 & 0.37 & 12.8 & 0.71 & 16.9 & 0.93 & 19.6 & 0.92 \\
\hline 40 & 6.2 & 0.22 & 21.1 & 0.93 & 6.5 & 0.25 & 10.1 & 0.39 & 13.2 & 0.73 & 17.5 & 0.96 & 20.3 & 0.95 \\
\hline 46 & 6.4 & 0.23 & 21.8 & 0.96 & 6.7 & 0.26 & 10.5 & 0.40 & 13.6 & 0.75 & 18.1 & 1.00 & 21.0 & 0.99 \\
\hline 50 & 6.5 & 0.21 & 22.2 & 0.97 & 6.8 & 0.29 & 10.6 & 0.36 & 13.9 & 0.76 & 18.4 & 1.01 & 21.4 & 1.01 \\
\hline 54 & 6.6 & 0.24 & 22.4 & 0.99 & 6.9 & 0.27 & 10.7 & 0.39 & 14.0 & 0.77 & 18.7 & 1.03 & 21.7 & 0.99 \\
\hline 58 & 6.7 & 0.33 & 22.6 & 0.88 & 7.0 & 0.25 & 10.8 & 0.41 & 14.1 & 0.78 & 18.8 & 1.04 & 22.0 & 1.03 \\
\hline 62 & 6.7 & 0.24 & 22.7 & 1.22 & 7.0 & 0.27 & 10.9 & 0.45 & 14.2 & 0.78 & 19.0 & 1.04 & 22.1 & 1.04 \\
\hline 65 & 6.7 & 0.31 & 22.9 & 1.01 & 7.1 & 0.22 & 11.0 & 0.42 & 14.3 & 0.79 & 19.1 & 1.05 & 22.2 & 1.05 \\
\hline 67 & 6.8 & 0.24 & 23.0 & 1.35 & 7.1 & 0.24 & 11.1 & 0.36 & 14.4 & 0.79 & 19.2 & 0.98 & 22.3 & 0.97 \\
\hline 72 & 6.9 & 0.25 & 23.3 & 0.98 & 7.2 & 0.28 & 11.2 & 0.42 & 14.6 & 0.80 & 19.4 & 1.07 & 22.8 & 1.07 \\
\hline 74 & 7.0 & 0.29 & 23.5 & 1.23 & 7.3 & 0.25 & 11.3 & 0.39 & 14.7 & 0.81 & 19.6 & 1.08 & 23.1 & 1.08 \\
\hline 78 & 7.0 & 0.25 & 23.6 & 1.04 & 7.4 & 0.29 & 11.3 & 0.43 & 14.7 & 0.81 & 19.8 & 1.09 & 23.3 & 1.10 \\
\hline
\end{tabular}




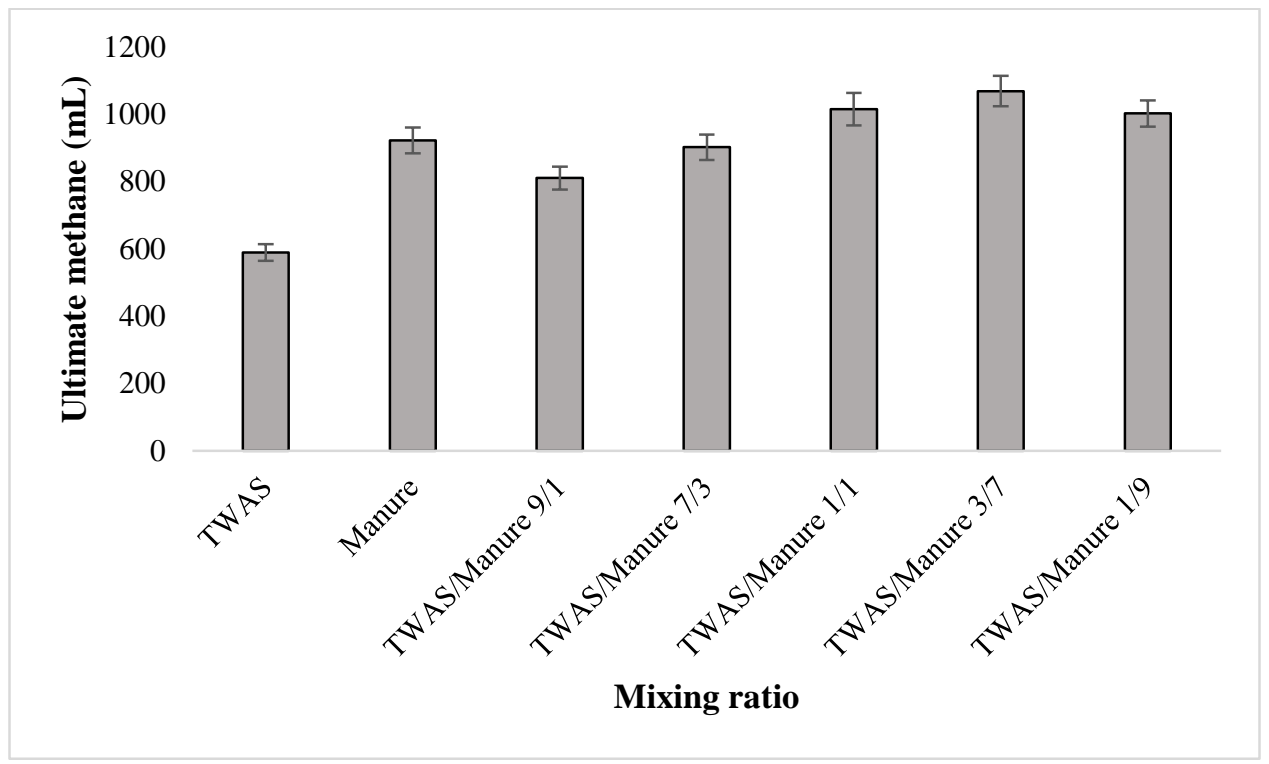

Figure B. 1. Ultimate methane production in AnCoD of TWAS and manure 
Table B. 6. Characteristics of the raw feedstocks for hydrolysis/acidification in AnCoD of TWAS and manure

\begin{tabular}{cccccccc}
\hline Parameters & Units & Manure & & TWAS & \multicolumn{3}{c}{ Seed } \\
\hline & & MEAN & SD & MEAN & SD & MEAN & SD \\
TCOD & $\mathrm{mg} / \mathrm{L}$ & 110433 & 4422 & 41200 & 1236 & 16652 & 552 \\
SCOD & $\mathrm{mg} / \mathrm{L}$ & 6560 & 228 & 2161 & 65 & 1255 & 50 \\
TSS & $\mathrm{mg} / \mathrm{L}$ & 52600 & 2530 & 37254 & 1118 & 15112 & 604 \\
VSS & $\mathrm{mg} / \mathrm{L}$ & 42800 & 2040 & 26521 & 796 & 10067 & 403 \\
TS & $\mathrm{mg} / \mathrm{L}$ & 68040 & 3202 & 39198 & 1176 & 36882 & 1475 \\
VS & $\mathrm{mg} / \mathrm{L}$ & 58520 & 2526 & 28562 & 857 & 26874 & 1075 \\
Ammonia & $\mathrm{mg} / \mathrm{L}$ & 13 & 1 & 327 & 10 & 307 & 12 \\
$\mathrm{pH}$ & - & 6.5 & 0.2 & 6.8 & 0.204 & 6 & 0.2 \\
Alkalinity & $\mathrm{mg} \mathrm{CaCO} / \mathrm{L}$ & 7689 & 384 & 4964 & 149 & 4671 & 187 \\
TN & $\mathrm{mg} / \mathrm{L}$ & 1435 & 72 & 2969 & 89 & 2794 & 112 \\
TSN & $\mathrm{mg} / \mathrm{L}$ & 68 & 3 & 422 & 13 & 397 & 16 \\
Total Carbs & $\mathrm{mg} / \mathrm{L}$ & 27116 & 1355.8 & 1327 & 40 & 503 & 20 \\
Total Proteins & $\mathrm{mg} / \mathrm{L}$ & 5123 & 256.15 & 3978 & 119 & 1510 & 60 \\
Total Lipids & $\mathrm{mg} / \mathrm{L}$ & 1360 & 68 & 530 & 16 & 201 & 8 \\
\hline
\end{tabular}


Table B. 7. Measured and theoretical VFAs concentrations over time

\begin{tabular}{cccccccc}
\hline \multicolumn{7}{c}{ Measured VFAs over time } \\
\hline Time & TWAS/Manure & TWAS/Manure & TWAS/Manure & TWAS/Manure & TWAS/Manure & Manure & TWAS \\
(hr) & $7 / 3$ & $1 / 1$ & $3 / 7$ & $1 / 9$ & $9 / 1$ & Only & Only \\
0 & 584 & 686 & 718 & 790 & 581 & 810 & 432 \\
6 & 596 & 719 & 793 & 831 & 593 & 849 & 458 \\
12 & 786 & 892 & 827 & 958 & 640 & 974 & 504 \\
24 & 912 & 1105 & 971 & 1230 & 681 & 1141 & 514 \\
48 & 942 & 1131 & 1299 & 1378 & 735 & 1366 & 528 \\
72 & 965 & 1175 & 1530 & 1491 & 775 & 1396 & 542 \\
& & & Theoretical VFAs over time & & & Mixture \\
Time & TWAS/Manure & TWAS/Manure & TWAS/Manure & TWAS/Manure & TWAS/Manure & Manure & TWAS \\
(hr) & $7 / 3$ & $1 / 1$ & $3 / 7$ & $1 / 9$ & $9 / 1$ & Only & Only \\
0 & 545 & 621 & 697 & 772 & 470 & 810 & 432 \\
6 & 575 & 654 & 732 & 810 & 497 & 849 & 458 \\
12 & 645 & 739 & 833 & 927 & 551 & 974 & 504 \\
24 & 702 & 827 & 953 & 1078 & 576 & 1141 & 514 \\
48 & 780 & 947 & 1115 & 1283 & 612 & 1366 & 528 \\
72 & 798 & 969 & 1140 & 1311 & 627 & 1396 & 542 \\
\hline
\end{tabular}


Table B. 8. Soluble and particulate COD concentrations over time

\begin{tabular}{|c|c|c|c|c|c|c|c|}
\hline \multicolumn{8}{|c|}{ Soluble COD concentrations $(\mathrm{mg} / \mathrm{L})$ over time } \\
\hline $\begin{array}{l}\text { Time } \\
\text { (hr) }\end{array}$ & $\begin{array}{c}\text { TWAS/Manure } \\
7 / 3\end{array}$ & $\begin{array}{c}\text { TWAS/Manure } \\
5 / 5\end{array}$ & $\begin{array}{c}\text { TWAS/Manure } \\
3 / 7\end{array}$ & $\begin{array}{c}\text { TWAS/Manure } \\
1 / 9\end{array}$ & $\begin{array}{c}\text { TWAS/Manure } \\
9 / 1\end{array}$ & $\begin{array}{c}\text { Manure } \\
\text { only }\end{array}$ & $\begin{array}{c}\text { TWAS } \\
\text { only }\end{array}$ \\
\hline 0 & 1880 & 1920 & 1611 & 1940 & 1929 & 1628 & 2181 \\
\hline 2 & 1920 & 1960 & 1817 & 2020 & 1972 & 1661 & 2254 \\
\hline 4 & 2040 & 2100 & 1868 & 2060 & 2471 & 1744 & 2377 \\
\hline 6 & 2260 & 2260 & 2005 & 2180 & 2536 & 1861 & 2524 \\
\hline 8 & 2380 & 2360 & 2159 & 2300 & 2644 & 1960 & 2548 \\
\hline 10 & 2420 & 2480 & 2228 & 2360 & 2904 & 1977 & 2597 \\
\hline 12 & 2540 & 2560 & 2451 & 2400 & 2969 & 2010 & 2769 \\
\hline 24 & 2780 & 2580 & 2622 & 2540 & 3099 & 2126 & 2988 \\
\hline 48 & 2920 & 2940 & 2896 & 2780 & 3208 & 2193 & 3087 \\
\hline 72 & 3990 & 3690 & 4190 & 4020 & 3860 & 3530 & 3120 \\
\hline \multicolumn{8}{|c|}{ Particulate COD concentrations $(\mathrm{mg} / \mathrm{L})$ over time } \\
\hline $\begin{array}{l}\text { Time } \\
(\mathrm{hr})\end{array}$ & $\begin{array}{c}\text { TWAS/Manure } \\
7 / 3\end{array}$ & $\begin{array}{c}\text { TWAS/Manure } \\
5 / 5\end{array}$ & $\begin{array}{c}\text { TWAS/Manure } \\
3 / 7\end{array}$ & $\begin{array}{c}\text { TWAS/Manure } \\
1 / 9\end{array}$ & $\begin{array}{c}\text { TWAS/Manure } \\
9 / 1\end{array}$ & $\begin{array}{l}\text { Manure } \\
\text { only }\end{array}$ & $\begin{array}{c}\text { TWAS } \\
\text { only }\end{array}$ \\
\hline 0 & 23155 & 22556 & 22486 & 21637 & 24388 & 21836 & 23635 \\
\hline 2 & 23015 & 22326 & 22467 & 21427 & 24545 & 21582 & 23115 \\
\hline 4 & 22895 & 22156 & 22219 & 21257 & 24186 & 21489 & 22696 \\
\hline 6 & 22675 & 22215 & 22081 & 21227 & 23712 & 21223 & 22468 \\
\hline 8 & 22555 & 22050 & 21527 & 20787 & 23893 & 21010 & 22276 \\
\hline 10 & 22515 & 21690 & 21399 & 20297 & 23543 & 20527 & 22083 \\
\hline 12 & 22485 & 21310 & 21016 & 20127 & 23228 & 20324 & 21945 \\
\hline 24 & 21155 & 20496 & 19691 & 19787 & 22801 & 20117 & 21697 \\
\hline 48 & 20615 & 20336 & 19300 & 19187 & 22203 & 19856 & 21563 \\
\hline 72 & 20445 & 20186 & 19056 & 19107 & 22177 & 19434 & 21508 \\
\hline
\end{tabular}




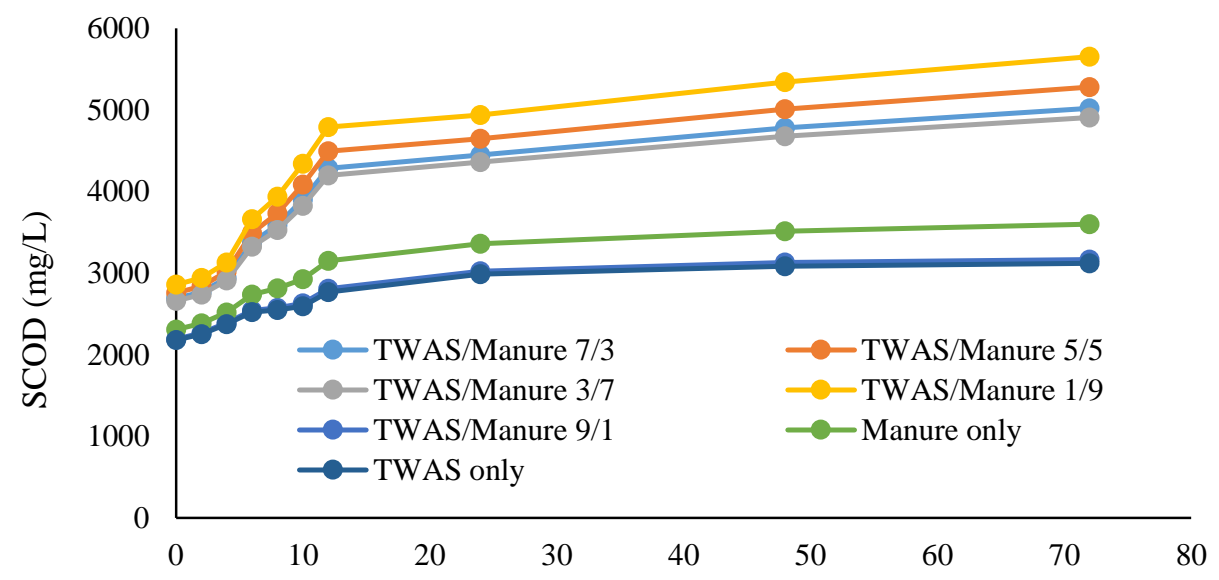

Time (h)

Figure B.2. Concentration of soluble COD over time at different mixing ratios of TWAS and manure 


\section{Analytical results for AnCoD of manure and SSO}

Table C. 1. Characteristics of raw feedstocks in AnCoD of manure and SSO

\begin{tabular}{cccccccc}
\hline \multirow{2}{*}{ Parameters } & \multirow{2}{*}{ Units } & \multicolumn{2}{c}{ Manure } & \multicolumn{2}{c}{ SSO } & \multicolumn{2}{c}{ Inoculum } \\
& & MEAN & SD & MEAN & SD & MEAN & SD \\
\hline TCOD & $\mathrm{mg} / \mathrm{L}$ & 105120 & 4795 & 115163 & 6090 & 17980 & 400 \\
SCOD & $\mathrm{mg} / \mathrm{L}$ & 44116 & 489 & 42932 & 92 & 722 & 16 \\
TSS & $\mathrm{mg} / \mathrm{L}$ & 54380 & 2252 & 62125 & 3063 & 15128 & 330 \\
VSS & $\mathrm{mg} / \mathrm{L}$ & 46420 & 2649 & 45584 & 2283 & 11687 & 160 \\
TS & $\mathrm{mg} / \mathrm{L}$ & 69785 & 3025 & 67987 & 1011 & 16160 & 503 \\
VS & $\mathrm{mg} / \mathrm{L}$ & 58590 & 2761 & 48535 & 682 & 12380 & 308 \\
Ammonia & $\mathrm{mg} / \mathrm{L}$ & 20 & 68.0 & 1073 & 5.87 & 745 & 36 \\
pH & & 6.8 & 0.030 & 5.8 & 0.050 & 7.2 & 0.05 \\
Alkalinity & $\mathrm{mg} \mathrm{CaCO} / \mathrm{L}$ & 4924 & 522 & 6176 & 339 & 4186 & 460 \\
TN & $\mathrm{mg} / \mathrm{L}$ & 2510 & 751 & 3498 & 202 & 1940 & 186 \\
TSN & $\mathrm{mg} / \mathrm{L}$ & 105.00 & 38 & 1046 & 21 & 716 & 93 \\
Total Carbs & $\mathrm{mg} / \mathrm{L}$ & 28796 & 3580 & 13495 & 687 & 453 & 68 \\
Total & & & & & & & \\
Proteins & $\mathrm{mg} / \mathrm{L}$ & 5930 & 198 & 2087 & 98 & 2598 & 38 \\
Total Lipids & $\mathrm{mg} / \mathrm{L}$ & 1400 & 2420 & 1103 & 92 & 225 & 43 \\
\hline
\end{tabular}


Table C. 2. Average $\mathrm{CH}_{4}$ measurements in $\mathrm{AnCoD}$ of manure and SSO

\begin{tabular}{|c|c|c|c|c|c|c|c|c|c|c|c|c|c|c|}
\hline \multicolumn{15}{|c|}{ Average $\mathrm{CH}_{4}$ Measurement (ml) } \\
\hline $\begin{array}{l}\text { Time } \\
\text { (day) }\end{array}$ & Manure & SD & SSO & $\mathrm{SD}$ & $\begin{array}{c}\text { M/SSO } \\
9 / 1\end{array}$ & SD & $\begin{array}{c}\mathrm{M} / \mathrm{SSO} \\
7 / 3\end{array}$ & SD & $\begin{array}{c}\mathrm{M} / \mathrm{SSO} \\
5 / 5\end{array}$ & $\mathrm{SD}$ & $\begin{array}{c}\mathrm{M} / \mathrm{SSO} \\
1 / 9\end{array}$ & $\mathrm{SD}$ & $\begin{array}{c}\mathrm{M} / \mathrm{SSO} \\
3 / 7\end{array}$ & SD \\
\hline 0 & 0 & 0 & 0 & 0 & 0 & 0 & 0 & 0 & 0 & 0 & 0 & 0 & 0 & 0 \\
\hline 1 & 44 & 1.7 & 24 & 1.0 & 28 & 1.2 & 33 & 1.5 & 32 & 1.7 & 30 & 1.6 & 25 & 1.1 \\
\hline 2 & 47 & 1.8 & 59 & 2.5 & 69 & 3.0 & 59 & 2.7 & 68 & 3.7 & 70 & 3.6 & 64 & 2.7 \\
\hline 3 & 66 & 2.5 & 102 & 4.3 & 118 & 5.1 & 72 & 3.3 & 92 & 5.0 & 104 & 5.4 & 110 & 4.7 \\
\hline 4 & 66 & 2.5 & 90 & 3.8 & 104 & 4.5 & 64 & 3.0 & 74 & 4.0 & 82 & 4.3 & 93 & 4.0 \\
\hline 5 & 75 & 2.9 & 103 & 4.3 & 119 & 5.1 & 74 & 3.4 & 88 & 4.7 & 94 & 4.9 & 106 & 4.6 \\
\hline 6 & 90 & 3.4 & 111 & 4.6 & 128 & 5.5 & 108 & 4.9 & 111 & 6.0 & 117 & 6.1 & 118 & 5.1 \\
\hline 7 & 45 & 1.7 & 84 & 3.5 & 97 & 4.2 & 85 & 3.9 & 89 & 4.8 & 89 & 4.6 & 95 & 4.1 \\
\hline 8 & 44 & 1.7 & 65 & 2.7 & 75 & 3.2 & 57 & 2.6 & 58 & 3.1 & 58 & 3.0 & 68 & 2.9 \\
\hline 9 & 40 & 1.5 & 70 & 2.9 & 81 & 3.5 & 56 & 2.6 & 56 & 3.0 & 54 & 2.8 & 67 & 2.9 \\
\hline 11 & 51 & 2.0 & 70 & 2.9 & 81 & 3.5 & 64 & 3.0 & 65 & 3.5 & 60 & 3.1 & 69 & 3.0 \\
\hline 12 & 32 & 1.2 & 33 & 1.4 & 38 & 1.6 & 40 & 1.8 & 38 & 2.0 & 34 & 1.8 & 33 & 1.4 \\
\hline 14 & 33 & 1.3 & 30 & 1.3 & 35 & 1.5 & 48 & 2.2 & 48 & 2.6 & 37 & 1.9 & 34 & 1.5 \\
\hline 15 & 23 & 0.9 & 17 & 0.7 & 19 & 0.8 & 28 & 1.3 & 24 & 1.3 & 21 & 1.1 & 19 & 0.8 \\
\hline 16 & 23 & 0.9 & 12 & 0.5 & 14 & 0.6 & 22 & 1.0 & 17 & 0.9 & 14 & 0.7 & 13 & 0.6 \\
\hline 17 & 21 & 0.8 & 14 & 0.6 & 16 & 0.7 & 19 & 0.9 & 15 & 0.8 & 13 & 0.7 & 11 & 0.5 \\
\hline 18 & 16 & 0.6 & 14 & 0.6 & 16 & 0.7 & 21 & 0.9 & 17 & 0.9 & 14 & 0.7 & 11 & 0.5 \\
\hline 20 & 27 & 1.0 & 16 & 0.7 & 19 & 0.8 & 31 & 1.4 & 25 & 1.4 & 22 & 1.1 & 18 & 0.8 \\
\hline 21 & 14 & 0.5 & 11 & 0.5 & 13 & 0.6 & 23 & 1.0 & 18 & 0.9 & 14 & 0.7 & 12 & 0.5 \\
\hline 22 & 13 & 0.5 & 11 & 0.5 & 13 & 0.6 & 15 & 0.7 & 12 & 0.7 & 10 & 0.5 & 9 & 0.4 \\
\hline 23 & 12 & 0.5 & 9 & 0.4 & 11 & 0.5 & 14 & 0.6 & 11 & 0.6 & 9 & 0.5 & 7 & 0.3 \\
\hline 24 & 12 & 0.5 & 9 & 0.4 & 10 & 0.4 & 14 & 0.7 & 11 & 0.6 & 9 & 0.5 & 7 & 0.3 \\
\hline 25 & 13 & 0.5 & 9 & 0.4 & 10 & 0.4 & 13 & 0.6 & 12 & 0.7 & 12 & 0.6 & 10 & 0.4 \\
\hline 26 & 16 & 0.6 & 10 & 0.4 & 11 & 0.5 & 13 & 0.6 & 12 & 0.7 & 9 & 0.5 & 7 & 0.3 \\
\hline 28 & 9 & 0.3 & 11 & 0.5 & 13 & 0.5 & 19 & 0.9 & 20 & 1.1 & 15 & 0.8 & 12 & 0.5 \\
\hline 29 & 8 & 0.3 & 7 & 0.3 & 9 & 0.4 & 14 & 0.6 & 11 & 0.6 & 10 & 0.4 & 8 & 0.4 \\
\hline 30 & 6 & 0.2 & 7 & 0.3 & 9 & 0.4 & 13 & 0.6 & 11 & 0.6 & 9 & 0.5 & 8 & 0.3 \\
\hline 31 & 7 & 0.3 & 6 & 0.3 & 7 & 0.3 & 7 & 0.3 & 5 & 0.3 & 5 & 0.2 & 3 & 0.1 \\
\hline 32 & 7 & 0.3 & 9 & 0.4 & 10 & 0.4 & 11 & 0.5 & 9 & 0.5 & 9 & 0.5 & 5 & 0.2 \\
\hline 35 & 10 & 0.4 & 10 & 0.4 & 12 & 0.5 & 21 & 0.9 & 18 & 0.9 & 15 & 0.8 & 12 & 0.5 \\
\hline 37 & 10 & 0.4 & 13 & 0.5 & 15 & 0.6 & 17 & 0.8 & 21 & 1.1 & 11 & 0.6 & 23 & 1.0 \\
\hline 40 & 22 & 0.8 & 10 & 0.4 & 12 & 0.5 & 19 & 0.9 & 17 & 0.9 & 15 & 0.8 & 12 & 0.5 \\
\hline 43 & 10 & 0.4 & 6 & 0.2 & 7 & 0.3 & 10 & 0.5 & 9 & 0.5 & 8 & 0.4 & 6 & 0.3 \\
\hline 46 & 3 & 0.1 & 3 & 0.1 & 4 & 0.2 & 9 & 0.3 & 8 & 0.4 & 7 & 0.4 & 6 & 0.2 \\
\hline 49 & 2 & 0.1 & 3 & 0.1 & 3 & 0.1 & 8 & 0.4 & 8 & 0.4 & 7 & 0.3 & 6 & 0.1 \\
\hline 52 & 4 & 0.2 & 4 & 0.2 & 5 & 0.2 & 7 & 0.3 & 7 & 0.4 & 6 & 0.3 & 5 & 0.2 \\
\hline
\end{tabular}

*M: manure 
Table C. 3. Cumulative $\mathrm{CH}_{4}$ production in $\mathrm{AnCoD}$ of manure and $\mathrm{SSO}$

\begin{tabular}{|c|c|c|c|c|c|c|c|c|c|c|c|c|c|c|}
\hline \multicolumn{15}{|c|}{ Cumulative $\mathrm{CH}_{4}$ production $(\mathrm{mL})$} \\
\hline $\begin{array}{l}\text { Time } \\
\text { (day) }\end{array}$ & Manure & $\mathrm{SD}$ & SSO & SD & $\begin{array}{c}\mathrm{M}^{*} / \mathrm{SSO} \\
9 / 1\end{array}$ & SD & $\begin{array}{c}\mathrm{M} / \mathrm{SSO} \\
7 / 3\end{array}$ & $\mathrm{SD}$ & $\begin{array}{c}\mathrm{M} / \mathrm{SSO} \\
5 / 5\end{array}$ & SD & $\begin{array}{c}\mathrm{M} / \mathrm{SSO} \\
1 / 9\end{array}$ & SD & $\begin{array}{c}\mathrm{M} / \mathrm{SSO} \\
3 / 7\end{array}$ & SD \\
\hline 0 & 0 & 0 & 0 & 0 & 0 & 0 & 0 & 0 & 0 & 0.0 & 0 & 0.0 & 0 & 0.0 \\
\hline 1 & 44 & 2.0 & 24 & 1.3 & 28 & 1.3 & 33 & 1.8 & 32 & 1.5 & 30 & 1.3 & 25 & 1.0 \\
\hline 2 & 90 & 4.1 & 83 & 4.6 & 93 & 4.5 & 93 & 5.0 & 101 & 4.5 & 100 & 4.2 & 89 & 3.4 \\
\hline 3 & 156 & 7.0 & 186 & 10.2 & 206 & 9.9 & 164 & 8.9 & 193 & 8.7 & 204 & 8.6 & 199 & 7.6 \\
\hline 4 & 222 & 10.0 & 276 & 15.2 & 305 & 14.6 & 229 & 12.3 & 267 & 12.0 & 287 & 12.0 & 291 & 11.1 \\
\hline 5 & 297 & 13.4 & 379 & 20.8 & 418 & 20.1 & 303 & 16.4 & 355 & 16.0 & 381 & 16.0 & 397 & 15.1 \\
\hline 6 & 387 & 17.4 & 489 & 26.9 & 540 & 25.9 & 410 & 22.2 & 466 & 21.0 & 498 & 20.9 & 516 & 19.6 \\
\hline 7 & 432 & 19.4 & 573 & 31.5 & 632 & 30.3 & 496 & 26.8 & 554 & 24.9 & 587 & 24.7 & 611 & 23.2 \\
\hline 8 & 476 & 21.4 & 638 & 35.1 & 703 & 33.7 & 553 & 29.9 & 612 & 27.5 & 645 & 27.1 & 679 & 25.8 \\
\hline 9 & 516 & 23.2 & 708 & 38.9 & 780 & 37.5 & 609 & 32.9 & 668 & 30.0 & 699 & 29.4 & 746 & 28.3 \\
\hline 11 & 567 & 25.5 & 778 & 42.8 & 857 & 41.1 & 673 & 36.3 & 732 & 33.0 & 759 & 31.9 & 815 & 31.0 \\
\hline 12 & 599 & 26.9 & 810 & 44.6 & 893 & 42.9 & 713 & 38.5 & 770 & 34.7 & 793 & 33.3 & 849 & 32.3 \\
\hline 14 & 632 & 28.4 & 840 & 46.2 & 926 & 44.4 & 761 & 41.1 & 818 & 36.8 & 830 & 34.9 & 883 & 33.5 \\
\hline 15 & 654 & 29.5 & 857 & 47.1 & 944 & 45.3 & 789 & 42.6 & 842 & 37.9 & 851 & 35.7 & 902 & 34.3 \\
\hline 16 & 677 & 30.5 & 870 & 47.8 & 958 & 46.0 & 811 & 43.8 & 859 & 38.7 & 866 & 36.4 & 915 & 34.8 \\
\hline 17 & 698 & 31.4 & 883 & 48.6 & 973 & 46.7 & 829 & 44.8 & 875 & 39.4 & 878 & 36.9 & 926 & 35.2 \\
\hline 18 & 714 & 32.1 & 897 & 49.3 & 987 & 47.4 & 850 & 45.9 & 892 & 40.1 & 893 & 37.5 & 937 & 35.6 \\
\hline 20 & 741 & 33.3 & 913 & 50.2 & 1001 & 48.0 & 881 & 47.6 & 917 & 41.3 & 914 & 38.4 & 955 & 36.3 \\
\hline 21 & 755 & 34.0 & 924 & 50.8 & 1021 & 49.0 & 904 & 48.8 & 934 & 42.0 & 929 & 39.0 & 967 & 36.8 \\
\hline 22 & 768 & 34.5 & 936 & 51.5 & 1035 & 49.7 & 919 & 49.6 & 947 & 42.6 & 939 & 39.4 & 976 & 37.1 \\
\hline 23 & 780 & 35.1 & 945 & 52.0 & 1045 & 50.2 & 933 & 50.4 & 957 & 43.1 & 948 & 39.8 & 984 & 36.3 \\
\hline 24 & 792 & 35.6 & 954 & 52.4 & 1055 & 50.6 & 947 & 51.1 & 968 & 43.6 & 957 & 40.2 & 991 & 37.6 \\
\hline 25 & 804 & 36.2 & 962 & 52.9 & 1065 & 51.1 & 960 & 51.9 & 980 & 44.1 & 969 & 40.7 & 1001 & 38.0 \\
\hline 26 & 820 & 36.9 & 972 & 53.5 & 1076 & 51.6 & 974 & 52.6 & 992 & 44.7 & 978 & 41.1 & 1008 & 35.4 \\
\hline 28 & 829 & 37.3 & 983 & 54.1 & 1088 & 52.2 & 993 & 53.6 & 1013 & 45.6 & 993 & 41.7 & 1020 & 38.8 \\
\hline 29 & 837 & 37.7 & 991 & 54.5 & 1099 & 52.7 & 1007 & 54.4 & 1024 & 46.1 & 1003 & 42.1 & 1029 & 39.1 \\
\hline 30 & 844 & 38.0 & 998 & 54.9 & 1110 & 53.3 & 1019 & 55.0 & 1035 & 46.6 & 1012 & 42.5 & 1036 & 40.6 \\
\hline 31 & 851 & 38.3 & 1004 & 55.2 & 1120 & 53.7 & 1027 & 55.5 & 1040 & 46.8 & 1017 & 42.7 & 1039 & 39.5 \\
\hline 32 & 858 & 38.6 & 1013 & 55.7 & 1132 & 54.3 & 1038 & 56.0 & 1049 & 47.2 & 1026 & 43.1 & 1044 & 38.4 \\
\hline 35 & 867 & 39.0 & 1023 & 56.3 & 1143 & 54.9 & 1058 & 57.1 & 1067 & 48.0 & 1041 & 43.7 & 1056 & 39.7 \\
\hline 37 & 877 & 39.5 & 1036 & 57.0 & 1157 & 55.5 & 1075 & 58.1 & 1087 & 48.9 & 1052 & 44.2 & 1080 & 41.0 \\
\hline 40 & 899 & 40.5 & 1046 & 57.6 & 1168 & 56.1 & 1094 & 59.1 & 1104 & 49.7 & 1067 & 44.8 & 1092 & 40.2 \\
\hline 43 & 910 & 40.9 & 1052 & 57.9 & 1175 & 56.4 & 1104 & 59.6 & 1114 & 50.1 & 1076 & 43.8 & 1098 & 41.7 \\
\hline 46 & 912 & 41.1 & 1056 & 58.1 & 1179 & 56.6 & 1113 & 60.1 & 1121 & 49.2 & 1082 & 45.5 & 1104 & 42.0 \\
\hline 49 & 915 & 41.2 & 1059 & 58.2 & 1182 & 56.7 & 1121 & 58.3 & 1129 & 50.8 & 1089 & 44.3 & 1110 & 43.5 \\
\hline 52 & 919 & 41.3 & 1063 & 58.5 & 1186 & 56.9 & 1129 & 61.0 & 1136 & 51.1 & 1095 & 46.0 & 1115 & 42.4 \\
\hline
\end{tabular}

*M: manure 
Table C. 4. Cumulative $\mathrm{CH}_{4}$ production per mass of $\mathrm{COD}$ added in $\mathrm{AnCoD}$ of manure and SSO

\begin{tabular}{|c|c|c|c|c|c|c|c|c|c|c|c|c|c|c|}
\hline \multicolumn{15}{|c|}{ Cumulative $\mathrm{CH}_{4}$ yield (mL CH4/g COD added) } \\
\hline $\begin{array}{l}\text { Time } \\
\text { (day) }\end{array}$ & Manure & $\mathrm{SD}$ & SSO & $\mathrm{SD}$ & $\begin{array}{c}\mathrm{M}^{*} / \mathrm{SSO} \\
9 / 1\end{array}$ & $\mathrm{SD}$ & $\begin{array}{c}\mathrm{M} / \mathrm{SSO} \\
7 / 3\end{array}$ & SD & $\begin{array}{c}\mathrm{M} / \mathrm{SSO} \\
5 / 5\end{array}$ & SD & $\begin{array}{c}\mathrm{M} / \mathrm{SSO} \\
1 / 9\end{array}$ & SD & $\begin{array}{c}\text { M/SSO } \\
3 / 7\end{array}$ & SD \\
\hline 0 & 0 & 0 & 0 & 0 & 0 & 0 & 0 & 0 & 0 & 0 & 0 & 0 & 0 & 0 \\
\hline 1 & 14 & 0.6 & 7 & 0.3 & 9 & 0.3 & 10 & 0.4 & 9 & 0.3 & 9 & 0.5 & 7 & 0.3 \\
\hline 2 & 28 & 1.2 & 24 & 0.9 & 29 & 1.1 & 28 & 1.0 & 29 & 1.0 & 30 & 1.5 & 25 & 0.9 \\
\hline 3 & 49 & 2.0 & 53 & 2.0 & 63 & 2.5 & 50 & 1.8 & 56 & 2.0 & 61 & 3.1 & 56 & 2.0 \\
\hline 4 & 69 & 2.9 & 79 & 3.0 & 93 & 3.7 & 70 & 2.4 & 77 & 2.7 & 86 & 4.4 & 82 & 3.0 \\
\hline 5 & 93 & 3.9 & 108 & 4.1 & 128 & 5.1 & 92 & 3.2 & 103 & 3.6 & 114 & 5.8 & 112 & 4.0 \\
\hline 6 & 121 & 5.1 & 140 & 5.3 & 165 & 6.6 & 125 & 4.4 & 135 & 4.7 & 149 & 7.6 & 146 & 5.3 \\
\hline 7 & 135 & 5.7 & 163 & 6.2 & 193 & 7.7 & 151 & 5.3 & 161 & 5.6 & 175 & 8.9 & 173 & 6.2 \\
\hline 8 & 149 & 6.2 & 182 & 6.9 & 215 & 8.6 & 169 & 5.9 & 178 & 6.2 & 193 & 9.8 & 192 & 6.9 \\
\hline 9 & 161 & 6.8 & 202 & 7.7 & 239 & 9.6 & 186 & 6.5 & 194 & 5.8 & 209 & 10.6 & 211 & 7.6 \\
\hline 11 & 177 & 7.4 & 222 & 8.4 & 262 & 10.5 & 205 & 7.2 & 213 & 7.4 & 226 & 11.5 & 231 & 8.3 \\
\hline 12 & 187 & 7.9 & 231 & 8.8 & 273 & 10.6 & 217 & 7.6 & 224 & 7.8 & 237 & 12.1 & 240 & 8.6 \\
\hline 14 & 197 & 8.3 & 240 & 9.1 & 283 & 11.3 & 232 & 8.1 & 238 & 8.3 & 248 & 11.6 & 250 & 9.0 \\
\hline 15 & 204 & 7.5 & 244 & 9.3 & 289 & 10.4 & 241 & 8.6 & 245 & 7.6 & 254 & 13.0 & 255 & 8.2 \\
\hline 16 & 211 & 8.9 & 248 & 9.4 & 293 & 11.7 & 247 & 7.7 & 250 & 8.7 & 258 & 12.2 & 259 & 9.3 \\
\hline 17 & 218 & 9.2 & 252 & 10.1 & 298 & 10.3 & 253 & 8.9 & 254 & 8.9 & 262 & 13.4 & 262 & 9.8 \\
\hline 18 & 223 & 9.4 & 256 & 9.7 & 302 & 11.2 & 259 & 9.1 & 259 & 9.1 & 266 & 12.3 & 265 & 8.6 \\
\hline 20 & 231 & 8.6 & 260 & 9.9 & 306 & 12.3 & 269 & 9.4 & 266 & 8.3 & 273 & 13.9 & 270 & 7.7 \\
\hline 21 & 236 & 9.9 & 264 & 10.0 & 312 & 12.5 & 276 & 8.7 & 271 & 9.5 & 277 & 14.1 & 274 & 8.9 \\
\hline 22 & 240 & 10.1 & 267 & 10.1 & 317 & 12.7 & 280 & 9.8 & 275 & 8.6 & 280 & 13.2 & 276 & 9.9 \\
\hline 23 & 244 & 9.7 & 269 & 9.4 & 320 & 11.6 & 285 & 10.0 & 278 & 9.7 & 283 & 14.4 & 278 & 9.3 \\
\hline 24 & 247 & 10.4 & 272 & 10.3 & 323 & 12.9 & 289 & 10.6 & 281 & 9.8 & 286 & 13.6 & 280 & 9.1 \\
\hline 25 & 251 & 10.6 & 274 & 9.7 & 326 & 13.0 & 293 & 10.3 & 285 & 10.0 & 289 & 14.7 & 283 & 10.5 \\
\hline 26 & 256 & 10.8 & 277 & 10.5 & 329 & 13.2 & 297 & 9.8 & 288 & 9.1 & 292 & 12.9 & 285 & 9.3 \\
\hline 28 & 259 & 10.9 & 280 & 9.8 & 333 & 13.3 & 303 & 10.6 & 294 & 10.3 & 296 & 15.1 & 289 & 10.4 \\
\hline 29 & 262 & 11.0 & 283 & 10.7 & 336 & 13.4 & 307 & 10.7 & 297 & 9.4 & 299 & 14.3 & 291 & 9.5 \\
\hline 30 & 264 & 10.4 & 285 & 9.3 & 340 & 13.6 & 311 & 10.9 & 301 & 10.5 & 302 & 15.4 & 293 & 10.6 \\
\hline 31 & 266 & 11.2 & 286 & 10.9 & 343 & 12.7 & 313 & 11.0 & 302 & 9.6 & 303 & 13.5 & 294 & 9.6 \\
\hline 32 & 268 & 10.7 & 289 & 12.3 & 346 & 13.9 & 317 & 9.7 & 305 & 10.7 & 306 & 15.6 & 295 & 10.6 \\
\hline 35 & 271 & 11.4 & 292 & 11.1 & 350 & 14.0 & 323 & 11.3 & 310 & 9.8 & 311 & 13.8 & 299 & 9.8 \\
\hline 37 & 274 & 11.5 & 295 & 10.5 & 354 & 13.8 & 328 & 9.8 & 316 & 11.1 & 314 & 14.9 & 306 & 11.0 \\
\hline 40 & 281 & 11.8 & 298 & 11.3 & 358 & 14.5 & 334 & 11.7 & 321 & 9.8 & 318 & 16.2 & 309 & 10.1 \\
\hline 43 & 284 & 11.9 & 300 & 10.2 & 360 & 13.2 & 337 & 10.2 & 323 & 11.3 & 321 & 15.1 & 311 & 11.2 \\
\hline 46 & 285 & 11.6 & 301 & 11.4 & 361 & 14.4 & 340 & 11.9 & 326 & 10.5 & 323 & 16.5 & 312 & 10.4 \\
\hline 49 & 286 & 10.2 & 302 & 10.6 & 362 & 13.1 & 342 & 10.9 & 328 & 9.2 & 325 & 14.6 & 314 & 9.7 \\
\hline 52 & 287 & 12.1 & 303 & 11.5 & 363 & 14.5 & 344 & 11.6 & 330 & 11.5 & 327 & 16.7 & 316 & 10.6 \\
\hline
\end{tabular}


Table C. 5. Cumulative $\mathrm{CH}_{4}$ production per mass of VSS added in AnCoD of manure and SSO

\begin{tabular}{|c|c|c|c|c|c|c|c|c|c|c|c|c|c|c|}
\hline \multirow{3}{*}{$\begin{array}{l}\text { Time } \\
\text { (day) }\end{array}$} & \multicolumn{14}{|c|}{ Cumulative $\mathrm{CH}_{4}$ yield (mL CH4/g VSS added) } \\
\hline & & & & & $\mathrm{M}^{*} / \mathrm{SSO}$ & & $\mathrm{M} / \mathrm{SSO}$ & & $\mathrm{M} / \mathrm{SSC}$ & & $\mathrm{M} / \mathrm{SSO}$ & & M/SSO & \\
\hline & Manure & SD & SSO & SD & $9 / 1$ & $\mathrm{SD}$ & $7 / 3$ & SD & $5 / 5$ & $\mathrm{SD}$ & & $\mathrm{SD}$ & & SD \\
\hline 0 & 0 & 0 & 0 & 0 & 0 & 0 & 0 & 0 & 0 & 0 & 0 & 0 & 0 & 0.0 \\
\hline 1 & 31 & 1.4 & 18 & 0.6 & 20 & 0.8 & 25 & 0.9 & 23 & 0.8 & 23 & 0.9 & 19 & 0.9 \\
\hline 2 & 64 & 2.9 & 62 & 2.2 & 67 & 2.8 & 69 & 2.5 & 73 & 2.5 & 76 & 2.9 & 65 & 3.1 \\
\hline 3 & 111 & 5.0 & 139 & 4.9 & 147 & 6.2 & 121 & 4.5 & 139 & 4.9 & 155 & 5.9 & 146 & 6.9 \\
\hline 4 & 158 & 7.1 & 206 & 7.2 & 218 & 9.1 & 169 & 6.2 & 193 & 6.8 & 218 & 8.3 & 214 & 10.1 \\
\hline 5 & 212 & 9.5 & 282 & 9.9 & 298 & 12.5 & 224 & 8.3 & 257 & 9.0 & 289 & 11.0 & 292 & 13.7 \\
\hline 6 & 276 & 12.4 & 365 & 12.8 & 385 & 16.2 & 303 & 11.2 & 337 & 11.8 & 379 & 14.4 & 379 & 17.8 \\
\hline 7 & 308 & 13.9 & 428 & 15.0 & 451 & 19.0 & 366 & 13.6 & 401 & 14.0 & 446 & 17.0 & 448 & 21.1 \\
\hline 8 & 339 & 15.2 & 476 & 16.7 & 502 & 21.1 & 408 & 15.1 & 443 & 15.5 & 491 & 18.6 & 499 & 23.4 \\
\hline 9 & 367 & 16.5 & 528 & 18.5 & 557 & 23.4 & 450 & 16.6 & 483 & 16.9 & 532 & 20.2 & 548 & 25.8 \\
\hline 11 & 404 & 18.2 & 580 & 20.3 & 612 & 25.7 & 497 & 18.4 & 530 & 18.5 & 577 & 21.9 & 599 & 28.2 \\
\hline 12 & 426 & 19.2 & 605 & 21.2 & 638 & 26.8 & 526 & 19.5 & 557 & 19.5 & 603 & 22.9 & 624 & 29.3 \\
\hline 14 & 450 & 20.3 & 627 & 21.9 & 661 & 27.8 & 562 & 20.8 & 592 & 20.7 & 631 & 24.0 & 648 & 30.5 \\
\hline 15 & 466 & 21.0 & 640 & 22.4 & 674 & 28.3 & 583 & 21.6 & 609 & 21.3 & 647 & 24.6 & 662 & 31.1 \\
\hline 16 & 482 & 21.7 & 649 & 22.7 & 684 & 28.7 & 599 & 22.2 & 622 & 21.8 & 658 & 23.7 & 672 & 31.6 \\
\hline 17 & 497 & 20.5 & 659 & 22.1 & 695 & 28.2 & 613 & 23.7 & 633 & 19.3 & 668 & 25.4 & 680 & 29.5 \\
\hline 18 & 508 & 22.9 & 669 & 23.4 & 705 & 29.6 & 628 & 23.2 & 645 & 22.6 & 679 & 25.8 & 689 & 32.4 \\
\hline 20 & 528 & 23.7 & 681 & 23.8 & 715 & 30.0 & 651 & 24.1 & 663 & 21.5 & 695 & 26.4 & 702 & 33.0 \\
\hline 21 & 538 & 24.2 & 690 & 24.1 & 729 & 29.6 & 668 & 24.7 & 676 & 23.7 & 706 & 26.8 & 710 & 31.9 \\
\hline 22 & 547 & 24.6 & 698 & 24.4 & 739 & 31.1 & 679 & 25.1 & 685 & 24.0 & 714 & 27.1 & 717 & 33.7 \\
\hline 23 & 555 & 25.0 & 705 & 23.5 & 747 & 30.4 & 689 & 25.5 & 693 & 23.2 & 721 & 25.8 & 722 & 34.0 \\
\hline 24 & 564 & 24.4 & 711 & 24.9 & 753 & 31.6 & 700 & 24.2 & 700 & 24.5 & 728 & 27.7 & 728 & 34.2 \\
\hline 25 & 573 & 25.8 & 718 & 25.1 & 760 & 30.9 & 709 & 26.2 & 709 & 24.8 & 737 & 28.0 & 735 & 33.5 \\
\hline 26 & 584 & 26.3 & 725 & 25.4 & 768 & 32.3 & 719 & 25.6 & 718 & 25.1 & 744 & 26.8 & 740 & 34.8 \\
\hline 28 & 591 & 24.6 & 734 & 24.7 & 777 & 32.6 & 733 & 27.1 & 733 & 24.6 & 755 & 28.7 & 749 & 35.2 \\
\hline 29 & 596 & 26.8 & 739 & 25.9 & 785 & 33.0 & 743 & 26.5 & 741 & 25.9 & 763 & 29.0 & 755 & 34.5 \\
\hline 30 & 601 & 25.5 & 745 & 25.1 & 792 & 33.3 & 753 & 27.9 & 749 & 26.2 & 770 & 29.2 & 761 & 35.8 \\
\hline 31 & 606 & 27.3 & 749 & 26.2 & 800 & 33.6 & 758 & 28.1 & 753 & 25.7 & 773 & 27.9 & 763 & 35.9 \\
\hline 32 & 611 & 26.5 & 756 & 24.4 & 808 & 32.9 & 766 & 27.4 & 759 & 26.6 & 780 & 29.6 & 767 & 34.9 \\
\hline 35 & 618 & 27.8 & 763 & 26.7 & 816 & 34.3 & 782 & 28.9 & 772 & 25.3 & 792 & 30.1 & 776 & 36.5 \\
\hline 37 & 625 & 28.1 & 773 & 27.1 & 826 & 34.7 & 794 & 28.4 & 787 & 27.5 & 800 & 28.4 & 793 & 37.3 \\
\hline 40 & 641 & 28.8 & 781 & 27.3 & 835 & 35.1 & 808 & 29.9 & 799 & 28.0 & 811 & 30.8 & 802 & 36.8 \\
\hline 43 & 648 & 29.2 & 785 & 255.0 & 839 & 34.2 & 815 & 30.2 & 806 & 26.2 & 818 & 29.3 & 807 & 37.9 \\
\hline 46 & 650 & 27.9 & 788 & 27.6 & 842 & 35.4 & 822 & 30.4 & 811 & 28.4 & 823 & 31.3 & 811 & 38.1 \\
\hline 49 & 652 & 28.3 & 790 & 25.6 & 844 & 34.5 & 828 & 29.7 & 817 & 27.6 & 828 & 30.5 & 815 & 37.3 \\
\hline 52 & 654 & 29.4 & 793 & 27.8 & 847 & 35.6 & 834 & 30.8 & 822 & 28.8 & 833 & 31.6 & 819 & 38.5 \\
\hline
\end{tabular}


Table C. 6. Cumulative $\mathrm{CH}_{4}$ production per volume of substrate added in AnCoD of manure and SSO

\begin{tabular}{|c|c|c|c|c|c|c|c|c|c|c|c|c|c|c|}
\hline \multirow{3}{*}{$\begin{array}{l}\text { Time } \\
\text { (day) }\end{array}$} & \multirow[b]{3}{*}{ Manure } & \multirow[b]{3}{*}{ SD } & \multirow[b]{3}{*}{$\mathrm{SSO}$} & \multicolumn{7}{|c|}{ Cumulative $\mathrm{CH}_{4}$ yield $(\mathrm{mL} \mathrm{CH} 4 / \mathrm{mL}$ substrate added) } & \multirow{3}{*}{$\begin{array}{l}\mathrm{M} / \mathrm{SSO} \\
1 / 9 \\
\end{array}$} & \multirow[b]{3}{*}{ SD } & \multirow{3}{*}{$\begin{array}{l}\mathrm{M} / \mathrm{SSO} \\
3 / 7 \\
\end{array}$} & \multirow[b]{3}{*}{ SD } \\
\hline & & & & & $\mathrm{M}^{*} / \mathrm{SSO}$ & & $\mathrm{M} / \mathrm{SSO}$ & & $\mathrm{M} / \mathrm{SSO}$ & & & & & \\
\hline & & & & SD & $9 / 1$ & $\mathrm{SD}$ & $7 / 3$ & $\mathrm{SD}$ & $5 / 5$ & $\mathrm{SD}$ & & & & \\
\hline 0 & 0 & 0 & 0 & 0 & 0 & 0 & 0 & 0 & 0 & 0 & 0 & 0 & 0 & 0 \\
\hline 1 & 1.45 & 0.06 & 1.4 & 0.05 & 1.0 & 0.04 & 1.4 & 0.06 & 1.5 & 0.07 & 1.6 & 0.07 & 1.4 & 0.08 \\
\hline 2 & 3.02 & 0.12 & 4.9 & 0.16 & 3.3 & 0.14 & 3.9 & 0.18 & 4.6 & 0.21 & 5.3 & 0.22 & 5.0 & 0.27 \\
\hline 3 & 5.21 & 0.20 & 10.9 & 0.35 & 7.3 & 0.31 & 6.8 & 0.32 & 8.8 & 0.40 & 10.8 & 0.45 & 11.0 & 0.61 \\
\hline 4 & 7.40 & 0.29 & 16.2 & 0.52 & 10.9 & 0.46 & 9.5 & 0.44 & 12.1 & 0.56 & 15.1 & 0.63 & 16.2 & 0.89 \\
\hline 5 & 9.90 & 0.39 & 22.3 & 0.71 & 14.9 & 0.63 & 12.6 & 0.58 & 16.1 & 0.74 & 20.0 & 0.84 & 22.1 & 1.21 \\
\hline 6 & 12.91 & 0.50 & 28.8 & 0.92 & 19.3 & 0.81 & 17.1 & 0.79 & 21.2 & 0.97 & 26.2 & 1.10 & 28.7 & 1.58 \\
\hline 7 & 14.41 & 0.56 & 33.7 & 1.08 & 22.6 & 0.95 & 20.7 & 0.95 & 25.2 & 1.16 & 30.9 & 1.30 & 33.9 & 1.87 \\
\hline 8 & 15.86 & 0.62 & 37.5 & 1.20 & 25.1 & 1.05 & 23.0 & 1.06 & 27.8 & 1.28 & 34.0 & 1.43 & 37.7 & 2.07 \\
\hline 9 & 17.19 & 0.67 & 41.7 & 1.33 & 27.9 & 1.17 & 25.4 & 1.17 & 30.3 & 1.40 & 36.8 & 1.55 & 41.4 & 2.28 \\
\hline 11 & 18.90 & 0.74 & 45.7 & 1.46 & 30.6 & 1.29 & 28.0 & 1.29 & 33.3 & 1.53 & 39.9 & 1.88 & 45.3 & 1.94 \\
\hline 12 & 19.96 & 0.78 & 47.7 & 1.53 & 31.9 & 1.34 & 29.7 & 1.37 & 35.0 & 1.61 & 41.8 & 1.75 & 47.2 & 2.59 \\
\hline 14 & 21.06 & 0.82 & 49.4 & 1.58 & 33.1 & 1.39 & 31.7 & 1.46 & 37.2 & 1.71 & 43.7 & 2.03 & 49.0 & 1.97 \\
\hline 15 & 21.81 & 0.95 & 50.4 & 1.61 & 33.7 & 1.42 & 32.9 & 1.51 & 38.3 & 1.76 & 44.8 & 1.88 & 50.1 & 2.76 \\
\hline 16 & 22.57 & 0.88 & 51.2 & 1.64 & 34.2 & 1.44 & 33.8 & 1.55 & 39.1 & 1.80 & 45.6 & 1.91 & 50.8 & 1.80 \\
\hline 17 & 23.27 & 0.91 & 52.0 & 1.66 & 34.7 & 1.46 & 34.6 & 1.59 & 39.8 & 1.83 & 46.2 & 1.94 & 51.5 & 2.83 \\
\hline 18 & 23.79 & 0.83 & 52.8 & 1.69 & 35.2 & 1.48 & 35.4 & 1.63 & 40.5 & 1.86 & 47.0 & 1.97 & 52.1 & 1.86 \\
\hline 20 & 24.69 & 0.96 & 53.7 & 1.72 & 35.7 & 1.50 & 36.7 & 1.69 & 41.7 & 1.92 & 48.1 & 2.02 & 53.1 & 2.92 \\
\hline 21 & 25.16 & 0.98 & 54.4 & 1.74 & 36.5 & 1.53 & 37.7 & 1.73 & 42.5 & 1.95 & 48.9 & 2.05 & 53.7 & 1.96 \\
\hline 22 & 25.59 & 1.00 & 55.0 & 1.76 & 37.0 & 1.55 & 38.3 & 1.76 & 43.0 & 1.98 & 49.4 & 2.08 & 54.2 & 2.98 \\
\hline 23 & 25.99 & 1.01 & 55.6 & 1.78 & 37.3 & 1.57 & 38.9 & 1.79 & 43.5 & 2.00 & 49.9 & 2.75 & 54.6 & 3.01 \\
\hline 24 & 26.39 & 9.83 & 56.1 & 1.79 & 37.7 & 1.58 & 39.5 & 1.82 & 44.0 & 2.02 & 50.4 & 2.12 & 55.0 & 2.93 \\
\hline 25 & 26.81 & 1.05 & 56.6 & 1.81 & 38.0 & 1.60 & 40.0 & 1.84 & 44.6 & 2.05 & 51.0 & 2.53 & 55.6 & 3.06 \\
\hline 26 & 27.35 & 9.87 & 57.2 & 1.83 & 38.4 & 1.61 & 40.6 & 1.87 & 45.1 & 2.08 & 51.5 & 2.16 & 56.0 & 2.98 \\
\hline 28 & 27.65 & 1.08 & 57.8 & 1.85 & 38.8 & 1.63 & 41.4 & 1.90 & 46.0 & 2.12 & 52.3 & 1.92 & 56.7 & 3.12 \\
\hline 29 & 27.91 & 1.09 & 58.3 & 1.86 & 39.2 & 1.65 & 41.9 & 1.93 & 46.5 & 2.14 & 52.8 & 2.22 & 57.1 & 2.94 \\
\hline 30 & 28.13 & 1.10 & 58.7 & 1.88 & 39.6 & 1.66 & 42.5 & 1.95 & 47.0 & 1.98 & 53.3 & 1.94 & 57.6 & 3.17 \\
\hline 31 & 28.36 & 1.11 & 59.1 & 1.89 & 40.0 & 1.68 & 42.8 & 1.97 & 47.3 & 2.17 & 53.5 & 2.25 & 57.7 & 2.98 \\
\hline 32 & 28.58 & 1.11 & 59.6 & 1.91 & 40.4 & 1.70 & 43.2 & 1.99 & 47.7 & 1.92 & 54.0 & 1.27 & 58.0 & 3.19 \\
\hline 35 & 28.92 & 9.83 & 60.2 & 1.93 & 40.8 & 1.71 & 44.1 & 2.03 & 48.5 & 2.23 & 54.8 & 2.30 & 58.7 & 3.23 \\
\hline 37 & 29.25 & 1.14 & 60.9 & 1.95 & 41.3 & 1.74 & 44.8 & 2.06 & 49.4 & 1.72 & 55.4 & 1.33 & 60.0 & 2.30 \\
\hline 40 & 29.98 & 1.17 & 61.6 & 1.97 & 41.7 & 1.75 & 45.6 & 2.10 & 50.2 & 2.31 & 56.2 & 2.36 & 60.7 & 3.34 \\
\hline 43 & 30.32 & 1.18 & 61.9 & 1.98 & 42.0 & 1.76 & 46.0 & 2.12 & 50.6 & 1.63 & 56.6 & 1.83 & 61.0 & 2.36 \\
\hline 46 & 30.41 & 1.19 & 62.1 & 1.99 & 42.1 & 1.77 & 46.4 & 2.13 & 51.0 & 2.34 & 57.0 & 2.39 & 61.3 & 3.37 \\
\hline 49 & 30.49 & 1.23 & 62.3 & 1.99 & 42.2 & 1.77 & 46.7 & 2.15 & 51.3 & 1.36 & 57.3 & 1.94 & 61.6 & 3.39 \\
\hline 52 & 30.63 & 1.19 & 62.5 & 2.00 & 42.4 & 1.78 & 47.0 & 2.16 & 51.6 & 2.37 & 57.6 & 2.42 & 61.9 & 2.41 \\
\hline
\end{tabular}




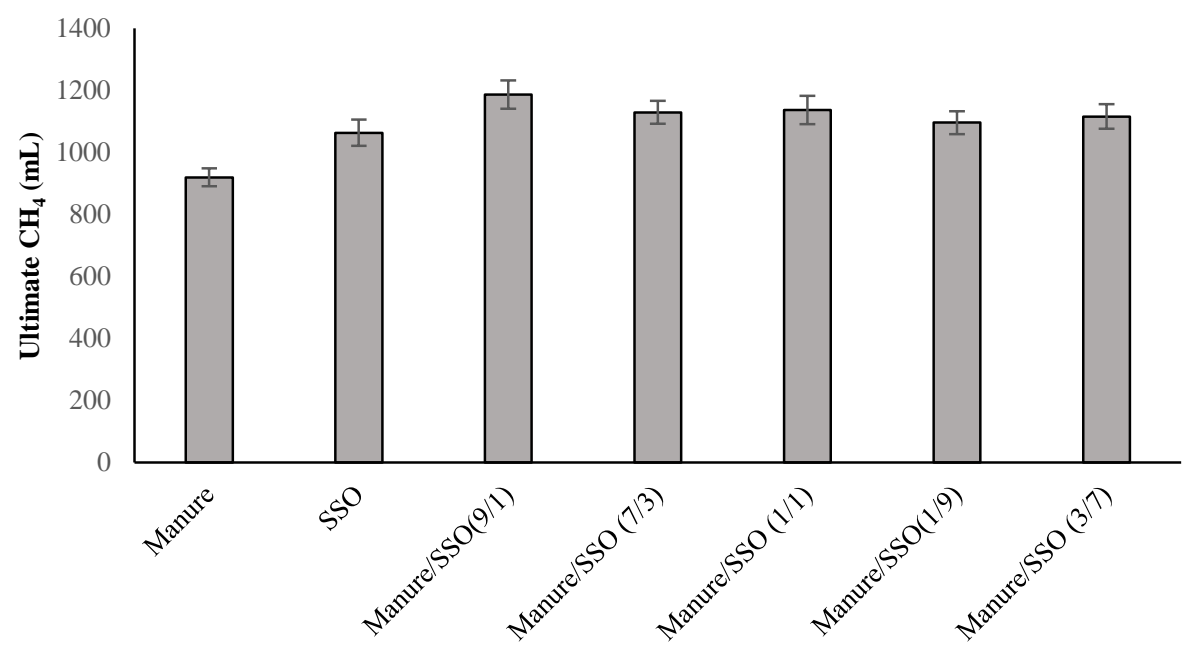

Mixing ratio

Figure C. 1. Ultimate methane production in AnCoD of TWAS and manure 
Table C. 7. Characteristics of the raw feedstocks for hydrolysis/acidification of manure and SSO

\begin{tabular}{cccccccc}
\hline \multirow{2}{*}{ Parameters } & \multirow{2}{*}{ Units } & \multicolumn{2}{c}{ Manure } & \multicolumn{2}{c}{ SSO } & \multicolumn{2}{c}{ Inoculum } \\
& & MEAN & SD & MEAN & SD & MEAN & SD \\
\hline TCOD & $\mathrm{mg} / \mathrm{L}$ & 101125 & 4551 & 115163 & 4607 & 17100 & 400 \\
SCOD & $\mathrm{mg} / \mathrm{L}$ & 10480 & 472 & 42932 & 1717 & 986 & 16 \\
TSS & $\mathrm{mg} / \mathrm{L}$ & 54600 & 2457 & 47112 & 1884 & 17143 & 330 \\
VSS & $\mathrm{mg} / \mathrm{L}$ & 32115 & 1445 & 39940 & 1598 & 11200 & 160 \\
TS & $\mathrm{mg} / \mathrm{L}$ & 68540 & 3084 & 77987 & 3119 & 21870 & 503 \\
VS & $\mathrm{mg} / \mathrm{L}$ & 58520 & 2633 & 63537 & 2541 & 13380 & 308 \\
Ammonia & $\mathrm{mg} / \mathrm{L}$ & 24.3 & 1 & 1396 & 55.84 & 1505 & 36 \\
$\mathrm{pH}$ & - & 6.7 & 0.302 & 5.8 & 0.2 & 7.2 & 0.1 \\
& $\mathrm{mg}$ & & & & & & \\
Alkalinity & $\mathrm{CaCO} / \mathrm{L}$ & 7224 & 325 & 6720 & 269 & 3986 & 460 \\
$\mathrm{TN}$ & $\mathrm{mg} / \mathrm{L}$ & 1740 & 78 & 4198 & 167.92 & 2048 & 186 \\
TSN & $\mathrm{mg} / \mathrm{L}$ & 107 & 5 & 1146 & 46 & 716 & 93 \\
Total Carbs & $\mathrm{mg} / \mathrm{L}$ & 26796 & 1206 & 14125 & 565 & 595 & 68 \\
Total Proteins & $\mathrm{mg} / \mathrm{L}$ & 5408 & 243 & 2156 & 86.24 & 1680 & 38 \\
Total Lipids & $\mathrm{mg} / \mathrm{L}$ & 1703 & 77 & 1468 & 59 & 168 & 93 \\
\hline
\end{tabular}


Table C. 8. Measured and theoretical VFAs over time in hydrolysis/acidification of manure and SSO

\begin{tabular}{|c|c|c|c|c|c|c|c|}
\hline \multicolumn{8}{|c|}{ Measured VFAs concentrations (mg/L)over time } \\
\hline & Mixture 1 & Mixture 2 & Mixture 3 & Mixture 4 & Mixture 5 & Mixture 6 & Mixture 7 \\
\hline $\begin{array}{l}\text { Time } \\
(\mathrm{hr})\end{array}$ & Manure & SSO & $\begin{array}{c}\text { Manure/SSO } \\
9 / 1\end{array}$ & $\begin{array}{c}\text { Manure/SSO } \\
7 / 3\end{array}$ & $\begin{array}{c}\text { Manure/SSO } \\
5 / 5\end{array}$ & $\begin{array}{c}\text { Manure/SSO } \\
3 / 7\end{array}$ & $\begin{array}{c}\text { Manure/SSO } \\
1 / 9\end{array}$ \\
\hline 0 & 668 & 635 & 646 & 649 & 666 & 740 & 722 \\
\hline 6 & 783 & 790 & 661 & 688 & 749 & 861 & 803 \\
\hline 12 & 803 & 1000 & 864 & 72 & 855 & 917 & 973 \\
\hline 24 & 1119 & 1178 & 983 & 843 & 1092 & 1054 & 1178 \\
\hline 48 & 1204 & 1365 & 1025 & 975 & 1143 & 1299 & 2756 \\
\hline 72 & 1114 & 1102 & 1227 & 1237 & 1258 & 1338 & 1408 \\
\hline \multicolumn{8}{|c|}{ Theoretical VFAs concentrations (mg/L)over time } \\
\hline $\begin{array}{l}\text { Time } \\
(\mathrm{hr})\end{array}$ & Manure & SSO & $\begin{array}{c}\text { Manure/SSO } \\
9 / 1\end{array}$ & $\begin{array}{c}\text { Manure/SSO } \\
7 / 3\end{array}$ & $\begin{array}{c}\text { Manure/SSO } \\
5 / 5\end{array}$ & $\begin{array}{c}\text { Manure/SSO } \\
3 / 7\end{array}$ & $\begin{array}{c}\text { Manure/SSO } \\
1 / 9\end{array}$ \\
\hline 0 & 668 & 635 & 665 & 658 & 651 & 645 & 638 \\
\hline 6 & 783 & 790 & 784 & 785 & 786 & 788 & 789 \\
\hline 12 & 803 & 1000 & 823 & 862 & 902 & 941 & 980 \\
\hline 24 & 1119 & 1178 & 1125 & 1137 & 1148 & 1160 & 1172 \\
\hline 48 & 1204 & 1365 & 1220 & 1252 & 1284 & 1317 & 1349 \\
\hline 72 & 1114 & 1102 & 1113 & 1110 & 1108 & 1106 & 1103 \\
\hline
\end{tabular}


Table C. 9. Concentration of soluble and particulate COD over time in hydrolysis/acidification of manure and SSO

\begin{tabular}{|c|c|c|c|c|c|c|c|}
\hline \multicolumn{8}{|c|}{ Soluble COD concentrations ( $\mathrm{mg} / \mathrm{L}$ ) ocer time } \\
\hline $\begin{array}{l}\text { Time } \\
(\mathrm{hr})\end{array}$ & $\begin{array}{c}\text { Manure/SSO } \\
7 / 3\end{array}$ & $\begin{array}{c}\text { Manure/SSO } \\
5 / 5\end{array}$ & $\begin{array}{c}\text { Manure/SSO } \\
3 / 7\end{array}$ & $\begin{array}{c}\text { Manure/SSO } \\
1 / 9\end{array}$ & $\begin{array}{c}\text { Manure/SSO } \\
9 / 1\end{array}$ & $\begin{array}{c}\text { Manure } \\
\text { only }\end{array}$ & $\begin{array}{l}\text { SSO } \\
\text { only }\end{array}$ \\
\hline 0 & 3986 & 4710 & 5624 & 6555 & 3460 & 2860 & 6980 \\
\hline 2 & 4580 & 5159 & 5941 & 6771 & 3860 & 2940 & 6992 \\
\hline 4 & 5336 & 6273 & 6273 & 6887 & 4440 & 3131 & 7009 \\
\hline 6 & 5559 & 6379 & 6379 & 7004 & 4725 & 3659 & 7015 \\
\hline 8 & 5751 & 6469 & 6469 & 7105 & 4927 & 3936 & 7026 \\
\hline 10 & 5974 & 6500 & 6469 & 7105 & 5280 & 4338 & 7040 \\
\hline 12 & 6127 & 6530 & 6509 & 7149 & 5525 & 4790 & 7043 \\
\hline 24 & 6326 & 6547 & 6547 & 7193 & 6268 & 4935 & 7052 \\
\hline 48 & 6543 & 6639 & 6639 & 7295 & 6675 & 5340 & 7061 \\
\hline 72 & 7189 & 7120 & 6934 & 7418 & 7355 & 5650 & 7080 \\
\hline \multicolumn{8}{|c|}{ Particulate COD concentrations (mg/L) over time } \\
\hline $\begin{array}{l}\text { Time } \\
(\mathrm{hr})\end{array}$ & $\begin{array}{c}\text { Manure/SSO } \\
7 / 3\end{array}$ & $\begin{array}{c}\text { Manure/SSO } \\
5 / 5\end{array}$ & $\begin{array}{c}\text { Manure/SSO } \\
3 / 7\end{array}$ & $\begin{array}{c}\text { Manure/SSO } \\
1 / 9\end{array}$ & $\begin{array}{c}\text { Manure/SSO } \\
9 / 1\end{array}$ & $\begin{array}{l}\text { Manure } \\
\text { only }\end{array}$ & $\begin{array}{l}\text { SSO } \\
\text { only }\end{array}$ \\
\hline 0 & 23605 & 22489 & 20787 & 19466 & 25746 & 26535 & 18565 \\
\hline 2 & 23298 & 21940 & 20539 & 19250 & 25160 & 26155 & 18446 \\
\hline 4 & 22780 & 21426 & 20176 & 19085 & 24726 & 25940 & 18279 \\
\hline 6 & 22489 & 21270 & 20099 & 18957 & 24141 & 25766 & 18165 \\
\hline 8 & 22385 & 21130 & 19887 & 18895 & 23749 & 25665 & 18050 \\
\hline 10 & 22205 & 21045 & 19830 & 18796 & 23470 & 25560 & 17955 \\
\hline 12 & 21935 & 20989 & 19695 & 18752 & 23255 & 25340 & 17915 \\
\hline 24 & 21325 & 20542 & 19280 & 18508 & 22410 & 24795 & 17755 \\
\hline 48 & 20895 & 20255 & 18965 & 18430 & 21978 & 24535 & 17646 \\
\hline 72 & 20809 & 20195 & 18925 & 18405 & 21860 & 24400 & 17614 \\
\hline
\end{tabular}




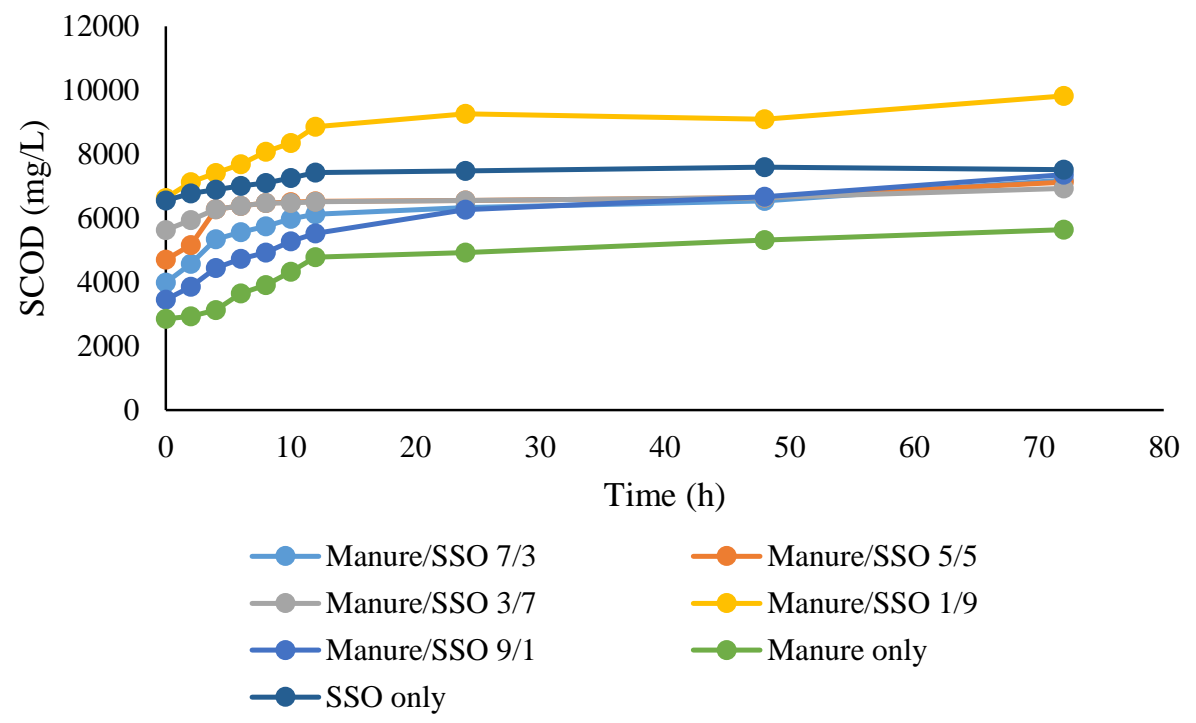

Figure C.2. Concentration of soluble COD over time at different mixing ratios 


\section{Analytical results for AnCoD of TWAS, manure and SSO}

Table D. 1. Characteristics of raw feedstocks in AnCoD of TWAS, Manure, and SSO

\begin{tabular}{cccccccccc}
\hline \multirow{2}{*}{ Parameters } & \multirow{2}{*}{ Units } & \multicolumn{2}{c}{ TWAS } & \multicolumn{2}{c}{ Manure } & \multicolumn{2}{c}{ SSO } & \multicolumn{2}{c}{ Seed } \\
& & MEAN & SD & MEAN & SD & MEAN & SD & MEAN & SD \\
\hline TCOD & $\mathrm{mg} / \mathrm{L}$ & 39977 & 1199 & 100126 & 3004 & 108560 & 3257 & 17105 & 513 \\
SCOD & $\mathrm{mg} / \mathrm{L}$ & 1354 & 41 & 42116 & 1263 & 40855 & 1225.65 & 761 & 23 \\
TSS & $\mathrm{mg} / \mathrm{L}$ & 31445 & 943 & 52380 & 1571 & 62136 & 1864 & 16055 & 482 \\
VSS & $\mathrm{mg} / \mathrm{L}$ & 26480 & 794 & 45420 & 1363 & 46990 & 1410 & 12781 & 383 \\
TS & $\mathrm{mg} / \mathrm{L}$ & 38944 & 1168 & 67785 & 2034 & 66985 & 2010 & 17125 & 514 \\
VS & $\mathrm{mg} / \mathrm{L}$ & 35155 & 1055 & 55590 & 1668 & 49585 & 1488 & 14380 & 431 \\
Ammonia & $\mathrm{mg} / \mathrm{L}$ & 218 & 7 & 18 & 1 & 1289 & 39 & 800 & 24 \\
pH & & 6.5 & 0.2 & 6.7 & 0.2 & 5.7 & 0.17 & 7.2 & 0.2 \\
Alkalinity & $\mathrm{mg} \mathrm{CaCO3/L}$ & 1888 & 57 & 5224 & 157 & 6227 & 187 & 4215 & 126 \\
TN & $\mathrm{mg} / \mathrm{L}$ & 2754 & 83 & 2110 & 63 & 3970 & 119 & 1975 & 59 \\
TSN & $\mathrm{mg} / \mathrm{L}$ & 376 & 11 & 125 & 4 & 965 & 29 & 716 & 21 \\
Total Carbs & $\mathrm{mg} / \mathrm{L}$ & 1524 & 46 & 27842 & 835 & 14126 & 424 & 839 & 25 \\
Total & $\mathrm{mg} / \mathrm{L}$ & 3892 & 117 & 5762 & 173 & 2420 & 73 & 1917 & 58 \\
Proteins & & & & & & & & \\
Total Lipids & $\mathrm{mg} / \mathrm{L}$ & 396 & 12 & 1356 & 41 & 1494 & 45 & 191 & 6 \\
\hline
\end{tabular}


Table D. 2. Average $\mathrm{CH}_{4}$ measurements $(\mathrm{mL})$ in AnCoD of TWAS/Mnaure/SSO (T: TWAS, M: Manure)

\begin{tabular}{|c|c|c|c|c|c|c|c|c|c|c|c|c|}
\hline Time (day) & TWAS & Manure & SSO & $\begin{array}{c}\text { T/M/SSO } \\
8 / 1 / 1 \\
\end{array}$ & $\begin{array}{c}\mathrm{T} / \mathrm{M} / \mathrm{SSO} \\
1 / 8 / 1 \\
\end{array}$ & $\begin{array}{c}\mathrm{T} / \mathrm{M} / \mathrm{SSO} \\
1 / 1 / 8 \\
\end{array}$ & $\begin{array}{c}\mathrm{T} / \mathrm{M} / \mathrm{SSO} \\
0.5 / 0.25 / 0.25 \\
\end{array}$ & $\begin{array}{c}\mathrm{T} / \mathrm{M} / \mathrm{SSO} \\
0.25 / 0.5 / 0.25\end{array}$ & $\begin{array}{c}\mathrm{T} / \mathrm{M} / \mathrm{SSO} \\
0.25 / 0.25 / 0.5 \\
\end{array}$ & $\begin{array}{c}\mathrm{T} / \mathrm{M} / \mathrm{SSO} \\
0.4 / 0.4 / 0.2 \\
\end{array}$ & $\begin{array}{c}\mathrm{T} / \mathrm{M} / \mathrm{SSO} \\
0.2 / 0.4 / 0.4\end{array}$ & $\begin{array}{c}\mathrm{T} / \mathrm{M} / \mathrm{SSO} \\
0.4 / 0.2 / 0.4 \\
\end{array}$ \\
\hline 0 & 0 & 0 & 0 & 0 & 0 & 0 & 0 & 0 & 0 & 0 & 0 & 0 \\
\hline 1 & 5 & 47 & 6 & 12 & 22 & 21 & 9 & 52 & 25 & 7 & 37 & 8 \\
\hline 3 & 21 & 73 & 48 & 32 & 71 & 68 & 55 & 95 & 60 & 54 & 64 & 42 \\
\hline 4 & 39 & 101 & 63 & 34 & 104 & 97 & 77 & 118 & 79 & 71 & 97 & 79 \\
\hline 5 & 52 & 101 & 98 & 57 & 143 & 136 & 105 & 124 & 115 & 104 & 118 & 113 \\
\hline 6 & 34 & 116 & 113 & 67 & 106 & 114 & 94 & 141 & 112 & 102 & 123 & 104 \\
\hline 7 & 33 & 139 & 112 & 69 & 105 & 108 & 97 & 98 & 116 & 106 & 125 & 106 \\
\hline 8 & 19 & 79 & 120 & 71 & 86 & 81 & 92 & 102 & 120 & 110 & 133 & 103 \\
\hline 10 & 25 & 77 & 128 & 76 & 83 & 85 & 81 & 95 & 107 & 97 & 106 & 95 \\
\hline 12 & 23 & 70 & 121 & 71 & 79 & 81 & 77 & 96 & 101 & 92 & 101 & 90 \\
\hline 14 & 19 & 91 & 90 & 54 & 65 & 66 & 63 & 63 & 81 & 74 & 84 & 73 \\
\hline 16 & 17 & 56 & 60 & 35 & 60 & 61 & 61 & 65 & 72 & 65 & 81 & 64 \\
\hline 19 & 21 & 58 & 54 & 32 & 61 & 63 & 52 & 51 & 59 & 53 & 61 & 55 \\
\hline 22 & 16 & 40 & 56 & 33 & 52 & 53 & 49 & 47 & 58 & 53 & 62 & 53 \\
\hline 25 & 13 & 40 & 47 & 28 & 42 & 43 & 38 & 36 & 46 & 41 & 47 & 42 \\
\hline 28 & 9 & 32 & 33 & 19 & 31 & 32 & 30 & 56 & 36 & 33 & 39 & 32 \\
\hline 31 & 29 & 24 & 78 & 46 & 78 & 80 & 58 & 29 & 67 & 61 & 62 & 66 \\
\hline 34 & 12 & 22 & 28 & 16 & 33 & 34 & 26 & 27 & 29 & 27 & 30 & 28 \\
\hline 37 & 10 & 19 & 30 & 10 & 29 & 30 & 24 & 24 & 29 & 26 & 20 & 27 \\
\hline 40 & 9 & 15 & 30 & 9 & 26 & 27 & 22 & 18 & 27 & 24 & 16 & 25 \\
\hline 43 & 5 & 11 & 26 & 6 & 17 & 17 & 16 & 9 & 21 & 19 & 10 & 15 \\
\hline 46 & 3 & 3 & 18 & 4 & 11 & 11 & 12 & 6 & 16 & 15 & 4 & 11 \\
\hline 49 & 2 & 3 & 9 & 2 & 6 & 6 & 6 & 3 & 8 & 7 & 3 & 6 \\
\hline 52 & 1 & 0 & 5 & 1 & 3 & 4 & 4 & 1 & 5 & 4 & 1 & 3 \\
\hline 58 & 0 & 0 & 2 & 0 & 0 & 0 & 1 & 1 & 1 & 1 & 1 & 1 \\
\hline
\end{tabular}

* M: Manure 
Table D. 3. Cumulative $\mathrm{CH}_{4}$ production (mL) in AnCoD of TWAS/Mnaure/SSO (T: TWAS, M:Manure)

\begin{tabular}{|c|c|c|c|c|c|c|c|c|c|c|c|c|}
\hline $\begin{array}{l}\text { Time } \\
\text { (day) }\end{array}$ & TWAS & Manure & SSO & $\begin{array}{c}\mathrm{T} / \mathrm{M} / \mathrm{SSO} \\
8 / 1 / 1 \\
\end{array}$ & $\begin{array}{c}\mathrm{T} / \mathrm{M} / \mathrm{SSO} \\
1 / 8 / 1 \\
\end{array}$ & $\begin{array}{c}\mathrm{T} / \mathrm{M} / \mathrm{SSO} \\
1 / 1 / 8\end{array}$ & $\begin{array}{c}\mathrm{T} / \mathrm{M} / \mathrm{SSO} \\
0.5 / 0.25 / 0.25\end{array}$ & $\begin{array}{c}\mathrm{T} / \mathrm{M} / \mathrm{SSO} \\
0.25 / 0.5 / 0.25\end{array}$ & $\begin{array}{c}\mathrm{T} / \mathrm{M} / \mathrm{SSO} \\
0.25 / 0.25 / 0.5\end{array}$ & $\begin{array}{c}\mathrm{T} / \mathrm{M} / \mathrm{SSO} \\
0.4 / 0.4 / 0.2\end{array}$ & $\begin{array}{c}\mathrm{T} / \mathrm{M} / \mathrm{SSO} \\
0.2 / 0.4 / 0.4\end{array}$ & $\begin{array}{c}\mathrm{T} / \mathrm{M} / \mathrm{SSO} \\
0.4 / 0.2 / 0.4\end{array}$ \\
\hline 0 & 0 & 0 & $\overline{0}$ & 0 & 0 & 0 & 0 & 0 & 0 & 0 & 0 & 0 \\
\hline 1 & 5 & 48 & 6 & 12 & 18 & 24 & 9 & 52 & 25 & 8 & 35 & 8 \\
\hline 3 & 27 & 120 & 54 & 44 & 89 & 93 & 64 & 147 & 85 & 62 & 99 & 50 \\
\hline 4 & 65 & 222 & 116 & 78 & 194 & 189 & 141 & 265 & 164 & 132 & 196 & 129 \\
\hline 5 & 117 & 323 & 215 & 135 & 336 & 325 & 246 & 389 & 278 & 237 & 314 & 242 \\
\hline 6 & 152 & 440 & 327 & 202 & 442 & 439 & 340 & 530 & 391 & 339 & 437 & 346 \\
\hline 7 & 185 & 579 & 440 & 271 & 548 & 547 & 437 & 628 & 507 & 445 & 562 & 452 \\
\hline 8 & 203 & 658 & 559 & 342 & 633 & 628 & 529 & 730 & 627 & 554 & 696 & 555 \\
\hline 10 & 228 & 735 & 687 & 417 & 717 & 713 & 610 & 825 & 734 & 652 & 802 & 650 \\
\hline 12 & 251 & 805 & 808 & 489 & 796 & 793 & 687 & 921 & 836 & 744 & 903 & 741 \\
\hline 14 & 270 & 895 & 898 & 542 & 860 & 859 & 750 & 984 & 917 & 818 & 986 & 813 \\
\hline 16 & 287 & 951 & 958 & 577 & 920 & 921 & 811 & 1049 & 989 & 883 & 1068 & 877 \\
\hline 19 & 307 & 1010 & 1012 & 609 & 981 & 983 & 862 & 1101 & 1047 & 937 & 1129 & 932 \\
\hline 22 & 324 & 1049 & 1067 & 642 & 1034 & 1037 & 911 & 1148 & 1105 & 989 & 1191 & 985 \\
\hline 25 & 337 & 1089 & 1115 & 670 & 1075 & 1079 & 948 & 1184 & 1151 & 1031 & 1238 & 1027 \\
\hline 28 & 346 & 1121 & 1148 & 690 & 1107 & 1111 & 978 & 1239 & 1187 & 1063 & 1277 & 1059 \\
\hline 31 & 375 & 1146 & 1225 & 736 & 1185 & 1191 & 1037 & 1268 & 1254 & 1125 & 1339 & 1125 \\
\hline 34 & 387 & 1167 & 1253 & 752 & 1218 & 1225 & 1063 & 1295 & 1283 & 1151 & 1369 & 1153 \\
\hline 37 & 397 & 1186 & 1284 & 762 & 1247 & 1255 & 1087 & 1319 & 1312 & 1177 & 1389 & 1180 \\
\hline 40 & 406 & 1201 & 1314 & 771 & 1273 & 1281 & 1109 & 1336 & 1338 & 1202 & 1405 & 1205 \\
\hline 43 & 411 & 1212 & 1339 & 777 & 1290 & 1298 & 1125 & 1345 & 1360 & 1221 & 1415 & 1220 \\
\hline 46 & 414 & 1215 & 1357 & 781 & 1301 & 1310 & 1137 & 1351 & 1376 & 1236 & 1419 & 1232 \\
\hline 49 & 416 & 1218 & 1366 & 783 & 1307 & 1316 & 1142 & 1354 & 1384 & 1243 & 1422 & 1237 \\
\hline 52 & 417 & 1218 & 1371 & 784 & 1310 & 1320 & 1146 & 1355 & 1388 & 1247 & 1423 & 1241 \\
\hline 58 & 417 & 1218 & 1373 & 784 & 1311 & 1320 & 1146 & 1355 & 1390 & 1248 & 1424 & 1241 \\
\hline
\end{tabular}

* M: Manure 
Table D. 4. Cumulative $\mathrm{CH}_{4}$ production per mass of COD added in AnCoD of TWAS/Mnaure/SSO (T: TWAS, M: Manure)

\begin{tabular}{|c|c|c|c|c|c|c|c|c|c|c|c|c|}
\hline $\begin{array}{l}\text { Time } \\
\text { (day) }\end{array}$ & TWAS & Manure & SSO & $\begin{array}{c}\mathrm{T} / \mathrm{M} / \mathrm{SSO} \\
8 / 1 / 1\end{array}$ & $\begin{array}{c}\mathrm{T} / \mathrm{M} / \mathrm{SSO} \\
1 / 8 / 1\end{array}$ & $\begin{array}{c}\mathrm{T} / \mathrm{M} / \mathrm{SSO} \\
1 / 1 / 8\end{array}$ & $\begin{array}{c}\mathrm{T} / \mathrm{M} / \mathrm{SSO} \\
0.5 / 0.25 / 0.25\end{array}$ & $\begin{array}{c}\mathrm{T} / \mathrm{M} / \mathrm{SSO} \\
0.25 / 0.5 / 0.25\end{array}$ & $\begin{array}{c}\mathrm{T} / \mathrm{M} / \mathrm{SSO} \\
0.25 / 0.25 / 0.5\end{array}$ & $\begin{array}{c}\mathrm{T} / \mathrm{M} / \mathrm{SSO} \\
0.4 / 0.4 / 0.2\end{array}$ & $\begin{array}{c}\mathrm{T} / \mathrm{M} / \mathrm{SSO} \\
0.2 / 0.4 / 0.4\end{array}$ & $\begin{array}{c}\mathrm{T} / \mathrm{M} / \mathrm{SSO} \\
0.4 / 0.2 / 0.4\end{array}$ \\
\hline 0 & 0 & 0 & 0 & 0 & 0 & 0 & 0 & 0 & 0 & 0 & 0 & 0 \\
\hline 1 & 2 & 12 & 1 & 4 & 4 & 6 & 2 & 13 & 6 & 2 & 9 & 2 \\
\hline 3 & 9 & 30 & 13 & 13 & 22 & 23 & 17 & 37 & 21 & 16 & 25 & 13 \\
\hline 4 & 21 & 54 & 28 & 23 & 48 & 46 & 37 & 67 & 41 & 34 & 49 & 33 \\
\hline 5 & 38 & 79 & 52 & 39 & 83 & 80 & 65 & 98 & 70 & 62 & 79 & 63 \\
\hline 6 & 49 & 108 & 79 & 59 & 110 & 108 & 90 & 134 & 98 & 88 & 109 & 89 \\
\hline 7 & 59 & 142 & 106 & 79 & 136 & 134 & 116 & 159 & 128 & 116 & 141 & 117 \\
\hline 8 & 65 & 162 & 135 & 99 & 157 & 154 & 140 & 185 & 158 & 144 & 174 & 144 \\
\hline 10 & 73 & 180 & 166 & 121 & 178 & 175 & 162 & 209 & 185 & 169 & 201 & 168 \\
\hline 12 & 80 & 198 & 195 & 142 & 198 & 195 & 182 & 233 & 210 & 193 & 226 & 191 \\
\hline 14 & 87 & 220 & 217 & 157 & 214 & 211 & 199 & 249 & 231 & 213 & 247 & 210 \\
\hline 16 & 92 & 234 & 231 & 168 & 228 & 226 & 215 & 265 & 249 & 230 & 267 & 227 \\
\hline 19 & 99 & 248 & 244 & 177 & 244 & 241 & 228 & 278 & 263 & 243 & 283 & 241 \\
\hline 22 & 104 & 258 & 258 & 186 & 257 & 254 & 241 & 290 & 278 & 257 & 298 & 255 \\
\hline 25 & 108 & 267 & 269 & 195 & 267 & 265 & 251 & 299 & 290 & 268 & 310 & 266 \\
\hline 28 & 111 & 275 & 277 & 200 & 275 & 272 & 259 & 314 & 299 & 276 & 320 & 274 \\
\hline 31 & 120 & 281 & 296 & 214 & 294 & 292 & 275 & 321 & 315 & 292 & 335 & 291 \\
\hline 34 & 124 & 287 & 303 & 218 & 302 & 300 & 282 & 328 & 323 & 299 & 343 & 298 \\
\hline 37 & 127 & 291 & 310 & 221 & 310 & 308 & 288 & 334 & 330 & 306 & 348 & 305 \\
\hline 40 & 130 & 295 & 317 & 224 & 316 & 314 & 294 & 338 & 337 & 312 & 352 & 312 \\
\hline 43 & 132 & 298 & 324 & 225 & 320 & 318 & 298 & 340 & 342 & 317 & 354 & 315 \\
\hline 46 & 133 & 298 & 328 & 227 & 323 & 321 & 301 & 342 & 346 & 321 & 355 & 318 \\
\hline 49 & 133 & 299 & 330 & 227 & 324 & 323 & 303 & 343 & 348 & 323 & 356 & 320 \\
\hline 52 & 134 & 299 & 331 & 228 & 325 & 324 & 303 & 343 & 349 & 324 & 356 & 321 \\
\hline 58 & 134 & 299 & 332 & 228 & 325 & 324 & 304 & 343 & 350 & 324 & 356 & 321 \\
\hline
\end{tabular}

*T: TWAS

* M: Manure 
Table D. 5. Cumulative $\mathrm{CH}_{4}$ production per mass of VSS added in AnCoD of TWAS/Mnaure/SSO (T: TWAS, M: Manure)

\begin{tabular}{|c|c|c|c|c|c|c|c|c|c|c|c|c|}
\hline $\begin{array}{l}\text { Time } \\
\text { (day) }\end{array}$ & $\begin{array}{c}\text { TWAS } \\
\text { Only }\end{array}$ & $\begin{array}{c}\text { Manure } \\
\text { Only }\end{array}$ & $\begin{array}{l}\text { SSO } \\
\text { only } \\
\end{array}$ & $\begin{array}{c}\text { T/M/SSO } \\
8 / 1 / 1 \\
\end{array}$ & $\begin{array}{c}\text { T/M/SSO } \\
1 / 8 / 1 \\
\end{array}$ & $\begin{array}{c}\mathrm{T} / \mathrm{M} / \mathrm{SSO} \\
1 / 1 / 8 \\
\end{array}$ & $\begin{array}{c}\mathrm{T} / \mathrm{M} / \mathrm{SSO} \\
0.5 / 0.25 / 0.25\end{array}$ & $\begin{array}{c}\mathrm{T} / \mathrm{M} / \mathrm{SSO} \\
0.25 / 0.5 / 0.25\end{array}$ & $\begin{array}{c}\mathrm{T} / \mathrm{M} / \mathrm{SSO} \\
0.25 / 0.25 / 0.5 \\
\end{array}$ & $\begin{array}{c}\mathrm{T} / \mathrm{M} / \mathrm{SSO} \\
0.4 / 0.4 / 0.2 \\
\end{array}$ & $\begin{array}{c}\text { T/M/SSO } \\
0.2 / 0.4 / 0.4 \\
\end{array}$ & $\begin{array}{c}\mathrm{T} / \mathrm{M} / \mathrm{SSO} \\
0.4 / 0.2 / 0.4 \\
\end{array}$ \\
\hline 0 & 0 & 0 & 0 & 0 & 0 & 0 & 0 & 0 & 0 & 0 & 0 & 0 \\
\hline 1 & 3 & 26 & 27 & 6 & 10 & 13 & 5 & 28 & 14 & 4 & 24 & 4 \\
\hline 3 & 13 & 65 & 67 & 22 & 48 & 51 & 34 & 79 & 46 & 33 & 68 & 27 \\
\hline 4 & 32 & 120 & 124 & 39 & 104 & 105 & 74 & 142 & 89 & 70 & 135 & 69 \\
\hline 5 & 57 & 175 & 181 & 68 & 181 & 180 & 129 & 209 & 151 & 125 & 217 & 129 \\
\hline 6 & 73 & 238 & 245 & 102 & 239 & 242 & 179 & 285 & 212 & 179 & 302 & 185 \\
\hline 7 & 89 & 313 & 323 & 137 & 296 & 302 & 230 & 337 & 275 & 235 & 388 & 241 \\
\hline 8 & 98 & 356 & 367 & 172 & 342 & 347 & 278 & 392 & 340 & 294 & 480 & 296 \\
\hline 10 & 110 & 398 & 410 & 210 & 387 & 394 & 321 & 443 & 398 & 345 & 553 & 347 \\
\hline 12 & 122 & 436 & 449 & 246 & 429 & 438 & 361 & 494 & 453 & 394 & 623 & 395 \\
\hline 14 & 131 & 485 & 500 & 273 & 464 & 475 & 395 & 528 & 497 & 433 & 681 & 434 \\
\hline 16 & 139 & 515 & 531 & 291 & 496 & 508 & 426 & 563 & 536 & 468 & 737 & 468 \\
\hline 19 & 149 & 546 & 564 & 307 & 530 & 543 & 454 & 591 & 567 & 496 & 779 & 498 \\
\hline 22 & 157 & 568 & 586 & 324 & 558 & 572 & 479 & 616 & 599 & 524 & 822 & 526 \\
\hline 25 & 163 & 589 & 608 & 338 & 580 & 596 & 499 & 636 & 623 & 546 & 854 & 548 \\
\hline 28 & 168 & 607 & 626 & 348 & 597 & 614 & 515 & 665 & 643 & 563 & 881 & 566 \\
\hline 31 & 182 & 620 & 640 & 371 & 639 & 658 & 545 & 681 & 679 & 596 & 924 & 601 \\
\hline 34 & 187 & 632 & 652 & 379 & 657 & 676 & 559 & 695 & 695 & 610 & 944 & 616 \\
\hline 37 & 192 & 642 & 662 & 384 & 673 & 693 & 572 & 708 & 711 & 623 & 958 & 630 \\
\hline 40 & 196 & 650 & 671 & 389 & 687 & 708 & 583 & 718 & 725 & 636 & 969 & 643 \\
\hline 43 & 199 & 656 & 677 & 392 & 696 & 717 & 592 & 722 & 737 & 647 & 976 & 651 \\
\hline 46 & 200 & 658 & 678 & 394 & 702 & 723 & 598 & 726 & 745 & 654 & 979 & 657 \\
\hline 49 & 201 & 659 & 680 & 395 & 705 & 727 & 601 & 727 & 750 & 658 & 981 & 660 \\
\hline 52 & 202 & 659 & 680 & 395 & 707 & 729 & 603 & 727 & 752 & 661 & 982 & 662 \\
\hline 58 & 202 & 659 & 680 & 395 & 707 & 729 & 603 & 728 & 753 & 661 & 982 & 663 \\
\hline
\end{tabular}

* M: Manure 
Table D. 6. Cumulative $\mathrm{CH}_{4}$ production per volume of substrate added in AnCoD of TWAS/Mnaure/SSO (T: TWAS, M: Manure)

\begin{tabular}{|c|c|c|c|c|c|c|c|c|c|c|c|c|}
\hline $\begin{array}{l}\text { Time } \\
\text { (day) }\end{array}$ & $\begin{array}{l}\text { TWAS } \\
\text { Only }\end{array}$ & $\begin{array}{l}\text { Manure } \\
\text { Only }\end{array}$ & $\begin{array}{l}\text { SSO } \\
\text { only }\end{array}$ & $\begin{array}{c}\text { T/M/SSO } \\
8 / 1 / 1\end{array}$ & $\begin{array}{c}\mathrm{T} / \mathrm{M} / \mathrm{SSO} \\
1 / 8 / 1\end{array}$ & $\begin{array}{c}\mathrm{T} / \mathrm{M} / \mathrm{SSO} \\
1 / 1 / 8\end{array}$ & $\begin{array}{c}\mathrm{T} / \mathrm{M} / \mathrm{SSO} \\
0.5 / 0.25 / 0.25\end{array}$ & $\begin{array}{c}\mathrm{T} / \mathrm{M} / \mathrm{SSO} \\
0.25 / 0.5 / 0.25\end{array}$ & $\begin{array}{c}\mathrm{T} / \mathrm{M} / \mathrm{SSO} \\
0.25 / 0.25 / 0.5\end{array}$ & $\begin{array}{c}\mathrm{T} / \mathrm{M} / \mathrm{SSO} \\
0.4 / 0.4 / 0.2\end{array}$ & $\begin{array}{c}\mathrm{T} / \mathrm{M} / \mathrm{SSO} \\
0.2 / 0.4 / 0.4\end{array}$ & $\begin{array}{c}\text { T/M/SSO } \\
0.4 / 0.2 / 0.4\end{array}$ \\
\hline 0 & 0 & 0 & 0 & 0 & 0 & 0 & 0 & 0 & 0 & 0 & 0 & 0 \\
\hline 1 & 0 & 1 & 0 & 0 & 0 & 1 & 0 & 1 & 1 & 0 & 1 & 0 \\
\hline 3 & 0 & 3 & 1 & 1 & 2 & 2 & 1 & 3 & 2 & 1 & 2 & 1 \\
\hline 4 & 1 & 5 & 3 & 1 & 5 & 5 & 1 & 6 & 4 & 3 & 4 & 3 \\
\hline 5 & 2 & 8 & 6 & 2 & 8 & 8 & 2 & 9 & 6 & 5 & 7 & 5 \\
\hline 6 & 2 & 11 & 9 & 3 & 10 & 11 & 3 & 12 & 9 & 7 & 10 & 7 \\
\hline 7 & 2 & 14 & 12 & 4 & 13 & 14 & 4 & 14 & 11 & 9 & 13 & 9 \\
\hline 8 & 3 & 16 & 15 & 5 & 15 & 16 & 5 & 16 & 14 & 11 & 16 & 11 \\
\hline 10 & 3 & 18 & 18 & 6 & 17 & 18 & 6 & 18 & 16 & 13 & 18 & 13 \\
\hline 12 & 3 & 20 & 21 & 7 & 19 & 20 & 7 & 20 & 19 & 15 & 21 & 15 \\
\hline 14 & 3 & 22 & 24 & 8 & 20 & 21 & 8 & 22 & 21 & 17 & 23 & 17 \\
\hline 16 & 4 & 23 & 25 & 9 & 22 & 23 & 8 & 23 & 22 & 18 & 24 & 18 \\
\hline 19 & 4 & 25 & 27 & 9 & 23 & 24 & 9 & 24 & 24 & 19 & 26 & 19 \\
\hline 22 & 4 & 26 & 28 & 10 & 24 & 26 & 9 & 25 & 25 & 20 & 27 & 20 \\
\hline 25 & 4 & 27 & 29 & 10 & 25 & 27 & 10 & 26 & 26 & 21 & 28 & 21 \\
\hline 28 & 4 & 28 & 30 & 11 & 26 & 27 & 10 & 27 & 27 & 21 & 29 & 22 \\
\hline 31 & 5 & 28 & 32 & 11 & 28 & 29 & 10 & 28 & 28 & 23 & 31 & 23 \\
\hline 34 & 5 & 29 & 33 & 12 & 29 & 30 & 11 & 29 & 29 & 23 & 31 & 24 \\
\hline 37 & 5 & 29 & 34 & 12 & 29 & 31 & 11 & 29 & 29 & 24 & 32 & 24 \\
\hline 40 & 5 & 30 & 34 & 12 & 30 & 32 & 11 & 29 & 30 & 24 & 32 & 25 \\
\hline 43 & 5 & 30 & 35 & 12 & 30 & 32 & 11 & 30 & 31 & 25 & 32 & 25 \\
\hline 46 & 5 & 30 & 36 & 12 & 31 & 32 & 11 & 30 & 31 & 25 & 32 & 25 \\
\hline 49 & 5 & 30 & 36 & 12 & 31 & 33 & 11 & 30 & 31 & 25 & 33 & 25 \\
\hline 52 & 5 & 30 & 36 & 12 & 31 & 33 & 12 & 30 & 31 & 25 & 33 & 25 \\
\hline 58 & 5 & 30 & 36 & 12 & 31 & 33 & 12 & 30 & 31 & 25 & 33 & 25 \\
\hline
\end{tabular}

*T: TWAS

* M: Manure 


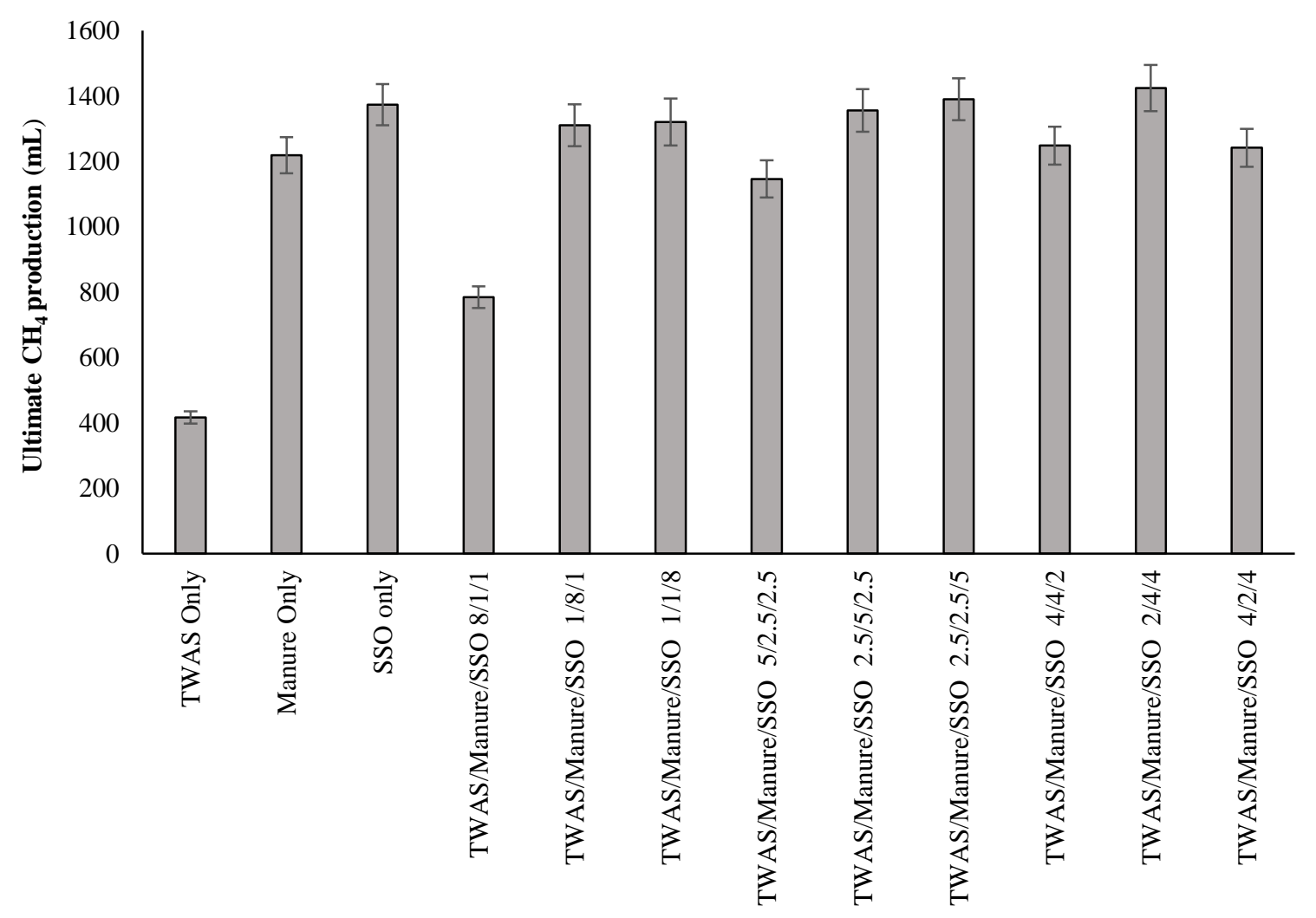

Mixing ratios

Figure D. 1. Ultimate $\mathrm{CH} 4$ production in AnCoD of TWAS/Manure/SSO 
Table D. 7. Characteristics of raw feedstocks for hydrolysis/acidification in AnCoD of TWAS/Manure/SSO

\begin{tabular}{cccccccccc}
\hline \multirow{2}{*}{ Parameters } & \multirow{2}{*}{ Units } & \multicolumn{2}{c}{ TWAS } & \multicolumn{2}{c}{ Manure } & \multicolumn{2}{c}{ SSO } & \multicolumn{2}{c}{ Inoculum } \\
& & MEAN & SD & MEAN & SD & MEAN & SD & MEAN & SD \\
\hline TCOD & $\mathrm{mg} / \mathrm{L}$ & 36875 & 2853 & 100240 & 4014 & 102180 & 4120 & 16854 & 389 \\
SCOD & $\mathrm{mg} / \mathrm{L}$ & 1931 & 26 & 10145 & 405 & 37350 & 1861 & 786 & 22 \\
TSS & $\mathrm{mg} / \mathrm{L}$ & 28934 & 1006 & 54100 & 2164 & 49510 & 2093 & 15022 & 326 \\
VSS & $\mathrm{mg} / \mathrm{L}$ & 22983 & 2216 & 45216 & 1289 & 44130 & 1743 & 10175 & 298 \\
TS & $\mathrm{mg} / \mathrm{L}$ & 35705 & 6859 & 67680 & 2707 & 76878 & 3460 & 20650 & 425 \\
VS & $\mathrm{mg} / \mathrm{L}$ & 31719 & 27 & 57830 & 2313 & 62948 & 2833 & 12988 & 396 \\
Ammonia & $\mathrm{mg} / \mathrm{L}$ & 235 & 30 & 22.3 & 1 & 1326 & 60 & 1478 & 32 \\
pH & - & 6 & 0.1 & 6.8 & 0.2 & 5.8 & 0.2 & 7.1 & 0.2 \\
Alkalinity & $\mathrm{mg} \mathrm{CaCO} / \mathrm{L}$ & 1797 & 148 & 6984 & 279 & 6415 & 289 & 3867 & 135 \\
TN & $\mathrm{mg} / \mathrm{L}$ & 2668 & 400 & 1690 & 68 & 4086 & 184 & 1983 & 69 \\
TSN & $\mathrm{mg} / \mathrm{L}$ & 386 & 64 & 119 & 4 & 1096 & 49 & 679 & 24 \\
Total Carbs & $\mathrm{mg} / \mathrm{L}$ & 849 & 112 & 25968 & 1039 & 13575 & 611 & 548 & 19 \\
Total Proteins & $\mathrm{mg} / \mathrm{L}$ & 2547 & 298 & 5126 & 205 & 2087 & 94 & 1596 & 56 \\
Total Lipids & $\mathrm{mg} / \mathrm{L}$ & 266 & 27 & 1652 & 66 & 1395 & 63 & 153 & 5 \\
\hline
\end{tabular}


Table D. 8. Measured and theoretical VFAs concentrations (mg/L) over time in hydrolysis/acidification of TWAS/Manure/SSO

\begin{tabular}{|c|c|c|c|c|c|c|c|c|c|c|c|c|}
\hline \multicolumn{13}{|c|}{ Measured VFAs concentrations (mg/L) over time } \\
\hline & TWAS & Manure & $\mathrm{SSO}$ & T/M/SSO 8/1/1 & $\begin{array}{c}\mathrm{T} / \mathrm{M} / \mathrm{SSO} \\
1 / 8 / 1\end{array}$ & $\begin{array}{c}\mathrm{T} / \mathrm{M} / \mathrm{SSO} \\
1 / 1 / 8\end{array}$ & $\begin{array}{l}\mathrm{T} / \mathrm{M} / \mathrm{SSO} \\
5 / 2.5 / 2.5\end{array}$ & $\begin{array}{l}\mathrm{T} / \mathrm{M} / \mathrm{SSO} \\
2.5 / 5 / 2.5\end{array}$ & $\begin{array}{l}\mathrm{T} / \mathrm{M} / \mathrm{SSO} \\
2.5 / 2.5 / 5\end{array}$ & $\begin{array}{c}\mathrm{T} / \mathrm{M} / \mathrm{SSO} \\
4 / 4 / 2\end{array}$ & $\begin{array}{c}\mathrm{T} / \mathrm{M} / \mathrm{SSO} \\
2 / 4 / 4\end{array}$ & $\begin{array}{c}\mathrm{T} / \mathrm{M} / \mathrm{SSO} \\
4 / 2 / 4\end{array}$ \\
\hline $\begin{array}{c}\text { Time } \\
\text { (hr) }\end{array}$ & 1 & 2 & 3 & 4 & 5 & 6 & 7 & 8 & 9 & 10 & 11 & 12 \\
\hline 0 & 428 & 1096 & 1538 & 673 & 1353 & 1660 & 977 & 1258 & 1357 & 1055 & 1402 & 1127 \\
\hline 6 & 340 & 1286 & 1914 & 657 & 1580 & 2032 & 1086 & 1459 & 1609 & 1188 & 1658 & 1297 \\
\hline 12 & 441 & 1319 & 2423 & 807 & 1690 & 2537 & 1295 & 1664 & 1949 & 1367 & 1949 & 1578 \\
\hline 24 & 672 & 1837 & 2854 & 1117 & 2296 & 3041 & 1690 & 2178 & 2424 & 1811 & 2474 & 1991 \\
\hline 48 & 744 & 1976 & 3309 & 1247 & 2503 & 3503 & 1897 & 2422 & 2755 & 2012 & 2783 & 2258 \\
\hline 72 & 925 & 2196 & 2671 & 1361 & 2667 & 2939 & 1881 & 2417 & 2497 & 2050 & 2622 & 2103 \\
\hline time $(\mathrm{hr})$ & TWAS & Manure & $\mathrm{SSO}$ & T/M/SSO 8/1/1 & $\begin{array}{l}\text { Theoretic } \\
\text { T/SSO } \\
1 / 8 / 1\end{array}$ & $\begin{array}{l}\text { VFAs concen } \\
\text { T/SSO 1/1/8 }\end{array}$ & $\begin{array}{l}\text { ations (mg/L) ove } \\
\text { T/SSO 5/2.5/2.5 }\end{array}$ & $\begin{array}{l}\text { ne } \\
\text { T/SSO } \\
2.5 / 5 / 2.5\end{array}$ & $\begin{array}{c}\text { T/SSO } \\
2.5 / 2.5 / 5\end{array}$ & $\begin{array}{c}\text { T/SSO } \\
4 / 4 / 2\end{array}$ & $\begin{array}{c}\text { T/SSO } \\
2 / 4 / 4\end{array}$ & $\begin{array}{c}\text { T/SSO } \\
4 / 2 / 4\end{array}$ \\
\hline 0 & 428 & 1096 & 1538 & 606 & 1074 & 1383 & 873 & 1040 & 1150 & 917 & 1140 & 1006 \\
\hline 6 & 340 & 1286 & 1914 & 592 & 1254 & 1694 & 970 & 1206 & 1363 & 1033 & 1348 & 1158 \\
\hline 12 & 441 & 1319 & 2423 & 727 & 1341 & 2114 & 1156 & 1375 & 1651 & 1188 & 1585 & 1409 \\
\hline 24 & 672 & 1837 & 2854 & 1007 & 1823 & 2534 & 1509 & 1800 & 2054 & 1575 & 2011 & 1778 \\
\hline 48 & 744 & 1976 & 3309 & 1124 & 1986 & 2919 & 1693 & 2001 & 2334 & 1750 & 2263 & 2016 \\
\hline 72 & 925 & 2196 & 2671 & 1227 & 2117 & 2449 & 1679 & 1997 & 2116 & 1783 & 2132 & 1878 \\
\hline
\end{tabular}


Table D. 9. Soluble and particulate COD concentrations (mg/L) over time in hydrolysis/acidification of TWAS/Manure/SSO

\begin{tabular}{|c|c|c|c|c|c|c|c|c|c|c|c|c|}
\hline \multicolumn{13}{|c|}{ Soluble COD concentrations $(\mathrm{mg} / \mathrm{L})$ ocer time } \\
\hline Time & TWAS & Manure & SSO & $\begin{array}{c}\mathrm{T} / \mathrm{M} / \mathrm{SSO} \\
8 / 1 / 1\end{array}$ & $\begin{array}{c}\text { T/M/SSO } \\
1 / 8 / 1\end{array}$ & $\begin{array}{c}\mathrm{T} / \mathrm{M} / \mathrm{SSO} \\
1 / 1 / 8\end{array}$ & $\begin{array}{l}\mathrm{T} / \mathrm{M} / \mathrm{SSO} \\
5 / 2.5 / 2.5\end{array}$ & $\begin{array}{l}\mathrm{T} / \mathrm{M} / \mathrm{SSO} \\
2.5 / 5 / 2.5\end{array}$ & $\begin{array}{l}\mathrm{T} / \mathrm{M} / \mathrm{SSO} \\
2.5 / 2.5 / 5\end{array}$ & $\begin{array}{c}\mathrm{T} / \mathrm{M} / \mathrm{SSO} \\
4 / 4 / 2\end{array}$ & $\begin{array}{c}\mathrm{T} / \mathrm{M} / \mathrm{SSO} \\
2 / 4 / 4\end{array}$ & $\begin{array}{c}\mathrm{T} / \mathrm{M} / \mathrm{SSO} \\
4 / 2 / 4\end{array}$ \\
\hline 0 & 1050 & 2803 & 9906 & 2322 & 4106 & 8975 & 4109 & 4886 & 7040 & 3945 & 5955 & 5536 \\
\hline 2 & 1700 & 3169 & 9933 & 2936 & 4548 & 9100 & 4576 & 5299 & 7353 & 4404 & 6274 & 5917 \\
\hline 4 & 1968 & 3375 & 9948 & 3198 & 4787 & 9172 & 4790 & 5507 & 7509 & 4622 & 6438 & 6094 \\
\hline 6 & 2175 & 3945 & 9956 & 3443 & 5374 & 9263 & 5065 & 5906 & 7745 & 4972 & 6745 & 6319 \\
\hline 8 & 2557 & 4243 & 9972 & 3814 & 5716 & 9350 & 5364 & 6200 & 7956 & 5280 & 6972 & 6564 \\
\hline 10 & 2868 & 4677 & 9992 & 4137 & 6184 & 9448 & 5662 & 6553 & 8189 & 5618 & 7246 & 6808 \\
\hline 12 & 3147 & 5163 & 9996 & 4437 & 6697 & 9534 & 5953 & 6923 & 8420 & 5962 & 7530 & 7044 \\
\hline 24 & 3282 & 5320 & 10009 & 4574 & 6870 & 9576 & 6075 & 7059 & 8514 & 6095 & 7636 & 7146 \\
\hline 48 & 3317 & 5757 & 10121 & 4665 & 7317 & 9725 & 6247 & 7361 & 8721 & 6332 & 7891 & 7310 \\
\hline 72 & 3368 & 6837 & 11168 & 4944 & 8516 & 10751 & 6866 & 8322 & 9681 & 7074 & 8145 & 8044 \\
\hline \multicolumn{13}{|c|}{ Particulate COD concentrations (mg/L) over time } \\
\hline Time & TWAS & Manure & SSO & $\begin{array}{c}\mathrm{T} / \mathrm{M} / \mathrm{SSO} \\
8 / 1 / 1\end{array}$ & $\begin{array}{c}\text { T/SSO } \\
1 / 8 / 1\end{array}$ & $\begin{array}{c}\text { T/SSO } \\
1 / 1 / 8\end{array}$ & $\begin{array}{c}\text { T/SSO } \\
5 / 2.5 / 2.5\end{array}$ & $\begin{array}{c}\text { T/SSO } \\
2.5 / 5 / 2.5\end{array}$ & $\begin{array}{c}\text { T/SSO } \\
2.5 / 2.5 / 5\end{array}$ & $\mathrm{~T} / \mathrm{SSO} 4 / 4 / 2$ & $\mathrm{~T} / \mathrm{SSO} 2 / 4 / 4$ & $\begin{array}{c}\mathrm{T} / \mathrm{SSO} \\
4 / 2 / 4\end{array}$ \\
\hline 0 & 23602 & 31182 & 23728 & 25372 & 29385 & 23980 & 26557 & 27793 & 25255 & 27501 & 22242 & 25756 \\
\hline 2 & 22053 & 30915 & 23581 & 24958 & 28895 & 23746 & 26090 & 27380 & 24983 & 27042 & 21923 & 25576 \\
\hline 4 & 21784 & 30659 & 23497 & 24496 & 28656 & 23573 & 25877 & 27072 & 24787 & 26824 & 21779 & 25198 \\
\hline 6 & 21577 & 30240 & 23319 & 24350 & 28265 & 23482 & 25601 & 26472 & 24451 & 26574 & 21452 & 24974 \\
\hline 8 & 21395 & 29942 & 23165 & 23990 & 27923 & 23395 & 25502 & 26279 & 24339 & 25966 & 21225 & 24329 \\
\hline 10 & 21184 & 29788 & 22885 & 23837 & 27555 & 22998 & 25375 & 25726 & 23906 & 25828 & 20951 & 24084 \\
\hline 12 & 20985 & 29586 & 22605 & 23755 & 27382 & 22770 & 25130 & 25455 & 23856 & 25584 & 20467 & 23948 \\
\hline 24 & 20780 & 28875 & 21920 & 23120 & 25960 & 22349 & 24320 & 24919 & 23182 & 24550 & 19461 & 22750 \\
\hline 48 & 20515 & 27730 & 21590 & 22776 & 25141 & 21880 & 23950 & 24108 & 22774 & 24124 & 19181 & 22110 \\
\hline 72 & 20404 & 27511 & 21496 & 22665 & 24990 & 21794 & 23830 & 23978 & 22675 & 23972 & 19055 & 21955 \\
\hline
\end{tabular}

$* \mathrm{~T}:$ TWAS
$* \mathrm{M}$ : Manure 


\begin{tabular}{|c|c|c|c|c|c|c|c|c|c|c|c|c|}
\hline \multicolumn{13}{|c|}{ Soluble COD concentrations (mg/L) over time } \\
\hline & & & & $\mathrm{T} / \mathrm{M} / \mathrm{SSO}$ & $\mathrm{T} / \mathrm{M} / \mathrm{SSO}$ & $\mathrm{T} / \mathrm{M} / \mathrm{SSO}$ & $\mathrm{T} / \mathrm{M} / \mathrm{SSO}$ & $\mathrm{T} / \mathrm{M} / \mathrm{SSO}$ & $\mathrm{T} / \mathrm{M} / \mathrm{SSO}$ & $\mathrm{T} / \mathrm{M} / \mathrm{SSO}$ & $\mathrm{T} / \mathrm{M} / \mathrm{SSO}$ & \\
\hline Time & IWAS & Manure & $\mathrm{SSO}$ & $8 / 1 / 1$ & $1 / 8 / 1$ & $1 / 1 / 8$ & $5 / 2.5 / 2.5$ & $2.5 / 5 / 2.5$ & $2.5 / 2.5 / 5$ & $4 / 4 / 2$ & $2 / 4 / 4$ & 17M/SSU 4/2/4 \\
\hline 0 & 1050 & 2803 & 9906 & 2322 & 4106 & 8975 & 4109 & 4886 & 7040 & 3945 & 5955 & 5536 \\
\hline 2 & 1700 & 3169 & 9933 & 2936 & 4548 & 9100 & 4576 & 5299 & 7353 & 4404 & 6274 & 5917 \\
\hline 4 & 1968 & 3375 & 9948 & 3198 & 4787 & 9172 & 4790 & 5507 & 7509 & 4622 & 6438 & 6094 \\
\hline 6 & 2175 & 3945 & 9956 & 3443 & 5374 & 9263 & 5065 & 5906 & 7745 & 4972 & 6745 & 6319 \\
\hline 8 & 2557 & 4243 & 9972 & 3814 & 5716 & 9350 & 5364 & 6200 & 7956 & 5280 & 6972 & 6564 \\
\hline 10 & 2868 & 4677 & 9992 & 4137 & 6184 & 9448 & 5662 & 6553 & 8189 & 5618 & 7246 & 6808 \\
\hline 12 & 3147 & 5163 & 9996 & 4437 & 6697 & 9534 & 5953 & 6923 & 8420 & 5962 & 7530 & 7044 \\
\hline 24 & 3282 & 5320 & 10009 & 4574 & 6870 & 9576 & 6075 & 7059 & 8514 & 6095 & 7636 & 7146 \\
\hline 48 & 3317 & 5757 & 10121 & 4665 & 7317 & 9725 & 6247 & 7361 & 8721 & 6332 & 7891 & 7310 \\
\hline 72 & 3368 & 6837 & 11168 & 4944 & 8516 & 10751 & 6866 & 8322 & 9681 & 7074 & 8145 & 8044 \\
\hline \multicolumn{13}{|c|}{ Particulate COD concentrations $(\mathrm{mg} / \mathrm{L})$ over time } \\
\hline Time & TWAS & Manure & SSO & $\mathrm{T} / \mathrm{M} / \mathrm{SSO}$ & $\mathrm{T} / \mathrm{SSO}$ & $\mathrm{T} / \mathrm{SSO}$ & $\mathrm{T} / \mathrm{SSO}$ & $\mathrm{T} / \mathrm{SSO}$ & $\mathrm{T} / \mathrm{SSO}$ & $\mathrm{T} / \mathrm{SSO}$ & $\mathrm{T} / \mathrm{SSO}$ & $\mathrm{T} / \mathrm{SSO} 4 / 2 / 4$ \\
\hline lime & 1 W AS & vianure & sso & 8/1/1 & $1 / 8 / 1$ & $1 / 1 / 8$ & $5 / 2.5 / 2.5$ & $2.5 / 5 / 2.5$ & $2.5 / 2.5 / 5$ & $4 / 4 / 2$ & $2 / 4 / 4$ & $1 / 3 \mathrm{SU} 4 / 2 / 4$ \\
\hline 0 & 23602 & 31182 & 23728 & 25372 & 29385 & 23980 & 26557 & 27793 & 25255 & 27501 & 22242 & 25756 \\
\hline 2 & 22053 & 30915 & 23581 & 24958 & 28895 & 23746 & 26090 & 27380 & 24983 & 27042 & 21923 & 25576 \\
\hline 4 & 21784 & 30659 & 23497 & 24496 & 28656 & 23573 & 25877 & 27072 & 24787 & 26824 & 21779 & 25198 \\
\hline 6 & 21577 & 30240 & 23319 & 24350 & 28265 & 23482 & 25601 & 26472 & 24451 & 26574 & 21452 & 24974 \\
\hline 8 & 21395 & 29942 & 23165 & 23990 & 27923 & 23395 & 25502 & 26279 & 24339 & 25966 & 21225 & 24329 \\
\hline 10 & 21184 & 29788 & 22885 & 23837 & 27555 & 22998 & 25375 & 25726 & 23906 & 25828 & 20951 & 24084 \\
\hline 12 & 20985 & 29586 & 22605 & 23755 & 27382 & 22770 & 25130 & 25455 & 23856 & 25584 & 20467 & 23948 \\
\hline 24 & 20780 & 28875 & 21920 & 23120 & 25960 & 22349 & 24320 & 24919 & 23182 & 24550 & 19461 & 22750 \\
\hline 48 & 20515 & 27730 & 21590 & 22776 & 25141 & 21880 & 23950 & 24108 & 22774 & 24124 & 19181 & 22110 \\
\hline 72 & 20404 & 27511 & 21496 & 22665 & 24990 & 21794 & 23830 & 23978 & 22675 & 23972 & 19055 & 21955 \\
\hline
\end{tabular}




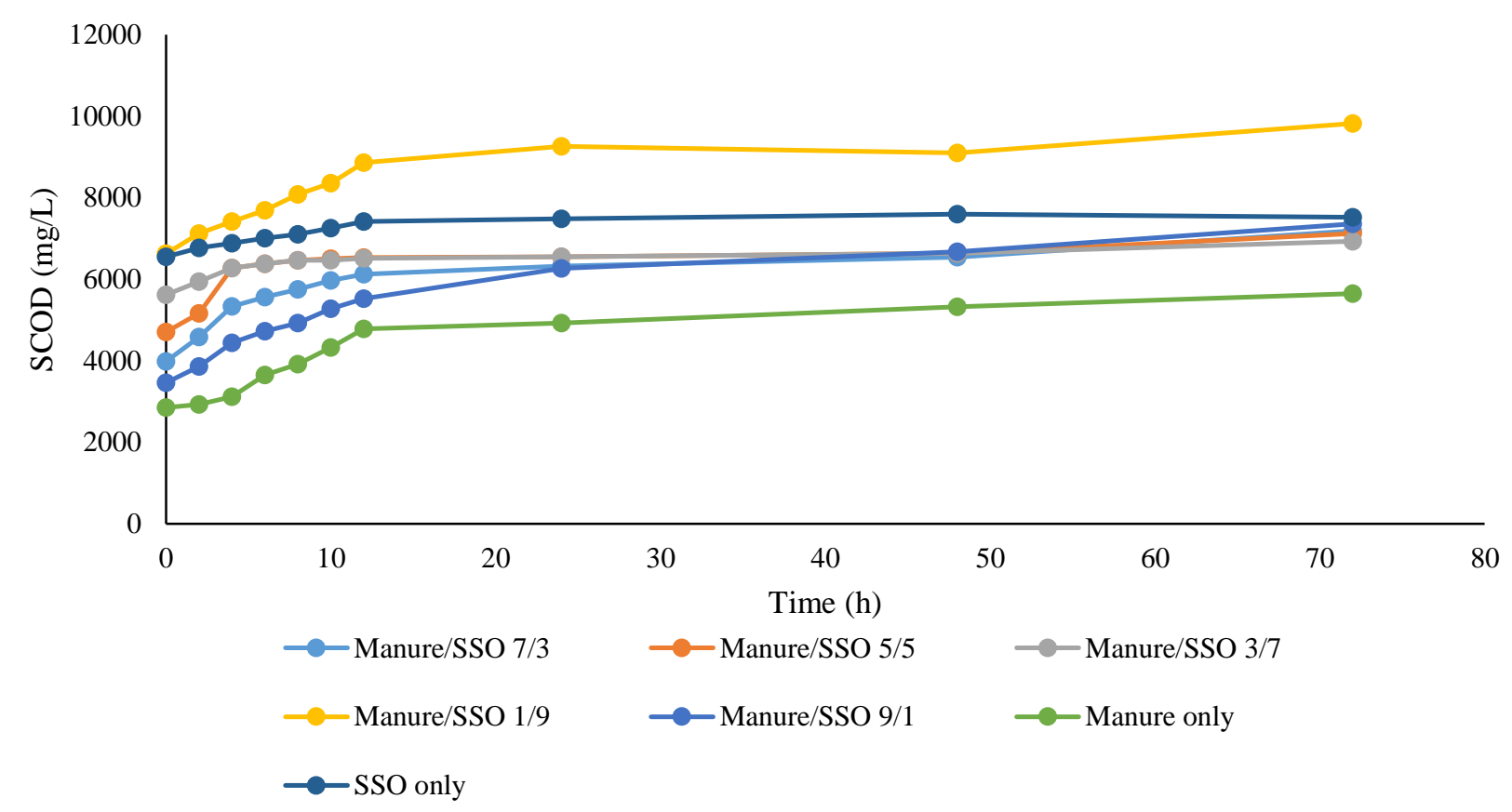

Figure D.2. Concentration of soluble COD over time at different mixing ratios 


\section{References}

Abudi, Z.N., Hu, Z., Sun, N., Xiao, B., Rajaa, N., Liu, C., Guo, D., 2016a. Batch anaerobic codigestion of OFMSW (organic fraction of municipal solid waste), TWAS (thickened waste activated sludge) and RS (rice straw): Influence of TWAS and RS pretreatment and mixing ratio. Energy. https://doi.org/10.1016/j.energy.2016.03.141

Abudi, Z.N., Hu, Z., Xiao, B., Abood, A.R., Rajaa, N., Laghari, M., 2016b. Effects of pretreatments on thickened waste activated sludge and rice straw co-digestion: Experimental and modeling study. J. Environ. Manage. https://doi.org/10.1016/j.jenvman.2016.04.028

Ahn, K.H., Park, K.Y., Maeng, S.K., Hwang, J.H., Lee, J.W., Song, K.G., Choi, S., 2002. Ozonation of wastewater sludge for reductio and recycling, in: Water Science and Technology. https://doi.org/10.1001/jama.288.11.1364

Ahring, B.K., Sandberg, M., Angelidaki, I., 1995. Volatile fatty acids as indicators of process imbalance in anaerobic digestors. Appl. Microbiol. Biotechnol. 43, 559-565. https://doi.org/10.1007/BF00218466

Akyol, Ç., Ozbayram, E.G., Ince, O., Kleinsteuber, S., Ince, B., 2016. Anaerobic co-digestion of cow manure and barley: Effect of cow manure to barley ratio on methane production and digestion stability. Environ. Prog. Sustain. Energy 35, 589-595. https://doi.org/10.1002/ep.12250

Angelidaki, I., Alves, M., Bolzonella, D., Borzacconi, L., Campos, J.L., Guwy, A.J., Kalyuzhnyi, S., Jenicek, P., Van Lier, J.B., 2009a. Defining the biomethane potential (BMP) of solid organic wastes and energy crops: A proposed protocol for batch assays. Water Sci. Technol. https://doi.org/10.2166/wst.2009.040

Angelidaki, I., Alves, M., Bolzonella, D., Borzacconi, L., Campos, J.L., Guwy, A.J., Kalyuzhnyi, S., Jenicek, P., Van Lier, J.B., 2009b. Defining the biomethane potential (BMP) of solid organic wastes and energy crops: A proposed protocol for batch assays. Water Sci. Technol. https://doi.org/10.2166/wst.2009.040

Anwar, Z., Gulfraz, M., Irshad, M., 2014. Agro-industrial lignocellulosic biomass a key to unlock the future bio-energy: A brief review. J. Radiat. Res. Appl. Sci. https://doi.org/10.1016/j.jrras.2014.02.003

Appels, L., Baeyens, J., Degrève, J., Dewil, R., 2008. Principles and potential of the anaerobic digestion of waste-activated sludge. Prog. Energy Combust. Sci. https://doi.org/10.1016/j.pecs.2008.06.002

Appels, L., Lauwers, J., Degrve, J., Helsen, L., Lievens, B., Willems, K., Van Impe, J., Dewil, R., 2011. Anaerobic digestion in global bio-energy production: Potential and research challenges. Renew. Sustain. Energy Rev. 15, 4295-4301. https://doi.org/10.1016/j.rser.2011.07.121

Azbar, N., Speece, R.E., 2001. Two-Phase, Two-Stage, and Single-Stage Anaerobic Process 
Comparison. J. Environ. Eng. 127, 240-248. https://doi.org/10.1061/(ASCE)07339372(2001)127:3(240)

Azman, S., Khadem, A., Zeeman, G., van Lier, J., Plugge, C., 2015a. Mitigation of Humic Acid Inhibition in Anaerobic Digestion of Cellulose by Addition of Various Salts. Bioengineering. https://doi.org/10.3390/bioengineering2020054

Azman, S., Khadem, A.F., Van Lier, J.B., Zeeman, G., Plugge, C.M., 2015b. Presence and role of anaerobic hydrolytic microbes in conversion of lignocellulosic biomass for biogas production. Crit. Rev. Environ. Sci. Technol. https://doi.org/10.1080/10643389.2015.1053727

Bali, G., Meng, X., Deneff, J.I., Sun, Q., Ragauskas, A.J., 2015. The effect of alkaline pretreatment methods on cellulose structure and accessibility. ChemSusChem. https://doi.org/10.1002/cssc.201402752

Bayr, S., Ojanperä, M., Kaparaju, P., Rintala, J., 2014. Long-term thermophilic mono-digestion of rendering wastes and co-digestion with potato pulp. Waste Manag. 34, 1853-1859. https://doi.org/10.1016/j.wasman.2014.06.005

Beccari, M., Majone, M., Torrisi, L., 1998. Two-reactor system with partial phase separation for anaerobic treatment of olive oil mill effluents, in: Water Science and Technology. pp. 5360. https://doi.org/10.1016/S0273-1223(98)00497-1

Benn, N., Zitomer, D., 2018. Pretreatment and Anaerobic Co-digestion of Selected PHB and PLA Bioplastics. Front. Environ. Sci. https://doi.org/10.3389/fenvs.2017.00093

Bertin, L., Grilli, S., Spagni, A., Fava, F., 2013. Innovative two-stage anaerobic process for effective codigestion of cheese whey and cattle manure. Bioresour. Technol. 128, 779-783. https://doi.org/10.1016/j.biortech.2012.10.118

Bitton, G., 2010. Wastewater Microbiology: Fourth Edition, Wastewater Microbiology: Fourth Edition. https://doi.org/10.1002/9780470901243

Björnsson, L., Murto, M., Mattiasson, B., 2000. Evaluation of parameters for monitoring an anaerobic co-digestion process. Appl. Microbiol. Biotechnol. 54, 844-849. https://doi.org/10.1007/s002530000471

Bordeleau, É.L., Droste, R.L., 2011. Comprehensive review and compilation of pretreatments for mesophilic and thermophilic anaerobic digestion. Water Sci. Technol. https://doi.org/10.2166/wst.2011.052

Bordoloi, R., Lilly, S.J., Kacprzak, G.G., Churchill, C.W., 2014. Modeling the distribution of Mg II absorbers around galaxies using background galaxies and quasars. Astrophys. J. 784. https://doi.org/10.1088/0004-637X/784/2/108

Bougrier, C., Delgenès, J.P., Carrère, H., 2007. Impacts of thermal pre-treatments on the semicontinuous anaerobic digestion of waste activated sludge. Biochem. Eng. J. https://doi.org/10.1016/j.bej.2006.11.013

Chandra, R., Vijay, V.K., Subbarao, P.M.V., Khura, T.K., 2012. Production of methane from anaerobic digestion of jatropha and pongamia oil cakes. Appl. Energy. 
https://doi.org/10.1016/j.apenergy.2010.10.049

Cioabla, A.E., Ionel, I., Dumitrel, G.A., Popescu, F., 2012. Comparative study on factors affecting anaerobic digestion of agricultural vegetal residues. Biotechnol. Biofuels. https://doi.org/10.1186/1754-6834-5-39

City of Toronto, 2019. Biosolids Management [WWW Document]. City Toronto.

City of Toronto, 2018. Ashbridges Bay Wastewater Treatment Plant: 2017 Annual Report. City.

Cornelissen, S., Koper, M., Deng, Y.Y., 2012. The role of bioenergy in a fully sustainable global energy system. Biomass and Bioenergy 41, 21-33.

https://doi.org/10.1016/j.biombioe.2011.12.049

Cuetos, M.J., Gómez, X., Escapa, A., Morán, A., 2007. Evaluation and simultaneous optimization of bio-hydrogen production using 32factorial design and the desirability function. J. Power Sources 169, 131-139. https://doi.org/10.1016/j.jpowsour.2007.01.050

Cuetos, M.J., Gómez, X., Otero, M., Morán, A., 2010. Anaerobic digestion and co-digestion of slaughterhouse waste (SHW): Influence of heat and pressure pre-treatment in biogas yield. Waste Manag. https://doi.org/10.1016/j.wasman.2010.01.034

Dareioti, M.A., Kornaros, M., 2015. Anaerobic mesophilic co-digestion of ensiled sorghum, cheese whey and liquid cow manure in a two-stage CSTR system: Effect of hydraulic retention time. Bioresour. Technol. 175, 553-562. https://doi.org/10.1016/j.biortech.2014.10.102

Demeyer, D.I., Henderickx, H.K., 1967. The effect of C18 unsaturated fatty acids on methane production in vitro by mixed rumen bacteria. Biochim. Biophys. Acta (BBA)/Lipids Lipid Metab. 137, 484-497. https://doi.org/10.1016/0005-2760(67)90130-0

Demirel, B., Scherer, P., 2008. The roles of acetotrophic and hydrogenotrophic methanogens during anaerobic conversion of biomass to methane: A review. Rev. Environ. Sci. Biotechnol. https://doi.org/10.1007/s11157-008-9131-1

Deng, Y., Dai, B., Xu, Jiaxing, Liu, X., Xu, Jiming, 2018. Anaerobic co-digestion of rice straw and soybean straw to increase biogas production by pretreatment with trichoderma reesei RUT C30. Environ. Prog. Sustain. Energy. https://doi.org/10.1002/ep.12782

Dinsdale, R.M., Premier, G.C., Hawkes, F.R., Hawkes, D.L., 2000. Two-stage anaerobic codigestion of waste activated sludge and fruit/vegetable waste using inclined tubular digesters. Bioresour. Technol. 72, 159-168. https://doi.org/10.1016/S0960-8524(99)001054

Divya, D., Gopinath, L.R., Merlin Christy, P., 2015. A review on current aspects and diverse prospects for enhancing biogas production in sustainable means. Renew. Sustain. Energy Rev. https://doi.org/10.1016/j.rser.2014.10.055

Droste, R.L., 1997. Theory and Practice of Water and Wastewater TreatmentJohn Wiley and Sons. Inc. New York, Pp754-757.

Duran, M., Tepe, N., Yurtsever, D., Punzi, V.L., Bruno, C., Mehta, R.J., 2006. Bioaugmenting 
anaerobic digestion of biosolids with selected strains of Bacillus, Pseudomonas, and Actinomycetes species for increased methanogenesis and odor control. Appl. Microbiol. Biotechnol. https://doi.org/10.1007/s00253-006-0548-6

Eastman, J.A., Ferguson, J.F., 1981. Solubilization of Particulate Organic Carbon during the Acid Phase of Anaerobic Digestion. Source J. (Water Pollut. Control Fed. Part I 53, 352 366. https://doi.org/10.2307/25041085

Eddy, M., 2003. Wastewater Engineering-Treatment, Disposal, Reuse. INC,.

Edström, M., Nordberg, ^̊., Thyselius, L., 2003. Anaerobic treatment of animal byproducts from slaughterhouses at laboratory and pilot scale, in: Applied Biochemistry and Biotechnology Part A Enzyme Engineering and Biotechnology. https://doi.org/10.1385/ABAB:109:1-3:127

Elbeshbishy, E., Dhar, B.R., Nakhla, G., Lee, H.S., 2017. A critical review on inhibition of dark biohydrogen fermentation. Renew. Sustain. Energy Rev. https://doi.org/10.1016/j.rser.2017.05.075

Elbeshbishy, E., Hafez, H., Dhar, B.R., Nakhla, G., 2011. Single and combined effect of various pretreatment methods for biohydrogen production from food waste. Int. J. Hydrogen Energy. https://doi.org/10.1016/j.ijhydene.2011.02.067

Elbeshbishy, E., Nakhla, G., 2012. Batch anaerobic co-digestion of proteins and carbohydrates. Bioresour. Technol. https://doi.org/10.1016/j.biortech.2012.04.052

Elbeshbishy, E., Nakhla, G., Hafez, H., 2012. Biochemical methane potential (BMP) of food waste and primary sludge: Influence of inoculum pre-incubation and inoculum source. Bioresour. Technol. https://doi.org/10.1016/j.biortech.2012.01.025

Esposito, G., Frunzo, L., Giordano, A., Liotta, F., Panico, A., Pirozzi, F., 2012. Anaerobic codigestion of organic wastes. Rev. Environ. Sci. Bio/Technology 11, 325-341. https://doi.org/10.1007/s11157-012-9277-8

Feng, J., Li, Y., Zhang, E., Zhang, J., Wang, W., He, Y., Liu, G., Chen, C., 2018. Solid-State Codigestion of NaOH-Pretreated Corn Straw and Chicken Manure Under Mesophilic Condition. Waste and Biomass Valorization. https://doi.org/10.1007/s12649-017-9834-z

Fernandes, T. V., van Lier, J.B., Zeeman, G., 2015. Humic Acid-Like and Fulvic Acid-Like Inhibition on the Hydrolysis of Cellulose and Tributyrin. Bioenergy Res. https://doi.org/10.1007/s12155-014-9564-z

Foladori, P., Velho, V.F., Costa, R.H.R., Bruni, L., Quaranta, A., Andreottola, G., 2015. Concerning the role of cell lysis-cryptic growth in anaerobic side-stream reactors: The single-cell analysis of viable, dead and lysed bacteria. Water Res. https://doi.org/10.1016/j.watres.2015.01.042

Frigon, J.C., Guiot, S.R., 1995. Impact of liquid-to-gas hydrogen mass transfer on substrate conversion efficiency of an upflow anaerobic sludge bed and filter reactor. Enzyme Microb. Technol. 17, 1080-1086. https://doi.org/10.1016/0141-0229(95)00040-2

Gaby, J.C., Zamanzadeh, M., Horn, S.J., 2017. The effect of temperature and retention time on methane production and microbial community composition in staged anaerobic digesters 
fed with food waste. Biotechnol. Biofuels. https://doi.org/10.1186/s13068-017-0989-4

Gelegenis, J., Georgakakis, D., Angelidaki, I., Christopoulou, N., Goumenaki, M., 2007. Optimization of biogas production from olive-oil mill wastewater, by codigesting with diluted poultry-manure. Appl. Energy 84, 646-663. https://doi.org/10.1016/j.apenergy.2006.12.001

Gerardi, M.H., 2003. The Microbiology of Anaerobic Digesters, John Wiley \& Sons, Inc. https://doi.org/10.1002/0471468967

Giordano, G.E.L.F.A., 2012. Anaerobic co-digestion of organic wastes. Rev. Environ. Sci. Bio/Technology. https://doi.org/10.1007/s11157-012-9277-8

Gonzalez-Avila, S.R., Klaseboer, E., Khoo, B.C., Ohl, C.D., 2011. Cavitation bubble dynamics in a liquid gap of variable height. J. Fluid Mech. https://doi.org/10.1017/jfm.2011.212

Gonzalez, A., Hendriks, A.T.W.M., van Lier, J.B., de Kreuk, M., 2018. Pre-treatments to enhance the biodegradability of waste activated sludge: Elucidating the rate limiting step. Biotechnol. Adv. https://doi.org/10.1016/j.biotechadv.2018.06.001

Gould, M.C., 2014. Bioenergy and Anaerobic Digestion, in: Bioenergy. https://doi.org/10.1016/b978-0-12-407909-0.00018-3

Government of Canada, 2013. Technical Document on Municipal Solid Waste Organics Processing, Ministry of the Environment in Canada.

Gujer, W., Zehnder, A.J.B., 1983. Conversion processes in anaerobic digestion. Water Sci. Technol. 15, 127-167. https://doi.org/10.2166/wst.1983.0164

Guo, J., Wang, W., Liu, X., Lian, S., Zheng, L., 2014. Effects of thermal pre-treatment on anaerobic co-digestion of municipal biowastes at high organic loading rate. Chemosphere. https://doi.org/10.1016/j.chemosphere.2013.12.007

Guo, M., Song, W., Buhain, J., 2015. Bioenergy and biofuels: History, status, and perspective. Renew. Sustain. Energy Rev. https://doi.org/10.1016/j.rser.2014.10.013

Hagos, K., Zong, J., Li, D., Liu, C., Lu, X., 2017. Anaerobic co-digestion process for biogas production: Progress, challenges and perspectives. Renew. Sustain. Energy Rev. https://doi.org/10.1016/j.rser.2016.11.184

Hanaki, K., Matsuo, T., Nagase, M., 1981. Mechanism of inhibition caused by long-chain fatty acids in anaerobic digestion process. Biotechnol. Bioeng. 23, 1591-1610. https://doi.org/10.1002/bit.260230717

He, Y., Pang, Y., Li, X., Liu, Y., Li, R., Zheng, M., 2009. Investigation on the changes of main compositions and extractives of rice straw pretreated with sodium hydroxide for biogas production. Energy and Fuels. https://doi.org/10.1021/ef8007486

Heffez, A., 2009. Our sewage plant is (unfortunately) bigger than yours [WWW Document]. Green Space, SPACINGMONTREAL, Spacing.ca.

Henze, M., van Loosdrecht, M.C.M., Ekama, G.A., Brdjanovic, D., 2015. Biological Wastewater Treatment: Principles, Modelling and Design. Water Intell. Online. 
https://doi.org/10.2166/9781780401867

Heo, N.H., Park, S.C., Kang, H., 2004. Effects of mixture ratio and hydraulic retention time on single-stage anaerobic co-digestion of food waste and waste activated sludge, in: Journal of Environmental Science and Health - Part A Toxic/Hazardous Substances and Environmental Engineering. pp. 1739-1756. https://doi.org/10.1081/ESE-120037874

Hidalgo, D., Martín-Marroquín, J.M., Sastre, E., 2014. Single-Phase and Two-Phase Anaerobic Co-Digestion of Residues from the Treatment Process of Waste Vegetable Oil and Pig Manure. Bioenergy Res. 7, 670-680. https://doi.org/10.1007/s12155-013-9396-2

Himmel, M.E., Ding, S.Y., Johnson, D.K., Adney, W.S., Nimlos, M.R., Brady, J.W., Foust, T.D., 2007. Biomass recalcitrance: Engineering plants and enzymes for biofuels production. Science (80-. ). https://doi.org/10.1126/science.1137016

Ike, M., Inoue, D., Miyano, T., Liu, T.T., Sei, K., Soda, S., Kadoshin, S., 2010. Microbial population dynamics during startup of a full-scale anaerobic digester treating industrial food waste in Kyoto eco-energy project. Bioresour. Technol. 101, 3952-3957. https://doi.org/10.1016/j.biortech.2010.01.028

Jenkins, S.R., Morgan, J.M., Zhang, X., 1991. Measuring the Usable Carbonate Alkalinity of Operating Anaerobic Digesters. Res. J. Water Pollut. Control Fed. 63, 28-34. https://doi.org/10.2307/25043948

Jihen, T., Baligh, M., Amel, F., Said, N., Moktar, H., Maher, G., Hassib, B., 2015. Microbial ecology overview during anaerobic codigestion of dairy wastewater and cattle manure and use in agriculture of obtained bio-fertilisers. Bioresour. Technol. 198, 141-149. https://doi.org/10.1016/j.biortech.2015.09.004

Kafle, G.K., Chen, L., 2016. Comparison on batch anaerobic digestion of five different livestock manures and prediction of biochemical methane potential (BMP) using different statistical models. Waste Manag. https://doi.org/10.1016/j.wasman.2015.10.021

Kangle, K.M., V, K.S., S, K. V, S, K.G., 2012. Recent Trends in Anaerobic Codigestion: A Review. Univers. J. Environ. Res. 2, 210-219.

Karagiannidis, A., Perkoulidis, G., 2009. A multi-criteria ranking of different technologies for the anaerobic digestion for energy recovery of the organic fraction of municipal solid wastes. Bioresour. Technol. 100, 2355-2360. https://doi.org/10.1016/j.biortech.2008.11.033

Kelleher Robins, 2013. Canadian Biogas Study Technical Document, Canadian Biogas Association.

Kim, J.K., Oh, B.R., Chun, Y.N., Kim, S.W., 2006. Effects of temperature and hydraulic retention time on anaerobic digestion of food waste. J. Biosci. Bioeng. 102, 328-332. https://doi.org/10.1263/jbb.102.328

Kim, S., Bae, J., Choi, O., Ju, D., Lee, J., Sung, H., Park, S., Sang, B.I., Um, Y., 2014. A pilot scale two-stage anaerobic digester treating food waste leachate (FWL): Performance and microbial structure analysis using pyrosequencing. Process Biochem. 49, 301-308. https://doi.org/10.1016/j.procbio.2013.10.022 
Kim, S., Shin, H., 2010. Enhanced Lipid Degradation in an Upflow Anaerobic Sludge Blanket Reactor by Integration with an Acidogenic Reactor. Water Environ. Res. 82, 267-272. https://doi.org/10.2175/106143009X442899

Kim, S.H., Han, S.K., Shin, H.S., 2004. Kinetics of LCFA Inhibition on Acetoclastic Methanogenesis, Propionate Degradation and ??-Oxidation, in: Journal of Environmental Science and Health - Part A Toxic/Hazardous Substances and Environmental Engineering. pp. 1025-1037. https://doi.org/10.1081/ESE-120028411

Kinnunen, M., Hilderbrandt, D., Grimberg, S., Rogers, S., Mondal, S., 2015. Comparative study of methanogens in one- and two-stage anaerobic digester treating food waste. Renew. Agric. Food Syst. 30, 515-523. https://doi.org/10.1017/S1742170514000350

Klocke, M., Nettmann, E., Bergmann, I., Mundt, K., Souidi, K., Mumme, J., Linke, B., 2008. Characterization of the methanogenic Archaea within two-phase biogas reactor systems operated with plant biomass. Syst. Appl. Microbiol. 31, 190-205. https://doi.org/10.1016/j.syapm.2008.02.003

Kwietniewska, E., Tys, J., 2014. Process characteristics, inhibition factors and methane yields of anaerobic digestion process, with particular focus on microalgal biomass fermentation. Renew. Sustain. Energy Rev. 34, 491-500. https://doi.org/10.1016/j.rser.2014.03.041

Lafitte-Trouqué, S., Forster, C.F., 2000. Dual anaerobic co-digestion of sewage sludge and confectionery waste. Bioresour. Technol. 71, 77-82. https://doi.org/10.1016/S09608524(99)00043-7

Lalman, J., Bagley, D.M., 2002. Effects of C18 long chain fatty acids on glucose, butyrate and hydrogen degradation. Water Res. 36, 3307-3313. https://doi.org/10.1016/S00431354(02)00014-3

Lay, J.J., Lee, Y.J., Noike, T., 1999. Feasibility of biological hydrogen production from organic fraction of municipal solid waste. Water Res. https://doi.org/10.1016/S00431354(98)00483-7

Lema, J.M., Suarez, S., 2017. Innovative Wastewater Treatment \&amp; Resource Recovery Technologies: Impacts on Energy, Economy and Environment. Water Intell. Online. https://doi.org/10.2166/9781780407876

Li, A.J., Li, X.Y., Yu, H.Q., 2011. Effect of the food-to-microorganism (F/M) ratio on the formation and size of aerobic sludge granules. Process Biochem. https://doi.org/10.1016/j.procbio.2011.09.007

Li, Y., Park, S.Y., Zhu, J., 2011. Solid-state anaerobic digestion for methane production from organic waste. Renew. Sustain. Energy Rev. 15, 821-826. https://doi.org/10.1016/j.rser.2010.07.042

Liew, L.N., Shi, J., Li, Y., 2012. Methane production from solid-state anaerobic digestion of lignocellulosic biomass. Biomass and Bioenergy 46, 125-132. https://doi.org/10.1016/j.biombioe.2012.09.014

Lin, J., Zuo, J., Ji, R., Chen, X., Liu, F., Wang, K., Yang, Y., 2012. Methanogenic community dynamics in anaerobic co-digestion of fruit and vegetable waste and food waste. J. Environ. 
Sci. (China) 24, 1288-1294. https://doi.org/10.1016/S1001-0742(11)60927-3

Lin, L., Xu, F., Ge, X., Li, Y., 2018. Improving the sustainability of organic waste management practices in the food-energy-water nexus: A comparative review of anaerobic digestion and composting. Renew. Sustain. Energy Rev. https://doi.org/10.1016/j.rser.2018.03.025

Lin, Y., Wang, D., Wu, S., Wang C, 2009. Alkali pretreatment enhances biogas production in the anaerobic digestion of pulp and paper sludge. J. Hazard. Mater. 170, 366-73. https://doi.org/10.1016/j.jhazmat.2009.04.086. Epub 2009 May 3.

Long, J.H., Aziz, T.N., Reyes, F.L.D.L., Ducoste, J.J., 2012. Anaerobic co-digestion of fat, oil, and grease (FOG): A review of gas production and process limitations. Process Saf. Environ. Prot. https://doi.org/10.1016/j.psep.2011.10.001

Luk, G.K., Bekmuradov, V., 2014. Energy products from source-separated organic waste. WIT Trans. Ecol. Environ. https://doi.org/10.2495/WM140411

Lynd, L.R., Weimer, P.J., van Zyl, W.H., Pretorius, I.S., 2002. Microbial Cellulose Utilization: Fundamentals and Biotechnology. Microbiol. Mol. Biol. Rev. 66, 506-577. https://doi.org/10.1128/MMBR.66.4.739.2002

Madigan, M.T., 2014. Brock Biology of Microorganisms, 14th Edition- Madigan, Pearson. https://doi.org/10.1007/s13398-014-0173-7.2

Mata-Alvarez, J., Dosta, J., Romero-Güiza, M.S., Fonoll, X., Peces, M., Astals, S., 2014. A critical review on anaerobic co-digestion achievements between 2010 and 2013. Renew. Sustain. Energy Rev. https://doi.org/10.1016/j.rser.2014.04.039

Mata-Alvarez, J., Macé, S., Llabrés, P., 2000a. Anaerobic digestion of organic solid wastes. An overview of research achievements and perspectives. Bioresour. Technol. https://doi.org/10.1016/S0960-8524(00)00023-7

Mata-Alvarez, J., Macé, S., Llabrés, P., 2000b. Anaerobic digestion of organic solid wastes. An overview of research achievements and perspectives. Bioresour. Technol. https://doi.org/10.1016/S0960-8524(00)00023-7

Mittal, A., 2011. Biological Wastewater Treatment, Chemical Engineering.

Moletta, R., Escoffier, Y., Ehlinger, F., Coudert, J.P., Leyris, J.P., 1994. On-line automatic control system. For monitoring an anaerobic fluidized-bed reactor: Response to organic overload, in: Water Science and Technology. pp. 11-20.

Monnet, F., 2003. A final report on “An Introduction to Anaerobic Digestion of Organic Wastes." Carbon N. Y.

Montusiewicz, A., 2014. Co-digestion of sewage sludge and mature landfill leachate in prebioaugmented system. J. Ecol. Eng. https://doi.org/10.12911/22998993.1125463

Moody, L., Burns, R., Wu-Haan, W., Spajić, R., 2009. Use of biochemical methane potential (BMP) assays for predicting and enhancing anaerobic digester performance. Proc. 4th Int. 44th Croat. Symp. Agric.

Naran, E., Toor, U.A., Kim, D.J., 2016. Effect of pretreatment and anaerobic co-digestion of 
food waste and waste activated sludge on stabilization and methane production. Int. Biodeterior. Biodegrad. https://doi.org/10.1016/j.ibiod.2016.04.011

Naroznova, I., Møller, J., Larsen, B., Scheutz, C., 2016. Evaluation of a new pulping technology for pre-treating source-separated organic household waste prior to anaerobic digestion. Waste Manag. https://doi.org/10.1016/j.wasman.2016.01.042

Nathao, C., Sirisukpoka, U., Pisutpaisal, N., 2013. Production of hydrogen and methane by one and two stage fermentation of food waste, in: International Journal of Hydrogen Energy. pp. 15764-15769. https://doi.org/10.1016/j.ijhydene.2013.05.047

Noike, T., Endo, G., Chang, J. -E, Yaguchi, J. -I, Matsumoto, J. -I, 1985. Characteristics of carbohydrate degradation and the rate-limiting step in anaerobic digestion. Biotechnol. Bioeng. 27, 1482-1489. https://doi.org/10.1002/bit.260271013

Nopharatana, A., Clarke, W.P., Pullammanappallil, P.C., Silvey, P., Chynoweth, D.P., 1998. Evaluation of methanogenic activities during anaerobic digestion of municipal solid waste. Bioresour. Technol. 64, 169-174. https://doi.org/10.1016/S0960-8524(97)00190-9

OECD/EIA, 2011. International Energy Outlook 2011, Center for Strategic and International Studies. https://doi.org/DOE/EIA-0484(2011)

Owen, W.F., Stuckey, D.C., Healy, J.B., Young, L.Y., McCarty, P.L., 1979. Bioassay for monitoring biochemical methane potential and anaerobic toxicity. Water Res. https://doi.org/10.1016/0043-1354(79)90043-5

P., V., D., S., M., L., 2014. Options for Reduction of Maize Silage in Biogas Plant Drazenci. Food Agric. Org. 121-130. https://doi.org/10.2507/daaam.scibook.2014.09

Paavola, T., Syväsalo, E., Rintala, J., 2006. Co-digestion of manure and biowaste according to the EC animal by-products regulation and Finnish national regulations. Water Sci. Technol. https://doi.org/10.2166/wst.2006.253

Park, Y.C., Kim, J.S., 2012. Comparison of various alkaline pretreatment methods of lignocellulosic biomass. Energy. https://doi.org/10.1016/j.energy.2012.08.010

Parra-Orobio, B.A., Torres-Lozada, P., Marmolejo-Rebellón, L.F., 2016. Influence of the mixing ratio on the anaerobic co-digestion of municipal biowaste with domestic wastewater sludge on methane production. Dyna. https://doi.org/http://10.0.60.86/dyna.v83n199.57382

Pauss, A., Andre, G., Perrier, M., Guiot, S.R., 1990. Liquid-to-Gas mass transfer in anaerobic processes: Inevitable transfer limitations of methane and hydrogen in the biomethanation process. Appl. Environ. Microbiol. 56, 1636-1644.

Peter Wright, Scott Inglis, Jianguo Ma, Curt Gooch, Brian Aldrich, Alex Meister and Norman Scott, 2013. Comparison of Five Anaerobic Digestion Systems on Dairy Farms. https://doi.org/10.13031/2013.16760

Poliafico, M., Murphy, J.., 2007. Anaerobic digestion in Ireland : Decision support system. Dep. Civil, Struct. Environ. Eng. Cork Inst. Technol. Irel.

Pouget, E.R., Hagan, H., Des Jarlais, D.C., 2012. Meta-analysis of hepatitis C seroconversion in 
relation to shared syringes and drug preparation equipment, Addiction.

https://doi.org/10.1111/j.1360-0443.2011.03765.x

R, A., K, K., R, D., M, S., 2017. Biogas Recovery from Hyper-Thermophilic Anaerobic CoDigestion of Thickened Waste Activated Sludge, Organic Fraction of Municipal Solid Waste and Fat, Oil and Grease. J. Bioremediation Biodegrad. 08. https://doi.org/10.4172/2155-6199.1000408

Rajesh Banu, J., Arulazhagan, P., Adish Kumar, S., Kaliappan, S., Lakshmi, A.M., 2015. Anaerobic co-digestion of chemical- and ozone-pretreated sludge in hybrid upflow anaerobic sludge blanket reactor. Desalin. Water Treat. https://doi.org/10.1080/19443994.2014.912156

Ratanatamskul, C., Wattanayommanaporn, O., Yamamoto, K., 2015. An on-site prototype twostage anaerobic digester for co-digestion of food waste and sewage sludge for biogas production from high-rise building. Int. Biodeterior. Biodegrad. 102, 143-148. https://doi.org/10.1016/j.ibiod.2015.03.019

Razavi, A.S., Hosseini Koupaie, E., Azizi, A., Hafez, H., Elbeshbishy, E., 2019. Hydrothermal pretreatment of source separated organics for enhanced solubilization and biomethane recovery. Bioresour. Technol. https://doi.org/10.1016/j.biortech.2018.12.024

Razavi, S.A.S., 2019. No Title. Ryerson University.

Ren, H., Jiang, N., Wang, T., Omar, M.M., Ruan, W., Ghafoor, A., 2018. Enhanced Biogas Production in the Duckweed Anaerobic Digestion Process. J. Energy Resour. Technol. 140. https://doi.org/10.1115/1.4039782

Rivière, D., Desvignes, V., Pelletier, E., Chaussonnerie, S., Guermazi, S., Weissenbach, J., Li, T., Camacho, P., Sghir, A., 2009. Towards the definition of a core of microorganisms involved in anaerobic digestion of sludge. ISME J. https://doi.org/10.1038/ismej.2009.2

Rodriguez-Verde, I., Regueiro, L., Lema, J.M., Carballa, M., 2017. Blending based optimisation and pretreatment strategies to enhance anaerobic digestion of poultry manure. Waste Manag. https://doi.org/10.1016/j.wasman.2017.11.002

Rodriguez, C., El-Hassan, Z., Olabi, A.G., 2017. Enhanced Methane Production from Waste Paper Through Anaerobic Co-Digestion with Macroalgae, in: 10th International Conference on Sustainable Energy \& Environmental Protection: Bioenergy and Biofuels. pp. 1-9. https://doi.org/https://doi.org/10.18690/978-961-286-048-6

Rozzi, A., Di Pinto, A.C., Limoni, N., Tomei, M.C., 1994. Start-up and operation of anaerobic digesters with automatic bicarbonate control. Bioresour. Technol. 48, 215-219. https://doi.org/10.1016/0960-8524(94)90149-X

Saha, M., Eskicioglu, C., Marin, J., 2011a. Microwave, ultrasonic and chemo-mechanical pretreatments for enhancing methane potential of pulp mill wastewater treatment sludge. Bioresour. Technol. 102, 7815-7826. https://doi.org/10.1016/j.biortech.2011.06.053

Saha, M., Eskicioglu, C., Marin, J., 2011b. Microwave, ultrasonic and chemo-mechanical pretreatments for enhancing methane potential of pulp mill wastewater treatment sludge. Bioresour. Technol. https://doi.org/10.1016/j.biortech.2011.06.053 
Saini, J.K., Saini, R., Tewari, L., 2015. Lignocellulosic agriculture wastes as biomass feedstocks for second-generation bioethanol production: concepts and recent developments. 3 Biotech. https://doi.org/10.1007/s13205-014-0246-5

Salminen, E., Rintala, J., 2002. Anaerobic digestion of organic solid poultry slaughterhouse waste - A review. Bioresour. Technol. https://doi.org/10.1016/S0960-8524(01)00199-7

Schievano, A., Tenca, A., Scaglia, B., Merlino, G., Rizzi, A., Daffonchio, D., Oberti, R., Adani, F., 2012. Two-stage vs single-stage thermophilic anaerobic digestion: Comparison of energy production and biodegradation efficiencies. Environ. Sci. Technol. 46, 8502-8510. https://doi.org/10.1021/es301376n

Schuetzle, D., Schuetzle, R., Kent Hoekman, S., Zielinska, B., 2015. The effect of oxygen on formation of syngas contaminants during the thermochemical conversion of biomass. Int. J. Energy Environ. Eng. 6, 405-417. https://doi.org/10.1007/s40095-015-0187-8

Shah, F.A., Mahmood, Q., Rashid, N., Pervez, A., Raja, I.A., Shah, M.M., 2015. Co-digestion, pretreatment and digester design for enhanced methanogenesis. Renew. Sustain. Energy Rev. https://doi.org/10.1016/j.rser.2014.10.053

Shen, F., Yuan, H., Pang, Y., Chen, S., Zhu, B., Zou, D., Liu, Y., Ma, J., Yu, L., Li, X., 2013. Performances of anaerobic co-digestion of fruit \& vegetable waste (FVW) and food waste (FW): Single-phase vs. two-phase. Bioresour. Technol. 144, 80-85. https://doi.org/10.1016/j.biortech.2013.06.099

Sheng, G.P., Yu, H.Q., Li, X.Y., 2010. Extracellular polymeric substances (EPS) of microbial aggregates in biological wastewater treatment systems: A review. Biotechnol. Adv. https://doi.org/10.1016/j.biotechadv.2010.08.001

Shin, S.G., Han, G., Lim, J., Lee, C., Hwang, S., 2010. A comprehensive microbial insight into two-stage anaerobic digestion of food waste-recycling wastewater. Water Res. 44, 48384849. https://doi.org/10.1016/j.watres.2010.07.019

Silvestre, G., Illa, J., Fernández, B., Bonmatí, A., 2014. Thermophilic anaerobic co-digestion of sewage sludge with grease waste: Effect of long chain fatty acids in the methane yield and its dewatering properties. Appl. Energy. https://doi.org/10.1016/j.apenergy.2013.11.075

Smith, P.H., Mah, R.A., 1966. Kinetics of acetate metabolism during sludge digestion. Appl. Microbiol.

Song, Z. lin, Yag, G. he, Feng, Y. zhong, Ren, G. xin, Han, X. hui, 2013. Pretreatment of rice straw by hydrogen peroxide for enhanced methane yield. J. Integr. Agric. https://doi.org/10.1016/S2095-3119(13)60355-X

Song, Z., Yang, G., Liu, X., Yan, Z., Yuan, Y., Liao, Y., 2014. Comparison of seven chemical pretreatments of corn straw for improving methane yield by anaerobic digestion. PLoS One. https://doi.org/10.1371/journal.pone.0093801

Sosnowski, P., Wieczorek, A., Ledakowicz, S., 2003. Anaerobic co-digestion of sewage sludge and organic fraction of municipal solid wastes. Adv. Environ. Res. 7, 609-616. https://doi.org/10.1016/S1093-0191(02)00049-7 
Sterling, M.C., Lacey, R.E., Engler, C.R., Ricke, S.C., 2001. Effects of ammonia nitrogen on H2 and $\mathrm{CH} 4$ production during anaerobic digestion of dairy cattle manure. Bioresour. Technol. https://doi.org/10.1016/S0960-8524(00)00138-3

Stuckey, D.C., McCarty, P.L., 1984. The effect of thermal pretreatment on the anaerobic biodegradability and toxicity of waste activated sludge. Water Res. https://doi.org/10.1016/0043-1354(84)90002-2

Sung, S., Contact, P., Bazylinski, D. a, Raskin, L., 2003. Biohydrogen Production from Renewable Organic Wastes. Challenges.

Sung, S., Dague, R.R., 1995. Laboratory studies on the anaerobic sequencing batch reactor. Water Environ. Res. https://doi.org/10.2175/106143095x131501

Supaphol, S., Jenkins, S.N., Intomo, P., Waite, I.S., Donnell, A.G.O., O’Donnell, A.G., 2011. Microbial community dynamics in mesophilic anaerobic co-digestion of mixed waste. Bioresour. Technol. 102, 4021-7. https://doi.org/10.1016/j.biortech.2010.11.124

Tedesco, S., Benyounis, K.Y., Olabi, A.G., 2013. Mechanical pretreatment effects on macroalgae-derived biogas production in co-digestion with sludge in Ireland. Energy 61, 27-33. https://doi.org/10.1016/j.energy.2013.01.071

Themelis, N.J., 2002. Anaerobic Digestion of Biodegradable Organics in Municipal Solid Wastes. Found. Sch. Eng. Appl. Sci. Columbia Univ. https://doi.org/10.1016/j.biotechadv.2010.10.005

Thies, E., Jenkins, T., Stutzenberger, F., 1994. Effects of the detergent Tween 80 on Thermomonospora curvata. World J. Microbiol. Biotechnol. 10, 657-663. https://doi.org/10.1007/BF00327954

Towey, F., 2013. The power of two. Lancet. Respir. Med. 1, 368. https://doi.org/10.1016/j.procbio.2004.03.007

Turovskiy, I.S., Mathai, P.K., 2005. Wastewater Sludge Processing, Wastewater Sludge Processing. https://doi.org/10.1002/047179161X

Uggetti, E., Passos, F., Solé, M., Garfí, M., Ferrer, I., 2017. Recent Achievements in the Production of Biogas from Microalgae. Waste and Biomass Valorization. https://doi.org/10.1007/s12649-016-9604-3

Varel, V.H., Hashimoto, A.G., Chen, Y.R., 1980. Effect of temperature and retention time on methane production from beef cattle waste. Appl. Environ. Microbiol.

Veeken, A., Hamelers, B., 1999. Effect of temperature on hydrolysis rates of selected biowaste components. Bioresour. Technol. https://doi.org/10.1016/S0960-8524(98)00188-6

Viotti, P., Di Genova, P., Falcioli, F., 2004. Numerical analysis of the anaerobic co-digestion of the organic fraction from municipal solid waste and wastewater: Prediction of the possible performances at Olmeto plant in Perugia (Italy). Waste Manag. Res. 22, 115-128. https://doi.org/10.1177/0734242X04043892

Wei, Y., Li, X., Yu, L., Zou, D., Yuan, H., 2015. Mesophilic anaerobic co-digestion of cattle 
manure and corn stover with biological and chemical pretreatment. Bioresour. Technol. https://doi.org/10.1016/j.biortech.2015.09.035

Wett, B., Phothilangka, P., Eladawy, A., 2010. Systematic comparison of mechanical and thermal sludge disintegration technologies. Waste Manag. https://doi.org/10.1016/j.wasman.2009.12.011

Wilcox, S.J., Hawkes, D.L., Hawkes, F.R., Guwy, A.J., 1995. A neural network, based on bicarbonate monitoring, to control anaerobic digestion. Water Res. 29, 1465-1470. https://doi.org/10.1016/0043-1354(94)00314-W

Yang, Z.H., Xu, R., Zheng, Y., Chen, T., Zhao, L.J., Li, M., 2016. Characterization of extracellular polymeric substances and microbial diversity in anaerobic co-digestion reactor treated sewage sludge with fat, oil, grease. Bioresour. Technol. 212, 164-173. https://doi.org/10.1016/j.biortech.2016.04.046

Yu, H.W., Samani, Z., Hanson, A., Smith, G., 2002. Energy recovery from grass using twophase anaerobic digestion. Waste Manag. 22, 1-5. https://doi.org/10.1016/S0956053X(00)00121-5

Yu, Z., Wen, X., Xu, M., Huang, X., 2012. Characteristics of extracellular polymeric substances and bacterial communities in an anaerobic membrane bioreactor coupled with online ultrasound equipment. Bioresour. Technol. 117, 333-340. https://doi.org/10.1016/j.biortech.2012.04.075

Yuan, X., Cao, Y., Li, J., Wen, B., Zhu, W., Wang, X., Cui, Z., 2012. Effect of pretreatment by a microbial consortium on methane production of waste paper and cardboard. Bioresour. Technol. https://doi.org/10.1016/j.biortech.2012.05.058

Zamanzadeh, M., Hagen, L.H., Svensson, K., Linjordet, R., Horn, S.J., 2017. Biogas production from food waste via co-digestion and digestion- effects on performance and microbial ecology. Sci. Rep. https://doi.org/10.1038/s41598-017-15784-w

Zhang, J., Li, W., Lee, J., Loh, K.C., Dai, Y., Tong, Y.W., 2017a. Enhancement of biogas production in anaerobic co-digestion of food waste and waste activated sludge by biological co-pretreatment. Energy. https://doi.org/10.1016/j.energy.2017.02.163

Zhang, J., Loh, K.C., Lee, J., Wang, C.H., Dai, Y., Wah Tong, Y., 2017b. Three-stage anaerobic co-digestion of food waste and horse manure. Sci. Rep. 7. https://doi.org/10.1038/s41598017-01408-w

Zhang, J., Wei, Y., Xiao, W., Zhou, Z., Yan, X., 2011. Performance and spatial community succession of an anaerobic baffled reactor treating acetone-butanol-ethanol fermentation wastewater. Bioresour. Technol. 102, 7407-7414. https://doi.org/10.1016/j.biortech.2011.05.035

Zhao, M., Wang, Y., Zhang, C., Li, S., Huang, Z., Ruan, W., 2014. Synergistic and pretreatment effect on anaerobic co-digestion from rice straw and municipal sewage sludge. BioResources. https://doi.org/10.15376/biores.9.4.5871-5882

Zhong, W., Zhang, Z., Luo, Y., Sun, S., Qiao, W., Xiao, M., 2011. Effect of biological pretreatments in enhancing corn straw biogas production. Bioresour. Technol. 
There will come a time when you believe everything is finished.

That will be the beginning...

Luis L'Amour

\section{Author's Declaration for Electronic Submission of a Dissertation}

I hereby declare that I am the sole author of this dissertation. This is a true copy of the dissertation, including any required final revisions, as accepted by my examiners.

I authorize Ryerson University to lend this dissertation to other institutions or individuals for the purpose of scholarly research.

I further authorize Ryerson University to reproduce this dissertation by photocopying or by other means, in total or in part, at the request of other institutions or individuals for the purpose of scholarly research. I understand that my dissertation may be made electronically available to the public. 


\title{
ANAEROBIC CO-DIGESTION OF MULTIPLE FEEDSTOCKS FOR BIOMETHANE RECOVERY- THE IMPACT OF LIPIDS:PROTEINS:CARBOHYDRATES RATIO
}

\author{
Anahita Rabii, Doctor of Philosophy, 2020 \\ Department of Civil Engineering, Ryerson University
}

\begin{abstract}
Municipalities are facing increasing challenges regarding management and disposal of solid waste. Anaerobic digestion (AD) of municipal biowaste enables waste reduction and biogas production that can be utilized as a renewable source of energy for heat and power generation. Anaerobic co-digestion (AnCoD) enhances the performance of conventional mono-digestion. The mixing ratio of the feedstocks is an important criterion in AnCoD design which is typically determined based on the optimum carbon to nitrogen $(\mathrm{C}: \mathrm{N})$ ratio within the range of 25-30 or COD:N ratio in the range of 50140. However, literature has shown contradictory results for the optimum C:N and COD:N ratios. Therefore, the main objective of this study was to primarily investigate the influence of the mixing ratio of the feedstocks including thickened waste activated sludge (TWAS), manure and source separated organics (SSO) on improving biomethane production and introducing a new methodology for optimizing the mixing ratio in $\mathrm{AnCoD}$ based on the lipids, proteins, and carbohydrates contents as the three main compounds existing in biowaste. The hydrolysis/acidification performance in $\mathrm{AnCoD}$ of manure, TWAS and SSO individually and in different combinations was also investigated. This study has introduced an empirical model to explain the relationship between the biomethane production and lipids: proteins: carbohydrates ratio of the feedstocks in anaerobic co-digestion of TWAS, manure and SSO. Among the binary ad ternary combinations, the ternary co-digestion of TWAS/manure/ SSO at the mixing ratio of 2:4:4 and lipids: proteins: carbohydrates ratio of 1:3:12 resulted in the maximum ultimate methane production. The maximum methane yield of $363 \mathrm{ml} \mathrm{CH}_{4} / \mathrm{g} \mathrm{COD}$ added corresponded to co-digestion of manure/SSO at the mixing ratio of 7:3. The maximum hydrolysis rate corresponded to the co-digestion of TWAS/manure at the ratio of 9:1. Overall, the best performance in both hydrolysis and methanogenesis was achieved by the co-digestion of TWAS with SSO at the ratio of 3:7 as well as TWAS/manure/SSO at the ratio of 2:4:4 compared to other feedstock mixes. It was observed that the proposed second order polynomial model could describe the relationship between biomethane production and lipids, proteins, and carbohydrates content of the feedstock.
\end{abstract}




\section{ACKNOWLEDGMENTS}

I am extremely grateful to almighty God, who has been there every step of the way, for giving me the strength to keep going and for his blessing throughout my life. My doctoral study at Ryerson University was a journey of learning, research, and teaching, full of inspiration. I definitely owe the success of this journey to the people who made it possible for me.

I would like to express my sincere gratitude to my $\mathrm{PhD}$ advisors, Dr. Elsayed Elbeshbishy, Dr. Yaser Dahman, and Dr. Saad Aldin for supporting me during these past few years. I owe a great deal to them for their guidance and support through this process. Their ideas and feedback have been absolutely invaluable.

I am profoundly grateful to my dear supervisor, Dr. Elsayed Elbeshbishy for his invaluable guidance and his continued support. I have had the great privilege to work in his research group; in fact, I have been very fortunate to learn from his knowledge and expertise during my research. While being always supportive and helpful at different stages of my work, he granted me the liberty to express and pursue my own ideas. He has been much more than my supervisor, rather a mentor and a great leader. I hope these precious experiences continue throughout my career as I still have a great deal to explore through collaborations with him.

My sincere thanks go to my committee members, Dr. Jiangning Wu, Dr. Ahmed Shaker, Dr Songnian Li, and Dr. Hisham Hafez. In addition, I also appreciate Dr. Darko Joksimovic and Dr. Grace Luk for their suggestions on my research proposal. I greatly appreciate their support and invaluable suggestions for improving this work. I would like to extend my gratitude to Dr. Younggy Kim at McMaster University for reviewing this dissertation and for providing invaluable comments and suggestions for improving my dissertation.

My teaching experiences as a Teaching Assistant at Ryerson University were gratifying and full of inspiration. I am thankful that I had the opportunity to contribute to the learning of students in the civil engineering department at Ryerson University. I appreciate the help and support of the faculty whom I had the opportunity of working with; many thanks to Dr. Darko Joksimovic, Dr. Soheil Kashi, Dr. Ehsan Hoseini Koupaye, and Dr. Rania Hamza. I also greatly appreciate the Teaching Development team, Michelle Schwarts, Curtis Maloley, and Dr. John Paul Foxe for their support and valuable resources throughout this journey. I would also like to thank my students in 
my tutorial and laboratory sessions, particularly those who inspired me by their enthusiasm, attention, and effort. A genuine thanks go to the administrative staff of the civil engineering department, Rachel Peluso, Kim Kritzer, Helen Rusan, Tamar Svadjian, and Annie Jiang for their continued support throughout these years.

I would like to thank my colleagues in Environmental Research for Resource Recovery (ER3) group, specifically Frances Okoye, Ahmed El Sayed, Devarshi Sevak, Salomeh Chegini, Dr. Valeriy Bekmuradov, and Hassan Mahdi for all their support and assistance. My gratitude extends to my amazing colleagues Farokhlaqa Kakar, Neha Purohit, Umme Sharmeen Hyder, and Abeer Hamzeh.

I would also thank Dr. Amir Bazyar Lakeh and Dr. Ehsan Hoseini Koupaye for their guidance and valuable suggestions during my research. In addition, I truly appreciate Robin Loung for his hard work and for providing continued support in conducting my research.

The financial contribution from the Natural Sciences and Engineering Research Council of Canada (NSERC) to this research is gratefully acknowledged. I would also like to acknowledge the City of Toronto's Ashbridges Bay Treatment Plant and Disco Road Organic Processing Facilty for all their support and collaborations throughout this work.

I would like to appreciate my dear parents who taught me how to work hard, how to be brave and pursue my dreams. Thanks to my sister Alaleh and my brother Joseph who have been always encouraging, supportive and caring during the challenging stages of my life. My dear thanks go to the heavenly gift of my life, who has been always by my side, whose smile has given me the strength to keep going, and who I am always grateful for, my son, Armin. Thank you for being so great, thoughtful, and understanding. 
Q Dedication

Ta the light of my eyes

Ta my heavenly gift

Aft to all of my

inspiration

Premix

xix 


\section{Table of Contents}

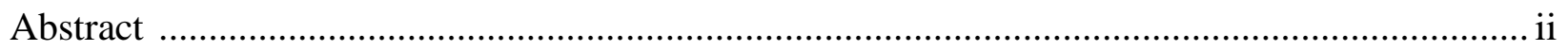

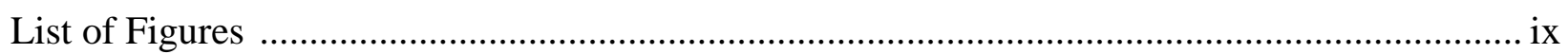

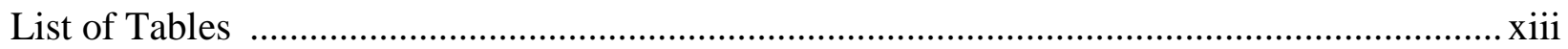

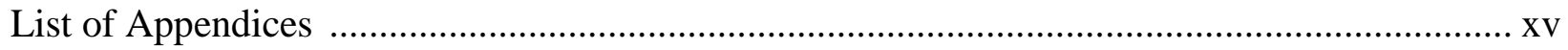

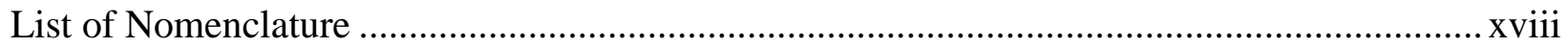

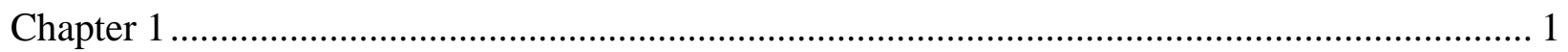

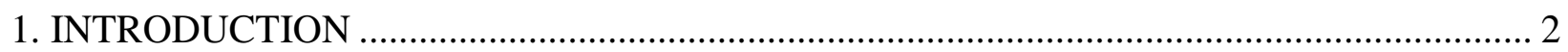

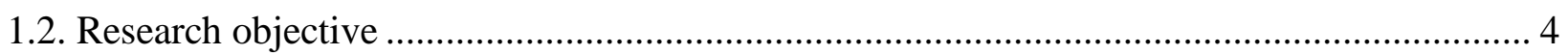

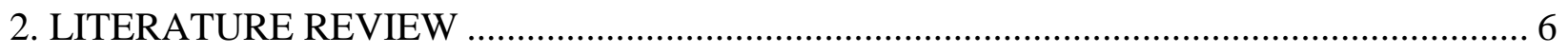

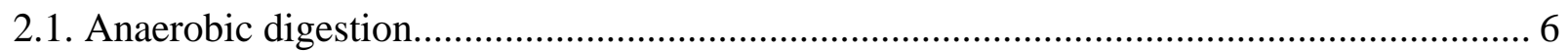

2.2. Processparameters of anaerobic digestion..................................................................... 11

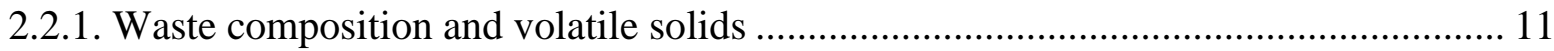

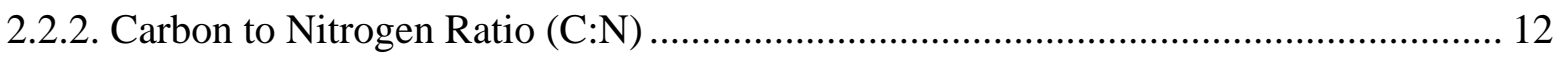

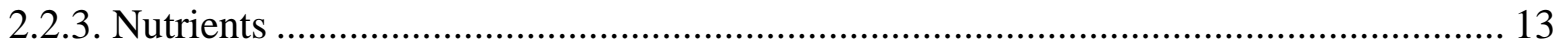

2.2.4. Total solids content (TS)/Organic loading rate (OLR) ................................................ 13

2.2.5. $\mathrm{pH}$, alkalinity and volatile acids/alkalinity ratio....................................................... 14

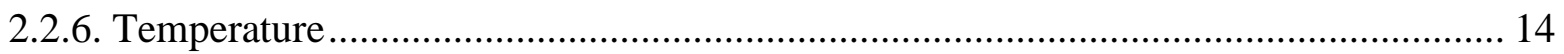

2.2.7. Solids and hydraulic retention time ……….............................................................. 15

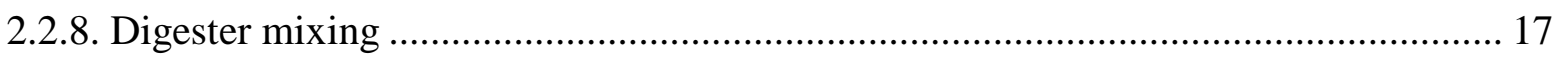

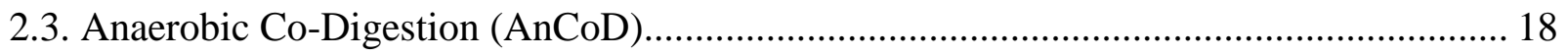

2.3.1. Microbial Diversity and Synergy in AnCoD ............................................................... 25

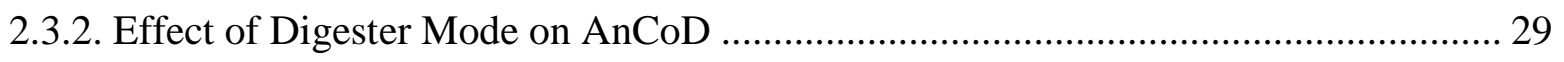

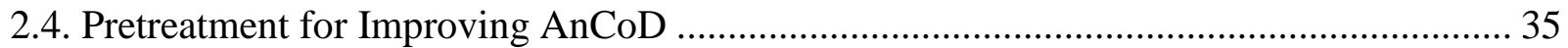

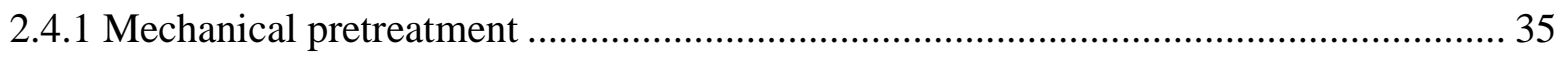

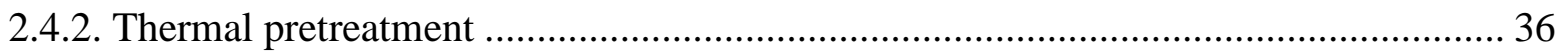

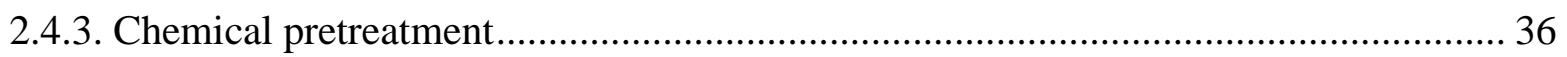




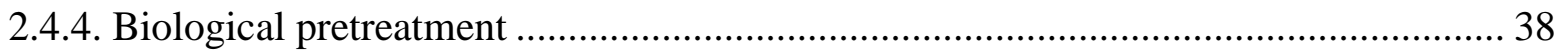

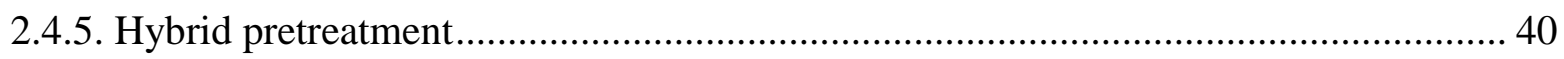

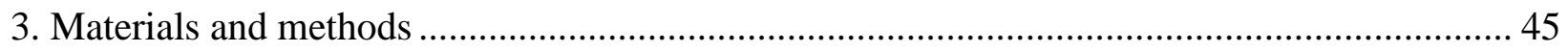

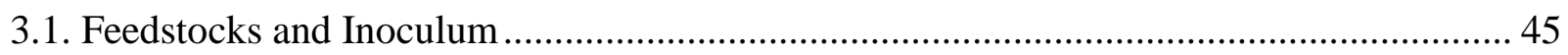

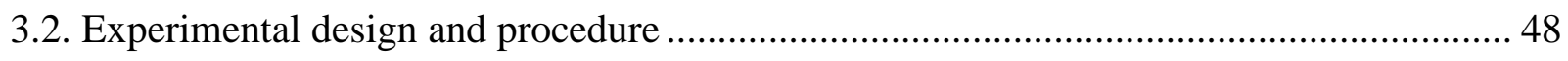

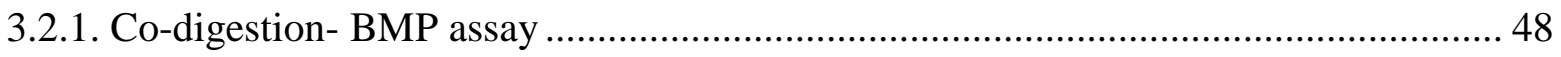

3.2.2. Co-digestion- hydrolysis/acidification experiment .................................................... 51

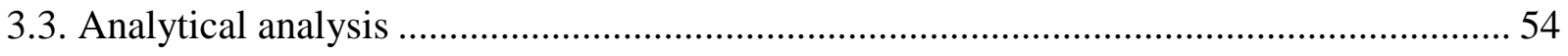

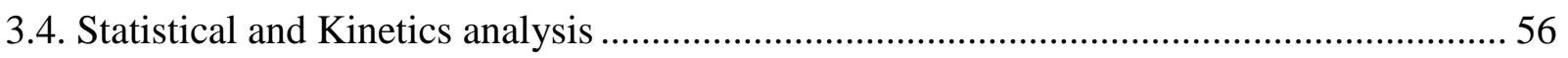

4. Results and discussion- TWAS/SSO co-digestion ................................................................ 58

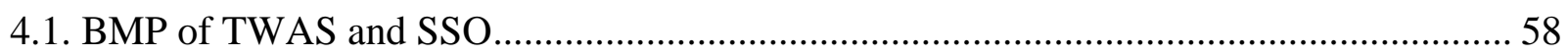

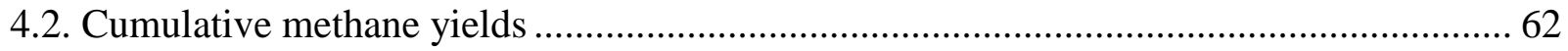

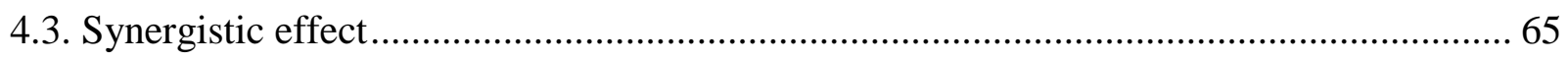

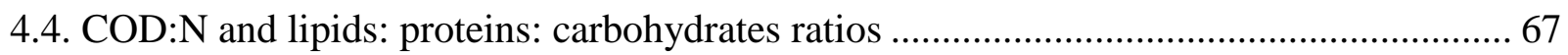

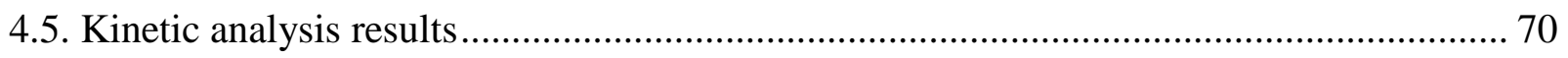

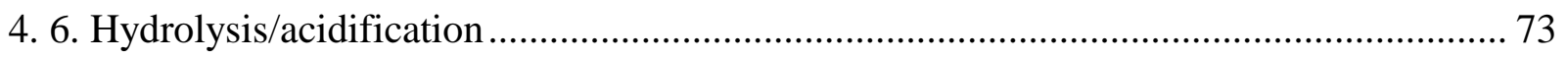

5. Results and discussion- TWAS and manure co-digestion ...................................................... 80

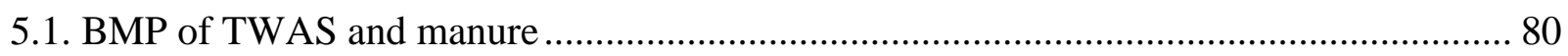

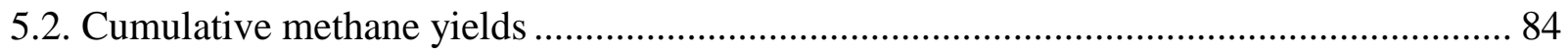

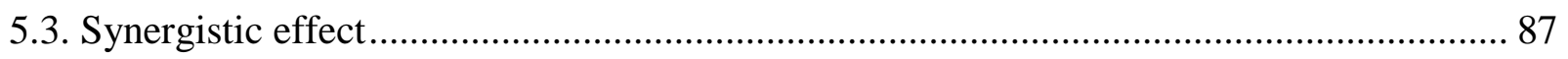

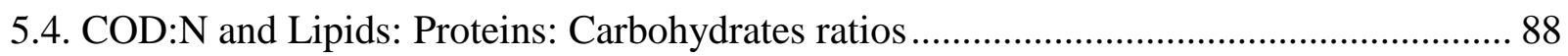

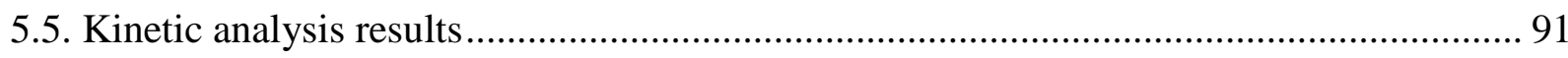

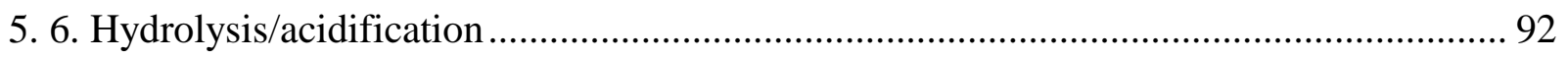

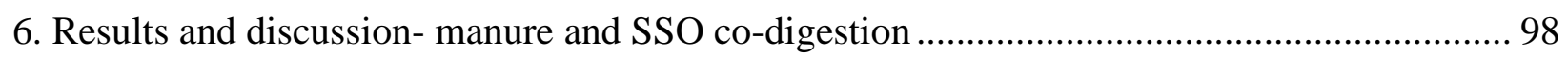

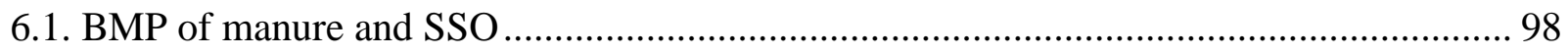

6.2. Cumulative methane yields .......................................................................................... 102

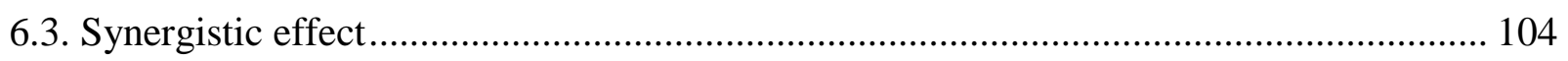

6.4. COD:N and lipids: proteins: carbohydrates ratios ....................................................... 105 


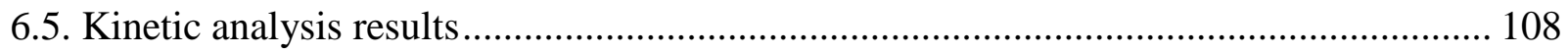

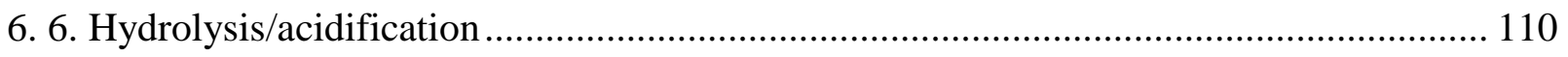

7. Results and discussion- TWAS, manure, SSO co-digestion................................................ 116

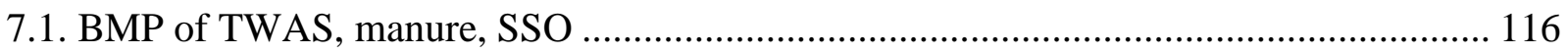

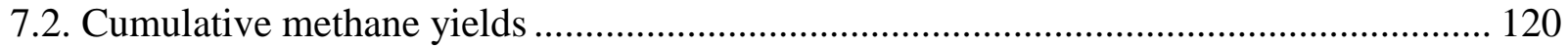

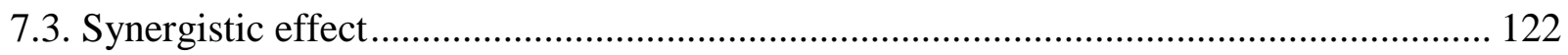

7.4. COD:N and Lipids: Proteins: Carbohydrates ratios ....................................................... 123

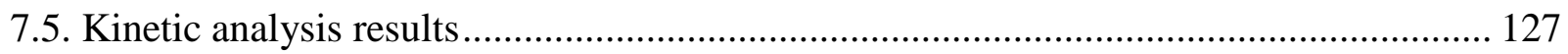

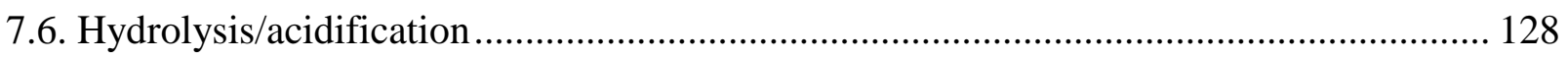

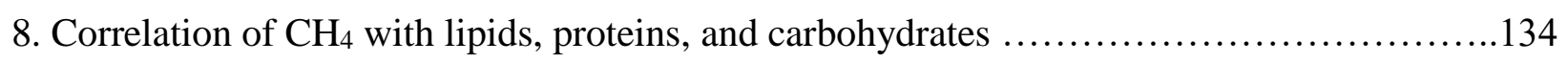

8.1 Correlation of organic compositions and biomethane yield ............................................ 135

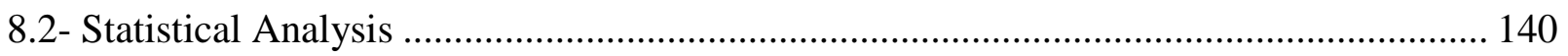

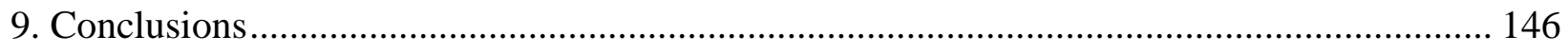

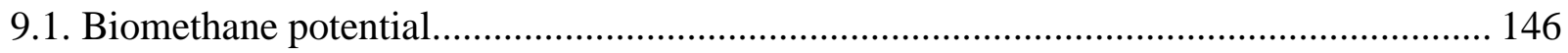

9.1.1. Binary co-digestion of TWAS, manure, and SSO ................................................... 146

9.1.2. Ternary co-digestion of TWAS, manure and SSO ................................................. 147

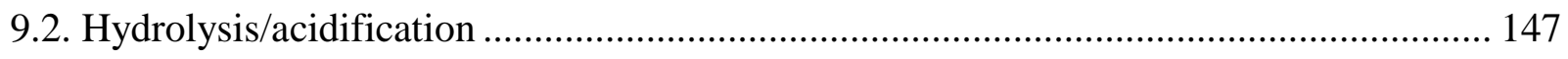

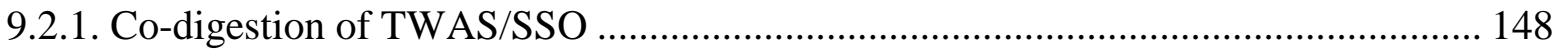

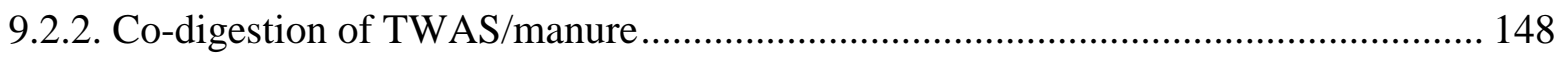

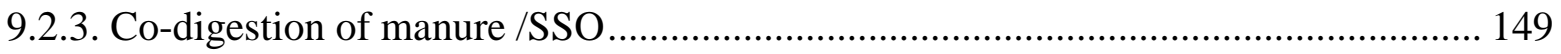

9.2.4. Co-digestion of TWAS/manure /SSO ................................................................... 149

9.3. Correlation of biomethane production with the organic content..................................... 150

9.3.1. COD:N and lipids:proteinscarbohydrates ratios ..................................................... 150

9.3.2. Anaerobic co-digestion model ................................................................................ 151

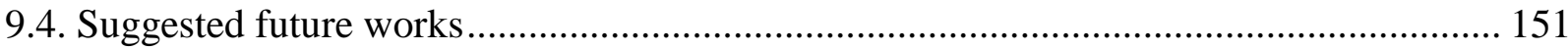




\section{List of Figures}

Figure 2.1. Pathways of anaerobic digestion (Salminen and Rintala, 2002) f.............................. 8

Figure 2.2. Schematic relationship between SRT and degree of digestion (Appels et al., 2008). 17 Figure 2.3. Types of mixing methods for digesters (a) external, pumped recirculation mixing, (b) internal mechanical mixing, and (c) external gas recirculation mixing (Appels et al., 2008)..... 18

Figure 2.4. Co- digestion of multi feedstocks for waste reduction and energy recovery ............. 20 Figure 2.5. Advantages of anaerobic co-digestion systems ................................................... 22 Figure 2.6. Types of digester configuration: (a) Single- stage; (b) Two- stage; and (c) Three-

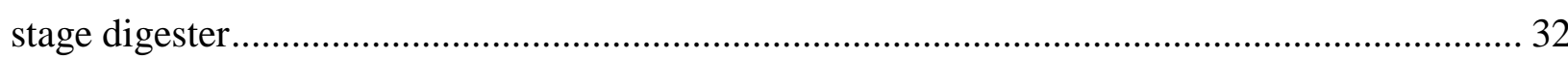

Figure 2.7. Mechanical methods of pretreatment used for AnCoD improvement........................ 35 Figure 3.1. The flow diagram of the Ashbridges Bay Wastewater Treatment Plant (City of

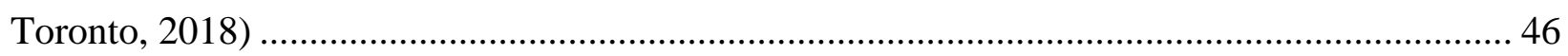

Figure 3.2. Source separated organics processing in Disco Road Facility ................................... 47

Figure 3.3. Experimental set-up for biomethane potential experiment ........................................ 51

Figure 3.4. Experimental set-up for hydrolysis/acidification experiment ................................... 54

Figure 3.5. The spectra and flourometric data for different total lipids concentrations ............... 55

Figure 4.1. Cumulative methane production for different mixing ratios of TWAS and SSO ...... 60

Figure 4.2. Methane production rate $(\mathrm{mL} / \mathrm{d})$ for different mixing ratios of TWAS and SSO ....... 61 Figure 4.3. Maximum methane production rate $(\mathrm{mL} / \mathrm{d})$ for different mixing ratios of TWAS and SSO

Figure 4.4. Methane yields: a) per unit mass TCOD added, b) per unit mass of VSS added and c) per unit volume of substrate added at different mixing ratio of TWAS and SSO 64

Figure 4.5. Biodegradable fraction of the feedstocks for different mixture ratios of TWAS and SSO

Figure 4.6. Synergetic effect of co-digestion at different mixing ratios of TWAS and SSO ....... 66 Figure 4.7. Main effects polot for $\mathrm{CH} 4$ yield data means in response to feedstock ratios at different lipids:proteins:carbohydrates ratios

Figure 4.8. Matrix effects plot for: a. ultimate $\mathrm{CH}_{4}$ and b. $\mathrm{CH}_{4}$ yield at different COD:N and Lipids: Proteins; Lipids:Carbohydrates and Proteins:Cabohydrates for the same corresponding mixing ratios of TWAS:SSO 70

Figure 4.9. Degree of COD solubilization at different mixing ratios of TWAS and SSO 75 
Figure 4.10. Synergistic effect on solubilization at different mixing ratios of TWAS and SSO.. 75 Figure 4.11. Total VFAs yield at different mixing ratios of TWAS/SSO ............................... 76 Figure 5.1. Cumulative methane production for different mixing ratios of TWAS and manure . 81 Figure 5.2. Methane production rate $(\mathrm{mL} / \mathrm{d})$ for different mixing ratios of TWAS and Manure. 82 Figure 5.3. Maximum methane production rate $(\mathrm{mL} / \mathrm{d})$ for different mixing ratios of TWAS and manure.

Figure 5.4. COD mass balance in co-digestion of TWAS and manure for different mixing ratios

Figure 5.5. Methane yields a) per unit mass TCOD added, b) per unit mass of VSS added and c) per volume substrate added at different mixing ratio of TWAS and manure 85

Figure 5.6. Biodegradable fraction of the feedstocks for different mixture ratios of TWAS and manure 86

Figure 5.7. Synergetic effect of co-digestion at different mixing ratios of TWAS and SSO ...... 87

Table 5.2. Ultimate $\mathrm{CH}_{4}$ and yield at different ratios of the substrates, COD:N, and Lipids:Proteins:Carbohydrates 89

Figure 5.8. Main effect plot for $\mathrm{CH}_{4}$ yield data means in response to feedstock and lipids:proteins:carbohydrates ratios at different feedstock mixing ratios in AnCoD of TWAS/manure . 89

Figure 5.9. Matrix plot for: a. ultimate $\mathrm{CH}_{4}$ and b. $\mathrm{CH}_{4}$ yield at different $\mathrm{COD} / \mathrm{N}$ and Lipids:

Proteins, Lipids: Carbohydrates, and Proteins: Carbohydrates ratios....................................... 90

Figure 5.10. Degree of COD solubilization at different mixing ratios of TWAS:Manure.......... 94

Figure 5.11. Synergistic effect on solubilization at different mixing ratios of TWAS:Manure ... 94

Figure 5.12. Total VFAs yield at different mixing ratios of TWAS/Manure ........................... 95

Figure 6.1. Cumulative methane production for different mixing ratios of manure and SSO .... 99 Figure 6.2. Methane production rate $(\mathrm{mL} / \mathrm{d})$ for different mixing ratios of Manure and SSO ... 100 Figure 6.3. Maximum methane production rate $(\mathrm{mL} / \mathrm{d})$ for different mixing ratios manure of and SSO 101

Figure 6.4. COD mass balance in co-digestion of manure and SSO for different mixing ratios 101 Figure 6.5. Methane yields a) per unit mass TCOD added, b) per unit mass of VSS added and c) per volume substrate added at different mixing ratio of TWAS and manure 103 
Figure 6.6. Biodegradable fraction of the feedstocks for different mixture ratios of TWAS and manure 104

Figure 6.7. Synergetic effect of co-digestion at different mixing ratios of manure and SSO ... 105

Figure 6.8. Main effect plot for $\mathrm{CH}_{4}$ yield data mean in response to feedstock and

lipids:proteins:carbohydrates ratios in $\mathrm{AnCoD}$ of manure/SSO. 107

Figure 6.9. Matrix plot for: a. ultimate $\mathrm{CH}_{4}$ and b. $\mathrm{CH}_{4}$ yield at different COD:N and Lipids:

Proteins, Lipids: Carbohydrates, and Proteins: Carbohydrates Ratios ................................... 108

Figure 6.10. Degree of COD solubilization at different mixing ratios of Manure/SSO ............ 112

Figure 6.11. Synergistic effect on solubilization at different mixing ratios of Manure/SSO ..... 112

Figure 6.12. Total VFAs yield at different mixing ratios of Manure/SSO ............................ 113

Figure 7.1. Cumulative methane production for different mixing ratios of TWAS/manure/SSO

Figure 7.2. Methane production rate $(\mathrm{mL} / \mathrm{d})$ for different mixing ratios of TWAS/Manure/SSO 118

Figure 7.3. Maximum methane production rate $(\mathrm{mL} / \mathrm{d})$ for different mixing ratios TWAS/manure/SSO.

Figure 7.4. COD mass balance in co-digestion of manure and SSO for different mixing ratios 4

Figure 7.5. Methane yields a) per unit mass TCOD added, b) per unit mass of VSS added and c) per volume substrate added at different mixing ratio of TWAS and manure 121

Figure 7.6. Biodegradable fraction of the feedstocks for different mixture ratios of TWAS and manure.

Figure 7.7. Synergetic effect of co-digestion at different mixing ratios of manure and SSO .... 123

Figure

Figure 7.8. Main effect plot for $\mathrm{CH}_{4}$ yield data mean in response to feedstock and

lipids:proteins:carbohydrates ratio in AnCoD of TWAS/manure/SSO

Figure 7.9. Matrix plot for: a. ultimate $\mathrm{CH}_{4}$ and b. $\mathrm{CH}_{4}$ yield at different $\mathrm{COD} / \mathrm{N}$ and Lipids:

Proteins, Lipids: Carbohydrates, and Proteins: Carbohydrates Ratios 126

Figure 7.10. Degree of COD solubilization at different mixing ratios of TWAS/Manure/SSO 130 Figure 7.11. Synergistic effect on solubilization at different mixing ratios of TWAS/Manure/SSO 
Figure 7.12. Total VFAs yield at different mixing ratios of TWAS/Manure/SSO.................. 132

Figure 8.1. Fitted plot of response variable to lipids, Lipids ${ }^{2}$, proteins, and carbohydrate ........ 137

Figure 8.2. Residuals vursus fits plot (residuals versus estimated response) .......................... 139

Figure 8.3. a. Main effect plot for $\mathrm{CH}_{4}$ yield data mean..................................................... 141

Figure 8.3. b. Main effect plot for $\mathrm{CH}_{4}$ yield data mean for the lag phase data mean............... 141

Figure 8.3. c. Main effect plot for $\mathrm{CH}_{4}$ yield data mean for the $\mathrm{CH}_{4}$ maximum rate data mean 142

Figure 8.4. Interval plot of $\mathrm{CH}_{4}$ yield with $95 \%$ confidence interval for the mean................. 143

Figure 8.5. a. Fisher individual $95 \%$ confidence interval differences of means for $\mathrm{CH}_{4}$ yields in

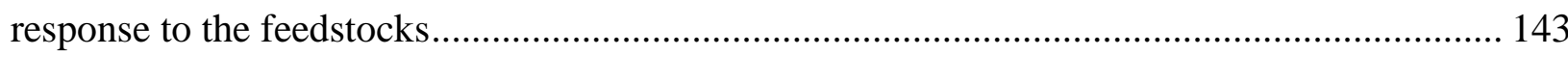

Figure 8.5. b. Fisher individual $95 \%$ confidence interval differences of means for $\mathrm{CH}_{4}$ yields in

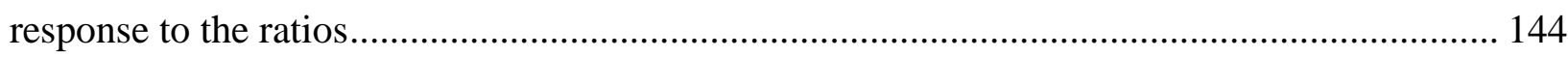




\section{List of Tables}

Table 2.1. Potential feedstocks for co-digestion to balance nutrient with regard to $\mathrm{C} / \mathrm{N}$ ratio

[(Hagos et al., 2017),(R et al., 2017)-(Sosnowski et al., 2003)]

Table 2.2. Microbial consortia diversity in various AnCoD systems for biogas and methane improvement 28

Table 2.3. Comparison of single-stage and two-stage digestion in AnCoD systems 34

Table 2.4. Biological pretreatment for enhanced AnCoD 40

Table 2.5. Hybrid pretreatment for enhanced AnCoD. 42

Table 3.1. Initial Characteristics of the feedstocks and inoculum used in this study 48 Table 3.2 Proportions of digesters' feed for binary co-digestion of TWAS/ SSO, TWAS/manure, and manure/ SSO in BMP experiment. 49

Table 3.3 Proportions of digesters' feed for ternary co-digestion of TWAS, SSO, and manure in BMP experiment 50

Table 3.4 Proportions of digesters' feed for binary co-digestion of TWAS/ SSO, TWAS/manure, and manure/ SSO in hydrolysis/acidification experiment 52 Table 3.5 Proportions of digesters' feed for ternary co-digestion of TWAS, SSO, and manure in in hydrolysis/acidification experiment. 53

Table 4.1. Mean values of the feed characteristics in digesters with different mixing ratios of TWAS and SSO 58

Table 4.2. Ultimate $\mathrm{CH}_{4}$ at different ratios of the substrates, COD:N, and

Lipids:Proteins:Carbohydrates. 68

Table 4.3. Summary of results of kinetic study using modified Gompertz models..................... 71

Table 4.4. Characteristics of the feedstocks at different mixing ratios of TWAS and SSO ........ 74

Table 4.5. Hydrolysis rate coefficient for COD, lipids, proteins, and carbohydrate content in codigesion of TWAS and SSO at different mixing ratios .................................................. 78

Table 5.1. Characteristics of feed to digesters with different mixing ratios .............................. 80

Table 5.2. Ultimate $\mathrm{CH}_{4}$ and yield at different ratios of the substrates, COD:N, and

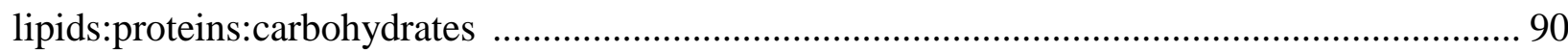

Table 5.3. Summary of results of kinetic study using modified Gompertz model .................... 91

Table 5.4. Characteristics of the feedstocks at different mixing ratios of TWAS and manure .... 93 
Table 5.5. Hydrolysis rate coefficients for COD, lipids, proteins, and carbohydrate content in co-

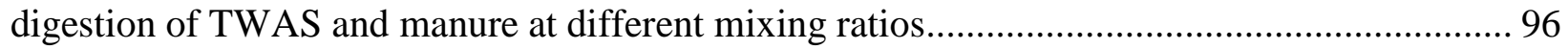
Table 6.1. Characteristics of feed to digesters with different mixing ratios of manure and SSO. 98 Table 6.2. Ultimate $\mathrm{CH}_{4}$ and yield at different ratios of the substrates, COD:N, and

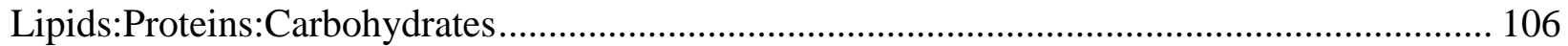

Table 6.3. Summary of results of kinetic study using modified Gompertz model ................... 109 Table 6.4. Characteristics of the feedstocks at different mixing ratios of manure and SSO ..... 111 Table 6.5. Hydrolysis rate coefficients for COD, lipids, proteins, and carbohydrate content in codigestion of SSO and manure at different mixing ratios

Table 7.1. Characteristics of feed to digesters with different mixing ratios of TWAS/ manure/SSO

Table 7.2. Ultimate $\mathrm{CH}_{4}$ and yield at different ratios of the substrates, COD:N, and Lipids:Proteins:Carbohydrates

Table 7.3. Summary of results of kinetic study using modified Gompertz model for

TWAS/manure/SSO co-digestion.

Table 7.4. Characteristics of the feedstocks at different mixing ratios of TWAS/manure/SSO 129 Table 7.5. Hydrolysis rate coefficients for COD, lipids, proteins, and carbohydrate content in co-

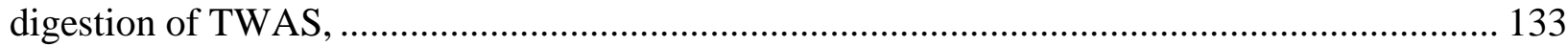

Manure, and SSO at different mixing ratios 133

Table 8.1.Coefficients of variables in the second-order polynomial model and the results of ANOVA analysis

Table 8.2. Experimental set-ups and the corresponding ratios of the feedstock and lipids:

proteins: carbohydrates 140 


\section{List of Appendices}

A. Analytical results for AnCoD of TWAS and SSO ...................................................... 152

Table A. 1. Characteristics of raw feedstocks in AnCoD of TWAS and SSO ........................ 152

Table A. 2. Average $\mathrm{CH}_{4}$ production in AnCoD of TWAS and SSO ................................... 153

Table A. 3. Cumulative $\mathrm{CH}_{4}$ production in AnCoD of TWAS and SSO .............................. 154

Table A. 4. Cumulative methane yield per unit mass of COD added in AnCoD of TWAS and

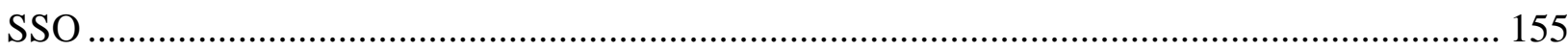

Table A. 5. Cumulative methane yield per unit mass of VSS added in AnCoD of TWAS and SSO 156

Table A. 6. Cumulative methane yield per unit volume of VSS added in AnCoD of TWAS and SSO 157

Figure A.1. Ultimate $\mathrm{CH}_{4}$ production in $\mathrm{AnCoD}$ of TWAS and SSO 158

Table A. 7. Characteristics of raw feedstocks for hydrolysis/acidification in AnCoD of TWAS/SSO 159

Table A. 8. Soluble and particulate COD concentrations (mg/L) over time 160

Figure A.2. Concentration of soluble COD over time for different mixing ratios 161

B. Analytical results for AnCoD of TWAS and manure 162

Table B.1 Characteristics of raw feedstocks in AnCoD of TWAS and manure 162

Table B. 2. Cumulative $\mathrm{CH}_{4}$ production in $\mathrm{AnCoD}$ of TWAS and manure 163

Table B. 3. Cumulative methane yield per unit mass of COD added in AnCoD of TWAS and manure.

Table B. 4. Cumulative methane yield per unit mass of VSS added in AnCoD of TWAS and manure

Table B. 5. Cumulative methane yield per unit volume of substrate added in AnCoD of TWAS and manure. 166

Figure B. 1. Ultimate methane production in AnCoD of TWAS and manure. 167 
Table B. 6. Characteristics of the raw feedstocks for hydrolysis/acidification in AnCoD of

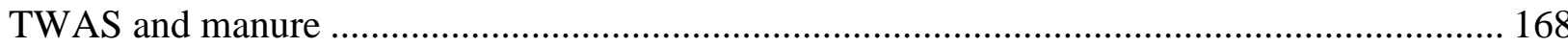

Table B. 7. Measured and theoretical VFAs concentrations over time .................................. 169

Table B. 8. Soluble and particulate COD concentrations over time .................................... 170

Figure B.2. Concentration of soluble COD over time at different mixing ratios of TWAS and

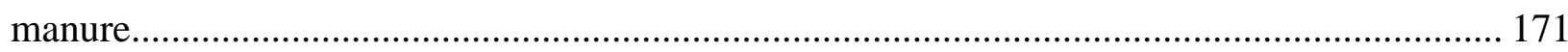

C. Analytical results for AnCoD of manure and SSO ................................................... 172

Table C. 1. Characteristics of raw feedstocks in AnCoD of manure and SSO ........................ 172

Table C. 2. Average $\mathrm{CH}_{4}$ measurements in $\mathrm{AnCoD}$ of manure and $\mathrm{SSO}$.............................. 173

Table C. 3. Cumulative $\mathrm{CH}_{4}$ production in $\mathrm{AnCoD}$ of manure and SSO .............................. 174

Table C. 4. Cumulative $\mathrm{CH}_{4}$ production per mass of COD added in AnCoD of manure and SSO

Table C. 5. Cumulative $\mathrm{CH}_{4}$ production per mass of VSS added in AnCoD of manure and SSO

Table C. 6. Cumulative $\mathrm{CH}_{4}$ production per volume of substrate added in AnCoD of manure and $\mathrm{SSO}$

Figure C. 1. Ultimate methane production in AnCoD of TWAS and manure. 178

Table C. 7. Characteristics of the raw feedstocks for hydrolysis/acidification of manure and SSO

Table C. 8. Measured and theoretical VFAs over time in hydrolysis/acidification of manure and SSO 180

Table C. 9. Concentration of soluble and particulate COD over time in hydrolysis/acidification of manure and SSO 181

Figure C.2. Concentration of soluble COD over time at different mixing ratios ..................... 182

D. Analytical results for AnCoD of TWAS, manure and SSO .......................................... 183

Table D. 1. Characteristics of raw feedstocks in AnCoD of TWAS, Manure, and SSO........... 183 
Table D. 2. Average $\mathrm{CH}_{4}$ measurements (mL) in AnCoD of TWAS/Mnaure/SSO (T:TWAS,

M:Manure)

Table D. 3. Cumulative $\mathrm{CH}_{4}$ production $(\mathrm{mL})$ in AnCoD of TWAS/Mnaure/SSO (T:TWAS,

M:Manure)

Table D. 4. Cumulative $\mathrm{CH}_{4}$ production per mass of COD added in AnCoD of

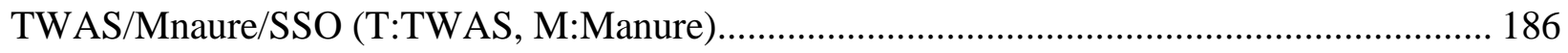

Table D. 5. Cumulative $\mathrm{CH}_{4}$ production per mass of VSS added in AnCoD of

TWAS/Mnaure/SSO (T:TWAS, M:Manure)

Table D. 6. Cumulative $\mathrm{CH}_{4}$ production per volume of substrate added in AnCoD of

TWAS/Mnaure/SSO (T:TWAS, M:Manure) .................................................................. 188

Figure D. 1. Ultimate CH4 production in AnCoD of TWAS/Manure/SSO ........................... 189

Table D. 7. Characteristics of raw feedstocks for hydrolysis/acidification in AnCoD of

TWAS/Manure/SSO

Table D. 8. Measured and theoretical VFAs concentrations (mg/L) over time in

hydrolysis/acidification of TWAS/Manure/SSO

Table D. 9. Soluble and particulate COD concentrations (mg/L) over time in

hydrolysis/acidification of TWAS/Manure/SSO

Figure D.2. Concentration of soluble COD over time at different mixing ratios

References 


\section{List of Nomenclature}

AD

AnCoD

TWAS

SSO

$\mathrm{F}: \mathrm{M}$

COD

TCOD

SCOD

VSS

TSS

VS

TS

VFAs

GC

S Proteins

S Lipids

S Carbohydrates

LCFAs

TN

TSN

$\mathrm{C}: \mathrm{N}$

COD:N
Anaerobic digestion

Anaerobic co-digestion

Thickened waste activated sludge

Source separated organics

Food to microorganism ratio

Chemical Oxygen Demand

Total Chemical Oxygen Demand

Soluble Chemical Oxygen Demand

Volatile suspended solids

Total suspended solids

Volatile solids

Total solids

Volatile fatty aids

Gas chromatograph

Soluble proteins

Soluble lipids

Soluble carbohydrates

Long chain fatty acids

Total nitrogen

Total soluble nitrogen

Carbon to nitrogen ratio

COD to nitrogen ratio 


\section{Chapter 1}

Introduction 


\section{INTRODUCTION}

Waste materials including biowaste are constantly being generated due to inevitable human activities. Different techniques have been employed to manage and reduce the growing amount of biowaste. However, such technologies result in secondary environmental impact. Landfilling can lead to soil and groundwater contamination imposing further actions and cost to remediate the secondary contamination. If not well managed and maintained, incineration will cause air pollution and subsequent environmental and health impact (Elbeshbishy et al., 2017; Feng et al., 2018). Mitigation of climate change and fossil fuel consumption demands a shift to alternative, renewable energy sources (Cornelissen et al., 2012; Kwietniewska and Tys, 2014). As reported by International Energy Outlook, 2011, total world energy consumption will increases from 505 quadrillion British thermal units (Btu) in 2008 to an estimated number of 619 quadrillion Btu in 2020, and it is expected to rise to 770 quadrillion Btu in 2035 which is equivalent to $53 \%$ increase in spam of 27 years (OECD/EIA, 2011). Energy obtained from biomass is regarded as an important future renewable source, as it is capable of providing a continuous power generation and it is also an essential part of the current $\mathrm{CO}_{2}$-mitigation policy (Appels et al., 2011; Kwietniewska and Tys, 2014).

Production of biofuel from biomass, has received increasing attention during recent years. Several treatment processes and technologies have been established to obtain sustainable and affordable biofuel which includes syngas (SNG). SNG is a synthetic gas produced by gasification of a carbon containing fuel that has some energy value. However, production of SNG is narrowly practiced due to its cost (Guo et al., 2015; Schuetzle et al., 2015). Anaerobic digestion (AD) which is widely used for the treatment of wet residual biomass is considered to be one of the most favorable processes for biofuel production from biomass.

In Canada, more than 27 million tons of food waste are disposed yearly. The produced organic waste can lead to serious health and environmental issues. Municipalities have taken different management actions to manage the organic fraction of municipal solid waste (OFMSW). Biological processes for the treatment and/or conversion of OFMSW to value-added products has aroused significant attention due to its financial benefits and less environmental impacts compared to the other waste disposal methods such as landfilling, incineration, gasification, etc. (Luk and Bekmuradov, 2014; Naroznova et al., 2016; Razavi et al., 2019). Organic matters in waste includes

food scraps, yard trimmings, wood waste, paper and cardboard products which normally make up 
around $33 \%$ by weight of the municipal solid waste. Source Separated Organics (SSO) refers to the organic waste which is segregated from other waste materials at the source for separate collection. SSO comprises mostly of food waste which is separated from the residential waste (Kelleher Robins, 2013).The study by Kelleher Environmental have indicated that almost 23\% of waste from non-residential industrial, commercial and institutional (IC\&I) sector is food waste which is generated by institutions and businesses in communities across Canada. The majority of food waste from this sector is created by restaurants, hotels, food processing facilities and hospitals. A considerable amount of biogas through anaerobic digestion process can be produced by food wastes from all of these sources (Government of Canada, 2013; Kelleher Robins, 2013). In areas with vast numbers of large-scale livestock farms, the development of a treatment process for manure is necessary to properly handle the high amount of produced waste. Animal manure provides adequate nutrients for anaerobic digestion to produce biogas as a source of renewable energy. High degradability of manure when flushed and fibrous materials are separated makes its anaerobic digestion a good treatment option to minimize waste above and beyond bioenergy recovery. However, in some farms manure is collected in lagoons or in the open areas which can result in air and water pollution. Manure stockpiling can lead to serious health environmental issues as a result of emissions such as methane, nitrous oxide, ammonia, hydrogen sulfide, volatile organic compounds and particulate matter (Lin et al., 2018; Peter Wright et al., 2013). Co-digestion of manure with additional substrates provided that appropriate mixture ratios are applied can improve digestion process and increase biogas production.

In addition, in Canada more than 660,000 metric tons of dry stabilized biosolids is produced each year. Biosolids are produced during the treatment of wastewater due to the removal of the solids content (sludge) from the liquid effluent. Biosolids management is responsible for almost $50 \%$ of the total operating annual cost of wastewater management. According to Canadian Council of Ministers of the Environment (CCME), a Canada-wide approach for the management of wastewater biosolids was approved on October 11, 2012 which encourages the favorable and thorough management of biosolids. Biosolids are the nutrient-rich, organic materials which makes them a useful resource, containing necessary plant nutrients, and organic matter. They can be recycled as a fertilizer and soil improvement for agricultural utilization. Annually, around 195,000 tons of biosolids is processed by the Ashbridges Bay and Highland Creek wastewater treatment 
plants in City of Toronto. The City's biosoilds management can be classified as the following options (City of Toronto, 2019):

- Land application for agricultural and other purposes

- Pelletization for fertilizer production

- Alkaline stabilization for producing fertilizer, landfill cover, or for the $\mathrm{pH}$ adjustment of acidic soil

- Landfilling

- Incineration

There are some pros and cons associated with any of the above options. However, Anaerobic Digestion (AD) for producing bioenergy from organic waste has shown to be an energy-efficient technology while causing less environmental footprint compared to other technologies such as composting that requires large land and can release uncontrolled odorous volatile organic compounds and pathogens (Bordeleau and Droste, 2011; Lin et al., 2018; Razavi et al., 2019). Nevertheless, in conventional anaerobic digestion of single feedstock some problems such as nutrient imbalance, low biodegradability, toxic substances, etc. can hinder the process causing low production of biogas. Such problems can be modified by simultaneous digestion of two or more feedstocks referred to as anaerobic co-digestion (AnCoD).

\subsection{Research objective}

Anaerobic digestion involves sequential phases comprising hydrolysis, acidogenesis, acetogenesis and methanogenesis. Anaerobic digestion of multi feedstocks has been advantageous compared to conventional anaerobic digestion of single feedstock. Literature has showed contradictory values for the optimum range of the $\mathrm{C}: \mathrm{N}$ or COD:N ratio for optimizing the mixing ratio of the feedstocks in anaerobic co-digestion systems. In addition, hydrolysis of particulate organic matter which is the rate-limiting stage, remains as the least well-defined phase of the process. Therefore, this research focused on the three main following objectives:

4- Study biomethane potential in AnCoD of TWAS, Manure, and SSO. This part of the study included the following purposes: 
Evaluate the influence of the mixing ratio on improving biomethane production in anaerobic co-digestion of TWAS, manure, and SSO.

Investigate the impact of lipids: proteins: carbohydrates on improving biomethane production in anaerobic co-digestion of TWAS, manure, and SSO.

$>$ Compare the impacts of COD:N ratio, lipids:proteins, lipids:carbohydrates, and proteins:carbohydrates on biomethane production in anaerobic co-digestion of TWAS, manure, and SSO.

5- Study the hydrolysis/acidification in AnCoD of TWAS, Manure, and SSO. This part of the present research comprised the following areas:

Monitor the dynamic changes of soluble and particulate COD, lipids, proteins, and carbohydrate content in co-digestion of TWAS, manure, and SSO.

Evaluate the influence of the mixing ratio on hydrolysis/acidification performance in co-digestion of TWAS, manure, and SSO.

Study the hydrolysis kinetics of lipids, proteins, carbohydrates and COD and calculate their hydrolysis rate coefficients for co-digestion of TWAS, manure, and SSO.

6- Investigate the relationship between biomethane production and the lipids, proteins, and carbohydrates contents of the feedstocks aiming to:

Propose an empirical model that explains the relationship between biomethane yield and concentration of lipids, proteins, and carbohydrates at different mixing ratios in anaerobic digestion of manure, TWAS and SSO individually and in their codigestion.

an empirical model that explains the relationship between biomethane yield and concentration of lipids, proteins, and carbohydrates at different mixing ratios in anaerobic digestion of manure, TWAS and SSO individually and in their co-digestion. 


\section{Chapter 2}

\section{Literature review}




\section{LITERATURE REVIEW}

\subsection{Anaerobic digestion}

Biological treatment is an integral part of any wastewater treatment plant that treats wastewater from either municipality or industry which contains soluble organic contaminants or a mix of both source types. There are clear economic advantages of biological systems in terms of capital investment and operating costs when compared to other methods such as chemical oxidation, thermal oxidation, etc. In addition, mostly biological treatment converts toxic contaminants to end products that are less harmful or non-harmful with the help of microorganisms (Mittal, 2011). The two types of biological treatment: aerobic and anaerobic processes are directly related to first the type of microorganisms involved in the degradation of organic compounds in a particular wastewater and second the operating conditions of the bioreactor. Aerobic treatment processes occur in the presence of air and consume those microorganisms also called aerobes, which use molecular/free oxygen to assimilate organic contaminant. They convert them in to carbon dioxide, water and biomass (Eddy, 2003). The anaerobic processes, on other side occur in the absence of air (molecular/free oxygen) by those microorganisms also called anaerobes which do not require air or molecular/free oxygen to assimilate organic contaminants. The end products of organic assimilation in anaerobic treatment contains methane $\left(\mathrm{CH}_{4}\right)$ and carbon dioxide $\left(\mathrm{CO}_{2}\right)$ and biomass.

Anaerobic digestion of municipal wastewater sludge has been widely developed since the early $1900 \mathrm{~s}$ and is the most applied sludge treatment method. Throughout the process about $40 \%$ to $60 \%$ of the organic solids is converted to methane and carbon dioxide. The chemical composition of the produced gas contains 60-65\% methane, 30-35\% carbon dioxide, and small quantities of $\mathrm{H}_{2}, \mathrm{~N}_{2}$, $\mathrm{H}_{2} \mathrm{~S}$, and $\mathrm{H}_{2} \mathrm{O}$. Among these, methane has the most value since it is a hydrocarbon fuel producing $36.5 \mathrm{MJ} / \mathrm{m}^{3}$ in combustion (Lema and Suarez, 2017). The residual organic matter is chemically stable, nearly odorless, and contains considerably reduced levels of pathogens.

Hydrogen production by the use of anaerobic microbial communities also referred to as dark fermentation from organic waste has raised attention due to its ability to produce an environmentally benign energy source, while it stabilizes waste material simultaneously (Sung et al., 2003). Albert Lea facility in Minnesota with 12 million gallons/day of sewage and 4.5 million gallons/day sludge produces $75,000 \mathrm{ft}^{3} /$ day of biogas. Their facility which consists of 4 Capstone 
microturbines, each of them $30 \mathrm{~kW}$, is producing 2,500 $\mathrm{kWh}$ per day electricity at peak production in addition to $28,000 \mathrm{Btu} /$ day of heat.

According to Canadian Biogas Study conducted by Canadian Biogas Association in 2013, biogas production has significant potential for extension and development. All biogas sources excluding energy crops, could meet nearly 3\% of Canada's natural gas demand as biogas contribution is $2,420 \mathrm{Mm}^{3}$ /year of renewable natural gas (RNG) or $1.3 \%$ of its electricity demand where biogas contribution is $810 \mathrm{MW}$. Anaerobic digestion (AD) is a multi-step process during which organic material is converted to biogas and digestate in the absence of oxygen and presence of anaerobic microorganisms. The AD process occurs in four stages including hydrolysis/liquefaction, acidogenesis, acetogenesis, and methanogenesis. The pathways in anaerobic degradation is shown in figure 1.

The first stage of the process is hydrolysis/liquefaction. This stage is very important in AD process since polymers cannot be directly consumed by the fermentative microorganisms. Therefore, hydrolysis makes the substrate available for the following conversion steps. In hydrolysis stage, insoluble complex organic materials are decomposed into their constituents. This allows them transport through microbial cell membrane (Madigan, 2014). Hydrolysis is accomplished due to the function of hydrolytic enzymes. In the first step of hydrolysis, or liquefaction, fermentative bacteria convert the insoluble complex organic compounds like cellulose, into soluble molecules such as sugars, fatty acids, and amino acids.

Hydrolysis/Liquefaction reactions

$$
\begin{aligned}
& \text { Lipids } \rightarrow \text { Fatty Acids } \\
& \text { Polysaccharides } \rightarrow \text { Monosaccharides } \\
& \text { Protein } \rightarrow \text { Amino Acids } \\
& \text { Nucleic Acids } \rightarrow \text { Purines and Pyrimidines }
\end{aligned}
$$

The next stage is acidogenesis/fermentation through which facultative and anaerobic bacteria convert sugars, amino acids and fatty acids to hydrogen, acetate, carbon dioxide, VFAs such as propionic, butyric and acetic acid, ketones, alcohols and lactic acid. Although a simple substrate like glucose can be fermented, various products are created by the diverse bacterial community. 
Conversion of glucose to acetate, ethanol and propionate are shown in the reactions 1, 2 and 3 below respectively (Kangle et al., 2012).

$$
\begin{aligned}
& \mathrm{C}_{6} \mathrm{H}_{12} \mathrm{O}_{6}+2 \mathrm{H}_{2} \mathrm{O} \rightarrow 2 \mathrm{CH}_{3} \mathrm{COOH}+2 \mathrm{CO}_{2}+4 \mathrm{H}_{2} \\
& \mathrm{C}_{6} \mathrm{H}_{12} \mathrm{O}_{6} \rightarrow 2 \mathrm{CH}_{3} \mathrm{CH}_{2} \mathrm{OH}+2 \mathrm{CO}_{2} \\
& \mathrm{C}_{6} \mathrm{H}_{12} \mathrm{O}_{6}+2 \mathrm{H}_{2} \rightarrow 2 \mathrm{CH}_{3} \mathrm{CH}_{2} \mathrm{COOH}+2 \mathrm{H}_{2} \mathrm{O}
\end{aligned}
$$

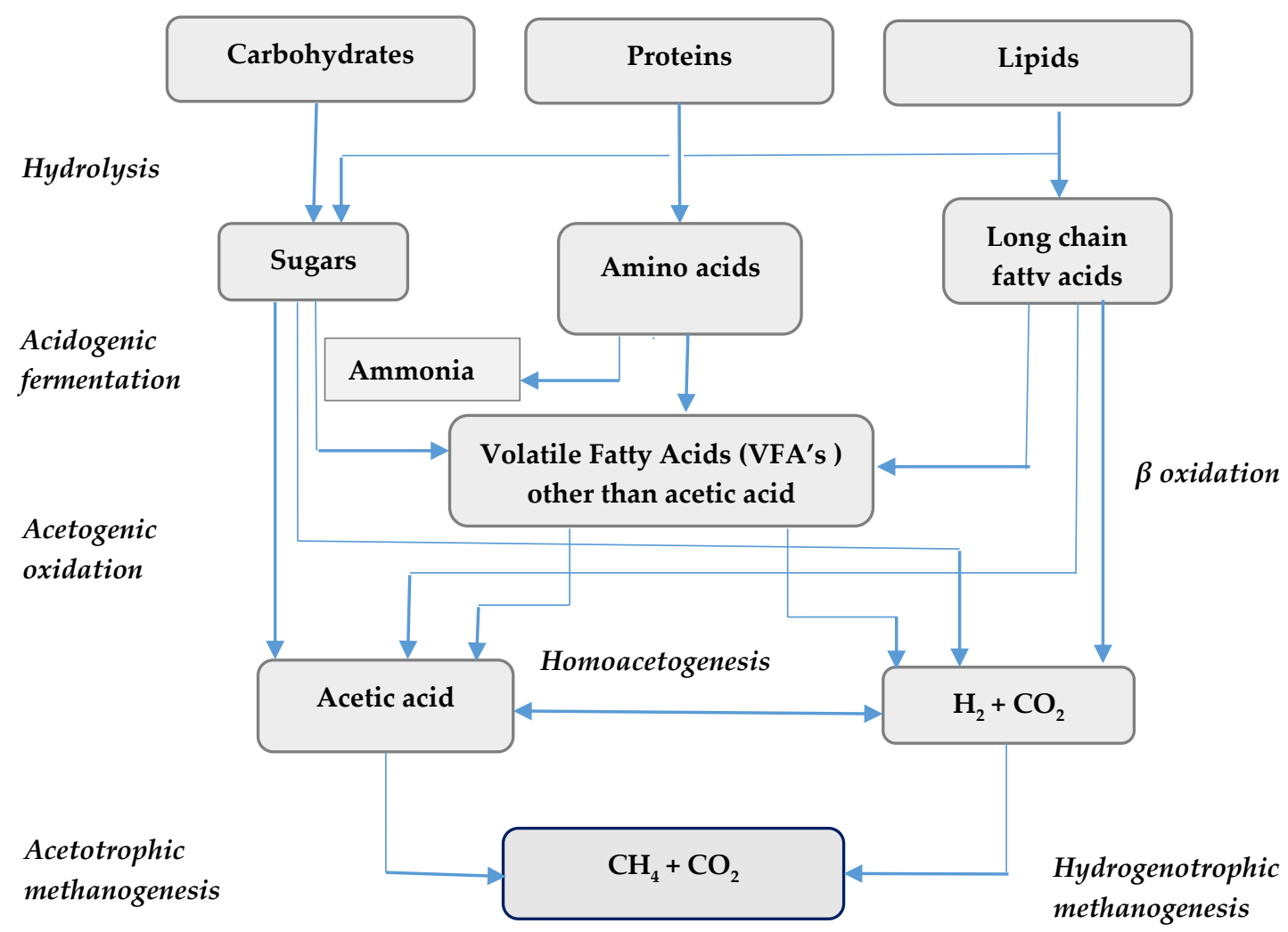

Figure 2.1. Pathways of anaerobic digestion (Salminen and Rintala, 2002)

In equilibrium condition, most of the organic material is converted into substrates such as acetate, hydrogen and carbon dioxide that are readily available for methanogenic microbes. However, a significant portion of about $30 \%$ is converted to short chain fatty acids or alcohols. Degradable organic material is eliminated in this step (Angelidaki et al., 2009a; Kangle et al., 2012). The 
byproduct of amino acids fermentation, ammonia and hydrogen sulfide are released. These compounds can be inhibitory for AD process (Kangle et al., 2012; Salminen and Rintala, 2002).

During the third stage, acetogenesis, certain fermentation products including VFAs with more than two atoms of carbon, alcohols and aromatic fatty acids are converted into acetate and hydrogen these conversions take place via obligate hydrogen producing bacteria (Boe, 2006, Kangle et al., 2012). In this step, the products of the first phase are converted to simple organic acids, carbon dioxide and hydrogen by acetogenic bacteria, also called acid formers. The main acids produced include acetic acid $\left(\mathrm{CH}_{3} \mathrm{COOH}\right)$, propionic acid $\left(\mathrm{CH}_{3} \mathrm{CH}_{2} \mathrm{COOH}\right)$, butyric acid $\left(\mathrm{CH}_{3} \mathrm{CH}_{2} \mathrm{CH}_{2} \mathrm{COOH}\right)$, and ethanol $\left(\mathrm{C}_{2} \mathrm{H}_{5} \mathrm{OH}\right)$. The activities of different microorganisms cause the formation of the products that are created during acetogenesis. These microorganisms include syntrophobacter wolinii, a propionate decomposer and sytrophomonos wolfei, a butyrate decomposer. Also other acid formers include clostridium spp., peptococcus anerobus, lactobacillus, and actinomyces (Kangle et al., 2012; Themelis, 2002). Hydrogen-producing acetogenic bacteria yield acetate, $\mathrm{H}_{2}$ and $\mathrm{CO}_{2}$ from volatile fatty acids and alcohol, whereas, homoacetogenic bacteria produce acetate from $\mathrm{CO}_{2}$ and $\mathrm{H}_{2}$ (Sterling et al., 2001). However, most of the acetate is formed via hydrogen-producing acetogenic bacteria (Angelidaki et al., 2009b). A reaction that occurs during acetogenesis is presented below:

$$
\mathrm{C}_{6} \mathrm{H}_{2} \mathrm{O}_{6} \rightarrow 2 \mathrm{C}_{2} \mathrm{H}_{5} \mathrm{OH}+2 \mathrm{CO}_{2}
$$

The final stage of $\mathrm{AD}$ process is methanogenesis. Various methane-forming bacteria are required in an $\mathrm{AD}$ system. That is because a single species is not able to degrade all the existing substrates. The methanogenic bacteria comprise methanobacterium, methanobacillus, methanococcus, and methanosarcina. Methanogenesis can be classified into two groups. These two groups involves acetate and $\mathrm{H}_{2} / \mathrm{CO}_{2}$ consumers. Methanosarcina spp. and methanothrix spp. Also known as methanosaeta are important in $\mathrm{AD}$ process both as $\mathrm{H}_{2} / \mathrm{CO}_{2}$ and acetate consumers. Almost $70 \%$ of the methane is produced from acetate. The remaining $30 \%$ is produced due to the reduction of carbon dioxide via hydrogen and other electron donors (Smith and Mah, 1966; Varel et al., 1980). With regard to the type of the substrate consumed by the methanogens, methanogenesis can be classified into two main types (Bitton, 2010; Kangle et al., 2012):

- Hydrogenotrophic methanogenesis 
In this type of methanogenesis, hydrogen and carbon dioxide are converted into methane through the following reaction:

$$
\mathrm{CO}_{2}+4 \mathrm{H}_{2} \rightarrow \mathrm{CH}_{4}+2 \mathrm{H}_{2} \mathrm{O}
$$

- Acetotrophic or aceticlastic methanogenesis

This type of methanogenesis involves the formation of methane from the conversion of acetate by the following reaction:

\section{$\mathrm{CH}_{3} \mathrm{COOH} \rightarrow \mathrm{CH}_{4}+\mathrm{CO}_{2}$}

Any substrate that can be converted to methane by anaerobic bacteria is referred to as feedstock. The main components of organic wastes (feedstock) are carbon, oxygen, nitrogen, hydrogen and phosphorus, and microbial cell material of those elements is approximately 50, 20, 12, 8 and $2 \%$ respectively (Gerardi, 2003). Sulfur is also required to synthesize vital proteins in metabolic and anabolic pathways (Madigan, 2014). Feedstocks can be a range of different waste materials from easily degradable wastewater to complex high-solid waste.

AD technologies have shown sufficient adaptability to different feedstocks (Bordoloi et al., 2014). Although $\mathrm{AD}$ is a commercial reality for a range of wastes, anaerobic digestion of single waste may be associated with certain drawbacks such as unbalanced nutrients, rapid acidogenesis, poor buffering capacity, high ammonia nitrogen concentration, inhibition of long chain fatty acids which can inhibit methanogenesis and lead to severe instability and process disruption (Bayr et al., 2014; Silvestre et al., 2014).

The growth rate of anaerobic microorganisms and subsequent biogas production depends highly on the composition of the organic matter in feedstock. The constituents of the feedstock added to the digester are consumed selectively by a range of different microbial consortia. In addition, the existence of nitrogen in the feedstock is necessary for the synthesis of amino acids, proteins and nucleic acids. It is also required for ammonia formation to neutralize VFAs produced during the fermentation process and to maintain neutral $\mathrm{pH}$ condition for cell growth. However, an excess of nitrogen in the feedstocks can result in toxic effects to bacteria by extreme ammonia formation. Therefore, a suitable amount of nitrogen is required to provide sufficient nutrient while avoiding ammonia toxicity (Hagos et al., 2017; P. et al., 2014). 
During anaerobic digestion, a series of complex biological degradation pathways are involved which are influenced by numerous factors. Therefore, a profound understanding of the biochemical activities of anaerobic microorganisms in the AD system is required to support an effective control of the governing factors in anaerobic digestion process (Viotti et al., 2004).

\subsection{Process parameters of anaerobic digestion}

A number of parameters affect the rate of the different steps of the digestion within the anaerobic environment. Overall, two groups of parameters can affect the anaerobic digestion performance including environmental and operational parameters. Environmental factors comprise temperature, $\mathrm{pH}$, alkalinity, waste characteristics such as the amounts of volatile solids (VS) carbon to nitrogen $(\mathrm{C}: \mathrm{N})$ ratio, total solids (TS), nutrients, organic loading rate (OLR), and volatile fatty acids (VFAs). The performance of anaerobic digesters can be reduced by various environmental factors including low $\mathrm{pH}$, accumulation of ammonia and volatile fatty acids (VFAs) which inhibit the activity of methanogenic microorganisms (Heo et al., 2004; Towey, 2013). VS consists of both biodegradable volatile solids (BVS) and the refractory volatile solids (RVS) fractions. BVS fraction of substrate is helpful in better biodegradability of the waste, organic loading rate, $\mathrm{C}: \mathrm{N}$ ratio, and biogas production. Apart from the environmental factors, a number of operational factors including solid retention time (SRT), hydraulic retention time (HRT), digestion mode in terms of single or multistage approaches, digester design being batch or continuous types, and digester mixing also affect the AD performance. These parameters are individually discussed in the following sections.

\subsubsection{Waste composition and volatile solids}

The wastes to be treated by $\mathrm{AD}$ process can be comprised of a biodegradable organic fraction, a combustible and an inert fraction. Waste materials containing high VS and low non- biodegradable material, or refractory volatile solids, are the most suited to AD treatment. Kitchen waste, food waste, and garden waste are biodegradable organic fraction of the waste. The combustible fraction can be slowly degrading lignocellulosic organic compounds such as coarser wood, paper, and cardboard. The lignocellulosic organic content does not readily degrade under anaerobic conditions. Therefore, they are more suitable for waste-to-energy plants. The inert fraction of the waste may contain stones, glass, sand, metal, etc. that can ideally be removed, recycled or used at 
land fill. It is important to remove the inert fraction before digestion, otherwise it causes the increase of digester volume and wear of equipment. The Volatile Solids (VS) of organic wastes are measured as total solids excluding the ash content which is obtained by complete combustion of the feed wastes. The VS contains the Biodegradable Volatile Solids (BVS) fraction and the Refractory Volatile Solids (RVS). Knowing the BVS fraction of substrate can be helpful in better estimation of the biodegradability of the waste, of biogas production, organic loading rate and $\mathrm{C}: \mathrm{N}$ ratio. Lignin is a complex organic compound which is not easily degraded by anaerobic bacteria and forms refractory volatile solids (RVS) in organic matter. Waste materials containing high VS and low non-biodegradable material, or RVS, are the most suited to AD treatment (Elbeshbishy et al., 2017; Kangle et al., 2012).

\subsubsection{Carbon to Nitrogen Ratio (C:N)}

The amount of carbon and nitrogen present in feedstock or $\mathrm{C}: \mathrm{N}$ ratio is a very important parameter of the AD process. A high $\mathrm{C}: \mathrm{N}$ ratio indicates rapid consumption of nitrogen by methanogens and leads to lower gas production. On the contrary, a lower $\mathrm{C}: \mathrm{N}$ ratio results in accumulation of ammonia and exceeding $\mathrm{pH}$ values which is toxic to methanogens. Low $\mathrm{C}: \mathrm{N}$ ratios occur when too much nitrogen is present. On the other side, a high $\mathrm{C}: \mathrm{N}$ ratio leads to deficiency in AD system (Mata-Alvarez et al., 2000a; Poliafico and Murphy, 2007). According to a study (Gerardi, 2003), the optimal gas production can be achieved by feedstock with C:N ratio of 25:1. Other studies reported that the optimum range falls within 25-30 and can be achieved by the co-digestion of different waste streams (Gonzalez-Avila et al., 2011; Monnet, 2003). Some other studies even though reported that the optimum $\mathrm{C}: \mathrm{N}$ ratios in anaerobic digesters are between 20 and 30 while some other studies even reported lower values than 20. Although, a very low C:N ratios occurs when too much nitrogen is present and leads ammonia $\left(\mathrm{NH}_{3}\right)$ to be accumulated which leads to either high pH values or methanogenic inhibition (Salminen et al., 2002, Kangle et al, 2012). On

the contrary, the high $\mathrm{C}: \mathrm{N}$ ratio indicates that nitrogen is rapidly depleted by methanogens and leads to lower gas production. A lower $\mathrm{C}: \mathrm{N}$ ratio results in accumulation of ammonia and exceeding $\mathrm{pH}$ values to over 8.5, which is toxic to methanogens. Mixing materials of high and low $\mathrm{C}: \mathrm{N}$ ratios, such as organic solid waste mixed with animal manure or sewage can help achieve optimum C:N ratios (Poliafico, 2007, Kangle et al, 2012). 


\subsubsection{Nutrients}

Some nutrient elements are needed for the growth of methane-forming bacteria. Particular metals comprising nickel, iron, cobalt, and molybdenum are essential for optimal growth and methane production. Trace metals stimulate methanogenic activity. Some metals including selenium, molybdenum, manganese, aluminum, and boron have been suggested as additional components in media. Addition of metal ions solutions to anaerobic digesters can improve the performance of the AD system. The amounts of 0.002, 0.004, 0.003, $0.02 \mathrm{mg} / \mathrm{g}$ are suggested for iron, cobalt, nickel, and zinc respectively. The requirement for nickel is quite unusual for biological systems and can exclusively characterize methanogenic bacteria. Addition of metal ions solutions to anaerobic digesters can improve the performance of the AD system (Azbar and Speece, 2001; Kangle et al., 2012; Mata-Alvarez et al., 2000a).

\subsubsection{Total solids content (TS)/Organic loading rate (OLR)}

The increase of the total solids (TS) fraction leads a corresponding decrease in the reactor volume. High solids (HS) AD system contain $22 \%$ to $40 \%$ TS, medium solids (MS) system about 15 to $20 \%$ and low solids (LS) AD systems contain less than $10 \%$ of TS. The organic loading rate (OLR) is defined as the organic matter flowing into the digester per time which is expressed as mass of organic matter over digester volume over time (Appels et al., 2008; Kangle et al., 2012). OLR is also defined as measure of the biological conversion capacity of the AD system. Typical values of OLR ranges between 0.5 and $3 \mathrm{~kg} \mathrm{VS} / \mathrm{m}^{3} / \mathrm{d}$. OLR is particularly important parameter in continuous systems. System failures as a result of overloading have been reported by numerous plants (Kangle et al., 2012; Poliafico and Murphy, 2007).

When feeding the system above its sustainable OLR, low biogas yield is obtained. This is caused by the accumulation of the inhibiting substances such as fatty acids in the digester slurry. Any substrate that can be converted to methane by anaerobic bacteria is referred to as feedstock. The main components of feedstock are carbon, oxygen, nitrogen, hydrogen and phosphorus, and microbial cell material of those elements is reported to be approximately 50, 20,12,8 and $2 \%$ respectively. Feedstocks can be a range of different waste materials from easily degradable wastewater to complex high-solid waste (Gerardi, 2003). 


\subsection{5. $\mathrm{pH}$, alkalinity and volatile acids/alkalinity ratio}

There is a different optimum $\mathrm{pH}$ range for each group of micro-organisms. Methanogenic bacteria are very sensitive to $\mathrm{pH}$. The optimum range for them is between 6.5 and 7.2. The fermentative microorganisms are relatively less sensitive and can tolerate a wider range of $\mathrm{pH}$ between 4.0 and 8.5. At low $\mathrm{pH}$, mainly acetic and butyric acids are produced, while at a $\mathrm{pH}$ of around 8.0, acetic and propionic acids are mainly produced. The VFAs produced during AD process result in a $\mathrm{pH}$ reduction. This reduction is normally adjusted by methanogenic bacteria, which produce alkalinity in the form of ammonia, carbon dioxide, and bicarbonate (Appels et al., 2008).

The $\mathrm{pH}$ of the system is controlled by the $\mathrm{CO}_{2}$ in the gas phase and the $\mathrm{HCO}_{3}$-alkalinity of the liquid phase. If the concentration of $\mathrm{CO}_{2}$ remains constant, the addition of $\mathrm{HCO}_{3}$-alkalinity can increase the $\mathrm{pH}$ of digester. In order to maintain a stable and well-buffered digestion process a buffering capacity of 70 meq $\mathrm{CaCO}_{3} / \mathrm{L}$ or a molar ratio of at least $1.4: 1$ of bicarbonate/VFA is required. However, previous studies have shown that particularly the stability of the ratio is very significant and not so much its level.

Except for ammonia, other factors such as sulfide, sodium and potassium, heavy metals, volatile fatty acids, long-chain fatty acids, and hydrogen can also affect the activity of methanogens. Molecular hydrogen is formed throughout different stages of anaerobic digestion. Inhibition can occur due to the lack of balance between the rates of hydrolysis and methanogenesis. A suitable balance between those rates is essential for higher methane production. Rapid methanogenesis is required to prevent accumulation of organic acid lowering $\mathrm{pH}$ to an extent that inhibits methanogenesis (Appels et al., 2008; Pouget et al., 2012).

\subsubsection{Temperature}

The temperature has an important effect on the physicochemical properties of the substrate. Moreover, it is effective on the growth rate and metabolism of micro-organisms and the population dynamics in the reactor. A stable operating temperature is very important to be maintained in the digester, since fluctuations in temperature affect the bacteria particularly the methanogens. Acetotrophic methanogens are one of the most sensitive groups of bacteria to increasing temperatures. The degradation of propionate and butyrate is also sensitive to temperatures above $70^{\circ} \mathrm{C}$. (The propionate or propanoate ion is $\mathrm{C}_{2} \mathrm{H}_{5} \mathrm{COO}-$ the conjugate base of propionic acid- $\mathrm{A}$ propionic or propanoic compound is a small salt or ester of propionic acid. Butyric acid, also 
known under the systematic name butanoic acid, abbreviated BTA, is a carboxylic acid with the structural formula $\mathrm{CH}_{3} \mathrm{CH}_{2} \mathrm{CH}_{2}-\mathrm{COOH}$. Salts and esters of butyric acid are known as butyrates or butanoates) (Appels et al., 2008; Turovskiy and Mathai, 2005).

The temperature has also a substantial effect on the partial pressure of $\mathrm{H}_{2}$ in digesters and therefore, it affects the kinetics of the syntrophic metabolism. Thermodynamic studies show that endergonic reactions under standard conditions, for example the decomposition of propionate into acetate, $\mathrm{CO}_{2}$, and $\mathrm{H}_{2}$, would be more favorable energetically at higher temperature, whereas the exergonic reactions such as hydrogenotrophic methanogenesis are less favored at higher temperatures. Increasing temperature can be favorable for several reasons. It can increase solubility of the organic compounds, enhance chemical and biological reaction rates, and an increase the rate of pathogens' death in thermophilic conditions. Although, the application of high temperatures (thermophilic) has some adverse effects as there will be an increase of the fraction of free ammonia which can be an inhibiting factor for the microorganisms. The increasing pKa of the VFA will make the process more susceptible to inhibition. Therefore, temperature control is a very important concern for thermophilic digestion in comparison with mesophilic digestion. A stable operating temperature is very important to be maintained in the digester, since fluctuations in temperature affect the bacteria particularly the methanogens. Process failure can occur at changes in temperature over $1{ }^{\circ} \mathrm{C} /$ day and changes of more than $0.6{ }^{\circ} \mathrm{C} /$ day in temperature should be avoided Process failure can occur at changes in temperature over $1{ }^{\circ} \mathrm{C} /$ day, and changes of more than $0.6^{\circ} \mathrm{C} /$ day in temperature should be avoided (Appels et al., 2008; Turovskiy and Mathai, 2005).

\subsubsection{Solids and hydraulic retention time}

The average time that the solids spend in a digester is referred to as solids retention time (SRT), and the average time that the liquid sludge is held in the digester is referred to as hydraulic retention time (HRT). SRT and HRT are an important design and operating parameter for all anaerobic processes. Reduction of SRT decreases the extent of the reactions and vice versa. A fraction of the bacterial population is removed each time when the sludge is withdrawn. Therefore, the cell growth must at least compensate the cell removal to maintain steady state and to prevent from process failure. 
The ratio between the solid content of the reactor by the solid flow rate indicates the average SRT. HRT specifies the average time that waste and wastewater are exposed to microorganisms for degradation. In a conventional mixed reactor, the SRT and HRT are equal while in a retained biomass reactor the SRT is usually higher than HRT. Higher SRT can be achieved by increasing the digester volume, increasing the solid content or using a retained biomass reactor. Equation (1.1) shows the calculation for HRT and equation (1.2) shows the calculation for SRT in continues stirred tank reactor (CSTR).

$\mathrm{HRT}=$ Volume of the reactor $(\mathrm{V}) /$ Influent flow rate $=\mathrm{L} / \mathrm{L} / \mathrm{d}=\mathrm{d}$

Eq. 2.1

$\mathrm{SRT}=$ Mass of the biomass in reactor/ mass of the biomass leaving

$=\left(\mathrm{V}^{\times} \mathrm{VSS}\right) /\left(\mathrm{Q}_{\text {out }}{ }^{\times} \mathrm{VSS}\right)=\mathrm{V} / \mathrm{Q}_{\text {out }}=H R T d$

The effect of the retention time on the gas production is mostly studied on laboratory scale. Studies indicated that in a semi-CSTR system retention times shorter than 5 days are insufficient for a stable digestion. VFA concentrations are increasing due to washout of methanogenic bacteria. VFA concentrations are relatively high for 5-8 days SRT. There is an incomplete breakdown of compounds, especially of the lipids for this SRT. It has also been indicated that stable digestion is obtained after 8-10 days. There is low VFA concentrations and the breakdown of lipids starts. According to the studies, the breakdown curve stabilizes at SRT $>10$ days as all sludge compounds are significantly reduced. Figure 2 shows a schematic relationship between SRT and degree of digestion (Appels et al., 2008). 


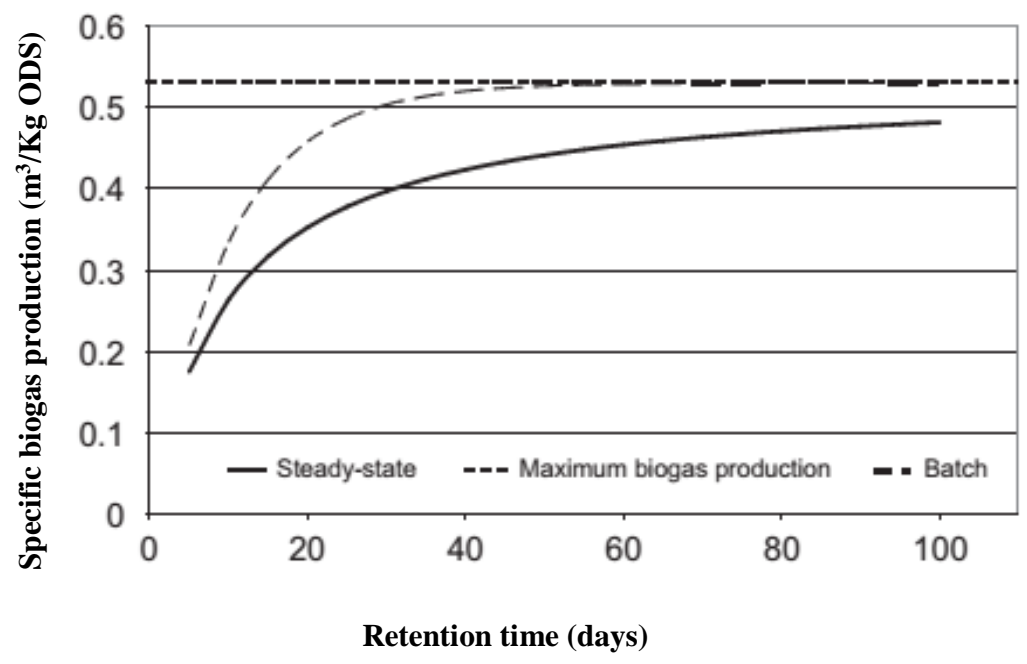

Figure 2.2. Schematic relationship between SRT and degree of digestion (Appels et al., 2008)

\subsubsection{Digester mixing}

To attain an optimum performance for an $\mathrm{AD}$ system, it is essential to maintain a proper mixing. Mixing causes near contact between the feed sludge and active biomass, yielding uniformity of several parameters including temperature, substrate concentration, other chemical, physical and biological aspects all through the digester. Mixing also prevents from the formation of surface scum layers and the sludge deposition on the bottom of the tank (Appels et al., 2008). The rise of gas bubbles and the thermal convection currents caused by the addition of heated sludge, results in some degree of natural mixing in the digestion tank. However, this is not adequate for an optimum performance and auxiliary mixing is required. There are some methods of auxiliary mixing including external pumped recirculation, internal mechanical mixing and internal gas mixing as it is shown in Fig. 3. 
a
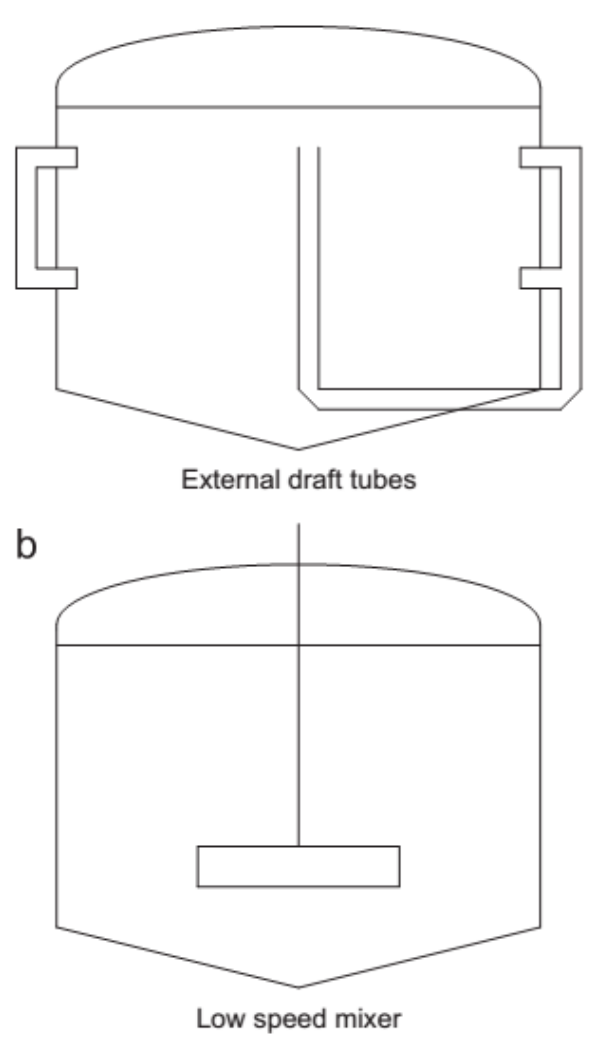

C
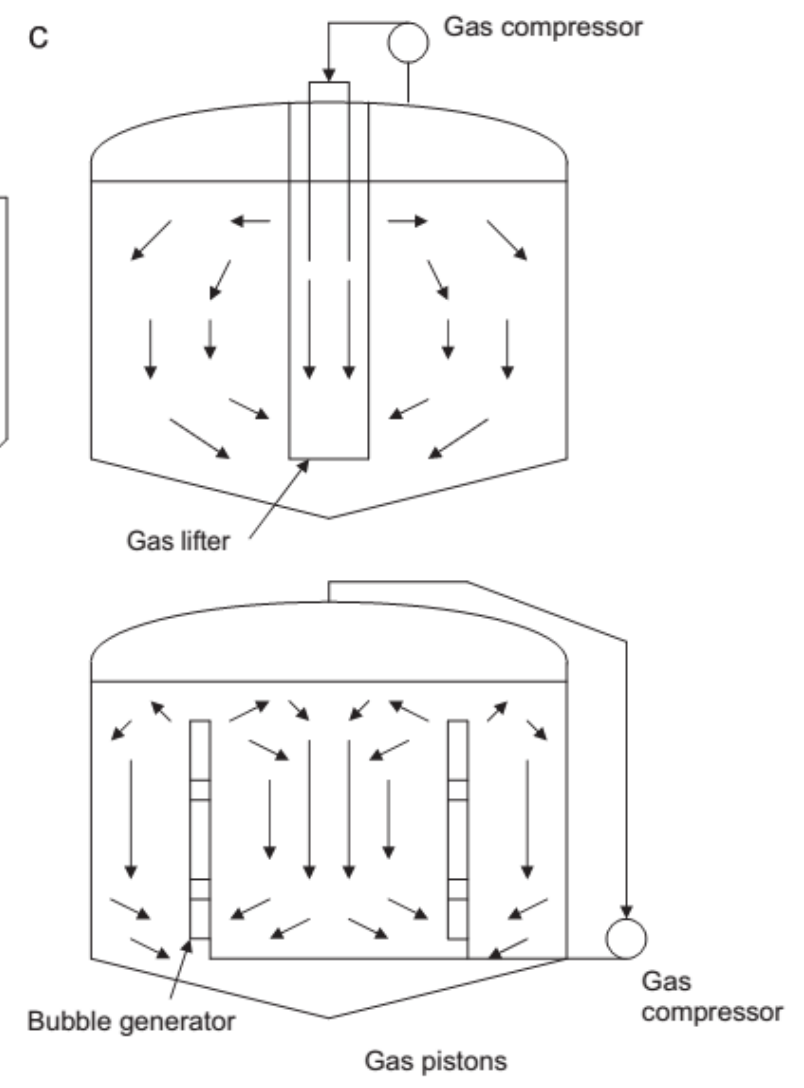

Figure 2.3. Types of mixing methods for digesters (a) external, pumped recirculation mixing, (b) internal mechanical mixing, and (c) external gas recirculation mixing (Appels et al., 2008).

\subsection{Anaerobic Co-Digestion (AnCoD)}

Anaerobic Co-digestion (AnCoD) involves the simultaneous anaerobic digestion of two or more organic waste feedstocks. The anaerobic co-digestion can be referred to as the simultaneous treatment of two or more organic biodegradable waste streams by anaerobic digestion. It provides a proper method of disposal for the organic fraction of solid waste which comes from source or from separate collection systems. This method of treatment, makes it possible to use the existing anaerobic reactors in wastewater treatment plants, with minor modifications and some additional requirements. By combining the treatments of two problematic wastes including for instance organic part of municipal solid waste and paper pulp sludge, higher yield in the production of biogas can be attained. Conventionally, anaerobic digestion was a single substrate and single purpose treatment. Recently, it has been indicated that when an increased variety of substrates 
applied at the same time, more stable AD is achieved. The most common state is when a major amount of a main basic substrate for example manure or sewage sludge is mixed and digested accompanied by minor amounts of a single, or a variety of additional substrate. The usage of cosubstrates usually improves the biogas yields from anaerobic digester due to positive interaction established in the digestion medium and the supply of missing nutrients by the co-substrates (Alvarez et al., 2008, Kangle et al, 2012). Anaerobic co-digestion offers several benefits including: improved nutrient balance and digestion, possible gate fees for waste treatment, additional biogas collection, and additional fertilizer i.e. soil conditioner (Elbeshbishy and Nakhla, 2012; Viotti et al., 2004).

Substrates with high C:N ratio have the poor buffering capacity and produce excessive amounts of VFAs during the fermentation. In contrast, substrates characterized by low $\mathrm{C}: \mathrm{N}$ ratio have high buffer capacity and the increased concentration of ammonia in the fermentation process leads to microbial growth inhibition. Anaerobic co-digestion ( $\mathrm{AnCoD})$, which entails the simultaneous digestion of two or more feedstocks has shown to be beneficial for its economic viability and increasing methane yields (Gelegenis et al., 2007; Karagiannidis and Perkoulidis, 2009; Kwietniewska and Tys, 2014). These problems have made AnCoD of multi feedstock to become a hot research area in the enhancement of conventional $\mathrm{AD}$ technology. The publications on AnCoD significantly increased within the last fifteen years indicating its capability for improving biogas production (Hagos et al., 2017). The main goal of anaerobic co-digestion is to increase biogas mainly biomethane for heat and electricity. As shown in Figure 2, a range of feedstocks can be co-digested at suitable blend ratio to maintain optimum condition required for metabolic activity and improved biogas production for thermal energy and power generation. Anaerobic co-digestion has shown to be a viable option to alleviate the drawbacks of mono-digestion while enhancing economic feasibility of the existing AD plants by increasing methane yields (Bayr et al., 2014; Mata-Alvarez et al., 2014, 2000b; Shah et al., 2015),(Mata-Alvarez et al., 2000a). 


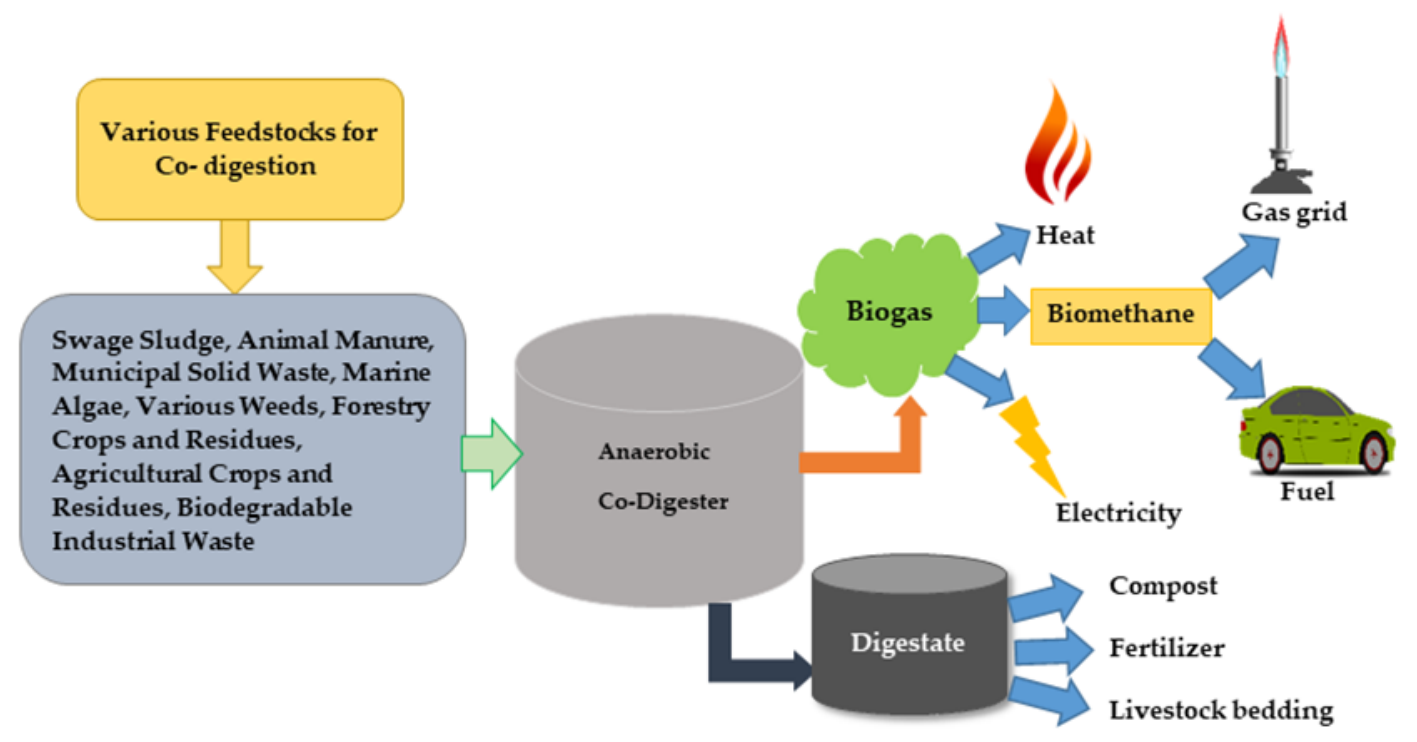

Figure 2.4. Co- digestion of multi feedstocks for waste reduction and energy recovery

Co-digestion can increase biogas production from $25 \%$ to $400 \%$ compared to the mono-digestion of the same substrates. Feedstocks characterized by higher $\mathrm{C}: \mathrm{N}$ ratio $(>50)$ such as rice and wheat straws, corn stalks, seaweed and algae can be co-digested by the feedstocks of lower C:N ratio for instance pig manure, poultry manure, food and kitchen wastes to achieve nutrient balance and to avoid the inhibitions which leads to system instability and reduced biogas production as a result of unsuited C:N ratio (Hagos et al., 2017; R et al., 2017; Sosnowski et al., 2003). Table 2.1 shows the possible feedstocks for co-digestion with regard to $\mathrm{C}: \mathrm{N}$ ratio.

Various advantages of AnCoD systems are presented in Figure 3. Multiple aspects are considered when applying AnCoD. Cost of transporting the co substrate from the generation point to the $\mathrm{AD}$ plant seems to be the primary consideration, the selection of the best co-substrate and blend ratio in order to enhance synergism, dilute disruptive compounds, optimization of methane production and digestate quality, are also important consideration that treatment plants need to evaluate when using the AnCoD (Divya et al., 2015; Mata-Alvarez et al., 2014). Operational and environmental factors as mentioned previously are factors that influence methane yield in anaerobic co-digestion of multi feedstocks (Long et al., 2012). 
Table 2.1. Potential feedstocks for co-digestion to balance nutrient with regard to C/N ratio [(Hagos et al., 2017),(R et al., 2017)-(Sosnowski et al., 2003)]

\begin{tabular}{|c|c|c|c|c|c|}
\hline $\begin{array}{l}\text { Feedstocks with } \max \\
\mathrm{C} / \mathrm{N} \text { ratio }<20\end{array}$ & $\mathrm{C} / \mathrm{N}$ ratio & $\begin{array}{l}\text { Feedstocks with } \max \\
\mathrm{C} / \mathrm{N} \text { ratio } \leq 40\end{array}$ & $\mathrm{C} / \mathrm{N}$ ratio & $\begin{array}{l}\text { Feedstocks with } \mathrm{C} / \mathrm{N} \\
\text { ratio around or }>50\end{array}$ & $\mathrm{C} / \mathrm{N}$ ratio \\
\hline TWAS $^{1}$ & $6-9$ & OFMSW $^{3}$ & 24 & Potatoes & $35-60$ \\
\hline $\mathrm{CSW}^{2}$ & 11 & Cow dung & $16-25$ & Oat straw & $48-50$ \\
\hline Poultry manure & $5-15$ & Horse manure & $20-25$ & Corn stalks/straw & $50-56$ \\
\hline Pig manure & $6-14$ & Kitchen Waste & $25-29$ & Fallen leaves & $50-53$ \\
\hline Goat manure & $10-17$ & Peanut shoots/ hulls & $20-31$ & Rice straw & $51-67$ \\
\hline $\begin{array}{l}\text { Grass/Grass } \\
\text { trimmings }\end{array}$ & $12-16$ & Slaughterhouse waste & $22-37$ & Seaweed & $70-79$ \\
\hline Alfalfa & $12-17$ & Mixed food waste & $15-32$ & Algae & $75-100$ \\
\hline \multirow[t]{3}{*}{ Food Waste } & $3-17$ & Waste cereal & $16-40$ & Sugar cane/ bagasse & $140-150$ \\
\hline & & Sugar beet/ Sugar foliage & $35-40$ & Sawdust & $200-500$ \\
\hline & & Waste cereals & $16-40$ & & \\
\hline
\end{tabular}

${ }^{1}$ Thickened Waste Activated Sludge,

${ }^{2}$ Caned Seafood Waste,

${ }^{3}$ Organic Fraction of Municipal Solid Wastes

However, for the implementation of $\mathrm{AnCoD}$, other than aforementioned factors that govern overall $\mathrm{AD}$ process, additional factors including the selection of co-substrates and their mixing ratio should be taken into consideration. For instance, mixing materials of high and low C:N ratios, such as organic solid waste mixed with animal manure or sewage can help achieve optimum C:N ratios (Akyol et al., 2016). In order to attain an improved co-digestion process, some precautions and suitable procedures are necessary. There may be requirements for supplementary digester equipment depending on the size of the operation, quality of waste, and characteristics of the wastes to be co-digested. Mainly, precautions or supplementary equipment would be required for: homogenization and mixing of co-substrates, delivery of the waste, prevention of excessive foaming and scum layer formation, and removal of sediments from the digester. 


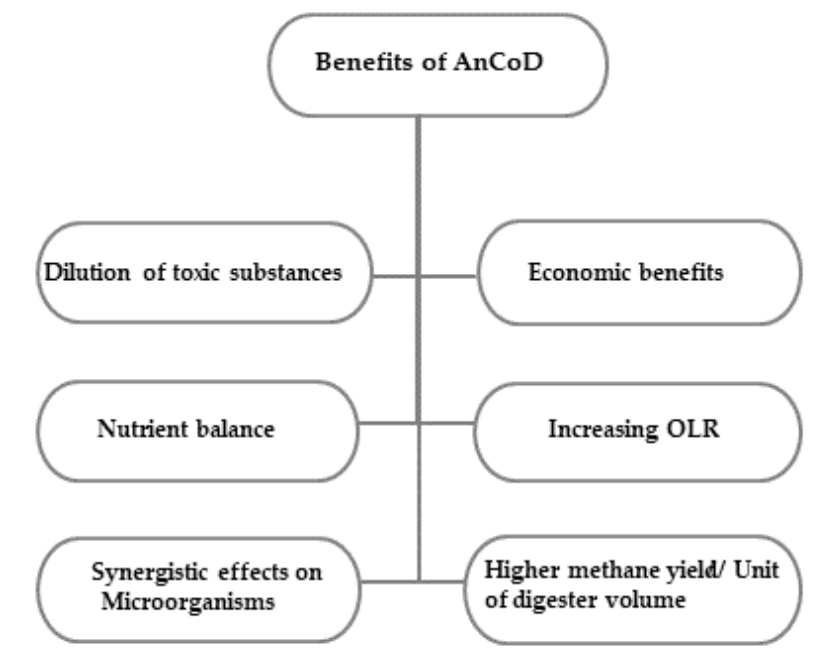

Figure 2.5. Advantages of anaerobic co-digestion systems

Furthermore, proper monitoring parameters should be determined to control and regulate the AnCoD digesters to help maintain an efficient performance when it is under operation. Applying suitable monitoring and control procedures when running the AnCoD process, allows for utilizing the full capacity of the system without overload risks. Monitoring can be performed by measuring indirectly the activity of different groups of organisms for example by measuring the rate of gas production, or the accumulation of intermediates of anaerobic degradation which reflect the existing metabolic status of the active organisms in the system (Gujer and Zehnder, 1983).

Several recommendations in the literature has been proposed specifying what control parameters should be chosen to be measured for this purpose. Some of the more common ones include $\mathrm{pH}$, alkalinity, volatile fatty acids (VFA), gas production rate and the amounts of hydrogen, methane and carbon dioxide in the gas (Ahring et al., 1995; Moletta et al., 1994).

Partial alkalinity (PA) has been considered as a reliable monitoring parameter (Jenkins et al., 1991; Rozzi et al., 1994; Supaphol et al., 2011; Wilcox et al., 1995). The applicability of pH as a process indicator was reported to be intensely dependent on the buffering capacity making it an unreliable monitoring parameter (Ahring et al., 1995). It is expected that the selection and applicability of a specific parameter could not be generalized depending on the individual process configuration and the waste characteristics. For a full-scale municipal system, co-digested excess sludge from the municipal wastewater treatment plant with carbohydrate-rich food processing waste, different parameters were assessed for monitoring and control of the system performance (Björnsson et al., 
2000). Those parameters included the volume of gas produced, $\mathrm{pH}$, VFA, and alkalinity. In addition, gas composition and the degradation of organic matter were also measured at steady state and during process changes. Both full-scale and lab-scale experiments were carried out by that research to evaluate the suitability of those parameters. The digester was run below maximum capacity in order to avoid overload. The only operational limit set for the plant, was that the $\mathrm{pH}$ should not have been below 6.8. So, the $\mathrm{pH}$ was compared with alkalinity, VFA concentration, gas production rate, and the gas composition. Alkalinity was measured as partial alkalinity (PA). OLR changes were monitored both in the full-scale digester and in the lab-scale models (Björnsson et al., 2000).

As indicated by the results of Björnsson et al's study, the load's fluctuations were reflected in the $\mathrm{pH}$, PA, and VFAs concentration. At overload condition, all the three parameters clearly demonstrated the process imbalance. The VFA concentrations proved to be a better indicator for an overload of the microbial system, although alkalinity and $\mathrm{pH}$ showed to be good monitoring parameters as well. The results indicated that gas-phase parameters demonstrated a slow response to load changes. The response of gas production and gas composition was behind and significant change occurred only after severe overload. Prior studies had observed that change in the gas phase parameters only takes place after well-developed imbalance. For that reason, the gas-phase concentration would not always reflect the actual concentration in the liquid, caused by limitations in liquid-to-gas mass transfer (Frigon and Guiot, 1995; Pauss et al., 1990).

At higher OLR, the process showed to be more sensitive to system disturbances. The changes in VFA concentration were not accurately reflected in $\mathrm{pH}$. The increased amounts of VFA were demonstrated in a lower $\mathrm{pH}$, because of the low buffering capacity of the process. Nevertheless, the $\mathrm{pH}$ was not presented as a reliable means of process monitoring, because of possible variation in buffering capacity as a result of variations in substrate composition. Therefore, a process imbalance causing significant accumulation of VFAs, could be unseen by this buffering effect. Therefore, relying on $\mathrm{pH}$ measurements for the process monitoring was not advised and the usage of $\mathrm{pH}$ measurements together with measurements of the PA or VFA was suggested by authors (Björnsson et al., 2000).

Different studies have been carried out to evaluate the impact of various effective factors on AnCoD processes. The main aim of the studies was to assess the influence of those parameters on biogas yield, biogas composition including biomethane or biohydrogen content. However, no 
comprehensive guidelines have been compiled so far to standardize the AnCoD systems. This would be firstly due to the complexity of the process caused by the variety of co-substrates and wastes composition, and secondly because it has not been broadly implemented in full scales.

A study for IEA Bioenergy has presented most important control parameters which regulate anaerobic co-digesters. The control parameters included the overall daily substrate flow $\left(\mathrm{m}^{3} / \mathrm{d}\right.$ or tones/d) and the amount of biogas produced daily $\left(\mathrm{m}^{3} / \mathrm{d}\right)$. Based on the results obtained by this study, for appropriate control of the process, the determination of the $\mathrm{CH}_{4}$ concentration was highly advised. In addition, a periodic calculation of the biogas yield referred to as daily biogas amount divided by daily substrate flow was recommended since it provides the efficiency of the digestion process. The analysis of the ammonia and the volatile fatty acids concentration were also suggested for large-scale operations. In addition, identifying the influences of co-substrates on the digester behavior was recommended. Particularly, detecting the formation of scum layers and bottom sediments was specified. It was suggested to maintain the record of the type and amount of separated contaminations in co-digestion. In the cases that sterilization is also involved, monitoring the type and the amount of waste streams and the treatment conditions such as time and temperature were also considered to be of necessary control parameters. The sampling frequency and methods for analysis required for quality assurance of the end product digestate or compost were also comprehensively defined in that study (Kim et al., 2006).

Although co-digestion of feedstocks such as poultry manure and kitchen waste with low $\mathrm{C}: \mathrm{N}$ ratio with those of higher $\mathrm{C}: \mathrm{N}$ ratio such as agricultural waste including rice and wheat straw is a solution to adjust its ratio at the optimum level, the existence of lignocellulosic material in the agricultural waste cause limitation during $\mathrm{AnCoD}$ as a result of long retention time and low biodegradability (Saha et al., 2011a).

Such problems still may demand for pretreatment techniques in order to speed up the hydrolysis which is the rate-limiting step in the anaerobic digestion process. The main purpose of the pretreatment is to increase the solublization by the breakdown of the complex substrates such as lignin in lingocellulosic feedstocks or tough cell wall in seaweed biomass, in order to accelerate the hydrolysis rate (Elbeshbishy et al., 2011; Giordano, 2012; Noike et al., 1985).

C. Rodriguez et al, 2017 studied the effect of using co-substrate on methane production in codigestion of waste paper (WP) with microalgal biomass (MA). Their study (Rodriguez et al., 2017) was carried out in batch mode and was intended to investigate the influence of the feedstocks 
mixing ratio (WP:MA) as well as feedstock to inoculum (F:I) ratio. They achieved the highest methane yield of $608 \mathrm{ml} \mathrm{CH}_{4} / \mathrm{gVS}$ at the F/I and WP:MA ratios of 0.2 and 50:50 respectively. At this mixing ratio of the feedstocks, the obtained methane yield was more than that of the feedstock's mono-digestion. The maximum $49.58 \%$ increase of the methane yield occurred at the same co-digestion ratio of 50:50 and F:I ratio of 0.4. Their study verified the synergetic effect at the feedstocks mixing ratio of 50:50 and all F:I ratios of 0.2, 0.3, and 0.4.

Pretreatment has proved successful at increasing the methane yield of numerous strains of microalgae in the digestion process. Most species of microalgae reduce the digestion rate due to their tough cell wall consisting of slowly biodegradable material (Uggetti et al., 2017).

With the increasing attention to anaerobic co-digestion, several researches have been allotted for co-digestion of various feedstocks and pre-treatment techniques from mechanical particle size reduction, thermal, chemical and ultrasonic treatment to enzymatic degradation and so on. For instance, mechanical pretreatment with Hollander beater in co-digestion of seaweed biomass with digester sludge increased biogas production by $20 \%$ at the ratio of $2: 3$ of algal pulp to sludge per reactor for $10 \mathrm{~min}$ beating time (Tedesco et al., 2013).

\subsubsection{Microbial Diversity and Synergy in AnCoD}

The selection of sludge inocula plays an important role in the effectiveness of biological anaerobic treatment of organic wastes. The analysis of microbial community dynamics has revealed that various waste streams and environmental factors can affect microbial community dynamics in an anaerobic co-digestion processes (Lin et al., 2012; Supaphol et al., 2011). Reportedly, mesophilic anaerobic co-digestion of mixed wastes allows for a better variety of substrates which in turn supports a wider diversity of bacteria and archaea. More diverse resource input results in more diverse communities and greater metabolic activity (Ike et al., 2010; Supaphol et al., 2011). However, there is limited awareness about the microbial consortia in anaerobic co-digestion process due to the lack of metabolic data on the microorganisms involved in the process. A comprehensive understanding of the microbial community is hindered by limitations of conventional molecular technology approaches that are restricted in terms of detecting sophisticated microbial diversity in the environment. Attempts for the analysis of the 16S rRNA gene sequencing have been carried out as an alternative to conventional culture techniques. This

method is used to identify and compare microorganisms present within a given sample and it is a 
well-established method for studying complex microbial community or environments that are difficult or impossible to study. The method of $16 \mathrm{~S}$ rRNA gene-based fingerprints could provide less biased and higher coverage information and can support many unknown details about the mechanism of microbial response to the digester enhancement. An improved understanding of the function and the metabolic role of microorganisms in the anaerobic co-digestion of various pollutants can be obtained b by the molecular inventories (Rivière et al., 2009; Zhang et al., 2011). Some of the results of these studies are presented in Table 2.2.

Organic matter in $\mathrm{AD}$ process, is decomposed synergistically by a bacterial consortium producing biogas including biomethane (Chandra et al., 2012; Liew et al., 2012). The process involves at least three functional groups of microorganisms that mainly regulate the mutual metabolic interactions under anaerobic conditions. The first microbial community hydrolyzes complex polymeric substances such as lipids, cellulose and protein to fundamental structural building blocks like glucose and amino acids. Subsequently, fermentation of these products to fatty acids, acetate and hydrogen is proceeded by the second community.

Among degradation processes involved in anaerobic digestion, the acidogenesis process has been shown to be the most important step. The third community develops methanogenesis process through which acetate and hydrogen are converted to methane and carbon dioxide. Therefore, microbial communities are vital to a stable and efficient transformation of organic matter to biogas (Zhang et al., 2011). General metabolism of microbial consortia involves extracellular polymeric substances (EPS) of sludge aggregates. It has been indicated that EPS is partly the result of the microbial metabolism that is affected by the microbial community structure and its activity. Growth conditions control the quantity of EPS which in turn affect the anaerobic digestibility and biogas production. It is not yet clear that how different microorganisms contribute to EPS secretion. A comparative study on the pathways of substrates degradation and the by-products of EPS sub-fractions could provide supplementary data on long-term impacts of microbial activity on anaerobic co-digestion reactors (Sheng et al., 2010; Yu et al., 2012). Monitoring qualitative and quantitative changes in a bacterial community structure, allows for the evaluation of the influence of the co-substrate on bacteria contributing to the biogas production.

However, there is not enough literature on this topic. Some attempts have been made to study microbial community structure and its influence on anaerobic co-digestion processes. Such studies as presented in Table 2.2 have been aimed to increase methane production by co-digestion of 
different organic-rich waste streams and they have been mostly developed with a view to the influencing parameters such as mixing ratio, organic loading rate (OLR), and carbon to nitrogen $(\mathrm{C}: \mathrm{N})$ ratio on the population of methanogenic archaea species (Benn and Zitomer, 2018; Gaby et al., 2017; A. J. Li et al., 2011).

It is reported that an even distribution of hydrogenotrophic and acetotrophic methanogen populations in a reactor is indicative of a stable operating condition (Demirel and Scherer, 2008; Kim et al., 2014; Yang et al., 2016). Yang et al. studied the performance of a co-digester for the treatment of sewage sludge with fat, oil and grease (FOG) using a mesophilic semi-continuous reactor and compared it to that of a mono-digester receiving only sewage sludge. It was indicated that the secretion of EPS increased by $40 \%$ in comparison with the mono-digester and that the improvement in co-digestion performance was stimulated due to the release of EPS. The analysis of microbial 16S rRNA gene showed the dynamic change of microbial community through the process. Both bacterial and archaeal community went through a progress with FOG addition, and a large amount of consortia such as Methanosaeta and N09 were involved in the process. As compared to sewer sludge mono-digestion, biogas production and TS removal efficiencies increased up to $35 \%$ and $26 \%$, respectively. It was shown that FOG addition resulted in nutrition balance and regulating microbial composition. Also, metabolic activities were stimulated, and more EPS were obtained with the progressive addition of FOG.

Jihen et al. investigated microbial community's structures in anaerobic co-digestion of dairy wastewater and cattle manure. A maximum volatile solids (VS) reduction of $88.6 \%$ and biogas production of $0.87 \mathrm{~L} / \mathrm{g}$ VS removed were obtained through their research corresponding to the C:N ratio of 24.7 at HRT of 20 days. The bacterial profile analysis showed a large quantity of Uncultured Bacteroidetes, Firmicutes and Synergistetes bacterium.

The Syntrophomonas strains associated with $\mathrm{H}_{2}$-using bacteria comprising Methanospirillum sp., Methanosphaera sp., and Methanobacterium formicicum were observed as well. These syntrophic associations are necessary in anaerobic digestion reactors allowing for maintaining a low hydrogen partial pressure. On the other hand, high concentrations of volatile fatty acids (VFAs) resulted from dairy wastes acidogenesis allowed the growth of Methanosarcina species. It was indicated that high concentrations of VFAs would result preferentially in the growth of the acetoclastic Methanosarcina species (Jihen et al., 2015). 
Table 2.2. Microbial consortia diversity in various AnCoD systems for biogas and methane improvement

\begin{tabular}{|c|c|c|c|c|}
\hline Feedstocks & Microbial Consortia & Digester mode & HRT & $\begin{array}{l}\text { Methane / Biogas } \\
\text { increase \% }\end{array}$ \\
\hline $\begin{array}{l}\text { Fruit vegetable } \\
\text { waste +Food waste } \\
(1: 1)\end{array}$ & $\begin{array}{l}\text { Methanoculleus, } \\
\text { Methanosaeta, } \\
\text { Methanosarcina }\end{array}$ & $\begin{array}{l}\text { CSTR } \\
\text { (mesophilic) }\end{array}$ & $\mathrm{NA}^{1}$ & $0.49 \mathrm{~m}^{3} \mathrm{CH}_{4} / \mathrm{kg} \mathrm{VS}$ \\
\hline \multirow{2}{*}{$\begin{array}{l}\text { Food, fruit, } \\
\text { vegetable + night } \\
\text { soil waste }\end{array}$} & \multirow{2}{*}{$\begin{array}{l}\text { Methanosaeta } \\
\text { (predominant methanogen) } \\
+ \text { hydrogenotrophs }\end{array}$} & \multirow[t]{2}{*}{$\begin{array}{l}\text { Full scale wet fed- } \\
\text { batch }\end{array}$} & $18-20 \mathrm{~d}$ & \multirow[t]{2}{*}{ NA } \\
\hline & & & C:N 8.6 & \\
\hline $\begin{array}{l}\text { Cow manure }+ \\
\text { grass silage }\end{array}$ & $\begin{array}{l}\text { Clostridia, unclassified } \\
\text { Bacteria, Bacteroidets }\end{array}$ & CSTR mesophilic & $20 \mathrm{~d}$ & NA \\
\hline $\begin{array}{l}\text { Cow manure + oat } \\
\text { straw }\end{array}$ & $\begin{array}{l}\text { Clostridia, unclassified } \\
\text { Bacteria, Bacteroidets, } \\
\text { Deltaproteobacteria }\end{array}$ & $\begin{array}{l}\text { CSTR } \\
\text { (mesophilic) }\end{array}$ & $20 \mathrm{~d}$ & NA \\
\hline $\begin{array}{l}\text { Cow manure }+ \\
\text { sugar beet tops }\end{array}$ & $\begin{array}{l}\text { unclassified } \\
\text { Bacteria,Clostridia, } \\
\text { Bacteroidets, Bacilli }\end{array}$ & $\begin{array}{l}\text { CSTR } \\
\text { (mesophilic) }\end{array}$ & $20 \mathrm{~d}$ & NA \\
\hline $\begin{array}{l}\text { STP-OGW + SC- } \\
\text { OFMSW (1:6) }\end{array}$ & $\begin{array}{l}\text { Methanobacterium, } \\
\text { Methanoculleus, } \\
\text { Methanothermobacter } \\
\text { uncultured archaea }\end{array}$ & $\begin{array}{l}\text { Batch } \\
\text { (thermophilic) }\end{array}$ & $14.4 \mathrm{~d}$ & $\begin{array}{l}52 \% \text { biogas and } \\
36 \% \text { methane } \\
\text { increase }\end{array}$ \\
\hline $\begin{array}{l}\text { biodiesel waste } \\
\text { glycerin + } \\
\text { municipal waste } \\
\text { sludge }(1.35: 0.65)\end{array}$ & $\begin{array}{l}\text { Dominated } \\
\text { by:Methanosaeta and } \\
\text { Methanomicrobium }\end{array}$ & $\begin{array}{l}\text { Two-stage CSTR } \\
\text { (mesophilic) }\end{array}$ & $20 \mathrm{~d}$ & $\begin{array}{l}100 \% \text { biogas and } \\
120 \% \text { methane } \\
\text { increase }\end{array}$ \\
\hline $\begin{array}{l}\text { Sewage sludge } \\
\text { +FOG }\end{array}$ & $\begin{array}{l}\text { Dominantly Methanosaeta, } \\
\text { and N09 }\end{array}$ & $\begin{array}{l}\text { Semi-continuous } \\
\text { (mesophilic) }\end{array}$ & $15 \mathrm{~d}$ & $\begin{array}{l}35 \% \text { biogas } \\
\text { increase }\end{array}$ \\
\hline $\begin{array}{l}\text { Dairy wastewater } \\
\text { + Cattle manure }\end{array}$ & $\begin{array}{l}\text { Uncultured Bacteroidetes, } \\
\text { Firmicutes, Synergistetes, } \\
\text { Syntrophomonas strains } \\
\text { Methanosarcina species }\end{array}$ & $\begin{array}{l}\text { ASBR } \\
\text { (mesophilic) }\end{array}$ & $\begin{array}{c}20 \mathrm{~d} \\
(\mathrm{C}: \mathrm{N} 24.7)\end{array}$ & $\begin{array}{l}\text { biogas produced: } \\
0.87 \mathrm{~L} / \mathrm{g} \text { VS } \\
\text { removed }\end{array}$ \\
\hline $\begin{array}{l}\text { Food wastewater } \\
\text { +WAS (3:1) }\end{array}$ & $\begin{array}{l}\text { Dominated by: } \\
\text { Methanothermobacter and } \\
\text { Methanosarcina }\end{array}$ & $\begin{array}{l}\text { CSTR } \\
\text { (thermophilic) }\end{array}$ & $20 \mathrm{~d}$ & $\begin{array}{l}\text { Max biogas: } 316.11 \\
\mathrm{~mL} \mathrm{CH}_{4} / \mathrm{g} \mathrm{COD} \\
\text { removed }\end{array}$ \\
\hline
\end{tabular}

${ }^{1} \overline{\text { Not available }}$

Former studies verify that the AnCoD of cellulosic materials such as grass silage, oat straw, and sugar beet tops to methane to be mediated by bacteria and methanogenic archaea. The polymers which are hydrolyzed into soluble compounds under fermentative condition, are converted to acetate and one-carbon constituents by acidogens and acetogens and these intermediates in turn 
can be transformed directly by methanogenic archaea into methane and carbon dioxide (Nopharatana et al., 1998; Veeken and Hamelers, 1999).

Anaerobic digestion process of cellulosic material including grass silage, oat straw and sugar beet tops, is a multistep process mediated by Bacteria and methanogenic Archaea to produce methane. The process involves the hydrolysis of polymers into soluble compounds under fermentative condition. Subsequently, Acidogenic and acetogenic bacteria convert these soluble intermediates to acetate and mono-carbon compounds. These compounds subsequently can be converted to methane and carbon dioxide by methanogenic Archaea. It was found that in anaerobic digestion of cellulytic feedstocks, significant cellulolytic competences occurs due to the existence of species belonging to the order Clostridiales (Lynd et al., 2002).

Martín-González et al. found that thermophilic conditions was superior to mesophilic conditions for the enhancement of $\mathrm{AnCoD}$ along with the use of sewage treatment plant fat, oil and grease wastes (STP-FOGW) as co-substrates in co-digestion with organic fraction of municipal waste (OFMSW). Monitoring of microbial structure demonstrated that the bacterial profiles clustered in two separate groups, before and after the extended contact with STP-FOGW, whereas, archaeal community structure remained relatively constant throughout the operation. Bacterial population structure showed a dynamic change determined to be due to introducing FOG residues to the reactor.

\subsubsection{Effect of Digester Mode on AnCoD}

In general, anaerobic digesters can be configured as one-stage, two-stage, or multi-stage reactors in which the hydrolysis/acidogenesis and acetogenesis/methanogenesis steps occur in either the same or separated digesters (Figure 4). Separating the digesters makes the process easier to control, and makes it possible to separately optimize the operational and environmental conditions for hydrolysis/acidogenesis and methanogenesis processes in order to enhance the overall reaction rate and biogas yield (Lalman and Bagley, 2002; Nathao et al., 2013).

Fluctuations in organic loading rate, heterogeneity of wastes, and or the presence of excessive inhibitors can lead to instability of the process, and multi-stage systems have shown to be more stable as compared to single-stage ones. Two or multi-stage systems allow for the selection and enrichment of different types of microorganisms in each digester which results in extending the possibility of processing different biomass constituents, improving substrate conversion, enhancing the chemical oxygen demand (COD) reduction, and increasing energy recovery. 
Although, multi-stage digesters are associated with greater construction and maintenance costs, multi-stage digesters provide higher performances as compared to single-stage systems (Azbar and Speece, 2001; Cuetos et al., 2007). Using two-stage digestion, controlled acidogenesis in the first digester, helps maintaining a high soluble feed to the second stage which subsequently enhances the biogas production (Dareioti and Kornaros, 2015).

In the two-stage anaerobic digestion systems, acid fermentation and methanogenesis are separated in two reactors in order to optimize reactor conditions for the different groups of microorganisms. The acidogenic stage is typically operated at a low HRT in the range of 2 to 3 days and a $\mathrm{pH}$ between 5 and 6. While the second stage, methanogenesis, is typically operated with a HRT of 20 to 30 days and a pH between 6 and 8 facilitating the development of slow-growing methanogenic archaea.

Moreover, acidogenesis phase allows for long chain fatty acids (LCFAs) saturation and degradation (Kangle et al., 2012; Kim et al., 2004). Owing to the bent molecular structure of unsaturated LCFAs because of the existence of double bonds, they have a greater cover area of cell wall per molecule as compared to saturated LCFAs. As a result, unsaturated LCFAs have demonstrated stronger inhibitory effects in comparison with saturated LCFAs (Demeyer and Henderickx, 1967; Thies et al., 1994). As such transformation of unsaturated to saturated LCFAs is beneficial. In addition, LCFA saturation would be necessary for the oxidative breakdown of fatty acid molecules and formation of acetic acid (Hanaki et al., 1981; Lalman and Bagley, 2002). The outcome of a study using a two-stage AD system treating a synthetic fat-containing wastewater comprised of glucose and LCFAs mixture revealed that, 19\% of LCFAs were degraded and $12 \%$ of unsaturated LCFAs were saturated in the acidogenic phase (Kim et al., 2004). Acidogenic phase also can convert the unsaturated LCFAs to palmitic acid which reduces the lipid inhibition of methanogenesis in the second stage (Beccari et al., 1998). Food waste, which composes a large portion of OFMSW, contains a substantial amount of organic soluble compounds which can be simply converted to VFA.

Therefore, it can be an ideal substrate for biogas production. Nevertheless, formation of excessive amount of VFA at initial digestion stages can result in a remarkable $\mathrm{pH}$ reduction and subsequently methanogenesis inhibition. Utilization of two-stage anaerobic digestion systems for food waste has shown to be an effective solution for the $\mathrm{pH}$ inhibition of one stage systems (Dinsdale et al., 2000; Kinnunen et al., 2015; Klocke et al., 2008; Y. Li et al., 2011; Shen et al., 2013; Shin et al., 
2010). Most of the studies on anaerobic co-digestion process have aimed to evaluate the digesters performance and optimal operating conditions for a particular type of waste.

Lafitte-Trouque et al. found two-stage thermophilic/mesophilic AnCoD systems to be effective for the co-digestion of sewage sludge and confectionery waste. The system with the second digester operating at a HRT of 12 days provided the best performance in terms of stability, VS reductions, and specific methane yield corresponding to an average $82 \%$ methane in the gas composition. However, a HRT of 8 days in the second stage digester was not able to assimilate high concentrations of volatile acid and low $\mathrm{pH}$ from the first digester. This was related to the insufficient retention time for maintaining a substantial methanogenic population. In a single-stage digester, a HRT of less than 20 days may cause methanogens to be washed out of the digester. Therefore, HRT is one of the important design parameters for the single-phase operations (LafitteTrouqué and Forster, 2000).

The study by Ratanatamskul et al. was conducted in pilot-scale on the development of an energy recovery system using a novel prototype single-stage anaerobic digester. Their system (Ratanatamskul et al., 2015) co-digested food waste with sewage sludge from a high-rise building for on-site biogas production.

The food waste to sewage sludge mixing ratio of $10: 1$ by weight was selected according to the result of lab experiments. Different HRTs of 27, 22, 19 days corresponding to 7.9, 10.8 and 14.0 $\mathrm{kgCOD} / \mathrm{m}^{3} \mathrm{~d}$ OLRs were applied and the optimal methane yield of $76.8 \%$ was achieved by their proposed single-stage reactor when the digester was operated at an HRT of 27 days. Although maximum biogas production occurred at the shortest HRT of 19 days. This indicated that the improvement of methane content of biogas could be attained by adequate operating HRT.

Although, the advantages of two-stage over single-stage digestion systems are addressed in the literature (Bertin et al., 2013; Shen et al., 2013; Yu et al., 2002) there exists a lack of adequate research available in terms of comparing the performances of single, two and or multi-phase codigestion systems. Kim et al. developed a two-stage system comprised of a continuously stirred tank reactor for acidogenesis and a methanogenic up-flow bed reactor for the treatment of a high lipid wastewater from a milk and ice cream factory co-digested with slaughterhouse wastewater. They obtained 1.2 times increase in the COD removal, 1.9 times increase in lipids removal, and 1.4 times increase in the methane production compared to the single phase system (Kim and Shin, 2010). 


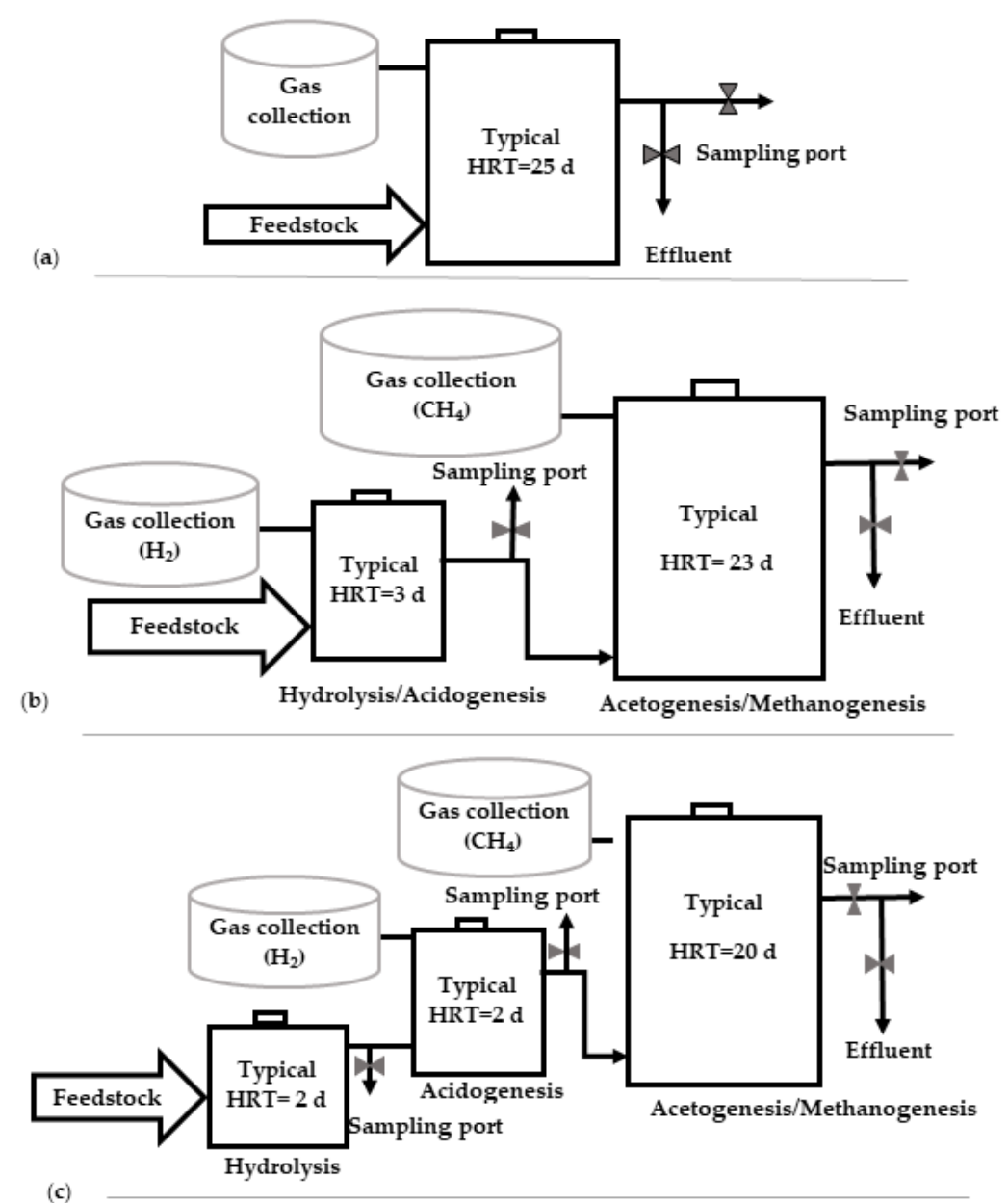

Figure 2.6. Types of digester configuration: (a) Single-stage; (b) Two-stage; and (c) Three-stage digester

Contrary to the result of that research (Kim and Shin, 2010), no significant increase in the overall energy recovery was attained by other study (Schievano et al., 2012) using a two-stage digester co-digesting swine manure and market biowaste in comparison with a single-stage one. Volumetric biogas productions were found to be 0.1 and $0.079 \mathrm{~m}^{3} / \mathrm{L}$ reactor for the single-stage and the twostage systems, respectively. Even though, the average biogas methane content of the two-stage system showed $25 \%$ increase over that of the single-stage digester. The accumulation of undegraded intermediate metabolites such as volatile fatty acids, ketones, amines, amino acids, and phenols was believed to be responsible for the reduced efficiency of the two-stage digester though. It was concluded that although the two-stage system could be capable of a higher bioenergy production, certain incompetent fermentative pathways may lead to formation of recalcitrant and toxic metabolites. 
Hidalgo et al. compared a single-phase with a two-phase reactor for the co-digestion of residues from the used vegetable oil processing industry and pig manure. The maximum methane production of $1.06 \mathrm{~m}^{3} / \mathrm{kg}$ VS removed in the single-stage digestion corresponding to a methane production of $0.69 \mathrm{~m}^{3} \mathrm{CH}_{4} / \mathrm{kg}$ VS removed $\left(65 \% \mathrm{CH}_{4}\right)$ was obtained at the end of first 50-day operational period. The average biogas productions of 0.46 and $0.33 \mathrm{~m}^{3} / \mathrm{kgVS}$ removed were observed for the second and third operational period with methane productions of $0.30(65.5 \%$ $\left.\mathrm{CH}_{4}\right)$ and $0.22\left(66 \% \mathrm{CH}_{4}\right) \mathrm{m}^{3} \mathrm{CH}_{4} / \mathrm{kg} \mathrm{VS}$ removed, respectively (Hidalgo et al., 2014).

The two-phase anaerobic digestion improved VS removal efficiencies and process stability in comparison with the single-phase reactor. Although the single-stage system produced more biogas, a higher methane content in produced biogas was obtained by the two-phase system and the latter was deemed to be more beneficial. Table 2.3 summarizes the results achieved by a number of studies on single and two-stage AnCoD systems.

The results obtained by applying a novel compact three-stage anaerobic digester in co-digestion of food waste and horse manure verified the advantages of the three-phase digester over single and two-stage ones as controls. By using three compartments in the three-stage anaerobic digester, three separated functional zones hydrolysis, acidogenesis and methanogenesis were created. This configuration significantly accelerated the solublization of solid organic matters and the formation of volatile fatty acids leading to an increase of 11 and $23 \%$ in methane yield in the two-stage and three-stage digesters in comparison with the single-stage one respectively. The analysis of $16 \mathrm{~S}$ rDNA showed that different microbial communities comprising hydrolyzing bacteria, acidogenic bacteria and methanogenic archaea were selectively enriched in the three separate reactors of the three-stage digester. It was also indicated that the abundance of the methanogenic archaea was increased by 0.8 and 1.28 times in the two-stage and the three-stage digesters compared to the single stage one correspondingly (Zhang et al., 2017b).

Despite its benefits, AnCoD implementation can be limited owing to long retention time and low biodegradability (Saha et al., 2011b). Therefore, certain techniques are required in order to overcome these obstacles. With the increasing interest in anaerobic co-digestion, a number of researches have been conducted during recent years with the purpose of improving co-digestion of various substrates. Part of those studies has been allotted for pre-treatment techniques from mechanical particle size reduction, thermal and ultrasonic treatment to enzymatic degradation and so on. The main purpose of the pre-treatment methods is to increase the solublization of the 
complex substrates by the breakdown of the complex substrates such as lignin in lingocellulosic feedstocks or tough cell wall in seaweed biomass, in order to accelerate the hydrolysis rate as hydrolysis is the limiting step in the anaerobic digestion process (Eastman and Ferguson, 1981; Esposito et al., 2012; Noike et al., 1985).

Table 2.3. Comparison of single-stage and two-stage digestion in AnCoD systems

\begin{tabular}{|c|c|c|c|c|}
\hline Digester mode & Feedstocks & $\begin{array}{l}\text { Mixing } \\
\text { ratio }\end{array}$ & HRT & Biogas / Methane content \\
\hline $\begin{array}{l}\text { Single- stage (CSTR, } \\
\text { mesophilic) }\end{array}$ & \multirow{2}{*}{$\begin{array}{l}\text { Sewage sludge } \\
+ \text { confectionery } \\
\text { waste }\end{array}$} & \multirow{2}{*}{$\mathrm{NA}^{1}$} & $20 \mathrm{~d}$ & $\begin{array}{l}\text { Methane yield: } 0.36-0.28 \mathrm{~m} 3 / \mathrm{kg} \\
\text { VS applied (76- } 82 \% \text { methane) })^{2}\end{array}$ \\
\hline $\begin{array}{l}\text { Two- stage (CSTR, } \\
\text { thermophilic/mesophilic) }\end{array}$ & & & $12 \mathrm{~d}$ & $\begin{array}{l}\text { Methane yield: } 0.3-0.34 \mathrm{~m} 3 / \mathrm{kg} \\
\text { VS applied }(66-76 \% \text { methane })^{2}\end{array}$ \\
\hline \multirow[t]{2}{*}{ Single stage (plug flow) } & \multirow[t]{2}{*}{$\begin{array}{l}\text { Food waste } \\
\text { +sewage sludge }\end{array}$} & \multirow[t]{2}{*}{$\begin{array}{l}10: 1 \\
\text { (weight) }\end{array}$} & $27 \mathrm{~d}$ & $\begin{array}{l}\text { Biogas production: } \\
1045 \pm 52.81 \mathrm{~L} / \mathrm{d}\end{array}$ \\
\hline & & & $19 \mathrm{~d}$ & $1662.58 \pm 37.32 \mathrm{~L} / \mathrm{d}$ \\
\hline Single- stage (UASB) & \multirow[b]{2}{*}{$\begin{array}{l}\text { Slaughter house } \\
+ \text { milk } \\
\text { wastewater }\end{array}$} & \multirow[b]{2}{*}{ NA } & $2.14 \mathrm{~d}$ & \multirow[b]{2}{*}{$\begin{array}{l}40 \% \text { Methane increase by two- } \\
\text { stage reactor }\end{array}$} \\
\hline Two- stage (CSTR/ UASB) & & & $2.9 \mathrm{~d}$ & \\
\hline $\begin{array}{l}\text { Single- stage (CSTR, } \\
\text { thermophilic) }\end{array}$ & \multirow{2}{*}{$\begin{array}{l}\text { Market biowaste } \\
+ \text { swine manure }\end{array}$} & \multirow{2}{*}{$\begin{array}{l}1: 4 \\
\text { (weight) }\end{array}$} & $25 \mathrm{~d}$ & $0.55 \mathrm{dm} 3 / \mathrm{L}$ digester $\mathrm{d}$ \\
\hline $\begin{array}{l}\text { Two- stage (CSTR, } \\
\text { thermophilic) }\end{array}$ & & & $3 / 22 \mathrm{~d}$ & $0.54 \mathrm{dm} 3 / \mathrm{L}$ digester $\mathrm{d}$ \\
\hline Single- stage (Batch) & \multirow{2}{*}{$\begin{array}{l}\text { Oil processing } \\
\text { wastewater }+ \\
\text { pig manure }\end{array}$} & \multirow{2}{*}{$\begin{array}{l}1: 3 \\
\text { (weight) }\end{array}$} & $20 \mathrm{~d}$ & $\begin{array}{l}\text { Average biogas: } 0.33 \mathrm{~m} 3 / \mathrm{kgVS} \\
\text { removed, }(0.66 \% \text { methane })\end{array}$ \\
\hline Two-stage (Batch) & & & $2 / 18 \mathrm{~d}$ & $\begin{array}{l}\text { Average biogas: } 0.4 \mathrm{~m} 3 / \mathrm{kgVS} \\
\text { removed, }(0.67 \% \text { methane })\end{array}$ \\
\hline Single-stage & \multirow{3}{*}{$\begin{array}{l}\text { Food waste/ } \\
\text { horse manure }\end{array}$} & \multirow{3}{*}{ NA } & $20 \mathrm{~d}$ & $\begin{array}{l}45.4 \text { L cumulative methane } \\
\text { production }\end{array}$ \\
\hline Two-stage & & & $4 / 16 \mathrm{~d}$ & 50.7 L cumulative methane \\
\hline Three-stage & & & $2 / 2 / 16$ & $\begin{array}{l}55.7 \mathrm{~L} \text { cumulative methane } \\
\text { production }\end{array}$ \\
\hline
\end{tabular}

${ }^{1}$ Not Available,

${ }^{2}$ Numbers are mean values after 70 - day period of phase 1 and phase 2 , respectively 


\subsection{Pretreatment for Improving AnCoD}

Pretreatment have been reported to improve the waste stabilization and the methane yield. However, given the additional costs of pretreatment, it mainly requires to be commercially viable (Esposito et al., 2012). Based on the existing literature, the pretreatment methods used for enhancing $\mathrm{AnCoD}$ fall into five main categories including mechanical, thermal/hydrothermal, chemical, biological, and hybrid (combined) pretreatment. As such, the different pretreatment methods that have been used for the enhancement of $\mathrm{AnCoD}$ of multi feedstocks are presented in the following sections.

\subsubsection{Mechanical pretreatment}

Mechanical pretreatment as a mean to improve the AnCoD process has been proposed by a number of researchers. The principal of the mechanical pretreatment technique is to breakdown and/or crushing the substrate particles reducing their particle size. The particle size reduction is proportional to the available specific surface area of the substrate constituents and therefore, it causes a more effective contact between the substrate and the anaerobic microorganisms during the AD process. As a result, the hydrolysis stage (as a known rate-limiting stage) is accelerated and the overall AD process is improved (Esposito et al., 2012).

In general, it has been observed that the smaller the size of the substrate particles is, the higher the methane production rate and yield are in the AD process. In addition to decreasing the particle size, the release of the intracellular components of the substrates has been reported by researchers specifically when the pretreatment was applied on waste activated sludge (WAS). Figure 2.4 illustrates different mechanical pretreatment techniques that have been practiced to enhance the AnCoD process.

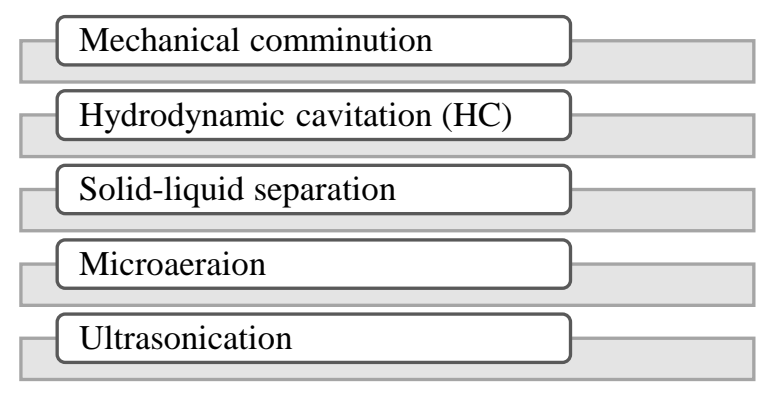

Figure 2.7. Mechanical methods of pretreatment used for AnCoD improvement 


\subsubsection{Thermal pretreatment}

Thermal pretreatment has been commonly applied to both raw and digested substrate to help with dewatering, sludge solublization, viscosity, and pathogen reduction. During thermal pretreatment and as a result of the increase in thermal energy (heat), the structure of the particulate (insoluble) matter changes in a way that it becomes more susceptible to biodegradation (Bougrier et al., 2007). Thermal pretreatment can be applied via conventional heating (conductive heating) or microwave hydrolysis. Edström et al. studied the effect of thermal pretreatment for enhanced co-digestion of animal by-products, food waste from restaurants and food distributors, and sludge from a slaughterhouse wastewater treatment plant. The authors reported a significant increase in the biogas yield from 0.31 to $1.14 \mathrm{~L} / \mathrm{g}$ VS-added when the animal by-product was exposed to conventional heating at a temperature of $70{ }^{\circ} \mathrm{C}$ for a duration of $1 \mathrm{~h}$ before the AnCoD process (Edström et al., 2003). In another study conducted by Paavola et al., thermal pretreatment via conductive heating was applied to a mixture of dairy manure and biowastes. An improvement in methane production at the extent of $14-18 \%$ was achieved compared to the process in the absence of thermal pretreatment (Paavola et al., 2006).

Despite these positive effect of thermal pretreatment on AD performance (including biogas production), the result of a study (Cuetos et al., 2010) indicated a 53\% decrease in methane production in the co-digestion of a mixture of solid slaughterhouse waste and OFMSW that was thermally pretreated at a temperature of $133^{\circ} \mathrm{C}$. The reduction of methane production was due to a foaming problem and accumulation of fats in the reactor that most likely occurred due to the formation of recalcitrant compounds at the elevated temperature of $133{ }^{\circ} \mathrm{C}$, exerting toxic effects on the AD process (Cuetos et al., 2010). Similarly, Guo et al. reported that during the co-digestion of food waste, fruit/vegetable residue, and thermally pretreated dewatered activated sludge, the methanogenesis process was inhibited by the accumulation of VFAs, which reduced the overall biogas production by $6 \%$ (Guo et al., 2014).

\subsubsection{Chemical pretreatment}

Chemical pretreatment can also lead to an improvement in the performance of AnCoD process through enhancement of the rate-limiting hydrolysis stage by facilitating the biodegradation of complex polymers via solubilizing the particulate fraction of the substrate. A variety of chemicals including acids, organic solvents, alkaline, and ionic liquids has shown a positive impact on 
disintegrating the structure of recalcitrant constituents of the substrates such as lignocellulosic compounds (Zhao et al., 2014).

Various types of alkaline compounds comprising $\mathrm{NaOH}, \mathrm{KOH}, \mathrm{Ca}(\mathrm{OH})_{2}$, hydrazine, and anhydrous ammonia can increase the internal surface area of biomass resulting in a decreased degree of polymerization. As a result, the complex structures can be decomposed and the strong bounds between carbohydrate molecules can be disrupted, which results in a higher availability of carbohydrates in the hetero matrix and enhanced reactivity of residual carbohydrates polymers in the biochemical process. Pretreatment by alkaline agents is able to eliminate acetyl and other uronic acid substitutions of hemicellulose that obstructs the accessibility of enzymes to cellulose surface. Alkali pretreatment has shown to be most successful for biomasses with lower lignin contents such as agricultural residues (Anwar et al., 2014; Saini et al., 2015).

Acidic pretreatment including dilute solutions of sulfuric acid, hydro-chloric acid, phosphoric acid, and free nitrous acid can be also employed to disintegrate the substrate structure prior to AnCoD process. Compared to the alkaline pretreatment, the application of concentrated acids is limited due to their corrosive nature and elevated costs. Acidic pretreatment has been effective in the hydrolysis of hemicelluloses to its monomeric units and increasing the bioavailability of cellulose. Acidic pretreatment may also be required to neutralize the hydrolysate providing a favorable environment for microbial activities (Lin et al., 2009). Some of the researches have suggested alkaline pretreatment using an ammonia solution or $\mathrm{NaOH}$ because of their simplicity, ease of operation, and high methane production efficiency (Bali et al., 2015; Guo et al., 2015; Park and Kim, 2012).

Among different substrates utilized in the co-digestion process, the lignocellulosic biomass as a complex mixture of cellulose, hemicelluloses, and lignin was found to be one of the most difficult to digest by compounds as it contains unbalanced carbon to nitrogen ratio as well as recalcitrant lingocellulosic structure. The lignocellulosic biomass is typically found in plant dry matter such as rice straw. Chemical pretreatment of rice straw prior to $\mathrm{AD}$ has been suggested in the literature as an effective way to improve the biodegradability of its constituents (Himmel et al., 2007; Zhao et al., 2014). For instance, the results obtained by Zhao et al. showed that alkali pretreatment using $\mathrm{NaOH}$ in co-digestion of rice straw and municipal waste sludge increased biogas yield by $20 \%$. In comparison, 40 and $45 \%$ increase in the removal rates of cellulose and hemicellulose were also reported, respectively due to the application of chemical pretreatment (Zhao et al., 2014). 
Wei et al. studied the chemical pretreatment using ammonia and $\mathrm{NaOH}$ during AnCoD of corn stover and cattle manure, resulting in improved cumulative biomethane production and solids removal rate. In the co-digestion of cattle manure and ammonia solution-treated corn stover, the required time to reach $80 \%$ of the ultimate methane yield (T80) was found to be $28 \pm 1$ days, which was $20 \%$ shorter than that of the control test with a T80 of $35 \pm 1$ days. Using sodium hydroxide, the T80 was determined to be $22 \pm 1$ days, $37 \%$ shorter as compared to that of the control digester (Wei et al., 2015).

\subsubsection{Biological pretreatment}

Biological pretreatment, which includes the addition of a particular strain, enzymes, or a consortium of microorganisms to the system, offers certain advantages over mechanical, thermal, and chemical pretreatment methods such as low energy requirements, avoiding toxic compounds generation, high yield of desired products, and the capability to target an specific compounds. Biological pretreatment methods such as bioaugmentation (stage 1) can result in the improvement of AD process by breaking complex polymers into simple monomers in hydrolysis stage which leads to increasing the rate of transformation of organic matter to biogas in the second stage. This process has shown to effectively increase both the methane yield and process stability (Song et al., 2014; Yuan et al., 2012; Zhong et al., 2011). Despite many researchers conducted on the application of biological pretreatment for improving conventional mono-digestion in AD systems, limited studies were performed on the application of these methods for enhancing biogas in AnCoD systems.

Table 2.4 summarizes the results of the studies conducted to enhance the AnCoD performance through biological pretreatment. In a study (Wei et al., 2015), biological pretreatment using liquid fraction of digestate (LFD) for AnCoD of cattle manure and corn stover was investigated. LFD obtained from anaerobic digester contains abundant microbes, inorganic substance as well as an organic substance such as amino acids, protein, a sugar that supplies nutrition substance for the process while acting as a microbial agent. As compared to untreated corn stover, pretreatment using LFD increased cumulative biomethane production (CBP) and VS removal rate by 25.40 and $30.12 \%$, respectively. In addition, it reduced T80 and improved buffer capacity of the anaerobic digestion system (Wei et al., 2015). 
Montusiewicz et al. studied the bioaugmentation pretreatment with a commercial product called Arkea ${ }^{\circledR}$ as a method for improving co-digestion of sewage sludge and mature landfill leachate. The co-digestion process was improved due to the enhanced activity of microorganisms involved in bioaugumenting system and their resistance to toxic elements (Montusiewicz, 2014). This was consistent with the results obtained by Duran et al. who used selected strains of Baccillus, Pseudomonas and Actinomycetes species for bioaugmentation (Duran et al., 2006).

In another study by Deng et al., the effect of enzymatic pretreatment on co-digestion of rice straw and soybean straw was evaluated. The research was conducted to investigate the effect of on-site generated cellulase produced by cultivation of Trichoderma reesei RUT C30 on lignocellulose to improve the hydrolysis of cellulose and hemicellulose content of the feedstock. They also investigated the influence of the pretreatment on biogas production via batch experiments. The authors reported more than $300 \%$ increase in the cumulative biogas yield compared to the untreated feedstock. They also achieved $40 \%$ shorter lag time in co-digestion of pretreated feedstock than the pretreated mono-digestion groups verifying a synergistic effect of rice and soybean straws codigestion. This study suggested enzymatic pretreatment using Trichoderma reesei RUT C30 as an effective method for improving biogas production (Deng et al., 2018).

Zhang et al, also investigated the effects of biological co-pretreatment on biogas production in AnCoD of food waste (FW) and waste activated sludge (WAS). They used co-pretreatment of FW and WAS followed by anaerobic co-digestion to improve hydrolysis efficiency. Their method was established based on the fact that a biological solublization process by mixing FW, water and microorganisms was effective as a result of size reduction of substrate particles and increase of solublization. They hypothesized that using a mixture of WAS and FW for biological solublization pretreatment (biological co-pretreatment), would improve the hydrolysis of FW and WAS for the reason that: 1) generation of the alkalis from WAS could buffer VFAs and maintain optimum $\mathrm{pH}$ for hydrolysis stage, and 2) a lower $\mathrm{pH}$ would enhance solublization of WAS through accelerating the hydrolysis of proteins and carbohydrates. Their method of pretreatment included a $2 \mathrm{~L}$ glass reactor with a mechanical stirrer at a mixing speed of $150 \mathrm{rpm}$ as a biological co-pretreatment reactor. They applied a blend ratio of 1:1 (weight) with different co-pretreatment time: $0 \mathrm{~h}, 15 \mathrm{~h}$, $24 \mathrm{~h}$ and $35 \mathrm{~h}$. The pretreated mixtures were used as the feed of the subsequent anaerobic digester. A mixture of fresh FW and WAS with $0 \mathrm{~h}$ co-pretreatment was used as the feed of the subsequent anaerobic digester as control. They achieved $24.6 \%$ higher methane production from co-digestion 
of co-pretreated substrates compared to control substrates without pretreatment. They also observed an increase of $10.1 \%$ in solids reduction under $24 \mathrm{~h}$ optimum pretreatment time (Zhang et al., 2017a).

Table 2.4. Biological pretreatment for enhanced AnCoD

\begin{tabular}{lllll}
\hline $\begin{array}{l}\text { Method of } \\
\text { Pretreatment }\end{array}$ & Feedstock & Digester mode & Mixing ratio & $\begin{array}{l}\text { Methane yield/ } \\
\text { Biogas increase\% }\end{array}$ \\
\hline \hline $\begin{array}{l}\text { Liquid fraction of } \\
\text { digestate }\end{array}$ & $\begin{array}{l}\text { Corn stover+ cattle } \\
\text { manure }\end{array}$ & $\begin{array}{l}\text { Batch } \\
\text { (mesophilic) }\end{array}$ & $3: 1$ (weight) & $\begin{array}{l}\text { 25\% methane } \\
\text { increase }\end{array}$ \\
\hline $\begin{array}{l}\text { Bioaugmentation } \\
\text { by Arkea }{ }^{\circ}\end{array}$ & $\begin{array}{l}\text { sewage sludge }{ }^{*}+ \\
\text { mature landfill }\end{array}$ & $\begin{array}{l}\text { CSTR } \\
\text { (mesophilic, }\end{array}$ & $\begin{array}{l}87: 4.3: 8.7 \\
(\mathrm{v} / \mathrm{v})\end{array}$ & $\begin{array}{l}5-8 \% \text { biogas } \\
\text { decrease }\end{array}$ \\
\hline $\begin{array}{l}\text { Biological } \\
\text { (Enzymatic) }\end{array}$ & Rice straw + & Batch & $1: 1$ TS ratio & $\begin{array}{l}318 \% \text { biogas } \\
\text { increase }\end{array}$ \\
\hline $\begin{array}{l}\text { biological co- } \\
\text { pretreatment }\end{array}$ & Foybean straw & (mesophilic) & & $\begin{array}{l}\text { 24.6\% methane } \\
\text { increase }\end{array}$ \\
\hline \hline
\end{tabular}

\subsubsection{Hybrid pretreatment}

Hybrid pretreatment is a combination two or more methods of mechanical, thermal, chemical, and biological pretreatment techniques. Of all the available hybrid pretreatment methods, thermoalkaline, $\mathrm{NaOH} / \mathrm{H}_{2} \mathrm{O}_{2}$, and Ozone/ $\mathrm{NaOH}$ have received more attention to enhance AnCoD. In a recent study performed by Benn et al. (2018), the usage of thermochemical bioplastic pretreatment was investigated in co-digestion with synthetic primary sludge in batch and continuous mode. The pretreatment experiments were conducted by applying different combinations of temperatures ranging from $35-90^{\circ} \mathrm{C}$ with alkaline conditions in a $\mathrm{pH}$ range from 8 to 12 (Benn and Zitomer, 2018). Percent conversion values for bioplastics to biomethane were calculated as the proportion of BMP value divided by the theoretical maximum methane production value indicated by the bioplastic theoretical oxygen demand loading. According to the authors, the thermos-alkaline pretreatment led to an increase in average BMP values up to over $100 \%$. The batch system codigesting synthetic primary sludge with bioplastic resulted in 80-100\% conversion of bioplastics to biomethane and a $50 \%$ biomethane production increase over the non-pretreated substrate. In comparison with the findings of the batch study, less than $20 \%$ increase in methane production was obtained by continuous co-digestion of pretreated bioplastics with the synthetic primary 
sludge in a CSTR system compared to the system fed with non-pretreated feedstock (Benn and Zitomer, 2018).

Another study conducted by Naran et al. revealed that the cumulative methane yield increased from 116.7 to $177.3 \mathrm{~mL} / \mathrm{g}$ VS added through the co-digestion of thermos-alkaline pretreated food waste from a food waste treatment plant and WAS generated at a municipal treatment compared to the non-pretreated sample (Naran et al., 2016). Similarly, the usage of thermo-alkaline pretreatment method to improve co-digestion of WAS and rice straw was studied and a biogas production of $409 \mathrm{~L} / \mathrm{kgVS}_{\text {added }}$ was obtained under the optimum condition equivalent to a $51 \%$ increase compared to the control test. The authors of that work also reported that the degree of WAS solublization was positively correlated with biogas production and VS removal. It was also observed that, following pretreatment, the cellulose and hemicellulose contents of rice straw decreased remarkably. According to the results of their research, the addition of $\mathrm{NaOH}$ caused $11 \%, 32 \%$, and $22 \%$ reduction in hemicellulose, cellulose, and lignin contents, respectively. The improvement of digestion performance in this study was related to the increased solublization and reduced particle size of the organic matter (Abudi et al., 2016b).

Abudi et al., employed $\mathrm{NaOH} / \mathrm{H}_{2} \mathrm{O}_{2}$ pretreatment on co-digestion of WAS and rice straw. The applied pretreatment resulted in a remarkable reduction in cellulose and hemicellulose contents of rice straw and hence improved the biogas production. $\mathrm{NaOH} / \mathrm{H}_{2} \mathrm{O}_{2}$ pretreatment was able to decrease hemicellulose, cellulose and lignin contents of rice straw by $16 \%, 41 \%$, and $7 \%$, respectively. Pretreatment was more effective in the solublization of hemicellulose content than cellulose and lignin contents of rice straw. Consistent results were obtained by others for single digestion of rice and corn straws using $\mathrm{NaOH} / \mathrm{H}_{2} \mathrm{O}_{2}$ pretreatment (He et al., 2009; Song et al., 2014, 2013).

Rajesh Banu et al. employed ozone/ $\mathrm{NaOH}$ pretreatment for the co-digestion of cow manure and WAS. This resulted in increasing biogas production from 17.9 to $18.8 \mathrm{~L} / \mathrm{d}$. Despite the fact that ozone utilization is considered to be costly, the combination of alkali and ozone not only increased the sludge disintegration efficiency but also saved a considerable amount of energy (Rajesh Banu et al., 2015). The increase in biogas production was most likely due to the particles decomposition resulting from ozone reaction with the organic fraction of sludge and the availability of extra carbon source (Ahn et al., 2002). 
In the study conducted by Ren et al., the effects of hot alkali pretreatment and mixing ratio on anaerobic co-digestion of duckweed and excess sludge were investigated. The result of their study primarily indicated that through co-digestion the delayed stage of gas generation reduced, and a cumulative gas yield of $2963 \mathrm{~mL}$ was obtained which was $11 \%$ higher than the calculated value. The methane content of the produced gas was 57\%, which was $13 \%$ higher than that of the duckweed and $9 \%$ higher than that of the excess sludge single digestion. Additionally, pretreatment of the duckweed in the mixture, improved the methane yield by $8 \%$ (Ren et al., 2018).

In co-digestion of poultry manure with pig manure in a batch system, the effect of a combined thermochemical pretreatment and ammonia stripping on the digester performance was assessed. The result revealed that the optimal blend ratio of poultry manure to pig manure was $24: 76$ on volumetric basis which was corresponding to highest methane production. The combined pretreatment improved the co-digestion system achieving an OLR of $4 \mathrm{~g}$ COD/L $\mathrm{d}$ with a HRT of 20 days (Rodriguez-Verde et al., 2017). The results of these studies are summarized in table 2.5.

Table 2.5. Hybrid pretreatment for enhanced AnCoD

\begin{tabular}{|c|c|c|c|c|}
\hline $\begin{array}{l}\text { Method of } \\
\text { Pretreatment }\end{array}$ & Feedstock & Digester mode & Mixing ratio & $\begin{array}{l}\text { Methane yield/ } \\
\text { Biogas increase \% }\end{array}$ \\
\hline Thermo-alkaline & $\begin{array}{l}\text { Synthetic municipal } \\
\text { primary sludge }+ \\
\text { bioplastics }\end{array}$ & $\begin{array}{l}\text { Batch } \\
\text { (mesophilic) }\end{array}$ & $10: 1(\mathrm{v} / \mathrm{v})$ & $6 \%$ methane increase \\
\hline Thermo-alkaline & WAS + food waste & $\begin{array}{l}\text { Batch } \\
\text { (mesophilic) }\end{array}$ & $7: 3(\mathrm{v} / \mathrm{v})$ & $\begin{array}{l}52 \% \text { methane } \\
\text { increase }\end{array}$ \\
\hline Thermo-alkaline & TWAS+ rice straw & $\begin{array}{l}\text { Batch } \\
\text { (mesophilic) }\end{array}$ & $1: 1(\mathrm{v} / \mathrm{v})$ & $51 \%$ biogas increase \\
\hline $\begin{array}{l}\text { Thermo- } \\
\text { alkaline/ } \mathrm{H}_{2} \mathrm{O}_{2}\end{array}$ & TWAS+ rice straw & $\begin{array}{l}\text { Batch } \\
\text { (mesophilic) }\end{array}$ & $1: 1(\mathrm{v} / \mathrm{v})$ & $56 \%$ biogas increase \\
\hline Ozone/NaOH & $\begin{array}{l}\text { Cow manure +dairy } \\
\text { wastewater }\end{array}$ & HUASB $* * *$ & $3: 1(\mathrm{v} / \mathrm{v})$ & $5 \%$ biogas increase \\
\hline Thermo-alkaline & $\begin{array}{l}\text { duckweed and waste } \\
\text { activated sludge }\end{array}$ & Batch mesophilic & NA & $8 \%$ \\
\hline Thermo-alkaline & $\begin{array}{l}\text { poultry manure and } \\
\text { pig manure }\end{array}$ & $\begin{array}{l}\text { Continuous } \\
\text { mesophilic }\end{array}$ & $24: 76$ & $37 \%$ \\
\hline
\end{tabular}

In summary, due to various advantages that co-digestion offers over conventional mono digestion, this area is attracted by several researchers and studies are still going on to better understand the 
system performance and to investigate different methods for improving the AnCoD systems. Such researches along with studies on control parameters, suitability of different feedstocks and their combinations and optimizing procedures would contribute in further improving this technology. 


\section{Chapter 3}

\section{Materials and methods}




\section{Materials and methods}

\subsection{Feedstocks and Inoculum}

This research was designed to evaluate the effect of mixing ratio of the feedstocks and its relationship with the lipids: proteins: carbohydrates content on anaerobic co-digestion process. The experiment included two sections including BMP assay and hydrolysis/acidification. The BMP was designed to assess the influence of the mixing ratio on biomethane production. Hydrolysis/acidification experiment was carried out to evaluate the effect of mixing ratio on hydrolysis kinetics. Different feedstocks including dairy manure, TWAS and SSO in different combinations were used as digester feedstocks. TWAS (3.8\% TS) and inoculum were collected from Ashbridges Bay Wastewater Treatment Plant Toronto, Ontario. The inoculum was obtained from the effluent of the anaerobic digesters operating at mesophilic condition at a temperature range of $34-38^{\circ} \mathrm{C}$, and receiving approximately $1600 \mathrm{~m}^{3} / \mathrm{d}$ TWAS and $6500 \mathrm{~m}^{3} / \mathrm{d}$ primary sludge. The average organic loading rate and SRT of the anaerobic digesters are $1.1 \mathrm{~kg} \mathrm{VS} / \mathrm{m}^{3}$ and $18 \mathrm{~d}$, respectively.

The Ashbridges Bay Wastewater Treatment Plant is the main wastewater treatment facility among the four treatment plants that service the city of Toronto. After Montreal's Jean-R. Marcotte facility, it is the second largest plant in Canada (Heffez, 2009) The plant treats the wastewater produced by approximately 1.4 million of the city of Toronto's residents and has a capacity of $818,000 \mathrm{~m}^{3} / \mathrm{d}$ (City of Toronto, 2018).

The influent to the treatment plant comes from Mid-Toronto, high level, low level and Lakefront interceptor sewers in addition to Coxwell and Queen Street trunk sewers. Biosolids generated at the plant was approximately 149733 wet tones in 2016 with $28.1 \%$ Total Solid (TS). The influent undergoes treatment processes which comprises preliminary treatment i.e. screening and grit removal, primary treatment, secondary treatment, nutrient removal, disinfection, Waste Activated Sludge (WAS) thickening, anaerobic digestion, biosolids dewatering and biosolids management. The influent Total Suspended Solids (TSS) and Biological Oxygen Demand (BOD) concentrations are $318.6 \mathrm{mg} / \mathrm{L}$ of $244.6 \mathrm{mg} / \mathrm{L}$, respectively (City of Toronto, 2018; Razavi, 2019).

The unit processes of the plant is shown in figure 3.1. The activated sludge system consists of three main stages including aeration tank, settling tank and return activated sludge. In the aeration tank, the atmospheric air is introduced to the primary treated wastewater using air blowers and the biological mass that produces biological flocs is called waste activated sludge (WAS). The 
produced sludge then goes through thickening process using different mechanisms to produce thickened waste activated sludge (TWAS).

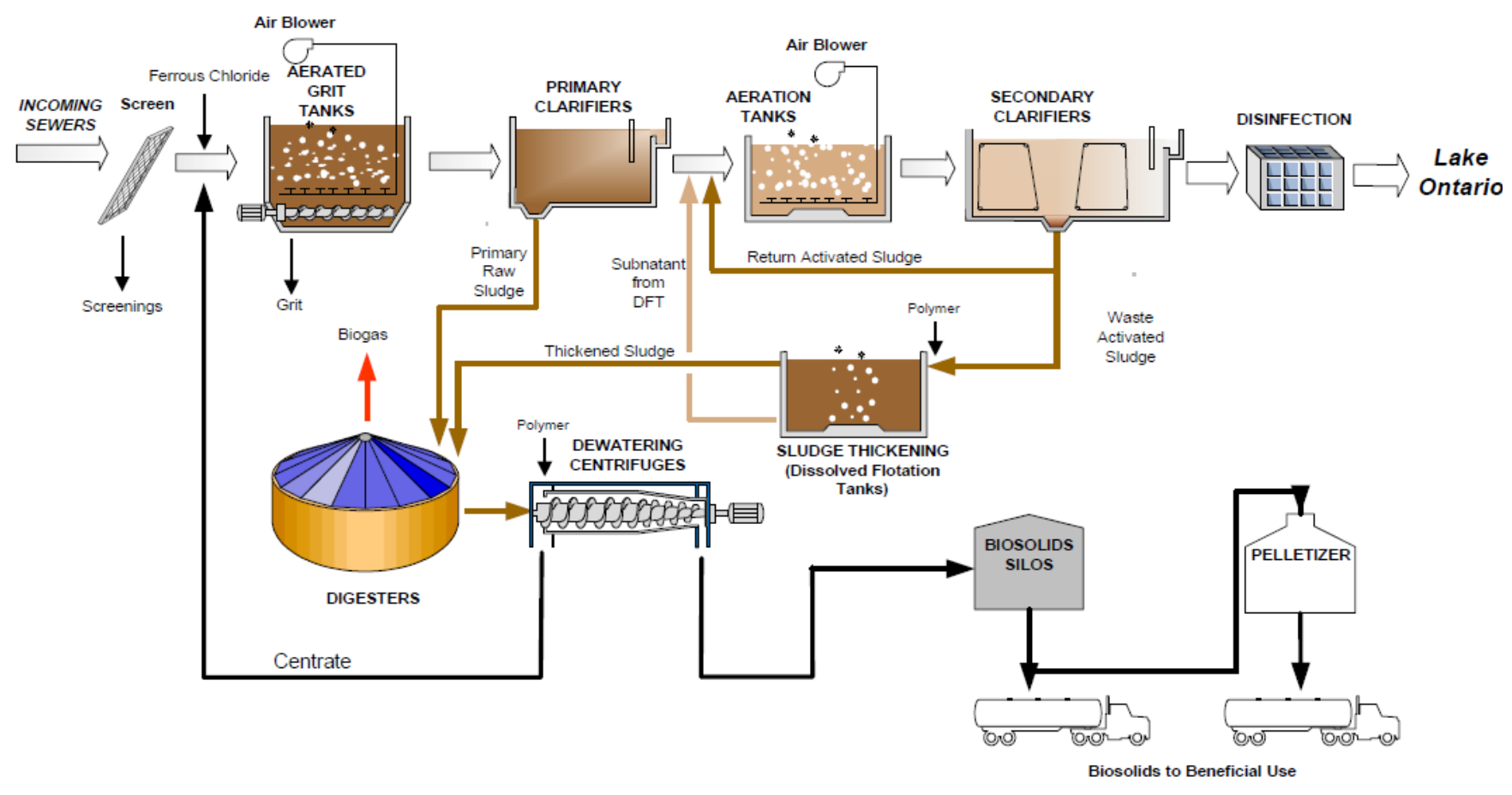

Figure 3.1. The flow diagram of the Ashbridges Bay Wastewater Treatment Plant (City of Toronto, 2018)

SSO was obtained from City of Toronto Disco Road Organics Processing Facility, Toronto, Ontario. The facility is located on 120 Disco Road and on a 1-hectare site and is the first full-scale plant in North America. It has been operated since 2014 for processing the source separated organics by anaerobic digestion. Being one of the municipality's diversion program, it receives almost half of the organics collected in Toronto and is capable of processing up to 75,000 tons of organic waste per year from homes and public buildings. The ultimate capacity of the facility is planned to rise to 130,000 tons in the near future. The acceptable materials to Disco Road facility includes food waste, paper food packaging, pet waste, diapers, houseplants, and biodegradable plastics. The organic processing at the facility is shown in figure 3.2. SSO first is delivered and stored and goes through a visual inspection to remove large unwanted items. At the next stage, the materials undergo the BTA ${ }^{\circledR}$ hydro-mechanical technology through which the organics will convert to a liquid (slurry) pulp. The BTA® consists of screens and hydropulpers for separating 
the unwanted materials including glass shards, plastic bags, metals and sand from the pulp (Razavi, 2019). In this study, the SSO samples were collected after the hydro-mechanical stage and transferred to the lab in the slurry form.
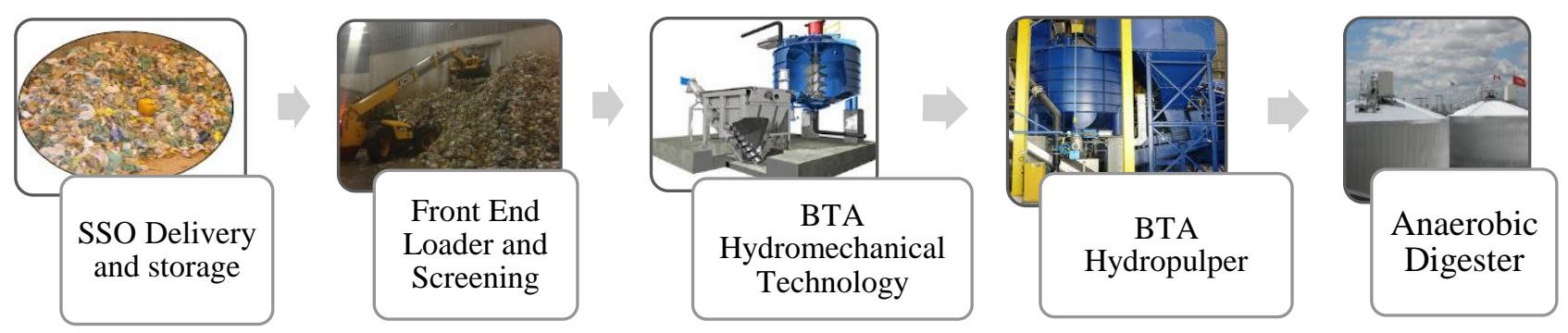

Figure 3.2. Source separated organics processing in Disco Road Facility

Cow manure was collected from a manure pit of a dairy farm located in Newmarket, Ontario. Manure slurry was prepared by addition and homogenization of cow manure with deionized distilled water using a blender followed by a VWR 400 DS bench top homogenizer. The reactors were fed with different combinations of the feedstocks.

The BMP included 3 binary co-digestion experiments using different feedstocks mixtures including different mixing ratios of TWAS/SSO, TWAS/manure, and Manure/SSO. The BMP of TWAS/SSO, TWAS/manure, and Manure/SSO were conducted individually in different periods. It also included a ternary co-digestion using the three feedstocks, TWAS/manure/SSO, at different mixing ratios. Each of the experiments continued until biogas production stopped or was negligible. A series of analysis for characterization of the inoculum, TWAS and SSO and manure was carried out primarily and presented in Table 3.1. Samples were transported and preserved according to Standard Method for Examination of Water and Wastewater (APHA, 2005). Feed of digesters as explained above were mixed at different mixture ratios on a volumetric basis. Batch reactors in working volume of $200 \mathrm{~mL}$ containing inoculum and feedstocks at different mixing ratios were prepared for the BMP essay. TWAS, manure, and SSO alone were also used as control reactor to assess the effect of co-digestion on the efficiency of the system in comparison with the single digestion of the feedstocks.

A series of analysis for characterization of the inoculum, TWAS and SSO was carried out primarily and are presented in table 3.1. The mean values are the average of four measurements on each of 
the parameters for the raw substrates and RSD is the ratio of standard deviation to the mean or relative standard deviation.

Table 3.1. Initial Characteristics of the feedstocks and inoculum used in this study

\begin{tabular}{|c|c|c|c|c|c|c|c|c|c|}
\hline \multirow[t]{2}{*}{ Parameters } & \multirow[t]{2}{*}{ Units } & \multicolumn{2}{|c|}{ SSO } & \multicolumn{2}{|c|}{ Manure } & \multicolumn{2}{|c|}{ TWAS } & \multicolumn{2}{|c|}{ Inoculum } \\
\hline & & MEAN & RSD & MEAN & RSD & MEAN & 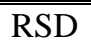 & MEAN & "RSD \\
\hline TCOD & $\mathrm{mg} / \mathrm{L}$ & 110000 & 0.07 & 198833 & 0.03 & 40000 & 0.07 & 16400 & 0.03 \\
\hline SCOD & $\mathrm{mg} / \mathrm{L}$ & 44400 & 0.002 & 10933 & 0.04 & 360 & 0.07 & 362 & 0.05 \\
\hline TSS & $\mathrm{mg} / \mathrm{L}$ & 53833 & 0.12 & 56520 & 0.03 & 31450 & 0.08 & 17033 & 0.02 \\
\hline VSS & $\mathrm{mg} / \mathrm{L}$ & 38478 & 0.095 & 29998 & 0.02 & 25600 & 0.09 & 10900 & 0.02 \\
\hline TS & $\mathrm{mg} / \mathrm{L}$ & 62187 & 0.02 & 73727 & 0.04 & 38810 & 0.12 & 21450 & 0.03 \\
\hline VS & $\mathrm{mg} / \mathrm{L}$ & 43493 & 0.02 & 38647 & 0.02 & 31205 & 0.01 & 13140 & 0.02 \\
\hline Ammonia & $\mathrm{mg} / \mathrm{L}$ & 1738 & 0.003 & 22 & 0.07 & 255 & 0.12 & 1495 & 0.03 \\
\hline pH & - & 5.6 & 0.001 & 6.4 & 0.01 & 6.3 & 0.005 & 7.2 & 0.001 \\
\hline Alkalinity & $\mathrm{mg} \mathrm{CaCO} 3 / \mathrm{L}$ & 7700 & 0.07 & 11133 & 0.05 & 1953 & 0.07 & 3943 & 0.12 \\
\hline TN & $\mathrm{mg} / \mathrm{L}$ & 4167 & 0.18 & 2200 & 0.12 & 2900 & 0.14 & 2025 & 0.10 \\
\hline TSN & $\mathrm{mg} / \mathrm{L}$ & 1793 & 0.02 & 104 & 0.09 & 420 & 0.15 & 696 & 0.16 \\
\hline Total Carbs & $\mathrm{mg} / \mathrm{L}$ & 40360 & 0.09 & 7398 & 0.06 & 1288 & 0.08 & 961 & 0.08 \\
\hline Total Proteins & $\mathrm{mg} / \mathrm{L}$ & 2021 & 0.09 & 5199 & 0.08 & 1459 & 0.18 & 1448 & 0.09 \\
\hline Total Lipids & $\mathrm{mg} / \mathrm{L}$ & 18620 & 0.12 & 7241 & 0.09 & 551 & 0.08 & 1920 & 0.10 \\
\hline
\end{tabular}

\subsection{Experimental design and procedure}

\subsubsection{Co-digestion- BMP assay}

The biochemical methane potential (BMP) assays were conducted according to the procedures described in the literature (Angelidaki et al., 2009a; Moody et al., 2009; Owen et al., 1979). The experiment was initiated by feeding the digesters with different mixing ratios of the feedstocks in triplicates. A 100\% SSO and 100\% TWAS, and 100\% manure as control reactors were assessed in triplicates as well. In this research, a substrate-to-biomass ratio $\left(\mathrm{S}^{0} / \mathrm{X}^{0}\right)$ of $2 \mathrm{~g} \mathrm{COD}$ substrate/g VSS inoculum was kept in all digesters which is within the range that has been suggested by the literature (Elbeshbishy et al., 2012). Substrate to inoculum ratio was selected for all of the batch reactors according to the procedure used in by Nasr et al., 2011 as following:

$\mathrm{S}^{0} / \mathrm{X}^{0}=\frac{\mathrm{g} \mathrm{TCOD}_{\text {substrate }}}{\mathrm{g} \mathrm{VSS}_{\text {inoculum }}}=\frac{\mathrm{V}_{\text {substrate }}{ }^{\times} \text {TCOD }_{\text {substrate }}}{\mathrm{V}_{\text {inoculum }}{ }^{\times} \text {VSS }_{\text {substrate }}}=2$ 
The TCOD of the mixture used in Eq. 3.1 was calculated considering the TCOD of the feedstocks and the mixing ratios. Accordingly, the volumes of the feedstocks were calculated based on the mixing ratios of substrates. Because of the heterogeneous composition of the feedstocks, all of the combinations were prepared in triplicates. The specific amount of the substrates along with the mesophilic inoculum as described above was added to $250 \mathrm{~mL}$ glass bottles. The headspaces in the bottles were flushed with nitrogen gas for 3 minutes at $10 \mathrm{psi}$ and subsequently the bottles were sealed to satisfy the anaerobic conditions. In addition, anaerobic systems require a $\mathrm{pH}$ within the range of 6.5-7.5 according to the literature (Cioabla et al., 2012; Droste, 1997). Therefore, the $\mathrm{pH}$ in each bottle were kept in a range of 7-7.4 using sulfuric acid and sodium hydroxide. The binary co-digestion of TWAS/SSO, TWAS/manure, and manure/SSO were conducted at the mixing ratios as presented in Table 3.2.

Table 3.2 Proportions of digesters'feed for binary co-digestion of TWAS/ SSO, TWAS/manure, and manure/ SSO in BMP experiment

\begin{tabular}{|c|c|c|c|}
\hline Feedstock & TWAS & Manure & SSO \\
\hline \multirow{20}{*}{$\begin{array}{l}\text { Proportion of the } \\
\text { feedstocks in } \\
\text { different runs of } \\
\text { Binary co-digestion } \\
\text { Volume: } 200 \mathrm{~mL}\end{array}$} & 0 & 1 & 0 \\
\hline & 1 & 0 & 0 \\
\hline & 9 & 1 & 0 \\
\hline & 7 & 3 & 0 \\
\hline & 5 & 5 & 0 \\
\hline & 3 & 7 & 0 \\
\hline & 1 & 9 & 0 \\
\hline & 0 & 2 & 4 \\
\hline & 0 & 0 & 1 \\
\hline & 0 & 9 & 1 \\
\hline & 0 & 7 & 3 \\
\hline & 0 & 5 & 5 \\
\hline & 0 & 3 & 7 \\
\hline & 0 & 1 & 9 \\
\hline & 0 & 0 & \\
\hline & 9 & 0 & 1 \\
\hline & 7 & 0 & 3 \\
\hline & 5 & 0 & 5 \\
\hline & 3 & 0 & 7 \\
\hline & 1 & 0 & 9 \\
\hline
\end{tabular}


The ternary co-digestion of TWAS/manure/SSO was carried out at different mixing ratios of TWAS, manure and SSO as presented in Table 3.3. As demonstrated in Table 3.2 and 3.3 reactors containing only manure, only TWAS and only SSO were also used in triplicates as control in each run.

Table 3.3 Proportions of digesters' feed for ternary co-digestion of TWAS, SSO, and manure in BMP experiment

\begin{tabular}{|l|c|c|c|}
\hline \multicolumn{1}{|c|}{ Feedstock } & TWAS & Manure & SSO \\
\hline \multirow{4}{*}{$\begin{array}{l}\text { Proportion of the } \\
\text { feedstocks in }\end{array}$} & 8 & 1 & 1 \\
\cline { 2 - 4 } different runs of \\
\cline { 2 - 4 } ternary co-digestion & 1 & 8 & 1 \\
\cline { 2 - 4 } Volume: 200 mL & 5 & 1 & 2.5 \\
\cline { 2 - 4 } & 2.5 & 5 & 2.5 \\
\cline { 2 - 4 } & 2.5 & 2.5 & 5 \\
\cline { 2 - 4 } & 4 & 4 & 2 \\
\cline { 2 - 4 } & 2 & 2 & 4 \\
\cline { 2 - 4 } & 1 & 4 & 0 \\
\cline { 2 - 4 } & 0 & 1 & 1 \\
\hline
\end{tabular}

The sets of 21 bottles for each of the three binary co-digestion experiments, and the 36 bottles for the ternary co-digestion experiment were placed in the Thermo Scientific MAXQ 4000 shakers and a rotational speed of 150 RPM was applied during the entire process. The incubator temperature was set at $37^{\circ} \mathrm{C}$ to satisfy mesophilic condition for the batch reactors. 


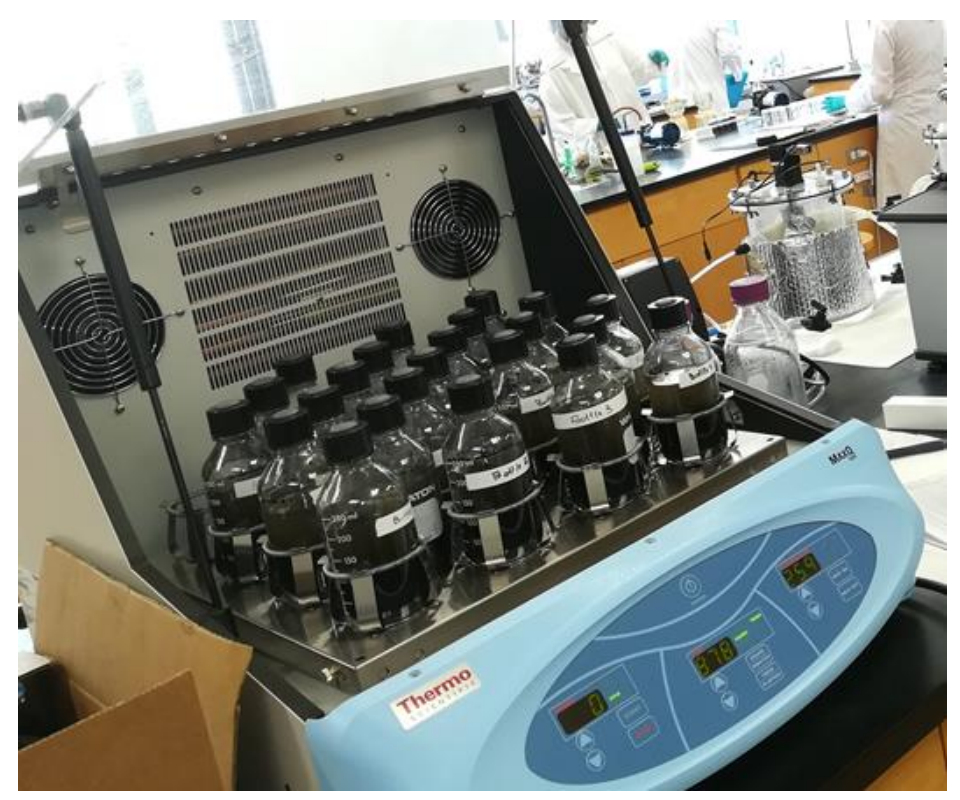

Figure 3.3- Experimental set-up for biomethane potential experiment

\subsubsection{Co-digestion- hydrolysis/acidification experiment}

This experiment was conducted to assess the influence of the mixing ratio and its relationship with the lipids: proteins: carbohydrates in co-digestion on hydrolysis/acidification rate. Similar to BMP experiment, the binary co-digestion of TWAS/ SSO, TWAS/manure, and manure/SSO were conducted at different combinations as presented in Table 3.4. The ternary co-digestion of TWAS/manure/SSO was carried out at the mixing ratios that are presented in Table 3.5. For each of the experiment's rectors containing only TWAS, only manure and only SSO were also used in triplicates as control reactors.

The bottles for each of the three binary co-digestion experiments, and for the ternary co-digestion experiment were placed in the Polyscience WB28 water bathes. Bottles were equipped with a mixer which maintained a rotational speed of 150 RPM the entire process. The temperature in water bathes was set at $37^{\circ} \mathrm{C}$ to satisfy mesophilic condition for the batch reactors.

Consistent with the BMP experiment, the substrate-to-biomass ratio $\left(\mathrm{S}^{0} / \mathrm{X}^{0}\right)$ of to $2 \mathrm{~g}$ COD substrate/g VSS inoculum was kept in all digesters. Feed of digesters as explained above were mixed at different mixture ratios on a volumetric basis. Batch reactors in working volume of $2000 \mathrm{~mL}$ containing inoculum and feedstocks at different mixing ratios were prepared for the BMP essay. In order to deactivate methanogens and to increase the accuracy of the hydrolysis/acidification experiment, 
the inoculum was heated to $70{ }^{\circ} \mathrm{c}$ for $30 \mathrm{~min}$ and $\mathrm{pH}$ was adjusted to a range between 5- 5.5 in all digesters. The experiment continued for a period of three days for each run of the binary codigestions and the ternary co-digestion experiment. Samples were collected with time to evaluate the solublization and hydrolysis rate of the mixtures and to assess the influence of the mixing ratios on them. Consistent with the BMP assay, The TCOD of the mixture in Eq. 3.1 was calculated considering the TCOD of the feedstocks and the mixing ratios. Accordingly, the volumes of the feedstocks were calculated based on the mixing ratios of substrates. Because of the heterogeneous composition of the feedstocks, all of the combinations were prepared in triplicates. The specific amount of the substrates along with the mesophilic inoculum as described above was added to $2500 \mathrm{~mL}$ glass bottles.

Table 3.4 Proportions of digesters' feed for binary co-digestion of TWAS/ SSO, TWAS/manure, and manure/ SSO in hydrolysis/acidification experiment

\begin{tabular}{|c|c|c|c|}
\hline Feedstock & TWAS & Manure & SSO \\
\hline \multirow{20}{*}{$\begin{array}{l}\text { Proportion of the } \\
\text { feedstocks in } \\
\text { different runs of } \\
\text { Binary co-digestion } \\
\text { Volume: } 2000 \mathrm{~mL}\end{array}$} & 0 & 1 & 0 \\
\hline & 1 & 0 & 0 \\
\hline & 9 & 1 & 0 \\
\hline & 7 & 3 & 0 \\
\hline & 5 & 5 & 0 \\
\hline & 3 & 7 & 0 \\
\hline & 1 & 9 & 0 \\
\hline & 0 & 2 & 4 \\
\hline & 0 & 0 & 1 \\
\hline & 0 & 9 & 1 \\
\hline & 0 & 7 & 3 \\
\hline & 0 & 5 & 5 \\
\hline & 0 & 3 & 7 \\
\hline & 0 & 1 & 9 \\
\hline & 0 & 0 & \\
\hline & 9 & 0 & 1 \\
\hline & 7 & 0 & 3 \\
\hline & 5 & 0 & 5 \\
\hline & 3 & 0 & 7 \\
\hline & 1 & 0 & 9 \\
\hline
\end{tabular}


Table 3.5 Proportions of digesters' feed for ternary co-digestion of TWAS, SSO, and manure in in hydrolysis/acidification experiment

\begin{tabular}{|l|c|c|c|}
\hline \multicolumn{1}{|c|}{ Feedstock } & TWAS & Manure & SSO \\
\hline \multirow{4}{*}{$\begin{array}{l}\text { Proportion of the } \\
\text { feedstocks in }\end{array}$} & 8 & 1 & 1 \\
\cline { 2 - 4 } different runs of \\
\cline { 2 - 4 } ternary co-digestion & 1 & 8 & 1 \\
\cline { 2 - 4 } Volume: 200 mL & 5 & 2.5 & 2.5 \\
\cline { 2 - 4 } & 2.5 & 5 & 2.5 \\
\cline { 2 - 4 } & 2.5 & 2.5 & 5 \\
\cline { 2 - 4 } & 4 & 4 & 4 \\
\cline { 2 - 4 } & 2 & 2 & 4 \\
\cline { 2 - 4 } & 1 & 4 & 0 \\
\cline { 2 - 4 } & 0 & 1 & 1 \\
\hline
\end{tabular}

The headspaces in the bottles were flushed with nitrogen gas for 3 minutes at 10 psi and subsequently the bottles were sealed to satisfy the anaerobic conditions. Gas production during the experiment was monitored using water displacement method and displayed real time. The percentage improvements in the soluble contents concentrations (degree of solubilization) (P) values was calculated using Eq 3.2.

$P(\%)=S C_{f}-S C_{i} / P C_{i} * 100 \%$

Eq. 3.2

Where $\mathrm{SC}_{\mathrm{i}}$ and $\mathrm{SC}_{\mathrm{f}}$ are the mass of soluble COD of the digester contents before and after the hydrolysis/acidification phase experiment $(\mathrm{mg})$ and $\mathrm{PC}_{\mathrm{i}}$ is the mass of initial particulate COD in the digesters $(\mathrm{mg})$. 


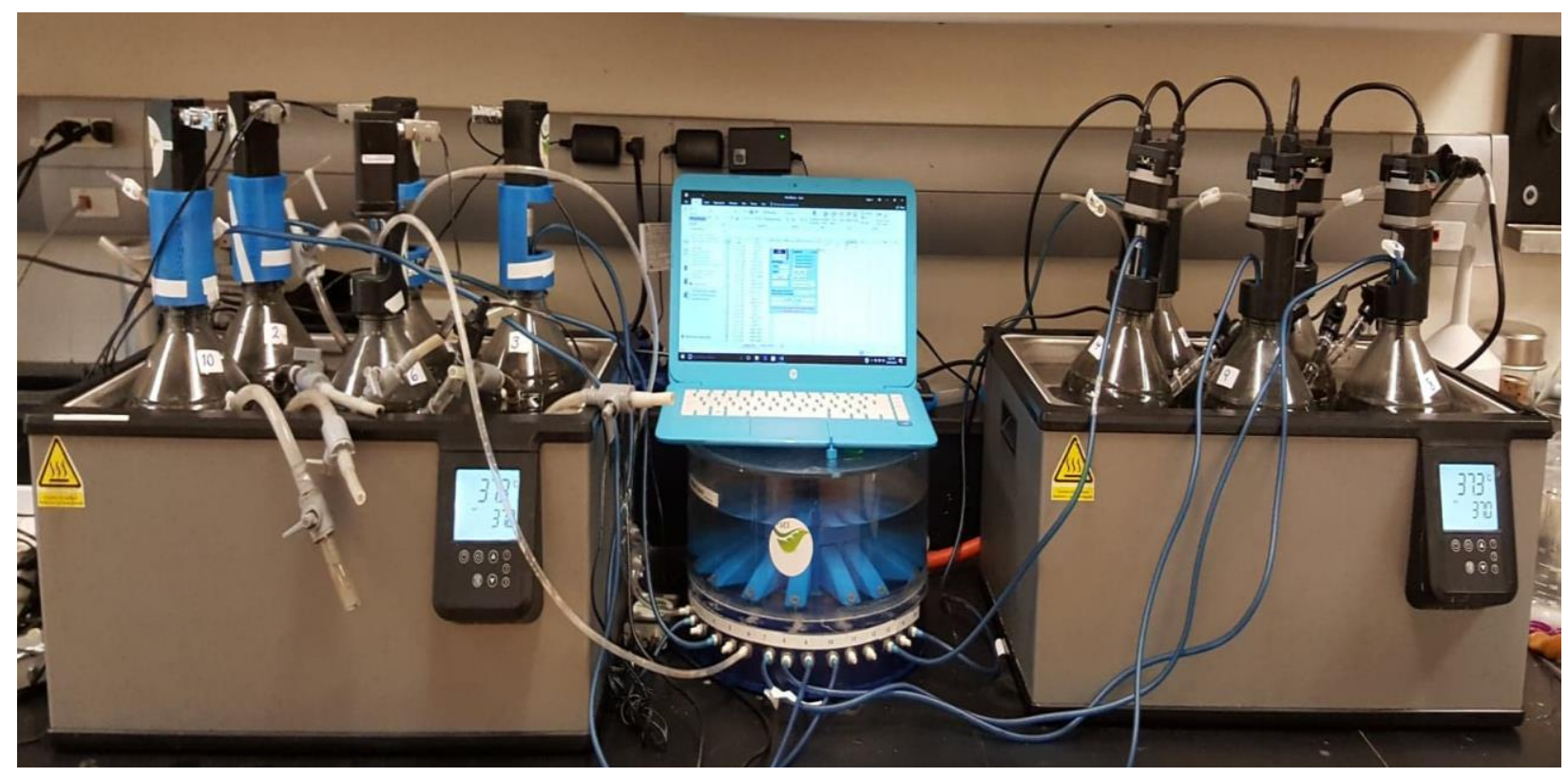

Figure 3.4. Experimental set-up for hydrolysis/acidification experiment

A first-order reaction model was applied using AquaSim 2.0 software to assess the effect of different mixing ratios on biodegradation rates of COD, proteins, lipids, and carbohydrates.

$\mathrm{r}_{\mathrm{su}}=\mathrm{dC} / \mathrm{dt}=-\mathrm{kC}$

Where $\mathrm{C}$ is the concentration $(\mathrm{mg} / \mathrm{L})$ of the parameters (TCOD, proteins, lipids, and carbohydrates) at time $t, k$ is first-order specific biodegradation rate constant $(1 / d)$ and $r_{\text {su }}$ is biodegradation rate (mg/L.d). Eq. 3.4 is derived by integration of Eq. (3.3).

$$
\mathrm{C}_{\mathrm{t}}=\mathrm{C}_{\mathrm{u}} \mathrm{e}^{-\mathrm{kt}}
$$

Where $t, C_{t}$ and $C_{u}$ are time $(d)$, concentration at time $t(\mathrm{mg} / \mathrm{L})$, ultimate particulate parameters (TCOD, proteins, lipids, and carbohydrates) in mg/L, respectively.

\subsection{Analytical analysis}

The analysis of solid contents of the feedstocks including total solids (TS), volatile solids (VS), total suspended solids (TSS) and volatile suspended solids (VSS) of the inoculum, TWAS, and SSO samples were determined according to the Standard Methods procedures (APHA, 2005). Chemical oxygen demand (TCOD) and (SCOD), ammonia, total nitrogen (TN) and total soluble nitrogen (TSN) were measured using a Hach spectrophotometer model 3900. For the measurement 
of the soluble content, samples were prepared by centrifuging at $9000 \mathrm{rpm}$ for $45 \mathrm{~min}$ and then the supernatant was filtered using microfiber filters with a pore size of $0.45 \mu \mathrm{m}$. The absorbance was set at the wavelengths of 600, 560 and $650 \mathrm{~nm}$ for the analysis of COD, ammonia, and alkalinity, respectively.

Total lipids concentration was measured by solvatochromatic method that rely upon a dye or mixture of dyes which change optical properties upon a change in condition of the solvent in which they are dissolved. In this test, there is an increase in fluorescence when there is an increased amount of dissolved lipids that form micelles or other structures. Solvatochromatic analysis was carried out at the fluorescence $405 \mathrm{~nm}$ mode using Vernier SpectroVis spectrometer and Logger Pro software. The data then exported as CSV files for further analysis. Figure 3.5 shows the graph created by Logger Pro at $405 \mathrm{~nm}$ wavelength and the absorbance for different concentrations of total lipids. The peak values of the curves corresponding to the lipids concentration of different standard samples with known concentrations were used to plot the calibration curve and get the curve equation. The measured absorbance values for the actual samples with unknown lipids concentrations were substituted into the calibration equation and was solved for the true value.

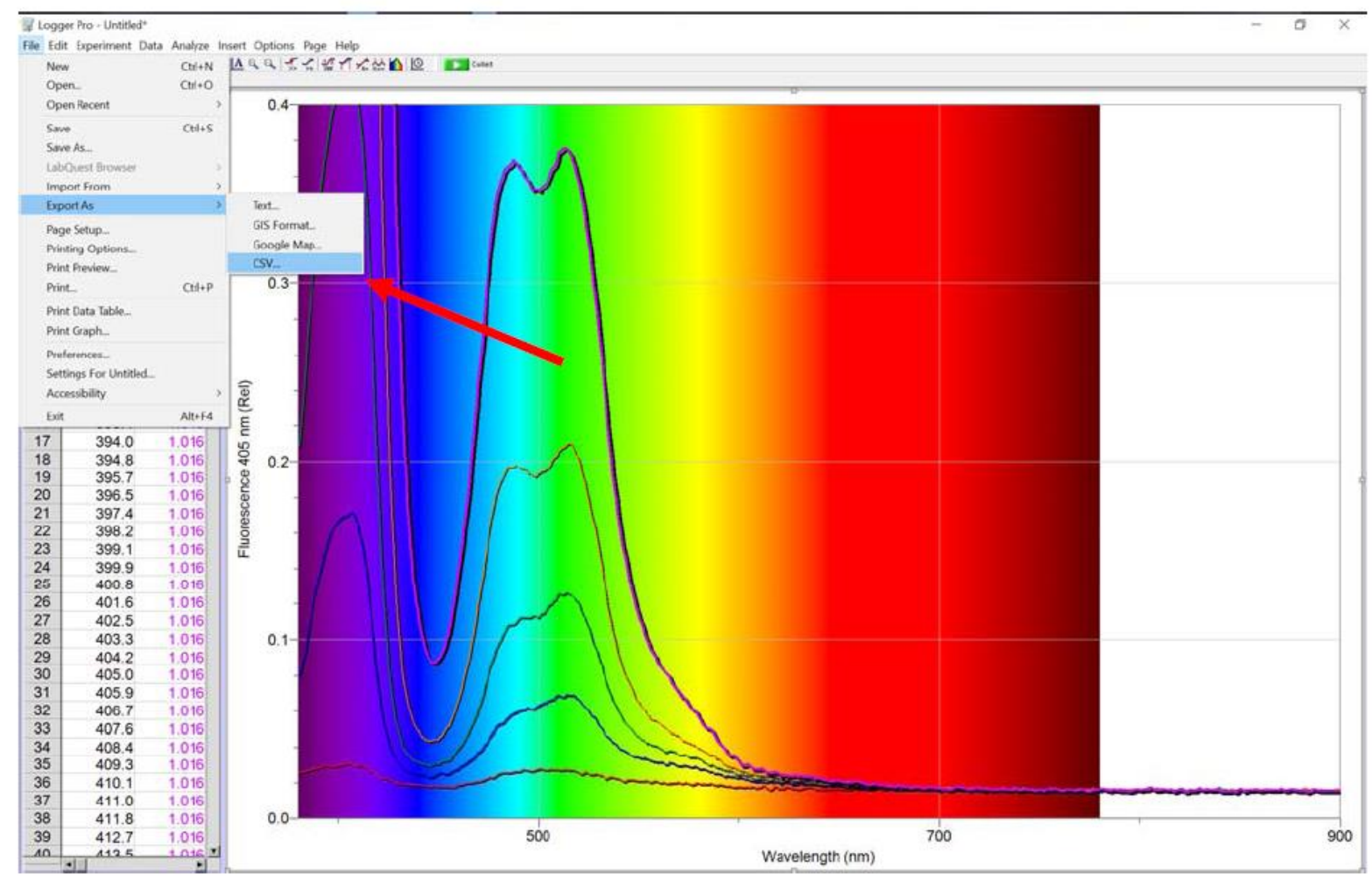

Figure 3.5. The spectra and flourometric data for different total lipids concentrations 
The volume of the produced gas was measured manually using a 100-mL Gastight Luer-Lock glass syringe daily at the beginning of the digestion period. The gas measurement was continued every couple of days later on when the gas production rate slowed down over time. The amount of biomethane in the produced biogas during the anaerobic digestion process was measured using a Thermo Scientific Trace 1310 gas chromatograph (GC). The GC was equipped with a thermal conductivity detector and the temperature of the oven, detector, and filament were set to 80, 100, and $250{ }^{\circ} \mathrm{C}$, respectively. The type of column used was a TG-Bond Msieve 5A model with a $30 \mathrm{~m}$ length and $0.53 \mathrm{~mm}$ diameter.

\subsection{Statistical and Kinetics analysis}

Statistical data including mean and standard deviation were calculated for the data obtained by the experiment. One way ANOVA for the analysis of variance was used to to find statistically significant differences between the group means and the results are presented in chapter 8 .

Gompertz equations provides a wide range of applications in process kinetics of anaerobic digestion and the methane potential studies. In this work, Modified Gompertz model (Elbeshbishy and Nakhla, 2012; Lay et al., 1999) was used to predict the biogas yield and to assess the kinetic parameters and to describe the progress of cumulative methane production through the batch process (eq. 3.52) where $\mathrm{CH}_{4}$ is the cumulative methane production $(\mathrm{mL}), \mathrm{P}$ is the ultimate methane production $(\mathrm{mL}), R_{m}^{e}$ is the maximum methane production rate $(\mathrm{mL} / \mathrm{d}), \lambda$ is the lag phase time (d), $t$ is the digestion time (d).

$\mathrm{CH}_{4}=\mathrm{p} \cdot \exp \left\{-\exp \left[\frac{R_{m}^{e}}{p}(\lambda-\mathrm{t})+1\right]\right\}$ 


\section{Chapter 4}

Results and discussion

\section{TWAS and SSO Co-digestion}




\section{Results and discussion- TWAS/SSO co-digestion}

\subsection{BMP of TWAS and SSO}

This experiment was designed to evaluate the effect of mixing ratio of TWAS with SSO and its relationship with the lipids: proteins: carbohydrates on anaerobic co-digestion process using TWAS and SSO in different combinations as digester feedstocks. Co-digestion of SSO with TWAS was conducted as explained in Chapter 3. The characteristics of the feed in each digester having different mixing ratios of the substrates are summarized in Table 4.1. As presented in Table 4.1, the amount of TCOD of SSO is remarkably higher than that of TWAS. Increasing the fractions of SSO, increased the TCOD of the feed to digesters. The total lipids and total carbohydrates contents of SSO are also significantly more than that of TWAS and therefore, by increasing the proportion of SSO in the co-digesters the concentrations of lipids and carbohydrates increased. $\mathrm{pH}$ was kept at neutral level from 7.0- 7.3 in all the mixtures to satisfy the favorable condition for methanogenesis.

Table 4.1. Mean values of the feed characteristics in digesters with different mixing ratios of TWAS and SSO

\begin{tabular}{|c|c|c|c|c|c|c|c|c|}
\hline & & TWAS Only & SSO Only & $\begin{array}{c}\text { TWAS:SSO } \\
9: 1\end{array}$ & $\begin{array}{c}\text { TWAS:SSO } \\
7: 3\end{array}$ & $\begin{array}{c}\text { TWAS:SSO } \\
1: 1\end{array}$ & $\begin{array}{c}\text { TWAS:SSO } \\
3: 7\end{array}$ & $\begin{array}{c}\text { TWAS:SSO } \\
1: 9\end{array}$ \\
\hline Parameters & Units & Mixture (1) & $\begin{array}{l}\text { Mixture } \\
\text { (2) }\end{array}$ & Mixture (3) & Mixture (4) & Mixture (5) & Mixture (6) & Mixture (7) \\
\hline TCOD & $\mathrm{g} / \mathrm{L}$ & 40 & 110 & 47 & 61 & 75 & 89 & 103 \\
\hline SCOD & $\mathrm{g} / \mathrm{L}$ & 1.4 & 44 & 5.7 & 14 & 23 & 32 & 40 \\
\hline TSS & $\mathrm{g} / \mathrm{L}$ & 32 & 54 & 34 & 38 & 43 & 47 & 52 \\
\hline VSS & $\mathrm{g} / \mathrm{L}$ & 26 & 39 & 27 & 30 & 32 & 35 & 37 \\
\hline TS & $\mathrm{g} / \mathrm{L}$ & 39 & 62 & 41 & 46 & 51 & 55 & 60 \\
\hline VS & $\mathrm{g} / \mathrm{L}$ & 35 & 44 & 35 & 37 & 39 & 41 & 43 \\
\hline Ammonia & $\mathrm{g} / \mathrm{L}$ & 0.3 & 1.1 & 0.3 & 0.5 & 0.7 & 0.9 & 1.0 \\
\hline $\mathrm{pH}$ & & 7.2 & 7.1 & 7.0 & 7.0 & 7.2 & 7.1 & 7.3 \\
\hline Alkalinity & $\mathrm{g} \mathrm{CaCO} 3 / \mathrm{L}$ & 2.0 & 6.1 & 2.4 & 3.2 & 4.0 & 4.8 & 5.7 \\
\hline $\mathrm{TN}$ & $\mathrm{g} / \mathrm{L}$ & 2.9 & 3.3 & 2.9 & 3.0 & 3.1 & 3.2 & 3.2 \\
\hline TSN & $\mathrm{g} / \mathrm{L}$ & 0.4 & 0.9 & 0.5 & 0.6 & 0.7 & 0.8 & 0.9 \\
\hline T-Carbs & $\mathrm{g} / \mathrm{L}$ & 1.1 & 144 & 2.4 & 5.1 & 7.7 & 10.4 & 13.0 \\
\hline T-Proteins & $\mathrm{g} / \mathrm{L}$ & 3.8 & 2.3 & 3.6 & 3.3 & 3.0 & 2.7 & 2.5 \\
\hline T-Lipids & $\mathrm{g} / \mathrm{L}$ & 0.4 & 1.7 & 0.5 & 0.8 & 1.0 & 1.3 & 1.6 \\
\hline
\end{tabular}


As shown in Fig. 4.1, no significant lag-phase occurred in the generation of biogas the reactors. Operation of the digesters continued until no significant biogas was produced. Fig. 4.1 shows the time-course profile of the cumulative biomethane production during the total operation period. As illustrated in the Figure 4.1, no significant lag time was observed for all the digesters. This no sign of significant inhibition would be due to the use of mesophilic inoculum acclimatized to municipal sludge streams similar to the one used in the experiment. The most lag-phase occurred at TWAS:SSO mixing ratios of $1: 1$, and 3:7, and 1:9 which could be due to the existence of particulate matters introduced by SSO to the mixture which could delay the hydrolysis phase and consequently affect methanogenesis.

The amount of biomethane produced by SSO was significantly higher than that of TWAS. Only $542 \mathrm{~mL}$ cumulative methane was produced by TWAS while the amount of cumulative methane obtained by SSO was $1101 \mathrm{~mL}$. This verified the low biodegradability of TWAS compared to SSO and would be due to the composition of TWAS as it mostly consists of proteins and humic substances with some bacterial biomass and carbohydrates. Although proteins, DNA and carbohydrates are anaerobically biodegradable, their biodegradability decreases when they are combined into an organized structure similar to TWAS (Gonzalez et al., 2018; Stuckey and McCarty, 1984). Microbial cells are difficult to break down under anaerobic digestion (Foladori et al., 2015; Wett et al., 2010) and similarly, the presence of humic substances affects enzymatic activity by immobilizing enzymes and as a result, lowers biodegradability (Azman et al., 2015a, 2015b; Fernandes et al., 2015).

Another reason for the low biodegradability of TWAS was investigated by a study, in which low digestibility was attributed to the slow hydrolysis process for the exterior polymeric component of the microbial culture within the sample. Furthermore, the study also found that the ratio of SCOD to TCOD was $34.6 \%$ for untreated TWAS compared to $63.6 \%$ and $68.1 \%$ for thermally and alkaline pretreated samples, respectively. The COD ratio was a clear indicator of the expected biogas production by the TWAS feedstock samples where lower biogas yield was reported for the untreated raw TWAS as compared to pretreated samples (Abudi et al., 2016a). 


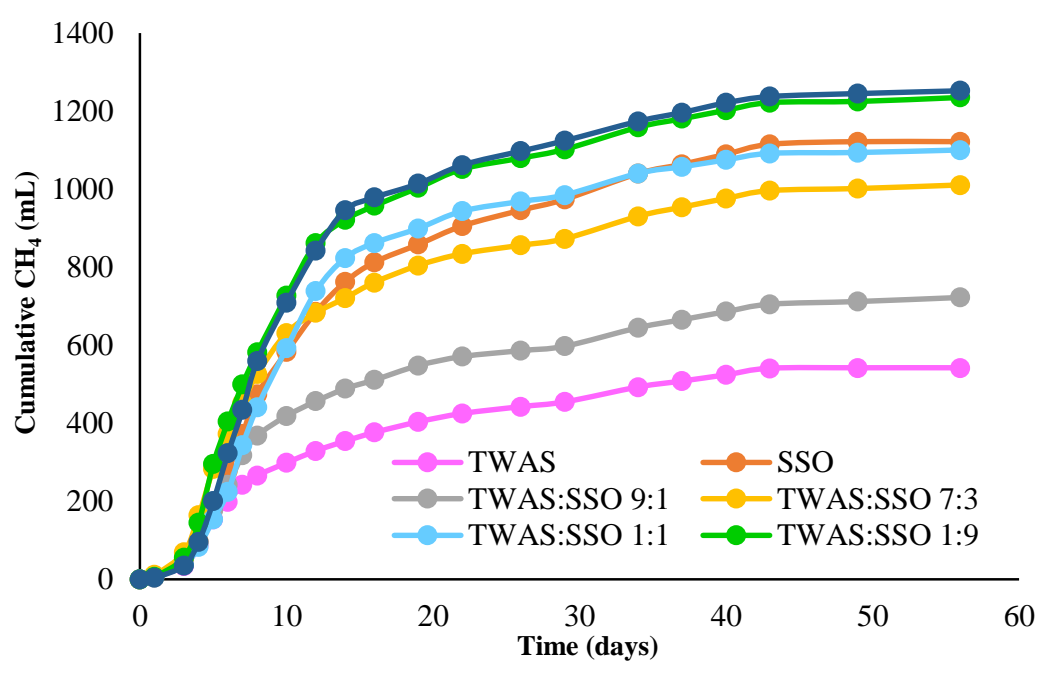

Figure 4.1. Cumulative methane production for different mixing ratios of TWAS and SSO

Addition of SSO to TWAS increased biodegradability and the methane yield compared to TWAS alone. However, as demonstrated in Figure 2, the trend showed an optimal mixing ratio of SSO with TWAS. The TWAS:SSO mixing ratio of 3:7 (V/V) delivered better results compared to TWAS:SSO mixing ratio of 1:9 (V/V) in terms of digestion process and methane enhancement.

Fig 4.2 shows the methane production rate in $\mathrm{mL} \mathrm{CH}_{4} / \mathrm{d}$ through the digestion period. Comparing TWAS and SSO, the methane production rate obtained by SSO was significantly higher than that of TWAS, although the maximum rate for the rectors digesting only TWAS occurred in earlier stage of the digestion process compared to the ones digested only SSO. Similar trend was observed in the digesters containing mixtures of TWAS and SSO so that increasing the fraction of SSO to the co-digesters, caused maximum methane production rate take place later than the co-digesters containing more fraction of TWAS. This could be as a result of abundant particulate matter that affects the hydrolysis rate and prolongs the entire process.

The biomethane data monitoring as presented in Figure 4.2, revealed that the highest portion of the biomethane was produced within the first month. The digesters generated 31-45\%, 65-76\% and $83-90 \%$ of their ultimate biogas productions during first 7, 14 and 30 days of operation, respectively. This would be due to the availability of sufficient nutrient right after the start of the 
operation which increases the metabolic activity of the microorganisms causing rapid conversion of substrate to biogas without inhibition in digesters (A. J. Li et al., 2011; Sung and Dague, 1995). The maximum methane production rate for mono and co-digestions is presented in Figure 4.3. The amount of maximum methane production rate of SSO mono digestion was $109 \mathrm{~mL} \mathrm{CH} / \mathrm{d}$ which was $60 \%$ higher that of TWAS mono digestion corresponding to $68 \mathrm{~mL} \mathrm{CH}_{4} / \mathrm{d}$. Comparing to TWAS mono digestion, increasing the percentage of SSO in the co-digesters containing TWAS:SSO mixtures from the ratio of $1: 9$ ratio to $3: 7$, increased the maximum methane production rate by $25 \%$ and $75 \%$, respectively. Further increasing the percentage of SSO in the co digesters at TWAS:SSO mixing ratios of 1:1, 1:9 and 3:7, significantly increased the maximum methane production rate both compared to TWAS and SSO mono digestion, although the maximum rate values were almost the same for the three mixing ratios.

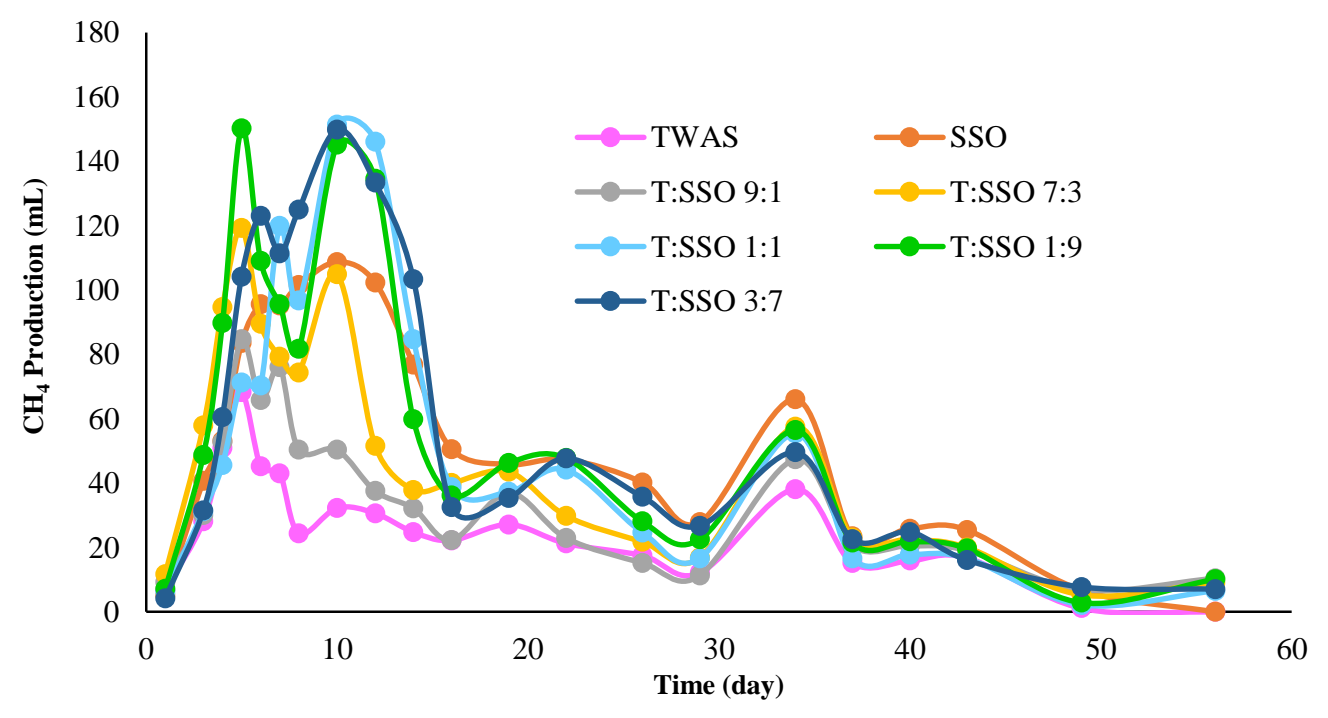

Figure 4.2. Methane production rate $(\mathrm{mL} / \mathrm{d})$ for different mixing ratios of TWAS and SSO

These findings as a matter of fact showed a close agreement to a previously conducted study (Abudi et al., 2016a), where the reported cumulative specific biogas yield (CSBY) for alternating ratios of organic fraction of municipal solid waste (OFMSW) and TWAS resulted in increasing biogas yield. As presented in Figure 4.3, the amount of maximum methane production rate was the highest for the TWAS:SSO mixing ratios of 1:1, 3:7, and 1:9 in a range between 150 to 151 
$\mathrm{mL} / \mathrm{d}$. However, the maximum biomethane production rate for the TWAS:SSO mixing ratio of 3:7 occurred on the day 5 of the operational period while it was observed on the day 10 for the mixing ratios of and 1:1 and 1:9. This would be an evident to the advantage of co-digestion while emphasizing the necessity of a proper mixing ratio of the substrates which fulfills both enhancing the methane production and the process kinetics.

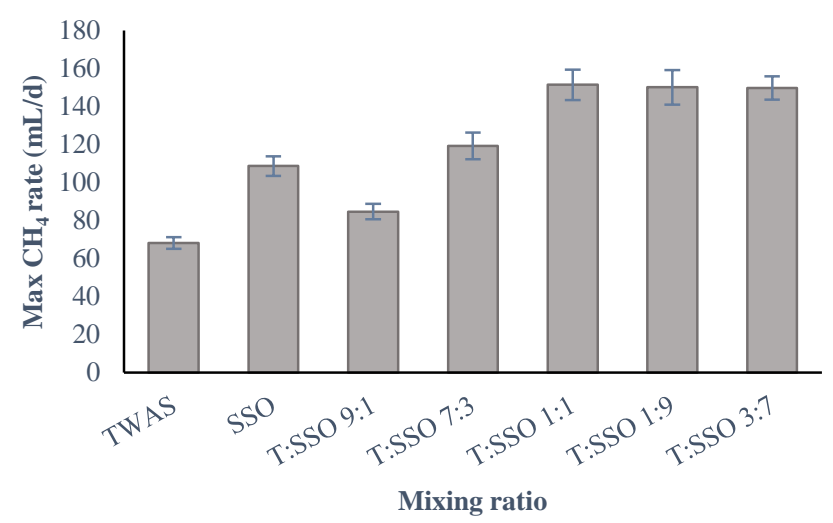

Figure 4.3. Maximum methane production rate $(\mathrm{mL} / \mathrm{d})$ for different mixing ratios of TWAS and SSO

A COD mass balance was conducted for all of the digesters to assess the accuracy of the experiment. The mass balance was carried out with reference to the initial and the final TCOD concentrations of the digester contents, and the theoretical methane production per unit mass of TCOD removed. Comparison between the experimental methane production attained by this research and that of determined by TCOD mass balance, verified a deviation of less than $10 \%$ for all the digesters.

\subsection{Cumulative methane yields}

Cumulative methane yields were calculated and presented in Figures 4.4 a), b) and c. The cumulative methane yield was normalized per substrate unit mass COD added (mLCH4/g TCOD added), per substrate unit mass VSS added ( $\mathrm{mLCH}_{4} / \mathrm{g}$ VSS added) and per unit volume of substrate added ( $\mathrm{mLCH}_{4} / \mathrm{mL}$ substrate added).

Figure 4.4 a) shows the result of cumulative methane yield per mass COD of substrate added for mono and co-digestions. Results showed that $192 \mathrm{mLCH}_{4} / \mathrm{g}$ TCOD added was obtained by TWAS mono digestion while a higher yield corresponding to $308 \mathrm{mLCH}_{4} / \mathrm{g}$ TCOD added was achieved 
by SSO alone. Mixing TWAS with SSO in co-digesters at all ratios excluding TWAS:SSO ratio of 9:1, increased the methane yield in comparison with both TWAS and SSO mono digestion. Even though, the ratio of 9:1 resulted in 27\% increase of the $\mathrm{CH}_{4}$ yield (mL) per mass of TCOD added compared to TWAS alone. The maximum cumulative methane yield was obtained by the 3:7 mixing ratio of TWAS:SSO corresponding to $358 \mathrm{~mL} \mathrm{CH}_{4} / \mathrm{g}$ TCOD added being 85\% and $16 \%$ higher than that of TWAS and SSO alone. It was observed that addition of SSO to co-digesters significantly increased the methane yield compared to mono digestion of TWAS while it did not remarkably increased the yield in comparison with SSO mono digestion.

\section{4. a)}

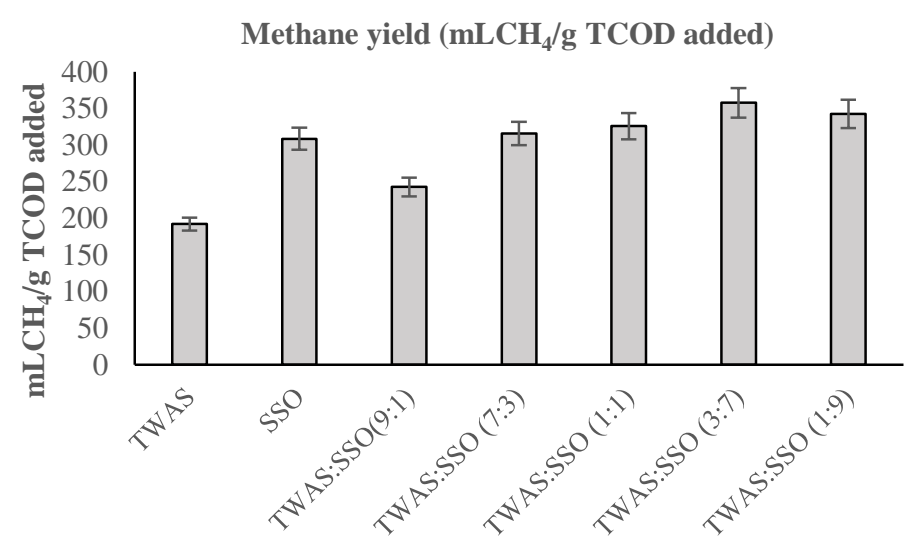

Mixing ratio

4.4. b)

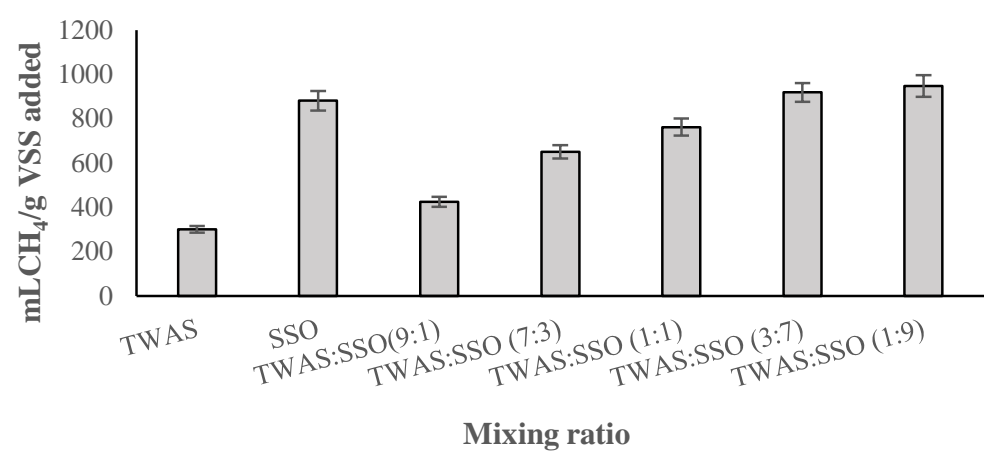


4.4. c)

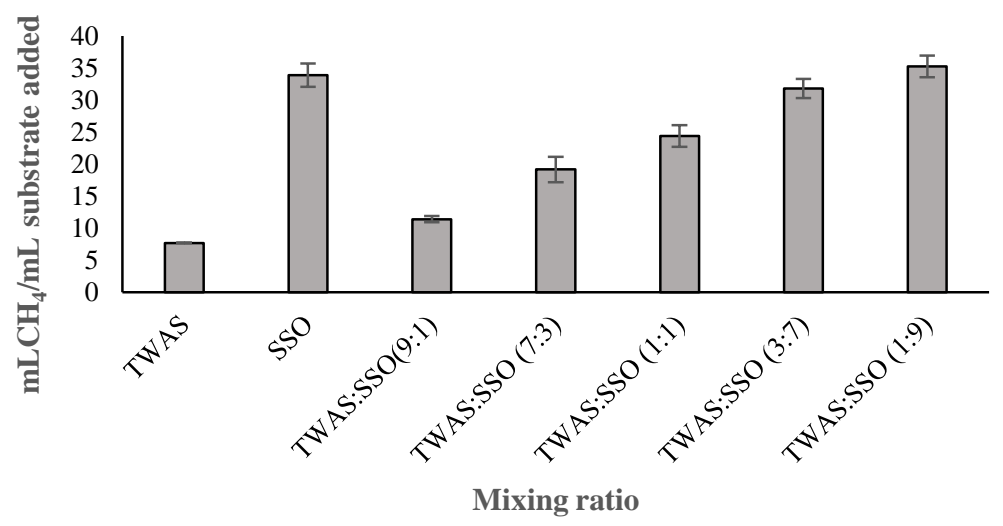

Figure 4.4. Methane yields: a) per unit mass TCOD added, b) per unit mass of VSS added and c) per unit volume of substrate added at different mixing ratio of TWAS and SSO

The yields of cumulative methane in terms of $\mathrm{mLCH}_{4} / \mathrm{g}$ VSS added have been presented in Figure 4.4. b). Similarly, the methane yield per mass of VSS added by SSO mono digestion was significantly higher than that of TWAS. A $300 \mathrm{mLCH}_{4} / \mathrm{g} \mathrm{VSS}$ added and $892 \mathrm{mLCH}_{4} / \mathrm{g}$ VSS added were attained by TWAS and SSO alone, respectively. All co-digesters produced higher methane yield per mass of VSS added compared to TWAS alone. However only the TWAS:SSO mixing ratios of 1:9 and 3:7 resulted in higher methane yields per mass of VSS added in comparison with both TWAS and SSO alone. The maximum methane yield of $975 \mathrm{mLCH}_{4} / \mathrm{g}$ VSS added occurred at the TWAS:SSO mixing ratio of 3:7.

It was revealed that addition of SSO to TWAS significantly increased biomethane production in the rectors co-digesting them compared to the control reactor digesting only TWAS. However, only the TWAS:SSO mixing ratio of 3:7 resulted in the increase of the methane yield compared to both TWAS and SSO mono digesters.

Eq. 4.1 was used for assessing the biodegradable fraction of the feedstocks for each of the rectors and the results are illustrated in Figure 4.5. 
Where $\mathrm{BF}$ is biodegradable fraction, $\mathrm{BM}$ experimental is the measured biomethane by the experiment and $\mathrm{BM}_{\text {theoretical }}$ is the theoretical biomethane production in $\mathrm{mL} \mathrm{CH}_{4}$ per $\mathrm{g}$ TCOD of the substrate in mesophilic condition and standard pressure.

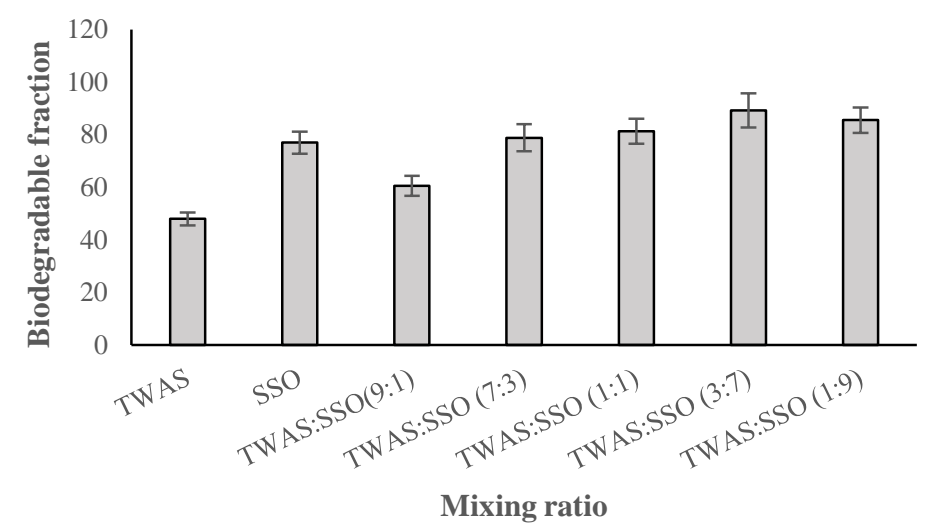

Figure 4.5. Biodegradable fraction of the feedstocks for different mixture ratios of TWAS and SSO

As shown in Figure 4.5, the TWAS:SSO mixing ratio of 3:7 corresponded to the maximum percentage of biodegradable fraction which complies with the maximum methane yield at the same mixing ratio. BF was $48 \%$ and $77 \%$ for TWAS and SSO, respectively. It was verified that addition of SSO as co-substrate increased biodegradability and enhanced the production of methane in the rectors co-digesting SSO with TWAS. All co-digesters had higher BF than TWAS alone. The reason would be the existence of readily biodegradable compounds in SSO that was introduced to the co-digesters. At the mixing ratio of 3:7, the BF was $89 \%$ which was $85 \%$ and $16 \%$ higher than that of TWAS and SSO alone respectively. The trend of BF changes in digesters complies with the methane yields obtained by the experimental results for the corresponding digesters.

\subsection{Synergistic effect}

In anaerobic digestion, production of biomethane develops through a syntrophic metabolism between both communities of methanogens including bacteria and archaea (Viotti et al., 2004). It is evident that both communities of bacteria and archaea are present in AnCoD systems. An 
improvement in the synergy and diverse microbial consortia is obtained when applying codigestion of multiple feedstocks (Zamanzadeh et al., 2017). The synergistic effect of co-digestion can be estimated as an additional methane production $(\mathrm{mL})$ for co-substrates over the weighted average of the methane production of individual substrates (Parra-Orobio et al., 2016). In this research, in order to investigate the synergetic effect of microbial populations on anaerobic codigestion of TWAS and SSO at different mixing ratios, the weighted methane production (MP) of co-substrates were calculated using Eq. (4.2):

$$
\text { Weighted } \mathrm{MP}=\mathrm{MP}_{\mathrm{SSO}} * \mathrm{P}_{\mathrm{SSO}}+\mathrm{MP}_{\mathrm{TWAS}} * \mathrm{P}_{\mathrm{TWAS}}
$$

Where weighted MP is the weighted average of methane production for co-substrates $\left(\mathrm{mLCH}_{4}\right)$; $\mathrm{MP}_{\text {SSO }}$ and $\mathrm{MP}_{\text {TWAS }}$ are the experimental methane production $\left(\mathrm{mLCH}_{4 /} \mathrm{mL}\right.$ substrate added) for SSO and TWAS; and PSSO and PTWAS are the volume $(\mathrm{mL})$ of SSO and TWAS in the substrate's mixture, respectively. When the percentage difference between experimental methane production for the mixtures and the calculated weighted average of methane production was positive, the synergistic effect could be concluded. Figure 4.6 shows the percentage of additional methane production for co-substrates over the weighted average of the methane production of individual substrates. As revealed in Fig 4.6, the maximum synergetic impact was observed in co-digestion of TWAS with SSO at the mixing ratio of 3:7. This is in good agreement with the maximum methane yield that occurred at the same mixing ratio of TWAS and SSO in their co-digestion.

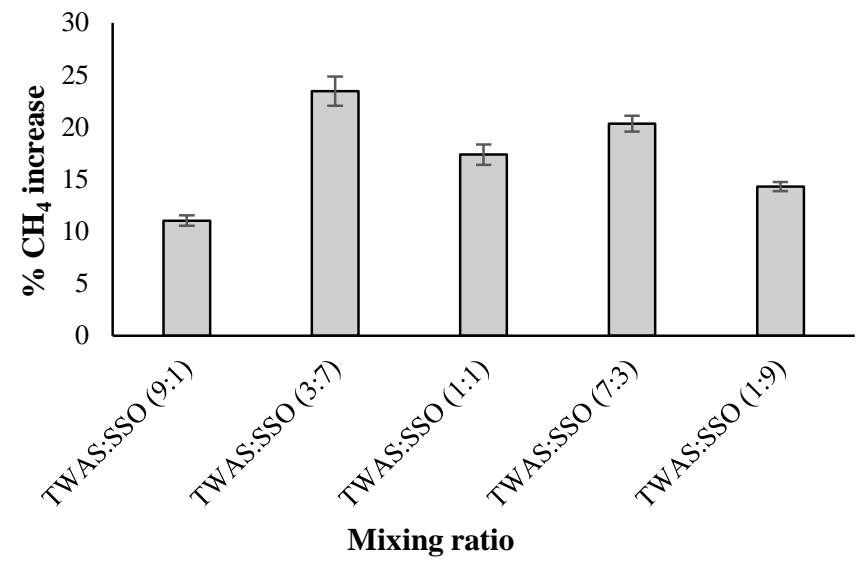

Figure 4.6. Synergetic effect of co-digestion at different mixing ratios of TWAS and SSO 
Although all of the mixings demonstrated the synergetic impact of co-digestion on improving biomethane production, no specific trend for the change of the synergetic effect and methane increase corresponding to the fraction of SSO in the co-digestion mixtures was observed. The percentage of methane increase due to the synergetic impact varied from 11 to 23 . The most percentage of biomethane increase of $23 \%$ as a result of the improved synergy occurred at the mixing ratio of 3:7. This is in good compliance with the results of maximum cumulative methane rate and the maximum methane yield which were achieved at the same mixing ratio. Although introducing fractions of SSO added more amounts of readily biodegradable materials to the codigesters, the more fractions of SSO did not necessarily satisfied the optimum condition for the process improvement.

The improvement of the synergy and biogas yield could be as a result of diverse microbial consortia introduced by applying co-digestion of multiple feedstocks. Nevertheless, enhanced synergy would be dependent on some factors relying on the proper mixing ratios which satisfy the optimal nutrients balance and effective conditions for microbial syntrophy. This could be the reason for the optimum fraction of TWAS and SSO at the mixing ratio of 3:7 in the co-digestion rather than other ratios.

\subsection{COD: $N$ and lipids: proteins: carbohydrates ratios}

The relationship between the COD:N ratios as well as lipids: proteins: carbohydrates ratios at different mixing ratios of TWAS and SSO with methane yield and ultimate methane production is presented in Table 4.2. The table also shows a comparison between the COD:N ratios with lipids: proteins: carbohydrates ratios for the digesters fed with different mixings of TWAS and SSO in volumetric basis. In co-digestion of TWAS and SSO, the COD:N ratio above 20 resulted in higher ultimate methane production, however, variation of the ratios from 28 to 34 caused a reduction of the amount of the produced methane. As shown in the table, the amounts of ultimate methane production were in a range between 542 to $1252 \mathrm{~mL}$ for different mixing ratios. The lowest ultimate methane production of $542 \mathrm{~mL}$ corresponded to a COD:N ratio of 14 and a lipids: proteins: carbohydrates ratio of 1:11:3 which occurred at TWAS:SSO mixing ratio of 1:0 in the reactors digesting TWAS alone. The maximum ultimate $\mathrm{CH}_{4}$ corresponded to COD:N ratio of 28 associated with the lipids: proteins: carbohydrates ratio of 1:2:8 for the reactors co-digesting TWAS and SSO at the mixing ratio of 3:7 (v/v). 
Considering the fact that lipids, proteins and carbohydrates are the main constituents of any organic material, considering the ratios of lipids: proteins: carbohydrates would be a good approach for optimizing the mixing ratios of the substrates. In addition, the optimal C:N or COD:N for some types of the feedstocks have shown to be very different from the generally observed the optimal values.

As presented in Table 4.2, the ratio of COD:N was 14 for TWAS alone while it was higher by 2.4 fold for SSO. The increase of the ratio of COD to $\mathrm{N}$ from 14 to 16, increased ultimate methane from 542 to $723 \mathrm{~mL}$. This increase of methane production could be due to the sufficient amounts of nutrient for microbial activities which led to enhanced biomethane production. On the other hand, an optimal nutrient synergy is required to ensure synergetic interactions of the microbial communities as the increase of COD:N ratio from 28 to 34, decreased the amount of ultimate $\mathrm{CH}_{4}$.

Table 4.2. Ultimate $\mathrm{CH}_{4}$ at different ratios of the substrates, COD:N, and lipids:proteins:carbohydrates

\begin{tabular}{ccccccc}
\hline Digester code & $\begin{array}{c}\text { TWAS: SSO } \\
\text { (V/V) }\end{array}$ & COD:N & $\begin{array}{c}\text { Feedstock } \\
\text { ratios code }\end{array}$ & $\begin{array}{c}\text { Lipids: Proteins: } \\
\text { Carbohydrates }\end{array}$ & $\begin{array}{c}\text { Ultimate } \\
\mathrm{CH}_{4}(\mathrm{~mL})\end{array}$ & $\begin{array}{c}\mathrm{mLCH}_{4} / \mathrm{g} \\
\text { TCOD added }\end{array}$ \\
\hline TWAS Only & $1: 0$ & 14 & AA & $1: 11: 3$ & 542 & 192 \\
SSO Only & $0: 1$ & 34 & BB & $1: 1.3: 8$ & 1122 & 308 \\
$\begin{array}{c}\text { TWAS:SSO } \\
\text { 9:1 }\end{array}$ & $9: 1$ & 16 & A & $1: 7: 5$ & 723 & 243 \\
$\begin{array}{c}\text { TWAS:SSO } \\
\text { 7:3 }\end{array}$ & $7: 3$ & 20 & B & $1: 4: 7$ & 1010 & 316 \\
$\begin{array}{c}\text { TWAS:SSO } \\
1: 1\end{array}$ & $1: 1$ & 24 & $\mathrm{C}$ & $1: 3: 7$ & 1100 & 326 \\
$\begin{array}{c}\text { TWAS:SSO } \\
\text { 3:7 }\end{array}$ & $3: 7$ & 28 & $\mathrm{D}$ & $1: 2: 8$ & 1252 & 358 \\
$\begin{array}{c}\text { TWAS:SSO } \\
1: 9\end{array}$ & $1: 9$ & 32 & $\mathrm{E}$ & $1: 1.5: 8$ & 1235 & 343 \\
\hline \hline
\end{tabular}

The main effect plot for $\mathrm{CH}_{4}$ yield data means in response to the feedstock ratios at different lipids:proteins:carbohydrates ratios is shown in Fig 4.7. As the trend shows, both type of the feedstock and their ratios have significant effect on the methane yield. Each of the feedstock ratios correspond to a different lipids:proteins:carbohydrates ratio. As shown in Figure 4.7, the minimum methane yield corresponds to the mono digestion of TWAS which occurred at the 
lipids:proteins:carbohydrates ratio of 1:11:3. The maximum $\mathrm{CH}_{4}$ yield corresponded to TWAS:SSO ratio of 3:7 corresponding to the lipids: proteins: carbohydrates ratio of 1:2:8.

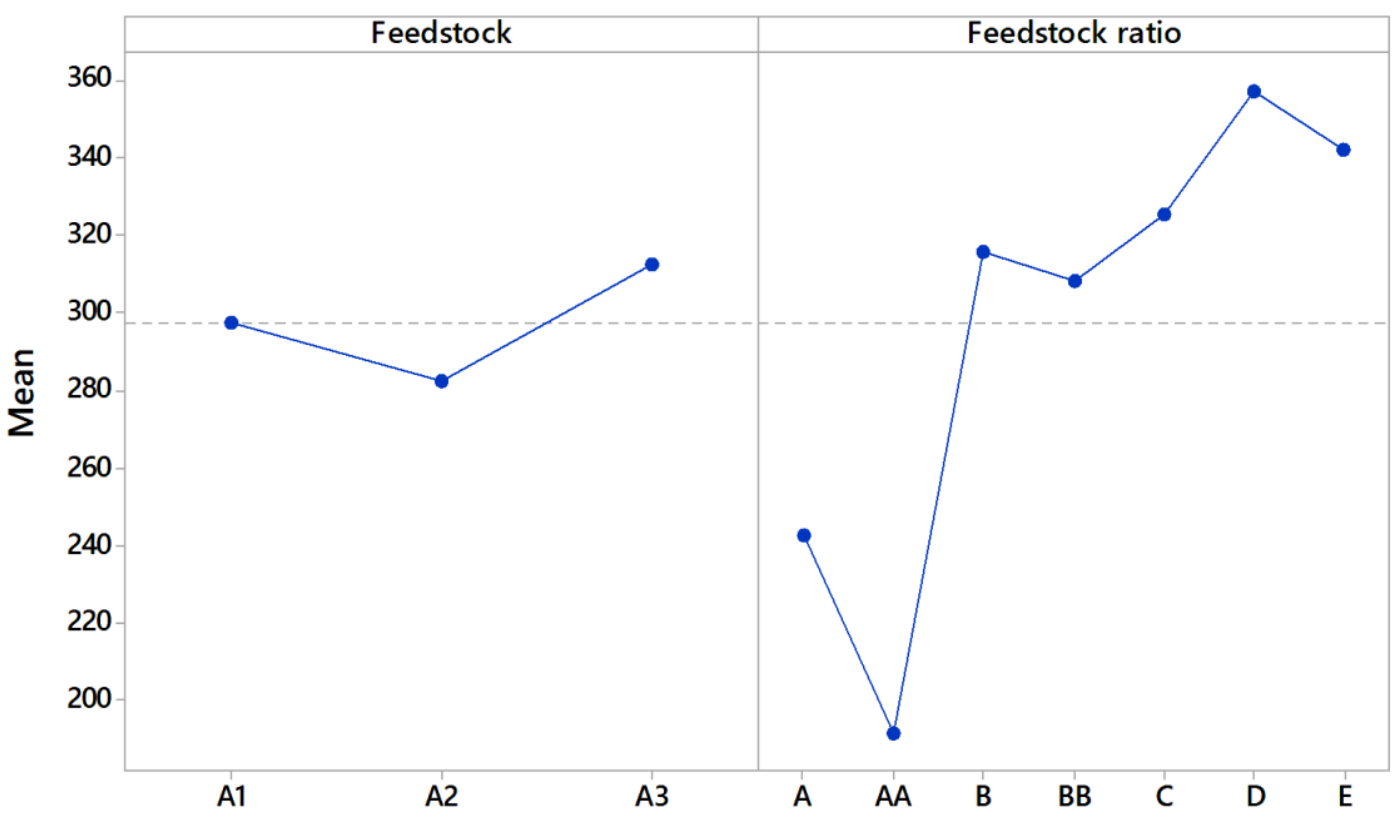

Figure 4.7. Main effects polot for $\mathrm{CH} 4$ yield data means in response to feedstock ratios at different lipids:proteins:carbohydrates ratios

A comparison between COD: $\mathrm{N}$ and the ratios of lipids: proteins, lipids: carbohydrates, and proteins:carbohydrates is presented in Figure 4.8. As illustrated in Figure 4.8 a) and 4.8 b), a same trend for the ultimate methane production in response to both COD:N ratios and lipids: proteins ratios was observed for the corresponding feedstocks mixing ratios. However, the COD:N ratio did not show the same trend as lipids: carbohydrates and proteins: carbohydrates ratios for the same feedstocks mixings, so that the minimum ultimate $\mathrm{CH}_{4}(542 \mathrm{~mL})$ corresponded to the minimum COD:N ratio of 14 while it corresponded to maximum lipids: carbohydrates of 0.32 as well as the maximum proteins: carbohydrates ratio of 3.48. Both lipids to carbohydrates and proteins to carbohydrates ratios showed the same trends at the corresponding feedstocks mixing ratios so that the maximum ultimate methane of $1252 \mathrm{~mL}$ occurred at the minimum lipids: carbohydrates of 0.12 and the minimum proteins: carbohydrates of 0.19 . Similar trends were observed for the $\mathrm{CH}_{4}$ yield ( $\mathrm{mLCH}_{4} / \mathrm{g}$ TCOD added) in response to the COD:N, lipids: proteins, lipids: carbohydrates and proteins: carbohydrates ratios. 


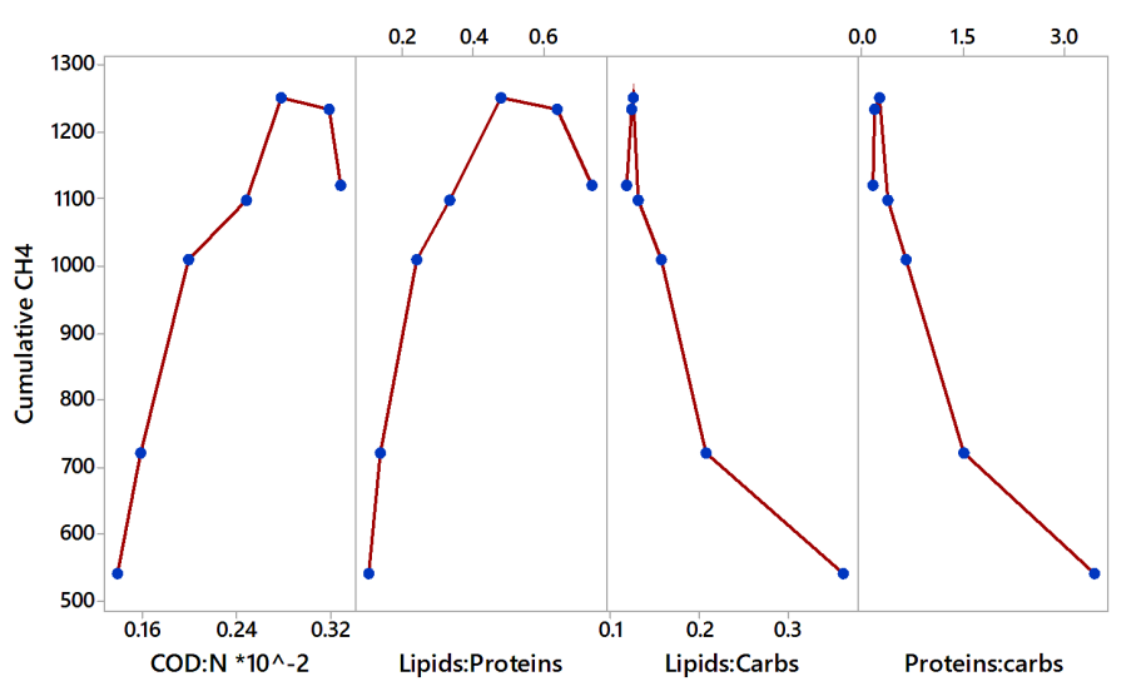

4.8. a

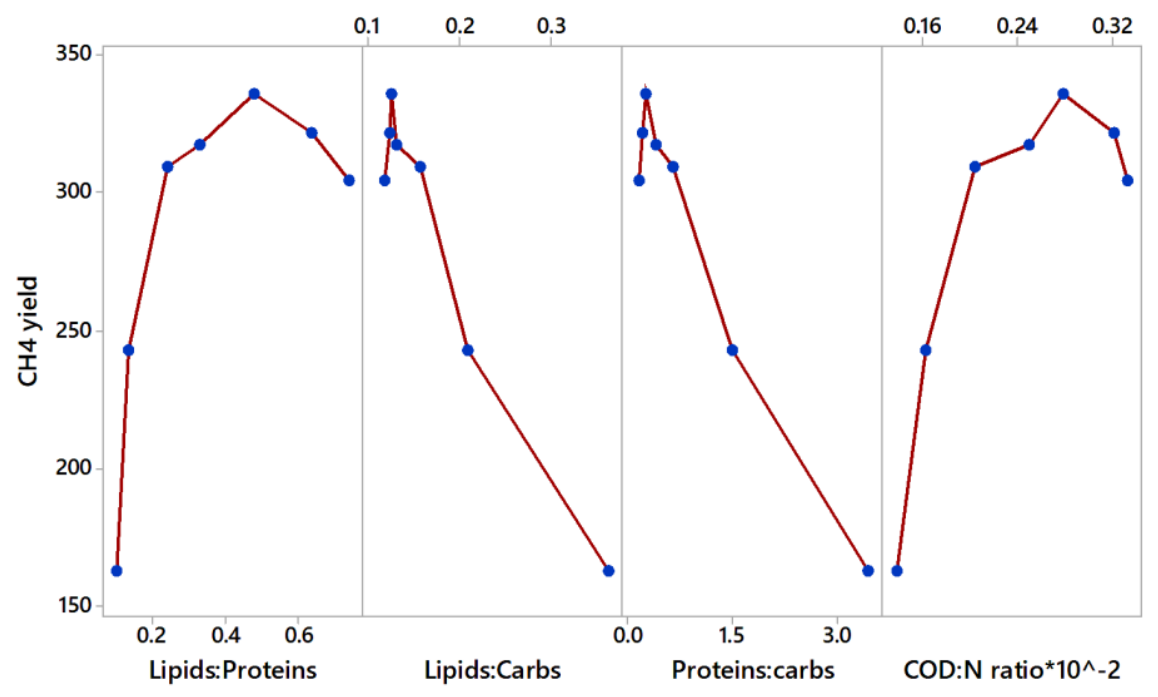

4.8. $\mathrm{b}$

Figure 4.8. Matrix effects plot for: a. ultimate $\mathrm{CH}_{4}$ and $b . \mathrm{CH}_{4}$ yield at different $\mathrm{COD:N}$ and Lipids: Proteins; lipids:carbohydrates and proteins:cabohydrates for the same corresponding mixing ratios of TWAS:SSO

\subsection{Kinetic analysis results}

The modified Gompertz model according to Eq. 3.5 was used to determine the coefficients for cumulated methane production and the results have been presented in Table 4.4. The modified Gompertz model could identify significant parameters related to anaerobic digestion including the maximum methane production rate, maximum methane production and lag phase, which 
underlines the time when the substrate is transformed and its correlation with the methane production phase. The volume data for the controls and for each mixing ratio collected during the experiment were used to apply the modified Gompertz model. The model corresponds to a sigmoid function expressing methane production in the reactor as a function of time (Lay et al., 1999; ParraOrobio et al., 2016).

Applying the collected data for cumulative methane production $\left(\mathrm{CH}_{4}\right)$ per unit substrate in $\mathrm{mL} / \mathrm{g}$ and the digestion time (t) in days to the Gompertz equation, the values of $\mathrm{P}$, representing the maximum methane production per unit substrate $(\mathrm{mL} / \mathrm{g}), R_{m}^{e}$, the maximum methane production rate $(\mathrm{ml} / \mathrm{g} \mathrm{h})$, and $\lambda$, the lag phase time (d) were calculated. The determination coefficient $\left(\mathrm{R}^{2}\right)$ as presented in Table 4 was used as criterion to evaluate the fitted models. The modified Gompertz model for mono- and co-digestions, respectively showed a good fit to the experimental results and the estimated parameters indicated that the co-digestion of SSO with TWAS improved the biogas production rate (Figure 1). The value of $\mathrm{R}^{2}=0.9998$ indicates that the proposed equations can accurately describe the variation of methane yield curves. Typically, S-shaped curves are obtained by the modified Gompertz model which shows a relatively slow upward trend related to the lag phase at the beginning of the curve. Therefore, the lag phase time for all of the TWAS/SSO codigestion mixtures could verify the suitability of the modified Gompertz model in estimated performance of the process.

Table 4.3. Summary of results of kinetic study using modified Gompertz models.

\begin{tabular}{ccccc}
\hline & $\mathrm{P}(\mathrm{mL})$ & $\boldsymbol{R}_{\boldsymbol{m}}^{\boldsymbol{e}}(\mathrm{mL} / \mathrm{d})$ & $\lambda(\mathrm{d})$ & $\mathrm{R}^{2}$ \\
\hline \hline TWAS & 510 & 28 & 0.1 & 0.999 \\
\hline SSO & 1063 & 68 & 2.0 & 0.999 \\
\hline TWAS:SSO 9:1 & 661 & 44 & 1.0 & 0.999 \\
\hline TWAS:SSO 7:3 & 938 & 68 & 1.1 & 0.999 \\
\hline TWAS:SSO 1:1 & 1046 & 84 & 3.1 & 0.999 \\
\hline TWAS:SSO 3:7 & 1182 & 95 & 2.7 & 0.999 \\
\hline TWAS:SSO 1:9 & 1165 & 89 & 1.9 & 0.999 \\
\hline
\end{tabular}


With reference to the adjustment to the Gompertz model, the lag phase varied from 0.1 to 3.1 days for different substrate mixing ratios. The shortest lag phase of $0.1 \mathrm{~d}$ corresponded to TWAS alone digestion. This result would be due to the existence of anaerobic inoculum that are easily assimilated by the microorganisms during this phase and cause rapid acclimatization to the substrate, which can be observed in the methane production. In theory, the inoculum activity, the amount of readily degradable constituent, and the initial $\mathrm{pH}$ of the feedstock affects the AD startup time (Kafle and Chen, 2016).

The results showed that with the increase of the proportion of SSO in TWAS/SSO co-digestion, a longer lag phase time occurred. This trend stopped with further increasing the SSO proportion to the mixing ratio of 1:9. However, the mixing ratio of 3:7 resulted in slightly higher value of $P$ which corresponds to the experimental results that showed the maximum methane production occurred at the mixing ratio of 3:7. Although, a long lag phase time is not favorable as it would increase the residence time and consequently, larger reactor volumes and higher costs during implementation and operation would be required.

The values of $\mathrm{P}$, varied from 509.5 to $1182 \mathrm{~mL}$ corresponding to TWAS alone and TWAS:SSO mixing ratio of 3:7. The trend of changes in the $\mathrm{P}$ values complies with the experimental results as it showed an increasing trend in methane production by increasing the proportion of SSO. Similar to the experimental results, the trend changed from TWAS:SSO mixing ratio of 3:7 to 1:9 so that the ratio of 3:7 corresponded a higher $P$ value for methane production than that $1: 9$. The values obtained for $\mathrm{R}_{\max }^{\mathrm{e}}$ were within a range from 28 to $95 \mathrm{ml} / \mathrm{d}$. The values of $\mathrm{R}_{\max }^{\mathrm{e}}$ and $\mathrm{P}_{\max }$ obtained by Gompertz for the TWAS:SSO ratios of 3:7 were $95 \mathrm{~mL} / \mathrm{d}$ and $1182 \mathrm{~mL}$, respectively.

In co-digestion of TWAS and SSO, the COD:N ratio above 20 resulted in higher ultimate methane production, however, the difference in the ratios from 21 to 26 did not have significant effect on the amounts of the produced methane. Therefore, considering lipids: proteins: carbohydrates ratios would be a good approach for optimizing the mixing ratios of the substrates. A lipids: proteins: carbohydrates ratio of 7:1:15 corresponding to COD:N ratio of 26 , resulted in an ultimate biomethane production of $1252 \mathrm{~mL}$ in TWAS and SSO co-digestion. It was verified that codigestion of TWAS and SSO improved methane production in comparison with conventional single digestion of the feedstocks. It was concluded that cumulative methane production and methane yields varied at different mixing ratios of TWAS and SSO. Co- digestion of TWAS and SSO at the mixing ratio of 3:7 resulted in a biomethane yield of $353 \mathrm{~mL} \mathrm{CH}_{4} / \mathrm{g}$ TCOD added which 
is corresponding to an increase of methane yield by $84 \%$ and $15 \%$ compared to TWAS and SSO alone. A 23\% increase in methane yield was observed as a result of synergetic effect of codigestion at TWAS:SSO mixing ratio of 3:7 (v/v) equivalent to lipids: proteins: carbohydrates ratio of 1:2:8. This study verified the advantage of co-digestion over conventional single digestion of the feedstocks in terms of biomethane improvement and increased microbial synergy resulting higher methane production. Further studies utilizing a range of various feedstocks is required to promote the viability of lipids: proteins: carbohydrates ratio for optimizing the anaerobic digestion process and for optimizing the mixing ratios in anaerobic co-digestion of multiple feedstocks.

\section{6. Hydrolysis/acidification}

This experiment was carried out to evaluate the hydrolysis/acidification stage in anaerobic codigestion of TWAS with SSO. In hydrolysis which is known to be the rate limiting stage in the entire anaerobic digestion process, the large complex organic polymers including lipids, proteins, and carbohydrates break down to simple smaller molecules such as fatty acids, amino acids and sugars. Subsequently, acidogenic microorganisms break down the by-product of hydrolysis to further smaller molecules in acidification step (Gould, 2014). Therefore, this part of the present research was aimed to investigate the degradation of organic compounds in hydrolysis stage through the analysis of the degree of solublization, synergetic effect of co-digestion at different mixing ratios on hydrolysis and liquefaction, volatile fatty acids (VFAs) yield, and kinetics of lipids, proteins, and carbohydrates in co-digestion of TWAS and SSO.

A series of the analysis for the characterization of the feedstocks was primarily carried out in triplicates and the mean values are summarized in Table 4.4. As presented in Table 4.4, the COD concentration of SSO is higher than that of TWAS. Adding SSO to TWAS in the co-digesters, increased the COD concentrations compared to TWAS mono digestion. SSO also contains higher amounts of carbohydrates and lipids than TWAS while the proteins concentration of TWAS is more than SSO by 30\%. The concentrations of total, soluble and particulate COD; total, soluble and particulate proteins; and total, soluble and particulate carbohydrates were monitored over time for calculating their hydrolysis rate coefficients. 
Table 4.4. Characteristics of the feedstocks at different mixing ratios of TWAS and SSO

\begin{tabular}{ccccccccc}
\hline & & TWAS & SSO & T/SSO & T/SSO & T/SSO & T/SSO & T/SSO \\
& & Only & Only & $9: 1$ & $7: 3$ & $1: 1$ & $3: 7$ & $1: 9$ \\
\hline \hline \multirow{2}{*}{ Parameters } & \multirow{2}{*}{ Units } & Mixture & Mixture & Mixture & Mixture & Mixture & Mixture & Mixture \\
& & $(1)$ & $(2)$ & $(3)$ & $(4)$ & $(5)$ & $(6)$ & $(7)$ \\
\hline TCOD & $\mathrm{g} / \mathrm{L}$ & 40 & 110 & 47 & 61 & 75 & 89 & 103 \\
\hline SCOD & $\mathrm{mg} / \mathrm{L}$ & 0.4 & 44.4 & 4.8 & 13.6 & 22.4 & 31.2 & 40.0 \\
\hline TSS & $\mathrm{mg} / \mathrm{L}$ & 31.5 & 53.8 & 33.7 & 38.2 & 42.6 & 47.1 & 51.6 \\
\hline VSS & $\mathrm{mg} / \mathrm{L}$ & 18.5 & 36.0 & 20.2 & 23.7 & 27.2 & 30.8 & 34.3 \\
\hline TS & $\mathrm{mg} / \mathrm{L}$ & 38.8 & 62.2 & 41.1 & 45.8 & 50.5 & 55.2 & 59.8 \\
\hline VS & $\mathrm{mg} / \mathrm{L}$ & 34.5 & 43.5 & 35.4 & 37.2 & 39.0 & 40.8 & 42.6 \\
\hline Ammonia & $\mathrm{mg} / \mathrm{L}$ & 0.3 & 1.1 & 0.3 & 0.5 & 0.7 & 0.9 & 1.0 \\
\hline $\mathrm{pH}$ & - & 5.6 & 5.6 & 5.8 & 5.7 & 5.7 & 5.8 & 5.8 \\
\hline Alkalinity & $\mathrm{mg} \mathrm{CaCO3/L}$ & 2.0 & 6.8 & 2.4 & 3.4 & 4.4 & 5.3 & 6.3 \\
\hline TN & $\mathrm{mg} / \mathrm{L}$ & 2.9 & 4.0 & 3.0 & 3.2 & 3.4 & 3.6 & 3.9 \\
\hline TSN & $\mathrm{mg} / \mathrm{L}$ & 0.4 & 1.1 & 0.5 & 0.6 & 0.7 & 0.9 & 1.0 \\
\hline TotalCarbs & $\mathrm{mg} / \mathrm{L}$ & 0.9 & 13.5 & 2.2 & 4.7 & 7.2 & 9.7 & 12.2 \\
\hline TotalProteins & $\mathrm{mg} / \mathrm{L}$ & 2.8 & 2.1 & 2.7 & 2.6 & 2.4 & 2.3 & 2.2 \\
\hline TotalLipids & $\mathrm{mg} / \mathrm{L}$ & 0.3 & 1.1 & 0.4 & 0.5 & 0.7 & 0.9 & 1.0 \\
\hline \hline
\end{tabular}

The data form total and soluble COD (SCOD) concentrations monitoring with time was used to obtain the degree of COD solublization for each mixture and the results are as follows. The degree of solublization was calculated using Eq. 3.1 and the result is shown in Figure 4.8. The degree of the COD solublization varied from $14 \%$ to $30 \%$. The maximum solublization of COD content was $30 \%$ for TWAS:SSO combination of 1:9 while the minimum value corresponded to the digester containing only TWAS. Except for TWAS:SSO mixing ratio of 9:1, other co-digesters achieved more than $25 \%$ improvement in solublization. The lower degree of solublization in TWAS mono digestion as well as TWAS:SSO mixing ratio of 9:1 with a high portion of TWAS would be due to the existence of slowly biodegradable content which slows down the hydrolysis and liquefaction. SSO contains sufficient amount of carbohydrates which decomposes more rapidly than proteins and lipids. This would be the reason for observing higher degree of solublization for SSO compared to TWAS which contains more proteins than carbohydrates.

However, by calculating the theoretical degree of solublization of each co-digester and comparing them to the ones obtained from the measured values based on the experimental data, it was revealed that co-digestion was effective for improving the solublization due to enhancing microbial synergy. As shown in Figure. 4.10, all co-digesters achieved an improvement in solublization due 
to synergistic effect of the microbial communities from 23 to $44 \%$ corresponding to TWAS:SSO mixing ratios of $3: 7$ and $7: 3$, respectively.

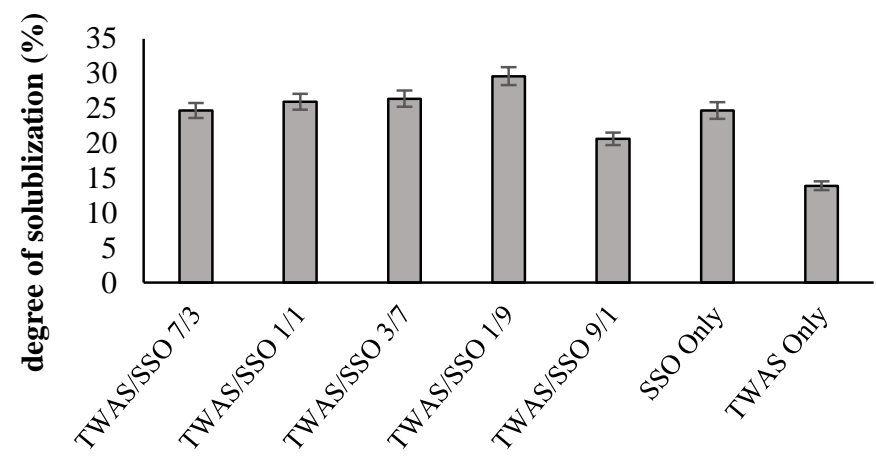

Mixing ratio

Figure 4.9. Degree of COD solublization at different mixing ratios of TWAS and SSO

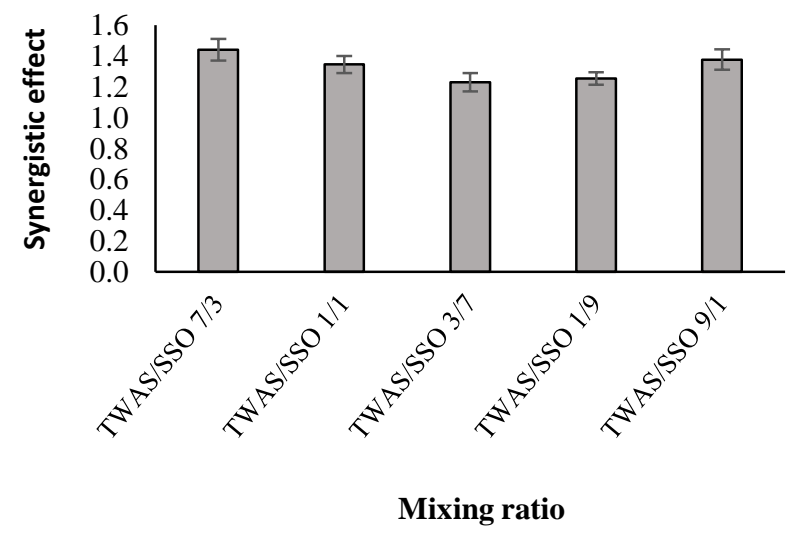

Figure 4.10. Synergistic effect on solublization at different mixing ratios of TWAS and SSO

As discussed earlier, the by-product of hydrolysis is subsequently broken down to smaller molecules by acidogenic bacterial communities. This leads to the formation of volatile fatty acids in acidogenesis. Therefore, monitoring VFAs concentration over time and calculating VFAs yield per mass of VSS added would be a good indicator of acidification progress. The VFAs concentrations monitoring showed an increasing trend during the $72-\mathrm{hr}$ of the hydrolysis/acidification process. The total VFAs concentration was used for calculating the VFAs yield in terms of mass of VFAs produced in mg per mass of VSS added in g. As indicated in Figure 
4.11, the VFAs yield decreased with the addition of TWAS in the digesters. This trend is similar to the trend of COD solublization as demonstrated in Figure 4.11. All of the reactors containing the mix of substrates had higher VFAs yield compared to the reactors containing only TWAS. The VFAs yields were $98 \mathrm{mg}$ VFAs/g VSS added for TWAS mono digestion and $213 \mathrm{mg}$ VFAs/g VSS added for TWAS:SSO ratio of 9:1, respectively. Other digesters resulted in higher amounts of VFAs production from 281 to $328 \mathrm{mg}$ VFAs/g VSS added.

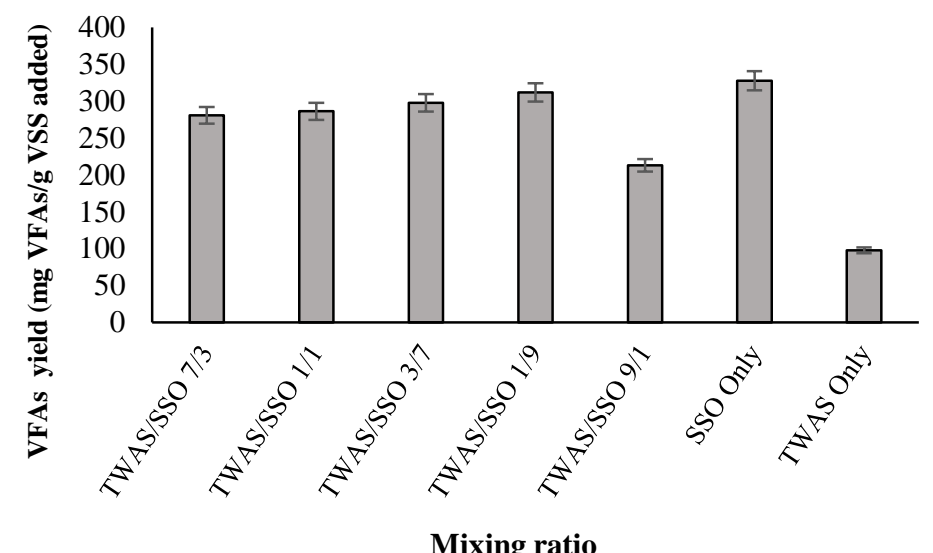

Figure 4.11. Total VFAs yield at different mixing ratios of TWAS/SSO

The analysis of soluble and particulate COD over time during the hydrolysis experimental period, showed an increasing trend in COD solublization and decreasing trend in particulate COD concentrations. Similarly, the analysis of soluble and particulate lipids, proteins, and carbohydrates over time demonstrated an increasing trend in solublization and particulate matter degradation. Although the trend is remarkably slower for lipids and proteins compared to carbohydrates. These results are summarized in the tables that are presented in the appendix. With applying the first order kinetics using equations 3.4., the hydrolysis rate coefficient $\left(\mathrm{K}_{\mathrm{h}}\right)$ was calculated in AquaSim 2.0 for COD, lipids, proteins and carbohydrates based on the particulate matter degradation and the results are summarized in Table 4.5.

As presented in Table 4.4, the hydrolysis rate for COD content of SSO was higher than that of TWAS by 1.8 folds. The $\mathrm{K}_{\mathrm{h}}$ values varied from 0.17 to 0.35 corresponding to TWAS alone and TWAS:SSO mixing ratio of 1:9, respectively. Hydrolysis rate coefficient increased in the reactors containing mixings of TWAS and SSO as compared to the reactors digesting only TWAS. The 
increase of COD hydrolysis rate could be the result of improved microbial synergy and good syntrophic interactions between the hydrolytic and acidogenic microbial communities through the process. The higher kinetic rate coefficient at the mixing ratio of 1:9 is in good agreement with the higher degree of solublization at the same mixing ratio compared to other co-digestion mixtures. Among lipids, proteins, and carbohydrates, lipids showed the lowest hydrolysis rate. In contrast, hydrolysis proceeded more rapidly for carbohydrates compared to lipids and proteins.

The higher hydrolysis rate of carbohydrates would be the result of more rapid biological metabolism of carbohydrates than lipids and proteins. $\mathrm{K}_{\mathrm{h}}$ varied from 0.03 to 0.08 corresponding to hydrolysis of the lipid content of TWAS alone and SSO alone, respectively. All co-digesters had a higher hydrolysis rate of the lipid content in comparison with the digesters containing only TWAS.

As shown in Table 4.5, the hydrolysis rate of proteins content of the digesters was higher than that of lipids content, however it was still lower compared to the hydrolysis rate of carbohydrates content of the feedstocks. $\mathrm{K}_{\mathrm{h}}$ for the proteins content was in a range between 0.19 to 0.34 corresponding to TWAS alone and TWAS:SSO mixings of 1:9, respectively. All of the reactors co-digesting TWAS with SSO demonstrated bigger hydrolysis rate coefficient than that of the TWAS mono digesters. The hydrolysis rate coefficient of the carbohydrates content was within a range between 0.32 to 0.68 corresponding to TWAS alone and the co-digestion of TWAS with SSO at the mixing ratio of 1:9. Contrary to expectation, the $\mathrm{K}_{\mathrm{h}}$ values of carbohydrates did not increase with the addition of SSO portion in the mixtures.

The variation of hydrolysis rate for the carbohydrates content did not show the same trend as the lipids and proteins contents. This verifies that the hydrolysis of the lipids, proteins and carbohydrates of the feedstocks occurs independently during the hydrolysis/acidification stage. It was observed that co-digestion had an effect on the hydrolysis rate by mostly improving the hydrolysis of proteins and carbohydrates content rather than lipids. 
Table 4.5. Hydrolysis rate coefficient for COD, lipids, proteins, and carbohydrate content in co-digesion of TWAS and SSO at different mixing ratios

\begin{tabular}{|c|c|c|c|c|c|c|c|}
\hline \multirow[t]{2}{*}{$\mathbf{K}_{\mathbf{h}}$} & TWAS:SSO & TWAS:SSO & TWAS:SSO & TWAS:SSO & TWAS:SSO & \multirow[t]{2}{*}{ TWAS } & \multirow[t]{2}{*}{ SSO } \\
\hline & $7: 3$ & $5: 5$ & $3: 7$ & & & & \\
\hline $\mathrm{Kh}_{\mathrm{COD}}$ & 0.30 & 0.32 & 0.32 & 0.25 & 0.35 & 0.17 & 0.30 \\
\hline Kh Lipids & 0.06 & 0.07 & 0.07 & 0.03 & 0.05 & 0.03 & 0.08 \\
\hline $\mathrm{Kh}$ Proteins & 0.28 & 0.27 & 0.33 & 0.25 & 0.34 & 0.19 & 0.28 \\
\hline $\mathrm{K}_{\mathrm{h} \text { Carbohydrates }}$ & 0.56 & 0.57 & 0.54 & 0.46 & 0.68 & 0.32 & 0.55 \\
\hline
\end{tabular}




\section{Chapter 5}

Results and discussion

\section{TWAS and Manure Co- digestion}




\section{Results and discussion- TWAS and manure co-digestion}

\subsection{BMP of TWAS and manure}

This experiment was designed to investigate the effect of mixing ratio of TWAS with manure and its correlation with the lipids: proteins: carbohydrates ratios on anaerobic co-digestion of TWAS and manure. The characteristics of the feed in each digester having different mixing ratios of the substrates are summarized in table 5.1. The COD concentration of manure slurry was significantly higher than that of TWAS. The average TCOD of manure was $122 \mathrm{~g} / \mathrm{L}$ while it was $45 \mathrm{~g} / \mathrm{L}$ for TWAS. Manure also contained significantly higher amount of total carbohydrates (27 g/L), proteins $(5.1 \mathrm{~g} / \mathrm{L})$, and lipids $(1.4 \mathrm{~g} / \mathrm{L})$ concentrations compared to TWAS. Mixtures of TWAS and manure as described in chapter 3 were prepared in different combinations and used as digester feedstocks. Addition of manure to TWAS increased the concentration of carbohydrates in codigesters by a large extent as compared to TWAS mono digesters. It also increased lipids and proteins content of the co-digesters in comparison with the digester containing TWAS alone.

Table 5.1. Characteristics of feed to digesters with different mixing ratios

\begin{tabular}{|c|c|c|c|c|c|c|c|c|}
\hline & & TWAS & Manure & $\mathrm{T}^{*}: \mathrm{M}^{* *} 9: 1$ & $\mathrm{~T}: \mathrm{M}$ 7:3 & $\mathrm{T}: \mathrm{M} 1: 1$ & T:M 3:7 & T:M 1:9 \\
\hline Parameters & Units & $\begin{array}{c}\text { Mixture } \\
\text { (1) }\end{array}$ & $\begin{array}{c}\text { Mixture } \\
\text { (2) }\end{array}$ & Mixture (3) & $\begin{array}{c}\text { Mixture } \\
\text { (4) }\end{array}$ & $\begin{array}{c}\text { Mixture } \\
\text { (5) }\end{array}$ & $\begin{array}{c}\text { Mixture } \\
\text { (6) }\end{array}$ & $\begin{array}{c}\text { Mixture } \\
\text { (7) }\end{array}$ \\
\hline TCOD & $\mathrm{g} / \mathrm{L}$ & 45 & 122 & 53 & 68 & 84 & 99 & 115 \\
\hline SCOD & $\mathrm{g} / \mathrm{L}$ & 2.0 & 8.9 & 2.7 & 4.1 & 5.5 & 6.7 & 8.2 \\
\hline TSS & $\mathrm{g} / \mathrm{L}$ & 36 & 80 & 40 & 49 & 58 & 67 & 75 \\
\hline VSS & $\mathrm{g} / \mathrm{L}$ & 27 & 77 & 38 & 42 & 52 & 62 & 72 \\
\hline $\mathrm{TS}$ & $\mathrm{g} / \mathrm{L}$ & 38 & 99 & 44 & 56 & 68 & 80 & 93 \\
\hline VS & $\mathrm{g} / \mathrm{L}$ & 28 & 86 & 33 & 45 & 57 & 68 & 80 \\
\hline Ammonia & $\mathrm{g} / \mathrm{L}$ & 0.32 & 0.02 & 0.29 & 0.23 & 0.17 & 0.11 & 0.05 \\
\hline $\mathrm{pH}$ & - & 7.1 & 7.0 & 6.9 & 7.0 & 7.1 & 7.2 & 7.4 \\
\hline Alkalinity & $\mathrm{g} \mathrm{CaCO} 3 / \mathrm{L}$ & 4.8 & 5.1 & 4.8 & 4.9 & 4.9 & 5.0 & 5.1 \\
\hline $\mathrm{TN}$ & $\mathrm{g} / \mathrm{L}$ & 2.9 & 2.2 & 2.8 & 2.7 & 2.5 & 2.4 & 2.3 \\
\hline TSN & $\mathrm{g} / \mathrm{L}$ & 0.4 & 0.1 & 0.4 & 0.3 & 0.3 & 0.2 & 0.1 \\
\hline T-Carbs & $\mathrm{g} / \mathrm{L}$ & 1.3 & 27.1 & 3.9 & 9.1 & 14.2 & 19.4 & 24.5 \\
\hline T-Proteins & $\mathrm{g} / \mathrm{L}$ & 3.9 & 5.1 & 4.1 & 4.3 & 4.5 & 4.8 & 5.0 \\
\hline T-Lipids & $\mathrm{g} / \mathrm{L}$ & 0.5 & 1.4 & 0.6 & 0.8 & 1.0 & 1.1 & 1.3 \\
\hline
\end{tabular}


The range of carbohydrates concentrations were within 3.9 to $24 \mathrm{~g} / \mathrm{L}$. Total proteins concentrations were 3.9 and 5.1 for TWAS and manure respectively and varied from 4.1 to $5 \mathrm{~g} / \mathrm{L}$ in the codigesters. The total lipids concentration of manure was $1.4 \mathrm{~g} / \mathrm{L}$ and it was higher by 2.8 fold than TWAS. Adding manure to TWAS increased the lipids content. In the reactors containing the mixtures of the TWAS and manure, lipids concentrations varied from 0.6 to $1.3 \mathrm{~g} / \mathrm{L}$.

Operation of the digesters continued until no significant biogas was produced. Fig. 5.1 shows the profile of the cumulative biomethane production versus time during the total digestion period. TWAS resulted in minimum cumulative methane production of $590 \mathrm{ml}$ while manure alone produced $838 \mathrm{~mL}$ of cumulative methane during the operation period.

As shown in figure 5.1, the maximum cumulative methane production was obtained by codigestion of TWAS:manure at the mixing ratio of 3:7 corresponding to the lipids: proteins: carbohydrates ratio of 1:4:17. Compared to mono digestion of manure, co-digestion at the mixing ratio of 9:1 did not increase methane production, although higher cumulative methane was obtained at that ratio in comparison with TWAS mono digestion. In fact TWAS alone with lipids: proteins: carbohydrates ratio of 1:7:2.5, produced the least amount of methane. Other co-digesters produced higher methane than that of TWAS and manure single digestion.

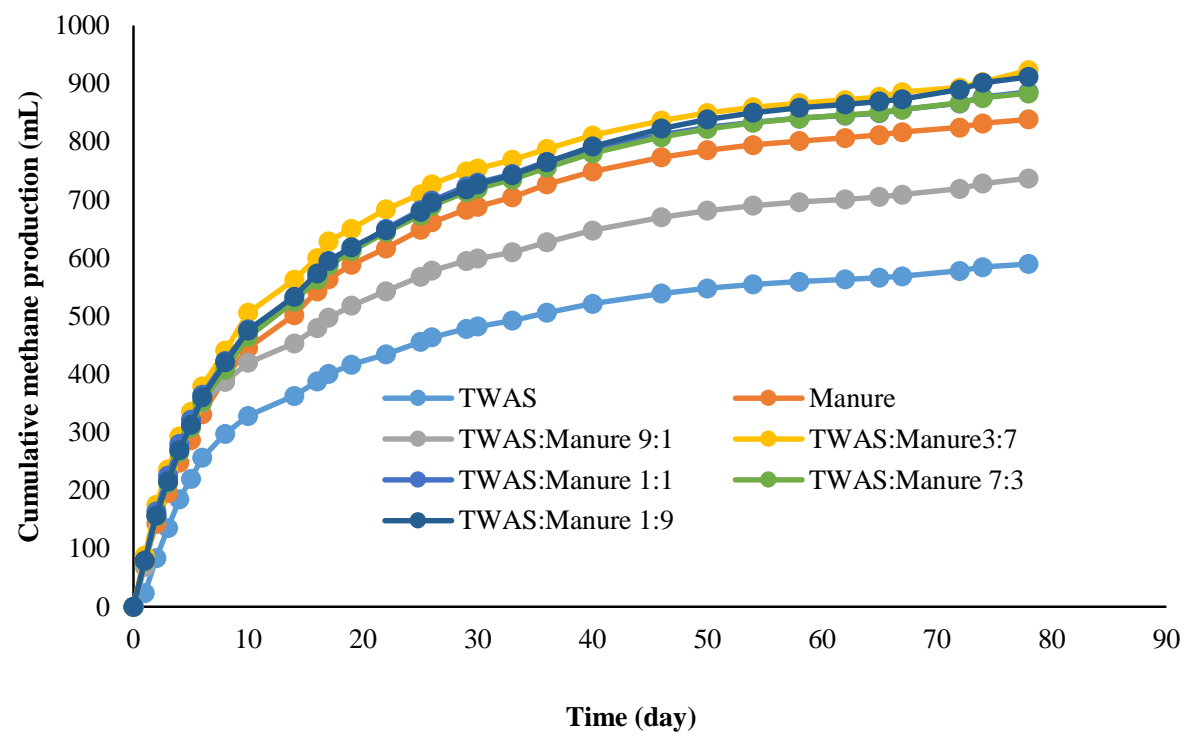

Figure 5.1. Cumulative methane production for different mixing ratios of TWAS and manure 
As illustrated in Figure 5.2, for all of the digesters, the maximum methane rate occurred in the first week of the operation period. The amount of maximum methane production rate of the digesters differed from each other, although a similar trend was observed for all of the co-digestion mixtures. The reactors generated $47 \%$ to $52 \%$ of their ultimate methane production at the first week of the digestion period and 59\% to $62 \%$ in two weeks operation. Compared to TWAS, manure alone resulted in higher methane rate. Addition of manure to TWAS increased partially the maximum methane production rate so that all of the co-digesters demonstrated slightly higher maximum methane rate compared to TWAS and manure mono digesters except for the reactor containing the TWAS and manure at the mixing ratios of 7:3 and 1:9 which had almost the same maximum methane rate as of manure alone. In comparison with TWAS alone, the maximum methane rate in co-digestion reactors were $30 \%$ to $47 \%$ higher.

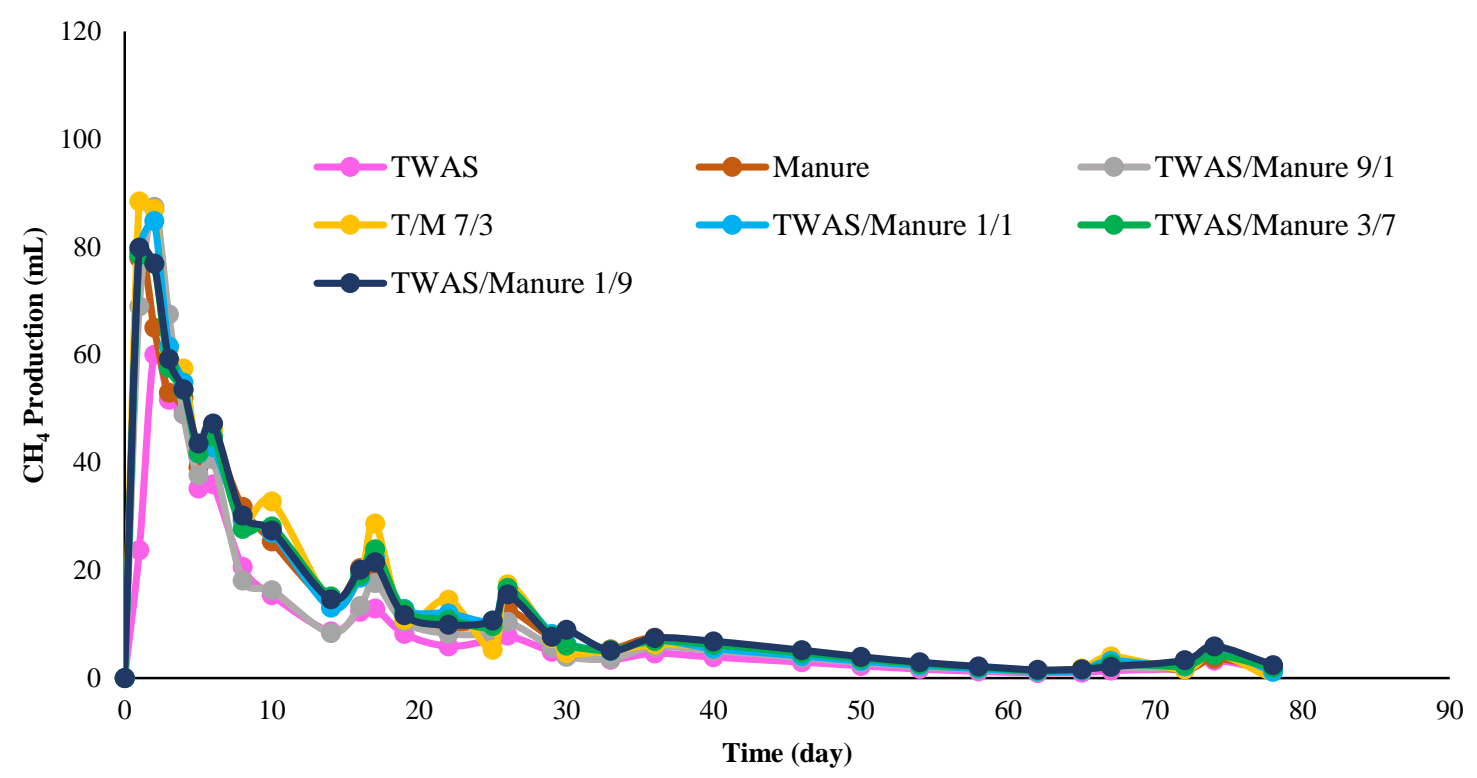

Figure 5.2. Methane production rate $(\mathrm{mL} / \mathrm{d})$ for different mixing ratios of TWAS and Manure

The maximum $\mathrm{CH}_{4}$ production rate at different mixing ratios is presented in Figure 5.3. As shown in Figure 5.3, the maximum rate was $100 \mathrm{~mL} /$ day that occurred at the first day of the process for TWAS/manure mixing ratio of 1:1 corresponding to the lipids: proteins: carbohydrates ratio of 1:25:78 and the minimum rate corresponded to TWAS mono digestion at the lipids: proteins: carbohydrates ratio of 1:7:2.5. 


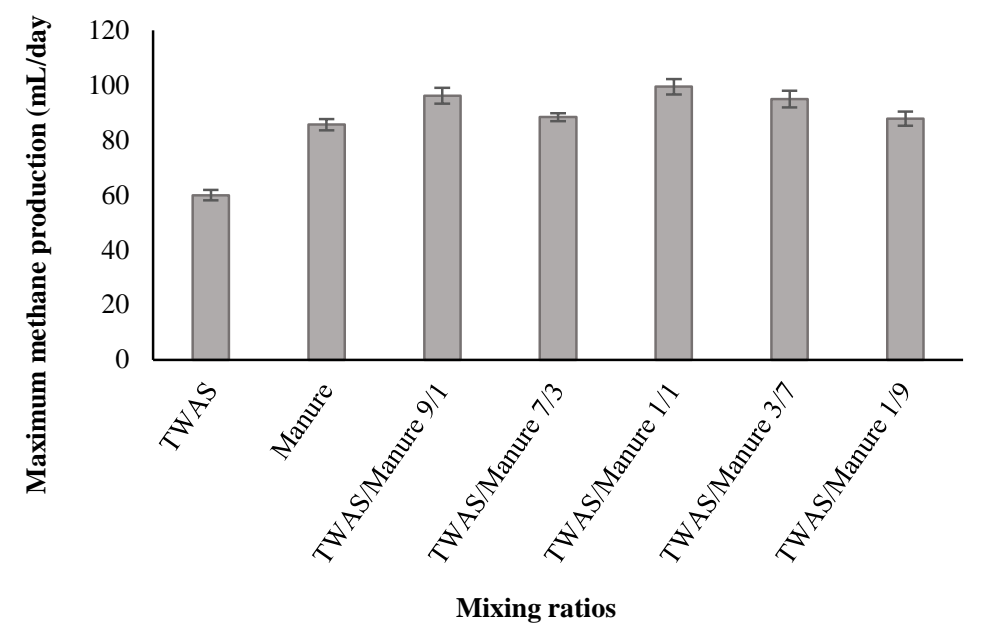

Figure 5.3. Maximum methane production rate $(\mathrm{mL} / \mathrm{d})$ for different mixing ratios of TWAS and manure

During the anaerobic process COD is only rearranged meaning that all COD that enters the system converts eventually to the end products methane minus the COD that incorporates in new microbial mass. Therefore, COD is generally taken as a useful control tool for the operation of anaerobic systems (Henze et al., 2015). A COD mass balance was conducted for all of the digesters to assess the accuracy of the experiment. The mass balance was carried out with reference to the initial and the final TCOD concentrations of the digester contents, and the theoretical methane production per unit mass of TCOD removed. Comparison between the experimental methane production attained by this research and that of determined by TCOD mass balance, as presented in figure 5.4 verified a deviation of less than $10 \%$ for all the digesters. The COD balance varied from $90 \%$ to $98 \%$ in all mono digestion and co-digestion reactors.

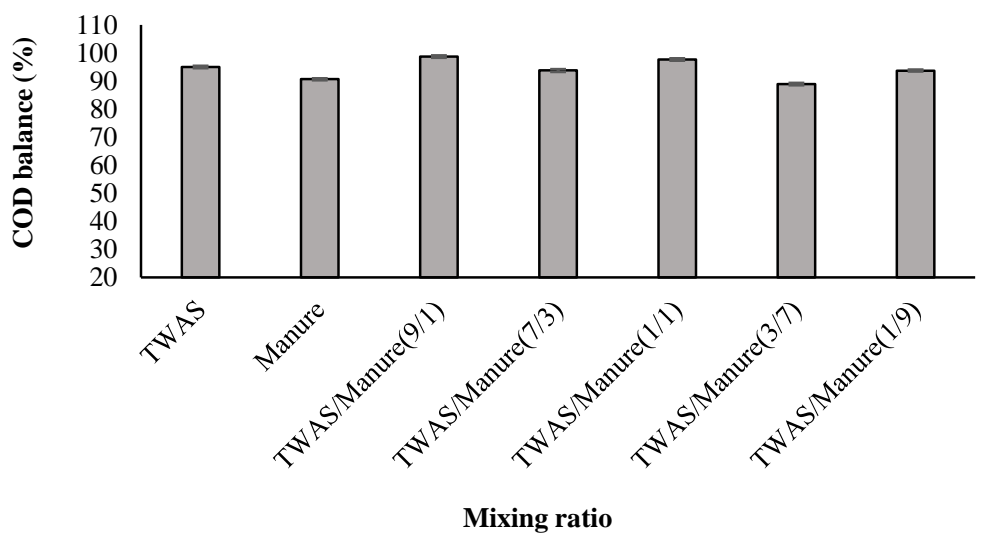

Figure 5.4. COD mass balance in co-digestion of TWAS and manure for different mixing ratios 


\subsection{Cumulative methane yields}

Cumulative methane yields including $\mathrm{mLCH}_{4} / \mathrm{g}$ TCOD added, $\mathrm{mLCH}_{4} / \mathrm{g}$ VSS added, and $\mathrm{mLCH}_{4} / \mathrm{mL}$ substrate added are presented in Figure 5.5. Figure 5.5 a. shows cumulative methane yield per mass COD of substrate added for mono and co-digestions. Manure produced a higher amount of biomethane compared to TWAS. The methane yield was $196 \mathrm{mLCH}_{4} / \mathrm{g}$ TCOD added and $298 \mathrm{mLCH}_{4} / \mathrm{g}$ TCOD added for TWAS and manure mono digestion, respectively. The addition of manure to TWAS, only increased the methane yield by $15 \%$ in co-digesters compared to digestion of manure alone. Even though, it increased the methane yield by $65 \%$ in comparison with mono digestion of TWAS. Results showed that 320 and $324 \mathrm{mLCH}_{4} / \mathrm{g}$ TCOD added was obtained by co-digestion at the mixing ratios of 1:9 and 3:7. Mixing TWAS with manure in codigesters except for TWAS:manure ratio of 9:1 and 7:3 enhanced the methane yield in comparison with both TWAS and manure mono digestion. Although, the ratios of 9:1 and 3:7, increased the methane yield per unit mass of COD added by $40 \%$ and $59 \%$ compared to TWAS alone. The maximum cumulative methane yield of $324 \mathrm{~mL} \mathrm{CH}_{4} / \mathrm{g}$ TCOD added at TWAS/SSO ratio of 3:7 corresponded to 1:4:17 lipids: proteins: carbohydrates ratio.

The methane yield per volume of substrate added in co-digesters were lower than that of manure alone. However, all of the co-digesters yielded higher methane per volume substrate added in comparison with TWAS mono digestion. Only $4 \mathrm{~mL}$ methane per unit volume of substrate added in single digestion of TWAS was obtained.

As shown in figure 5.5. b, cumulative methane yield in terms of $\mathrm{mLCH}_{4} / \mathrm{g}$ VSS added significantly increased compared to TWAS alone due to addition of manure as co-substrate. Although the yield $\left(\mathrm{mLCH}_{4} / \mathrm{g}\right.$ VSS added) only increased by $12 \%$ compared to manure alone. Co-digestion of manure with TWAS significantly increased the methane yield per unit mass of VSS added compared to TWAS alone. A $136 \mathrm{mLCH}_{4} / \mathrm{g}$ VSS added and $401 \mathrm{mLCH}_{4} / \mathrm{g}$ VSS added were attained by TWAS and manure alone, respectively. The amount of methane yield per unit mass of VSS added in codigesters varied from 206 to $448 \mathrm{mLCH}_{4} / \mathrm{g}$ VSS added. The maximum $\mathrm{CH}_{4}$ yield per unit mass of VSS added corresponded to lipids: proteins: carbohydrates ratio of 1:4:17 at TWAS:manure mixing ratio of $3: 7(\mathrm{v} / \mathrm{v})$. 


\section{5. a)}

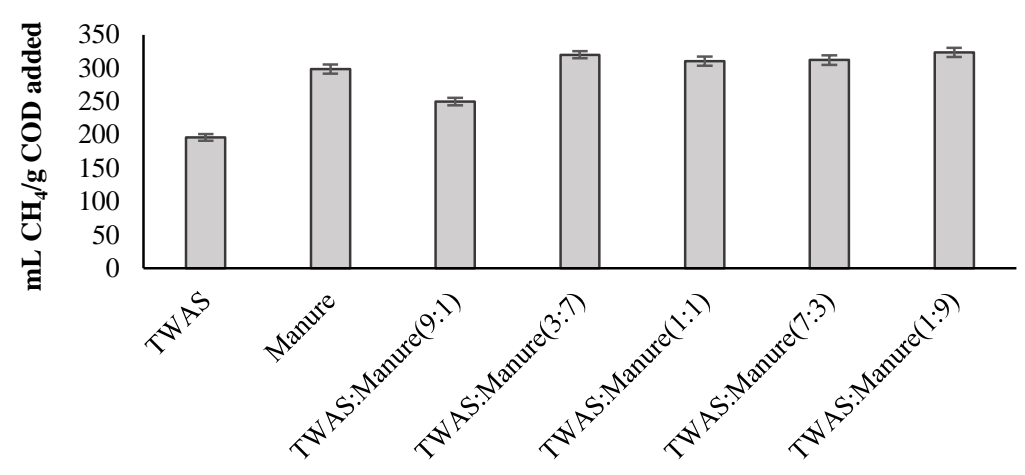

Mixing ratios

\section{5. b)}

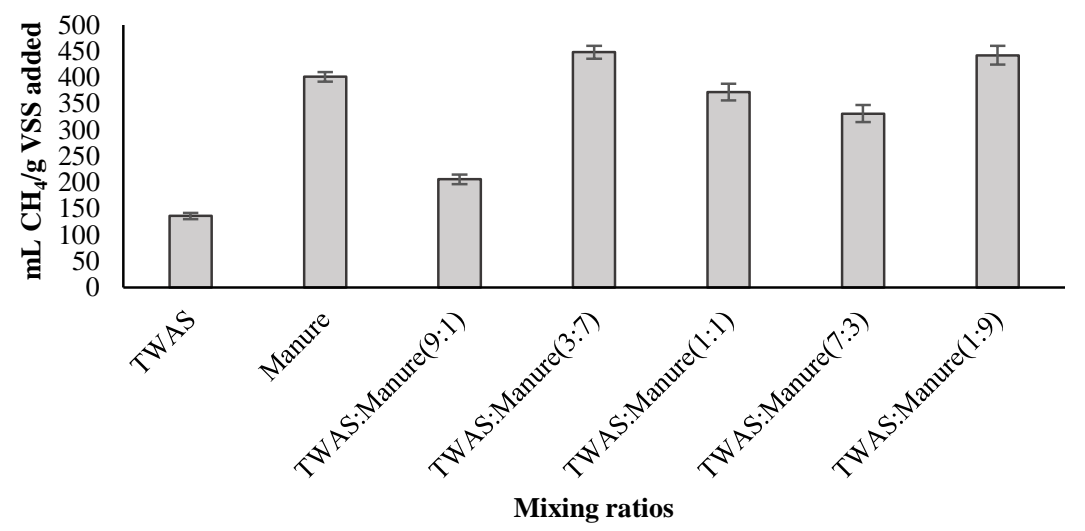

\section{5. c)}

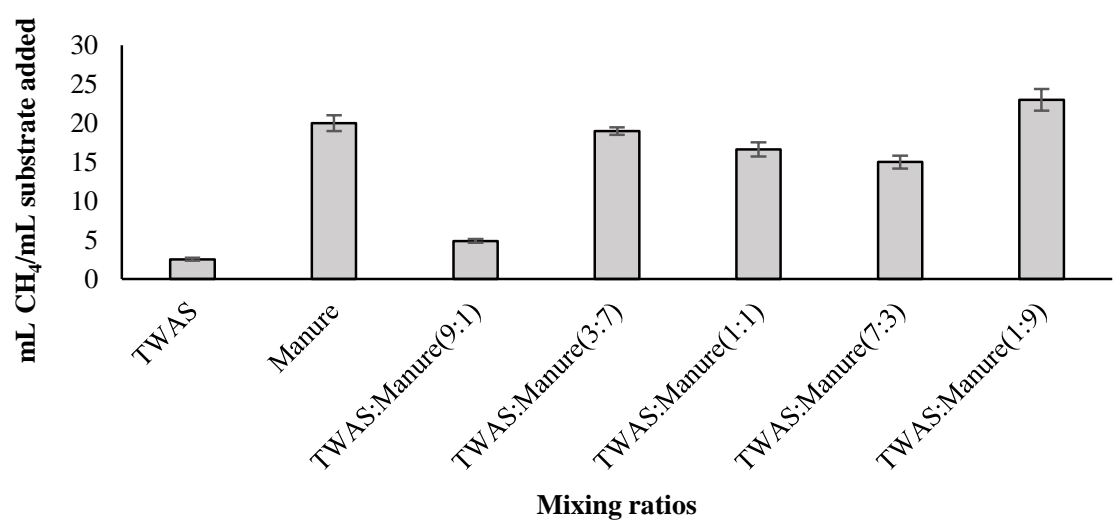

Figure 5.5. Methane yields a) per unit mass TCOD added, $b$ ) per unit mass of VSS added and c) per volume substrate added at different mixing ratio of TWAS and manure 
Assessing biodegradable fraction of the feedstocks with reference to Eq. 4.1, confirmed a 53\% more biodegradable fraction for manure than TWAS. This verifies the higher amount of biomethane obtained by manure compared to TWAS. Co- digestion enhanced biodegradability by $8 \%$ and $65 \%$ in comparison with the control reactors digesting only manure and TWAS, respectively.

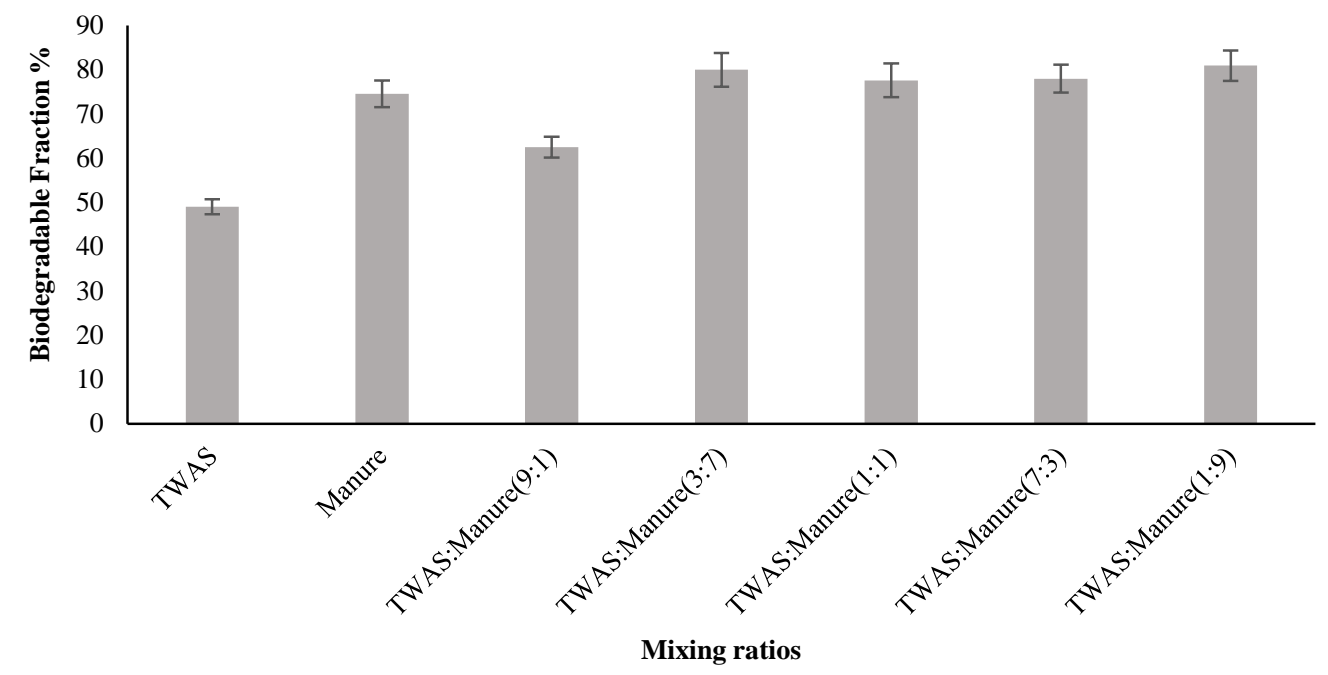

Figure 5.6. Biodegradable fraction of the feedstocks for different mixture ratios of TWAS and manure

As shown in Figure 5.6, biodegradable fraction was 49\% for TWAS while it was $75 \%$ for manure. The mixing ratios of $3: 7$ and 1:9 had the highest biodegradable fraction of almost $80 \%$ in TWAS/manure co-digestion. It was verified that addition of manure as co-substrate increased biodegradability and enhanced the production of methane in the rectors co-digesting manure with TWAS. All co-digesters had higher biodegradable fraction than TWAS alone. The reason would be the existence of readily biodegradable compounds in manure that was introduced to the codigesters. At the mixing ratio of 3:7, the biodegradable fraction was almost $10 \%$ and $0.53 \%$ higher than that of manure and TWAS alone. The trend of biodegradable fraction changes in digesters complies with the methane yields obtained by the experimental results for the corresponding digesters. 


\subsection{Synergistic effect}

Synergistic effect was evaluated with reference to Eq. 4.2 as described in chapter 4. Figure 5.7 shows the percentage of additional methane yield for co-substrates that was measured by the experiment, over the weighted average of the methane production of individual substrates per unit volume of substrate added. As shown in Fig 5.7, the synergistic effect varied from 10 to $24 \%$ in co-digesters. The maximum synergetic impact was observed in co-digestion of TWAS/manure at the mixing ratio of 3:7. This is in compliance with the maximum methane yield that was obtained at the same mixing ratio of TWAS and manure in the reactors co-digesting them. In co-digestion of TWAS and manure, $24 \%$ improvement was achieved due to synergistic effect at the mixing ratios of 3:7.

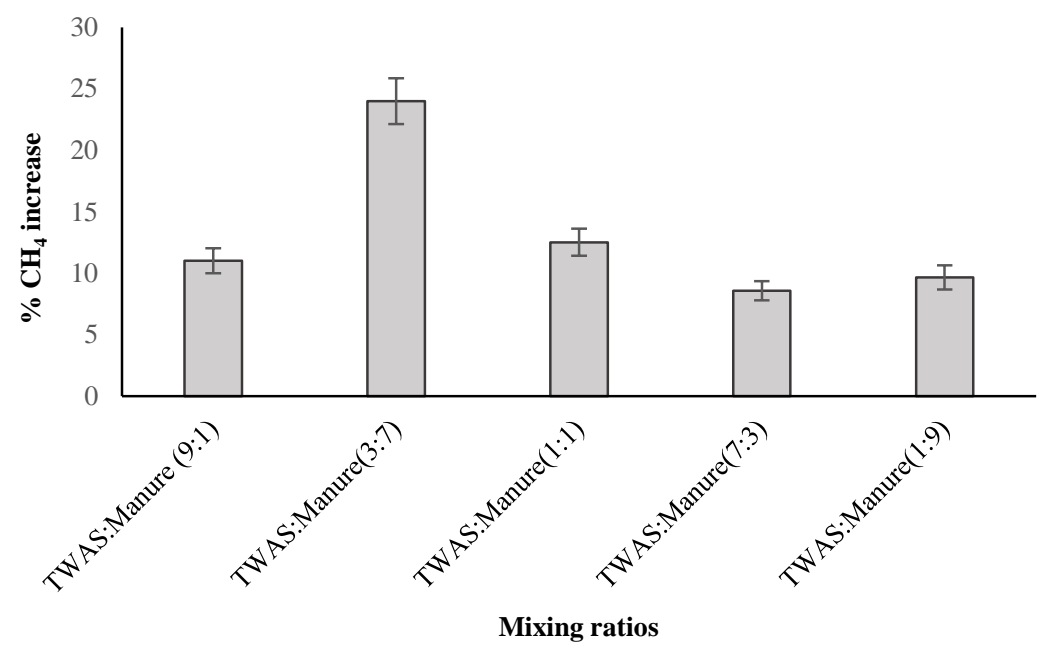

Figure 5.7. Synergetic effect of co-digestion at different mixing ratios of TWAS and SSO

Although all of the mixings demonstrated the synergetic impact of co-digestion on improving biomethane production, no specific trend for the change of the synergetic effect and methane increase corresponding to the fraction of manure in the co-digestion mixtures was observed. Similar to co-digestion of TWAS/SSO, adding fractions of manure as co-substrate enhanced the amounts of readily biodegradable materials to the co-digesters. The ratios with more fraction of carbohydrates produced more methane as the biodegradation of carbohydrate occurs more rapidly than lipids and proteins content. However, increasing the fractions of manure did not essentially resulted in the process improvement. 
The improvement of the synergy and biogas yield in TWAS/manure co-digestion could be due to the abundance of methanogenic populations and diversity of archaeal communities present in manure. Nevertheless, improved synergy would also depend on the optimal nutrients balance and effective conditions for microbial growth. This could explain the optimum fraction of TWAS and manure at the mixing ratio of 3:7 in the co-digestion rather than other ratios.

\subsection{COD:N and Lipids: Proteins: Carbohydrates ratios}

Table 5.2 presents the ultimate methane production and the methane yield per unit mass of COD added, the COD:N and lipids: proteins: carbohydrates ratios of the digesters. Table 5.2 provides a comparison between the COD: $\mathrm{N}$ ratios and the lipids: proteins: carbohydrates ratios of the digesters fed with different mixings of TWAS and manure. In co-digestion of TWAS and manure, the COD: $\mathrm{N}$ ratios between 33 and 56 resulted in higher ultimate methane production, however, the ratios below 20 resulted in lower methane yields. The ultimate methane production and methane yield ranged between 590 to $1069 \mathrm{~mL}$ and 196 to $324 \mathrm{~mL} / \mathrm{g}$ TCOD added for different mixing ratios, respectively.

As presented in Table 5.2, the ratios of COD:N and lipids: proteins: carbohydrates were 16 and 1:7:2.5 for TWAS alone while they were 56 and 1:4:20 for manure, respectively. The methane yield obtained by manure was $52 \%$ more than that of TWAS. The lowest ultimate methane production and methane yield of $590 \mathrm{~mL}$ and $196 \mathrm{~mL} / \mathrm{g}$ TCOD added, corresponded to the TWAS/manure mixing ratio of 1:0 in the control reactors digesting only TWAS. The maximum ultimate $\mathrm{CH}_{4}$ and $\mathrm{CH}_{4}$ yield corresponded to the COD:N ratio of 41 and the lipids: proteins: carbohydrates ratio of 1:4:17 for the reactors co-digesting TWAS and manure at the mixing ratio of $3: 7(\mathrm{v} / \mathrm{v})$.

Figure 5.8. shows the main effect plot for $\mathrm{CH}_{4}$ yield data means in response to feedstock and lipids:proteins:carbohydrates ratios at different feedstock mixing ratios in co-digestion of TWAS/manure. As illustrated in Figure 5.8, both feedstock and lipids:proteins:carbohydrates ratios has significant effect on the methane yield. The minimum methane yield corresponded to TWAS alone with lipids:proteins:carbohydrate ratio of 1:7:2.5 while the maximum yield occurred at the mixing ratio of 3:7 and lipids:proteins:carbohydrate ratio of 1:4:17 in co-digestion of TWAS with SSO. With reference to the results of the ANOVA test, the both COD:N and Lipids: Proteins: 
Carbohydrates ratios had statistically significant effects on the ultimate methane production $(\mathrm{P}<$ $0.05)$.

Table 5.2. Ultimate $\mathrm{CH}_{4}$ and yield at different ratios of the substrates, $\mathrm{COD}: \mathrm{N}$, and lipids:proteins:carbohydrates

\begin{tabular}{ccccccc}
\hline Digester code & $\begin{array}{c}\text { TWAS: } \\
\text { Manure (V/V) }\end{array}$ & COD:N & $\begin{array}{c}\text { Feedstock } \\
\text { ratios } \\
\text { code }\end{array}$ & $\begin{array}{c}\text { Lipids: } \\
\text { Proteins: } \\
\text { Carbohydrates }\end{array}$ & $\begin{array}{c}\text { Ultimate } \\
\text { CH}_{4}(\mathbf{m L})\end{array}$ & $\begin{array}{c}\text { mLCH4/g } \\
\text { TCOD } \\
\text { added }\end{array}$ \\
\hline \hline TWAS Only & $1: 0$ & 16 & $\mathrm{AA}$ & $1: 7: 2.5$ & 590 & 196 \\
$\begin{array}{c}\text { Manure Only } \\
\text { TWAS/Manure } \\
\text { 9/1 }\end{array}$ & $0: 1$ & 56 & $\mathrm{CC}$ & $1: 4: 20$ & 922 & 298 \\
$\begin{array}{c}\text { TWAS/Manure } \\
\text { 7/3 }\end{array}$ & $9: 1$ & 19 & $\mathrm{~A}$ & $1: 7: 6$ & 811 & 250 \\
$\begin{array}{c}\text { TWAS/Manure } \\
\text { 1/1 }\end{array}$ & $1: 1$ & 26 & $\mathrm{~B}$ & $1: 5.5: 12$ & 902 & 320 \\
$\begin{array}{c}\text { TWAS/Manure } \\
\text { 3/7 }\end{array}$ & $3: 7$ & 33 & $\mathrm{C}$ & $1: 25: 78$ & 1015 & 310 \\
$\begin{array}{c}\text { TWAS/Manure } \\
\text { 1/9 }\end{array}$ & $1: 9$ & 41 & $\mathrm{D}$ & $1: 4: 17$ & 1069 & 324 \\
\hline \hline
\end{tabular}

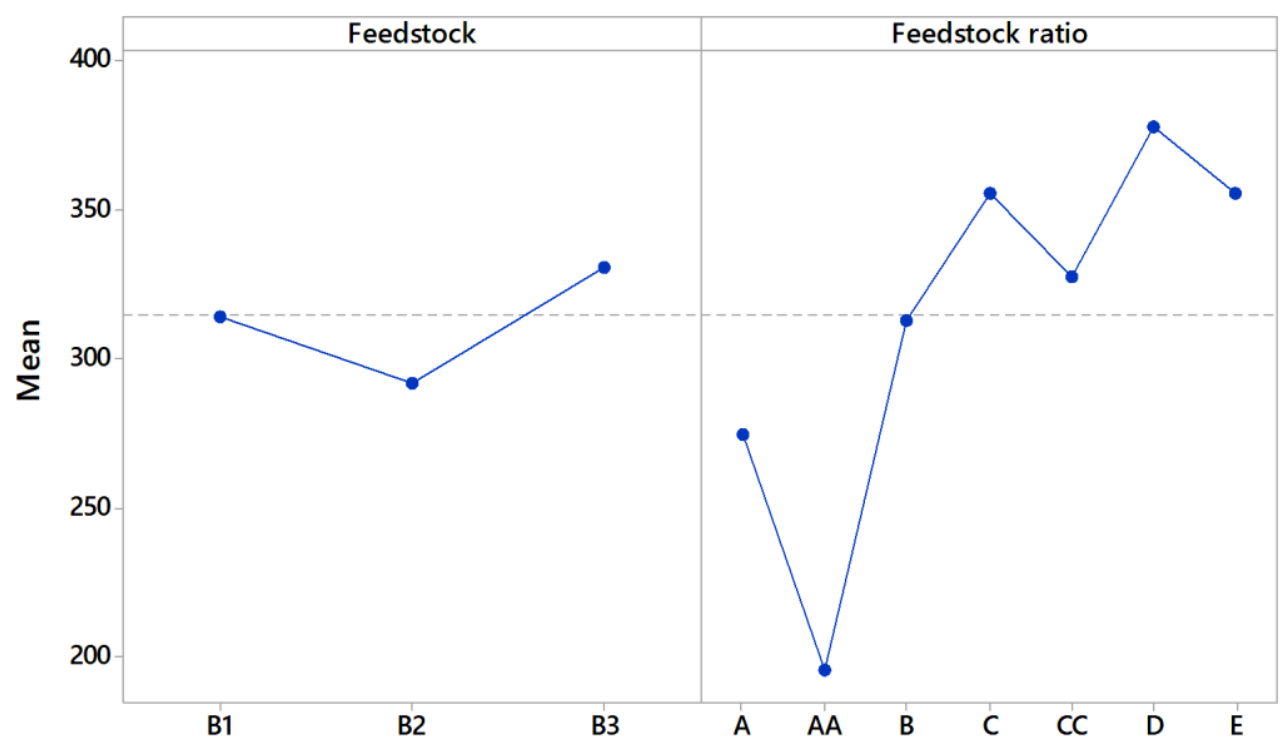

Figure 5.8. Main effect plot for $\mathrm{CH}_{4}$ yield data means in response to feedstock and lipids:proteins:carbohydrates ratios at different feedstock mixing ratios in AnCoD of TWAS/manure 
Figures 5.9. a and 5.9. $\mathrm{b}$ show the variation of ultimate methane and the methane yield versus COD:N ratio, lipids:proteins ratio, and proteins:carbohydrates ratios. As illustrated in Figure 5.9, different trends for the variations of methane yield and the ultimate methane versus COD:N, lipids: proteins, lipids: carbohydrates, and proteins: carbohydrates ratios were observed. For the corresponding feedstocks mixing ratios, a similar trend for the variations of methane versus COD: $N$ and lipids: proteins ratios was observed. However, the COD:N ratio did not show the same trend as lipids: carbohydrates and proteins: carbohydrates ratios for the same feedstocks mixings.

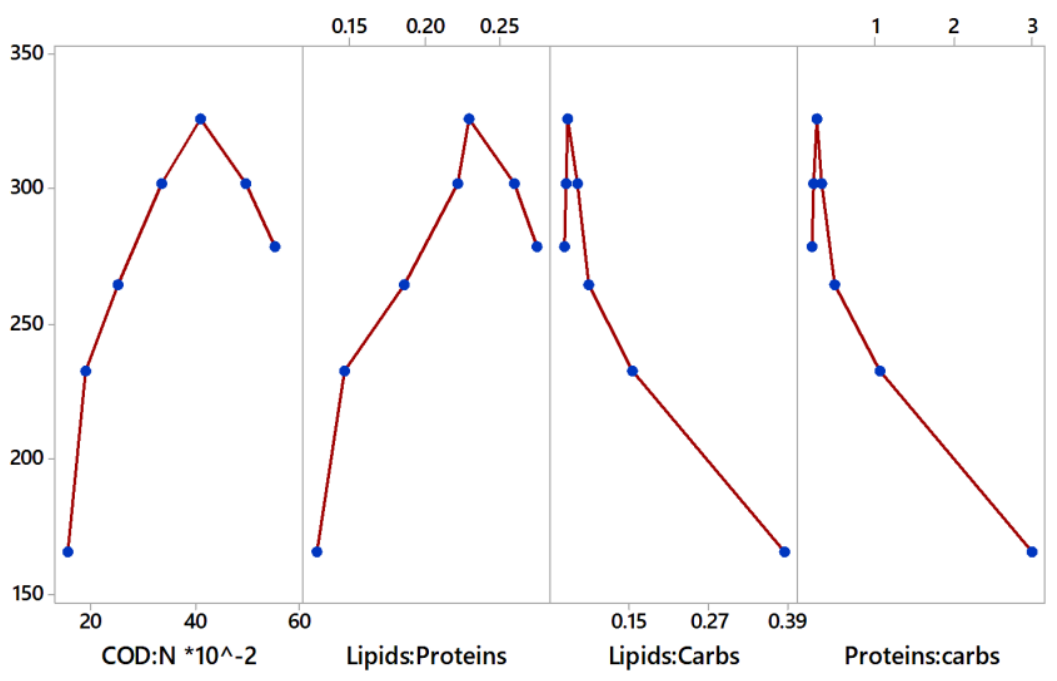

5.9. a

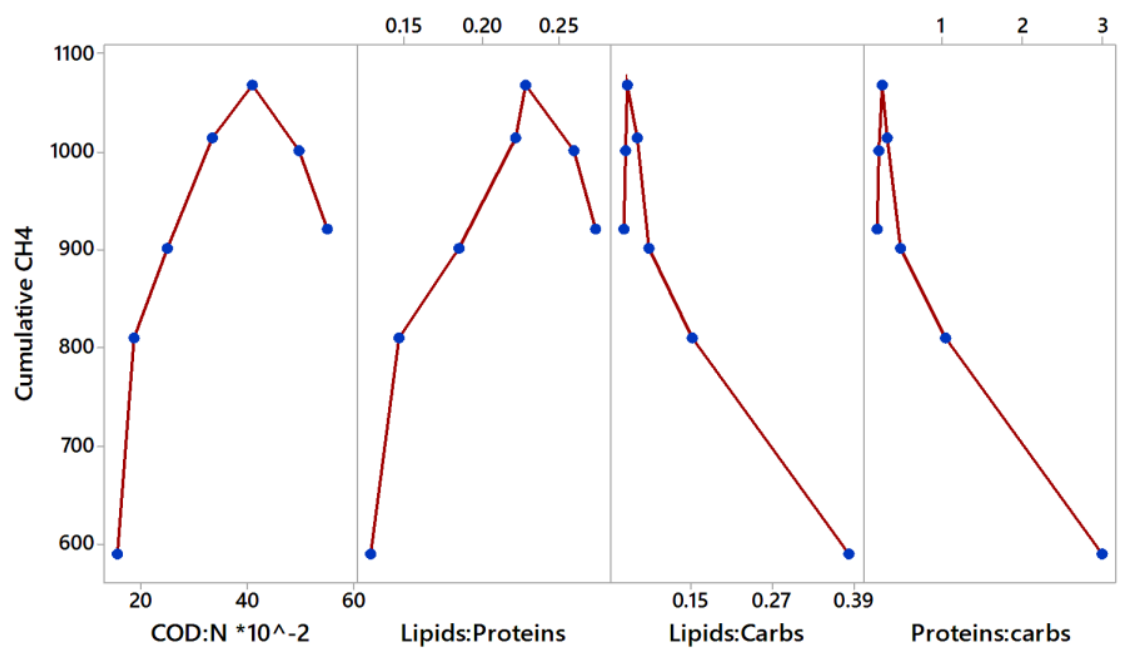

5.9 b.

Figure 5.10. Matrix plot for: a. ultimate $\mathrm{CH}_{4}$ and $b . \mathrm{CH}_{4}$ yield at different $\mathrm{COD} / \mathrm{N}$ and lipids: proteins, lipids: carbohydrates, and proteins: carbohydrates ratios 


\subsection{Kinetic analysis results}

Table 5.3 presents the results of analyzing biomethane production according to the modified Gompertz model using Eq. 3.5. The data collected by the experiment from the control reactors and from each co-digester was applied to the model. The experimental data for cumulative methane production $\left(\mathrm{CH}_{4}\right)$ per unit substrate in $\mathrm{mL} / \mathrm{g}$ and the digestion time $(\mathrm{t})$ in days were applied to the Gompertz equation to calculate the values of $\mathrm{P}$, representing the maximum methane production per unit substrate $(\mathrm{mL} / \mathrm{g}), R_{m}^{e}$, the maximum methane production rate $(\mathrm{mL} / \mathrm{g} \mathrm{h})$, and $\lambda$, the lag phase time (d). The modified Gompertz model for mono- and co-digestions of TWAS and manure, showed a good fit to the experimental results with less than 5\% diversion from the experimental values. The estimated parameters indicated that the co-digestion of manure with TWAS enhanced the biogas production rate. Such performance $\left(R^{2}=0.9998\right)$ shows that the proposed equations can accurately describe the variation of methane yield curves.

Table 5.3. Summary of results of kinetic study using modified Gompertz model

\begin{tabular}{ccccc}
\hline & $\mathbf{P}(\mathbf{m L})$ & $\boldsymbol{R}_{\boldsymbol{m}}^{\boldsymbol{e}}(\mathbf{m L} / \mathbf{d})$ & $\lambda(\mathbf{d})$ & $\mathbf{R}^{2}$ \\
\hline \hline TWAS & 554 & 21 & 0.09 & 0.999 \\
\hline Manure & 880 & 31 & 0.38 & 0.999 \\
\hline TWAS/Manure 9/1 & 763 & 27 & 0.06 & 0.999 \\
\hline TWAS/Manure 7/3 & 861 & 32 & 0.04 & 0.999 \\
\hline TWAS/Manure 5/5 & 969 & 36 & 0.04 & 0.999 \\
\hline TWAS/Manure 3/7 & 1016 & 35 & 0.04 & 0.999 \\
\hline TWAS/Manure 1/9 & 947 & 31 & 0.05 & 0.999 \\
\hline
\end{tabular}

With reference to the adjustment to the nonlinear regression Gompertz model, the lag phase varied from 0.04 to 0.06 days for the co-digesters with different substrate mixing ratios. By increasing the proportion of manure higher values of $\mathrm{P}$ was estimated. The mixing ratio of 7:3 and 1:9 corresponded to the higher $\mathrm{P}$ values. These findings were in good agreement with the data collected from the experiment. As verified by the experiment, a longer lag phase was observed in codigestion of TWAS:manure compared to co-digestion of TWAS:SSO. As mentioned before, a long lag phase time is unfavorable as it demands for a higher residence time and consequently, larger 
reactor volumes which increases the operational costs of the anaerobic system. The values of $\mathrm{P}$, varied from 554 to $1016 \mathrm{~mL}$ corresponding to TWAS alone and TWAS:manure mixing ratio of 3:7. The trend of changes in the $\mathrm{P}$ values complies with the experimental data as it showed an increasing trend in methane production by increasing the proportion of manure. The values of $\mathrm{R}_{\max }^{\mathrm{e}}$ were within a range from 21.3 to $36.2 \mathrm{~mL} / \mathrm{d}$. The values of $\mathrm{R}_{\max }^{\mathrm{e}}$ and $\mathrm{P}_{\max }$ obtained by Gompertz for the TWAS/manure ratios of 3:7, were $35.1 \mathrm{~mL} / \mathrm{d}$ and $1016 \mathrm{~mL}$, respectively.

\section{6. Hydrolysis/acidification}

This experiment was conducted to investigate the hydrolysis/acidification phase in anaerobic codigestion of TWAS and manure. The degradation of organic compounds in hydrolysis stage was evaluated using a series of analysis such as degree of solublization, synergetic effect of codigestion at different mixing ratios on hydrolysis and liquefaction, volatile fatty acids (VFAs) yield, and hydrolysis kinetics of lipids, proteins, and carbohydrates .

Characterization of the feedstocks was initially carried out in triplicates and the mean values are summarized in table 5.4. As presented in the table, the amount of COD concentration is remarkably higher in manure than TWAS. Adding manure to TWAS in the co-digesters, increased the COD concentrations compared to the reactor digesting only TWAS. Manure also contains higher amounts of carbohydrates, lipids, and proteins than TWAS. The concentrations of total, soluble and particulate COD; total, soluble and particulate proteins; and total, soluble and particulate carbohydrates were monitored over time to obtain their hydrolysis rate coefficients.

The data form total and soluble COD (SCOD) concentrations monitoring over time during a 72- $\mathrm{h}$ experimental period was used to obtain the degree of COD solublization for each mixture. The degree of solublization was calculated using Eq. 3.1 and the result is summarized in Figure 5.10. The degree of the COD solublization varied from $21 \%$ to $34 \%$. The maximum solublization $34 \%$ occurred at TWAS/manure combination of 3:7 while the minimum value corresponded to the digesters containing only TWAS. Except for TWAS/manure mixing ratio of 9:1, other co-digesters demonstrated an increase of solublization compared to both TWAS and manure alone. A $31 \%$ and $62 \%$ improvement was achieved by TWAS/Manure co-digestion at the mixing ratio of 3:7 compared to manure and TWAS single digestion, respectively. Manure contains sufficient amount of rapidly biodegradable materials than TWAS. The lower degree of solublization in TWAS mono digestion as well as TWAS:manure mixing ratio of 9:1 with a high portion of TWAS could be due 
to the existence of slowly biodegradable materials which slows down the hydrolysis and liquefaction process and decreases the degree of solublization.

Table 5.4. Characteristics of the feedstocks at different mixing ratios of TWAS and manure

\begin{tabular}{|c|c|c|c|c|c|c|c|c|}
\hline & & TWAS & Manure & $\begin{array}{c}\mathrm{T}^{*}: \mathrm{M}^{* *} \\
9: 1\end{array}$ & $\mathrm{~T}: \mathrm{M}$ 7:3 & $\mathrm{T}: \mathrm{M} \mathrm{1:1}$ & $\mathrm{T}: \mathrm{M} 3: 7$ & $\mathrm{~T}: \mathrm{M} 1: 9$ \\
\hline Parameters & Units & $\begin{array}{c}\text { Mixture } \\
(1)\end{array}$ & $\begin{array}{c}\text { Mixture } \\
\text { (2) }\end{array}$ & $\begin{array}{c}\text { Mixture } \\
(3)\end{array}$ & $\begin{array}{c}\text { Mixture } \\
(4)\end{array}$ & $\begin{array}{c}\text { Mixture } \\
(5)\end{array}$ & $\begin{array}{c}\text { Mixture } \\
(6)\end{array}$ & Mixture (7) \\
\hline TCOD & $\mathrm{g} / \mathrm{L}$ & 41 & 110 & 48 & 62 & 76 & 90 & 104 \\
\hline SCOD & $\mathrm{g} / \mathrm{L}$ & 2.2 & 6.6 & 2.6 & 3.5 & 4.4 & 5.2 & 6.1 \\
\hline TSS & $\mathrm{g} / \mathrm{L}$ & 37.3 & 52.6 & 38.8 & 41.9 & 44.9 & 48.0 & 51.1 \\
\hline VSS & $\mathrm{g} / \mathrm{L}$ & 26.5 & 42.8 & 28.1 & 31.4 & 34.7 & 37.9 & 41.2 \\
\hline $\mathrm{TS}$ & $\mathrm{g} / \mathrm{L}$ & 39.2 & 68.0 & 42.1 & 47.9 & 53.6 & 59.4 & 65.2 \\
\hline VS & $\mathrm{g} / \mathrm{L}$ & 28.6 & 58.5 & 31.6 & 37.5 & 43.5 & 49.5 & 55.5 \\
\hline Ammonia & $g / L$ & 0.3 & 0.0 & 0.3 & 0.2 & 0.2 & 0.1 & 0.0 \\
\hline $\mathrm{pH}$ & - & 5.8 & 5.8 & 5.6 & 5.7 & 5.8 & 5.8 & 5.8 \\
\hline Alkalinity & $\mathrm{g} \mathrm{CaCO}_{3} / \mathrm{L}$ & 5.0 & 7.7 & 5.2 & 5.8 & 6.3 & 6.9 & 7.4 \\
\hline $\mathrm{TN}$ & $\mathrm{g} / \mathrm{L}$ & 3.0 & 1.4 & 2.8 & 2.5 & 2.2 & 1.9 & 1.6 \\
\hline $\mathrm{TSN}$ & $\mathrm{g} / \mathrm{L}$ & 0.4 & 0.1 & 0.4 & 0.3 & 0.2 & 0.2 & 0.1 \\
\hline T-Carbs & $\mathrm{g} / \mathrm{L}$ & 1.3 & 27.1 & 3.9 & 9.1 & 14.2 & 19.4 & 24.5 \\
\hline T-Proteins & $\mathrm{g} / \mathrm{L}$ & 4.0 & 5.1 & 4.1 & 4.3 & 4.6 & 4.8 & 5.0 \\
\hline T-Lipids & $\mathrm{g} / \mathrm{L}$ & 0.5 & 1.4 & 0.6 & 0.8 & 0.9 & 1.1 & 1.3 \\
\hline
\end{tabular}

${ }^{*}$ T: TWAS

** M: Manure

Manure contains a large portion of carbohydrates which decomposes more rapidly than proteins and lipids. This would lead to a higher degree of solublization of manure than TWAS which contains more proteins than carbohydrates.

Comparing the theoretical degree of solublization of each co-digester to the ones obtained from the experimental data, showed that co-digestion improved solublization due to enhancing microbial synergy. As shown in Figure. 5.11, all co-digesters demonstrated an improvement in solublization due to synergistic effect of the microbial communities from 11 to $38 \%$ corresponding to TWAS:manure mixing ratios of 1:1 and 3:7, respectively. 


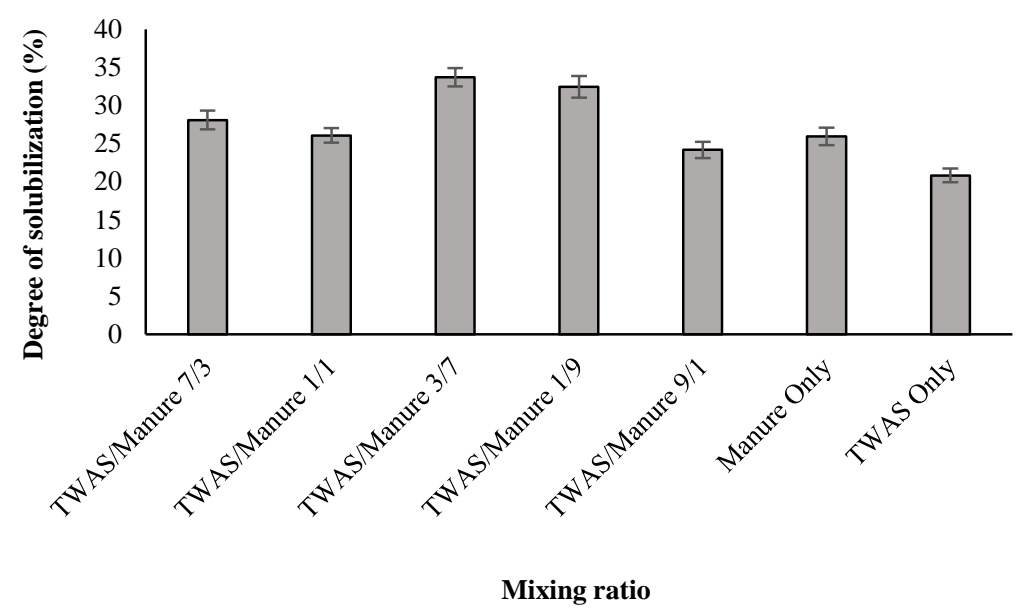

Figure 5.10. Degree of COD solublization at different mixing ratios of TWAS:Manure

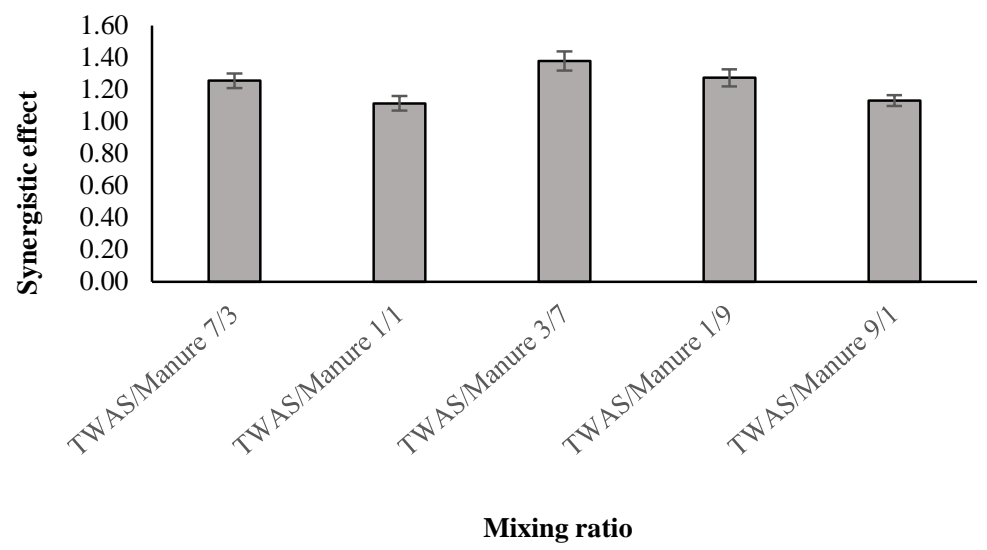

Figure 5.11. Synergistic effect on solublization at different mixing ratios of TWAS:Manure

The VFAs concentrations monitoring showed an increasing trend over the 72-hr of the hydrolysis/acidification experimental period. The total VFAs yield is presented in terms of mass of VFAs produced in mg per mass of VSS added in g. As indicated in Figure 5.12, manure alone had a significantly higher VFAs yields than TWAS alone. The VFAs yield in the co-digester was correlated to the mixing ratios of the feedstocks. The trend of VFAs yield did not conform to the trend of COD solublization of the corresponding mixing ratios (Figure 5.10). Therefore, hydrolysis and liquefaction showed a different trend from acidification. All of the reactors containing the mix of substrates had higher VFAs yield compared to the reactors containing only TWAS. The VFAs yields were $95 \mathrm{mg}$ VFAs/g VSS added and $260 \mathrm{mg}$ VFAs/g VSS added for TWAS and manure 
mono digestions, respectively. A VFAs yield of $307 \mathrm{mg}$ VFAs/g VSS added was achieved for TWAS:manure ratio of 3:7.

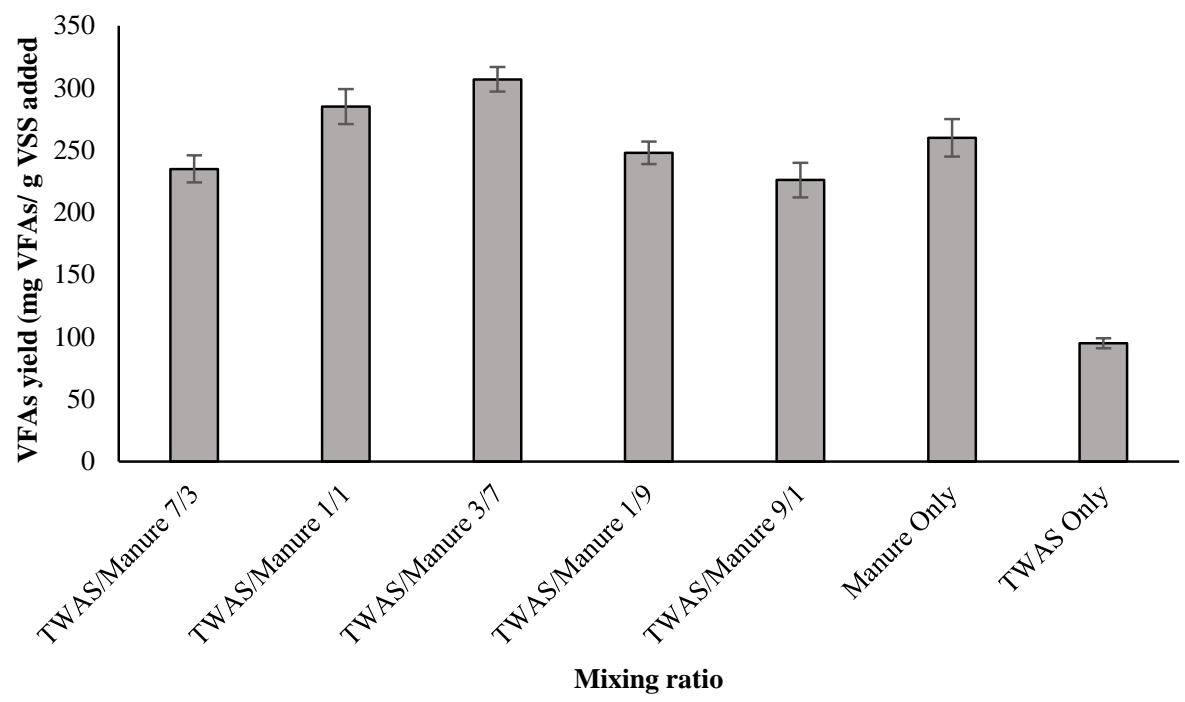

Figure 5.12. Total VFAs yield at different mixing ratios of TWAS/Manure

Monitoring COD over time, showed an increasing trend in solublization of COD and decreasing particulate COD concentrations. In addition, the analysis of soluble and particulate lipids, proteins, and carbohydrates over time showed an increasing trend in solublization and particulate matter degradation. For lipids and proteins, the hydrolysis rate was slower than carbohydrate. These results are summarized in the tables in the appendix. The hydrolysis rate coefficient $\left(\mathrm{K}_{\mathrm{h}}\right)$ was calculated by applying first order kinetics using AquaSim 2.0 for COD, lipids, proteins and carbohydrates based on the particulate degradation and results are summarized in Table 5.5.

As presented in Table 5.5, the hydrolysis rate for COD content of TWAS was higher than that of manure by $35 \%$. The $\mathrm{K}_{\mathrm{h}}$ values in the co-digesters varied from 0.21 to 0.33 corresponding to TWAS and manure mixing ratios of 9:1 and 3:7, respectively. The maximum hydrolysis rate coefficient corresponded to the reactors containing mixings of TWAS and manure at the ratio of $3: 7$.

Lipids showed the lowest hydrolysis rate compared to proteins, and carbohydrates. On the contrary, the most rapid hydrolysis rate was observed for carbohydrates as a result of more rapid biological metabolism of carbohydrates than lipids and proteins. $\mathrm{K}_{\mathrm{h}}$ varied from 0.4 to 0.09 in the 
reactors co-digesting TWAS with manure. The maximum hydrolysis rate coefficient of the lipids contents also corresponded to TWAS:manure mixing of 7:3. TWAS alone has the minimum $\mathrm{K}_{\mathrm{h}}$ for the lipids. TWAS alone and manure alone had $\mathrm{K}_{\mathrm{h}}$ values of 0.03 and 0.07 , respectively. As presented in Table 5.5, the hydrolysis rate of proteins was slightly higher than that of lipids content of the digesters, although it was still lower than the hydrolysis rate of carbohydrates. $\mathrm{K}_{\mathrm{h}}$ for the proteins content of the feedstocks was within a range between 0.22 to 0.27 corresponding to TWAS:manure mixings of $1: 9$ and 3:7, respectively. The hydrolysis rate coefficient of the carbohydrates varied from 0.38 to 0.59 corresponding to the digestion of TWAS:manure with the mixing ratios of 9:1 and 3:7, respectively. The carbohydrates content of the manure showed more rapid biodegradability than TWAS. The hydrolysis rate variation of lipids did not show the same trend as the proteins and carbohydrates of the feedstocks. This revealed that the hydrolysis of the lipids, proteins and carbohydrates of the feedstocks developed independently during the hydrolysis/acidification stage. This independent hydrolysis of the lipids, proteins, and carbohydrates was observed in co-digestion of TWAS and SSO as well.

Table 5.5. Hydrolysis rate coefficients for COD, lipids, proteins, and carbohydrate content in co-digestion of TWAS and manure at different mixing ratios

\begin{tabular}{lccccccc}
\hline $\mathbf{K}_{\mathbf{h}}$ & $\mathrm{T} / \mathrm{M} \mathrm{7/3}$ & $\mathrm{T} / \mathrm{M} 5 / 5$ & $\mathrm{~T} / \mathrm{M} 3 / 7$ & $\mathrm{~T} / \mathrm{M}$ 1/9 & $\mathrm{T} / \mathrm{M}$ 9/1 & TWAS & Manure \\
\hline \hline Kh $\mathrm{COD}$ & 0.26 & 0.24 & 0.33 & 0.28 & 0.21 & 0.17 & 0.23 \\
\hline $\mathrm{Kh}_{\text {Lipids }}$ & 0.04 & 0.05 & 0.09 & 0.07 & 0.04 & 0.03 & 0.07 \\
\hline $\mathrm{Kh}_{\text {Proteins }}$ & 0.23 & 0.22 & 0.27 & 0.25 & 0.22 & 0.19 & 0.21 \\
\hline $\mathrm{K}_{\mathrm{h} \text { Carbohydrates }}$ & 0.49 & 0.44 & 0.59 & 0.48 & 0.38 & 0.32 & 0.43 \\
\hline
\end{tabular}

${ }^{*} \mathrm{~T}$ : TWAS

${ }^{* *} \mathrm{M}$ : Manure 


\section{Chapter 6}

\section{Results and discussion}

\section{Manure and SSO Co- digestion}




\section{Results and discussion- manure and SSO co-digestion}

\subsection{BMP of manure and SSO}

In this experiment co-digestion of manure and SSO was investigated. Manure slurry was prepared as discussed in chapter 3 and was fed to the reactors in different combinations with SSO. The influence of the feedstocks mixing ratios and their correlation with the lipids: proteins: carbohydrates ratios on biomethane production in anaerobic co-digestion of manure and SSO was evaluated. The characteristics of the feed in each digester containing different mixing ratios of the substrates are summarized in table 6.1. The values are the average of each parameter that was measured in triplicate. As presented in Table 6.1, both manure and SSO have high amount of COD concentrations and as a result, the amount of COD in the digesters are high and exceed $100 \mathrm{~g} / \mathrm{L}$. Both VSS and COD values did not vary significantly (less than $8 \%$ ) in the digesters. The amount of carbohydrates and proteins of manure is significantly higher than that of SSO. Therefore, addition of manure increased the carbohydrates and proteins content of the co-digesters compared to the rectors digesting only SSO.

Table 6.1. Characteristics of feed to digesters with different mixing ratios of manure and SSO

\begin{tabular}{ccccccccc}
\hline & & Manure & \multirow{2}{*}{ SSO Only } & M/SSO & M/SSO & M/SSO & M/SSO & \multicolumn{2}{c}{ M/SSO } \\
& & Only & & $9 / 1$ & $7 / 3$ & $5 / 5$ & $3 / 7$ & $1 / 9$ \\
\hline \hline \multirow{2}{*}{ Parameters } & Units & Mixture & Mixture & Mixture & Mixture & Mixture & Mixture & Mixture \\
& & $(1)$ & $(2)$ & $(3)$ & $(4)$ & $(5)$ & $(6)$ & $(7)$ \\
TCOD & $\mathrm{g} / \mathrm{L}$ & 105 & 115 & 106 & 108 & 110 & 112 & 114 \\
SCOD & $\mathrm{g} / \mathrm{L}$ & 44 & 43 & 44 & 44 & 44 & 43 & 43 \\
TSS & $\mathrm{g} / \mathrm{L}$ & 54 & 62 & 55 & 57 & 58 & 60 & 61 \\
VSS & $\mathrm{g} / \mathrm{L}$ & 46.4 & 45.6 & 46.3 & 46.2 & 46.0 & 45.8 & 45.7 \\
$\mathrm{TS}$ & $\mathrm{g} / \mathrm{L}$ & 70 & 68 & 70 & 69 & 69 & 69 & 68 \\
VS & $\mathrm{g} / \mathrm{L}$ & 59 & 49 & 58 & 56 & 54 & 52 & 50 \\
Ammonia & $\mathrm{g} / \mathrm{L}$ & 0.02 & 1.07 & 0.13 & 0.3 & 0.5 & 0.8 & 1.0 \\
pH & - & 6.6 & 5.8 & 6.9 & 7.0 & 7.2 & 7.1 & 7.0 \\
Alkalinity & $\mathrm{g}$ CaCO3/L & 4.9 & 6.2 & 5.0 & 5.3 & 5.6 & 5.8 & 6.1 \\
TN & $\mathrm{g} / \mathrm{L}$ & 2.5 & 3.5 & 2.6 & 2.8 & 3.0 & 3.2 & 3.4 \\
TSN & $\mathrm{g} / \mathrm{L}$ & 0.1 & 1.0 & 0.2 & 0.4 & 0.6 & 0.8 & 1.0 \\
T-Carbs & $\mathrm{g} / \mathrm{L}$ & 29 & 13 & 27 & 24 & 21 & 18 & 15 \\
T-Proteins & $\mathrm{g} / \mathrm{L}$ & 5.9 & 2.1 & 5.5 & 4.8 & 4.0 & 3.2 & 2.5 \\
T-Lipids & $\mathrm{g} / \mathrm{L}$ & 1.4 & 1.1 & 1.4 & 1.3 & 1.3 & 1.2 & 1.1 \\
\hline${ }^{*}$ M: Manure & & & & & & & &
\end{tabular}


Carbohydrates concentrations were within a range between 13 to $29 \mathrm{~g} / \mathrm{L}$ in the rectors. Total proteins concentrations of manure and SSO were 2.1 and 5.9, respectively and varied from 2.5 to $5.5 \mathrm{~g} / \mathrm{L}$ in the co-digesters. The total lipids concentrations varied from 1.1 to $1.4 \mathrm{~g} / \mathrm{L}$ in the digesters including the controls. Adding manure slightly increased the lipids content in the reactors containing the combination of manure and SSO.

Operation of the digesters proceeded until no significant amount of biogas was generated. Fig. 6.1 shows the profile of the cumulative biomethane production versus time during the digestion period of manure with SSO including the controls. The cumulative methane production generated by SSO was higher than that of manure in the control reactors. All co-digesters produced more biomethane than the control reactors containing only manure and only SSO.

SSO alone produced $15 \%$ more methane than manure alone. The amount of ultimate $\mathrm{CH}_{4}$ obtained by single digestion of SSO and manure was 1063 and $919 \mathrm{~mL}$, corresponding to the lipids: proteins: carbohydrates ratios of 1:2:12 and 1:4.2:21, respectively. As shown in figure 6.1, the maximum cumulative methane production of $1186 \mathrm{~mL}$ corresponded to the manure:SSO mixing ratio of 7:3 and lipids: proteins: carbohydrates ratio of 1:3.5:18.5.

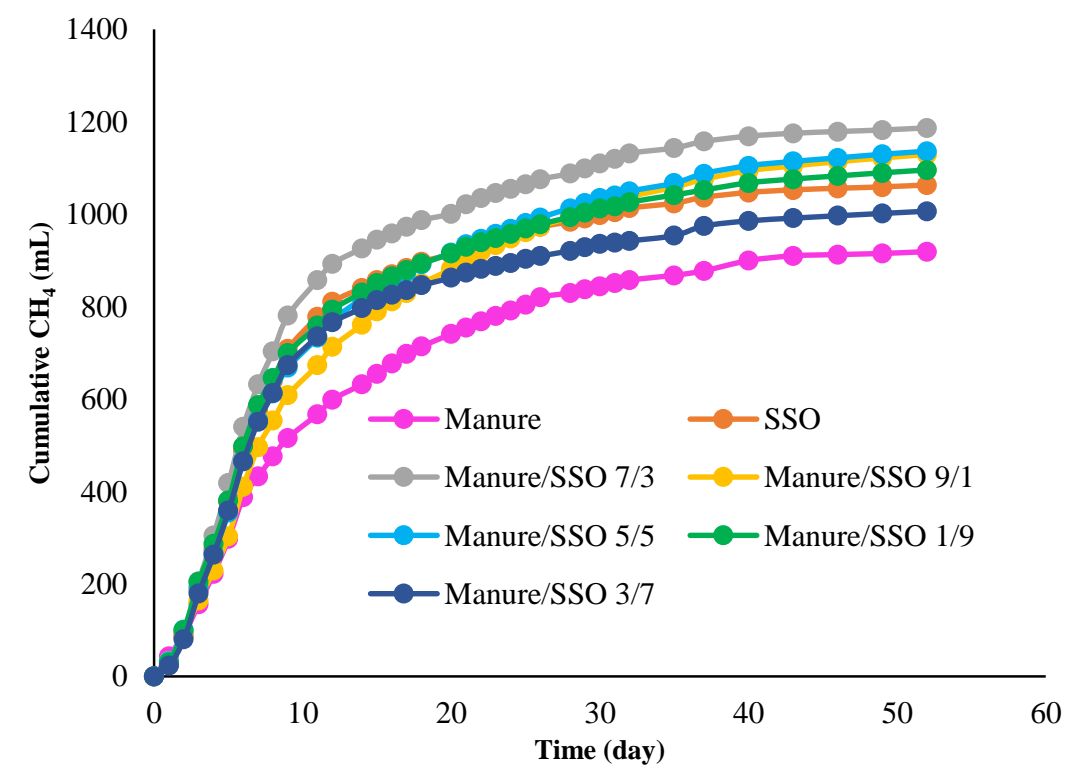

Figure 6.1. Cumulative methane production for different mixing ratios of manure and SSO

Figure 6.2, shows the methane rate in $\mathrm{mL} /$ day for all of the feedstocks combinations including the control reactors. For all of the digesters, the maximum methane rate occurred in the first week of 
the operation period. The maximum methane production rate of the digesters containing the mixings of manure and SSO was higher compared to the control reactors. The reactors generated $44 \%$ to $55 \%$ of their ultimate methane production at the first week of the digestion period and $69 \%$ to $79 \%$ of it in two weeks of operation. Compared to SSO alone, a higher $\mathrm{CH}_{4}$ rate was observed for single digestion of manure. Addition of manure to SSO increased the maximum methane production rate so that all of the co-digester demonstrated a higher maximum methane rate compared to the control rectors. The manure/SSO mixing ratio of $7: 3$ achieved the highest maximum biomethane rate in comparison with other combinations and the lowest value for the

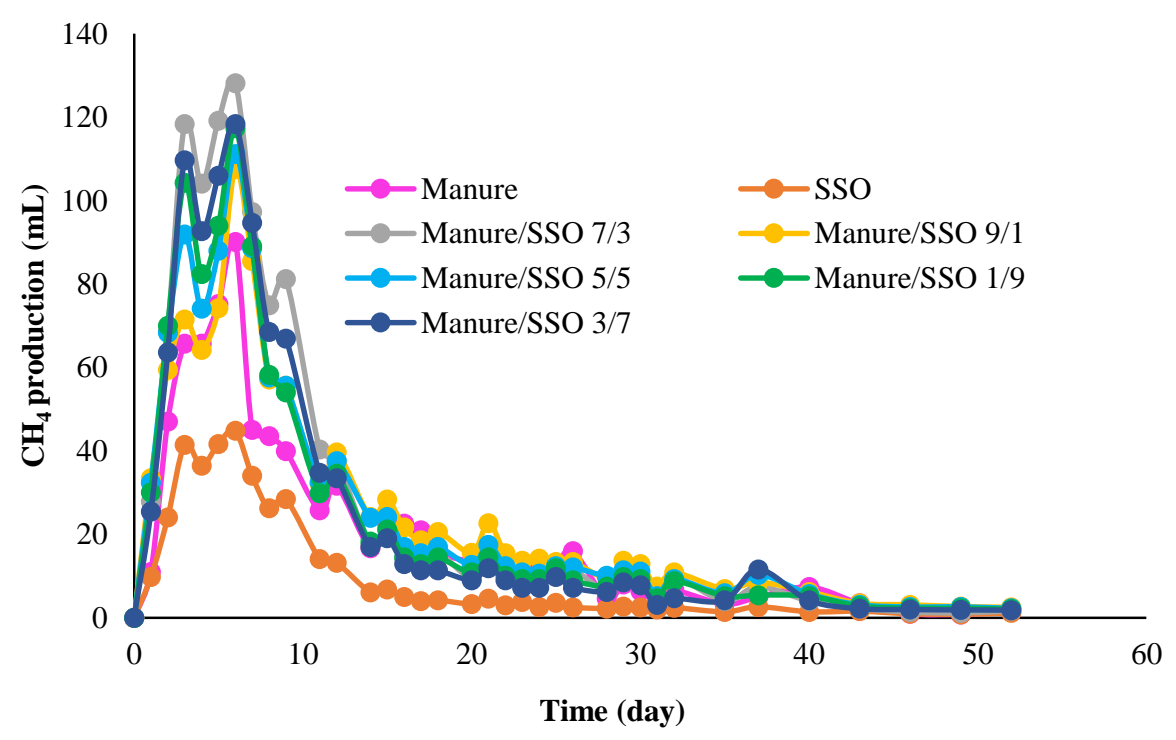

Figure 6.2. Methane production rate $(\mathrm{mL} / \mathrm{d})$ for different mixing ratios of Manure and SSO

maximum biomethane rate corresponded to single digestion of $\mathrm{SSO}$. The maximum $\mathrm{CH}_{4}$ production rate at different mixing ratios is shown in Figure 6.3. As presented in the figure, the most maximum rate was $128 \mathrm{~mL} /$ day corresponding to the mixing ratio of $7: 3$. The lowest value of $45 \mathrm{~mL} /$ day corresponded to single digestion of SSO. Manure alone had the maximum $\mathrm{CH}_{4}$ rate of $90 \mathrm{~mL} /$ day which it was higher than that of SSO by 2 fold. The maximum $\mathrm{CH}_{4}$ rate varied from 108 to $128 \mathrm{~mL} /$ day for the digesters containing combinations of manure and SSO. 


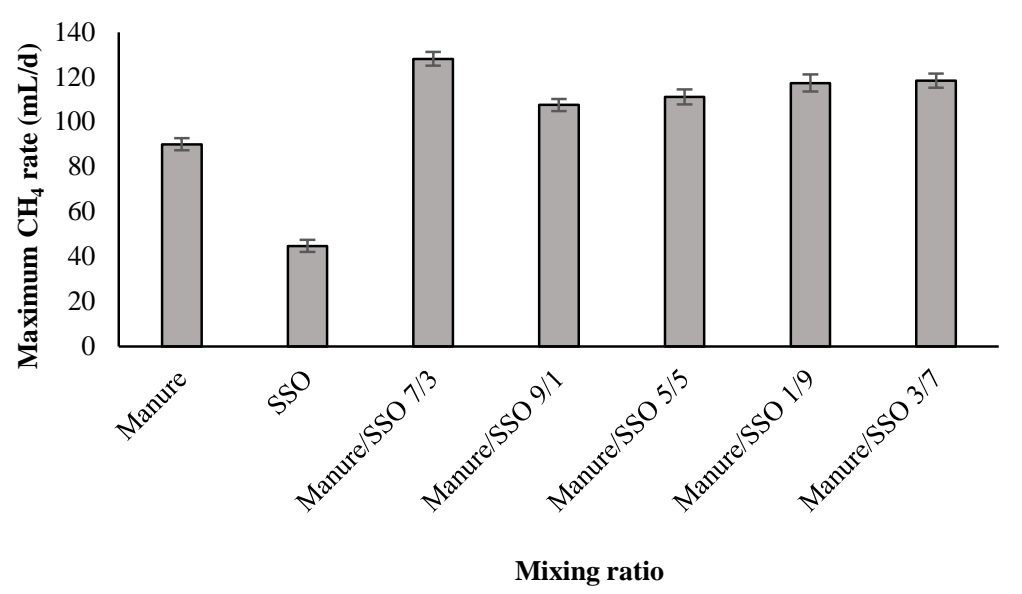

Figure 6.3. Maximum methane production rate $(\mathrm{mL} / \mathrm{d})$ for different mixing ratios manure of and SSO

The COD mass balance was conducted for all of the digesters with reference to the initial and the final TCOD concentrations of the digester contents, and the theoretical methane production per unit mass of TCOD removed. Comparison between the experimental methane production data and that of obtained by TCOD mass balance, showed a deviation of less than $8 \%$ for all the digesters. As shown in Figure 6.4, the COD balance varied from almost 90\% to $97 \%$ in all mono and codigesters.

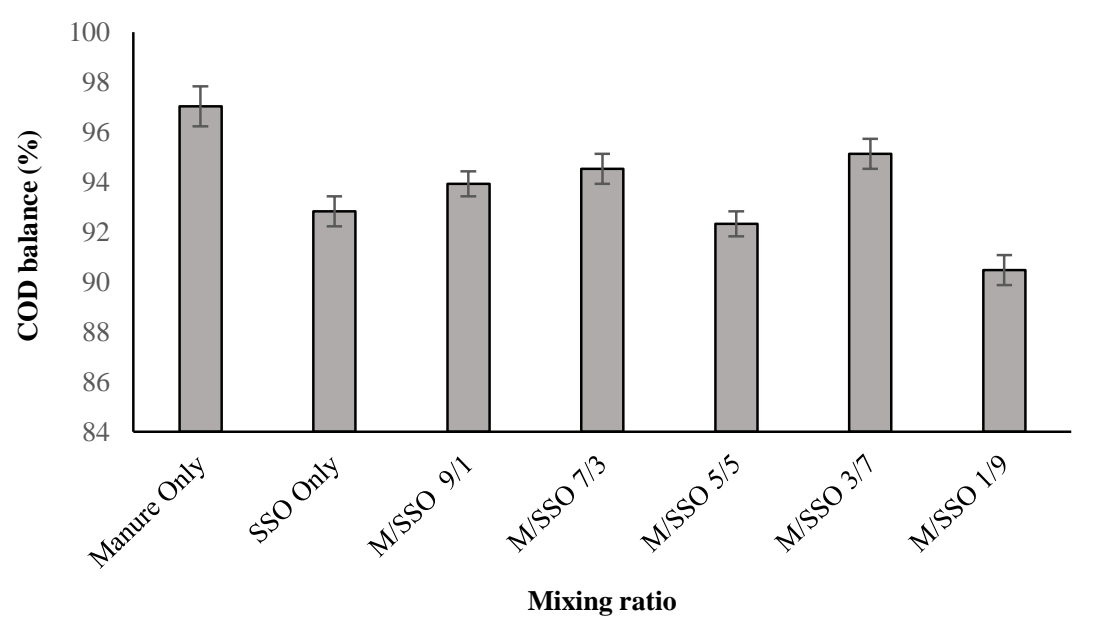

Figure 6.4. COD mass balance in co-digestion of manure and SSO for different mixing ratios 


\subsection{Cumulative methane yields}

Cumulative methane yields including $\mathrm{mLCH}_{4} / \mathrm{g}$ TCOD added, $\mathrm{mLCH}_{4} / \mathrm{g}$ VSS added, and $\mathrm{mLCH}_{4} / \mathrm{mL}$ substrate added are presented in Figure 6.5. It was observed that cumulative methane yield per mass COD of substrate added increased in co-digesters in comparison with the control reactors. As shown in Figure 6.5. a), SSO and manure alone produced $303 \mathrm{mLCH}_{4} / \mathrm{g}$ TCOD added and $287 \mathrm{mLCH}_{4} / \mathrm{g}$ TCOD added, respectively. The addition of manure with SSO, increased the methane yield in the co-digesters. The amounts of the biomethane yields were within a range between 316 and $362 \mathrm{mLCH}_{4} / \mathrm{g}$ TCOD added in the co-digesters. The highest yield of 362 $\mathrm{mLCH}_{4} / \mathrm{g}$ TCOD added occurred at the manure/SSO mixing ratio of 7:3. $\mathrm{CH}_{4}$ yield increased by $26 \%$ and $20 \%$ compared to single digestion of manure and SSO, respectively.

Figure 6.5. b), shows the methane yields in $\mathrm{mLCH}_{4} / \mathrm{g}$ VSS added. Manure produced $654 \mathrm{mLCH}_{4} / \mathrm{g}$ VSS added. The yield was higher for SSO corresponding to $793 \mathrm{mLCH}_{4} / \mathrm{g}$ VSS added. The highest yield occurred at the manure/SSO mixing ratio of 7:3 corresponding to $876 \mathrm{mLCH}_{4} / \mathrm{g}$ VSS added. The $\mathrm{CH}_{4}$ yield improved by $30 \%$ compared to the digestion of manure alone. All of the reactors with mixings of manure and SSO resulted in higher amounts of methane yields per unit mass of VSS added. The methane yields of from 819 to $847 \mathrm{mLCH}_{4} / \mathrm{g}$ VSS added were obtained in codigestion of manure and SSO at different mixing ratios.

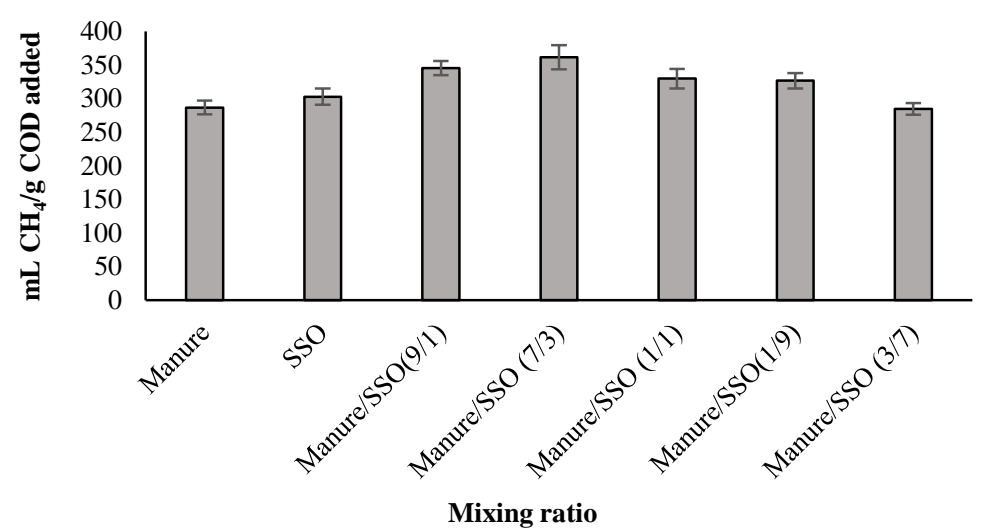

6.5. a)

Mixing ratio 


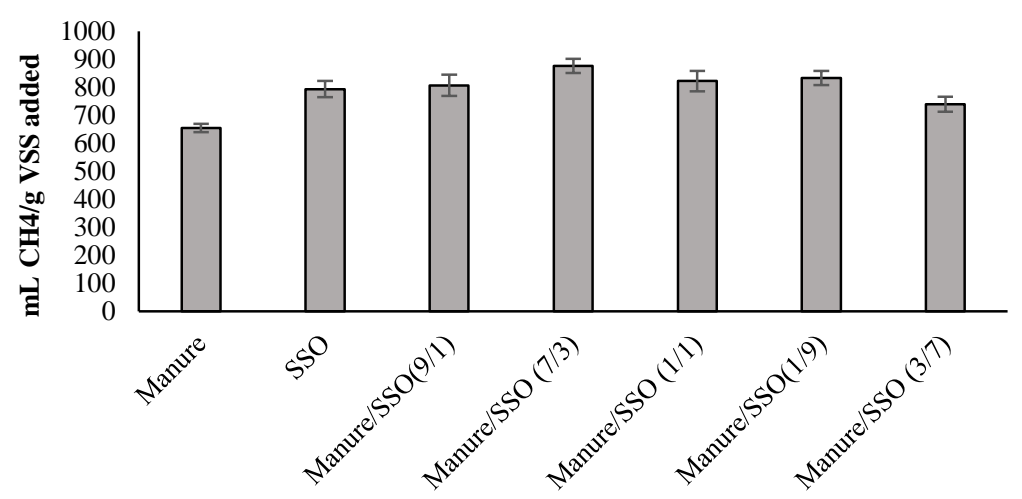

6.5. b)

Mixing ratio

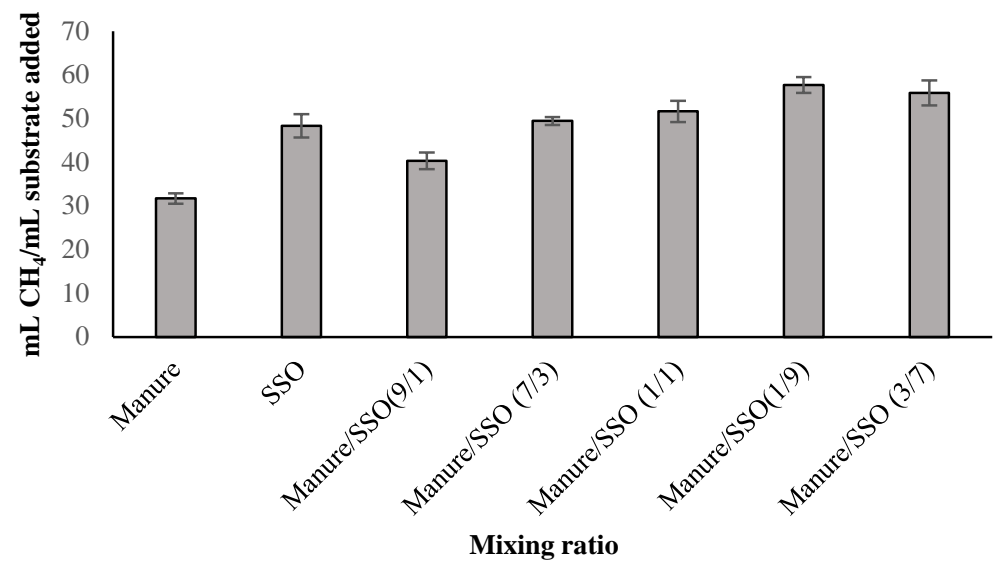

Figure 6.5. Methane yields a) per unit mass TCOD added, $b$ ) per unit mass of VSS added and c) per volume substrate added at different mixing ratio of TWAS and manure

Figure 6.5. c), shows the methane yield per unit volume of substrate added in $\mathrm{mLCH}_{4} / \mathrm{mL}$ substrate added. Manure and SSO individually produced $32 \mathrm{mLCH}_{4} / \mathrm{mL}$ substrate added and $48 \mathrm{mLCH}_{4} /$ $\mathrm{mL}$ substrate added, respectively. The reactors with the combinations of manure and SSO resulted in methane yields ranging from $40 \mathrm{mLCH}_{4} / \mathrm{mL}$ substrate added to $58 \mathrm{mLCH}_{4} / \mathrm{mL}$ substrate added. The most values of the $\mathrm{CH}_{4}$ yield in terms of unit volume of substrate added corresponded to manure/SSO co-digestion at the mixing ratios of 1:9 and 3:7.

Biodegradable fraction of the feedstocks was obtained using the Eq. 4.1 and the result is summarized in Figure 6.6. Manure and SSO individually had biodegradable fractions of 72\% and $76 \%$, respectively. Co- digestion increased biodegradable fraction of the feedstocks by $20 \%$ and $26 \%$ in comparison with the control reactors digesting only SSO and manure, respectively. The 
most percentage of biodegradable fraction occurred at the reactor co-digesting manure with SSO at the mixing ratio of $7: 3$. This in good compliance with the maximum methane production at the same mixing ratio.

As shown in Figure 6.6, the trend of biodegradable fraction variations in the digesters conforms to the trend of the methane yields obtained by the experimental results for the corresponding digesters. It was verified that addition of manure to SSO as co-substrate increased biodegradability and enhanced methane production in the rectors co-digesting manure and SSO. The reason would be the existence of abundant of methanogenic populations in manure that was introduced to the co-digesters and enhanced degradation of organic matters.

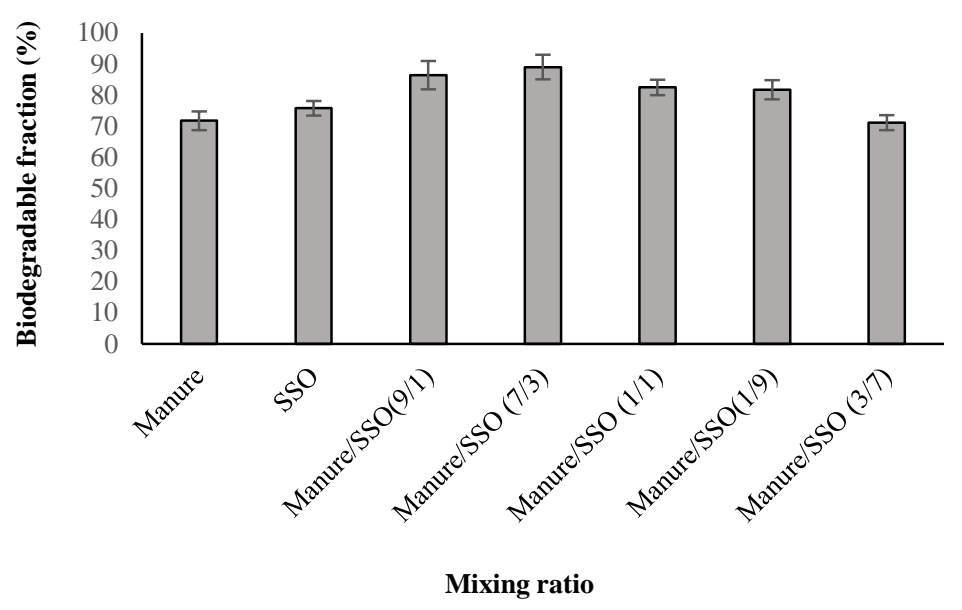

Figure 6.6. Biodegradable fraction of the feedstocks for different mixture ratios of TWAS and manure

\subsection{Synergistic effect}

Synergistic effect was assessed using Eq. 4.2 as presented in chapter 4 and the result is summarized in Figure 6.7. Synergistic effect represents the percentage of additional methane yield for cosubstrates that was measured by the experiment, over the weighted average of the methane yield of individual substrates per unit volume of substrate added. As demonstrated in Fig 6.7, the most synergetic impact corresponds to the co-digestion of manure/SSO at the mixing ratio of 7:3. In codigestion of manure with $\mathrm{SSO}$, the increase of $\mathrm{CH}_{4}$ yield due to synergistic effect ranged from $22 \%$ to $36 \%$ corresponding to the reactor co-digesting manure and SSO at the mixing ratio of 9:1 and 7:3, respectively. It was revealed that only adding the fraction of manure in co-digesters, did not lead to increasing synergy. Although increasing the fraction of manure would introduce more 
populations of methanogenic archaea and bacteria, a balance between the microbial populations and nutrient is necessary for the effective microbial growth and enhanced methanogenesis.

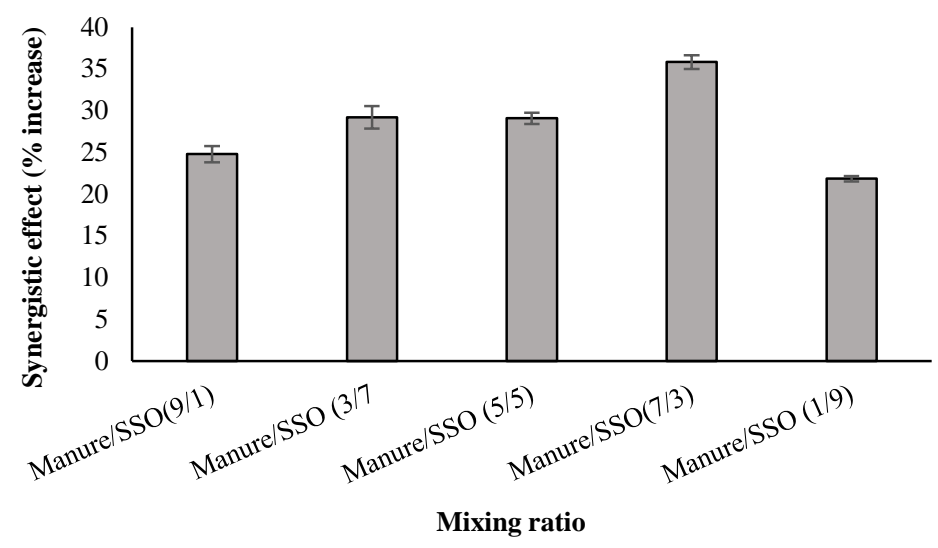

Figure 6.7. Synergetic effect of co-digestion at different mixing ratios of manure and SSO

\subsection{COD: $\mathrm{N}$ and lipids: proteins: carbohydrates ratios}

Table 6.2 presents the COD:N ratios, lipids: proteins: carbohydrates ratios, the ultimate methane production, and the methane yield per unit mass of COD added of the digesters with different mixing ratios. The COD:N ratios were 33 and 42 corresponding to SSO and manure alone, respectively. The values of COD:N varied from 34 to 41 in the co-digesters. The ultimate methane production and methane yield ranged from 919 to $1186 \mathrm{~mL}$, and 287 to $363 \mathrm{~mL} / \mathrm{g}$ TCOD added for different mixing ratios, respectively.

As shown in Table 6.2 The minimum ultimate methane and methane yield occurred at mono digestion of manure corresponding to the COD: $\mathrm{N}$ ratio of 42 and lipids: proteins: carbohydrates ratio of 1:4.2:21. However, the maximum ultimate methane and methane yield occurred at the mixing ratio of 7:3 corresponding to the COD:N ratio of 41 and lipids: proteins: carbohydrates ratio of 1:3.5:18.5. SSO alone with a lipids: proteins: carbohydrates ratio of 1:2:12 produced 15\% more ultimate methane than manure alone with the lipids: proteins: carbohydrates ratio of 1:4.2:21. Although, it only resulted in 6\% more methane yield per unit mass of COD added than manure. On the other side, the 1:4.2:21 lipids: proteins: carbohydrates ratios for manure alone and 1:4:20 for manure/SSO co-digestion at the mixing ratio of 9:1 had only a minor variation while the ultimate methane and the methane yield were $23 \%$ and $20 \%$ higher for the latter. 
Table 6.2. Ultimate $\mathrm{CH}_{4}$ and yield at different ratios of the substrates, $\mathrm{COD}: \mathrm{N}$, and Lipids:Proteins:Carbohydrates

\begin{tabular}{ccccccc}
\hline Digester code & $\begin{array}{c}\text { TWAS: Manure } \\
\text { (V/V) }\end{array}$ & COD:N & $\begin{array}{c}\text { Feedstock } \\
\text { ratio codes }\end{array}$ & $\begin{array}{c}\text { Lipids: Proteins: } \\
\text { Carbohydrates }\end{array}$ & $\begin{array}{c}\text { Ultimate } \\
\mathrm{CH}_{4}(\mathrm{~mL})\end{array}$ & $\begin{array}{c}\mathrm{mLCH} / \mathrm{g} \\
\text { TCOD added }\end{array}$ \\
\hline \hline Manure & $1: 0$ & 42 & $\mathrm{CC}$ & $1: 4.2: 21$ & 919 & 287 \\
SSO & $0: 1$ & 33 & $\mathrm{BB}$ & $1: 2: 12$ & 1063 & 303 \\
$\begin{array}{c}\text { Manure/SSO } \\
7: 3\end{array}$ & $7: 3$ & 41 & $\mathrm{~A}$ & $1: 3.5: 18.5$ & 1186 & 363 \\
$\begin{array}{c}\text { Manure/SSO } \\
\text { 9:1 }\end{array}$ & $9: 1$ & 39 & $\mathrm{~B}$ & $1: 4: 20$ & 1129 & 344 \\
$\begin{array}{c}\text { Manure/SSO } \\
5: 5\end{array}$ & $1: 1$ & 37 & $\mathrm{C}$ & $1: 3: 17$ & 1136 & 330 \\
$\begin{array}{c}\text { Manure/SSO } \\
\text { 3:7 }\end{array}$ & $3: 7$ & 35 & $\mathrm{D}$ & $1: 2.7: 15$ & 1095 & 327 \\
$\begin{array}{c}\text { Manure/SSO } \\
1: 9\end{array}$ & $1: 9$ & 34 & $\mathrm{E}$ & $1: 2: 13$ & 1115 & 316 \\
\hline \hline
\end{tabular}

Manure alone with lipids:proteins:carbohydrates ratio of 1:4.2:21 corresponded the minimum methane yield while the maximum yield occurred at manure:SSO mixing ratio of 7:3 and lipids:proteins:carbohydrates ratio of 1:3.5:18.5. This increase of the methane would be the result of microbial population diversity introduced by manure to the co-digesters and the synergetic impact of co-digestion rather than the ratio of lipids: proteins: carbohydrates.

Figure 6.8. shows the main effect plot for $\mathrm{CH}_{4}$ yield data mean in response to feedstock and lipids:proteins:carbohydrates ratios in $\mathrm{AnCoD}$ of manure/SSO. As shown in figure 6.8, the different lipids:proteins:carbohydrates ratios for the different mixing ratios of the feedstocks have significant effect on the methane yield. 


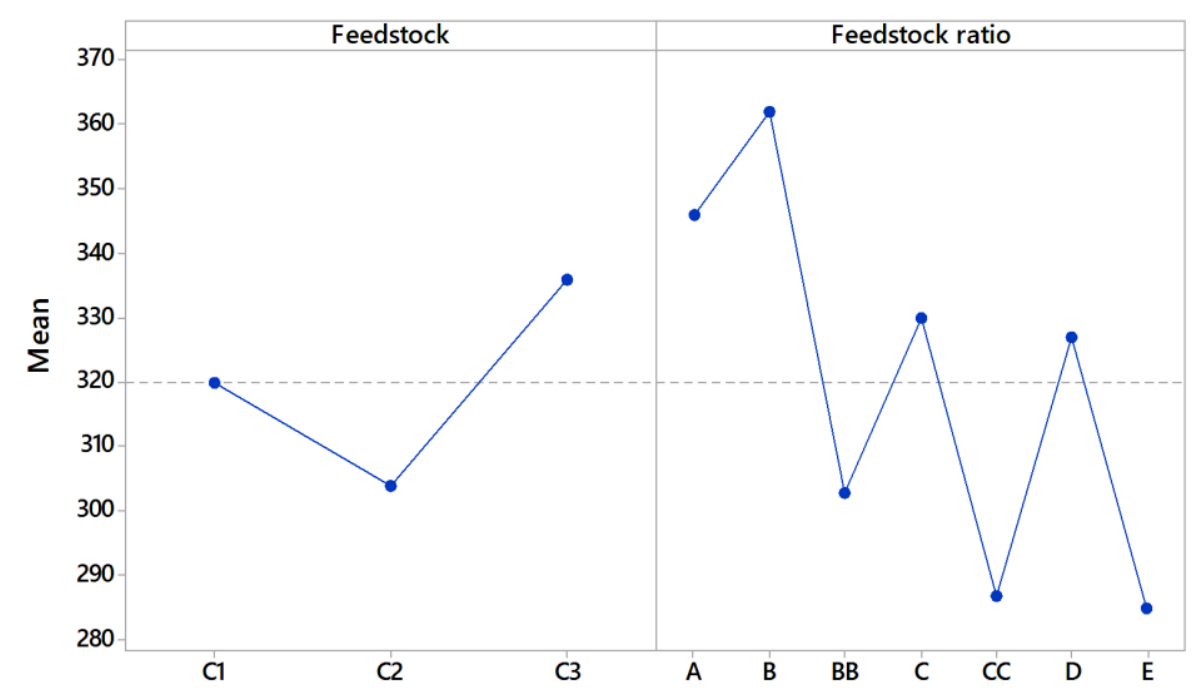

Figure 6.8. Main effect plot for $\mathrm{CH}_{4}$ yield data mean in response to feedstock and lipids:proteins:carbohydrates ratios in AnCoD of manure/SSO

Figure 6.9 shows the matrix plot for the variations of the methane yield and the ultimate methane versus COD:N ratio, proteins: lipids, carbohydrates: lipids, and carbohydrates: proteins ratios. As illustrated in Figure 6.9. a, the trend of variations of the methane yields versus COD:N and versus proteins: carbohydrates were similar. lipids: proteins and lipids: carbohydrates also showed a similar trend but for both of them the trend was the mirror image of COD:N and proteins: carbohydrates. The ultimate methane as illustrated in Figure 6.9. $\mathrm{b}$ also demonstrated a similar response to those ratios. These observations were contrary to the results of TWAS:SSO and TWAS/Manure co-digestion. The reason would be the minor variations of proteins: lipids ratios at the different combinations of manure and SSO. As mentioned earlier, in co-digestion of manure/SSO the methane yield in the co-digesters could more depend on the microbial diversity than the ratio of the lipids: proteins: carbohydrates. 

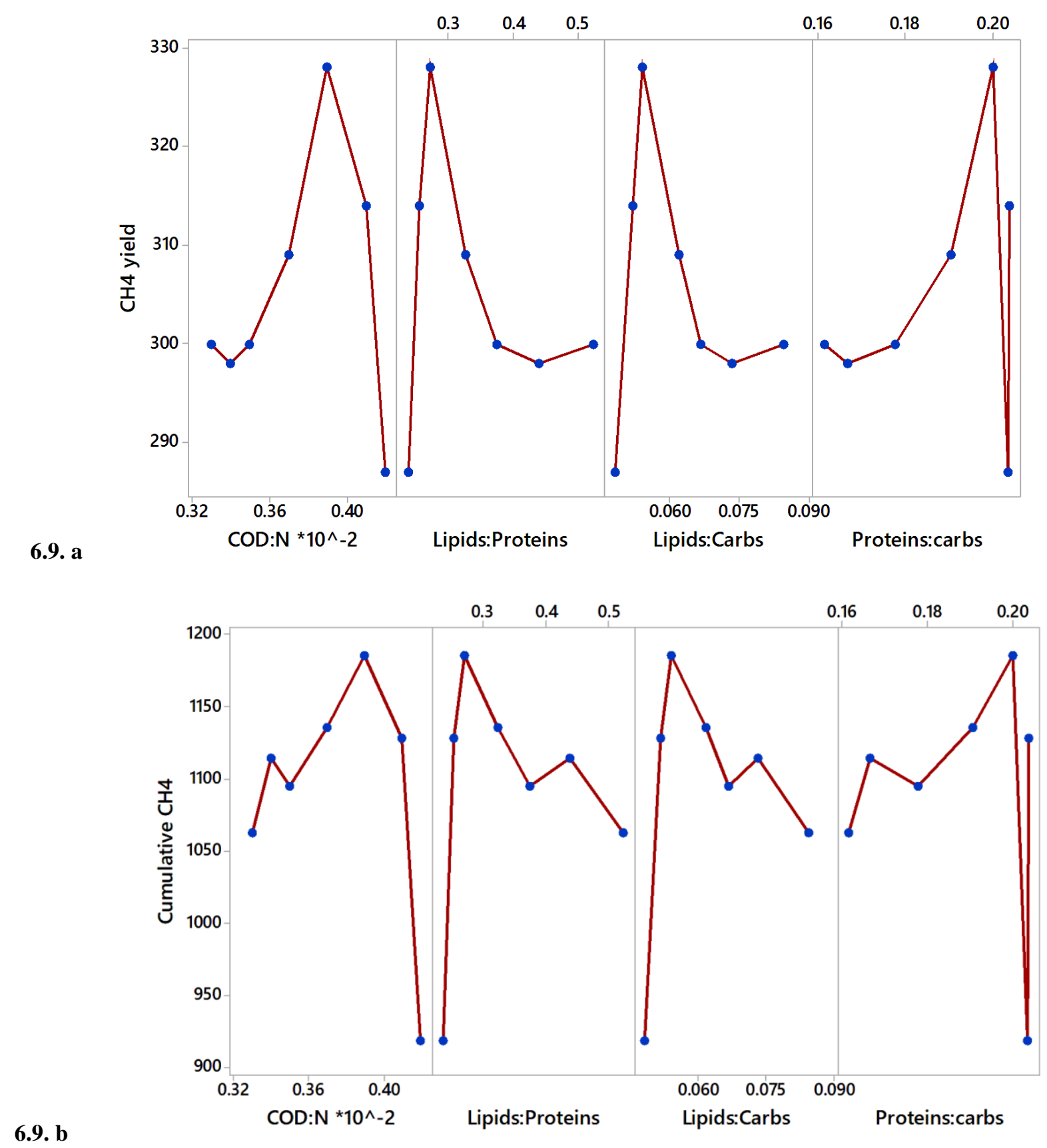

Figure 6.10. Matrix plot for: a. ultimate $\mathrm{CH}_{4}$ and b. $\mathrm{CH}_{4}$ yield at different COD:N and Lipids: Proteins, Lipids:

Carbohydrates, and Proteins: Carbohydrates Ratios

\subsection{Kinetic analysis results}

The results of kinetic study by modified Gompertz model using Eq. 3.5 is summarized in Table 6.3. The model was applied to the experimental data from mono and co-digestion of manure and 
SSO. The values of $\mathrm{P}$ for the maximum methane production per unit substrate $(\mathrm{mL} / \mathrm{g}), R_{m}^{e}$, the maximum methane production rate $(\mathrm{mL} / \mathrm{g} \mathrm{h})$, and $\lambda$, the lag phase time (d) were calculated and the results values are summarized in Table 6.3. The modified Gompertz model for mono- and codigestions of manure and SSO, rather showed a good fit to the experimental results with less than $10 \%$ diversion from the measured values. The estimated values and their correlation with the mixing ratio of the feedstock were in good compliance with the data obtained by the experiment. An increasing in $\mathrm{P}$ values was observed in co-digestion of manure with SSO which conformed to the experimental results.

Table 6.3. Summary of results of kinetic study using modified Gompertz model

\begin{tabular}{ccccc}
\hline & $\mathbf{P}(\mathbf{m L})$ & $\boldsymbol{R}_{\boldsymbol{m}}^{\boldsymbol{e}}\left(\frac{\mathbf{m L}}{\mathbf{D}}\right)$ & $\lambda(\mathbf{d})$ & $\mathbf{R}^{2}$ \\
\hline \hline Manure & 875 & 48 & 0.4 & 0.999 \\
\hline SSO & 998 & 83 & 1.6 & 0.999 \\
\hline Manure/SSO 7/3 & 1112 & 89 & 0.9 & 0.999 \\
\hline Manure/SSO 9/1 & 1065 & 57 & 0.7 & 0.999 \\
\hline Manure/SSO 5/5 & 1060 & 66 & 0.9 & 0.999 \\
\hline Manure/SSO 1/9 & 1018 & 76 & 1.1 & 0.999 \\
\hline Manure/SSO 3/7 & 936 & 81 & 0.8 & 0.999 \\
\hline
\end{tabular}

The trend of $\mathrm{P}$ variations complied with the experimental data as it showed the same trend in response to the corresponding mixing ratios. For instance, the highest $\mathrm{P}$ value of $1112 \mathrm{~mL}$ corresponded to the mixing ratio of 7:3. The lag phase varied from 0.01 to 0.7 days for different substrate mixing ratios. The lag phase time was quite short and less than 1 day for all the digesters. A short lag phase is advantageous as it does not demand for a long residence time and therefore, it does not require a large reactor volume which reduces the operational costs of the system. The values of $\mathrm{R}_{\max }^{\mathrm{e}}$ ranged from 48 to $89 \mathrm{~mL} / \mathrm{d}$ corresponding to manure mono digestion and manure/SSO mixing ratio of 7:3, respectively. 


\section{6. Hydrolysis/acidification}

This experiment was carried out for evaluating the hydrolysis/acidification phase in anaerobic codigestion of manure and SSO. A series of analysis such as degree of solublization, synergetic effect of co-digestion at different mixing ratios on hydrolysis and liquefaction, volatile fatty acids (VFAs) yield, and hydrolysis kinetics of lipids, proteins, and carbohydrates in co-digestion of manure and SSO was carried out and the results are summarized below.

Initially, characterization of the feedstocks in triplicates was conducted and the mean values are presented in Table 6.4. As shown in Table 6.4, both manure and SSO have high amount of COD concentration which is above $100 \mathrm{~g} / \mathrm{L}$. Therefore, COD concentrations of the feed for all of the reactors containing the combinations of manure and SSO were above $100 \mathrm{~g} / \mathrm{L}$. Manure contains high amount of carbohydrates and proteins. Adding manure to SSO increased carbohydrates and proteins concentrations in the co-digesters compared to the reactor digesting SSO alone. In order to obtain the hydrolysis rate coefficients of COD, lipids, proteins, and manure, the concentrations of total, soluble and particulate COD; total, soluble and particulate proteins; and total, soluble and particulate carbohydrates were monitored over time.

The data form total and soluble COD (SCOD) concentrations monitoring in all the digesters over time during a 72-h hydrolysis/acidification period was used to calculate the degree of COD solublization for each digester. As explained earlier, the degree of solublization was calculated using Eq. 3.1 and the result is summarized in Figure 6.10.

As illustrated in figure 6.10, the degree of the COD solublization ranged from $24 \%$ to $35 \%$. The most solublization of the COD content was $35 \%$ which corresponded to manure:SSO combination of 3:7 and 1:9. The COD solublization in mono digestion of manure and SSO were $24 \%$ and $28 \%$, respectively. The reactors containing only manure had the lowest degree of solublization. Manure resulted in a relatively lower solublization than SSO which could be due to the presence of some recalcitrant contents such as fibers and cellulosic compounds in manure that delay its hydrolysis and liquefaction. 
Table 6.4. Characteristics of the feedstocks at different mixing ratios of manure and SSO

\begin{tabular}{ccccccccc}
\hline & & Manure & SSO & M $/$ SSO & M/SSO & M/SSO & M/SSO & M/SSO \\
& & & & $9 / 1$ & $7 / 3$ & $5 / 5$ & $3 / 7$ & $1 / 9$ \\
\hline Parameters & Units & Mixture & Mixture & Mixture & Mixture & Mixture & Mixture & Mixture \\
& & $(1)$ & $(2)$ & $(3)$ & $(4)$ & $(5)$ & $(6)$ & $(7)$ \\
TCOD & & Ave. & & & & & & \\
SCOD & $\mathrm{g} / \mathrm{L}$ & 101 & 115 & 103 & 105 & 108 & 111 & 114 \\
TSS & $\mathrm{g} / \mathrm{L}$ & 55 & 47 & 54 & 52 & 51 & 49 & 48 \\
VSS & $\mathrm{g} / \mathrm{L}$ & 32.1 & 39.9 & 32.9 & 34.5 & 36.0 & 37.6 & 39.2 \\
TS & $\mathrm{g} / \mathrm{L}$ & 69 & 78 & 69 & 71 & 73 & 75 & 77 \\
VS & $\mathrm{g} / \mathrm{L}$ & 59 & 64 & 59 & 60 & 61 & 62 & 63 \\
Ammonia & $\mathrm{g} / \mathrm{L}$ & 0.02 & 1.40 & 0.16 & 0.4 & 0.7 & 1.0 & 1.3 \\
pH & & 5.6 & 5.8 & 5.9 & 5.7 & 5.7 & 5.6 & 5.8 \\
Alkalinity & $\mathrm{g}$ CaCO3/L & 7.2 & 6.7 & 7.2 & 7.1 & 7.0 & 6.9 & 6.8 \\
TN & $\mathrm{g} / \mathrm{L}$ & 1.7 & 4.2 & 2.0 & 2.5 & 3.0 & 3.5 & 4.0 \\
TSN & $\mathrm{g} / \mathrm{L}$ & 0.1 & 1.1 & 0.2 & 0.4 & 0.6 & 0.8 & 1.0 \\
T-Carbs & $\mathrm{g} / \mathrm{L}$ & 26.8 & 14 & 25.5 & 23.0 & 20.5 & 17.9 & 15.4 \\
T-Proteins & $\mathrm{g} / \mathrm{L}$ & 5.4 & 2 & 5.1 & 4.4 & 3.8 & 3.1 & 2.5 \\
T-Lipids & $\mathrm{g} / \mathrm{L}$ & 1.7 & 1.5 & 1.7 & 1.6 & 1.6 & 1.5 & 1.5 \\
\hline \hline M: Manure & & & & & & & &
\end{tabular}

It was observed that co-digestion increased solublization as all of the reactors containing the mixings of manure and SSO achieved a higher degree of solublization than the control reactors.

A $46 \%$ and $25 \%$ improvement in solublization was achieved in the co-digestion of manure and SSO in comparison with the control reactors digesting only manure and only SSO. Although SSO demonstrated more solublization than manure, no correlation between the portion of SSO and the degree of solublization at different mixing ratio was observed. This could verify that a proper mixing ratio is requires to enhance the microbial synergy for the process improvement.

The synergistic effect on the solublization of the feedstocks was evaluated by comparing the theoretical degree of solublization of each co-digester to the measured data from the experiment. The results indicated that co-digestion improved solublization by improving microbial synergy. As shown in Figure 6.11, all co-digesters achieved an improvement in solublization due to the synergistic effect of the microbial communities. The synergistic effect varied from 18 to $34 \%$ corresponding to manure/SSO mixing ratios of 5:5 and 9:1, respectively. 


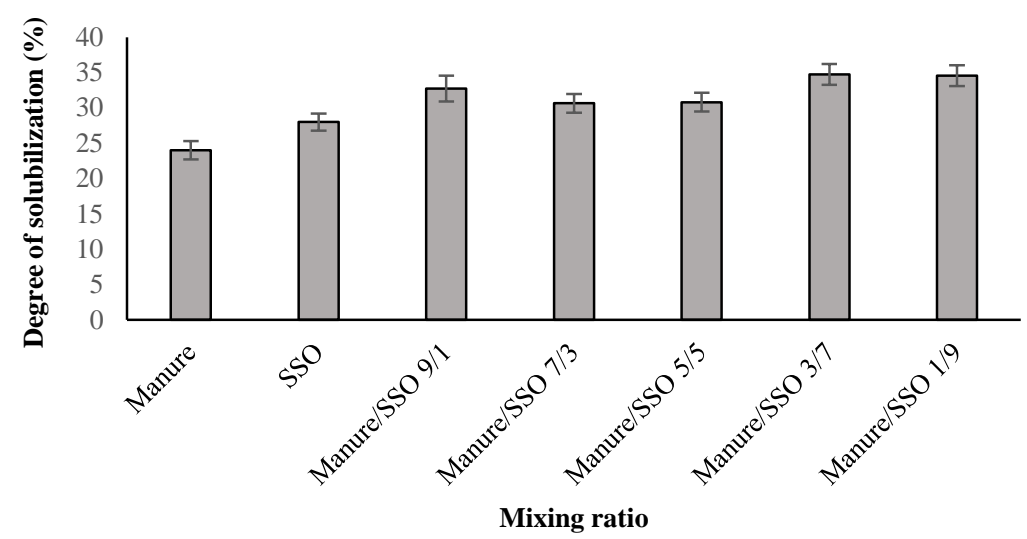

Figure 6.10. Degree of COD solublization at different mixing ratios of Manure/SSO

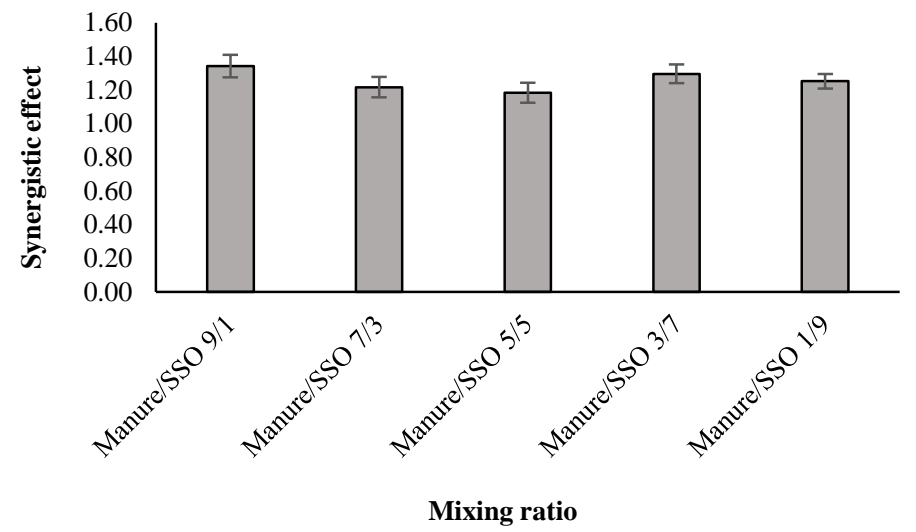

Figure 6.11. Synergistic effect on solublization at different mixing ratios of Manure/SSO

The monitoring of VFAs concentrations over time showed an increasing trend during the 72-hr of the hydrolysis/acidification process. The total VFAs yields were calculated in terms of mass of produced VFAs per mass of VSS added (mg VFAs/g VSS added). As illustrated in Figure 6.12, SSO alone had more VFAs yields than manure alone. The VFAs yield in the co-digester was correlated to the mixing ratios of the feedstocks so that the VFAs yield increased by increasing the fraction of SSO in the co-digesters. The trend of VFAs yield did not comply with the trend of COD solublization of the corresponding mixing ratios (Figure 6.10). Hence, hydrolysis/liquefaction occurred independently of acidification and followed a different trend from acidification. All of the reactors containing the mix of manure and SSO had higher VFAs yield than the control reactors. The VFAs yields were $231 \mathrm{mg}$ VFAs/g VSS added and $325 \mathrm{mg}$ VFAs/g VSS added for the manure and SSO mono digestions, respectively. The maximum VFAs yield of $400 \mathrm{mg}$ VFAs/g VSS added was achieved by manure/SSO ratio of 1:9. 


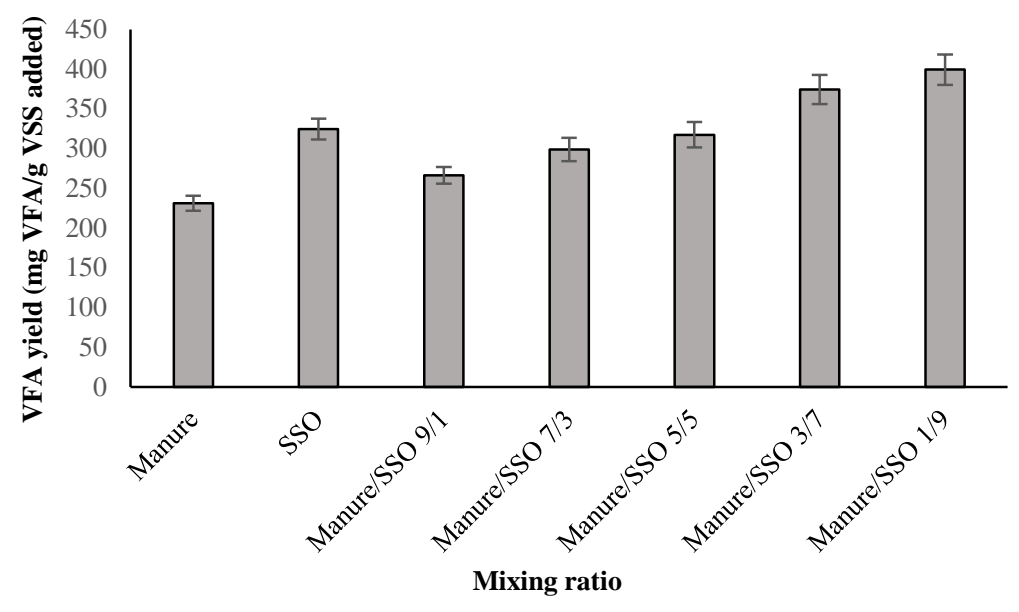

Figure 6.12. Total VFAs yield at different mixing ratios of Manure/SSO

Monitoring soluble and particulate COD over time, indicated an increasing trend in solublization of COD and particulate COD degradation. Furthermore, the monitoring of soluble and particulate lipids, proteins, and carbohydrates over time also demonstrated an increasing trend in solublization and particulate matter degradation. Similar to the previous experiments, in codigestion of manure and SSO, the hydrolysis and solublization rates of the lipids and proteins, were slower than carbohydrate. These results are summarized in the tables presented in the appendix. The hydrolysis rate coefficient $\left(\mathrm{K}_{\mathrm{h}}\right)$ based on first order kinetics (equations 3.3 and 3.4) was calculated using AquaSim 2.0 for COD, lipids, proteins and carbohydrates based on the particulate degradation and results are summarized in Table 6.5.

In manure and SSO co-digestion, hydrolysis did not show the same trend as methanogenesis. The hydrolysis kinetics showed a higher rate in the manure:SSO mixing ratio of 3:7 while the most methane yield occurred at the mixing ratio of 7:3 with more fraction of manure. This would be due to the more microbial population of methanogens which was introduced by more fraction of manure in the co-digester. Similar to the co-digestion of TWAS with SSO and TWAS with manure, the improvement of the hydrolysis in co-digestion of manure with SSO resulted from the improvement of proteins and carbohydrates hydrolysis than that of lipids contents of the feedstocks. Unlike TWAS/manure and TWAS/SSO co-digestion, the fluctuations of hydrolysis rate coefficient of lipids and carbohydrates at different feedstocks mixing ratios showed similar 
trend in manure/SSO co-digestion. Nevertheless, in co-digestion of manure with SSO the variations of $\mathrm{K}_{\mathrm{h}}$ for proteins demonstrated a different trend from that of lipids and carbohydrates. The maximum hydrolysis rate of proteins corresponded to the manure:SSO mixing ratio of 1:9. However, the maximum $\mathrm{K}_{\mathrm{h}}$ values for lipids and carbohydrates were 0.11 and 0.68 corresponding to the mixing ratio of $3: 7$.

Table 6.5. Hydrolysis rate coefficients for COD, lipids, proteins, and carbohydrate content in co-digestion of SSO and manure at different mixing ratios

\begin{tabular}{lccccccc}
\hline \multicolumn{1}{c}{$\mathbf{K}_{\mathbf{h}}$} & $\mathrm{M}^{*} / \mathrm{SSO} 1 / 9$ & $\mathrm{M} / \mathrm{SSO} 3 / 7$ & $\mathrm{M} / \mathrm{SSO} 5 / 5$ & $\mathrm{M} / \mathrm{SSO} 7 / 3$ & $\mathrm{M} / \mathrm{SSO}$ 9/1 & Manure & SSO \\
\hline \hline $\mathrm{K}_{\mathrm{h} \text { COD }}$ & 0.37 & 0.38 & 0.31 & 0.32 & 0.35 & 0.23 & 0.30 \\
\hline $\mathrm{K}_{\mathrm{h} \text { Lipids }}$ & 0.09 & 0.11 & 0.08 & 0.07 & 0.08 & 0.07 & 0.08 \\
\hline $\mathrm{K}_{\mathrm{h} \text { Proteins }}$ & 0.35 & 0.33 & 0.25 & 0.29 & 0.32 & 0.21 & 0.28 \\
\hline $\mathrm{K}_{\mathrm{h} \text { Carbohydrates }}$ & 0.63 & 0.68 & 0.57 & 0.55 & 0.61 & 0.43 & 0.55
\end{tabular}

${ }^{*} \mathrm{M}$ : Manure 


\section{Chapter 7}

Results and discussion

$$
\begin{gathered}
\text { TWAS, Manure, SSO Co- } \\
\text { digestion }
\end{gathered}
$$




\section{Results and discussion- TWAS, manure, SSO co-digestion 7.1. BMP of TWAS, manure, SSO}

This experiment was conducted on ternary co-digestion of TWAS, manure, and SSO. Manure slurry was prepared as discussed in chapter 3 and was fed to the reactors in different combinations with SSO and TWAS in triplicates. Control reactors containing TWAS, manure, and SSO individually were also used in triplicates. The influence of feedstocks mixing ratios and their correlation with the lipids: proteins: carbohydrates ratios on biomethane production in anaerobic co-digestion of TWAS/manure/SSO was evaluated. The characteristics of the feed in each digester with different mixing ratios of the substrates for the average of three measurement of each parameter are summarized in Table 7.1.

As presented in Table 7.1, both manure and SSO have high amount of COD concentrations compared to TWAS. The COD concentrations of the feedstocks were 100, 109, and $101 \mathrm{~g} / \mathrm{L}$ for the digesters fed with manure, SSO and mixture of TWAS/manure/SSO at 1:1:8 ratio, respectively. Other reactors which contained the combinations of TWAS, manure, and SSO had a COD concentration ranging from 53 to $95 \mathrm{~g} / \mathrm{L}$. VSS concentrations were 26.5, 45.4, and 47.0 for TWAS, manure, and SSO respectively and ranged from 30.4 to 44.8 for the reactors containing the combinations of them. The amount of carbohydrates and lipids of manure and SSO were significantly more than that of TWAS. Therefore, addition of manure and SSO to TWAS increased the carbohydrates and lipids content of the co-digesters compared to the rectors digesting only TWAS.

Carbohydrates concentrations were within a range between 5.4 to $23.8 \mathrm{~g} / \mathrm{L}$ in the co-digesters. Total proteins concentrations of TWAS, manure, and SSO were 3.9, 5.8, and 2.4 respectively and varied from 2.9 to $4.5 \mathrm{~g} / \mathrm{L}$ in the co-digesters. The total lipids concentrations of TWAS was 0.4 $\mathrm{g} / \mathrm{L}$ while manure and SSO had lipids concentrations of 1.4 and $1.5 \mathrm{~g} / \mathrm{L}$ respectively. Adding manure and SSO increased the lipids content in the reactors containing the combination of TWAS, manure, and SSO compared to the reactors containing only TWAS. The total lipids concentrations varied from 0.6 to $1.3 \mathrm{~g} / \mathrm{L}$ in the co-digesters containing the mix of the three feedstocks. $\mathrm{pH}$ was around neutral point varying from 7 to 7.2 for all of the reactors. 
Table 7.1. Characteristics of feed to digesters with different mixing ratios of TWAS/ manure/SSO

\begin{tabular}{|c|c|c|c|c|c|c|c|c|c|c|c|c|c|}
\hline & & TWAS & Manure & SSO & $\begin{array}{c}\mathrm{T}^{*}: \mathrm{M}^{* *}: \mathrm{SSO} \\
8: 1: 1\end{array}$ & $\begin{array}{c}\text { T:M:SSO } \\
1: 8: 1\end{array}$ & $\begin{array}{c}\text { T/M/SSO } \\
1: 1: 8\end{array}$ & $\begin{array}{l}\text { T/M/SSO } \\
5: 2.5: 2.5\end{array}$ & $\begin{array}{l}\text { T/M/SSO } \\
2.5: 5: 2.5\end{array}$ & $\begin{array}{l}\text { T/M/SSO } \\
2.5: 2.5: 5\end{array}$ & $\begin{array}{c}\text { T/M/SSO } \\
4: 4: 2\end{array}$ & $\begin{array}{c}\text { T/M/SSO } \\
2: 4: 4\end{array}$ & $\begin{array}{c}\text { T/M/SSO } \\
4: 2: 4\end{array}$ \\
\hline Parameters & Units & $\begin{array}{c}\text { Mixture } \\
\text { (1) }\end{array}$ & $\begin{array}{c}\text { Mixture } \\
\text { (2) }\end{array}$ & $\begin{array}{c}\text { Mixture } \\
\text { (3) }\end{array}$ & Mixture (4) & $\begin{array}{c}\text { Mixture } \\
(5)\end{array}$ & $\begin{array}{c}\text { Mixture } \\
\text { (6) }\end{array}$ & $\begin{array}{c}\text { Mixture } \\
(7)\end{array}$ & $\begin{array}{c}\text { Mixture } \\
(8)\end{array}$ & $\begin{array}{c}\text { Mixture } \\
(9)\end{array}$ & $\begin{array}{c}\text { Mixture } \\
(10)\end{array}$ & $\begin{array}{c}\text { Mixture } \\
\text { (11) }\end{array}$ & $\begin{array}{c}\text { Mixture } \\
\text { (12) }\end{array}$ \\
\hline TCOD & $\mathrm{g} / \mathrm{L}$ & 40 & 100 & 109 & 53 & 95 & 101 & 72 & 87 & 89 & 78 & 71 & 79 \\
\hline SCOD & $\mathrm{g} / \mathrm{L}$ & 1.4 & 42 & 41 & 9 & 38 & 37 & 21 & 32 & 31 & 26 & 25 & 25 \\
\hline TSS & $\mathrm{g} / \mathrm{L}$ & 31 & 52 & 62 & 37 & 51 & 58 & 44 & 50 & 52 & 46 & 42 & 48 \\
\hline VSS & $\mathrm{g} / \mathrm{L}$ & 26.5 & 45.4 & 47.0 & 30.4 & 43.7 & 44.8 & 36.3 & 41.1 & 41.5 & 38.2 & 33.2 & 38.5 \\
\hline $\mathrm{TS}$ & $\mathrm{g} / \mathrm{L}$ & 38.9 & 67.8 & 67.0 & 44.6 & 64.8 & 64.3 & 53.2 & 60.4 & 60.2 & 56.1 & 48.1 & 55.9 \\
\hline VS & $\mathrm{g} / \mathrm{L}$ & 35.2 & 55.6 & 49.6 & 38.6 & 52.9 & 48.7 & 43.9 & 49.0 & 47.5 & 46.2 & 38.0 & 45.0 \\
\hline $\mathrm{pH}$ & - & 7.0 & 7.0 & 7.2 & 7.1 & 7.0 & 7.0 & 7.1 & 7.1 & 7.1 & 7.0 & 7.0 & 7.1 \\
\hline Alkalinity & $\begin{array}{c}\mathrm{g} \\
\mathrm{CaCO}_{3} / \mathrm{L}\end{array}$ & 1.9 & 5.2 & 6.2 & 2.7 & 5.0 & 5.7 & 3.8 & 4.6 & 4.9 & 4.1 & 3.9 & 4.3 \\
\hline $\mathrm{TN}$ & $\mathrm{g} / \mathrm{L}$ & 2.8 & 2.1 & 4.0 & 2.8 & 2.4 & 3.7 & 2.9 & 2.7 & 3.2 & 2.7 & 2.6 & 3.1 \\
\hline TSN & $\mathrm{g} / \mathrm{L}$ & 0.4 & 0.1 & 1.0 & 0.4 & 0.2 & 0.8 & 0.5 & 0.4 & 0.6 & 0.4 & 0.5 & 0.6 \\
\hline Total carbs & $\mathrm{g} / \mathrm{L}$ & 1.5 & 27.8 & 14.1 & 5.4 & 23.8 & 14.2 & 11.3 & 17.8 & 14.4 & 14.6 & 11.5 & 11.8 \\
\hline $\begin{array}{c}\text { Total } \\
\text { proteins }\end{array}$ & $\mathrm{g} / \mathrm{L}$ & 3.9 & 5.8 & 2.4 & 3.9 & 5.2 & 2.9 & 4.0 & 4.5 & 3.6 & 4.3 & 2.9 & 3.7 \\
\hline Total lipids & $\mathrm{g} / \mathrm{L}$ & 0.4 & 1.4 & 1.5 & 0.6 & 1.3 & 1.4 & 0.9 & 1.2 & 1.2 & 1.0 & 0.9 & 1.0 \\
\hline
\end{tabular}

${ }^{*}$ T: TWAS

** M: Manure 
Operation of the digesters carried on until no significant amount of biogas was produced. Fig. 7.1 shows the profile of the cumulative biomethane production versus time during the co-digestion of TWAS, manure and SSO including the control rectors. SSO alone produced more cumulative methane than TWAS in the control reactors. The amount of methane produced by SSO alone was $13 \%$ more methane than manure alone.

All co-digesters produced more biomethane than the control reactors containing only TWAS. The amounts of ultimate $\mathrm{CH}_{4}$ obtained by single digestion of SSO and manure were higher than that of TWAS alone by 3.2 and 2.9 fold. As illustrated in figure 7.1, all of the co-digesters generated higher amounts of $\mathrm{CH}_{4}$ than the control reactors containing only manure. The reactors with the mix of the three feedstocks at the ratios of $8: 1: 1$ and 5:2.5:2.5 produced more methane than TWAS alone however, they did not show any improvement in comparison with single digestion of manure and SSO. Other combinations produced more methane than TWAS and SSO alone, although only the three of them with the mixing ratios of 2.5:2.5:5, 4:2:2, and 4:2:4 resulted in higher cumulative $\mathrm{CH}_{4}$ production comparing to all of the control reactors. The maximum cumulative methane production of $1424 \mathrm{~mL}$ corresponded to the TWAS/manure/SSO mixing ratio of 2:4:4 and lipids: proteins: carbohydrates ratio of $1: 3: 12$.

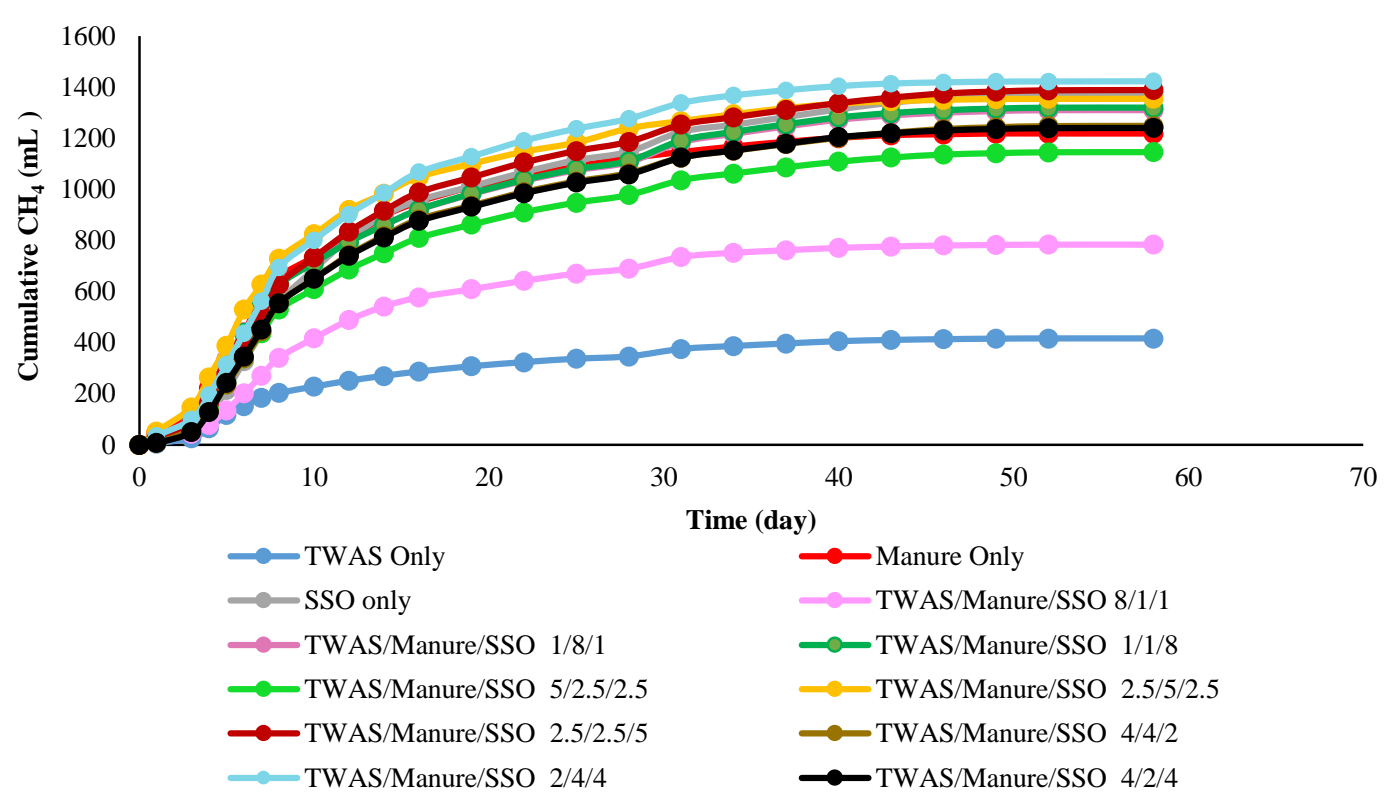

Figure 7.1. Cumulative methane production for different mixing ratios of TWAS/manure/SSO 
Figure 7.2, illustrates the methane rate in $\mathrm{mL} /$ day for all of the feedstocks combinations including the control reactors. The lowest maximum $\mathrm{CH}_{4}$ rate corresponded to TWAS mono digestion. Among control reactors, manure had the highest maximum $\mathrm{CH}_{4}$ production rate. All of the digesters, reached their maximum methane rate in less than 10 days of the process operation. The digesters containing the mixings of TWAS/manure/SSO at the ratio of 1:8:1 achieved the highest maximum methane production rate at the first week of the operational period. The digesters produced $32 \%$ to $47 \%$ of their ultimate $\mathrm{CH}_{4}$ production during the first week and $65 \%$ to $73 \%$ of it during the two weeks of the digestion period.

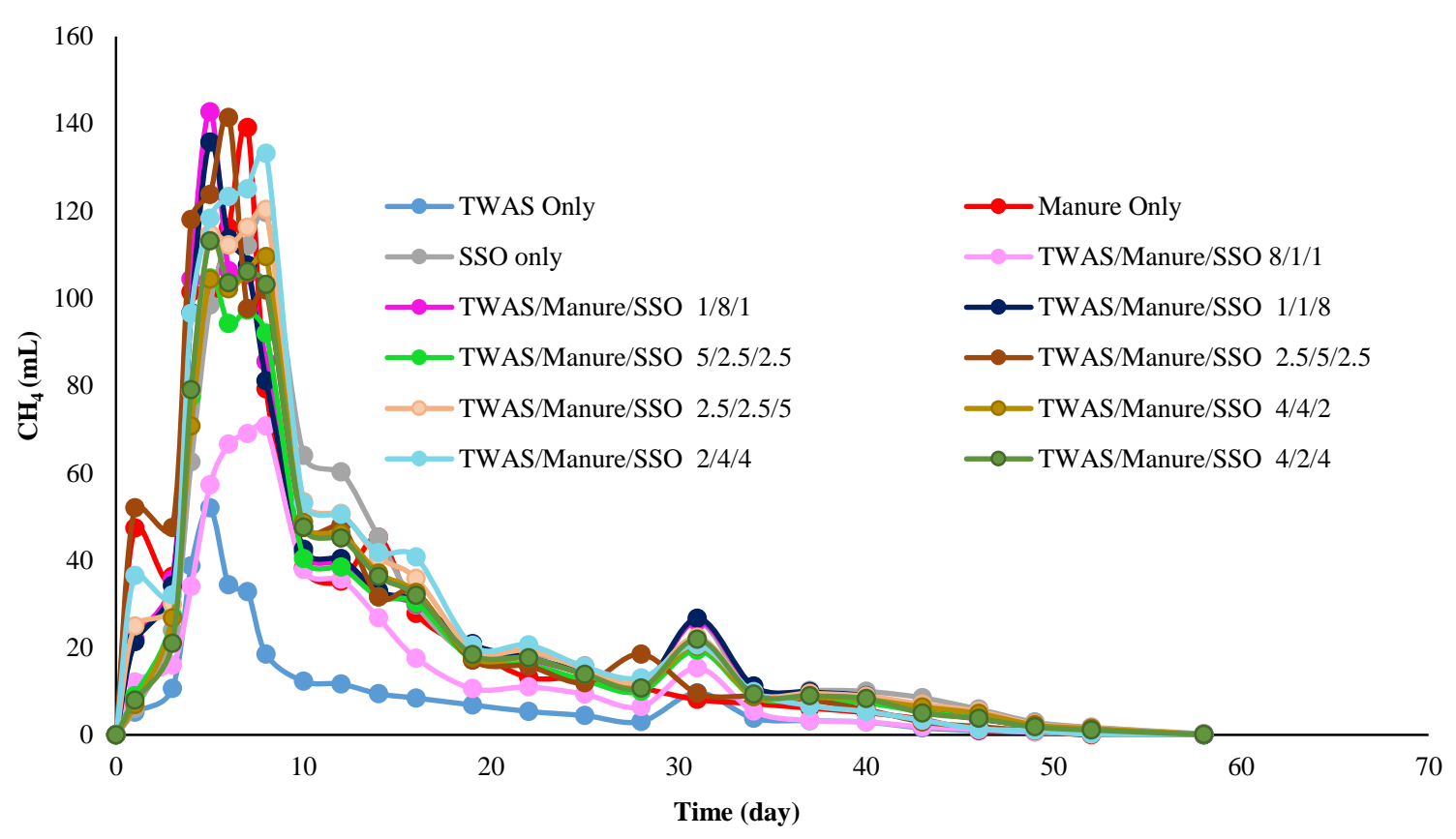

Figure 7.2. Methane production rate $(\mathrm{mL} / \mathrm{d})$ for different mixing ratios of TWAS/Manure/SSO

The maximum $\mathrm{CH}_{4}$ production rates at different mixing ratios including the controls are shown in Figure 7.3. The lowest maximum rate of $52 \mathrm{~mL} /$ day corresponded to single digestion of TWAS. The maximum $\mathrm{CH}_{4}$ rates of manure and SSO alone were $139 \mathrm{~mL} /$ day and $120 \mathrm{~mL} / \mathrm{day}$, respectively. The maximum $\mathrm{CH}_{4}$ rates of the co-digesters including the mix of the three feedstocks varied from 71 to $143 \mathrm{~mL} / \mathrm{day}$. As presented in the figure, the highest maximum rate of $143 \mathrm{~mL} /$ day corresponded to the TWAS/manure/SSO mixing ratio of 1:8:1. 


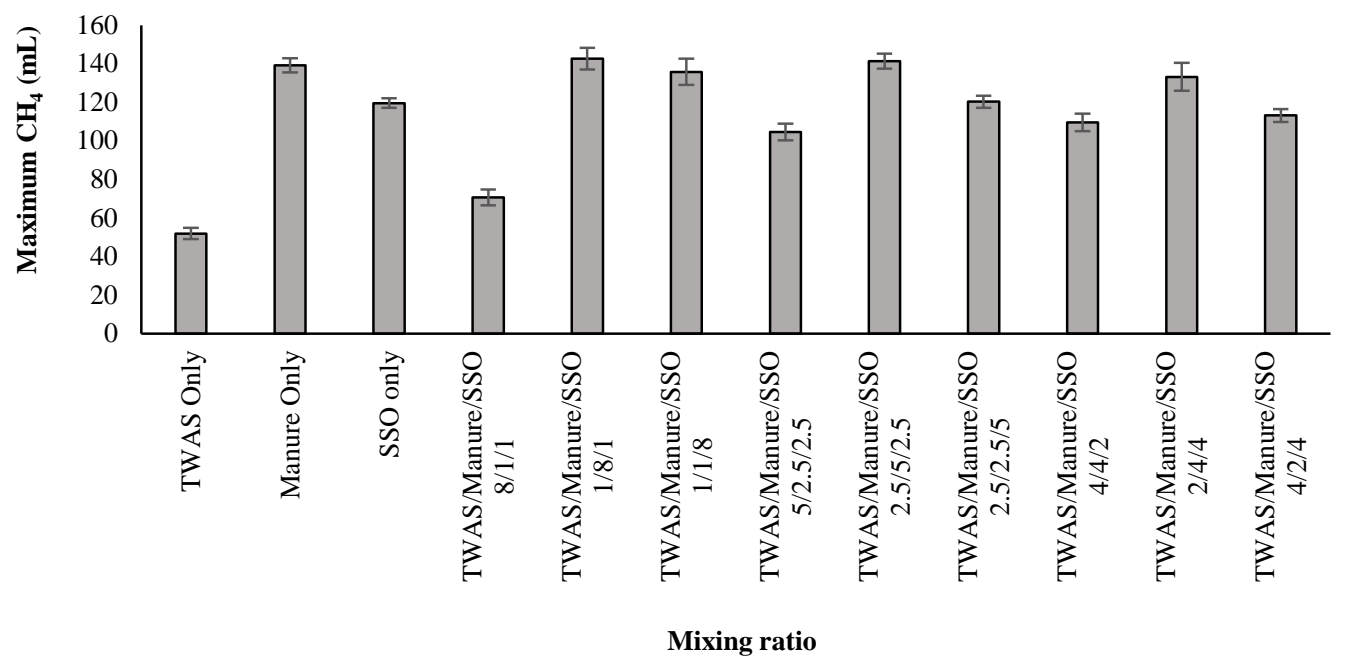

Figure 7.3. Maximum methane production rate $(\mathrm{mL} / \mathrm{d})$ for different mixing ratios TWAS/manure/SSO

The COD mass balance was conducted for all of the digesters and the result is summarized in Figure 7.4. The mass balance was calculated considering the initial and the final TCOD concentrations of the reactors' contents, and the theoretical methane production per unit mass of TCOD removed. Comparing the experimental methane production data with the ones obtained by the calculations, showed a deviation of less than $5 \%$ for all of the digesters.

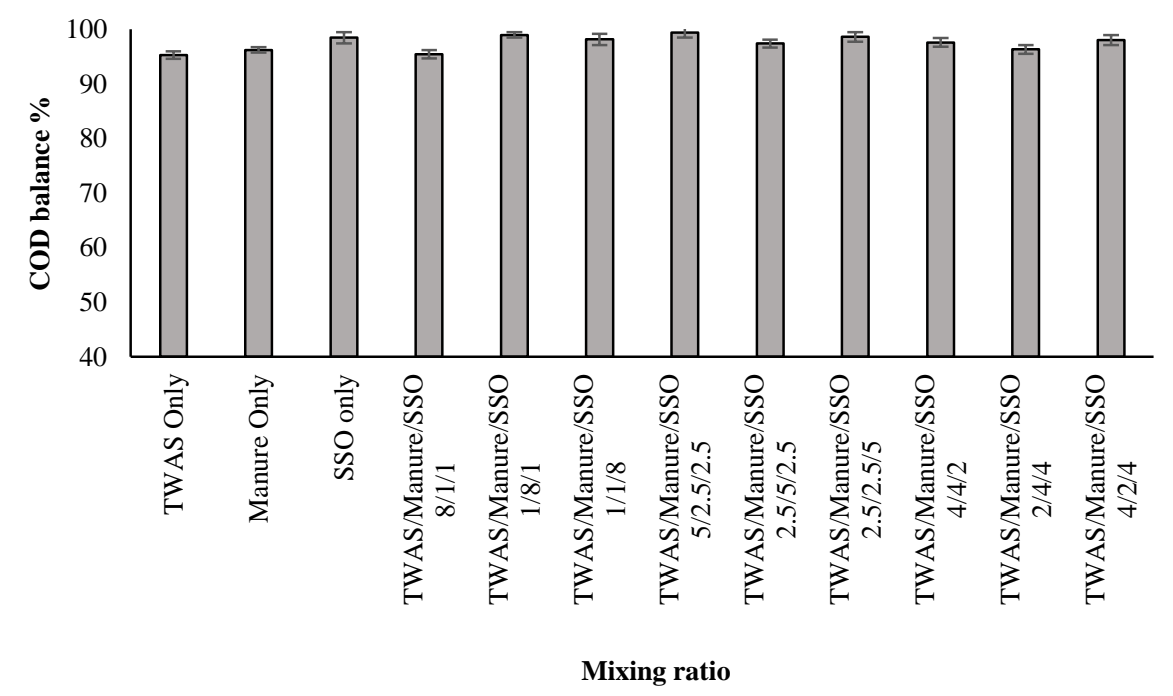

Figure 7.4. COD mass balance in co-digestion of manure and SSO for different mixing ratios 4 


\subsection{Cumulative methane yields}

Figure 7.5 shows the cumulative methane yields of the digesters including $\mathrm{mLCH}_{4} / \mathrm{g}$ TCOD added, $\mathrm{mLCH}_{4} / \mathrm{g}$ VSS added, and $\mathrm{mLCH}_{4} / \mathrm{mL}$ substrate added. The cumulative methane yield per mass COD of substrate added were 134, 299, and $332 \mathrm{mLCH}_{4} / \mathrm{g}$ TCOD added for the control reactors digesting only TWAS, manure, and SSO, respectively. As shown in Figure 7.5. a), the minimum and the maximum yield corresponded to mono digestion of TWAS and TWAS/manure/SSO mixing ratio of 2:4:4, respectively. The biomethane yields ranged from 228 to $356 \mathrm{mLCH}_{4} / \mathrm{g}$ TCOD added in the co-digesters. $\mathrm{CH}_{4}$ yield increased by $165 \%, 19 \%$ and $7 \%$ compared to single digestion of TWAS, manure, and SSO, respectively.

The methane yields in $\mathrm{mLCH}_{4} / \mathrm{g}$ VSS added are presented in Figure 7.5. b). The $\mathrm{CH}_{4}$ yields of 202, 659, and $766 \mathrm{mLCH}_{4} / \mathrm{g}$ VSS added corresponded TWAS, manure, and SSO for the control reactors, respectively. The $\mathrm{CH}_{4}$ yield varied from 395 to $982 \mathrm{mLCH}_{4} / \mathrm{g}$ VSS added in the reactors containing the combinations of the three feedstocks. The maximum $\mathrm{CH}_{4}$ yield of $395 \mathrm{mLCH}_{4} / \mathrm{g}$ VSS added was achieved by the co-digestion of TWAS/manure/SSO at the mixing ratio of 2:4:4. Figure 7.5. c), illustrates the methane yield per unit volume of substrate added. TWAS, Manure and SSO individually resulted in 5.3, 30 and $36 \mathrm{mLCH}_{4} / \mathrm{mL}$ substrate added, respectively. The reactors with the combinations of manure and SSO resulted in methane yields ranging from 12 $\mathrm{mLCH}_{4} / \mathrm{mL}$ substrate added to $32.6 \mathrm{mLCH}_{4} / \mathrm{mL}$ substrate added. The most $\mathrm{CH}_{4}$ yield per unit volume of substrate added corresponded to single digestion of SSO and TWAS/manure/SSO codigestion at the mixing ratios of 2:4:4.

Biodegradable fraction as mentioned earlier was calculated using the Eq. 4.1 and the result is summarized in Figure 7.6. The biodegradable fraction of the digesters ranged from 33\% to 89\%. TWAS, manure and SSO alone, corresponded to biodegradable fractions of 33\%, $75 \%$ and $83 \%$, respectively. The most percentage of biodegradable fraction occurred at the reactor co-digesting TWAS, manure and SSO at the mixing ratio of 2:4:4. This in good compliance with the maximum methane production that occurred at the same mixing ratio. The trend of variation in the methane

yields also conforms to the trend that was observed for the variations of biodegradable fractions at the different mixing ratios. All of the co-digesters demonstrated a more biodegradable fraction than TWAS alone, nevertheless not all of the combinations resulted in more biodegradable fraction than manure and SSO alone. 
7.5. a)

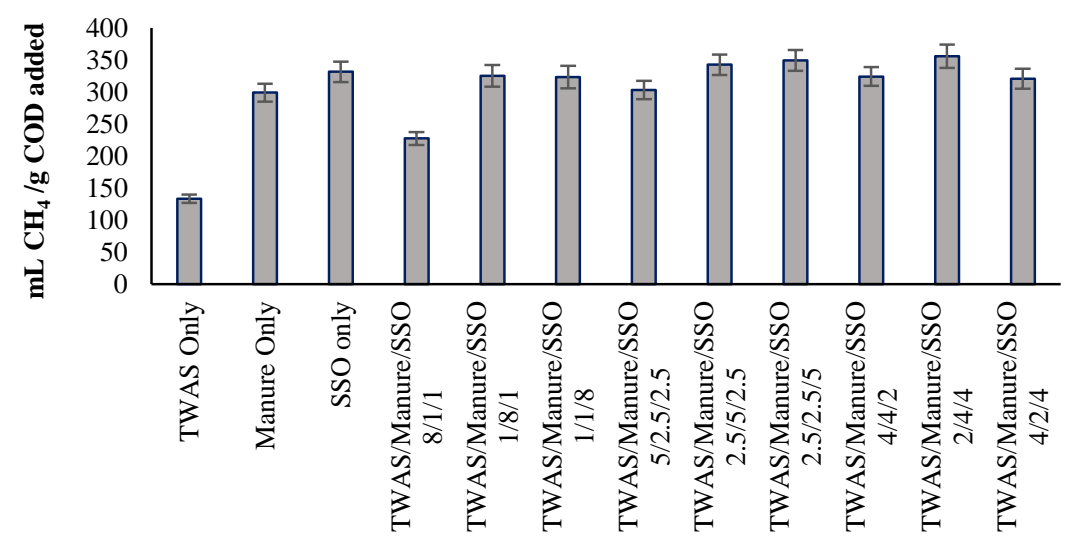

7.5. b)

Mixing ratios

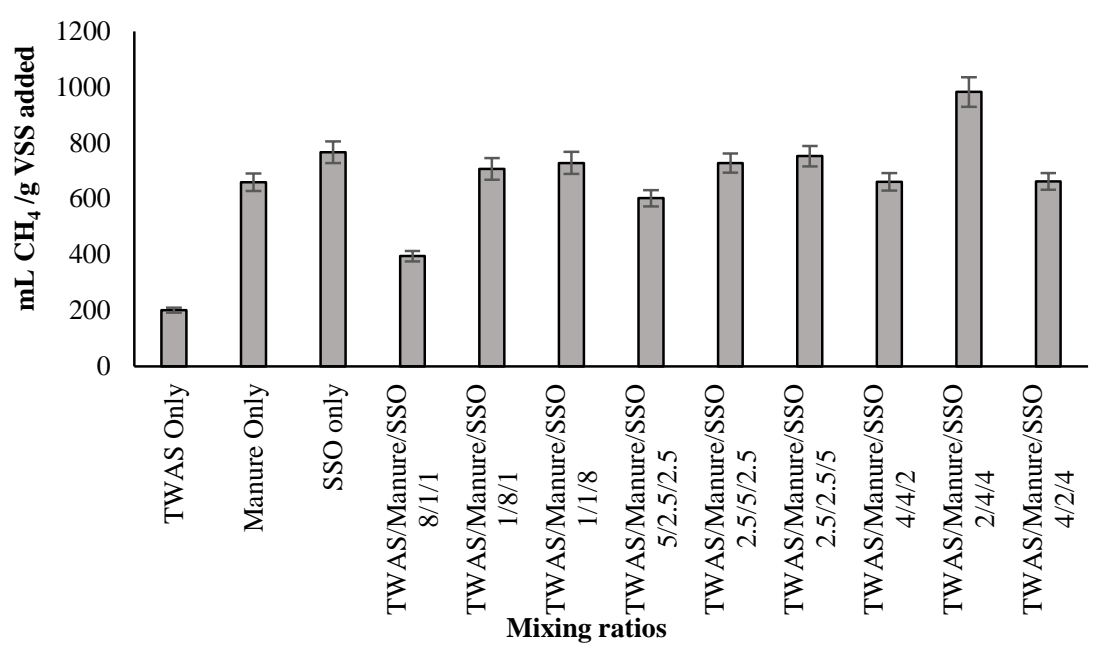

7.5. c)

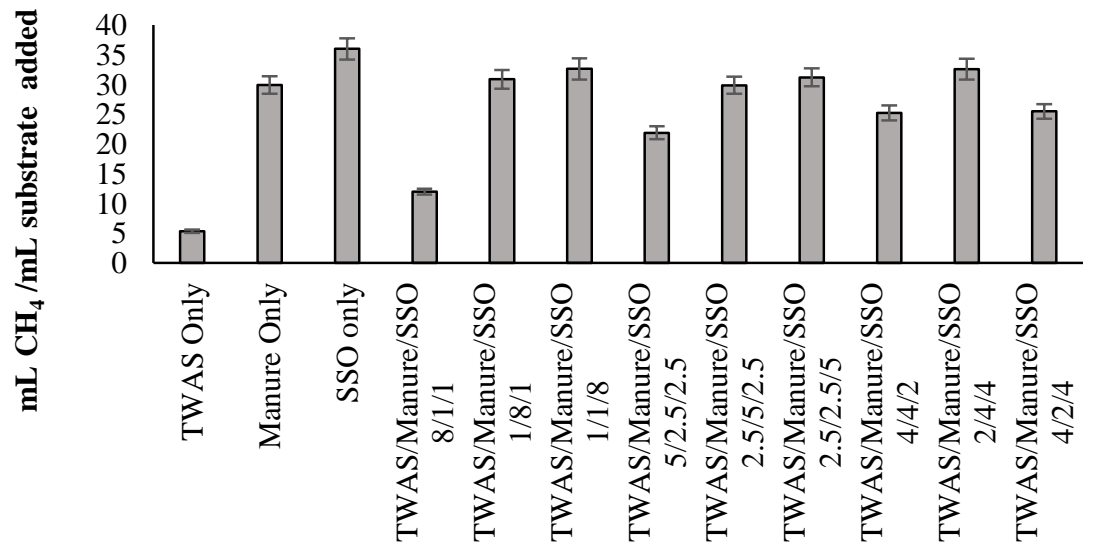

\section{Mixing ratios}

Figure 7.5. Methane yields a) per unit mass TCOD added, $b$ ) per unit mass of VSS added and c) per volume substrate added at different mixing ratio of TWAS and manure 
The reason would be that the balance of the nutrient with the microbial communities was not necessarily ideal for methanogenic populations in the reactors containing the mix of the three feedstocks at all of the mixing ratios.

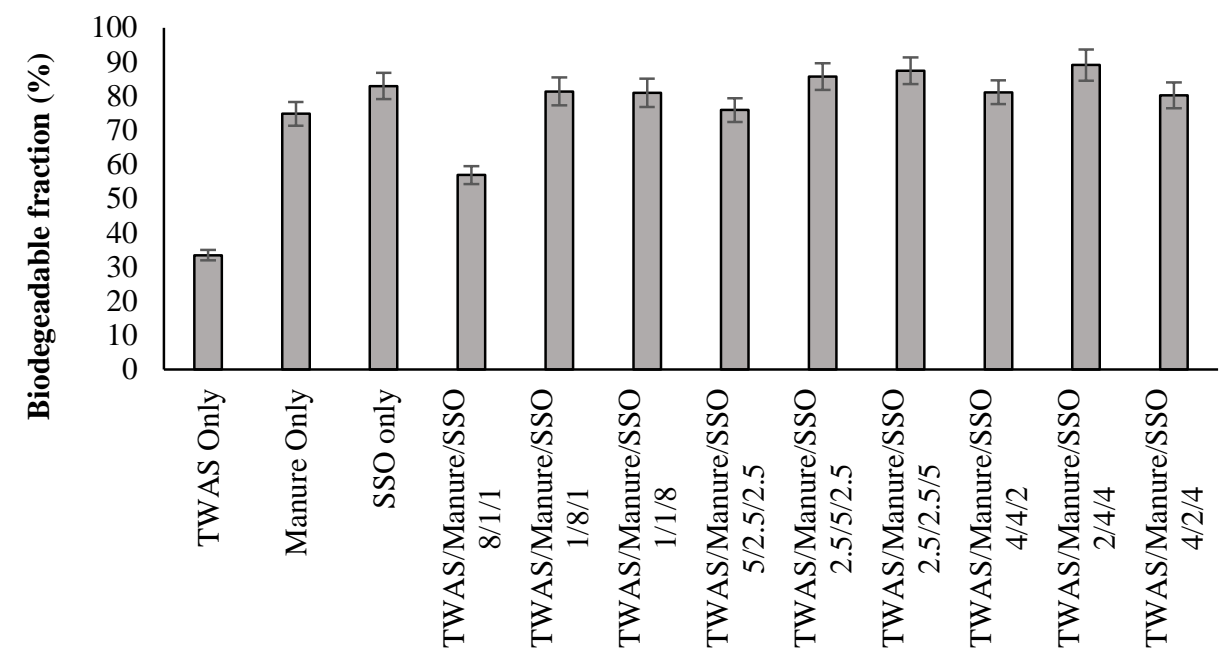

Mixing ratios

Figure 6.6. Biodegradable fraction of the feedstocks for different mixture ratios of TWAS and manure

\subsection{Synergistic effect}

Synergistic effect was obtained by calculating the percentage of additional methane yield achieved in co-digestion. This was done by dividing the measured yields, over the weighted average of the methane yield of individual substrates per unit volume of substrate added. Figure 7.7 shows the percentage improvement of biomethane production due to the synergistic impact. As demonstrated in Fig 7.7, in the ternary co-digestion of TWAS/manure/SSO, the most synergetic impact corresponds to the mixing ratio of 2:4:4. No significant improvement due to synergy was observed at the mixing ratio of 1:1:8. The maximum synergistic effect that was achieved by this experiment was $19 \%$ which was slightly lower than the result obtained by some of digesters in the binary codigestion. 


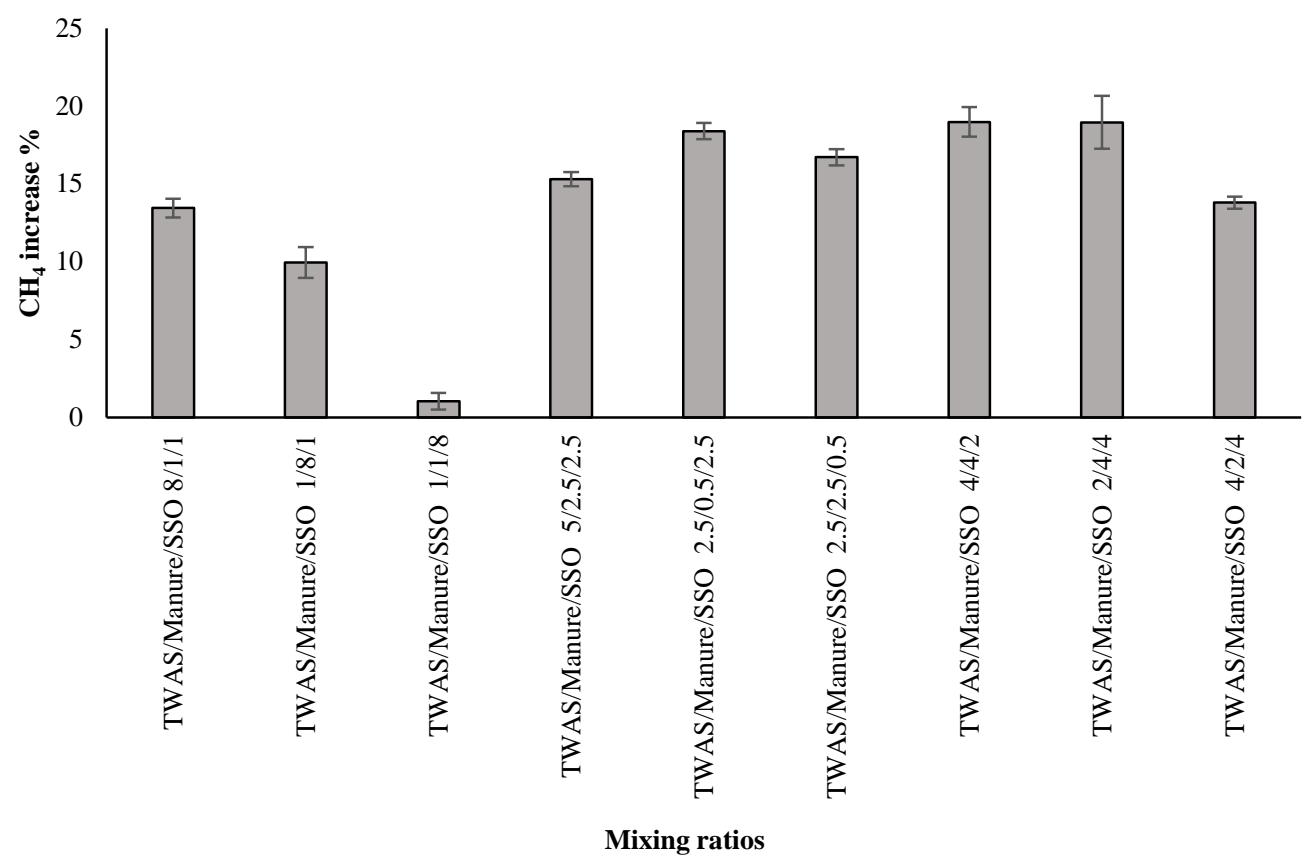

Figure 7.7. Synergetic effect of co-digestion at different mixing ratios of manure and SSO

\subsection{COD:N and Lipids: Proteins: Carbohydrates ratios}

The values of COD: $\mathrm{N}$ ratios, lipids: proteins: carbohydrates ratios, the ultimate methane production and the methane yield per unit mass of COD added at different mixing ratios are presented in Table 7.2. The COD:N ratios of TWAS, manure and SSO were 15, 47, and 27 respectively. For the co-digesters, COD:N varied from 19 to 40 . The lipids: proteins: carbohydrates ratios were 1:10:4, 1:4:20, and 1:1.6:9 for TWAS, manure, and SSO respectively. Among them SSO had the most ultimate methane production and methane yield corresponding to $1373 \mathrm{~mL}$ and $332 \mathrm{~mL} \mathrm{CH}_{4} / \mathrm{g}$ COD added. As shown in Table 7.2, the minimum ultimate methane production and the methane yield occurred at TWAS mono digestion corresponding to the COD:N ratio of 15 and lipids: proteins: carbohydrates ratio of 1:10:4. On the other side, the maximum ultimate methane production and yields occurred at the mixing ratios of 2:4:4 corresponding to the COD:N ratio of 28 and lipids: proteins: carbohydrates ratio of 1:3:12. 
Table 7.2. Ultimate $\mathrm{CH}_{4}$ and yield at different ratios of the substrates, $\mathrm{COD}: \mathrm{N}$, and lipids:proteins:carbohydrates

\begin{tabular}{|c|c|c|c|c|c|c|}
\hline $\begin{array}{l}\text { Digester } \\
\text { code }\end{array}$ & $\begin{array}{c}\text { TWAS: Manure: SSO } \\
(\mathrm{V} / \mathrm{V})\end{array}$ & COD:N & $\begin{array}{l}\text { Feedstock } \\
\text { ratio codes }\end{array}$ & $\begin{array}{l}\text { Lipids: Proteins: } \\
\text { Carbohydrates }\end{array}$ & $\begin{array}{c}\text { Ultimate } \\
\mathrm{CH}_{4} \\
(\mathrm{~mL})\end{array}$ & $\begin{array}{c}\mathrm{mLCH}_{4} / \mathrm{g} \\
\text { TCOD } \\
\text { added }\end{array}$ \\
\hline $\begin{array}{l}\text { TWAS } \\
\text { Only }\end{array}$ & 1:0:0 & 15 & AA & $1: 10: 4$ & 417 & 134 \\
\hline $\begin{array}{l}\text { Manure } \\
\text { Only }\end{array}$ & $0: 1: 0$ & 47 & $\mathrm{CC}$ & $1: 4: 20$ & 1218 & 299 \\
\hline SSO only & 0:0:1 & 27 & BB & 1:1.6:9 & 1373 & 332 \\
\hline $\begin{array}{c}\mathrm{T} / \mathrm{M} / \mathrm{SSO} \\
8 / 1 / 1\end{array}$ & $8: 1: 1$ & 19 & $\mathrm{~F}$ & $1: 6.5: 9$ & 784 & 228 \\
\hline $\begin{array}{c}\mathrm{T} / \mathrm{M} / \mathrm{SSO} \\
1 / 8 / 1\end{array}$ & $1: 8: 1$ & 40 & G & $1: 4: 19$ & 1311 & 325 \\
\hline $\begin{array}{c}\mathrm{T} / \mathrm{M} / \mathrm{SSO} \\
1 / 1 / 8\end{array}$ & $1: 1: 8$ & 28 & $\mathrm{H}$ & $1: 2: 10$ & 1320 & 324 \\
\hline $\begin{array}{l}\mathrm{T} / \mathrm{M} / \mathrm{SSO} \\
5 / 2.5 / 2.5\end{array}$ & $5: 2.5: 2.5$ & 25 & I & $1: 4: 12$ & 1146 & 304 \\
\hline $\begin{array}{l}\mathrm{T} / \mathrm{M} / \mathrm{SSO} \\
2.5 / 5 / 2.5\end{array}$ & $2.5: 5: 2.5$ & 32 & $\mathrm{~J}$ & $1: 3.8: 15.5$ & 1355 & 343 \\
\hline $\begin{array}{l}\mathrm{T} / \mathrm{M} / \mathrm{SSO} \\
2.5 / 2.5 / 5\end{array}$ & $2.5: 2.5: 5$ & 28 & $\mathrm{~K}$ & $1: 3: 12$ & 1390 & 350 \\
\hline $\begin{array}{c}\mathrm{T} / \mathrm{M} / \mathrm{SSO} \\
4 / 4 / 2\end{array}$ & $4: 4: 2$ & 28 & $\mathrm{~L}$ & $1: 4: 15$ & 1248 & 324 \\
\hline $\begin{array}{c}\mathrm{T} / \mathrm{M} / \mathrm{SSO} \\
2 / 4 / 4\end{array}$ & $2: 4: 4$ & 28 & M & $1: 3: 12$ & 1424 & 356 \\
\hline $\begin{array}{c}\mathrm{T} / \mathrm{M} / \mathrm{SSO} \\
4 / 2 / 4\end{array}$ & $4: 2: 4$ & 26 & $\mathrm{~N}$ & $1: 3.7: 11.8$ & 1241 & 321 \\
\hline
\end{tabular}

Figure 7.8. illustrates the main effect plot for $\mathrm{CH}_{4}$ yield data mean in response to feedstock and lipids:proteins:carbohydrates ratios in AnCoD of TWAS/manure/SSO. As shown in Figure 7.8, feedstocks and lipids:proteins:carbohydrates ratios have significant effect on $\mathrm{CH}_{4}$ yield. TWAS only with lipids:proteins:carbohydrates ratio of 1:10:4 has the minimum methane yield. TWAS/manure/SSO co-digestion at the mixing ratio of 2:4:4 and lipids:proteins:carbohydrates ratio of 1:3:12 corresponded to the maximum methane yield.

The matrix plot in Figure 7.9 shows the relationship of COD:N, proteins: lipids, carbohydrates: lipids, and carbohydrates: proteins ratios with the ultimate methane production $(\mathrm{mL})$ and with the 
yield (mg CH $4 / \mathrm{g}$ TCOD added). The responses of the ultimate methane production and the methane yield to the different ratios is illustrated in figures 7.9. a) and 7.9. b), respectively.

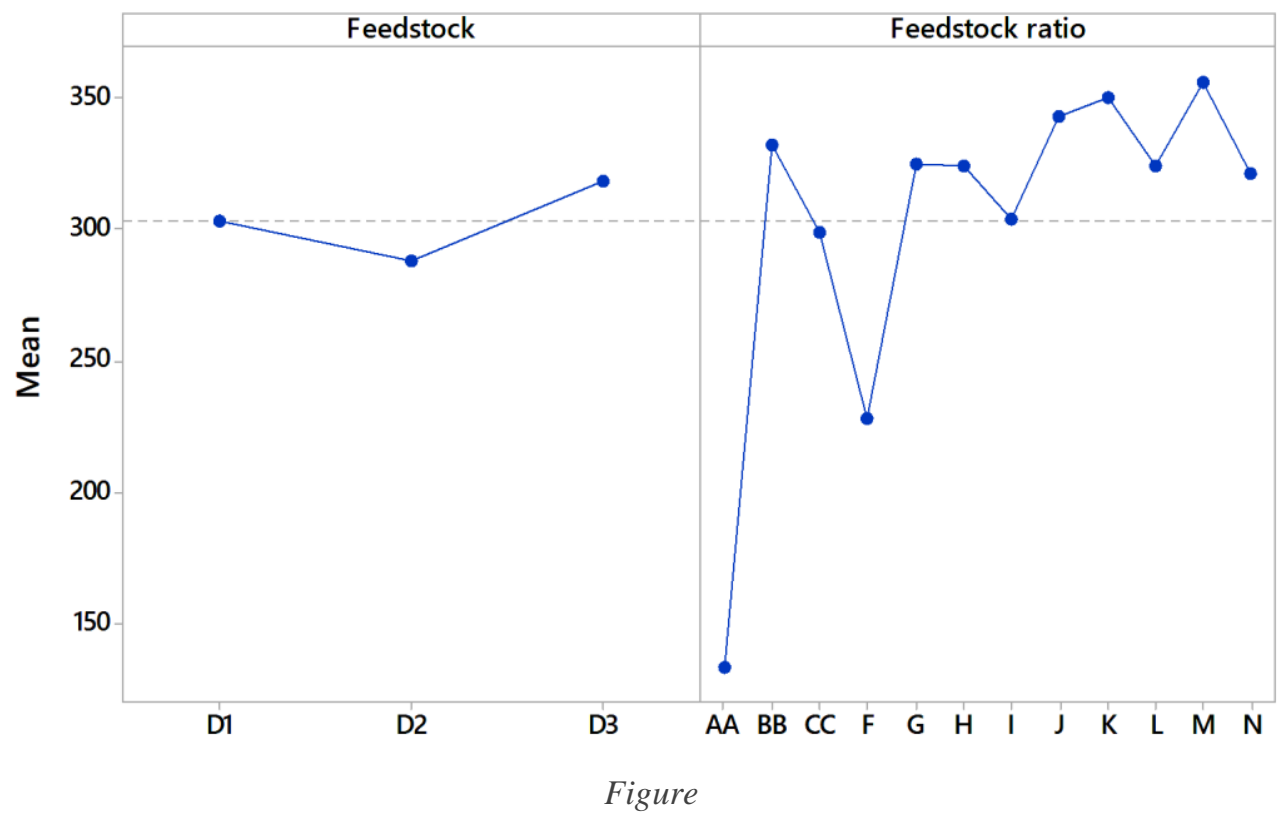

Figure 7.8. Main effect plot for $\mathrm{CH}_{4}$ yield data mean in response to feedstock and lipids:proteins:carbohydrates ratio in AnCoD of TWAS/manure/SSO

As shown in Figures 7.9. a) and 7.9. b) the trends of variations of methane versus COD:N, and lipids: proteins were similar. However, lipids: carbohydrates and proteins: carbohydrates did not conform to COD:N ratio. As illustrated in the Figures 7.8. a), the minimum ultimate methane production corresponds to the minimum COD:N ratio and the minimum lipids: proteins ratio while it corresponded to the maximum lipids: carbohydrates and maximum protein: carbohydrates ratios. The trend of the ultimate methane production variations in response to the COD:N and to the lipids: proteins ratios relatively conform to each other excluding some of the ratios. The ultimate methane production was higher at the COD:N ratios between 26 and 47 and at the lipids: proteins ratios between 0.23 and 0.62 . On the contrary, the increase of the lipids: carbohydrates and proteins: carbohydrates ratios, reduced the ultimate methane production. The most ultimate methane production occurred at the minimum lipids: carbohydrates and proteins: carbohydrates ratios of 0.05 and 0.17 , respectively. In contrast the minimum ultimate methane production corresponded to the maximum lipids: carbohydrates and proteins: carbohydrates ratios of 0.26 and 2.55 , respectively. The lipids: carbohydrates ratios above 0.1 and proteins: carbohydrates ratios above 
0.3 resulted in significant decrease in the ultimate methane production. Similar trend was observed for the methane yield in response to the COD:N, lipids: proteins and lipids: carbohydrates, and proteins: carbohydrates ratios.

7.9. a)

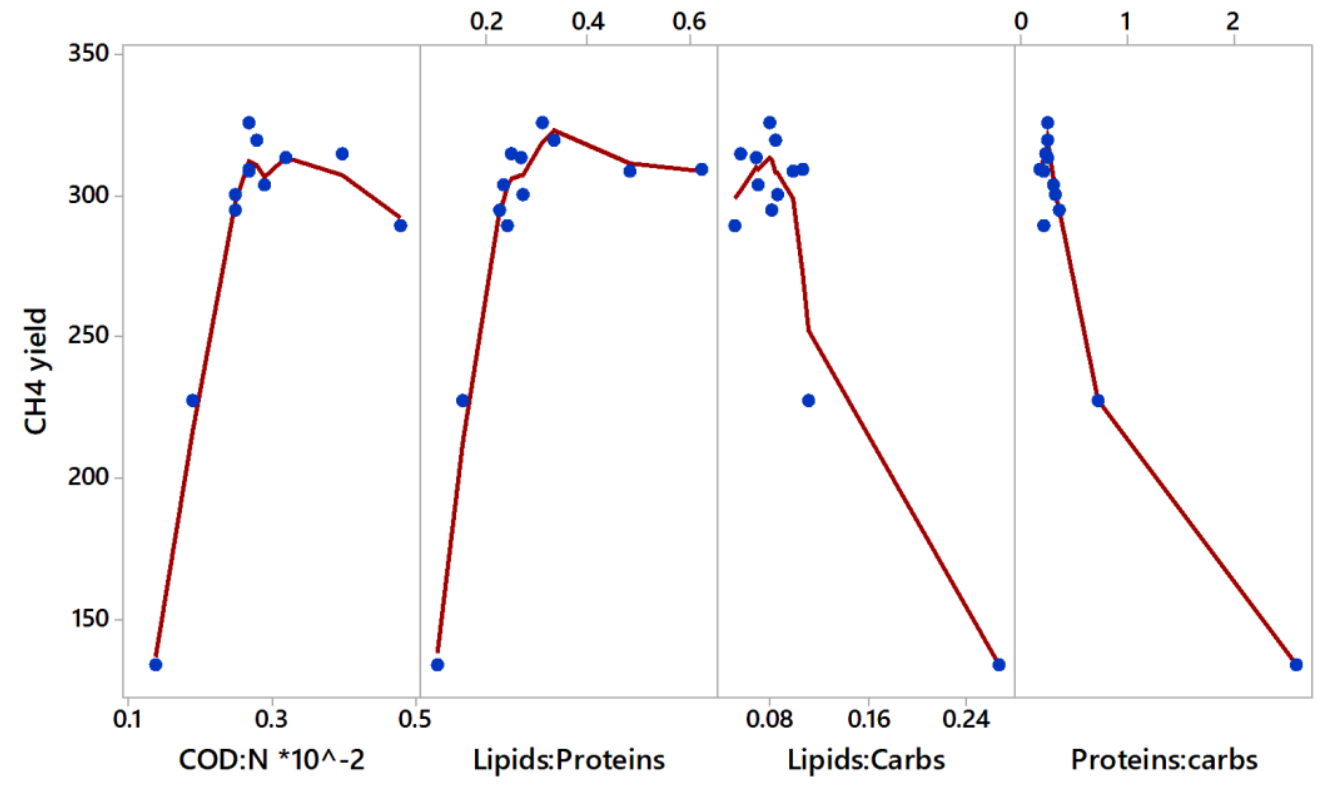

7.9. b)

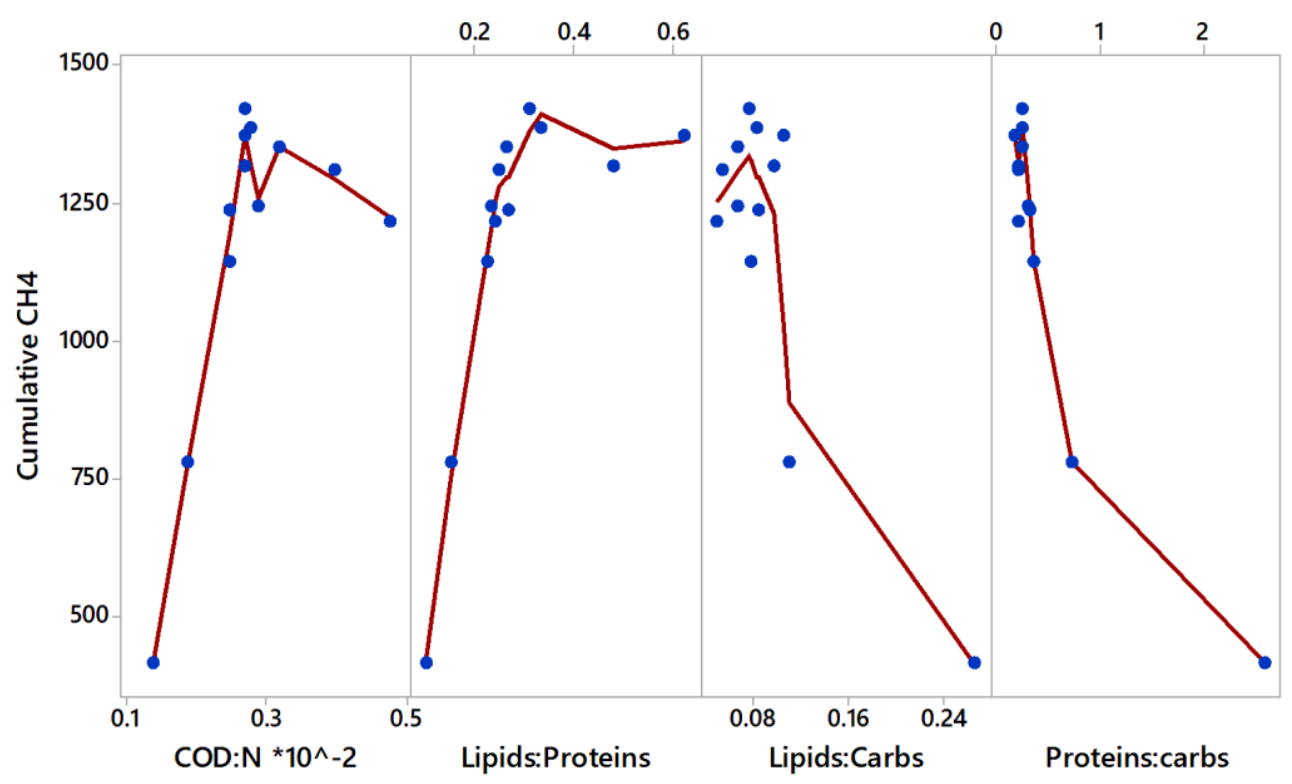

Figure 7.9. Matrix plot for: a. ultimate $\mathrm{CH}_{4}$ and b. $\mathrm{CH}_{4}$ yield at different $\mathrm{COD} / \mathrm{N}$ and Lipids: Proteins, Lipids:

Carbohydrates, and Proteins: Carbohydrates Ratios 


\subsection{Kinetic analysis results}

The results of kinetic by modified Gompertz model using Eq. 3.5 is summarized in Table 7.3. The model was applied to the experimental data from mono and co-digestion of manure and SSO. The values of $\mathrm{P}$ for the maximum methane production $(\mathrm{mL}), R_{m}^{e}$, the maximum methane production rate (mL/g day), and $\lambda$, the lag phase time $(\mathrm{d})$ were calculated and the estimated values are presented in Table 7.3. The modified Gompertz model for mono- and co-digestions of TWAS, manure and SSO, showed a good fit to the experimental results with less than $10 \%$ diversion from the measured values. The estimated values and their correlation with the mixing ratio of the feedstock showed compliance with the data obtained by the experiment. The trend of $\mathrm{P}$ variations showed the same trend as the trend observed by the experimental data in response to the corresponding mixing ratios.

Table 7.3. Summary of results of kinetic study using modified Gompertz model for TWAS/manure/SSO co-digestion

\begin{tabular}{|c|c|c|c|c|}
\hline Digester codes & $\mathbf{P}(\mathbf{m L})$ & $R_{m}^{e}(\mathrm{~mL} / \mathrm{d})$ & $\lambda(\mathbf{d})$ & $\mathbf{R}^{2}$ \\
\hline TWAS Only & 400 & 20.3 & 0.02 & 0.999 \\
\hline Manure Only & 1180 & 78.4 & 0.7 & 0.999 \\
\hline SSO only & 1307 & 73.5 & 1.76 & 0.999 \\
\hline $\mathrm{T}^{*}: \mathrm{M}^{* *}: \mathrm{SSO} 8: 1: 1$ & 760 & 46.6 & 1.7 & 0.999 \\
\hline $\mathrm{T}: \mathrm{M}: S S O$ 1:8:1 & 1257 & 66.9 & 0.2 & 0.999 \\
\hline $\mathrm{T}: \mathrm{M}: \mathrm{SSO} 1: 1: 8$ & 1267 & 66.1 & 0.2 & 0.999 \\
\hline $\mathrm{T}: \mathrm{M}: S S O$ 5:2.5:2.5 & 1099 & 60.7 & 0.8 & 0.999 \\
\hline T:M:SSO 2.5:5:2.5 & 1308 & 84.9 & 0.5 & 0.999 \\
\hline $\mathrm{T}: \mathrm{M}: S S O 2.5: 2.5: 5$ & 1330 & 74.4 & 1.0 & 0.999 \\
\hline T:M:SSO 4:4:2 & 1193 & 67.2 & 1.2 & 0.999 \\
\hline $\mathrm{T}: \mathrm{M}: \mathrm{SSO} 2: 4: 4$ & 1382 & 84.6 & 1.1 & 0.999 \\
\hline $\mathrm{T}: \mathrm{M}: \mathrm{SSO} 4: 2: 4$ & 1191 & 66.8 & 1.2 & 0.999 \\
\hline
\end{tabular}


The Maximum $\mathrm{P}$ value for the predicted cumulative methane production was $1382 \mathrm{~mL}$ and corresponded to the mixing ratio of 2:4:4. The lag phase varied from 0.0 to 1.7 days for different substrate mixing ratios. The lag phase time was less than 1 day for half of the digesters. The other half had a lag phase time from 1 to 1.7 days. The values of $\mathrm{R}_{\max }^{\mathrm{e}}$ ranged from 20 to $84.9 \mathrm{~mL} / \mathrm{g} \mathrm{d}$ corresponding to TWAS mono digestion and TWAS/manure/SSO mixing ratio of 2:4:4, respectively.

\subsection{Hydrolysis/acidification}

This experiment was carried out for evaluating the hydrolysis/acidification phase in anaerobic codigestion of TWAS, manure, and SSO. The analysis of degree of solublization, synergistic effect of co-digestion at different mixing ratios, volatile fatty acids (VFAs) yield, and hydrolysis kinetics of lipids, proteins, and carbohydrates was conducted by monitoring the soluble and particulate contents during a 72-hr period. Initially, characterization of the feedstocks in triplicates was carried out and the mean values are presented in Table 7.4. As shown in the table, both manure and SSO have higher amount of COD concentration than TWAS. Manure contains high amount of carbohydrates and proteins. The lipids and carbohydrates content of manure and SSO are also remarkably higher than that of TWAS. $\mathrm{pH}$ values of the digesters were adjusted to satisfy the required condition for hydrolysis/acidification and ranged from 5.6 to 5.8 in this experiment.

The data form total and soluble COD (SCOD) concentrations monitoring in all of the digesters over time during a 72-h hydrolysis/acidification period was used to obtain the degree of COD solublization for each digester. As explained earlier, the degree of solublization was calculated using Eq. 3.1 and the result is summarized in Figure 7.10.

As illustrated in figure 7.10, the degree of the COD solublization varied from $15 \%$ to $30 \%$. The most solublization of the COD content of $30 \%$ corresponded to TWAS/manure/SSO combinations of 1:8:1 and 2:4:4. For the control reactors, the COD solublization in mono digestion of TWAS, manure and SSO were $15 \%$ and $23 \%$, and $26 \%$ respectively. The reactors containing only TWAS had the lowest degree of solublization. Similar to the results of previous experiments, manure resulted in a relatively lower solublization than SSO which would be due to the presence of some recalcitrant contents such as fibers and cellulosic compounds that delay hydrolysis and liquefaction. 
Table 7.4. Characteristics of the feedstocks at different mixing ratios of TWAS/manure/SSO

\begin{tabular}{|c|c|c|c|c|c|c|c|c|c|c|c|c|c|}
\hline & & TWAS & Manure & SSO & $\begin{array}{c}\mathrm{T}^{*}: \mathrm{M}^{* *}: \mathrm{SSO} \\
8: 1: 1\end{array}$ & $\begin{array}{c}\text { T:M:SSO } \\
1: 8: 1\end{array}$ & $\begin{array}{c}\text { T:M:SSO } \\
1: 1: 8\end{array}$ & $\begin{array}{l}\text { T:M:SSO } \\
5: 2.5: 2.5\end{array}$ & $\begin{array}{l}\text { T:M:SSO } \\
2.5: 5: 2.5\end{array}$ & $\begin{array}{l}\text { T:M:SSO } \\
2.5: 2.5: 5\end{array}$ & $\begin{array}{c}\text { T:M:SSO } \\
4: 4: 2\end{array}$ & $\begin{array}{c}\text { T:M:SSO } \\
2: 4: 4\end{array}$ & $\begin{array}{c}\text { T:M:SSO } \\
4: 2: 4\end{array}$ \\
\hline Parameters & Units & $\begin{array}{c}\text { Mixture } \\
\text { (1) }\end{array}$ & $\begin{array}{c}\text { Mixture } \\
\text { (2) }\end{array}$ & $\begin{array}{c}\text { Mixture } \\
\text { (3) }\end{array}$ & Mixture (4) & $\begin{array}{c}\text { Mixture } \\
(5)\end{array}$ & $\begin{array}{c}\text { Mixture } \\
(6)\end{array}$ & $\begin{array}{c}\text { Mixture } \\
\text { (7) }\end{array}$ & $\begin{array}{c}\text { Mixture } \\
(8)\end{array}$ & $\begin{array}{c}\text { Mixture } \\
(9)\end{array}$ & $\begin{array}{c}\text { Mixture } \\
(10)\end{array}$ & $\begin{array}{c}\text { Mixture } \\
\text { (11) }\end{array}$ & $\begin{array}{c}\text { Mixture } \\
(12)\end{array}$ \\
\hline TCOD & $\mathrm{g} / \mathrm{L}$ & 37 & 100 & 102 & 50 & 94 & 95 & 69 & 85 & 85 & 75 & 68 & 76 \\
\hline SCOD & $\mathrm{g} / \mathrm{L}$ & 1.9 & 10 & 37 & 6 & 12 & 31 & 13 & 15 & 22 & 12 & 17 & 17.7 \\
\hline TSS & $\mathrm{g} / \mathrm{L}$ & 29 & 54 & 50 & 34 & 51 & 48 & 40 & 47 & 46 & 43 & 36 & 42 \\
\hline VSS & $\mathrm{g} / \mathrm{L}$ & 23 & 45 & 44 & 27 & 43 & 42 & 34 & 39 & 39 & 36 & 31 & 36 \\
\hline $\mathrm{TS}$ & $\mathrm{g} / \mathrm{L}$ & 36 & 68 & 77 & 43 & 65 & 72 & 54 & 62 & 64 & 57 & 51 & 59 \\
\hline VS & $\mathrm{g} / \mathrm{L}$ & 32 & 58 & 63 & 37 & 56 & 59 & 46 & 53 & 54 & 48 & 43 & 49 \\
\hline Ammonia & $\mathrm{g} / \mathrm{L}$ & 0.2 & 0.0 & 1.3 & 0.3 & 0.2 & 1.1 & 0.5 & 0.4 & 0.7 & 0.4 & 0.6 & 0.6 \\
\hline $\mathrm{pH}$ & - & 5.6 & 5.7 & 5.8 & 5.6 & 5.7 & 5.8 & 5.7 & 5.7 & 5.7 & 5.7 & 5.8 & 5.7 \\
\hline Alkalinity & $\mathrm{g} \mathrm{CaCO} 3 / \mathrm{L}$ & 1.8 & 7.0 & 6.4 & 2.8 & 6.4 & 6.0 & 4.2 & 5.5 & 5.4 & 4.8 & 4.3 & 4.7 \\
\hline TN & $\mathrm{g} / \mathrm{L}$ & 2.7 & 1.7 & 4.1 & 2.7 & 2.0 & 3.7 & 2.8 & 2.5 & 3.1 & 2.6 & 2.5 & 3.0 \\
\hline TSN & $\mathrm{g} / \mathrm{L}$ & 0.4 & 0.1 & 1.1 & 0.4 & 0.2 & 0.9 & 0.5 & 0.4 & 0.7 & 0.4 & 0.5 & 0.6 \\
\hline T-Carbs & $\mathrm{g} / \mathrm{L}$ & 0.8 & 26.0 & 13.6 & 4.6 & 22.2 & 13.5 & 10.3 & 16.6 & 13.5 & 13.4 & 10.8 & 11.0 \\
\hline T-Proteins & $\mathrm{g} / \mathrm{L}$ & 2.5 & 5.1 & 2.1 & 2.8 & 4.6 & 2.4 & 3.1 & 3.7 & 3.0 & 3.5 & 2.4 & 2.9 \\
\hline T-Lipids & $\mathrm{g} / \mathrm{L}$ & 0.3 & 1.7 & 1.4 & 0.5 & 1.5 & 1.3 & 0.9 & 1.2 & 1.2 & 1.0 & 0.9 & 1.0 \\
\hline
\end{tabular}

*T: TWAS

${ }^{* *} \mathrm{M}$ : Manure 


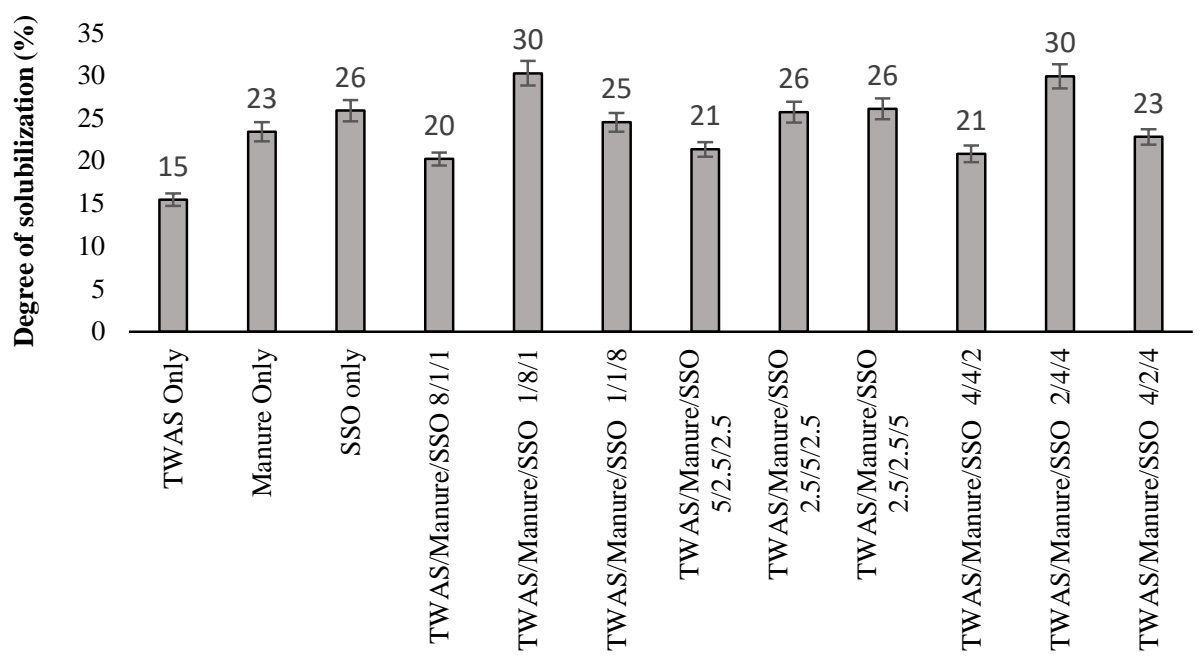

Mixing ratio

Figure 7.10. Degree of COD solublization at different mixing ratios of TWAS/Manure/SSO

It was observed that all of the reactors containing the mixings of TWAS, manure and SSO achieved a higher degree of solublization than the control reactors containing only TWAS. Solublization increased by $100 \%, 30 \%$ and $15 \%$ in the co-digestion of TWAS/manure/SSO in comparison with the control reactors digesting only TWAS, manure and SSO, respectively. Although SSO demonstrated more solublization than manure, no correlation between the portion of SSO and the degree of solublization at different mixing ratio was observed. This could verify that a proper mixing ratio is required to enhance the microbial synergy of the hydrolytic and acidogenic microbial communities for the process improvement.

The synergistic effect on the solublization of the feedstocks was evaluated by comparing the theoretical degree of solublization of each co-digester to the measured data from the experiment and the result is summarized in Figure 7.11. Although the improvement of synergy was not significant at some combinations, most of the co-digesters resulted in an increase of solublization due to the synergistic effect of the microbial communities. The synergistic effect varied from $2 \%$ to $35 \%$ in hydrolysis/acidification of TWAS/manure/SSO at different mixing ratios. 


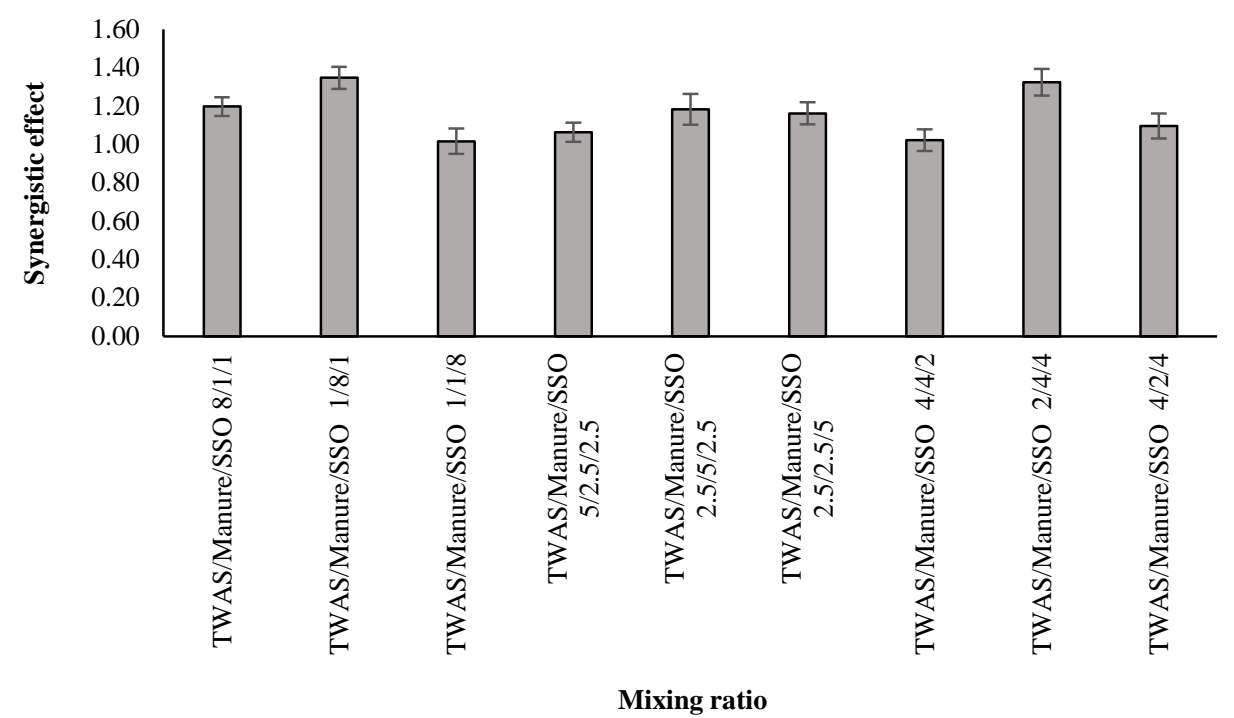

Figure 7.11. Synergistic effect on solublization at different mixing ratios of TWAS/Manure/SSO

The monitoring of VFAs concentrations over time indicated an increasing trend during the 72-hr of the hydrolysis/acidification period. The total VFAs yields were calculated in terms of mass of produced VFAs per mass of VSS added (mg VFAs/g VSS added). As illustrated in Figure 7.12, SSO alone had more VFAs yields than manure alone. Both manure and SSO had a higher VFAs yield than TWAS. The VFAs yield in the co-digester was correlated to the mixing ratios of the feedstocks so that the VFAs yield increased by increasing the fractions of manure and SSO in the co-digesters. Similar to the result of the previous experiments, the trend of VFAs yield did not comply with the trend of COD solublization of the corresponding mixing ratios (Figure 7.10). Therefore, it was assumed that hydrolysis/liquefaction would occur independently of acidification. All of the reactors containing the mix of manure and SSO had higher VFAs yield than the control reactors containing TWAS alone. The VFAs yields were $231 \mathrm{mg}$ VFAs/g VSS added and $325 \mathrm{mg}$ VFAs/g VSS added for the manure and SSO mono digestions, respectively. The maximum VFAs yield of $400 \mathrm{mg}$ VFAs/g VSS added was achieved by manure:SSO ratio of 1:9. 


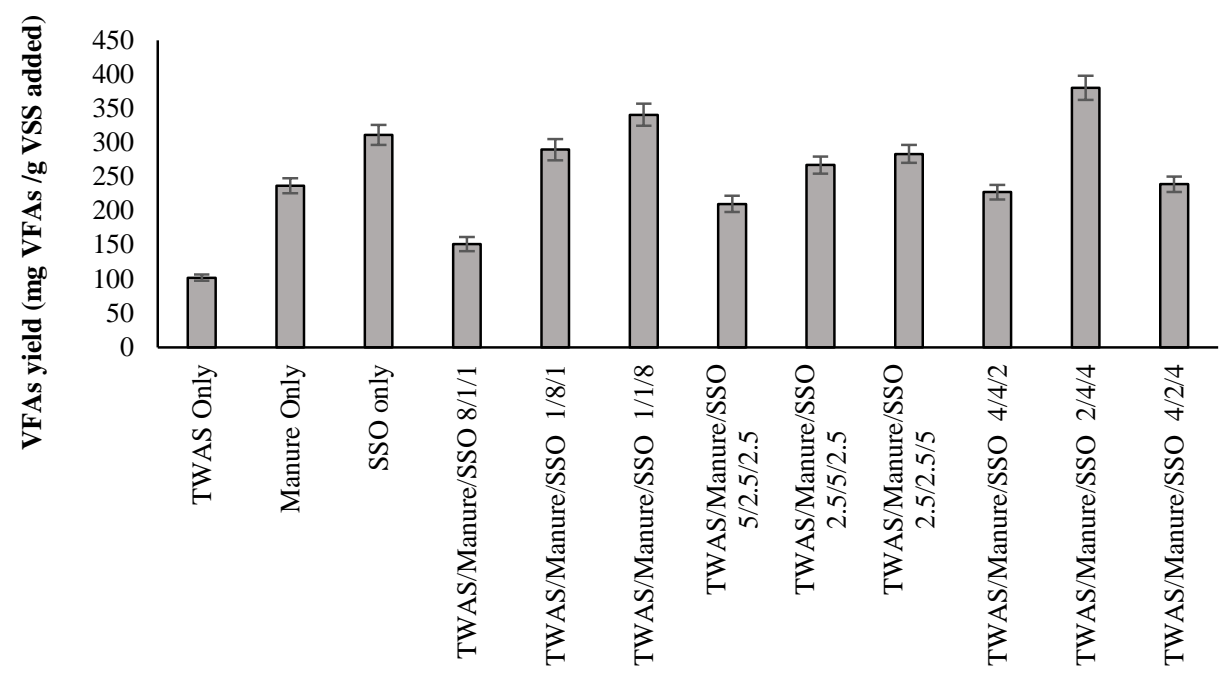

Mixing ratio

Figure 7.12. Total VFAs yield at different mixing ratios of TWAS/Manure/SSO

In co-digestion of TWAS/manure/SSO, by monitoring soluble and particulate COD over time, an increasing trend in solublization of COD and decreasing particulate COD concentrations was observed. Furthermore, monitoring soluble and particulate lipids, proteins, and carbohydrates over time also showed an increasing trend in solublization and particulate matter degradation of the feedstocks. Similar to the previous experiments, the hydrolysis rates of the lipids and proteins, were slower than carbohydrate. These results are summarized in the tables presented in the appendix. The hydrolysis rate coefficient $\left(\mathrm{K}_{\mathrm{h}}\right)$ was calculated according to first order kinetic (equations 3.3 and 3.4) for COD, lipids, proteins and carbohydrates based on the particulate degradation and results are summarized in Table 7.5.

Although the first order kinetics was compatible with the trend of particulate matters degradation, the hydrolysis kinetics coefficient did not show the same trend as solublization and acidification as well as methanogenesis. Hydrolysis rate coefficients of the co-digesters increased in the codigesters although, it did not change significantly in all of them compared to the single digestion of SSO. In some co-digesters the $\mathrm{K}_{\mathrm{h}}$ values were even lower than that of SSO alone. The maximum VFAs yield corresponded to TWAS:manure:SSO 2:4:4 and 1:1:8. However, the Kh for the mixing ratio of $1: 8: 1$ was higher than that of $1: 1: 8$. The maximum solublization corresponded to 
TWAS:manure:SSO mixing ratios of 2:4:4 and 1:8:1. However, the maximum methane yield occurred at the mixing ratios of 2:4:4 and 2.5:2.5:5 in the reactors co-digesting TWAS, manure, and SSO. Only the mixing ratio of 2:4:4 resulted in improving both methanogenesis and hydrolysis which would be due to the balance of the nutrients that favors both methanogenic and hydrolytic microbial consortia.

Table 7.5. Hydrolysis rate coefficients for COD, lipids, proteins, and carbohydrate content in co-digestion of TWAS,

Manure, and SSO at different mixing ratios

\begin{tabular}{|c|c|c|c|c|c|c|c|c|c|c|c|c|}
\hline$K$ & & & & $\mathrm{~T} / \mathrm{M} / \mathrm{SSO}$ & $\mathrm{T} / \mathrm{M} / \mathrm{SSO}$ & $\mathrm{T} / \mathrm{M} / \mathrm{SSO}$ & $\mathrm{T} / \mathrm{M} / \mathrm{SSO}$ & $\mathrm{T} / \mathrm{M} / \mathrm{SSO}$ & $\mathrm{T} / \mathrm{M} / \mathrm{SSO}$ & $\mathrm{T} / \mathrm{M} / \mathrm{SSO}$ & $\mathrm{T} / \mathrm{M} / \mathrm{SSO}$ & $\mathrm{T} / \mathrm{M} / \mathrm{SSO}$ \\
\hline $\mathbf{N h}$ & TWAS & Manure & SSO & $8 / 1 / 1$ & $1 / 8 / 1$ & $1 / 1 / 8$ & $5 / 2.5 / 2.5$ & $2.5 / 5 / 2.5$ & $2.5 / 2.5 / 5$ & $4 / 4 / 2$ & $2 / 4 / 4$ & $4 / 2 / 4$ \\
\hline $\mathrm{Kh}_{\mathrm{COD}}$ & 0.17 & 0.23 & 0.3 & 0.21 & 0.37 & 0.27 & 0.24 & 0.31 & 0.3 & 0.24 & 0.39 & 0.26 \\
\hline Kh Lipids & 0.03 & 0.07 & 0.08 & 0.05 & 0.12 & 0.06 & 0.05 & 0.09 & 0.08 & 0.09 & 0.14 & 0.05 \\
\hline $\mathrm{Kh}$ Proteins & 0.19 & 0.21 & 0.28 & 0.23 & 0.31 & 0.24 & 0.2 & 0.23 & 0.24 & 0.21 & 0.31 & 0.21 \\
\hline $\mathrm{K}_{\mathrm{h} \text { Carbs }}$ & 0.32 & 0.43 & 0.55 & 0.36 & 0.66 & 0.51 & 0.46 & 0.63 & 0.58 & 0.26 & 0.69 & 0.52 \\
\hline
\end{tabular}




\section{Chapter 8}

\section{Correlation of $\mathrm{CH}_{4}$}

\section{with lipids, proteins,}

and carbohydrates 


\subsection{Correlation of organic compositions and biomethane yield}

Lipids, proteins and carbohydrates make up the principal constituents of organic waste. Currently, there is limited information on how the three main components of organics impacts the AD performance. Therefore, assessing the correlation between the performance of anaerobic digestion system and the three main organic composition is required in order to enhance the efficiency of the AD process and to provide a tool for the prediction of the system performance.

In addition, in designing a co-digestion system this would provide a tool to optimize the system based on lipids, proteins, and carbohydrates fractions along with $\mathrm{C}: \mathrm{N}$ ratio as they are both important design parameters. In this work the influence of lipids, proteins and carbohydrates content on the anaerobic digestion of TWAS, SSO and manure was investigated. The feedstocks and their mixtures with different ratios as presented in Table 3.2- Table 3.5, were analysed to determine their total lipids, proteins, and carbohydrates contents. The results of biogas monitoring and the methane in biogas using GC from the sets of BMP essay were used to determine an empirical model based on the relationship between lipids, proteins, carbohydrates components and the system efficiency in terms of methane production and yield. The functional relationship between responses $\left(\mathrm{CH}_{4}\right)$ and the factors $(\mathrm{Lp}, \mathrm{Pr}$, and $\mathrm{Cr}$ ) were described by estimating the coefficients of the second-order polynomial model based on the experimental data according to Eq. 8.1 where $\mathrm{B}_{0}$ is a constant, $\mathrm{B}_{1}, \mathrm{~B}_{3}$, and $\mathrm{B}_{4}$ are linear coefficients and $\mathrm{B}_{2}$ is quadratic coefficient. The empirical model was used to predict the methane yield ( $\left.\mathrm{mLCH}_{4} / \mathrm{gCOD}_{\text {added }}\right)$ in terms of lipids, proteins, and carbohydrates components and the results are depicted by fit plots and residual plots as presented in figures 8.1 and 8.2.

$\mathrm{CH}_{4}=\mathrm{B}_{0}+\mathrm{B}_{1} \mathrm{Lp}+\mathrm{B}_{2} \mathrm{Lp}^{2}+\mathrm{B}_{3} \mathrm{Pr}+\mathrm{B}_{4} \mathrm{Cr}$

Each of the fit plots as shown in Figure 8.1 contains adequate number of observations throughout the entire range of the predictor values. Although for the lipids a few points on the top left corner of the plot seems to be outlier, the multiple $\mathrm{R}$ value of 0.7 indicates the model properly fits in the data. 
8.1. a)

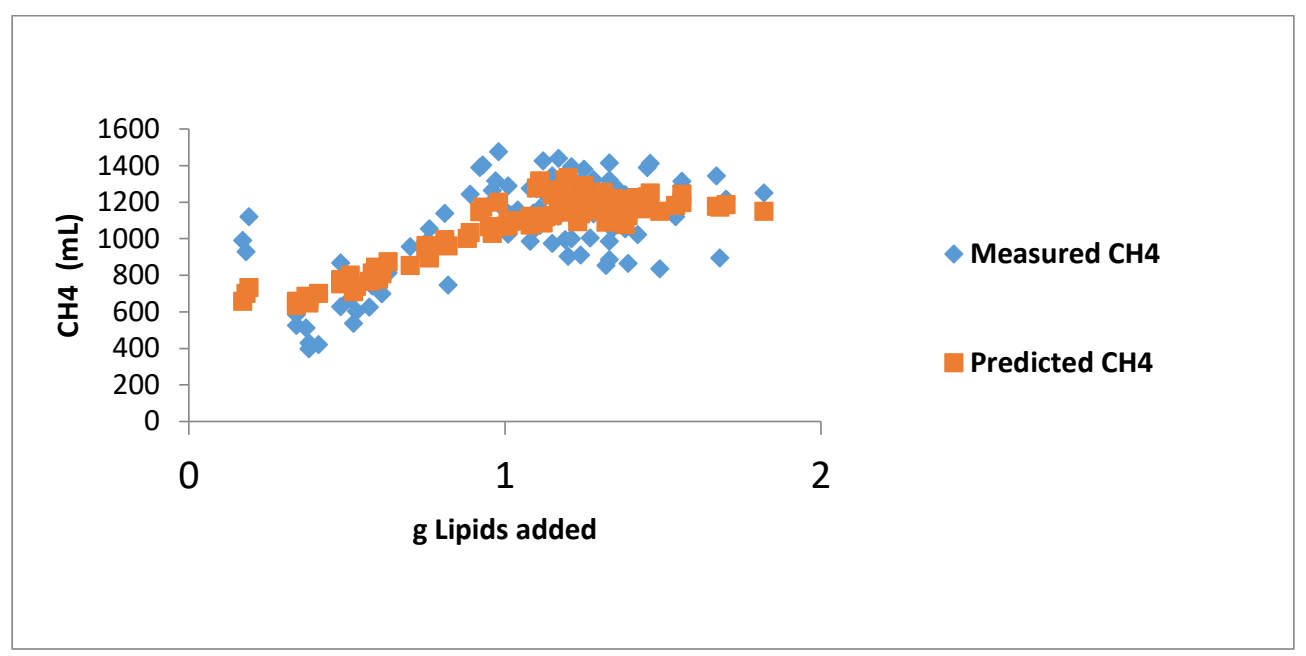

8.1.b)

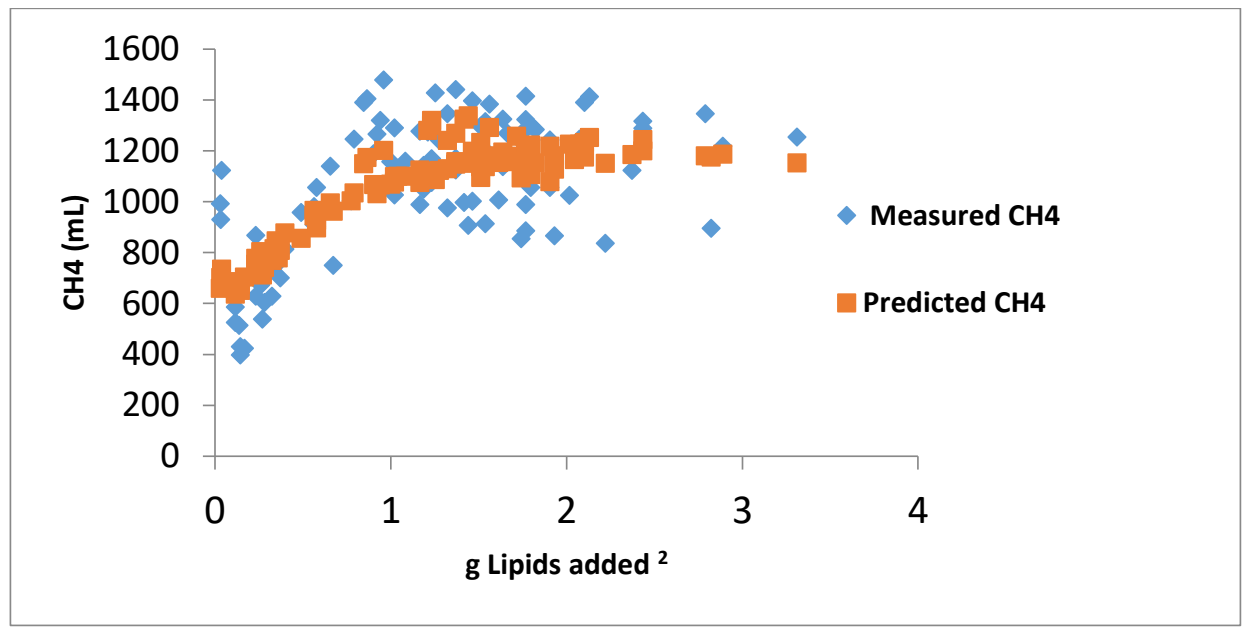


8.1.c)

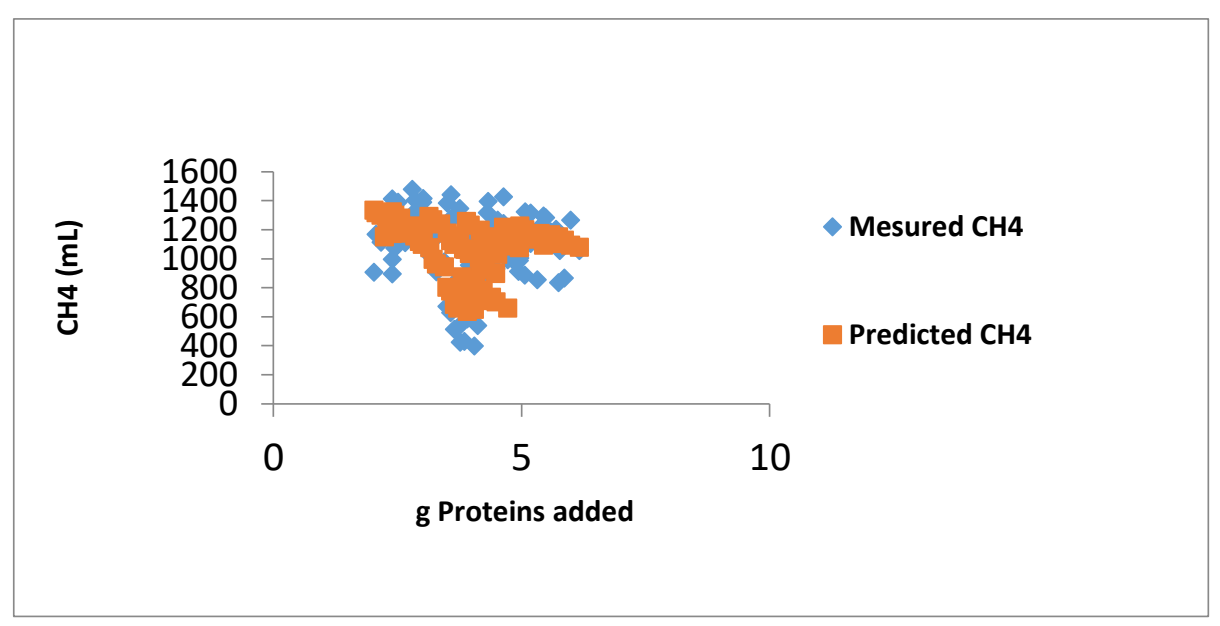

8.1. d)

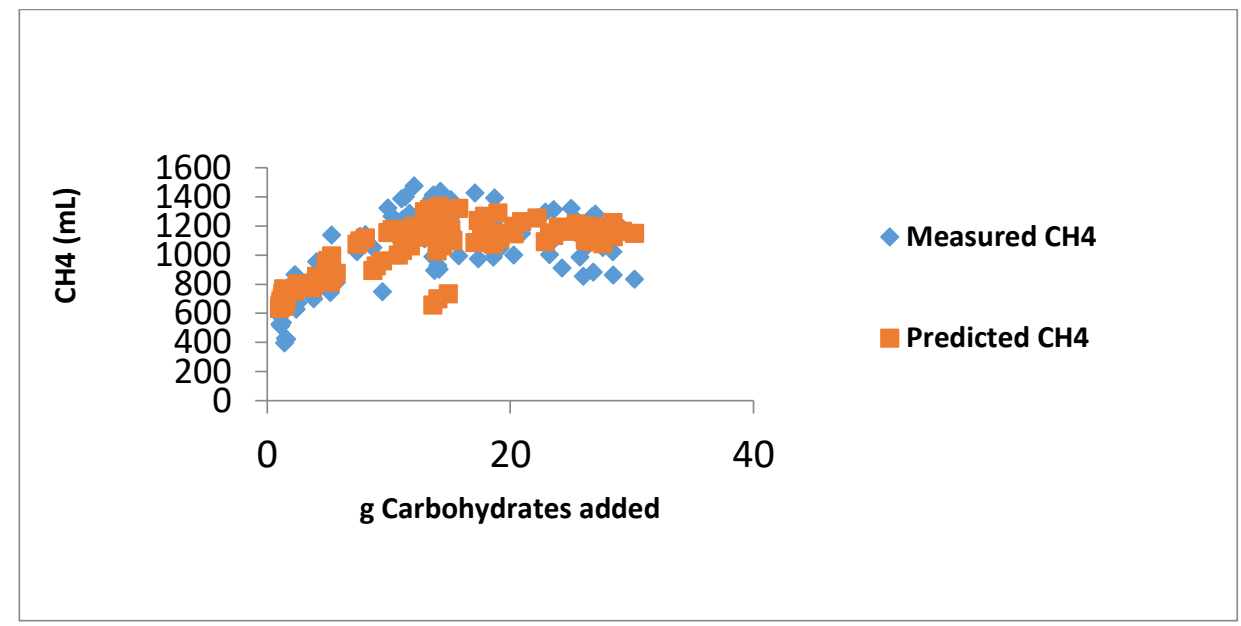

Figure 8.1. Fitted plot of response variable to lipids, Lipids², proteins, and carbohydrate

The residual plot was used to determine whether the model adequately meets the assumptions of the model. As the patterns in the points show, the points on the residual plots are randomly on both side of 0 which indicate that the model meets the model assumptions. 
8.2. a)

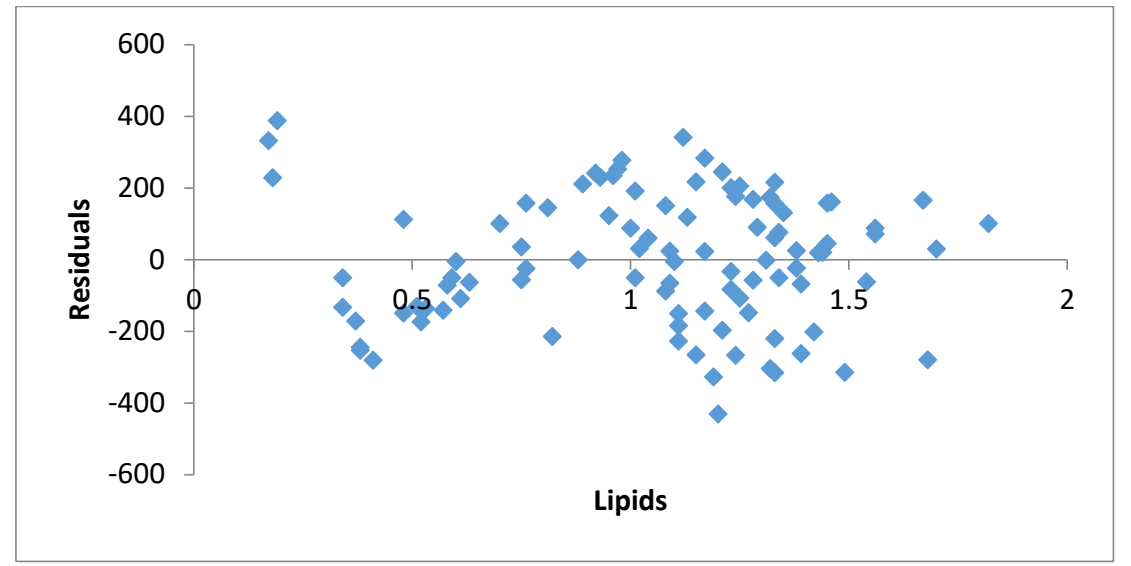

8.2. b)

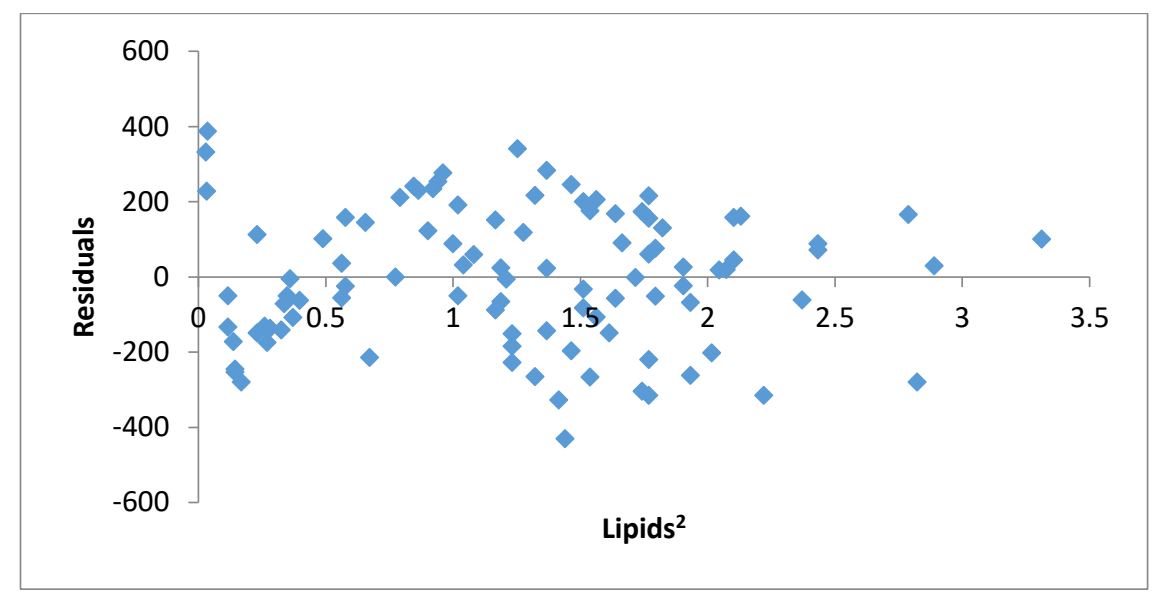

8.2. c)

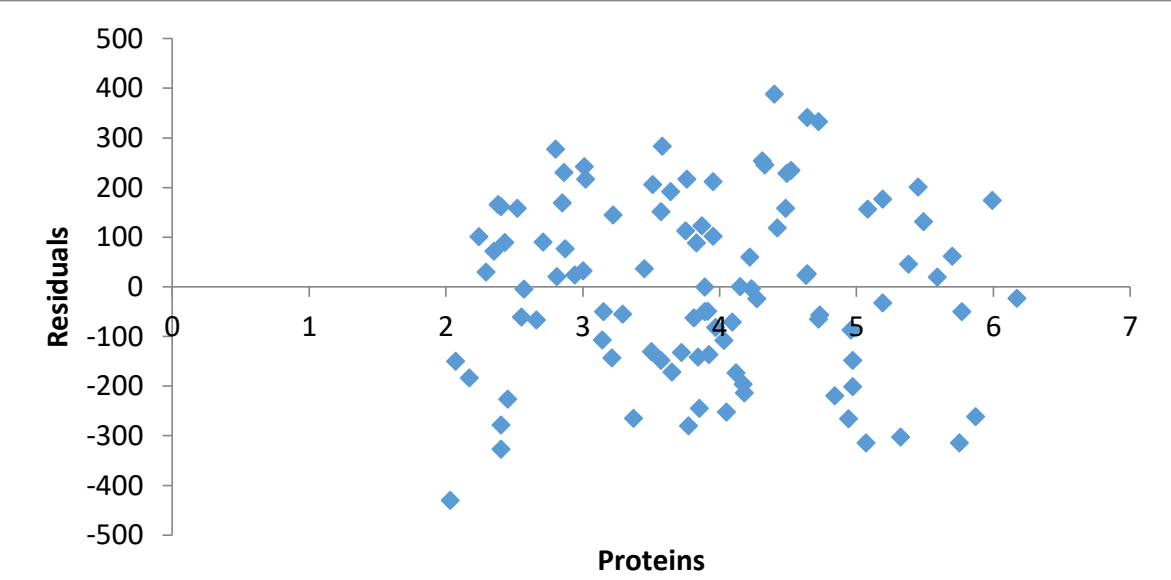


8.2.d.)

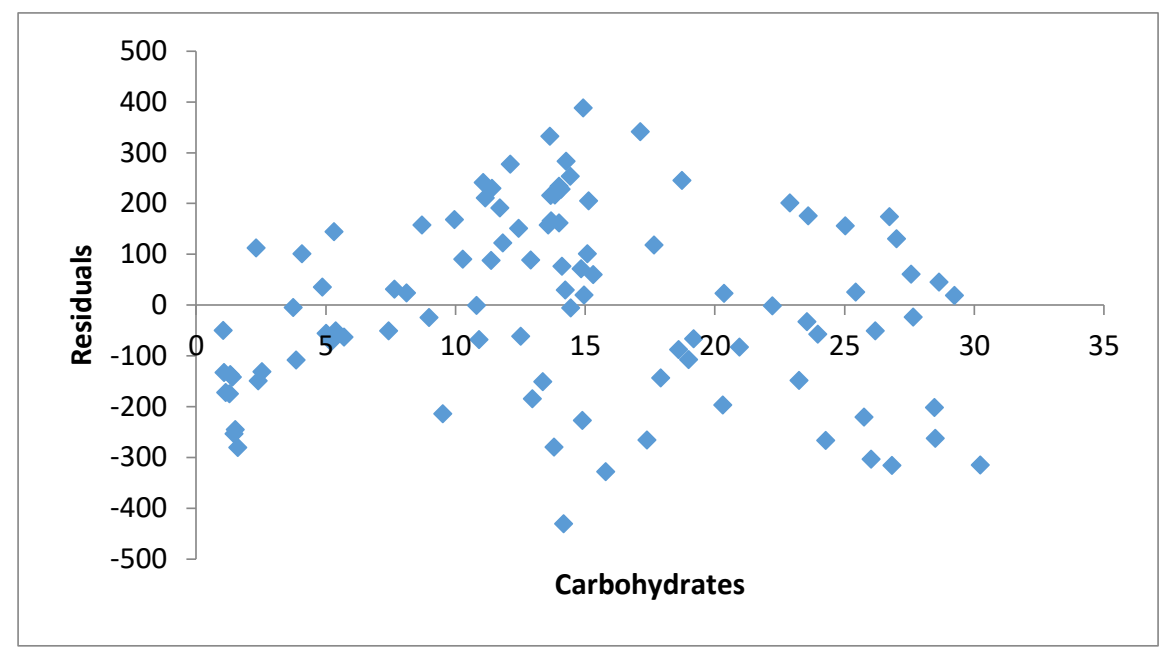

Figure 8.2. Residuals versus fits plot (residuals versus estimated response)

Table 8.1 includes the intercept, linear and quadratic coefficients of the Eq. 8.1 and summarizes some of the results of ANOVA analysis. The P-values $<0.05$ as shown in the table verifies that the correlation between the response and the variables is statistically significant. The findings suggest that the amount of lipids in substrate is directly proportional to the methane yield and its impact is significantly more than carbohydrates. While the lower fraction of proteins than lipids and carbohydrates increase the methane yield. Y. Li et al, 2017 also used a second-order polynomial model as a quick estimation of AD parameters, however their model only included proteins and lipids as variables. Their findings though verified that the more fraction of lipids significantly increases the methane production compared to proteins.

Table 8.1.Coefficients of variables in the second-order polynomial model and the results of ANOVA analysis

\begin{tabular}{lccccccc}
\hline & & & & Upper & Lower & Upper \\
& Coefficients & t Stat & P-value & Lower 95\% & $\mathbf{9 5 \%}$ & $\mathbf{9 5 . 0 \%}$ & $\mathbf{9 5 . 0 \%}$ \\
\hline Intercept & 825.279203 & 5.12631511 & $1.576 \mathrm{E}-06$ & 505.632211 & 1144.926 & 505.6322 & 1144.926 \\
Lipids & 862.890011 & 3.63241266 & 0.0004573 & 391.223242 & 1334.557 & 391.2232 & 1334.557 \\
Lipids $^{2}$ & -380.17074 & -3.2738253 & 0.0014856 & -610.738303 & -149.603 & -610.738 & -149.603 \\
Proteins & -117.12258 & -3.7852707 & 0.0002703 & -178.557985 & -55.6872 & -178.558 & -55.6872 \\
Carbs & 18.4329527 & 3.36910525 & 0.0010949 & 7.56981336 & 29.29609 & 7.569813 & 29.29609 \\
\hline
\end{tabular}




\section{2- Statistical Analysis}

Table 8.2 shows the experimental set-ups and the corresponding feedstock ratios and lipids: proteins: carbohydrates ratios. ANOVA was carried out for the $\mathrm{CH}_{4}$ yields in response to the feedstock ratios and lipids: proteins: carbohydrates ratios. The main effect plot for $\mathrm{CH}_{4}$ yield data mean is presented in Figure 8.3. The main effect plot shows the mean response of each level factors connected by the line. The steeper slope in the line explains the greater scale of the main effect. An effect is the variation in the methane yield when the factors i.e. the feedstock and lipids: proteins: carbohydrates ratios change from one level to another. The $\mathrm{P}$ value from the ANOVA results showed that the feedstock and lipids: proteins: carbohydrates ratios have significant effects on the $\mathrm{CH}_{4}$ yield.

Table 8.2. Experimental set-ups and the corresponding ratios of the feedstock and lipids: proteins: carbohydrates

\begin{tabular}{llcc}
\hline Experiment code & Feedstock ratios & Feedstock ratios code & Lipids: Proteins: Carbs \\
\hline A & TWAS Only & AA & $1: 11: 3$ \\
\hline A & SSO Only & BB & $1: 1.3: 8$ \\
\hline A & TWAS/SSO 9/1 & A & $1: 7: 5$ \\
\hline A & TWAS /SSO 7/3 & B & $1: 4: 7$ \\
\hline A & TWAS /SSO 1/1 & C & $1: 3: 7$ \\
\hline A & TWAS /SSO 3/7 & D & $1: 2: 8$ \\
\hline A & TWAS /SSO 1/9 & E & $1: 1.5: 8$ \\
\hline B & TWAS & AA & $1: 7: 2.5$ \\
\hline B & CC & $1: 4: 20$ \\
\hline B & Manure & A & $1: 7: 6$ \\
\hline B & TWAS / Manure 9/1 & B & $1: 5: 12$ \\
\hline B & TWAS / Manure 7/3 & C & $1: 4: 17$ \\
\hline B & TWAS / Manure 1/1 & D & $1: 4: 19$ \\
\hline B & TWAS / Manure 3/7 & E & $1: 4.2: 21$ \\
\hline C & TWAS / Manure 1/9 & CC & $1: 2: 12$ \\
\hline C & Manure & BB & $1: 3.5: 18.5$ \\
\hline C & SSO & A & $1: 4: 20$ \\
\hline C & Manure/SSO 9/1 & B & $1: 3: 17$ \\
\hline C & Manure /SSO 7/3 & D & $1: 2.7: 15$ \\
\hline C & Manure /SSO 5/5 & & \\
\hline
\end{tabular}




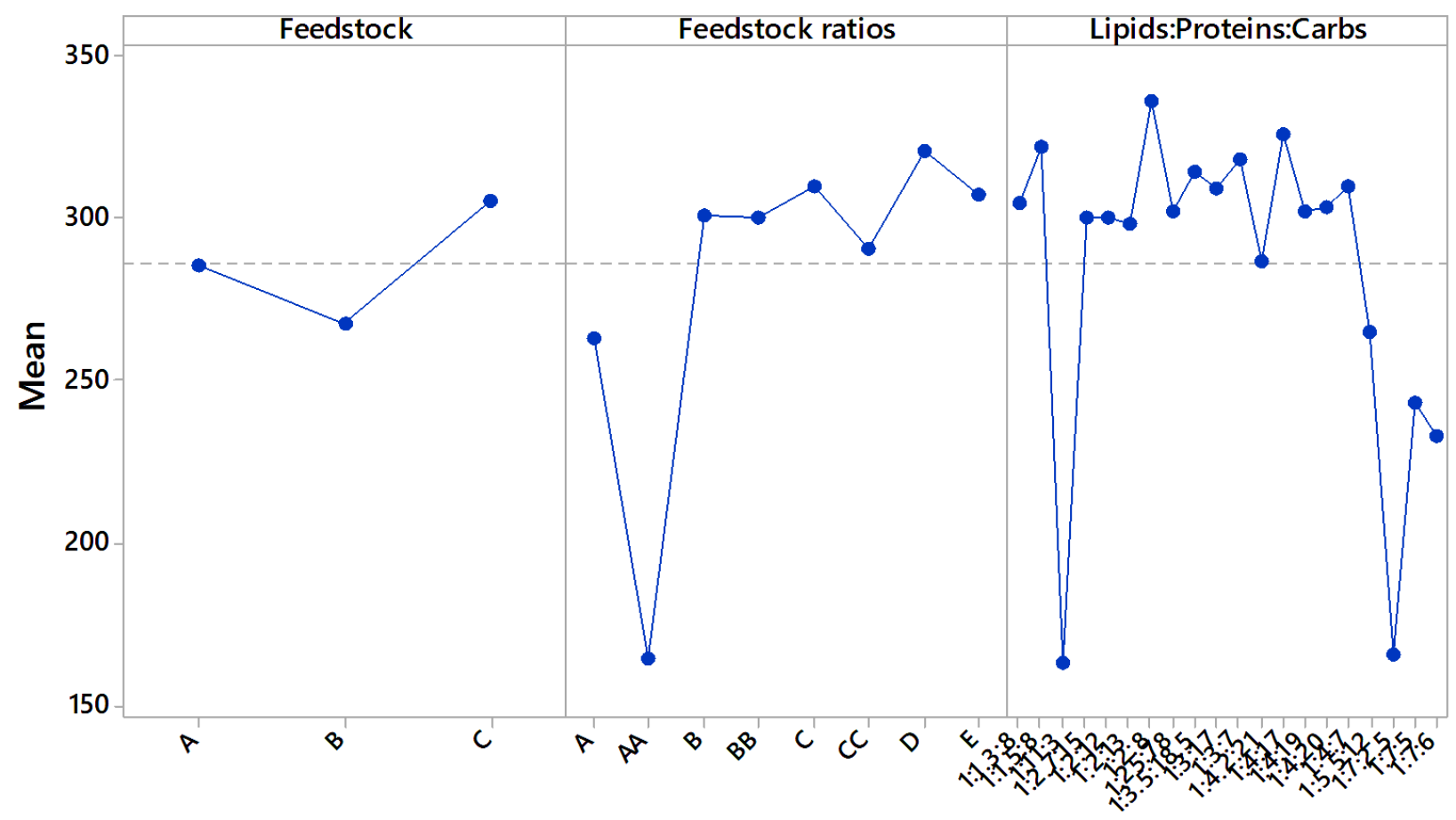

Figure 8.3. a. Main effect plot for $\mathrm{CH}_{4}$ yield data mean

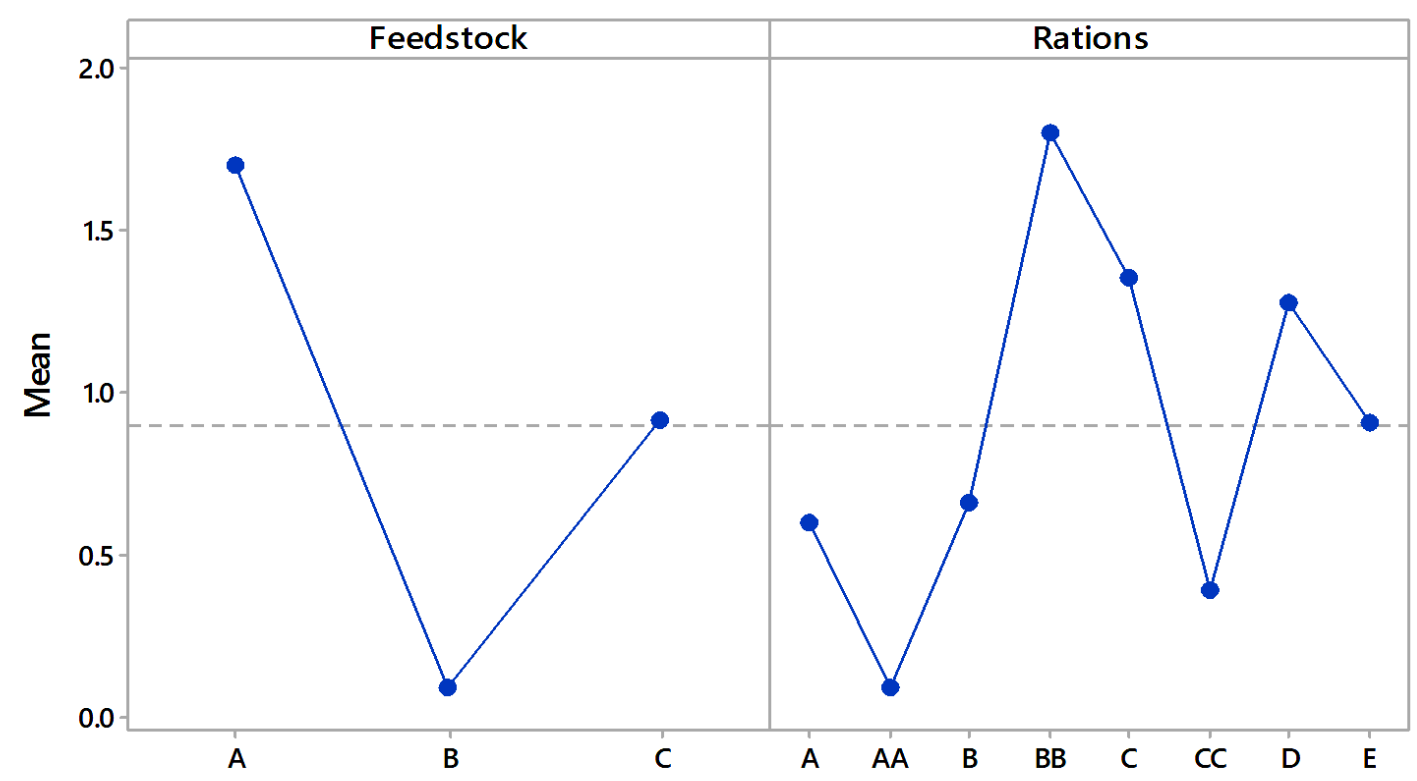

Figure 8.3. b. Main effect plot for $\mathrm{CH}_{4}$ yield data mean for the lag phase data mean 


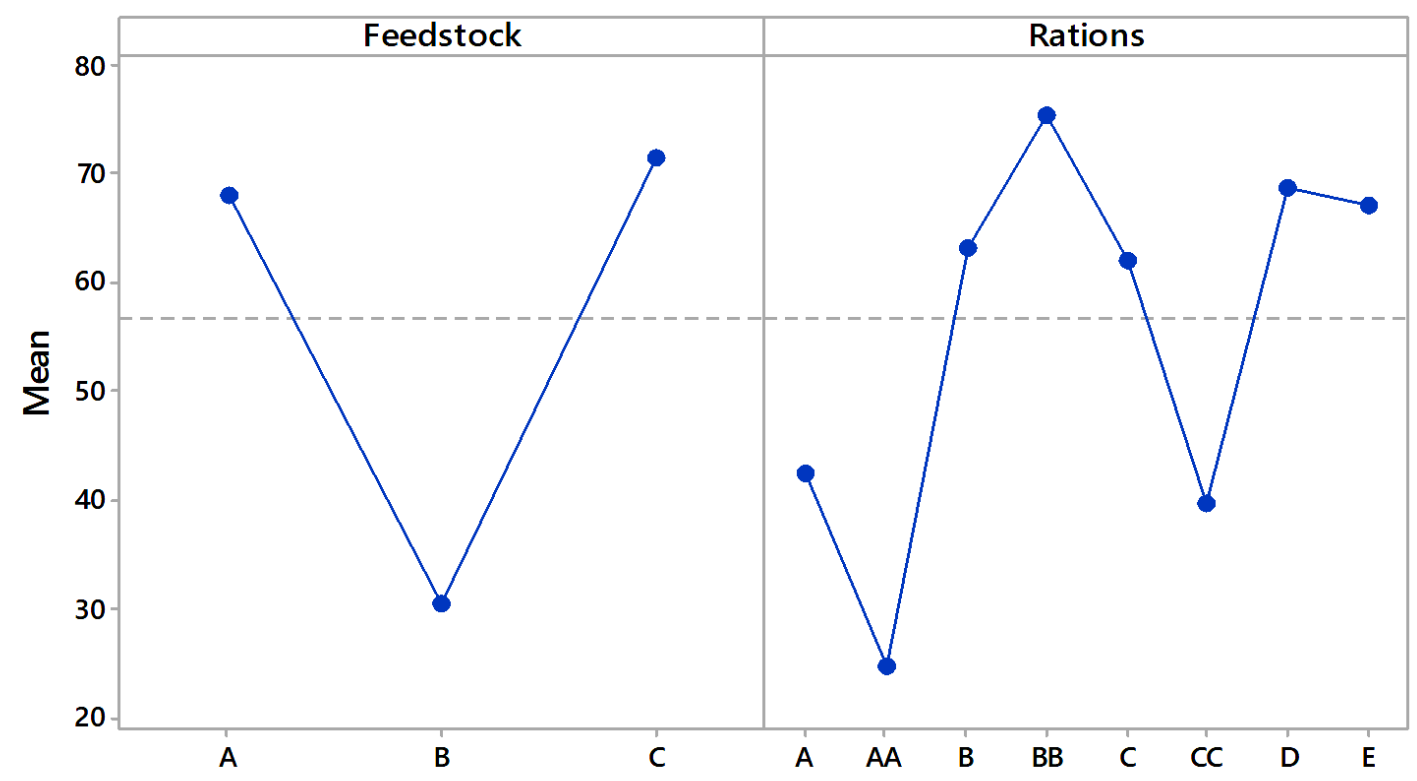

Figure 8.3. c. Main effect plot for $\mathrm{CH}_{4}$ yield data mean for the $\mathrm{CH}_{4}$ maximum rate data mean

The interval plot of $\mathrm{CH}_{4}$ yield with $95 \%$ confidence interval for the mean using individual standard deviations to calculate the intervals is shown in Figure 8.4. The width of the interval plot represents the extent of variation in the data. A small interval would indicate more consistent data and less variation. As shown in Figure 8.4 the variation is more for some ratios than the other ones. The variation in the methane yield for the feedstock ratios of 1:9 and 7:3 were more than the other feedstock mixing ratios. The data was quite consistent with that of SSO alone. As shown in Figure 8.5.a, all of the intervals include 0 which means that the corresponding $\mathrm{CH}_{4}$ yield data means are not significantly different in response to the feedstocks. However, according to Figure 8.5.b., for some of the feedstock mixing ratios, the corresponding means are significantly different. 


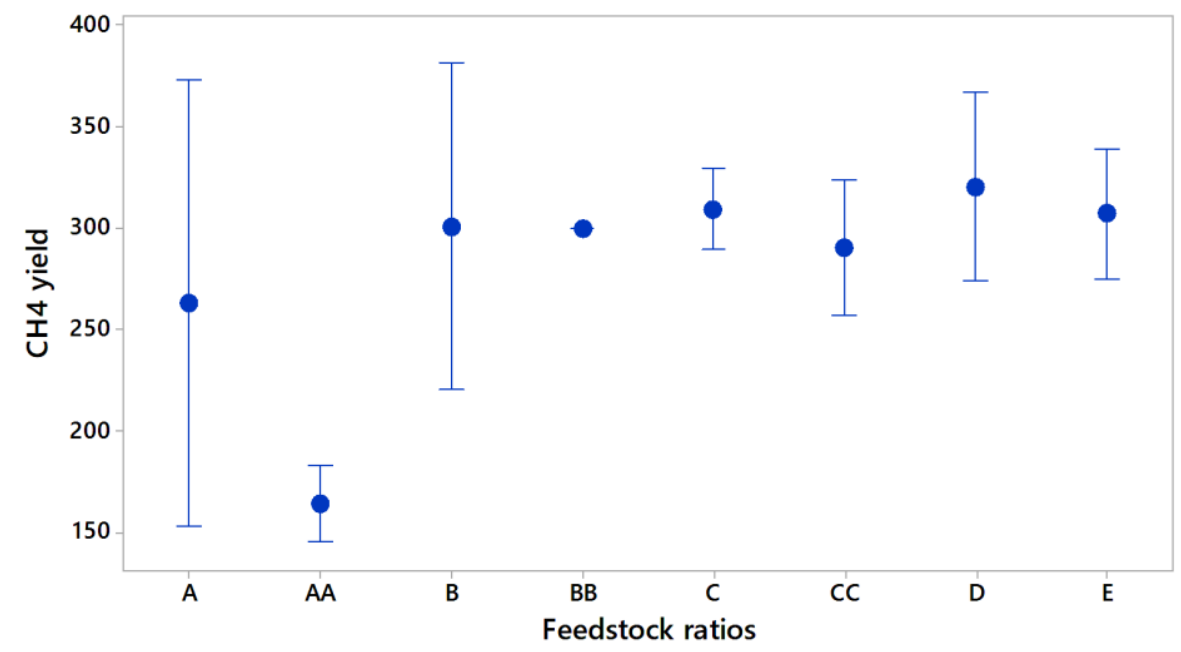

Figure 8.4. Interval plot of $\mathrm{CH}_{4}$ yield with $95 \%$ confidence interval for the mean

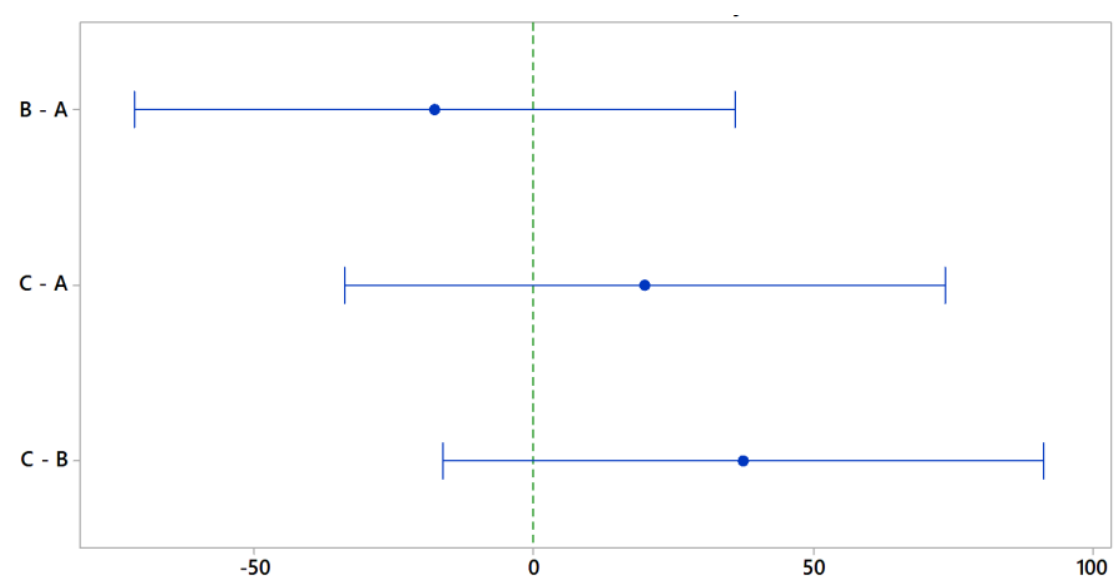

Figure 8.5. a. Fisher individual 95\% confidence interval differences of means for $\mathrm{CH}_{4}$ yields in response to the feedstocks 


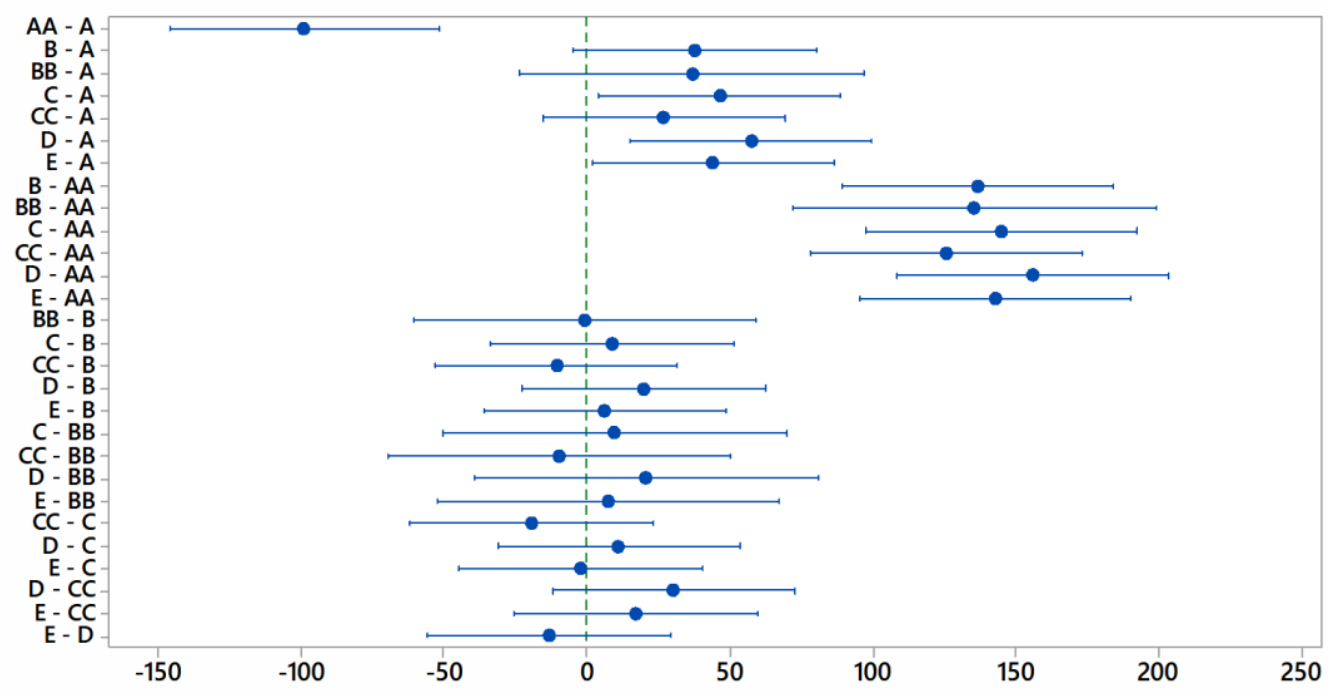

Figure 8.5. b. Fisher individual $95 \%$ confidence interval differences of means for $\mathrm{CH}_{4}$ yields in response to the ratios 


\section{Chapter 9}

Conclusions 


\section{Conclusions}

\subsection{Biomethane potential}

A series of binary and ternary anaerobic co-digestion was designed to evaluate the biomethane potential and to investigate the correlation between the biomethane production and the mixing ratios as well as lipids: proteins: carbohydrates ratios of the feedstock. Gompertz model was used to assess the compatibility of the model to the data obtained by the experiment. A first order kinetic model was applied using AquaSim 2.0 to assess the hydrolysis kinetics and its influence on the process. A simple empirical model was derived based on the correlation of biomethane production to the lipids, proteins and carbohydrates content of the feedstocks which included TWAS, manure and SSO.

\subsubsection{Binary co-digestion of TWAS, manure, and SSO}

$>$ The three sets of the binary co-digestion primarily verified the advantages of co-digestion over mono-digestion of TWAS, manure and SSO.

$>$ Among the three binary co-digestion including TWAS/SSO, TWAS/manure, and Manure/SSO, the most amount of the methane yield was achieved by co-digestion of Manure/SSO at the mixing ratio of 7:3 corresponding to $363 \mathrm{~mL} \mathrm{CH}_{4} / \mathrm{g}$ COD added.

$>$ The modified Gompertz model showed a good fit to the experimental data by a diversion ranging from $4 \%$ to $6 \%$.

$>$ The optimum ratios of the feedstocks as well as the lipids: proteins: carbohydrates varied by the different feedstocks.

$>$ In co-digestion of TWAS/SSO the maximum ultimate $\mathrm{CH}_{4}$ of $1252 \mathrm{~mL}^{2}$ and $\mathrm{CH}_{4}$ yield of $357 \mathrm{~mL} \mathrm{CH}_{4} / \mathrm{g} \mathrm{COD}$ added corresponded to the mixing ratio of 3:7 and lipids: proteins: carbohydrates ratio of $1: 2: 8$.

TWAS/manure co-digestion resulted in $1069 \mathrm{~mL}$ ultimate $\mathrm{CH}_{4}$ production and $324 \mathrm{~mL}$ $\mathrm{CH}_{4} / \mathrm{g}$ COD added biomethane yield at the mixing ratio of 3:7 and lipids: proteins: carbohydrates ratio of $1: 4: 17$.

$>$ For Manure/SSO co-digestion an ultimate $\mathrm{CH}_{4}$ of $1186 \mathrm{~mL}$ and a $\mathrm{CH}_{4}$ yield of $363 \mathrm{~mL}$ $\mathrm{CH}_{4} / \mathrm{g} \mathrm{COD}$ added was achieved corresponding to the mixing ratio of $7: 3$ and lipids: proteins: carbohydrate ratio of $1: 3.5: 18.5$. 


\subsubsection{Ternary co-digestion of TWAS, manure and SSO}

$>$ Among co-digestion of the three feedstocks at different ternary combinations, the most ultimate methane production and yield occurred at TWAS/manure/SSO mixing ratio of 2:4:4 and lipids: proteins: carbohydrate ratio of $1: 3: 12$.

$>$ The maximum methane production and yield in co-digestion of TWAS/manure/SSO were $1424 \mathrm{~mL} 356 \mathrm{~mL} \mathrm{CH}_{4} / \mathrm{g}$ COD added, respectively.

$>$ The modified Gompertz model showed a good fit to the results obtained by the experiment with less than $5 \%$ diversion.

$>$ A higher maximum ultimate methane and methane yield was achieved by ternary codigestion of TWAS/manure and SSO, however at some ratios the ternary co-digestion did not produce higher methane than some of the combinations in the binary co-digestion.

$>$ Synergistic effect did not demonstrate further improvement in the ternary co-digestion of TWAS, manure, and SSO compared to their combinations in the binary co-digestion.

$>$ The most synergistic effect in co-digestion of TWAS/manure/SSO was 19\% while the maximum synergistic effect was $36 \%$ in manure/SSO co-digestion.

\subsection{Hydrolysis/acidification}

Along with the biomethane potential assay, a series of hydrolysis/ acidification of the binary and ternary combinations of the feedstocks was designed to evaluate the hydrolysis and acidification process in co-digestion of TWAS, manure, and SSO. The binary and ternary anaerobic codigestion were set up with the same combination as the BMP experiments for investigating the correlation between the solublization, VFAs yield, and hydrolysis rate, with the mixing ratios as well as lipids: proteins: carbohydrates ratios of the feedstock. First-order kinetic model was used to assess the compatibility of the model to the data obtained by the experiment. The model was used to obtain the kinetic rare coefficients of COD, lipids, proteins and carbohydrates of the feedstocks at different mixing ratios. 


\subsubsection{Co-digestion of TWAS/SSO}

An improvement of hydrolysis/acidification was observed in co-digestion of the feedstocks at different mixing ratios

$>$ In TWAS/SSO co-digestion the maximum degree of solublization was $30 \%$ at the mixing ratio of 1:9.

$>$ The synergistic effect was the highest at TWAS/SSO mixing ratio of 7:3 corresponding to $44 \%$ improvement as a result of using co-substrate and improving synergy.

$>$ A maximum VFAs yield of $312 \mathrm{mg} / \mathrm{g}$ VSS added was attained by TWAS/SSO codigestion at the mixing ratio of 1:9.

$>$ The hydrolysis kinetic rate coefficient was compatible to the first order kinetic model with a diversion less than $10 \%$.

$>$ The maximum hydrolysis rate coefficient of COD, proteins, and carbohydrates in codigestion of TWAS/SSO were $0.35,0.07,0.34$ and $0.68 \mathrm{~d}^{-1}$, corresponding to the mixing ratios of 1:9, 3:7, 1:9, respectively.

$>$ The maximum hydrolysis rate coefficient of lipids was 0.07 which corresponded to the TWAS:SSO mixing ratios of 5:5 and 3:7.

\subsubsection{Co-digestion of TWAS/manure}

The percentage improvement of hydrolysis/acidification varied by different mixing ratios of TWAS and manure.

$>$ In co-digestion of TWAS/manure, the maximum degree of solublization was $34 \%$ corresponding to the TWAS/manure mixing ratio of 3:7.

$>$ A synergistic effect of $38 \%$ was achieved by TWAS/manure co-digestion at the mixing ratio of $3: 7$.

$>$ The maximum VFAs yield of $507 \mathrm{mg} / \mathrm{g}$ VSS added was achieved by TWAS/manure mixing ratio of 3:7.

$>$ The hydrolysis kinetic rate coefficient was compatible to the first order kinetic model with a diversion less than $10 \%$. 
The maximum hydrolysis rate coefficient of COD, lipids, proteins, and carbohydrates in co-digestion of TWAS/manure were $0.33,0.09,0.27$, and $0.59 \mathrm{~d}^{-1}$, corresponding to the mixing ratio of $3: 7$.

\subsubsection{Co-digestion of manure /SSO}

The hydrolysis/acidification improved to different extents in response to the different mixing ratios in co-digestion of TWAS and manure.

$>$ The most degree of solublization was $35 \%$ corresponding to manure/SSO mixing ratios of $3: 7$ and $1: 9$.

$>$ The highest synergistic effect of $34 \%$ in co-digestion of manure and SSO occurred at the mixing ratio of $9: 1$.

$>$ In manure/SSO co-digestion, the maximum VFAs yield of $400 \mathrm{mg} / \mathrm{g}$ VSS added was achieved at the mixing ratio of 1:9.

$>$ The hydrolysis kinetic rate coefficient was compatible to the first order kinetic model with a diversion less than $10 \%$

$>$ The maximum hydrolysis rate coefficient of COD, lipids, proteins, and carbohydrates in co-digestion of manure/SSO were $0.38,0.11,0.33$, and $0.68 \mathrm{~d}-1$, corresponding to the mixing ratio of $3: 7$.

\subsubsection{Co-digestion of TWAS/manure /SSO}

$>$ In the ternary co-digestion of TWAS, manure, and SSO the maximum solublization was $30 \%$ corresponding to TWAS/manure/SSO mixing ratio of 2:4:4 and 1:8:1.

$>$ The maximum synergistic effect of $35 \%$ corresponded to TWAS/manure/SSO mixing ratio of $1: 8: 1$.

$>$ In co-digestion of TWAS/manure/SSO the highest VFAs yield of $380 \mathrm{mg}$ VFAs/g VSS added was achieved at the mixing ratio of $2: 4: 4$.

$>$ The hydrolysis kinetic rate coefficient was compatible to the first order kinetic model with a diversion less than $10 \%$

$>$ The hydrolysis rate coefficient of COD were within the range of 0.21 to 0.39 for all of the co-digesters. 
$>$ The maximum hydrolysis rate coefficients of COD, lipids, and carbohydrates in codigestion of TWAS/manure/SSO were $0.39,0.14$, and $0.69 \mathrm{~d}^{-1}$ corresponding to the mixing ratio of $2: 4: 4$.

$>$ In co-digestion of TWAS/manure/SSO, the maximum hydrolysis rate coefficient for the proteins content was 0.23 which corresponded to the mixing ratios of $8: 1: 1$ and $2.5: 5: 2.5$.

\subsection{Correlation of biomethane production with the organic content}

\subsubsection{COD: $N$ and lipids:proteinscarbohydrates ratios}

$>$ In TWAS/SSO co-digestion the maximum ultimate $\mathrm{CH}_{4}$ corresponded to COD:N ratio of 28 corresponding to the lipids: proteins: carbohydrates ratio of $1: 2: 8$. The trend of variations of the methane yield versus COD: $\mathrm{N}$ was similar to the trend that was observed for its variation vursus lipids:proteins. The changes of methane yield versus lipids:carbohydrates and proteins:carbohydrates showed a similar trend while it did not comply with that of COD: $\mathrm{N}$ ratio.

$>$ In TWAS/manure co-digestion, the maximum $\mathrm{CH}_{4}$ yield occurred at COD:N ratio of 41 corresponding to the lipids: proteins: carbohydrates ratio of 1:4:17. Similar to the codigestion of TWAS/SSO, in TWAS/manure co-digestion the trend of variations of the methane yield versus COD: $\mathrm{N}$ was similar to the trend that was observed for its variation versus lipids:proteins. The changes of methane yield versus lipids:carbohydrates and proteins:carbohydrates did not comply with that of COD:N ratio although they both showed a simiar trend.

$>$ In Manure/SSO co-digestion, the maximum methane yield corresponded to the COD:N ratio of 41 and lipids: proteins: carbohydrates ratio of 1:3.5:18.5. The variations of methane yield versus COD:N and lipids: proteins did not show a similar trend. Although, in terms of the changes of the $\mathrm{CH}_{4}$ yield, both lipids: carbohydrates and proteins: carbohydrates ratios demonstrated similar trend.

In TWAS/manure/SSO co-digestion the maximum methane yield corresponded to COD:N tatio of 28 and the lipids:proteins:carbohydrates ratio of 1:3:12. The trend of variations of the methane yield vursus COD:N was similar to the trend that was observed for its variation versus lipids:proteins. The changes of methane yield vursus lipids:carbohydrates and 
proteins:carbohydrates showed a similar trend while it did not comply with that of COD:N. TWAS/manure/SSO co-digestion.

\subsubsection{Anaerobic co-digestion model}

An empirical model would be a useful approach to predict the methane yield $\left(\mathrm{mLCH}_{4} / \mathrm{gCOD}_{\text {added }}\right)$ based on the lipids, proteins, and carbohydrates components of the feedstock in anaerobic codigestion of multi substrate. As the three main components of any type of feedstocks in biowaste are lipids, proteins, and carbohydrates, an empirical model was presented that correlates the $\mathrm{CH}_{4}$ yields with lipids, proteins and carbohydrates content of the feedstock. The functional relationship between responses which was $\mathrm{CH}_{4}$ yield and the factors including lipids, proteins and carbohydrates (Lp, Pr, and Cr) were described by estimating the coefficients of the second-order polynomial model based on the experimental data. The fit plots together with the residual plots for lipids, proteins and carbohydrates showed that the model adequately fits to the data and meet the model assumption.

\subsection{Suggested future works}

Validating the batch study results for AnCoD of Manure/SSO and TWAS/Manure/SSO at 7:3 and 2:4:4 mixing ratios in CSTR mode

$>$ Investigating the validity of the proposed empirical model for a variety of the feedstocks including the industrial wastes

$>$ Investigating the validity of the proposed empirical model using CSTR mode.

$>$ Implementing a cost-benefit analysis for applying AnCoD of Manure/SSO and TWAS/Manure/SSO for GTA versus individual AD of manure and SSO.

Analyzing carbon footprint reduction by AnCoD of manure with TWAS as compared to conventional $\mathrm{AD}$ and composting 


\section{Appendices}

\section{A. Analytical results for AnCoD of TWAS and SSO}

Table A. 1. Characteristics of raw feedstocks in AnCoD of TWAS and SSO

\begin{tabular}{|c|c|c|c|c|c|c|c|}
\hline \multirow{2}{*}{ Parameters } & \multirow{2}{*}{ Units } & \multicolumn{2}{|c|}{ SSO } & \multicolumn{2}{|c|}{ TWAS } & \multicolumn{2}{|c|}{ Seed } \\
\hline & & MEAN & SD & MEAN & SD & MEAN & $\mathrm{SD}$ \\
\hline TCOD & $\mathrm{mg} / \mathrm{L}$ & 110000 & 7795 & 40000 & 2053 & 16400 & 444 \\
\hline SCOD & $\mathrm{mg} / \mathrm{L}$ & 44400 & 100 & 1360 & 26 & 762 & 19 \\
\hline TSS & $\mathrm{mg} / \mathrm{L}$ & 53833 & 6252 & 31450 & 1006 & 15033 & 400 \\
\hline VSS & $\mathrm{mg} / \mathrm{L}$ & 38478 & 3649 & 25600 & 2216 & 10900 & 200 \\
\hline TS & $\mathrm{mg} / \mathrm{L}$ & 62187 & 1025 & 38810 & 1859 & 17450 & 582 \\
\hline VS & $\mathrm{mg} / \mathrm{L}$ & 43493 & 761 & 34477 & 1027 & 13140 & 328 \\
\hline Ammonia & $\mathrm{mg} / \mathrm{L}$ & 1138 & 6 & 255 & 9 & 795 & 40 \\
\hline $\mathrm{pH}$ & $\begin{array}{c}- \\
\mathrm{mg}\end{array}$ & 5.6 & 0.2 & 6.3 & 0.2 & 7.2 & 0.2 \\
\hline Alkalinity & $\mathrm{CaCO} 3 / \mathrm{L}$ & 6080 & 522 & 1953 & 148 & 4943 & 465 \\
\hline $\mathrm{TN}$ & $\mathrm{mg} / \mathrm{L}$ & 3267 & 751 & 2900 & 400 & 1425 & 203 \\
\hline TSN & $\mathrm{mg} / \mathrm{L}$ & 910 & 38 & 420 & 64 & 696 & 112 \\
\hline Total Carbs & $\mathrm{mg} / \mathrm{L}$ & 14360 & 3580 & 1080 & 112 & 549 & 82 \\
\hline Total Proteins & $\mathrm{mg} / \mathrm{L}$ & 2308 & 198 & 3759 & 298 & 1648 & 56 \\
\hline Total Lipids & $\mathrm{mg} / \mathrm{L}$ & 1731 & 2420 & 351 & 27 & 163 & 52 \\
\hline
\end{tabular}


Table A. 2. Average $\mathrm{CH}_{4}$ production in AnCoD of TWAS and SSO

\begin{tabular}{|c|c|c|c|c|c|c|c|c|c|c|c|c|c|}
\hline \multicolumn{14}{|c|}{ Average $\mathrm{CH}_{4}$ Measurement $(\mathrm{mL})$} \\
\hline $\begin{array}{l}\text { Time } \\
\text { (day) }\end{array}$ & TWAS & SD & SSO & SD & $\begin{array}{c}\text { T/SSO } \\
9 / 1\end{array}$ & SD & $\begin{array}{c}\text { T/SSO } \\
7 / 3\end{array}$ & SD & $\begin{array}{c}\text { T/SSO } \\
1 / 1\end{array}$ & SD & $\begin{array}{c}\mathrm{T} / \mathrm{SSO} \\
1 / 9\end{array}$ & SD & $\begin{array}{c}\mathrm{T} / \mathrm{SSO} \\
3 / 7\end{array}$ \\
\hline 1 & 6 & 0.3 & 3 & 0.1 & 6 & 0.3 & 7 & 0.3 & 3 & 0.1 & 4 & 0.1 & 3 \\
\hline 3 & 24 & 1.2 & 20 & 1.0 & 20 & 1.0 & 33 & 1.7 & 17 & 0.7 & 27 & 1.0 & 20 \\
\hline 4 & 43 & 2.3 & 27 & 1.3 & 35 & 1.7 & 54 & 2.8 & 25 & 1.1 & 49 & 1.9 & 38 \\
\hline 5 & 58 & 3.0 & 42 & 2.0 & 57 & 2.8 & 68 & 3.5 & 39 & 1.7 & 82 & 3.1 & 65 \\
\hline 6 & 39 & 2.0 & 48 & 2.3 & 44 & 2.2 & 51 & 2.6 & 39 & 1.6 & 60 & 2.3 & 76 \\
\hline 7 & 37 & 1.9 & 48 & 2.3 & 51 & 2.5 & 45 & 2.3 & 66 & 2.8 & 52 & 2.0 & 69 \\
\hline 8 & 21 & 1.1 & 51 & 2.5 & 34 & 1.7 & 42 & 2.2 & 53 & 2.2 & 45 & 1.7 & 78 \\
\hline 10 & 28 & 1.4 & 55 & 2.7 & 34 & 1.7 & 60 & 3.0 & 84 & 3.5 & 80 & 3.0 & 93 \\
\hline 12 & 26 & 1.4 & 51 & 2.5 & 25 & 1.2 & 29 & 1.5 & 81 & 3.4 & 74 & 2.8 & 83 \\
\hline 14 & 21 & 1.1 & 39 & 1.9 & 22 & 1.1 & 22 & 1.1 & 47 & 2.0 & 33 & 1.2 & 64 \\
\hline 16 & 19 & 1.0 & 25 & 1.2 & 15 & 0.7 & 23 & 1.2 & 21 & 0.9 & 20 & 0.8 & 20 \\
\hline 19 & 23 & 1.2 & 23 & 1.1 & 25 & 1.2 & 25 & 1.3 & 21 & 0.9 & 25 & 1.0 & 22 \\
\hline 22 & 18 & 0.9 & 24 & 1.2 & 15 & 0.8 & 17 & 0.9 & 24 & 1.0 & 26 & 1.0 & 30 \\
\hline 26 & 15 & 0.8 & 20 & 1.0 & 10 & 0.5 & 12 & 0.6 & 14 & 0.6 & 15 & 0.6 & 22 \\
\hline 29 & 10 & 0.5 & 14 & 0.7 & 8 & 0.4 & 10 & 0.5 & 9 & 0.4 & 12 & 0.5 & 17 \\
\hline 34 & 33 & 1.7 & 33 & 1.6 & 32 & 1.6 & 33 & 1.7 & 31 & 1.3 & 31 & 1.2 & 31 \\
\hline 37 & 13 & 0.7 & 12 & 0.6 & 14 & 0.7 & 13 & 0.7 & 9 & 0.4 & 12 & 0.4 & 14 \\
\hline 40 & 14 & 0.7 & 13 & 0.6 & 14 & 0.7 & 13 & 0.7 & 10 & 0.4 & 12 & 0.5 & 15 \\
\hline 43 & 14 & 0.7 & 13 & 0.6 & 13 & 0.6 & 11 & 0.6 & 9 & 0.4 & 11 & 0.4 & 10 \\
\hline 49 & 1 & 0.1 & 3 & 0.2 & 5 & 0.2 & 3 & 0.2 & 1 & 0.1 & 2 & 0.1 & 5 \\
\hline 56 & 0 & 0.0 & 0 & 0.0 & 7 & 0.3 & 5 & 0.3 & 4 & 0.2 & 6 & 0.2 & 4 \\
\hline
\end{tabular}


Table A. 3. Cumulative $\mathrm{CH}_{4}$ production in AnCoD of TWAS and SSO

\begin{tabular}{|c|c|c|c|c|c|c|c|c|c|c|c|c|c|c|}
\hline \multicolumn{15}{|c|}{ Cumulative $\mathrm{CH}_{4}$ Production (mL) } \\
\hline $\begin{array}{l}\text { Time } \\
\text { (day) }\end{array}$ & TWAS & SD & SSO & SD & $\begin{array}{c}\mathrm{T} / \mathrm{SSO} \\
9 / 1\end{array}$ & SD & $\begin{array}{c}\text { T/SSO } \\
7 / 3\end{array}$ & SD & $\begin{array}{c}\mathrm{T} / \mathrm{SSO} \\
1 / 1\end{array}$ & SD & $\begin{array}{c}\text { T/SSO } \\
1 / 9\end{array}$ & SD & $\begin{array}{l}\text { T/SSO } \\
3 / 7\end{array}$ & SD \\
\hline 0 & 0 & 0 & 0 & 0 & 0 & 0 & 0 & 0 & 0 & 0 & 0 & 0 & 0 & 0.0 \\
\hline 1 & 7 & 0 & 5 & 0 & 9 & 0 & 12 & 1 & 6 & 0 & 7 & 0 & 4 & 0.2 \\
\hline 3 & 35 & 2 & 46 & 2 & 39 & 2 & 70 & 3 & 37 & 2 & 56 & 3 & 36 & 1.4 \\
\hline 4 & 86 & 4 & 99 & 5 & 92 & 5 & 164 & 8 & 83 & 4 & 146 & 8 & 96 & 3.8 \\
\hline 5 & 154 & 8 & 182 & 8 & 177 & 8 & 284 & 14 & 154 & 8 & 296 & 16 & 200 & 8.0 \\
\hline 6 & 199 & 10 & 278 & 13 & 243 & 13 & 373 & 18 & 225 & 11 & 405 & 22 & 323 & 12.9 \\
\hline 7 & 242 & 12 & 373 & 17 & 319 & 17 & 452 & 22 & 345 & 18 & 500 & 27 & 435 & 17.4 \\
\hline 8 & 267 & 13 & 475 & 22 & 369 & 22 & 527 & 26 & 441 & 23 & 582 & 31 & 560 & 22.3 \\
\hline 10 & 299 & 15 & 583 & 27 & 419 & 27 & 631 & 31 & 593 & 30 & 727 & 39 & 710 & 28.3 \\
\hline 12 & 330 & 16 & 685 & 31 & 457 & 31 & 683 & 34 & 739 & 38 & 862 & 46 & 843 & 34.5 \\
\hline 14 & 354 & 17 & 762 & 35 & 489 & 35 & 721 & 35 & 823 & 42 & 922 & 49 & 946 & 37.8 \\
\hline 16 & 377 & 18 & 813 & 37 & 511 & 37 & 761 & 37 & 862 & 44 & 958 & 51 & 979 & 39.1 \\
\hline 19 & 404 & 20 & 859 & 39 & 548 & 39 & 804 & 40 & 899 & 46 & 1004 & 54 & 1014 & 40.5 \\
\hline 22 & 425 & 21 & 906 & 42 & 571 & 42 & 834 & 41 & 944 & 48 & 1052 & 56 & 1062 & 42.4 \\
\hline 26 & 443 & 22 & 946 & 43 & 586 & 43 & 856 & 42 & 968 & 49 & 1080 & 58 & 1098 & 43.8 \\
\hline 29 & 455 & 22 & 974 & 45 & 598 & 45 & 873 & 43 & 985 & 50 & 1102 & 59 & 1124 & 44.9 \\
\hline 34 & 493 & 24 & 1040 & 48 & 645 & 48 & 930 & 46 & 1040 & 53 & 1159 & 62 & 1174 & 46.8 \\
\hline 37 & 508 & 25 & 1064 & 49 & 666 & 49 & 953 & 47 & 1057 & 54 & 1180 & 63 & 1196 & 47.7 \\
\hline 40 & 524 & 26 & 1089 & 50 & 686 & 50 & 976 & 48 & 1075 & 55 & 1202 & 64 & 1221 & 48.7 \\
\hline 43 & 541 & 26 & 1115 & 51 & 705 & 51 & 996 & 45 & 1091 & 56 & 1222 & 65 & 1237 & 45.4 \\
\hline 49 & 542 & 26 & 1122 & 48 & 712 & 49 & 1001 & 49 & 1094 & 55 & 1225 & 62 & 1245 & 49.7 \\
\hline 56 & 542 & 26 & 1122 & 47 & 723 & 50 & 1010 & 50 & 1100 & 57 & 1235 & 66 & 1252 & 50.0 \\
\hline
\end{tabular}


Table A. 4. Cumulative methane yield per unit mass of COD added in AnCoD of TWAS and SSO

\begin{tabular}{|c|c|c|c|c|c|c|c|c|c|c|c|c|c|c|}
\hline \multicolumn{15}{|c|}{ Cumulative $\mathrm{CH}_{4}$ yield (mLCH $4 / \mathrm{COD}$ added) } \\
\hline $\begin{array}{l}\text { Time } \\
\text { (day) }\end{array}$ & TWAS & SD & SSO & SD & $\begin{array}{l}\mathrm{T} / \mathrm{SSO} \\
9 / 1\end{array}$ & SD & $\begin{array}{l}\mathrm{T} / \mathrm{SSO} \\
7 / 3\end{array}$ & SD & $\begin{array}{l}\text { T/SSO } \\
1 / 1\end{array}$ & SD & $\begin{array}{l}\mathrm{T} / \mathrm{SSO} \\
3 / 7\end{array}$ & SD & $\begin{array}{l}\mathrm{T} / \mathrm{SSO} \\
1 / 9\end{array}$ & SD \\
\hline 0 & 0 & 0 & 0 & 0 & 0 & 0 & 0 & 0 & 0 & 0 & 0 & 0 & 0 & 0 \\
\hline 1 & 2 & 0.1 & 1 & 0.1 & 3 & 0.2 & 4 & 0.2 & 2 & 0.1 & 2 & 0.1 & 1 & 0.1 \\
\hline 3 & 12 & 0.6 & 13 & 0.7 & 13 & 0.6 & 22 & 1.1 & 11 & 0.5 & 16 & 0.9 & 10 & 0.4 \\
\hline 4 & 30 & 1.6 & 27 & 1.5 & 31 & 1.5 & 51 & 2.5 & 25 & 1.1 & 42 & 2.3 & 27 & 1.2 \\
\hline 5 & 55 & 2.8 & 50 & 2.7 & 59 & 2.9 & 88 & 4.3 & 46 & 2.1 & 84 & 4.6 & 56 & 2.4 \\
\hline 6 & 71 & 3.7 & 76 & 4.1 & 81 & 4.0 & 116 & 5.7 & 66 & 3.0 & 116 & 6.4 & 90 & 3.9 \\
\hline 7 & 86 & 4.5 & 103 & 5.5 & 107 & 5.2 & 141 & 6.9 & 102 & 4.6 & 143 & 7.9 & 121 & 5.2 \\
\hline 8 & 94 & 4.9 & 130 & 7.0 & 124 & 6.1 & 164 & 8.0 & 131 & 5.9 & 166 & 9.1 & 156 & 6.7 \\
\hline 10 & 106 & 5.5 & 160 & 8.7 & 141 & 6.9 & 197 & 9.6 & 175 & 7.9 & 208 & 11.4 & 197 & 8.5 \\
\hline 12 & 117 & 6.1 & 188 & 10.2 & 153 & 7.5 & 213 & 10.4 & 219 & 9.8 & 246 & 13.5 & 234 & 10.1 \\
\hline 14 & 126 & 6.5 & 209 & 11.3 & 164 & 8.0 & 224 & 11.0 & 244 & 11.0 & 263 & 14.5 & 263 & 10.5 \\
\hline 16 & 133 & 6.9 & 223 & 12.1 & 172 & 8.4 & 237 & 11.6 & 255 & 11.5 & 273 & 15.0 & 272 & 11.7 \\
\hline 19 & 143 & 7.4 & 236 & 12.7 & 184 & 9.0 & 250 & 12.3 & 266 & 12.0 & 287 & 15.8 & 282 & 12.1 \\
\hline 22 & 151 & 7.8 & 249 & 13.4 & 192 & 9.4 & 260 & 12.7 & 279 & 12.6 & 300 & 16.5 & 295 & 11.3 \\
\hline 26 & 157 & 8.2 & 260 & 13.8 & 197 & 9.6 & 266 & 13.1 & 287 & 12.9 & 308 & 17.0 & 305 & 13.1 \\
\hline 29 & 161 & 8.4 & 268 & 14.5 & 201 & 9.8 & 272 & 13.3 & 292 & 13.1 & 315 & 17.3 & 312 & 13.7 \\
\hline 34 & 175 & 9.1 & 286 & 15.4 & 217 & 10.6 & 290 & 14.2 & 308 & 13.9 & 331 & 18.2 & 326 & 14.0 \\
\hline 37 & 180 & 9.4 & 292 & 15.8 & 223 & 11.0 & 297 & 14.5 & 313 & 14.1 & 337 & 18.5 & 332 & 14.3 \\
\hline 40 & 186 & 9.7 & 299 & 14.8 & 230 & 10.3 & 304 & 14.9 & 318 & 12.7 & 343 & 18.9 & 339 & 13.5 \\
\hline 43 & 192 & 10.2 & 306 & 16.5 & 237 & 12.2 & 310 & 16.1 & 323 & 14.5 & 349 & 17.2 & 344 & 14.8 \\
\hline 49 & 192 & 9.8 & 308 & 15.5 & 239 & 11.7 & 312 & 15.3 & 324 & 13.2 & 350 & 16.3 & 346 & 15.2 \\
\hline 56 & 192 & 9.5 & 308 & 16.2 & 243 & 10.6 & 315 & 14.4 & 326 & 14.7 & 353 & 15.8 & 348 & 14.4 \\
\hline
\end{tabular}


Table A. 5. Cumulative methane yield per unit mass of VSS added in AnCoD of TWAS and SSO

\begin{tabular}{|c|c|c|c|c|c|c|c|c|c|c|c|c|c|c|}
\hline \multicolumn{15}{|c|}{ Cumulative $\mathrm{CH}_{4}$ yield (mLCH$/$ VSS added) } \\
\hline $\begin{array}{l}\text { Time } \\
\text { (day) }\end{array}$ & TWAS & SD & SSO & SD & $\begin{array}{l}\mathrm{T} / \mathrm{SSO} \\
9 / 1\end{array}$ & SD & $\begin{array}{l}\mathrm{T} / \mathrm{SSO} \\
7 / 3\end{array}$ & SD & $\begin{array}{l}\mathrm{T} / \mathrm{SSO} \\
1 / 1\end{array}$ & SD & $\begin{array}{l}\text { T/SSO } \\
3 / 7\end{array}$ & SD & $\begin{array}{l}\mathrm{T} / \mathrm{SSO} \\
1 / 9\end{array}$ & SD \\
\hline 0 & 0 & 0 & 0 & 0 & 0 & 0 & 0 & 0 & 0 & 0 & 0 & 0 & 0 & 0 \\
\hline 1 & 4 & 0.2 & 1 & 0.0 & 5 & 0.3 & 8 & 0.4 & 4 & 0.2 & 5 & 0.3 & 3 & 0.2 \\
\hline 3 & 19 & 0.9 & 10 & 0.4 & 23 & 1.2 & 45 & 2.2 & 26 & 1.3 & 41 & 2.0 & 28 & 1.3 \\
\hline 4 & 47 & 2.3 & 21 & 0.8 & 54 & 2.8 & 106 & 5.2 & 58 & 2.8 & 107 & 5.2 & 74 & 3.6 \\
\hline 5 & 85 & 4.1 & 39 & 1.6 & 104 & 5.4 & 183 & 9.0 & 107 & 5.2 & 217 & 10.6 & 154 & 7.6 \\
\hline 6 & 110 & 5.4 & 60 & 2.4 & 142 & 7.4 & 240 & 11.9 & 156 & 7.6 & 297 & 14.6 & 249 & 12.2 \\
\hline 7 & 134 & 6.5 & 81 & 3.2 & 187 & 9.7 & 291 & 14.4 & 239 & 11.6 & 367 & 18.0 & 335 & 16.4 \\
\hline 8 & 148 & 7.2 & 102 & 4.1 & 216 & 11.3 & 339 & 16.8 & 306 & 14.9 & 427 & 20.9 & 431 & 21.1 \\
\hline 10 & 165 & 8.0 & 126 & 5.0 & 246 & 12.8 & 407 & 20.1 & 411 & 20.0 & 534 & 26.2 & 546 & 26.8 \\
\hline 12 & 182 & 8.9 & 148 & 5.9 & 268 & 14.0 & 440 & 21.8 & 512 & 24.9 & 633 & 31.0 & 649 & 31.8 \\
\hline 14 & 196 & 9.5 & 165 & 6.6 & 287 & 15.0 & 465 & 23.0 & 571 & 27.8 & 677 & 33.2 & 728 & 35.7 \\
\hline 16 & 208 & 10.1 & 175 & 7.0 & 300 & 15.6 & 490 & 24.3 & 597 & 29.1 & 703 & 34.5 & 753 & 36.9 \\
\hline 19 & 224 & 10.9 & 185 & 7.4 & 322 & 16.8 & 518 & 25.7 & 623 & 30.4 & 737 & 36.1 & 781 & 38.2 \\
\hline 22 & 235 & 11.4 & 196 & 7.8 & 335 & 17.5 & 538 & 26.6 & 654 & 31.8 & 772 & 37.8 & 817 & 40.0 \\
\hline 26 & 245 & 11.9 & 204 & 8.1 & 344 & 17.9 & 552 & 26.5 & 671 & 32.7 & 793 & 38.8 & 845 & 41.4 \\
\hline 29 & 252 & 12.2 & 210 & 8.4 & 351 & 18.3 & 563 & 27.8 & 683 & 33.2 & 809 & 39.7 & 865 & 42.4 \\
\hline 34 & 273 & 13.3 & 225 & 8.9 & 379 & 19.7 & 600 & 29.7 & 721 & 35.1 & 851 & 41.7 & 903 & 44.3 \\
\hline 37 & 281 & 13.7 & 230 & 9.1 & 391 & 20.4 & 615 & 30.4 & 732 & 35.7 & 867 & 42.5 & 921 & 45.1 \\
\hline 40 & 290 & 15.4 & 235 & 9.4 & 403 & 21.0 & 629 & 32.3 & 745 & 36.3 & 883 & 43.3 & 940 & 46.1 \\
\hline 43 & 299 & 14.6 & 241 & 10.0 & 414 & 20.2 & 642 & 31.8 & 756 & 36.8 & 897 & 43.2 & 952 & 46.7 \\
\hline 49 & 300 & 13.8 & 242 & 9.9 & 418 & 19.8 & 645 & 30.5 & 758 & 35.4 & 899 & 45.4 & 958 & 45.1 \\
\hline 56 & 300 & 15.1 & 242 & 9.2 & 424 & 21.6 & 651 & 32.2 & 762 & 37.1 & 907 & 44.4 & 964 & 47.2 \\
\hline
\end{tabular}


Table A. 6. Cumulative methane yield per unit volume of VSS added in AnCoD of TWAS and SSO

\begin{tabular}{|c|c|c|c|c|c|c|c|c|c|c|c|c|c|c|}
\hline \multicolumn{15}{|c|}{ Cumulative $\mathrm{CH}_{4}$ yield (mLCH $4 / \mathrm{mL}$ substrate added) } \\
\hline $\begin{array}{l}\text { Time } \\
\text { (day) }\end{array}$ & TWAS & SD & SSO & SD & $\begin{array}{l}\mathrm{T} / \mathrm{SSO} \\
9 / 1\end{array}$ & SD & $\begin{array}{l}\text { T/SSO } \\
7 / 3\end{array}$ & SD & $\begin{array}{l}\mathrm{T} / \mathrm{SSO} \\
1 / 1\end{array}$ & SD & $\begin{array}{l}\text { T/SSO } \\
3 / 7\end{array}$ & SD & $\begin{array}{l}\mathrm{T} / \mathrm{SSO} \\
1 / 9\end{array}$ & SD \\
\hline 0 & 0.00 & 0.00 & 0.00 & 0.00 & 0.00 & 0.00 & 0.00 & 0.00 & 0.00 & 0.00 & 0.00 & 0.00 & 0.00 & 0.00 \\
\hline 1 & 0.10 & 0.00 & 0.15 & 0.01 & 0.14 & 0.01 & 0.22 & 0.01 & 0.14 & 0.01 & 0.18 & 0.01 & 0.12 & 0.00 \\
\hline 3 & 0.49 & 0.02 & 1.38 & 0.07 & 0.62 & 0.03 & 1.32 & 0.06 & 0.83 & 0.04 & 1.42 & 0.07 & 1.02 & 0.04 \\
\hline 4 & 1.22 & 0.05 & 2.99 & 0.14 & 1.45 & 0.08 & 3.12 & 0.15 & 1.84 & 0.09 & 3.70 & 0.18 & 2.76 & 0.10 \\
\hline 5 & 2.18 & 0.09 & 5.51 & 0.27 & 2.79 & 0.15 & 5.38 & 0.26 & 3.43 & 0.17 & 7.52 & 0.36 & 5.74 & 0.22 \\
\hline 6 & 2.82 & 0.12 & 8.40 & 0.41 & 3.83 & 0.21 & 7.08 & 0.34 & 4.99 & 0.25 & 10.29 & 0.49 & 9.26 & 0.35 \\
\hline 7 & 3.43 & 0.14 & 11.28 & 0.55 & 5.03 & 0.27 & 8.59 & 0.41 & 7.65 & 0.39 & 12.72 & 0.61 & 12.45 & 0.47 \\
\hline 8 & 3.78 & 0.16 & 14.34 & 0.70 & 5.82 & 0.32 & 10.00 & 0.48 & 9.80 & 0.50 & 14.79 & 0.71 & 16.02 & 0.61 \\
\hline 10 & 4.24 & 0.18 & 17.63 & 0.85 & 6.62 & 0.36 & 11.99 & 0.58 & 13.16 & 0.67 & 18.48 & 0.88 & 20.31 & 0.77 \\
\hline 12 & & 0.20 & 20 & 1.00 & & 0.39 & 12.97 & 0.62 & 16.40 & 0.84 & 21.90 & 1.05 & 24.12 & 92 \\
\hline 14 & 5.02 & 0.21 & 23.04 & 1.12 & 7.72 & 0.42 & 13.69 & 0.66 & 18.28 & 0.93 & 23.42 & 1.12 & 27.08 & 1.03 \\
\hline 16 & 5.34 & 0.22 & 24.57 & 1.19 & 8.07 & 0.44 & 14.45 & 0.69 & 19.14 & 0.98 & 24.34 & 1.16 & 28.01 & 1.06 \\
\hline 19 & 5.72 & 0.24 & 25.95 & 1.26 & 8.65 & 0.47 & 15.27 & 0.73 & 19.97 & 1.02 & 25.51 & 1.22 & 29.03 & 1.10 \\
\hline 22 & 6.02 & 0.25 & 27.39 & 1.33 & 9.01 & 0.49 & 15.84 & 0.76 & 20.95 & 1.07 & 26.73 & 1.28 & 30.39 & 1.15 \\
\hline 26 & 6.27 & 0.26 & 28.60 & 1.39 & 9.25 & 0.51 & 16.25 & 0.78 & 21.50 & 1.10 & 27.44 & 1.31 & 31.42 & 1.19 \\
\hline 29 & 6.45 & 0.27 & 29.44 & 1.43 & 9.43 & 0.51 & 16.57 & 0.80 & 21.87 & 1.12 & 28.02 & 1.34 & 32.18 & 1.22 \\
\hline 34 & 6.99 & 0.29 & 31.44 & 1.52 & 10.18 & 0.56 & 17.67 & 0.85 & 23.10 & 1.18 & 29.45 & 1.41 & 33.60 & 1.28 \\
\hline 37 & 7.20 & 0.30 & 32.15 & 1.56 & 10.50 & 0.57 & 18.11 & 0.87 & 23.47 & 1.20 & 30.00 & 1.48 & 34.24 & 1.30 \\
\hline 40 & 7.43 & 0.31 & 32.93 & 1.60 & 10.83 & 0.59 & 18.53 & 0.89 & 23.86 & 1.22 & 30.56 & 1.46 & 34.95 & 1.33 \\
\hline 43 & 7.67 & 0.29 & 33.70 & 1.63 & 11.13 & 0.61 & 18.91 & 0.78 & 24.23 & 1.24 & 31.06 & 1.48 & 35.42 & 1.45 \\
\hline 49 & 7.68 & 0.22 & 33.91 & 1.56 & 11.24 & 0.52 & 19.01 & 0.88 & 24.28 & 1.35 & 31.13 & 1.39 & 35.64 & 1.25 \\
\hline 56 & 7.68 & 0.32 & 33.91 & 1.70 & 11.40 & 0.65 & 19.18 & 0.92 & 24.42 & 1.25 & 31.39 & 1.50 & 35.84 & 1.36 \\
\hline
\end{tabular}




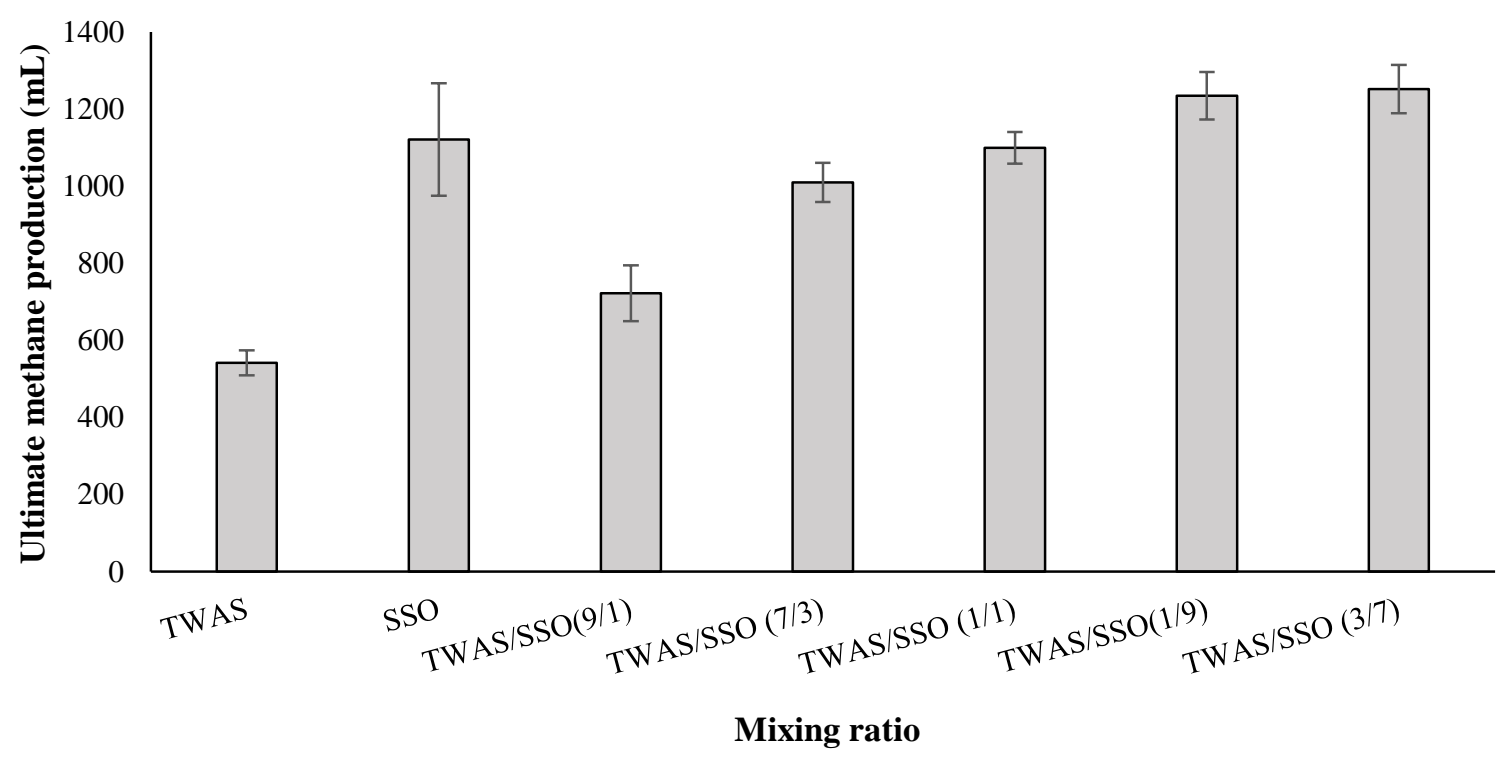

Figure A.1. Ultimate $\mathrm{CH}_{4}$ production in $\mathrm{AnCoD}$ of TWAS and SSO 
Table A. 7. Characteristics of raw feedstocks for hydrolysis/acidification in AnCoD of TWAS/SSO

\begin{tabular}{cccccccc}
\hline \multirow{2}{*}{ Parameters } & \multirow{2}{*}{ Units } & \multicolumn{2}{c}{ SSO } & \multicolumn{2}{c}{ TWAS } & \multicolumn{2}{c}{ Seed } \\
& & MEAN & SD & MEAN & SD & MEAN & SD \\
\hline TCOD & $\mathrm{mg} / \mathrm{L}$ & 110000 & 7795 & 40000 & 2853 & 16400 & 444 \\
SCOD & $\mathrm{mg} / \mathrm{L}$ & 44400 & 100 & 2360 & 26 & 960 & 19 \\
TSS & $\mathrm{mg} / \mathrm{L}$ & 53833 & 6252 & 31450 & 1006 & 17033 & 400 \\
VSS & $\mathrm{mg} / \mathrm{L}$ & 36030 & 3649 & 18460 & 2216 & 10900 & 200 \\
TS & $\mathrm{mg} / \mathrm{L}$ & 62187 & 1025 & 38810 & 6859 & 21450 & 582 \\
VS & $\mathrm{mg} / \mathrm{L}$ & 43493 & 761 & 34477 & 27 & 13140 & 328 \\
Ammonia & $\mathrm{mg} / \mathrm{L}$ & 1138 & 6 & 255 & 30 & 1495 & 40 \\
$\mathrm{pH}$ & - & 5.6 & 0.006 & 6.3 & 0.03 & 7.2 & 0.1 \\
Alkalinity & $\mathrm{mg} \mathrm{CaCO3/L}$ & 6800 & 522 & 1953 & 148 & 3943 & 465 \\
TN & $\mathrm{mg} / \mathrm{L}$ & 3967 & 751 & 2900 & 400 & 2025 & 203 \\
TSN & $\mathrm{mg} / \mathrm{L}$ & 1055 & 38 & 420 & 64 & 696 & 112 \\
Total Carbs & $\mathrm{mg} / \mathrm{L}$ & 13495 & 3580 & 923 & 112 & 545 & 82 \\
Total Proteins & $\mathrm{mg} / \mathrm{L}$ & 2087 & 198 & 2769 & 298 & 1635 & 56 \\
Total Lipids & $\mathrm{mg} / \mathrm{L}$ & 1103 & 2420 & 289 & 27 & 164 & 52 \\
\hline
\end{tabular}


Table A. 8. Soluble and particulate COD concentrations (mg/L) over time

\begin{tabular}{|c|c|c|c|c|c|c|c|}
\hline \multicolumn{8}{|c|}{ Soluble COD concentrations $(\mathrm{mg} / \mathrm{L}$ ) over time } \\
\hline Time & $\mathrm{T} / \mathrm{SSO} 7 / 3$ & T/SSO $1 / 1$ & T/SSO 3/7 & T/SSO $1 / 9$ & $\mathrm{~T} / \mathrm{SSO}$ 9/1 & SSO Only & TWAS Only \\
\hline 0 & 4590 & 5527 & 6525 & 7318 & 2281 & 7630 & 1905 \\
\hline 2 & 4698 & 6087 & 6959 & 7857 & 2529 & 8123 & 2254 \\
\hline 4 & 5036 & 6527 & 7233 & 8216 & 2727 & 8416 & 2377 \\
\hline 6 & 5284 & 6767 & 7557 & 8655 & 3345 & 8689 & 2524 \\
\hline 8 & 5802 & 7017 & 8011 & 8994 & 3503 & 9082 & 2548 \\
\hline 10 & 5990 & 7487 & 8275 & 9353 & 3961 & 9355 & 2597 \\
\hline 12 & 6198 & 7787 & 8599 & 9712 & 4209 & 9858 & 2769 \\
\hline 24 & 6616 & 8047 & 8933 & 10221 & 4397 & 10261 & 2988 \\
\hline 48 & 7534 & 8427 & 9337 & 10540 & 4715 & 11094 & 3087 \\
\hline 72 & 7732 & 9067 & 9981 & 11079 & 5435 & 10817 & 3120 \\
\hline \multicolumn{8}{|c|}{ Particulate COD concentrations $(\mathrm{mg} / \mathrm{L})$ over time } \\
\hline Time & $\mathrm{T} / \mathrm{SSO} 7 / 3$ & T/SSO 1/1 & T/SSO 3/7 & T/SSO $1 / 9$ & T/SSO 9/1 & SSO Only & TWAS Only \\
\hline 0 & 24029 & 24473 & 24182 & 24237 & 23558 & 13979 & 23635 \\
\hline 2 & 23817 & 24256 & 23905 & 24105 & 23416 & 13717 & 22604 \\
\hline 4 & 23681 & 23968 & 23495 & 23987 & 23203 & 13527 & 22518 \\
\hline 6 & 23432 & 22832 & 22843 & 23602 & 22825 & 13256 & 22418 \\
\hline 8 & 22948 & 22683 & 22747 & 23360 & 22635 & 12967 & 22325 \\
\hline 10 & 22629 & 22410 & 22684 & 23020 & 22438 & 12392 & 22223 \\
\hline 12 & 22379 & 22112 & 21558 & 22844 & 22289 & 12228 & 22125 \\
\hline 24 & 21256 & 21110 & 21222 & 21163 & 20870 & 11287 & 21897 \\
\hline 48 & 20684 & 20371 & 21021 & 20815 & 20376 & 10951 & 21581 \\
\hline 72 & 20484 & 20233 & 20574 & 20471 & 20154 & 10623 & 21508 \\
\hline
\end{tabular}




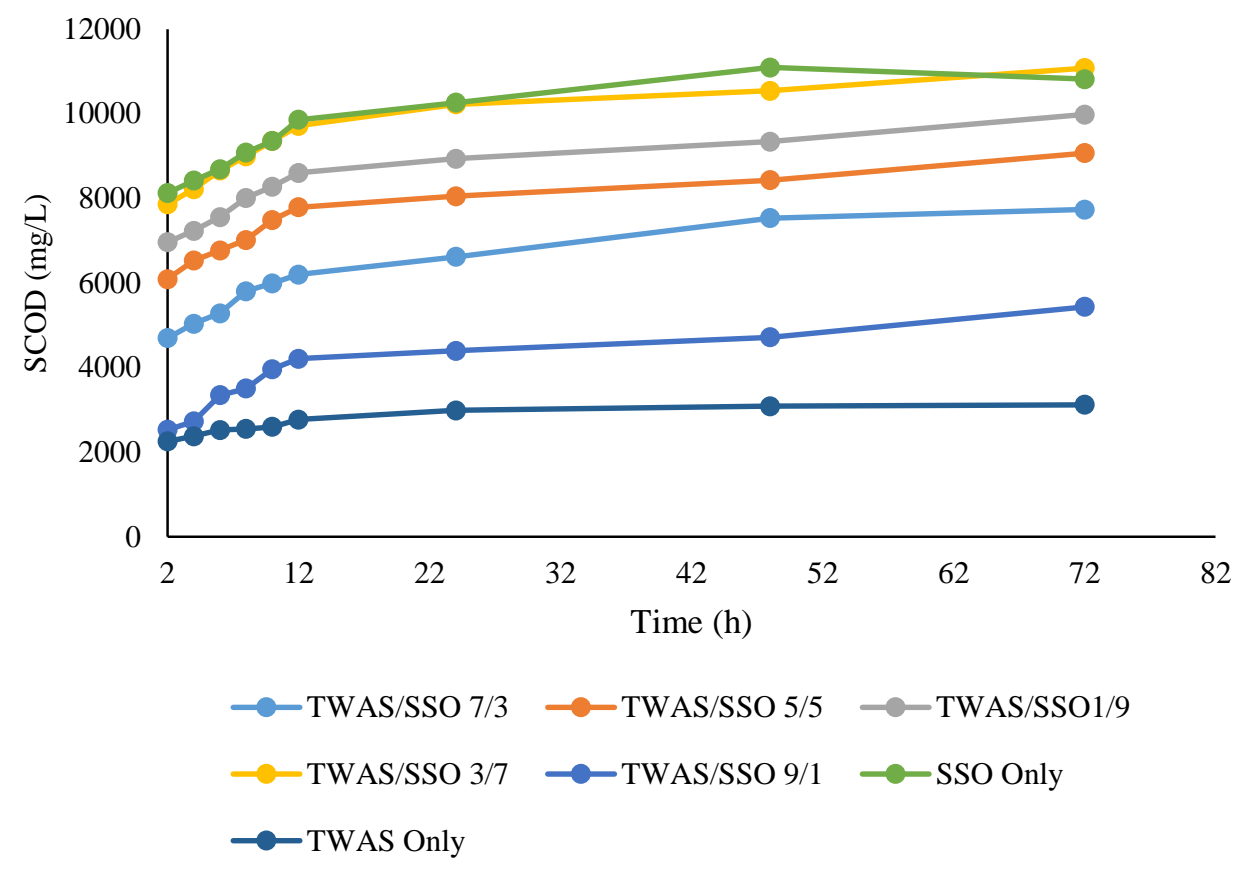

Figure A.2. Concentration of soluble COD over time for different mixing ratios 


\section{B. Analytical results for AnCoD of TWAS and manure}

Table B.1 Characteristics of raw feedstocks in AnCoD of TWAS and manure

\begin{tabular}{cccccccc}
\hline \multirow{2}{*}{ Parameters } & \multirow{2}{*}{ Units } & \multicolumn{2}{c}{ Manure } & \multicolumn{2}{c}{ TWAS } & \multicolumn{2}{c}{ Inoculum } \\
& & MEAN & SD & MEAN & SD & MEAN & SD \\
\hline TCOD & $\mathrm{mg} / \mathrm{L}$ & 122833 & 19553 & 45600 & 500 & 17167 & 306 \\
SCOD & $\mathrm{mg} / \mathrm{L}$ & 8933 & 503 & 2020 & 265 & 1250 & 173 \\
TSS & $\mathrm{mg} / \mathrm{L}$ & 79920 & 19060 & 36150 & 538 & 15580 & 459 \\
VSS & $\mathrm{mg} / \mathrm{L}$ & 76998 & 1789 & 26710 & 349 & 10313 & 284 \\
TS & $\mathrm{mg} / \mathrm{L}$ & 98727 & 1035 & 38053 & 1832 & 16590 & 302 \\
VS & $\mathrm{mg} / \mathrm{L}$ & 85647 & 1123 & 27730 & 1092 & 10180 & 286 \\
Ammonia & $\mathrm{mg} / \mathrm{L}$ & 22 & 3 & 317 & 20 & 497 & 68 \\
$\mathrm{pH}$ & - & 6.4 & 0.1 & 6.8 & 0.1 & 7 & 0.1 \\
Alkalinity & $\mathrm{mg} \mathrm{CaCO3/L}$ & 5133 & 902 & 4820 & 452 & 5517 & 306 \\
Total N & $\mathrm{mg} / \mathrm{L}$ & 2200 & 100 & 2883 & 278 & 2050 & 278 \\
Total Soluble N & $\mathrm{mg} / \mathrm{L}$ & 104 & 15 & 410 & 22 & 747 & 15 \\
Total Carbs & $\mathrm{mg} / \mathrm{L}$ & 27100 & 958 & 1322 & 104 & 508 & 23 \\
Total Proteins & $\mathrm{mg} / \mathrm{L}$ & 5115 & 244 & 3958 & 276 & 1498 & 50 \\
Total Lipids & $\mathrm{mg} / \mathrm{L}$ & 1355 & 54 & 536 & 44 & 205 & 8 \\
\hline
\end{tabular}


Table B. 2. Cumulative $\mathrm{CH}_{4}$ production in AnCoD of TWAS and manure

\begin{tabular}{|c|c|c|c|c|c|c|c|c|c|c|c|c|c|c|}
\hline \multicolumn{15}{|c|}{ Cumulative methane production $(\mathrm{mL})$} \\
\hline $\begin{array}{l}\text { time } \\
\text { (day) }\end{array}$ & TWAS & SD & Manure & SD & $\begin{array}{c}\mathrm{T} / \mathrm{M} \\
9 / 1\end{array}$ & SD & $\begin{array}{c}\mathrm{T} / \mathrm{M} \\
7 / 3\end{array}$ & SD & $\begin{array}{c}\mathrm{T} / \mathrm{M} \\
1 / 1\end{array}$ & SD & $\begin{array}{c}\mathrm{T} / \mathrm{M} \\
3 / 7\end{array}$ & SD & $\begin{array}{c}\mathrm{T} / \mathrm{M} \\
1 / 9\end{array}$ & SD \\
\hline 0 & 0 & 0 & 0 & 0 & 0 & 0 & 0 & 0 & 0 & 0 & 0 & 0 & 0 & 0 \\
\hline 1 & 24 & 1 & 86 & 5 & 76 & 3 & 88 & 5 & 100 & 5 & 95 & 5 & 88 & 4.39 \\
\hline 2 & 84 & 4 & 157 & 9 & 172 & 7 & 175 & 9 & 197 & 7 & 188 & 9 & 172 & 8.62 \\
\hline 3 & 135 & 7 & 215 & 12 & 246 & 11 & 237 & 12 & 266 & 12 & 258 & 13 & 238 & 11.9 \\
\hline 4 & 185 & 9 & 273 & 15 & 300 & 13 & 294 & 15 & 331 & 15 & 322 & 16 & 297 & 14.8 \\
\hline 5 & 220 & 11 & 316 & 17 & 341 & 15 & 336 & 17 & 378 & 17 & 373 & 18 & 344 & 17.2 \\
\hline 6 & 256 & 12 & 364 & 20 & 386 & 17 & 382 & 20 & 430 & 20 & 427 & 21 & 396 & 19.8 \\
\hline 8 & 298 & 14 & 434 & 24 & 426 & 19 & 441 & 23 & 496 & 23 & 494 & 24 & 463 & 23.1 \\
\hline 10 & 328 & 16 & 490 & 27 & 462 & 20 & 506 & 26 & 570 & 26 & 562 & 28 & 523 & 26.1 \\
\hline 14 & 363 & 18 & 552 & 30 & 498 & 22 & 562 & 29 & 633 & 29 & 635 & 31 & 587 & 29.4 \\
\hline 16 & 388 & 19 & 597 & 33 & 528 & 23 & 600 & 31 & 675 & 31 & 681 & 33 & 631 & 31.6 \\
\hline 17 & 400 & 19 & 620 & 34 & 547 & 24 & 629 & 33 & 708 & 33 & 710 & 35 & 655 & 32.7 \\
\hline 19 & 417 & 20 & 648 & 36 & 570 & 25 & 650 & 34 & 732 & 34 & 740 & 36 & 680 & 34 \\
\hline 22 & 434 & 21 & 678 & 37 & 597 & 26 & 694 & 36 & 781 & 36 & 780 & 38 & 713 & 35.6 \\
\hline 25 & 456 & 22 & 713 & 39 & 625 & 27 & 710 & 37 & 799 & 37 & 815 & 40 & 748 & 37.4 \\
\hline 26 & 464 & 22 & 728 & 40 & 636 & 28 & 727 & 38 & 818 & 38 & 835 & 41 & 765 & 38.2 \\
\hline 29 & 478 & 23 & 752 & 41 & 655 & 28 & 749 & 39 & 843 & 39 & 863 & 42 & 790 & 39.5 \\
\hline 30 & 482 & 23 & 757 & 42 & 659 & 29 & 754 & 39 & 848 & 39 & 870 & 43 & 800 & 39 \\
\hline 33 & 492 & 24 & 775 & 43 & 671 & 29 & 769 & 40 & 865 & 40 & 889 & 44 & 817 & 40.8 \\
\hline 36 & 506 & 24 & 799 & 44 & 690 & 30 & 788 & 41 & 886 & 41 & 914 & 45 & 841 & 42.1 \\
\hline 40 & 521 & 25 & 824 & 45 & 712 & 31 & 811 & 42 & 912 & 42 & 944 & 46 & 871 & 43.5 \\
\hline 46 & 539 & 26 & 851 & 47 & 737 & 32 & 836 & 43 & 941 & 43 & 978 & 48 & 905 & 45.2 \\
\hline 50 & 548 & 27 & 864 & 48 & 750 & 33 & 850 & 44 & 956 & 44 & 995 & 49 & 922 & 46.1 \\
\hline 54 & 555 & 27 & 874 & 48 & 759 & 33 & 859 & 45 & 967 & 42 & 1007 & 49 & 935 & 46.7 \\
\hline 58 & 559 & 27 & 881 & 48 & 766 & 33 & 866 & 42 & 975 & 45 & 1017 & 48 & 944 & 47.2 \\
\hline 62 & 563 & 27 & 887 & 49 & 771 & 34 & 872 & 44 & 981 & 41 & 1023 & 51 & 951 & 45 \\
\hline 65 & 566 & 27 & 893 & 49 & 776 & 34 & 877 & 46 & 987 & 43 & 1029 & 50 & 956 & 47.8 \\
\hline 67 & 569 & 28 & 898 & 49 & 780 & 34 & 885 & 45 & 996 & 44 & 1035 & 52 & 961 & 42 \\
\hline 72 & 578 & 28 & 907 & 50 & 791 & 34 & 893 & 46 & 1005 & 42 & 1049 & 48 & 979 & 48.9 \\
\hline 74 & 584 & 28 & 915 & 50 & 801 & 33 & 903 & 47 & 1016 & 45 & 1059 & 49 & 992 & 43 \\
\hline 78 & 590 & 29 & 922 & 51 & 811 & 35 & 902 & 43 & 1015 & 47 & 1069 & 51 & 1002 & 50.1 \\
\hline
\end{tabular}


Table B. 3. Cumulative methane yield per unit mass of COD added in AnCoD of TWAS and manure

\begin{tabular}{|c|c|c|c|c|c|c|c|c|c|c|c|c|c|c|}
\hline \multicolumn{15}{|c|}{ Cumulative methane yield ( $\mathrm{mL} \mathrm{CH}_{4} / \mathrm{g}$ COD added) } \\
\hline $\begin{array}{l}\text { time } \\
\text { (day) }\end{array}$ & TWAS & SD & Manure & SD & $\begin{array}{c}\mathrm{T} / \mathrm{M} \\
9 / 1\end{array}$ & $\mathrm{SD}$ & $\begin{array}{c}\mathrm{T} / \mathrm{M} \\
7 / 3\end{array}$ & SD & $\begin{array}{c}\mathrm{T} / \mathrm{M} \\
1 / 1\end{array}$ & SD & $\begin{array}{c}\mathrm{T} / \mathrm{M} \\
3 / 7\end{array}$ & SD & $\begin{array}{c}\text { T/M } \\
1 / 9\end{array}$ & SD \\
\hline 0 & 0 & 0 & 0 & 0 & 0 & 0 & 0 & 0 & 0 & 0 & 0 & 0 & 0 & 0 \\
\hline 1 & 8 & 0.3 & 30 & 1.6 & 26 & 1.2 & 31 & 1.4 & 35 & 1.3 & 34 & 1.8 & 34 & 1.2 \\
\hline 2 & 28 & 1.2 & 56 & 2.9 & 58 & 2.7 & 61 & 2.9 & 69 & 2.6 & 66 & 3.7 & 67 & 2.5 \\
\hline 3 & 45 & 1.9 & 77 & 4.0 & 84 & 3.8 & 82 & 3.9 & 93 & 3.5 & 91 & 5.0 & 91 & 3.4 \\
\hline 4 & 62 & 2.7 & 97 & 5.0 & 102 & 4.7 & 102 & 4.8 & 116 & 4.4 & 114 & 6.3 & 114 & 4.2 \\
\hline 5 & 73 & 3.2 & 112 & 5.8 & 116 & 5.3 & 116 & 5.5 & 133 & 5.0 & 132 & 7.2 & 132 & 4.9 \\
\hline 6 & 85 & 3.7 & 130 & 6.7 & 131 & 6.0 & 132 & 6.2 & 151 & 5.7 & 151 & 8.3 & 152 & 5.6 \\
\hline 8 & 99 & 4.3 & 154 & 8.0 & 144 & 6.6 & 153 & 7.2 & 174 & 6.6 & 175 & 9.6 & 175 & 6.5 \\
\hline 10 & 109 & 4.7 & 174 & 9.1 & 157 & 7.2 & 175 & 8.2 & 200 & 7.6 & 199 & 10.9 & 200 & 7.4 \\
\hline 14 & 121 & 5.2 & 196 & 10.2 & 169 & 7.8 & 195 & 9.2 & 222 & 8.4 & 224 & 12.3 & 225 & 8.3 \\
\hline 16 & 129 & 5.5 & 212 & 11.0 & 179 & 8.2 & 208 & 9.8 & 237 & 9.0 & 241 & 13.2 & 242 & 8.9 \\
\hline 17 & 133 & 5.7 & 221 & 11.5 & 186 & 8.5 & 218 & 10.2 & 248 & 9.4 & 251 & 13.8 & 252 & 9.3 \\
\hline 19 & 139 & 6.0 & 230 & 12.0 & 193 & 8.9 & 225 & 10.6 & 257 & 9.8 & 262 & 14.4 & 263 & 9.7 \\
\hline 22 & 145 & 6.2 & 241 & 12.6 & 202 & 9.3 & 241 & 11.3 & 274 & 10.4 & 275 & 15.2 & 277 & 10.2 \\
\hline 25 & 152 & 6.5 & 254 & 13.2 & 212 & 9.7 & 246 & 11.6 & 280 & 10.6 & 288 & 15.8 & 289 & 10.7 \\
\hline 26 & 154 & 6.6 & 259 & 13.5 & 216 & 9.9 & 252 & 11.8 & 287 & 10.9 & 295 & 16.2 & 296 & 11.0 \\
\hline 29 & 159 & 6.8 & 267 & 13.9 & 222 & 10.2 & 260 & 12.2 & 296 & 11.2 & 305 & 16.8 & 306 & 11.3 \\
\hline 30 & 160 & 6.9 & 269 & 14.0 & 224 & 10.3 & 261 & 12.3 & 298 & 10.2 & 307 & 16.9 & 309 & 11.4 \\
\hline 33 & 164 & 7.0 & 276 & 14.3 & 227 & 10.5 & 267 & 12.5 & 303 & 11.5 & 314 & 17.3 & 316 & 11.7 \\
\hline 36 & 168 & 7.2 & 284 & 14.8 & 234 & 10.8 & 273 & 12.8 & 311 & 11.8 & 323 & 17.8 & 325 & 13.3 \\
\hline 40 & 173 & 7.5 & 293 & 15.2 & 242 & 11.1 & 281 & 13.2 & 320 & 12.2 & 334 & 18.3 & 335 & 12.4 \\
\hline 46 & 179 & 7.7 & 303 & 15.7 & 250 & 11.5 & 290 & 13.6 & 330 & 12.5 & 345 & 17.6 & 347 & 12.8 \\
\hline 50 & 182 & 7.8 & 307 & 16.0 & 254 & 11.7 & 294 & 13.8 & 335 & 10.7 & 351 & 19.3 & 353 & 14.1 \\
\hline 54 & 184 & 7.9 & 311 & 16.2 & 257 & 11.8 & 298 & 14.0 & 339 & 12.9 & 356 & 17.5 & 358 & 13.2 \\
\hline 58 & 186 & 8.0 & 314 & 16.3 & 260 & 11.9 & 300 & 13.5 & 342 & 13.5 & 359 & 19.8 & 361 & 14.2 \\
\hline 62 & 187 & 7.5 & 316 & 15.7 & 261 & 12.0 & 302 & 14.2 & 344 & 12.9 & 362 & 19.3 & 363 & 13.4 \\
\hline 65 & 188 & 8.1 & 318 & 16.5 & 263 & 11.8 & 304 & 14.3 & 346 & 13.2 & 364 & 20.0 & 365 & 12.8 \\
\hline 67 & 189 & 7.9 & 320 & 15.5 & 265 & 12.2 & 307 & 13.8 & 349 & 14.1 & 366 & 19.2 & 368 & 14.5 \\
\hline 72 & 192 & 8.3 & 323 & 16.8 & 268 & 10.9 & 310 & 14.6 & 353 & 12.8 & 371 & 20.4 & 372 & 11.7 \\
\hline 74 & 194 & 7.6 & 326 & 15.3 & 272 & 11.5 & 313 & 13.5 & 356 & 13.5 & 374 & 18.9 & 376 & 13.9 \\
\hline 78 & 196 & 8.4 & 328 & 17.1 & 275 & 12.6 & 313 & 14.7 & 356 & 11.6 & 378 & 20.8 & 379 & 14.0 \\
\hline
\end{tabular}


Table B. 4. Cumulative methane yield per unit mass of VSS added in AnCoD of TWAS and manure

\begin{tabular}{|c|c|c|c|c|c|c|c|c|c|c|c|c|c|c|}
\hline \multicolumn{15}{|c|}{ Cumulative methane yield (mL CH4/g VSS added) } \\
\hline $\begin{array}{l}\text { time } \\
\text { (day) }\end{array}$ & TWAS & SD & Manure & $\mathrm{SD}$ & $\begin{array}{c}\mathrm{T} / \mathrm{M} \\
9 / 1\end{array}$ & $\mathrm{SD}$ & $\begin{array}{c}\mathrm{T} / \mathrm{M} \\
7 / 3\end{array}$ & SD & $\begin{array}{c}\mathrm{T} / \mathrm{M} \\
1 / 1\end{array}$ & SD & $\begin{array}{c}\mathrm{T} / \mathrm{M} \\
3 / 7\end{array}$ & $\mathrm{SD}$ & $\begin{array}{c}\mathrm{T} / \mathrm{M} \\
1 / 9\end{array}$ & SD \\
\hline 0 & 0 & 0 & 0 & 0 & 0 & 0 & 0 & 0 & 0 & 0 & 0 & 0 & 0 & 0 \\
\hline 1 & 16 & 0.7 & 127 & 5.8 & 62 & 2.4 & 92 & 4.2 & 119 & 5.1 & 136 & 4.8 & 117 & 4.208 \\
\hline 2 & 58 & 2.6 & 233 & 10.7 & 142 & 5.5 & 182 & 8.4 & 237 & 10.2 & 270 & 9.4 & 229 & 8.258 \\
\hline 3 & 94 & 4.2 & 320 & 14.7 & 203 & 7.9 & 245 & 11.3 & 319 & 13.7 & 370 & 13.0 & 316 & 11.38 \\
\hline 4 & 128 & 5.8 & 404 & 18.6 & 247 & 9.6 & 304 & 14.0 & 397 & 17.1 & 463 & 16.2 & 394 & 14.2 \\
\hline 5 & 153 & 6.9 & 468 & 21.5 & 281 & 11.0 & 348 & 16.0 & 453 & 19.5 & 536 & 18.7 & 458 & 16.49 \\
\hline 6 & 178 & 8.0 & 540 & 24.9 & 318 & 12.4 & 396 & 18.2 & 516 & 22.2 & 613 & 21.5 & 527 & 18.98 \\
\hline 8 & 206 & 9.3 & 644 & 29.6 & 351 & 13.7 & 456 & 21.0 & 595 & 25.6 & 710 & 24.8 & 615 & 22.15 \\
\hline 10 & 227 & 10.2 & 727 & 33.4 & 380 & 14.8 & 524 & 24.1 & 683 & 29.4 & 807 & 28.3 & 695 & 25.03 \\
\hline 14 & 251 & 11.3 & 819 & 37.7 & 410 & 15.6 & 582 & 26.8 & 759 & 32.6 & 912 & 31.9 & 781 & 28.11 \\
\hline 16 & 268 & 12.1 & 885 & 40.7 & 435 & 16.9 & 621 & 28.6 & 810 & 34.8 & 979 & 34.3 & 839 & 30.22 \\
\hline 17 & 277 & 12.5 & 919 & 42.3 & 451 & 17.6 & 651 & 29.9 & 849 & 36.5 & 1020 & 35.7 & 871 & 31.35 \\
\hline 19 & 289 & 13.0 & 961 & 44.2 & 469 & 18.3 & 673 & 31.0 & 878 & 37.7 & 1064 & 37.2 & 905 & 32.58 \\
\hline 22 & 301 & 13.5 & 1006 & 45.3 & 492 & 19.2 & 719 & 33.1 & 937 & 40.3 & 1120 & 39.2 & 948 & 34.13 \\
\hline 25 & 316 & 14.2 & 1058 & 48.7 & 514 & 20.1 & 735 & 33.8 & 958 & 39.1 & 1170 & 41.0 & 995 & 35.81 \\
\hline 26 & 321 & 14.5 & 1079 & 46.7 & 524 & 18.7 & 753 & 34.6 & 981 & 42.2 & 1199 & 42.0 & 1017 & 36.63 \\
\hline 29 & 331 & 14.9 & 1115 & 51.3 & 539 & 21.0 & 776 & 35.7 & 1011 & 43.5 & 1240 & 43.4 & 1051 & 39.2 \\
\hline 30 & 334 & 13.8 & 1123 & 49.5 & 543 & 22.4 & 781 & 34.2 & 1017 & 42.8 & 1250 & 43.8 & 1064 & 38.3 \\
\hline 33 & 341 & 15.4 & 1149 & 52.9 & 552 & 21.5 & 796 & 36.6 & 1038 & 44.6 & 1277 & 44.7 & 1086 & 39.1 \\
\hline 36 & 351 & 15.8 & 1186 & 54.5 & 568 & 20.3 & 815 & 37.5 & 1063 & 45.7 & 1313 & 46.0 & 1119 & 38.6 \\
\hline 40 & 361 & 16.3 & 1222 & 50.6 & 586 & 22.9 & 840 & 38.6 & 1094 & 47.1 & 1356 & 44.8 & 1158 & 41.7 \\
\hline 46 & 373 & 14.5 & 1261 & 58.0 & 607 & 23.7 & 866 & 39.8 & 1129 & 48.5 & 1404 & 49.2 & 1204 & 43.33 \\
\hline 50 & 380 & 17.1 & 1281 & 54.5 & 617 & 24.1 & 880 & 40.5 & 1147 & 45.2 & 1429 & 50.0 & 1226 & 44.15 \\
\hline 54 & 384 & 16.9 & 1296 & 59.6 & 625 & 23.8 & 890 & 39.3 & 1160 & 49.9 & 1447 & 48.7 & 1243 & 44.76 \\
\hline 58 & 387 & 15.2 & 1307 & 58.3 & 631 & 24.6 & 897 & 41.3 & 1169 & 50.3 & 1461 & 51.1 & 1256 & 46.3 \\
\hline 62 & 390 & 17.6 & 1316 & 60.5 & 635 & 25.2 & 903 & 41.5 & 1177 & 48.6 & 1470 & 52.6 & 1265 & 45.52 \\
\hline 65 & 392 & 16.8 & 1324 & 56.4 & 639 & 24.9 & 908 & 39.7 & 1184 & 50.9 & 1478 & 49.8 & 1272 & 45.78 \\
\hline 67 & 394 & 17.7 & 1332 & 61.3 & 642 & 23.5 & 917 & 40.9 & 1195 & 49.7 & 1487 & 51.3 & 1278 & 46 \\
\hline 72 & 400 & 16.7 & 1345 & 59.2 & 651 & 25.4 & 925 & 42.6 & 1206 & 51.8 & 1507 & 52.7 & 1302 & 45.2 \\
\hline 74 & 405 & 17.5 & 1357 & 58.1 & 660 & 24.8 & 935 & 43.0 & 1218 & 47.4 & 1522 & 50.2 & 1319 & 47.48 \\
\hline 78 & 408 & 18.4 & 1368 & 60.8 & 667 & 26.7 & 934 & 41.5 & 1217 & 50.2 & 1535 & 53.7 & 1333 & 47.98 \\
\hline
\end{tabular}


Table B. 5. Cumulative methane yield per unit volume of substrate added in AnCoD of TWAS and manure

\begin{tabular}{|c|c|c|c|c|c|c|c|c|c|c|c|c|c|c|}
\hline \multicolumn{15}{|c|}{ Cumulative methane yield ( $\mathrm{mL} \mathrm{CH}_{4} / \mathrm{mL}$ substrate added) } \\
\hline $\begin{array}{l}\text { time } \\
\text { (day) }\end{array}$ & TWAS & & Manure & & $\begin{array}{c}\mathrm{T} / \mathrm{M} \\
9 / 1\end{array}$ & & $\begin{array}{c}\mathrm{T} / \mathrm{M} \\
7 / 3\end{array}$ & & $\begin{array}{c}\mathrm{T} / \mathrm{M} \\
1 / 1\end{array}$ & & $\begin{array}{c}\mathrm{T} / \mathrm{M} \\
3 / 7\end{array}$ & & $\begin{array}{c}\mathrm{T} / \mathrm{M} \\
1 / 9\end{array}$ & \\
\hline 0 & 0 & 0 & 0 & 0 & 0 & 0 & 0 & 0.00 & 0 & 0.00 & 0 & 0.00 & 0 & 0.00 \\
\hline 1 & 0.3 & 0.01 & 2.2 & 0.10 & 0.7 & 0.03 & 1.1 & 0.04 & 1.4 & 0.08 & 1.8 & 0.10 & 2.0 & 0.10 \\
\hline 2 & 1.0 & 0.04 & 4.0 & 0.18 & 1.6 & 0.10 & 2.2 & 0.08 & 2.9 & 0.16 & 3.5 & 0.19 & 4.0 & 0.19 \\
\hline 3 & 1.6 & 0.06 & 5.5 & 0.24 & 2.2 & 0.09 & 3.0 & 0.11 & 3.9 & 0.21 & 4.8 & 0.26 & 5.5 & 0.26 \\
\hline 4 & 2.2 & 0.08 & 7.0 & 0.31 & 2.7 & 0.11 & 3.7 & 0.14 & 4.8 & 0.26 & 6.0 & 0.33 & 6.9 & 0.32 \\
\hline 5 & 2.6 & 0.09 & 8.1 & 0.36 & 3.1 & 0.12 & 4.2 & 0.16 & 5.5 & 0.30 & 6.9 & 0.38 & 8.0 & 0.38 \\
\hline 6 & 3.1 & 0.11 & 9.3 & 0.41 & 3.5 & 0.14 & 4.8 & 0.18 & 6.2 & 0.34 & 7.9 & 0.43 & 9.2 & 0.43 \\
\hline 8 & 3.5 & 0.13 & 11.1 & 0.49 & 3.9 & 0.13 & 5.5 & 0.21 & 7.2 & 0.40 & 9.1 & 0.50 & 10.8 & 0.51 \\
\hline 10 & 3.9 & 0.14 & 12.6 & 0.55 & 4.2 & 0.16 & 6.3 & 0.24 & 8.3 & 0.45 & 10.4 & 0.57 & 12.2 & 0.57 \\
\hline 14 & 4.3 & 0.16 & 14.2 & 0.62 & 4.5 & 0.18 & 7.0 & 0.27 & 9.2 & 0.50 & 11.8 & 0.65 & 13.7 & 0.64 \\
\hline 16 & 4.6 & 0.17 & 15.3 & 0.67 & 4.8 & 0.19 & 7.5 & 0.29 & 9.8 & 0.54 & 12.6 & 0.69 & 14.7 & 0.69 \\
\hline 17 & 4.8 & 0.15 & 15.9 & 0.70 & 5.0 & 0.15 & 7.9 & 0.30 & 10.3 & 0.56 & 13.1 & 0.72 & 15.2 & 0.72 \\
\hline 19 & 5.0 & 0.18 & 16.6 & 0.73 & 5.2 & 0.20 & 8.1 & 0.31 & 10.6 & 0.58 & 13.7 & 0.75 & 15.8 & 0.74 \\
\hline 22 & 5.2 & 0.19 & 17.4 & 0.77 & 5.4 & 0.21 & 8.7 & 0.33 & 11.3 & 0.62 & 14.4 & 0.79 & 16.6 & 0.78 \\
\hline 25 & 5.4 & 0.20 & 18.3 & 0.80 & 5.7 & 0.22 & 8.9 & 0.34 & 11.6 & 0.64 & 15.1 & 0.83 & 17.4 & 0.82 \\
\hline 26 & 5.5 & 0.19 & 18.7 & 0.82 & 5.8 & 0.23 & 9.1 & 0.35 & 11.9 & 0.65 & 15.5 & 0.85 & 17.8 & 0.84 \\
\hline 29 & 5.7 & 0.20 & 19.3 & 0.85 & 6.0 & 0.23 & 9.4 & 0.36 & 12.2 & 0.67 & 16.0 & 0.88 & 18.4 & 0.86 \\
\hline 30 & 5.7 & 0.21 & 19.4 & 0.85 & 6.0 & 0.27 & 9.4 & 0.36 & 12.3 & 0.68 & 16.1 & 0.89 & 18.6 & 0.87 \\
\hline 33 & 5.9 & 0.21 & 19.9 & 0.87 & 6.1 & 0.24 & 9.6 & 0.41 & 12.5 & 0.69 & 16.5 & 0.91 & 19.0 & 0.89 \\
\hline 36 & 6.0 & 0.20 & 20.5 & 0.90 & 6.3 & 0.20 & 9.8 & 0.37 & 12.8 & 0.71 & 16.9 & 0.93 & 19.6 & 0.92 \\
\hline 40 & 6.2 & 0.22 & 21.1 & 0.93 & 6.5 & 0.25 & 10.1 & 0.39 & 13.2 & 0.73 & 17.5 & 0.96 & 20.3 & 0.95 \\
\hline 46 & 6.4 & 0.23 & 21.8 & 0.96 & 6.7 & 0.26 & 10.5 & 0.40 & 13.6 & 0.75 & 18.1 & 1.00 & 21.0 & 0.99 \\
\hline 50 & 6.5 & 0.21 & 22.2 & 0.97 & 6.8 & 0.29 & 10.6 & 0.36 & 13.9 & 0.76 & 18.4 & 1.01 & 21.4 & 1.01 \\
\hline 54 & 6.6 & 0.24 & 22.4 & 0.99 & 6.9 & 0.27 & 10.7 & 0.39 & 14.0 & 0.77 & 18.7 & 1.03 & 21.7 & 0.99 \\
\hline 58 & 6.7 & 0.33 & 22.6 & 0.88 & 7.0 & 0.25 & 10.8 & 0.41 & 14.1 & 0.78 & 18.8 & 1.04 & 22.0 & 1.03 \\
\hline 62 & 6.7 & 0.24 & 22.7 & 1.22 & 7.0 & 0.27 & 10.9 & 0.45 & 14.2 & 0.78 & 19.0 & 1.04 & 22.1 & 1.04 \\
\hline 65 & 6.7 & 0.31 & 22.9 & 1.01 & 7.1 & 0.22 & 11.0 & 0.42 & 14.3 & 0.79 & 19.1 & 1.05 & 22.2 & 1.05 \\
\hline 67 & 6.8 & 0.24 & 23.0 & 1.35 & 7.1 & 0.24 & 11.1 & 0.36 & 14.4 & 0.79 & 19.2 & 0.98 & 22.3 & 0.97 \\
\hline 72 & 6.9 & 0.25 & 23.3 & 0.98 & 7.2 & 0.28 & 11.2 & 0.42 & 14.6 & 0.80 & 19.4 & 1.07 & 22.8 & 1.07 \\
\hline 74 & 7.0 & 0.29 & 23.5 & 1.23 & 7.3 & 0.25 & 11.3 & 0.39 & 14.7 & 0.81 & 19.6 & 1.08 & 23.1 & 1.08 \\
\hline 78 & 7.0 & 0.25 & 23.6 & 1.04 & 7.4 & 0.29 & 11.3 & 0.43 & 14.7 & 0.81 & 19.8 & 1.09 & 23.3 & 1.10 \\
\hline
\end{tabular}




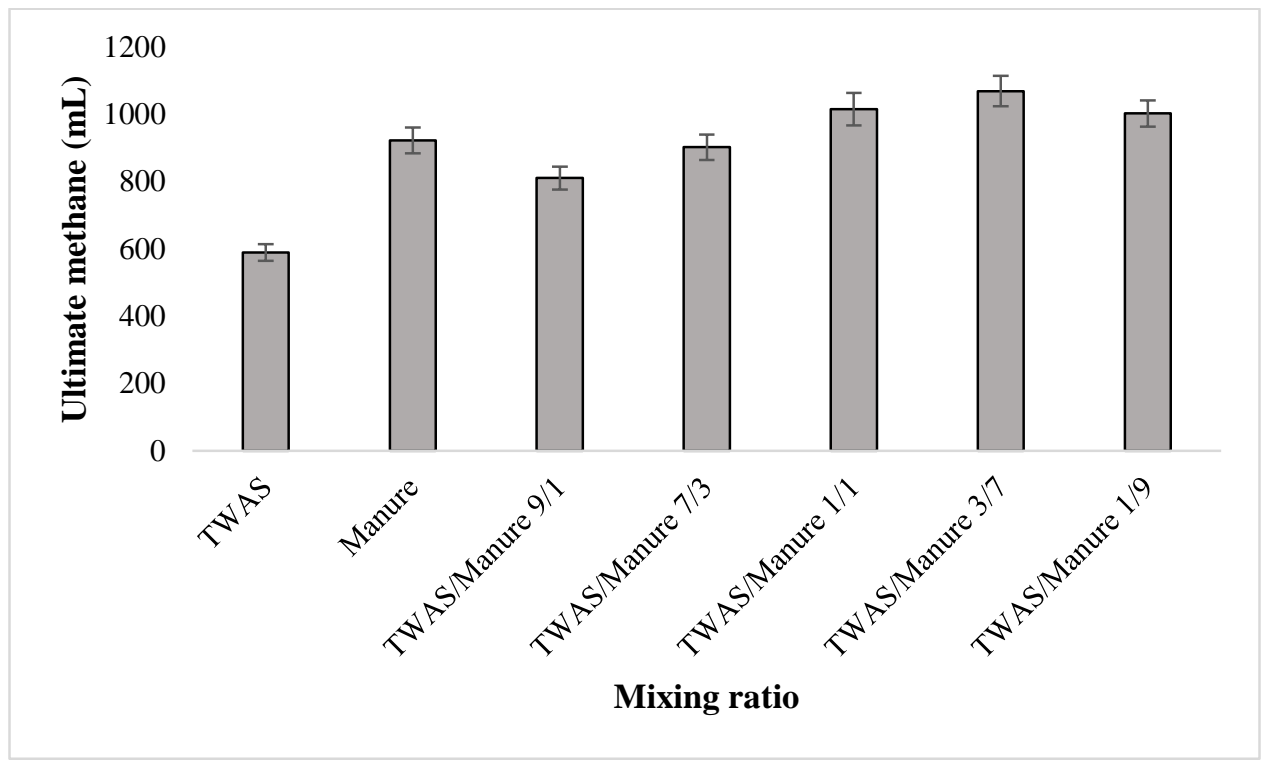

Figure B. 1. Ultimate methane production in AnCoD of TWAS and manure 
Table B. 6. Characteristics of the raw feedstocks for hydrolysis/acidification in AnCoD of TWAS and manure

\begin{tabular}{cccccccc}
\hline Parameters & Units & Manure & & TWAS & \multicolumn{3}{c}{ Seed } \\
\hline & & MEAN & SD & MEAN & SD & MEAN & SD \\
TCOD & $\mathrm{mg} / \mathrm{L}$ & 110433 & 4422 & 41200 & 1236 & 16652 & 552 \\
SCOD & $\mathrm{mg} / \mathrm{L}$ & 6560 & 228 & 2161 & 65 & 1255 & 50 \\
TSS & $\mathrm{mg} / \mathrm{L}$ & 52600 & 2530 & 37254 & 1118 & 15112 & 604 \\
VSS & $\mathrm{mg} / \mathrm{L}$ & 42800 & 2040 & 26521 & 796 & 10067 & 403 \\
TS & $\mathrm{mg} / \mathrm{L}$ & 68040 & 3202 & 39198 & 1176 & 36882 & 1475 \\
VS & $\mathrm{mg} / \mathrm{L}$ & 58520 & 2526 & 28562 & 857 & 26874 & 1075 \\
Ammonia & $\mathrm{mg} / \mathrm{L}$ & 13 & 1 & 327 & 10 & 307 & 12 \\
$\mathrm{pH}$ & - & 6.5 & 0.2 & 6.8 & 0.204 & 6 & 0.2 \\
Alkalinity & $\mathrm{mg} \mathrm{CaCO} / \mathrm{L}$ & 7689 & 384 & 4964 & 149 & 4671 & 187 \\
TN & $\mathrm{mg} / \mathrm{L}$ & 1435 & 72 & 2969 & 89 & 2794 & 112 \\
TSN & $\mathrm{mg} / \mathrm{L}$ & 68 & 3 & 422 & 13 & 397 & 16 \\
Total Carbs & $\mathrm{mg} / \mathrm{L}$ & 27116 & 1355.8 & 1327 & 40 & 503 & 20 \\
Total Proteins & $\mathrm{mg} / \mathrm{L}$ & 5123 & 256.15 & 3978 & 119 & 1510 & 60 \\
Total Lipids & $\mathrm{mg} / \mathrm{L}$ & 1360 & 68 & 530 & 16 & 201 & 8 \\
\hline
\end{tabular}


Table B. 7. Measured and theoretical VFAs concentrations over time

\begin{tabular}{cccccccc}
\hline \multicolumn{7}{c}{ Measured VFAs over time } \\
\hline Time & TWAS/Manure & TWAS/Manure & TWAS/Manure & TWAS/Manure & TWAS/Manure & Manure & TWAS \\
(hr) & $7 / 3$ & $1 / 1$ & $3 / 7$ & $1 / 9$ & $9 / 1$ & Only & Only \\
0 & 584 & 686 & 718 & 790 & 581 & 810 & 432 \\
6 & 596 & 719 & 793 & 831 & 593 & 849 & 458 \\
12 & 786 & 892 & 827 & 958 & 640 & 974 & 504 \\
24 & 912 & 1105 & 971 & 1230 & 681 & 1141 & 514 \\
48 & 942 & 1131 & 1299 & 1378 & 735 & 1366 & 528 \\
72 & 965 & 1175 & 1530 & 1491 & 775 & 1396 & 542 \\
& & & Theoretical VFAs over time & & & Mixture \\
Time & TWAS/Manure & TWAS/Manure & TWAS/Manure & TWAS/Manure & TWAS/Manure & Manure & TWAS \\
(hr) & $7 / 3$ & $1 / 1$ & $3 / 7$ & $1 / 9$ & $9 / 1$ & Only & Only \\
0 & 545 & 621 & 697 & 772 & 470 & 810 & 432 \\
6 & 575 & 654 & 732 & 810 & 497 & 849 & 458 \\
12 & 645 & 739 & 833 & 927 & 551 & 974 & 504 \\
24 & 702 & 827 & 953 & 1078 & 576 & 1141 & 514 \\
48 & 780 & 947 & 1115 & 1283 & 612 & 1366 & 528 \\
72 & 798 & 969 & 1140 & 1311 & 627 & 1396 & 542 \\
\hline
\end{tabular}


Table B. 8. Soluble and particulate COD concentrations over time

\begin{tabular}{|c|c|c|c|c|c|c|c|}
\hline \multicolumn{8}{|c|}{ Soluble COD concentrations $(\mathrm{mg} / \mathrm{L})$ over time } \\
\hline $\begin{array}{l}\text { Time } \\
\text { (hr) }\end{array}$ & $\begin{array}{c}\text { TWAS/Manure } \\
7 / 3\end{array}$ & $\begin{array}{c}\text { TWAS/Manure } \\
5 / 5\end{array}$ & $\begin{array}{c}\text { TWAS/Manure } \\
3 / 7\end{array}$ & $\begin{array}{c}\text { TWAS/Manure } \\
1 / 9\end{array}$ & $\begin{array}{c}\text { TWAS/Manure } \\
9 / 1\end{array}$ & $\begin{array}{c}\text { Manure } \\
\text { only }\end{array}$ & $\begin{array}{c}\text { TWAS } \\
\text { only }\end{array}$ \\
\hline 0 & 1880 & 1920 & 1611 & 1940 & 1929 & 1628 & 2181 \\
\hline 2 & 1920 & 1960 & 1817 & 2020 & 1972 & 1661 & 2254 \\
\hline 4 & 2040 & 2100 & 1868 & 2060 & 2471 & 1744 & 2377 \\
\hline 6 & 2260 & 2260 & 2005 & 2180 & 2536 & 1861 & 2524 \\
\hline 8 & 2380 & 2360 & 2159 & 2300 & 2644 & 1960 & 2548 \\
\hline 10 & 2420 & 2480 & 2228 & 2360 & 2904 & 1977 & 2597 \\
\hline 12 & 2540 & 2560 & 2451 & 2400 & 2969 & 2010 & 2769 \\
\hline 24 & 2780 & 2580 & 2622 & 2540 & 3099 & 2126 & 2988 \\
\hline 48 & 2920 & 2940 & 2896 & 2780 & 3208 & 2193 & 3087 \\
\hline 72 & 3990 & 3690 & 4190 & 4020 & 3860 & 3530 & 3120 \\
\hline \multicolumn{8}{|c|}{ Particulate COD concentrations $(\mathrm{mg} / \mathrm{L})$ over time } \\
\hline $\begin{array}{l}\text { Time } \\
(\mathrm{hr})\end{array}$ & $\begin{array}{c}\text { TWAS/Manure } \\
7 / 3\end{array}$ & $\begin{array}{c}\text { TWAS/Manure } \\
5 / 5\end{array}$ & $\begin{array}{c}\text { TWAS/Manure } \\
3 / 7\end{array}$ & $\begin{array}{c}\text { TWAS/Manure } \\
1 / 9\end{array}$ & $\begin{array}{c}\text { TWAS/Manure } \\
9 / 1\end{array}$ & $\begin{array}{l}\text { Manure } \\
\text { only }\end{array}$ & $\begin{array}{c}\text { TWAS } \\
\text { only }\end{array}$ \\
\hline 0 & 23155 & 22556 & 22486 & 21637 & 24388 & 21836 & 23635 \\
\hline 2 & 23015 & 22326 & 22467 & 21427 & 24545 & 21582 & 23115 \\
\hline 4 & 22895 & 22156 & 22219 & 21257 & 24186 & 21489 & 22696 \\
\hline 6 & 22675 & 22215 & 22081 & 21227 & 23712 & 21223 & 22468 \\
\hline 8 & 22555 & 22050 & 21527 & 20787 & 23893 & 21010 & 22276 \\
\hline 10 & 22515 & 21690 & 21399 & 20297 & 23543 & 20527 & 22083 \\
\hline 12 & 22485 & 21310 & 21016 & 20127 & 23228 & 20324 & 21945 \\
\hline 24 & 21155 & 20496 & 19691 & 19787 & 22801 & 20117 & 21697 \\
\hline 48 & 20615 & 20336 & 19300 & 19187 & 22203 & 19856 & 21563 \\
\hline 72 & 20445 & 20186 & 19056 & 19107 & 22177 & 19434 & 21508 \\
\hline
\end{tabular}




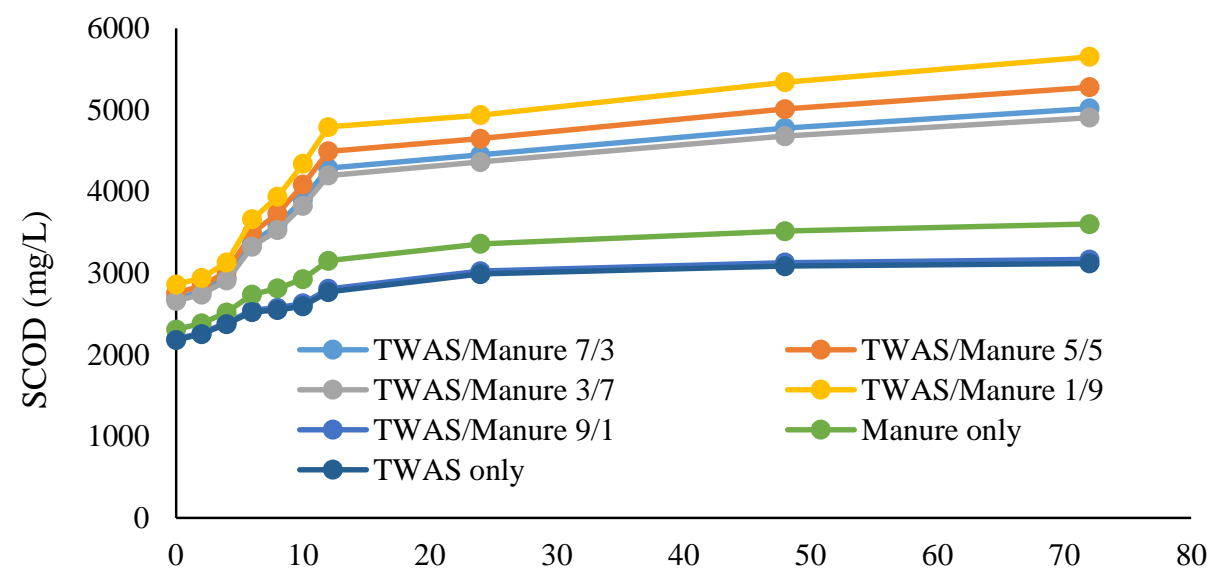

Time (h)

Figure B.2. Concentration of soluble COD over time at different mixing ratios of TWAS and manure 


\section{Analytical results for AnCoD of manure and SSO}

Table C. 1. Characteristics of raw feedstocks in AnCoD of manure and SSO

\begin{tabular}{cccccccc}
\hline \multirow{2}{*}{ Parameters } & \multirow{2}{*}{ Units } & \multicolumn{2}{c}{ Manure } & \multicolumn{2}{c}{ SSO } & \multicolumn{2}{c}{ Inoculum } \\
& & MEAN & SD & MEAN & SD & MEAN & SD \\
\hline TCOD & $\mathrm{mg} / \mathrm{L}$ & 105120 & 4795 & 115163 & 6090 & 17980 & 400 \\
SCOD & $\mathrm{mg} / \mathrm{L}$ & 44116 & 489 & 42932 & 92 & 722 & 16 \\
TSS & $\mathrm{mg} / \mathrm{L}$ & 54380 & 2252 & 62125 & 3063 & 15128 & 330 \\
VSS & $\mathrm{mg} / \mathrm{L}$ & 46420 & 2649 & 45584 & 2283 & 11687 & 160 \\
TS & $\mathrm{mg} / \mathrm{L}$ & 69785 & 3025 & 67987 & 1011 & 16160 & 503 \\
VS & $\mathrm{mg} / \mathrm{L}$ & 58590 & 2761 & 48535 & 682 & 12380 & 308 \\
Ammonia & $\mathrm{mg} / \mathrm{L}$ & 20 & 68.0 & 1073 & 5.87 & 745 & 36 \\
pH & & 6.8 & 0.030 & 5.8 & 0.050 & 7.2 & 0.05 \\
Alkalinity & $\mathrm{mg} \mathrm{CaCO} / \mathrm{L}$ & 4924 & 522 & 6176 & 339 & 4186 & 460 \\
TN & $\mathrm{mg} / \mathrm{L}$ & 2510 & 751 & 3498 & 202 & 1940 & 186 \\
TSN & $\mathrm{mg} / \mathrm{L}$ & 105.00 & 38 & 1046 & 21 & 716 & 93 \\
Total Carbs & $\mathrm{mg} / \mathrm{L}$ & 28796 & 3580 & 13495 & 687 & 453 & 68 \\
Total & & & & & & & \\
Proteins & $\mathrm{mg} / \mathrm{L}$ & 5930 & 198 & 2087 & 98 & 2598 & 38 \\
Total Lipids & $\mathrm{mg} / \mathrm{L}$ & 1400 & 2420 & 1103 & 92 & 225 & 43 \\
\hline
\end{tabular}


Table C. 2. Average $\mathrm{CH}_{4}$ measurements in $\mathrm{AnCoD}$ of manure and SSO

\begin{tabular}{|c|c|c|c|c|c|c|c|c|c|c|c|c|c|c|}
\hline \multicolumn{15}{|c|}{ Average $\mathrm{CH}_{4}$ Measurement (ml) } \\
\hline $\begin{array}{l}\text { Time } \\
\text { (day) }\end{array}$ & Manure & SD & SSO & $\mathrm{SD}$ & $\begin{array}{c}\text { M/SSO } \\
9 / 1\end{array}$ & SD & $\begin{array}{c}\mathrm{M} / \mathrm{SSO} \\
7 / 3\end{array}$ & SD & $\begin{array}{c}\mathrm{M} / \mathrm{SSO} \\
5 / 5\end{array}$ & $\mathrm{SD}$ & $\begin{array}{c}\mathrm{M} / \mathrm{SSO} \\
1 / 9\end{array}$ & $\mathrm{SD}$ & $\begin{array}{c}\mathrm{M} / \mathrm{SSO} \\
3 / 7\end{array}$ & SD \\
\hline 0 & 0 & 0 & 0 & 0 & 0 & 0 & 0 & 0 & 0 & 0 & 0 & 0 & 0 & 0 \\
\hline 1 & 44 & 1.7 & 24 & 1.0 & 28 & 1.2 & 33 & 1.5 & 32 & 1.7 & 30 & 1.6 & 25 & 1.1 \\
\hline 2 & 47 & 1.8 & 59 & 2.5 & 69 & 3.0 & 59 & 2.7 & 68 & 3.7 & 70 & 3.6 & 64 & 2.7 \\
\hline 3 & 66 & 2.5 & 102 & 4.3 & 118 & 5.1 & 72 & 3.3 & 92 & 5.0 & 104 & 5.4 & 110 & 4.7 \\
\hline 4 & 66 & 2.5 & 90 & 3.8 & 104 & 4.5 & 64 & 3.0 & 74 & 4.0 & 82 & 4.3 & 93 & 4.0 \\
\hline 5 & 75 & 2.9 & 103 & 4.3 & 119 & 5.1 & 74 & 3.4 & 88 & 4.7 & 94 & 4.9 & 106 & 4.6 \\
\hline 6 & 90 & 3.4 & 111 & 4.6 & 128 & 5.5 & 108 & 4.9 & 111 & 6.0 & 117 & 6.1 & 118 & 5.1 \\
\hline 7 & 45 & 1.7 & 84 & 3.5 & 97 & 4.2 & 85 & 3.9 & 89 & 4.8 & 89 & 4.6 & 95 & 4.1 \\
\hline 8 & 44 & 1.7 & 65 & 2.7 & 75 & 3.2 & 57 & 2.6 & 58 & 3.1 & 58 & 3.0 & 68 & 2.9 \\
\hline 9 & 40 & 1.5 & 70 & 2.9 & 81 & 3.5 & 56 & 2.6 & 56 & 3.0 & 54 & 2.8 & 67 & 2.9 \\
\hline 11 & 51 & 2.0 & 70 & 2.9 & 81 & 3.5 & 64 & 3.0 & 65 & 3.5 & 60 & 3.1 & 69 & 3.0 \\
\hline 12 & 32 & 1.2 & 33 & 1.4 & 38 & 1.6 & 40 & 1.8 & 38 & 2.0 & 34 & 1.8 & 33 & 1.4 \\
\hline 14 & 33 & 1.3 & 30 & 1.3 & 35 & 1.5 & 48 & 2.2 & 48 & 2.6 & 37 & 1.9 & 34 & 1.5 \\
\hline 15 & 23 & 0.9 & 17 & 0.7 & 19 & 0.8 & 28 & 1.3 & 24 & 1.3 & 21 & 1.1 & 19 & 0.8 \\
\hline 16 & 23 & 0.9 & 12 & 0.5 & 14 & 0.6 & 22 & 1.0 & 17 & 0.9 & 14 & 0.7 & 13 & 0.6 \\
\hline 17 & 21 & 0.8 & 14 & 0.6 & 16 & 0.7 & 19 & 0.9 & 15 & 0.8 & 13 & 0.7 & 11 & 0.5 \\
\hline 18 & 16 & 0.6 & 14 & 0.6 & 16 & 0.7 & 21 & 0.9 & 17 & 0.9 & 14 & 0.7 & 11 & 0.5 \\
\hline 20 & 27 & 1.0 & 16 & 0.7 & 19 & 0.8 & 31 & 1.4 & 25 & 1.4 & 22 & 1.1 & 18 & 0.8 \\
\hline 21 & 14 & 0.5 & 11 & 0.5 & 13 & 0.6 & 23 & 1.0 & 18 & 0.9 & 14 & 0.7 & 12 & 0.5 \\
\hline 22 & 13 & 0.5 & 11 & 0.5 & 13 & 0.6 & 15 & 0.7 & 12 & 0.7 & 10 & 0.5 & 9 & 0.4 \\
\hline 23 & 12 & 0.5 & 9 & 0.4 & 11 & 0.5 & 14 & 0.6 & 11 & 0.6 & 9 & 0.5 & 7 & 0.3 \\
\hline 24 & 12 & 0.5 & 9 & 0.4 & 10 & 0.4 & 14 & 0.7 & 11 & 0.6 & 9 & 0.5 & 7 & 0.3 \\
\hline 25 & 13 & 0.5 & 9 & 0.4 & 10 & 0.4 & 13 & 0.6 & 12 & 0.7 & 12 & 0.6 & 10 & 0.4 \\
\hline 26 & 16 & 0.6 & 10 & 0.4 & 11 & 0.5 & 13 & 0.6 & 12 & 0.7 & 9 & 0.5 & 7 & 0.3 \\
\hline 28 & 9 & 0.3 & 11 & 0.5 & 13 & 0.5 & 19 & 0.9 & 20 & 1.1 & 15 & 0.8 & 12 & 0.5 \\
\hline 29 & 8 & 0.3 & 7 & 0.3 & 9 & 0.4 & 14 & 0.6 & 11 & 0.6 & 10 & 0.4 & 8 & 0.4 \\
\hline 30 & 6 & 0.2 & 7 & 0.3 & 9 & 0.4 & 13 & 0.6 & 11 & 0.6 & 9 & 0.5 & 8 & 0.3 \\
\hline 31 & 7 & 0.3 & 6 & 0.3 & 7 & 0.3 & 7 & 0.3 & 5 & 0.3 & 5 & 0.2 & 3 & 0.1 \\
\hline 32 & 7 & 0.3 & 9 & 0.4 & 10 & 0.4 & 11 & 0.5 & 9 & 0.5 & 9 & 0.5 & 5 & 0.2 \\
\hline 35 & 10 & 0.4 & 10 & 0.4 & 12 & 0.5 & 21 & 0.9 & 18 & 0.9 & 15 & 0.8 & 12 & 0.5 \\
\hline 37 & 10 & 0.4 & 13 & 0.5 & 15 & 0.6 & 17 & 0.8 & 21 & 1.1 & 11 & 0.6 & 23 & 1.0 \\
\hline 40 & 22 & 0.8 & 10 & 0.4 & 12 & 0.5 & 19 & 0.9 & 17 & 0.9 & 15 & 0.8 & 12 & 0.5 \\
\hline 43 & 10 & 0.4 & 6 & 0.2 & 7 & 0.3 & 10 & 0.5 & 9 & 0.5 & 8 & 0.4 & 6 & 0.3 \\
\hline 46 & 3 & 0.1 & 3 & 0.1 & 4 & 0.2 & 9 & 0.3 & 8 & 0.4 & 7 & 0.4 & 6 & 0.2 \\
\hline 49 & 2 & 0.1 & 3 & 0.1 & 3 & 0.1 & 8 & 0.4 & 8 & 0.4 & 7 & 0.3 & 6 & 0.1 \\
\hline 52 & 4 & 0.2 & 4 & 0.2 & 5 & 0.2 & 7 & 0.3 & 7 & 0.4 & 6 & 0.3 & 5 & 0.2 \\
\hline
\end{tabular}

*M: manure 
Table C. 3. Cumulative $\mathrm{CH}_{4}$ production in $\mathrm{AnCoD}$ of manure and $\mathrm{SSO}$

\begin{tabular}{|c|c|c|c|c|c|c|c|c|c|c|c|c|c|c|}
\hline \multicolumn{15}{|c|}{ Cumulative $\mathrm{CH}_{4}$ production $(\mathrm{mL})$} \\
\hline $\begin{array}{l}\text { Time } \\
\text { (day) }\end{array}$ & Manure & $\mathrm{SD}$ & SSO & SD & $\begin{array}{c}\mathrm{M}^{*} / \mathrm{SSO} \\
9 / 1\end{array}$ & SD & $\begin{array}{c}\mathrm{M} / \mathrm{SSO} \\
7 / 3\end{array}$ & $\mathrm{SD}$ & $\begin{array}{c}\mathrm{M} / \mathrm{SSO} \\
5 / 5\end{array}$ & SD & $\begin{array}{c}\mathrm{M} / \mathrm{SSO} \\
1 / 9\end{array}$ & SD & $\begin{array}{c}\mathrm{M} / \mathrm{SSO} \\
3 / 7\end{array}$ & SD \\
\hline 0 & 0 & 0 & 0 & 0 & 0 & 0 & 0 & 0 & 0 & 0.0 & 0 & 0.0 & 0 & 0.0 \\
\hline 1 & 44 & 2.0 & 24 & 1.3 & 28 & 1.3 & 33 & 1.8 & 32 & 1.5 & 30 & 1.3 & 25 & 1.0 \\
\hline 2 & 90 & 4.1 & 83 & 4.6 & 93 & 4.5 & 93 & 5.0 & 101 & 4.5 & 100 & 4.2 & 89 & 3.4 \\
\hline 3 & 156 & 7.0 & 186 & 10.2 & 206 & 9.9 & 164 & 8.9 & 193 & 8.7 & 204 & 8.6 & 199 & 7.6 \\
\hline 4 & 222 & 10.0 & 276 & 15.2 & 305 & 14.6 & 229 & 12.3 & 267 & 12.0 & 287 & 12.0 & 291 & 11.1 \\
\hline 5 & 297 & 13.4 & 379 & 20.8 & 418 & 20.1 & 303 & 16.4 & 355 & 16.0 & 381 & 16.0 & 397 & 15.1 \\
\hline 6 & 387 & 17.4 & 489 & 26.9 & 540 & 25.9 & 410 & 22.2 & 466 & 21.0 & 498 & 20.9 & 516 & 19.6 \\
\hline 7 & 432 & 19.4 & 573 & 31.5 & 632 & 30.3 & 496 & 26.8 & 554 & 24.9 & 587 & 24.7 & 611 & 23.2 \\
\hline 8 & 476 & 21.4 & 638 & 35.1 & 703 & 33.7 & 553 & 29.9 & 612 & 27.5 & 645 & 27.1 & 679 & 25.8 \\
\hline 9 & 516 & 23.2 & 708 & 38.9 & 780 & 37.5 & 609 & 32.9 & 668 & 30.0 & 699 & 29.4 & 746 & 28.3 \\
\hline 11 & 567 & 25.5 & 778 & 42.8 & 857 & 41.1 & 673 & 36.3 & 732 & 33.0 & 759 & 31.9 & 815 & 31.0 \\
\hline 12 & 599 & 26.9 & 810 & 44.6 & 893 & 42.9 & 713 & 38.5 & 770 & 34.7 & 793 & 33.3 & 849 & 32.3 \\
\hline 14 & 632 & 28.4 & 840 & 46.2 & 926 & 44.4 & 761 & 41.1 & 818 & 36.8 & 830 & 34.9 & 883 & 33.5 \\
\hline 15 & 654 & 29.5 & 857 & 47.1 & 944 & 45.3 & 789 & 42.6 & 842 & 37.9 & 851 & 35.7 & 902 & 34.3 \\
\hline 16 & 677 & 30.5 & 870 & 47.8 & 958 & 46.0 & 811 & 43.8 & 859 & 38.7 & 866 & 36.4 & 915 & 34.8 \\
\hline 17 & 698 & 31.4 & 883 & 48.6 & 973 & 46.7 & 829 & 44.8 & 875 & 39.4 & 878 & 36.9 & 926 & 35.2 \\
\hline 18 & 714 & 32.1 & 897 & 49.3 & 987 & 47.4 & 850 & 45.9 & 892 & 40.1 & 893 & 37.5 & 937 & 35.6 \\
\hline 20 & 741 & 33.3 & 913 & 50.2 & 1001 & 48.0 & 881 & 47.6 & 917 & 41.3 & 914 & 38.4 & 955 & 36.3 \\
\hline 21 & 755 & 34.0 & 924 & 50.8 & 1021 & 49.0 & 904 & 48.8 & 934 & 42.0 & 929 & 39.0 & 967 & 36.8 \\
\hline 22 & 768 & 34.5 & 936 & 51.5 & 1035 & 49.7 & 919 & 49.6 & 947 & 42.6 & 939 & 39.4 & 976 & 37.1 \\
\hline 23 & 780 & 35.1 & 945 & 52.0 & 1045 & 50.2 & 933 & 50.4 & 957 & 43.1 & 948 & 39.8 & 984 & 36.3 \\
\hline 24 & 792 & 35.6 & 954 & 52.4 & 1055 & 50.6 & 947 & 51.1 & 968 & 43.6 & 957 & 40.2 & 991 & 37.6 \\
\hline 25 & 804 & 36.2 & 962 & 52.9 & 1065 & 51.1 & 960 & 51.9 & 980 & 44.1 & 969 & 40.7 & 1001 & 38.0 \\
\hline 26 & 820 & 36.9 & 972 & 53.5 & 1076 & 51.6 & 974 & 52.6 & 992 & 44.7 & 978 & 41.1 & 1008 & 35.4 \\
\hline 28 & 829 & 37.3 & 983 & 54.1 & 1088 & 52.2 & 993 & 53.6 & 1013 & 45.6 & 993 & 41.7 & 1020 & 38.8 \\
\hline 29 & 837 & 37.7 & 991 & 54.5 & 1099 & 52.7 & 1007 & 54.4 & 1024 & 46.1 & 1003 & 42.1 & 1029 & 39.1 \\
\hline 30 & 844 & 38.0 & 998 & 54.9 & 1110 & 53.3 & 1019 & 55.0 & 1035 & 46.6 & 1012 & 42.5 & 1036 & 40.6 \\
\hline 31 & 851 & 38.3 & 1004 & 55.2 & 1120 & 53.7 & 1027 & 55.5 & 1040 & 46.8 & 1017 & 42.7 & 1039 & 39.5 \\
\hline 32 & 858 & 38.6 & 1013 & 55.7 & 1132 & 54.3 & 1038 & 56.0 & 1049 & 47.2 & 1026 & 43.1 & 1044 & 38.4 \\
\hline 35 & 867 & 39.0 & 1023 & 56.3 & 1143 & 54.9 & 1058 & 57.1 & 1067 & 48.0 & 1041 & 43.7 & 1056 & 39.7 \\
\hline 37 & 877 & 39.5 & 1036 & 57.0 & 1157 & 55.5 & 1075 & 58.1 & 1087 & 48.9 & 1052 & 44.2 & 1080 & 41.0 \\
\hline 40 & 899 & 40.5 & 1046 & 57.6 & 1168 & 56.1 & 1094 & 59.1 & 1104 & 49.7 & 1067 & 44.8 & 1092 & 40.2 \\
\hline 43 & 910 & 40.9 & 1052 & 57.9 & 1175 & 56.4 & 1104 & 59.6 & 1114 & 50.1 & 1076 & 43.8 & 1098 & 41.7 \\
\hline 46 & 912 & 41.1 & 1056 & 58.1 & 1179 & 56.6 & 1113 & 60.1 & 1121 & 49.2 & 1082 & 45.5 & 1104 & 42.0 \\
\hline 49 & 915 & 41.2 & 1059 & 58.2 & 1182 & 56.7 & 1121 & 58.3 & 1129 & 50.8 & 1089 & 44.3 & 1110 & 43.5 \\
\hline 52 & 919 & 41.3 & 1063 & 58.5 & 1186 & 56.9 & 1129 & 61.0 & 1136 & 51.1 & 1095 & 46.0 & 1115 & 42.4 \\
\hline
\end{tabular}

*M: manure 
Table C. 4. Cumulative $\mathrm{CH}_{4}$ production per mass of $\mathrm{COD}$ added in $\mathrm{AnCoD}$ of manure and SSO

\begin{tabular}{|c|c|c|c|c|c|c|c|c|c|c|c|c|c|c|}
\hline \multicolumn{15}{|c|}{ Cumulative $\mathrm{CH}_{4}$ yield (mL CH4/g COD added) } \\
\hline $\begin{array}{l}\text { Time } \\
\text { (day) }\end{array}$ & Manure & $\mathrm{SD}$ & SSO & $\mathrm{SD}$ & $\begin{array}{c}\mathrm{M}^{*} / \mathrm{SSO} \\
9 / 1\end{array}$ & $\mathrm{SD}$ & $\begin{array}{c}\mathrm{M} / \mathrm{SSO} \\
7 / 3\end{array}$ & SD & $\begin{array}{c}\mathrm{M} / \mathrm{SSO} \\
5 / 5\end{array}$ & SD & $\begin{array}{c}\mathrm{M} / \mathrm{SSO} \\
1 / 9\end{array}$ & SD & $\begin{array}{c}\text { M/SSO } \\
3 / 7\end{array}$ & SD \\
\hline 0 & 0 & 0 & 0 & 0 & 0 & 0 & 0 & 0 & 0 & 0 & 0 & 0 & 0 & 0 \\
\hline 1 & 14 & 0.6 & 7 & 0.3 & 9 & 0.3 & 10 & 0.4 & 9 & 0.3 & 9 & 0.5 & 7 & 0.3 \\
\hline 2 & 28 & 1.2 & 24 & 0.9 & 29 & 1.1 & 28 & 1.0 & 29 & 1.0 & 30 & 1.5 & 25 & 0.9 \\
\hline 3 & 49 & 2.0 & 53 & 2.0 & 63 & 2.5 & 50 & 1.8 & 56 & 2.0 & 61 & 3.1 & 56 & 2.0 \\
\hline 4 & 69 & 2.9 & 79 & 3.0 & 93 & 3.7 & 70 & 2.4 & 77 & 2.7 & 86 & 4.4 & 82 & 3.0 \\
\hline 5 & 93 & 3.9 & 108 & 4.1 & 128 & 5.1 & 92 & 3.2 & 103 & 3.6 & 114 & 5.8 & 112 & 4.0 \\
\hline 6 & 121 & 5.1 & 140 & 5.3 & 165 & 6.6 & 125 & 4.4 & 135 & 4.7 & 149 & 7.6 & 146 & 5.3 \\
\hline 7 & 135 & 5.7 & 163 & 6.2 & 193 & 7.7 & 151 & 5.3 & 161 & 5.6 & 175 & 8.9 & 173 & 6.2 \\
\hline 8 & 149 & 6.2 & 182 & 6.9 & 215 & 8.6 & 169 & 5.9 & 178 & 6.2 & 193 & 9.8 & 192 & 6.9 \\
\hline 9 & 161 & 6.8 & 202 & 7.7 & 239 & 9.6 & 186 & 6.5 & 194 & 5.8 & 209 & 10.6 & 211 & 7.6 \\
\hline 11 & 177 & 7.4 & 222 & 8.4 & 262 & 10.5 & 205 & 7.2 & 213 & 7.4 & 226 & 11.5 & 231 & 8.3 \\
\hline 12 & 187 & 7.9 & 231 & 8.8 & 273 & 10.6 & 217 & 7.6 & 224 & 7.8 & 237 & 12.1 & 240 & 8.6 \\
\hline 14 & 197 & 8.3 & 240 & 9.1 & 283 & 11.3 & 232 & 8.1 & 238 & 8.3 & 248 & 11.6 & 250 & 9.0 \\
\hline 15 & 204 & 7.5 & 244 & 9.3 & 289 & 10.4 & 241 & 8.6 & 245 & 7.6 & 254 & 13.0 & 255 & 8.2 \\
\hline 16 & 211 & 8.9 & 248 & 9.4 & 293 & 11.7 & 247 & 7.7 & 250 & 8.7 & 258 & 12.2 & 259 & 9.3 \\
\hline 17 & 218 & 9.2 & 252 & 10.1 & 298 & 10.3 & 253 & 8.9 & 254 & 8.9 & 262 & 13.4 & 262 & 9.8 \\
\hline 18 & 223 & 9.4 & 256 & 9.7 & 302 & 11.2 & 259 & 9.1 & 259 & 9.1 & 266 & 12.3 & 265 & 8.6 \\
\hline 20 & 231 & 8.6 & 260 & 9.9 & 306 & 12.3 & 269 & 9.4 & 266 & 8.3 & 273 & 13.9 & 270 & 7.7 \\
\hline 21 & 236 & 9.9 & 264 & 10.0 & 312 & 12.5 & 276 & 8.7 & 271 & 9.5 & 277 & 14.1 & 274 & 8.9 \\
\hline 22 & 240 & 10.1 & 267 & 10.1 & 317 & 12.7 & 280 & 9.8 & 275 & 8.6 & 280 & 13.2 & 276 & 9.9 \\
\hline 23 & 244 & 9.7 & 269 & 9.4 & 320 & 11.6 & 285 & 10.0 & 278 & 9.7 & 283 & 14.4 & 278 & 9.3 \\
\hline 24 & 247 & 10.4 & 272 & 10.3 & 323 & 12.9 & 289 & 10.6 & 281 & 9.8 & 286 & 13.6 & 280 & 9.1 \\
\hline 25 & 251 & 10.6 & 274 & 9.7 & 326 & 13.0 & 293 & 10.3 & 285 & 10.0 & 289 & 14.7 & 283 & 10.5 \\
\hline 26 & 256 & 10.8 & 277 & 10.5 & 329 & 13.2 & 297 & 9.8 & 288 & 9.1 & 292 & 12.9 & 285 & 9.3 \\
\hline 28 & 259 & 10.9 & 280 & 9.8 & 333 & 13.3 & 303 & 10.6 & 294 & 10.3 & 296 & 15.1 & 289 & 10.4 \\
\hline 29 & 262 & 11.0 & 283 & 10.7 & 336 & 13.4 & 307 & 10.7 & 297 & 9.4 & 299 & 14.3 & 291 & 9.5 \\
\hline 30 & 264 & 10.4 & 285 & 9.3 & 340 & 13.6 & 311 & 10.9 & 301 & 10.5 & 302 & 15.4 & 293 & 10.6 \\
\hline 31 & 266 & 11.2 & 286 & 10.9 & 343 & 12.7 & 313 & 11.0 & 302 & 9.6 & 303 & 13.5 & 294 & 9.6 \\
\hline 32 & 268 & 10.7 & 289 & 12.3 & 346 & 13.9 & 317 & 9.7 & 305 & 10.7 & 306 & 15.6 & 295 & 10.6 \\
\hline 35 & 271 & 11.4 & 292 & 11.1 & 350 & 14.0 & 323 & 11.3 & 310 & 9.8 & 311 & 13.8 & 299 & 9.8 \\
\hline 37 & 274 & 11.5 & 295 & 10.5 & 354 & 13.8 & 328 & 9.8 & 316 & 11.1 & 314 & 14.9 & 306 & 11.0 \\
\hline 40 & 281 & 11.8 & 298 & 11.3 & 358 & 14.5 & 334 & 11.7 & 321 & 9.8 & 318 & 16.2 & 309 & 10.1 \\
\hline 43 & 284 & 11.9 & 300 & 10.2 & 360 & 13.2 & 337 & 10.2 & 323 & 11.3 & 321 & 15.1 & 311 & 11.2 \\
\hline 46 & 285 & 11.6 & 301 & 11.4 & 361 & 14.4 & 340 & 11.9 & 326 & 10.5 & 323 & 16.5 & 312 & 10.4 \\
\hline 49 & 286 & 10.2 & 302 & 10.6 & 362 & 13.1 & 342 & 10.9 & 328 & 9.2 & 325 & 14.6 & 314 & 9.7 \\
\hline 52 & 287 & 12.1 & 303 & 11.5 & 363 & 14.5 & 344 & 11.6 & 330 & 11.5 & 327 & 16.7 & 316 & 10.6 \\
\hline
\end{tabular}


Table C. 5. Cumulative $\mathrm{CH}_{4}$ production per mass of VSS added in AnCoD of manure and SSO

\begin{tabular}{|c|c|c|c|c|c|c|c|c|c|c|c|c|c|c|}
\hline \multirow{3}{*}{$\begin{array}{l}\text { Time } \\
\text { (day) }\end{array}$} & \multicolumn{14}{|c|}{ Cumulative $\mathrm{CH}_{4}$ yield (mL CH4/g VSS added) } \\
\hline & & & & & $\mathrm{M}^{*} / \mathrm{SSO}$ & & $\mathrm{M} / \mathrm{SSO}$ & & $\mathrm{M} / \mathrm{SSC}$ & & $\mathrm{M} / \mathrm{SSO}$ & & M/SSO & \\
\hline & Manure & SD & SSO & SD & $9 / 1$ & $\mathrm{SD}$ & $7 / 3$ & SD & $5 / 5$ & $\mathrm{SD}$ & & $\mathrm{SD}$ & & SD \\
\hline 0 & 0 & 0 & 0 & 0 & 0 & 0 & 0 & 0 & 0 & 0 & 0 & 0 & 0 & 0.0 \\
\hline 1 & 31 & 1.4 & 18 & 0.6 & 20 & 0.8 & 25 & 0.9 & 23 & 0.8 & 23 & 0.9 & 19 & 0.9 \\
\hline 2 & 64 & 2.9 & 62 & 2.2 & 67 & 2.8 & 69 & 2.5 & 73 & 2.5 & 76 & 2.9 & 65 & 3.1 \\
\hline 3 & 111 & 5.0 & 139 & 4.9 & 147 & 6.2 & 121 & 4.5 & 139 & 4.9 & 155 & 5.9 & 146 & 6.9 \\
\hline 4 & 158 & 7.1 & 206 & 7.2 & 218 & 9.1 & 169 & 6.2 & 193 & 6.8 & 218 & 8.3 & 214 & 10.1 \\
\hline 5 & 212 & 9.5 & 282 & 9.9 & 298 & 12.5 & 224 & 8.3 & 257 & 9.0 & 289 & 11.0 & 292 & 13.7 \\
\hline 6 & 276 & 12.4 & 365 & 12.8 & 385 & 16.2 & 303 & 11.2 & 337 & 11.8 & 379 & 14.4 & 379 & 17.8 \\
\hline 7 & 308 & 13.9 & 428 & 15.0 & 451 & 19.0 & 366 & 13.6 & 401 & 14.0 & 446 & 17.0 & 448 & 21.1 \\
\hline 8 & 339 & 15.2 & 476 & 16.7 & 502 & 21.1 & 408 & 15.1 & 443 & 15.5 & 491 & 18.6 & 499 & 23.4 \\
\hline 9 & 367 & 16.5 & 528 & 18.5 & 557 & 23.4 & 450 & 16.6 & 483 & 16.9 & 532 & 20.2 & 548 & 25.8 \\
\hline 11 & 404 & 18.2 & 580 & 20.3 & 612 & 25.7 & 497 & 18.4 & 530 & 18.5 & 577 & 21.9 & 599 & 28.2 \\
\hline 12 & 426 & 19.2 & 605 & 21.2 & 638 & 26.8 & 526 & 19.5 & 557 & 19.5 & 603 & 22.9 & 624 & 29.3 \\
\hline 14 & 450 & 20.3 & 627 & 21.9 & 661 & 27.8 & 562 & 20.8 & 592 & 20.7 & 631 & 24.0 & 648 & 30.5 \\
\hline 15 & 466 & 21.0 & 640 & 22.4 & 674 & 28.3 & 583 & 21.6 & 609 & 21.3 & 647 & 24.6 & 662 & 31.1 \\
\hline 16 & 482 & 21.7 & 649 & 22.7 & 684 & 28.7 & 599 & 22.2 & 622 & 21.8 & 658 & 23.7 & 672 & 31.6 \\
\hline 17 & 497 & 20.5 & 659 & 22.1 & 695 & 28.2 & 613 & 23.7 & 633 & 19.3 & 668 & 25.4 & 680 & 29.5 \\
\hline 18 & 508 & 22.9 & 669 & 23.4 & 705 & 29.6 & 628 & 23.2 & 645 & 22.6 & 679 & 25.8 & 689 & 32.4 \\
\hline 20 & 528 & 23.7 & 681 & 23.8 & 715 & 30.0 & 651 & 24.1 & 663 & 21.5 & 695 & 26.4 & 702 & 33.0 \\
\hline 21 & 538 & 24.2 & 690 & 24.1 & 729 & 29.6 & 668 & 24.7 & 676 & 23.7 & 706 & 26.8 & 710 & 31.9 \\
\hline 22 & 547 & 24.6 & 698 & 24.4 & 739 & 31.1 & 679 & 25.1 & 685 & 24.0 & 714 & 27.1 & 717 & 33.7 \\
\hline 23 & 555 & 25.0 & 705 & 23.5 & 747 & 30.4 & 689 & 25.5 & 693 & 23.2 & 721 & 25.8 & 722 & 34.0 \\
\hline 24 & 564 & 24.4 & 711 & 24.9 & 753 & 31.6 & 700 & 24.2 & 700 & 24.5 & 728 & 27.7 & 728 & 34.2 \\
\hline 25 & 573 & 25.8 & 718 & 25.1 & 760 & 30.9 & 709 & 26.2 & 709 & 24.8 & 737 & 28.0 & 735 & 33.5 \\
\hline 26 & 584 & 26.3 & 725 & 25.4 & 768 & 32.3 & 719 & 25.6 & 718 & 25.1 & 744 & 26.8 & 740 & 34.8 \\
\hline 28 & 591 & 24.6 & 734 & 24.7 & 777 & 32.6 & 733 & 27.1 & 733 & 24.6 & 755 & 28.7 & 749 & 35.2 \\
\hline 29 & 596 & 26.8 & 739 & 25.9 & 785 & 33.0 & 743 & 26.5 & 741 & 25.9 & 763 & 29.0 & 755 & 34.5 \\
\hline 30 & 601 & 25.5 & 745 & 25.1 & 792 & 33.3 & 753 & 27.9 & 749 & 26.2 & 770 & 29.2 & 761 & 35.8 \\
\hline 31 & 606 & 27.3 & 749 & 26.2 & 800 & 33.6 & 758 & 28.1 & 753 & 25.7 & 773 & 27.9 & 763 & 35.9 \\
\hline 32 & 611 & 26.5 & 756 & 24.4 & 808 & 32.9 & 766 & 27.4 & 759 & 26.6 & 780 & 29.6 & 767 & 34.9 \\
\hline 35 & 618 & 27.8 & 763 & 26.7 & 816 & 34.3 & 782 & 28.9 & 772 & 25.3 & 792 & 30.1 & 776 & 36.5 \\
\hline 37 & 625 & 28.1 & 773 & 27.1 & 826 & 34.7 & 794 & 28.4 & 787 & 27.5 & 800 & 28.4 & 793 & 37.3 \\
\hline 40 & 641 & 28.8 & 781 & 27.3 & 835 & 35.1 & 808 & 29.9 & 799 & 28.0 & 811 & 30.8 & 802 & 36.8 \\
\hline 43 & 648 & 29.2 & 785 & 255.0 & 839 & 34.2 & 815 & 30.2 & 806 & 26.2 & 818 & 29.3 & 807 & 37.9 \\
\hline 46 & 650 & 27.9 & 788 & 27.6 & 842 & 35.4 & 822 & 30.4 & 811 & 28.4 & 823 & 31.3 & 811 & 38.1 \\
\hline 49 & 652 & 28.3 & 790 & 25.6 & 844 & 34.5 & 828 & 29.7 & 817 & 27.6 & 828 & 30.5 & 815 & 37.3 \\
\hline 52 & 654 & 29.4 & 793 & 27.8 & 847 & 35.6 & 834 & 30.8 & 822 & 28.8 & 833 & 31.6 & 819 & 38.5 \\
\hline
\end{tabular}


Table C. 6. Cumulative $\mathrm{CH}_{4}$ production per volume of substrate added in AnCoD of manure and SSO

\begin{tabular}{|c|c|c|c|c|c|c|c|c|c|c|c|c|c|c|}
\hline \multirow{3}{*}{$\begin{array}{l}\text { Time } \\
\text { (day) }\end{array}$} & \multirow[b]{3}{*}{ Manure } & \multirow[b]{3}{*}{ SD } & \multirow[b]{3}{*}{$\mathrm{SSO}$} & \multicolumn{7}{|c|}{ Cumulative $\mathrm{CH}_{4}$ yield $(\mathrm{mL} \mathrm{CH} 4 / \mathrm{mL}$ substrate added) } & \multirow{3}{*}{$\begin{array}{l}\mathrm{M} / \mathrm{SSO} \\
1 / 9 \\
\end{array}$} & \multirow[b]{3}{*}{ SD } & \multirow{3}{*}{$\begin{array}{l}\mathrm{M} / \mathrm{SSO} \\
3 / 7 \\
\end{array}$} & \multirow[b]{3}{*}{ SD } \\
\hline & & & & & $\mathrm{M}^{*} / \mathrm{SSO}$ & & $\mathrm{M} / \mathrm{SSO}$ & & $\mathrm{M} / \mathrm{SSO}$ & & & & & \\
\hline & & & & SD & $9 / 1$ & $\mathrm{SD}$ & $7 / 3$ & $\mathrm{SD}$ & $5 / 5$ & $\mathrm{SD}$ & & & & \\
\hline 0 & 0 & 0 & 0 & 0 & 0 & 0 & 0 & 0 & 0 & 0 & 0 & 0 & 0 & 0 \\
\hline 1 & 1.45 & 0.06 & 1.4 & 0.05 & 1.0 & 0.04 & 1.4 & 0.06 & 1.5 & 0.07 & 1.6 & 0.07 & 1.4 & 0.08 \\
\hline 2 & 3.02 & 0.12 & 4.9 & 0.16 & 3.3 & 0.14 & 3.9 & 0.18 & 4.6 & 0.21 & 5.3 & 0.22 & 5.0 & 0.27 \\
\hline 3 & 5.21 & 0.20 & 10.9 & 0.35 & 7.3 & 0.31 & 6.8 & 0.32 & 8.8 & 0.40 & 10.8 & 0.45 & 11.0 & 0.61 \\
\hline 4 & 7.40 & 0.29 & 16.2 & 0.52 & 10.9 & 0.46 & 9.5 & 0.44 & 12.1 & 0.56 & 15.1 & 0.63 & 16.2 & 0.89 \\
\hline 5 & 9.90 & 0.39 & 22.3 & 0.71 & 14.9 & 0.63 & 12.6 & 0.58 & 16.1 & 0.74 & 20.0 & 0.84 & 22.1 & 1.21 \\
\hline 6 & 12.91 & 0.50 & 28.8 & 0.92 & 19.3 & 0.81 & 17.1 & 0.79 & 21.2 & 0.97 & 26.2 & 1.10 & 28.7 & 1.58 \\
\hline 7 & 14.41 & 0.56 & 33.7 & 1.08 & 22.6 & 0.95 & 20.7 & 0.95 & 25.2 & 1.16 & 30.9 & 1.30 & 33.9 & 1.87 \\
\hline 8 & 15.86 & 0.62 & 37.5 & 1.20 & 25.1 & 1.05 & 23.0 & 1.06 & 27.8 & 1.28 & 34.0 & 1.43 & 37.7 & 2.07 \\
\hline 9 & 17.19 & 0.67 & 41.7 & 1.33 & 27.9 & 1.17 & 25.4 & 1.17 & 30.3 & 1.40 & 36.8 & 1.55 & 41.4 & 2.28 \\
\hline 11 & 18.90 & 0.74 & 45.7 & 1.46 & 30.6 & 1.29 & 28.0 & 1.29 & 33.3 & 1.53 & 39.9 & 1.88 & 45.3 & 1.94 \\
\hline 12 & 19.96 & 0.78 & 47.7 & 1.53 & 31.9 & 1.34 & 29.7 & 1.37 & 35.0 & 1.61 & 41.8 & 1.75 & 47.2 & 2.59 \\
\hline 14 & 21.06 & 0.82 & 49.4 & 1.58 & 33.1 & 1.39 & 31.7 & 1.46 & 37.2 & 1.71 & 43.7 & 2.03 & 49.0 & 1.97 \\
\hline 15 & 21.81 & 0.95 & 50.4 & 1.61 & 33.7 & 1.42 & 32.9 & 1.51 & 38.3 & 1.76 & 44.8 & 1.88 & 50.1 & 2.76 \\
\hline 16 & 22.57 & 0.88 & 51.2 & 1.64 & 34.2 & 1.44 & 33.8 & 1.55 & 39.1 & 1.80 & 45.6 & 1.91 & 50.8 & 1.80 \\
\hline 17 & 23.27 & 0.91 & 52.0 & 1.66 & 34.7 & 1.46 & 34.6 & 1.59 & 39.8 & 1.83 & 46.2 & 1.94 & 51.5 & 2.83 \\
\hline 18 & 23.79 & 0.83 & 52.8 & 1.69 & 35.2 & 1.48 & 35.4 & 1.63 & 40.5 & 1.86 & 47.0 & 1.97 & 52.1 & 1.86 \\
\hline 20 & 24.69 & 0.96 & 53.7 & 1.72 & 35.7 & 1.50 & 36.7 & 1.69 & 41.7 & 1.92 & 48.1 & 2.02 & 53.1 & 2.92 \\
\hline 21 & 25.16 & 0.98 & 54.4 & 1.74 & 36.5 & 1.53 & 37.7 & 1.73 & 42.5 & 1.95 & 48.9 & 2.05 & 53.7 & 1.96 \\
\hline 22 & 25.59 & 1.00 & 55.0 & 1.76 & 37.0 & 1.55 & 38.3 & 1.76 & 43.0 & 1.98 & 49.4 & 2.08 & 54.2 & 2.98 \\
\hline 23 & 25.99 & 1.01 & 55.6 & 1.78 & 37.3 & 1.57 & 38.9 & 1.79 & 43.5 & 2.00 & 49.9 & 2.75 & 54.6 & 3.01 \\
\hline 24 & 26.39 & 9.83 & 56.1 & 1.79 & 37.7 & 1.58 & 39.5 & 1.82 & 44.0 & 2.02 & 50.4 & 2.12 & 55.0 & 2.93 \\
\hline 25 & 26.81 & 1.05 & 56.6 & 1.81 & 38.0 & 1.60 & 40.0 & 1.84 & 44.6 & 2.05 & 51.0 & 2.53 & 55.6 & 3.06 \\
\hline 26 & 27.35 & 9.87 & 57.2 & 1.83 & 38.4 & 1.61 & 40.6 & 1.87 & 45.1 & 2.08 & 51.5 & 2.16 & 56.0 & 2.98 \\
\hline 28 & 27.65 & 1.08 & 57.8 & 1.85 & 38.8 & 1.63 & 41.4 & 1.90 & 46.0 & 2.12 & 52.3 & 1.92 & 56.7 & 3.12 \\
\hline 29 & 27.91 & 1.09 & 58.3 & 1.86 & 39.2 & 1.65 & 41.9 & 1.93 & 46.5 & 2.14 & 52.8 & 2.22 & 57.1 & 2.94 \\
\hline 30 & 28.13 & 1.10 & 58.7 & 1.88 & 39.6 & 1.66 & 42.5 & 1.95 & 47.0 & 1.98 & 53.3 & 1.94 & 57.6 & 3.17 \\
\hline 31 & 28.36 & 1.11 & 59.1 & 1.89 & 40.0 & 1.68 & 42.8 & 1.97 & 47.3 & 2.17 & 53.5 & 2.25 & 57.7 & 2.98 \\
\hline 32 & 28.58 & 1.11 & 59.6 & 1.91 & 40.4 & 1.70 & 43.2 & 1.99 & 47.7 & 1.92 & 54.0 & 1.27 & 58.0 & 3.19 \\
\hline 35 & 28.92 & 9.83 & 60.2 & 1.93 & 40.8 & 1.71 & 44.1 & 2.03 & 48.5 & 2.23 & 54.8 & 2.30 & 58.7 & 3.23 \\
\hline 37 & 29.25 & 1.14 & 60.9 & 1.95 & 41.3 & 1.74 & 44.8 & 2.06 & 49.4 & 1.72 & 55.4 & 1.33 & 60.0 & 2.30 \\
\hline 40 & 29.98 & 1.17 & 61.6 & 1.97 & 41.7 & 1.75 & 45.6 & 2.10 & 50.2 & 2.31 & 56.2 & 2.36 & 60.7 & 3.34 \\
\hline 43 & 30.32 & 1.18 & 61.9 & 1.98 & 42.0 & 1.76 & 46.0 & 2.12 & 50.6 & 1.63 & 56.6 & 1.83 & 61.0 & 2.36 \\
\hline 46 & 30.41 & 1.19 & 62.1 & 1.99 & 42.1 & 1.77 & 46.4 & 2.13 & 51.0 & 2.34 & 57.0 & 2.39 & 61.3 & 3.37 \\
\hline 49 & 30.49 & 1.23 & 62.3 & 1.99 & 42.2 & 1.77 & 46.7 & 2.15 & 51.3 & 1.36 & 57.3 & 1.94 & 61.6 & 3.39 \\
\hline 52 & 30.63 & 1.19 & 62.5 & 2.00 & 42.4 & 1.78 & 47.0 & 2.16 & 51.6 & 2.37 & 57.6 & 2.42 & 61.9 & 2.41 \\
\hline
\end{tabular}




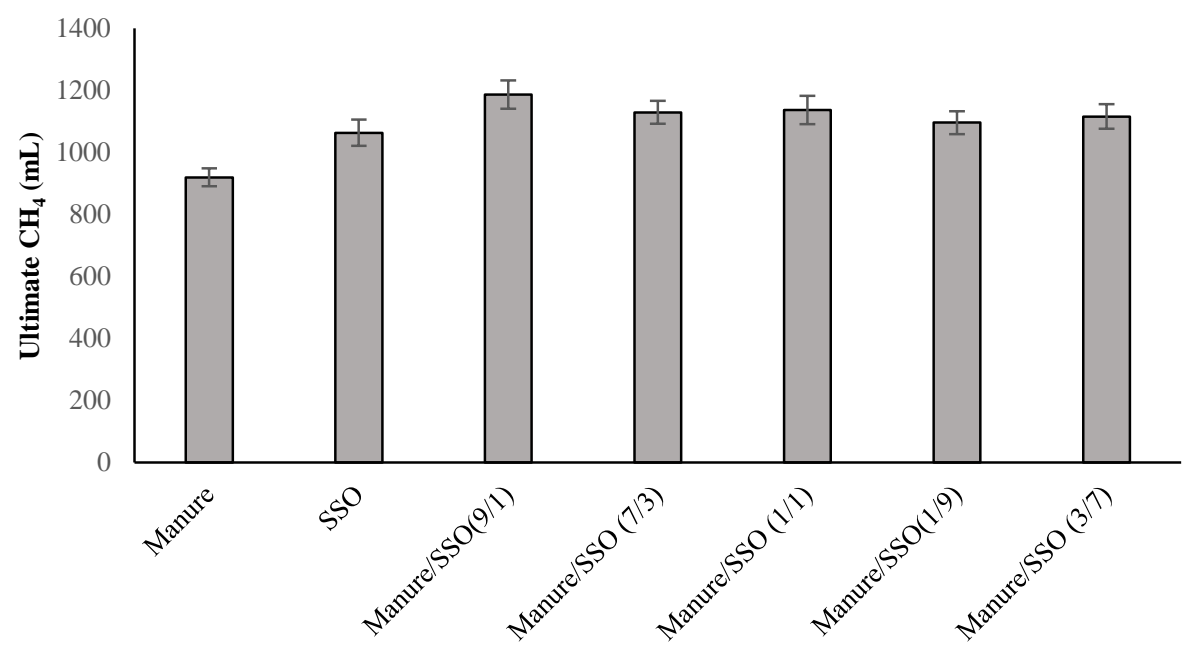

Mixing ratio

Figure C. 1. Ultimate methane production in AnCoD of TWAS and manure 
Table C. 7. Characteristics of the raw feedstocks for hydrolysis/acidification of manure and SSO

\begin{tabular}{cccccccc}
\hline \multirow{2}{*}{ Parameters } & \multirow{2}{*}{ Units } & \multicolumn{2}{c}{ Manure } & \multicolumn{2}{c}{ SSO } & \multicolumn{2}{c}{ Inoculum } \\
& & MEAN & SD & MEAN & SD & MEAN & SD \\
\hline TCOD & $\mathrm{mg} / \mathrm{L}$ & 101125 & 4551 & 115163 & 4607 & 17100 & 400 \\
SCOD & $\mathrm{mg} / \mathrm{L}$ & 10480 & 472 & 42932 & 1717 & 986 & 16 \\
TSS & $\mathrm{mg} / \mathrm{L}$ & 54600 & 2457 & 47112 & 1884 & 17143 & 330 \\
VSS & $\mathrm{mg} / \mathrm{L}$ & 32115 & 1445 & 39940 & 1598 & 11200 & 160 \\
TS & $\mathrm{mg} / \mathrm{L}$ & 68540 & 3084 & 77987 & 3119 & 21870 & 503 \\
VS & $\mathrm{mg} / \mathrm{L}$ & 58520 & 2633 & 63537 & 2541 & 13380 & 308 \\
Ammonia & $\mathrm{mg} / \mathrm{L}$ & 24.3 & 1 & 1396 & 55.84 & 1505 & 36 \\
$\mathrm{pH}$ & - & 6.7 & 0.302 & 5.8 & 0.2 & 7.2 & 0.1 \\
& $\mathrm{mg}$ & & & & & & \\
Alkalinity & $\mathrm{CaCO} / \mathrm{L}$ & 7224 & 325 & 6720 & 269 & 3986 & 460 \\
$\mathrm{TN}$ & $\mathrm{mg} / \mathrm{L}$ & 1740 & 78 & 4198 & 167.92 & 2048 & 186 \\
TSN & $\mathrm{mg} / \mathrm{L}$ & 107 & 5 & 1146 & 46 & 716 & 93 \\
Total Carbs & $\mathrm{mg} / \mathrm{L}$ & 26796 & 1206 & 14125 & 565 & 595 & 68 \\
Total Proteins & $\mathrm{mg} / \mathrm{L}$ & 5408 & 243 & 2156 & 86.24 & 1680 & 38 \\
Total Lipids & $\mathrm{mg} / \mathrm{L}$ & 1703 & 77 & 1468 & 59 & 168 & 93 \\
\hline
\end{tabular}


Table C. 8. Measured and theoretical VFAs over time in hydrolysis/acidification of manure and SSO

\begin{tabular}{|c|c|c|c|c|c|c|c|}
\hline \multicolumn{8}{|c|}{ Measured VFAs concentrations (mg/L)over time } \\
\hline & Mixture 1 & Mixture 2 & Mixture 3 & Mixture 4 & Mixture 5 & Mixture 6 & Mixture 7 \\
\hline $\begin{array}{l}\text { Time } \\
(\mathrm{hr})\end{array}$ & Manure & SSO & $\begin{array}{c}\text { Manure/SSO } \\
9 / 1\end{array}$ & $\begin{array}{c}\text { Manure/SSO } \\
7 / 3\end{array}$ & $\begin{array}{c}\text { Manure/SSO } \\
5 / 5\end{array}$ & $\begin{array}{c}\text { Manure/SSO } \\
3 / 7\end{array}$ & $\begin{array}{c}\text { Manure/SSO } \\
1 / 9\end{array}$ \\
\hline 0 & 668 & 635 & 646 & 649 & 666 & 740 & 722 \\
\hline 6 & 783 & 790 & 661 & 688 & 749 & 861 & 803 \\
\hline 12 & 803 & 1000 & 864 & 72 & 855 & 917 & 973 \\
\hline 24 & 1119 & 1178 & 983 & 843 & 1092 & 1054 & 1178 \\
\hline 48 & 1204 & 1365 & 1025 & 975 & 1143 & 1299 & 2756 \\
\hline 72 & 1114 & 1102 & 1227 & 1237 & 1258 & 1338 & 1408 \\
\hline \multicolumn{8}{|c|}{ Theoretical VFAs concentrations (mg/L)over time } \\
\hline $\begin{array}{l}\text { Time } \\
(\mathrm{hr})\end{array}$ & Manure & SSO & $\begin{array}{c}\text { Manure/SSO } \\
9 / 1\end{array}$ & $\begin{array}{c}\text { Manure/SSO } \\
7 / 3\end{array}$ & $\begin{array}{c}\text { Manure/SSO } \\
5 / 5\end{array}$ & $\begin{array}{c}\text { Manure/SSO } \\
3 / 7\end{array}$ & $\begin{array}{c}\text { Manure/SSO } \\
1 / 9\end{array}$ \\
\hline 0 & 668 & 635 & 665 & 658 & 651 & 645 & 638 \\
\hline 6 & 783 & 790 & 784 & 785 & 786 & 788 & 789 \\
\hline 12 & 803 & 1000 & 823 & 862 & 902 & 941 & 980 \\
\hline 24 & 1119 & 1178 & 1125 & 1137 & 1148 & 1160 & 1172 \\
\hline 48 & 1204 & 1365 & 1220 & 1252 & 1284 & 1317 & 1349 \\
\hline 72 & 1114 & 1102 & 1113 & 1110 & 1108 & 1106 & 1103 \\
\hline
\end{tabular}


Table C. 9. Concentration of soluble and particulate COD over time in hydrolysis/acidification of manure and SSO

\begin{tabular}{|c|c|c|c|c|c|c|c|}
\hline \multicolumn{8}{|c|}{ Soluble COD concentrations ( $\mathrm{mg} / \mathrm{L}$ ) ocer time } \\
\hline $\begin{array}{l}\text { Time } \\
(\mathrm{hr})\end{array}$ & $\begin{array}{c}\text { Manure/SSO } \\
7 / 3\end{array}$ & $\begin{array}{c}\text { Manure/SSO } \\
5 / 5\end{array}$ & $\begin{array}{c}\text { Manure/SSO } \\
3 / 7\end{array}$ & $\begin{array}{c}\text { Manure/SSO } \\
1 / 9\end{array}$ & $\begin{array}{c}\text { Manure/SSO } \\
9 / 1\end{array}$ & $\begin{array}{c}\text { Manure } \\
\text { only }\end{array}$ & $\begin{array}{l}\text { SSO } \\
\text { only }\end{array}$ \\
\hline 0 & 3986 & 4710 & 5624 & 6555 & 3460 & 2860 & 6980 \\
\hline 2 & 4580 & 5159 & 5941 & 6771 & 3860 & 2940 & 6992 \\
\hline 4 & 5336 & 6273 & 6273 & 6887 & 4440 & 3131 & 7009 \\
\hline 6 & 5559 & 6379 & 6379 & 7004 & 4725 & 3659 & 7015 \\
\hline 8 & 5751 & 6469 & 6469 & 7105 & 4927 & 3936 & 7026 \\
\hline 10 & 5974 & 6500 & 6469 & 7105 & 5280 & 4338 & 7040 \\
\hline 12 & 6127 & 6530 & 6509 & 7149 & 5525 & 4790 & 7043 \\
\hline 24 & 6326 & 6547 & 6547 & 7193 & 6268 & 4935 & 7052 \\
\hline 48 & 6543 & 6639 & 6639 & 7295 & 6675 & 5340 & 7061 \\
\hline 72 & 7189 & 7120 & 6934 & 7418 & 7355 & 5650 & 7080 \\
\hline \multicolumn{8}{|c|}{ Particulate COD concentrations (mg/L) over time } \\
\hline $\begin{array}{l}\text { Time } \\
(\mathrm{hr})\end{array}$ & $\begin{array}{c}\text { Manure/SSO } \\
7 / 3\end{array}$ & $\begin{array}{c}\text { Manure/SSO } \\
5 / 5\end{array}$ & $\begin{array}{c}\text { Manure/SSO } \\
3 / 7\end{array}$ & $\begin{array}{c}\text { Manure/SSO } \\
1 / 9\end{array}$ & $\begin{array}{c}\text { Manure/SSO } \\
9 / 1\end{array}$ & $\begin{array}{l}\text { Manure } \\
\text { only }\end{array}$ & $\begin{array}{l}\text { SSO } \\
\text { only }\end{array}$ \\
\hline 0 & 23605 & 22489 & 20787 & 19466 & 25746 & 26535 & 18565 \\
\hline 2 & 23298 & 21940 & 20539 & 19250 & 25160 & 26155 & 18446 \\
\hline 4 & 22780 & 21426 & 20176 & 19085 & 24726 & 25940 & 18279 \\
\hline 6 & 22489 & 21270 & 20099 & 18957 & 24141 & 25766 & 18165 \\
\hline 8 & 22385 & 21130 & 19887 & 18895 & 23749 & 25665 & 18050 \\
\hline 10 & 22205 & 21045 & 19830 & 18796 & 23470 & 25560 & 17955 \\
\hline 12 & 21935 & 20989 & 19695 & 18752 & 23255 & 25340 & 17915 \\
\hline 24 & 21325 & 20542 & 19280 & 18508 & 22410 & 24795 & 17755 \\
\hline 48 & 20895 & 20255 & 18965 & 18430 & 21978 & 24535 & 17646 \\
\hline 72 & 20809 & 20195 & 18925 & 18405 & 21860 & 24400 & 17614 \\
\hline
\end{tabular}




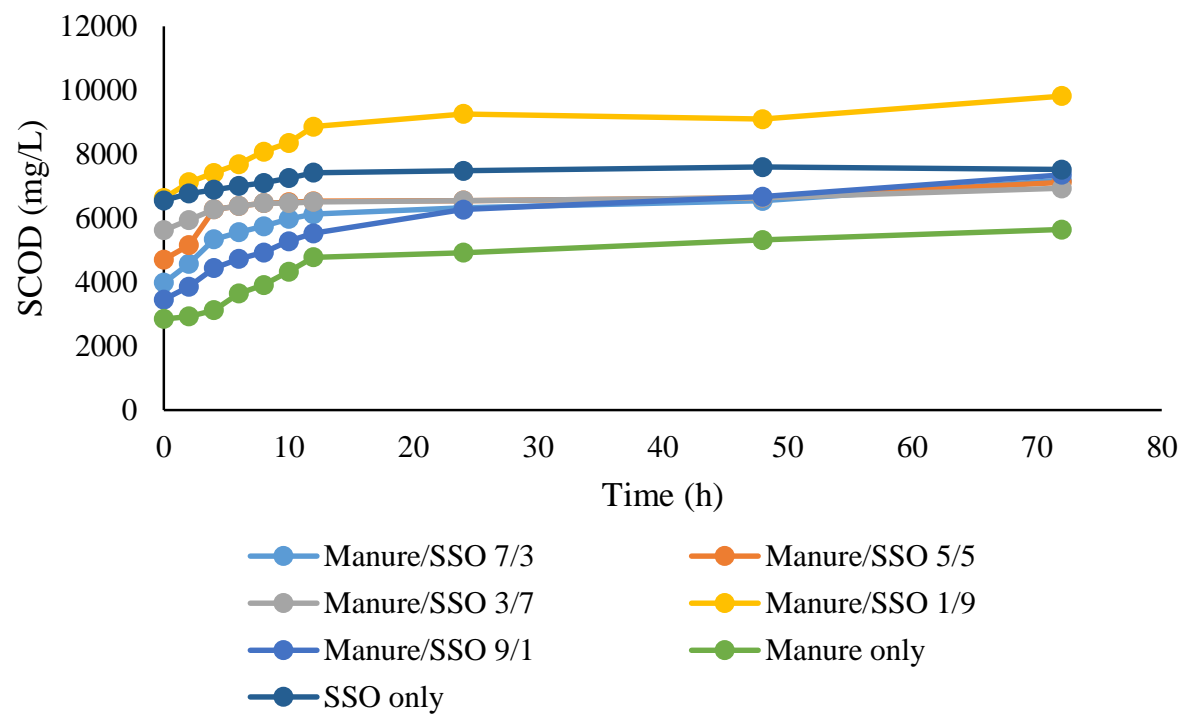

Figure C.2. Concentration of soluble COD over time at different mixing ratios 


\section{Analytical results for AnCoD of TWAS, manure and SSO}

Table D. 1. Characteristics of raw feedstocks in AnCoD of TWAS, Manure, and SSO

\begin{tabular}{cccccccccc}
\hline \multirow{2}{*}{ Parameters } & \multirow{2}{*}{ Units } & \multicolumn{2}{c}{ TWAS } & \multicolumn{2}{c}{ Manure } & \multicolumn{2}{c}{ SSO } & \multicolumn{2}{c}{ Seed } \\
& & MEAN & SD & MEAN & SD & MEAN & SD & MEAN & SD \\
\hline TCOD & $\mathrm{mg} / \mathrm{L}$ & 39977 & 1199 & 100126 & 3004 & 108560 & 3257 & 17105 & 513 \\
SCOD & $\mathrm{mg} / \mathrm{L}$ & 1354 & 41 & 42116 & 1263 & 40855 & 1225.65 & 761 & 23 \\
TSS & $\mathrm{mg} / \mathrm{L}$ & 31445 & 943 & 52380 & 1571 & 62136 & 1864 & 16055 & 482 \\
VSS & $\mathrm{mg} / \mathrm{L}$ & 26480 & 794 & 45420 & 1363 & 46990 & 1410 & 12781 & 383 \\
TS & $\mathrm{mg} / \mathrm{L}$ & 38944 & 1168 & 67785 & 2034 & 66985 & 2010 & 17125 & 514 \\
VS & $\mathrm{mg} / \mathrm{L}$ & 35155 & 1055 & 55590 & 1668 & 49585 & 1488 & 14380 & 431 \\
Ammonia & $\mathrm{mg} / \mathrm{L}$ & 218 & 7 & 18 & 1 & 1289 & 39 & 800 & 24 \\
pH & & 6.5 & 0.2 & 6.7 & 0.2 & 5.7 & 0.17 & 7.2 & 0.2 \\
Alkalinity & $\mathrm{mg} \mathrm{CaCO3/L}$ & 1888 & 57 & 5224 & 157 & 6227 & 187 & 4215 & 126 \\
TN & $\mathrm{mg} / \mathrm{L}$ & 2754 & 83 & 2110 & 63 & 3970 & 119 & 1975 & 59 \\
TSN & $\mathrm{mg} / \mathrm{L}$ & 376 & 11 & 125 & 4 & 965 & 29 & 716 & 21 \\
Total Carbs & $\mathrm{mg} / \mathrm{L}$ & 1524 & 46 & 27842 & 835 & 14126 & 424 & 839 & 25 \\
Total & $\mathrm{mg} / \mathrm{L}$ & 3892 & 117 & 5762 & 173 & 2420 & 73 & 1917 & 58 \\
Proteins & & & & & & & & \\
Total Lipids & $\mathrm{mg} / \mathrm{L}$ & 396 & 12 & 1356 & 41 & 1494 & 45 & 191 & 6 \\
\hline
\end{tabular}


Table D. 2. Average $\mathrm{CH}_{4}$ measurements $(\mathrm{mL})$ in AnCoD of TWAS/Mnaure/SSO (T: TWAS, M: Manure)

\begin{tabular}{|c|c|c|c|c|c|c|c|c|c|c|c|c|}
\hline Time (day) & TWAS & Manure & SSO & $\begin{array}{c}\text { T/M/SSO } \\
8 / 1 / 1 \\
\end{array}$ & $\begin{array}{c}\mathrm{T} / \mathrm{M} / \mathrm{SSO} \\
1 / 8 / 1 \\
\end{array}$ & $\begin{array}{c}\mathrm{T} / \mathrm{M} / \mathrm{SSO} \\
1 / 1 / 8 \\
\end{array}$ & $\begin{array}{c}\mathrm{T} / \mathrm{M} / \mathrm{SSO} \\
0.5 / 0.25 / 0.25 \\
\end{array}$ & $\begin{array}{c}\mathrm{T} / \mathrm{M} / \mathrm{SSO} \\
0.25 / 0.5 / 0.25\end{array}$ & $\begin{array}{c}\mathrm{T} / \mathrm{M} / \mathrm{SSO} \\
0.25 / 0.25 / 0.5 \\
\end{array}$ & $\begin{array}{c}\mathrm{T} / \mathrm{M} / \mathrm{SSO} \\
0.4 / 0.4 / 0.2 \\
\end{array}$ & $\begin{array}{c}\mathrm{T} / \mathrm{M} / \mathrm{SSO} \\
0.2 / 0.4 / 0.4\end{array}$ & $\begin{array}{c}\mathrm{T} / \mathrm{M} / \mathrm{SSO} \\
0.4 / 0.2 / 0.4 \\
\end{array}$ \\
\hline 0 & 0 & 0 & 0 & 0 & 0 & 0 & 0 & 0 & 0 & 0 & 0 & 0 \\
\hline 1 & 5 & 47 & 6 & 12 & 22 & 21 & 9 & 52 & 25 & 7 & 37 & 8 \\
\hline 3 & 21 & 73 & 48 & 32 & 71 & 68 & 55 & 95 & 60 & 54 & 64 & 42 \\
\hline 4 & 39 & 101 & 63 & 34 & 104 & 97 & 77 & 118 & 79 & 71 & 97 & 79 \\
\hline 5 & 52 & 101 & 98 & 57 & 143 & 136 & 105 & 124 & 115 & 104 & 118 & 113 \\
\hline 6 & 34 & 116 & 113 & 67 & 106 & 114 & 94 & 141 & 112 & 102 & 123 & 104 \\
\hline 7 & 33 & 139 & 112 & 69 & 105 & 108 & 97 & 98 & 116 & 106 & 125 & 106 \\
\hline 8 & 19 & 79 & 120 & 71 & 86 & 81 & 92 & 102 & 120 & 110 & 133 & 103 \\
\hline 10 & 25 & 77 & 128 & 76 & 83 & 85 & 81 & 95 & 107 & 97 & 106 & 95 \\
\hline 12 & 23 & 70 & 121 & 71 & 79 & 81 & 77 & 96 & 101 & 92 & 101 & 90 \\
\hline 14 & 19 & 91 & 90 & 54 & 65 & 66 & 63 & 63 & 81 & 74 & 84 & 73 \\
\hline 16 & 17 & 56 & 60 & 35 & 60 & 61 & 61 & 65 & 72 & 65 & 81 & 64 \\
\hline 19 & 21 & 58 & 54 & 32 & 61 & 63 & 52 & 51 & 59 & 53 & 61 & 55 \\
\hline 22 & 16 & 40 & 56 & 33 & 52 & 53 & 49 & 47 & 58 & 53 & 62 & 53 \\
\hline 25 & 13 & 40 & 47 & 28 & 42 & 43 & 38 & 36 & 46 & 41 & 47 & 42 \\
\hline 28 & 9 & 32 & 33 & 19 & 31 & 32 & 30 & 56 & 36 & 33 & 39 & 32 \\
\hline 31 & 29 & 24 & 78 & 46 & 78 & 80 & 58 & 29 & 67 & 61 & 62 & 66 \\
\hline 34 & 12 & 22 & 28 & 16 & 33 & 34 & 26 & 27 & 29 & 27 & 30 & 28 \\
\hline 37 & 10 & 19 & 30 & 10 & 29 & 30 & 24 & 24 & 29 & 26 & 20 & 27 \\
\hline 40 & 9 & 15 & 30 & 9 & 26 & 27 & 22 & 18 & 27 & 24 & 16 & 25 \\
\hline 43 & 5 & 11 & 26 & 6 & 17 & 17 & 16 & 9 & 21 & 19 & 10 & 15 \\
\hline 46 & 3 & 3 & 18 & 4 & 11 & 11 & 12 & 6 & 16 & 15 & 4 & 11 \\
\hline 49 & 2 & 3 & 9 & 2 & 6 & 6 & 6 & 3 & 8 & 7 & 3 & 6 \\
\hline 52 & 1 & 0 & 5 & 1 & 3 & 4 & 4 & 1 & 5 & 4 & 1 & 3 \\
\hline 58 & 0 & 0 & 2 & 0 & 0 & 0 & 1 & 1 & 1 & 1 & 1 & 1 \\
\hline
\end{tabular}

* M: Manure 
Table D. 3. Cumulative $\mathrm{CH}_{4}$ production (mL) in AnCoD of TWAS/Mnaure/SSO (T: TWAS, M:Manure)

\begin{tabular}{|c|c|c|c|c|c|c|c|c|c|c|c|c|}
\hline $\begin{array}{l}\text { Time } \\
\text { (day) }\end{array}$ & TWAS & Manure & SSO & $\begin{array}{c}\mathrm{T} / \mathrm{M} / \mathrm{SSO} \\
8 / 1 / 1 \\
\end{array}$ & $\begin{array}{c}\mathrm{T} / \mathrm{M} / \mathrm{SSO} \\
1 / 8 / 1 \\
\end{array}$ & $\begin{array}{c}\mathrm{T} / \mathrm{M} / \mathrm{SSO} \\
1 / 1 / 8\end{array}$ & $\begin{array}{c}\mathrm{T} / \mathrm{M} / \mathrm{SSO} \\
0.5 / 0.25 / 0.25\end{array}$ & $\begin{array}{c}\mathrm{T} / \mathrm{M} / \mathrm{SSO} \\
0.25 / 0.5 / 0.25\end{array}$ & $\begin{array}{c}\mathrm{T} / \mathrm{M} / \mathrm{SSO} \\
0.25 / 0.25 / 0.5\end{array}$ & $\begin{array}{c}\mathrm{T} / \mathrm{M} / \mathrm{SSO} \\
0.4 / 0.4 / 0.2\end{array}$ & $\begin{array}{c}\mathrm{T} / \mathrm{M} / \mathrm{SSO} \\
0.2 / 0.4 / 0.4\end{array}$ & $\begin{array}{c}\mathrm{T} / \mathrm{M} / \mathrm{SSO} \\
0.4 / 0.2 / 0.4\end{array}$ \\
\hline 0 & 0 & 0 & $\overline{0}$ & 0 & 0 & 0 & 0 & 0 & 0 & 0 & 0 & 0 \\
\hline 1 & 5 & 48 & 6 & 12 & 18 & 24 & 9 & 52 & 25 & 8 & 35 & 8 \\
\hline 3 & 27 & 120 & 54 & 44 & 89 & 93 & 64 & 147 & 85 & 62 & 99 & 50 \\
\hline 4 & 65 & 222 & 116 & 78 & 194 & 189 & 141 & 265 & 164 & 132 & 196 & 129 \\
\hline 5 & 117 & 323 & 215 & 135 & 336 & 325 & 246 & 389 & 278 & 237 & 314 & 242 \\
\hline 6 & 152 & 440 & 327 & 202 & 442 & 439 & 340 & 530 & 391 & 339 & 437 & 346 \\
\hline 7 & 185 & 579 & 440 & 271 & 548 & 547 & 437 & 628 & 507 & 445 & 562 & 452 \\
\hline 8 & 203 & 658 & 559 & 342 & 633 & 628 & 529 & 730 & 627 & 554 & 696 & 555 \\
\hline 10 & 228 & 735 & 687 & 417 & 717 & 713 & 610 & 825 & 734 & 652 & 802 & 650 \\
\hline 12 & 251 & 805 & 808 & 489 & 796 & 793 & 687 & 921 & 836 & 744 & 903 & 741 \\
\hline 14 & 270 & 895 & 898 & 542 & 860 & 859 & 750 & 984 & 917 & 818 & 986 & 813 \\
\hline 16 & 287 & 951 & 958 & 577 & 920 & 921 & 811 & 1049 & 989 & 883 & 1068 & 877 \\
\hline 19 & 307 & 1010 & 1012 & 609 & 981 & 983 & 862 & 1101 & 1047 & 937 & 1129 & 932 \\
\hline 22 & 324 & 1049 & 1067 & 642 & 1034 & 1037 & 911 & 1148 & 1105 & 989 & 1191 & 985 \\
\hline 25 & 337 & 1089 & 1115 & 670 & 1075 & 1079 & 948 & 1184 & 1151 & 1031 & 1238 & 1027 \\
\hline 28 & 346 & 1121 & 1148 & 690 & 1107 & 1111 & 978 & 1239 & 1187 & 1063 & 1277 & 1059 \\
\hline 31 & 375 & 1146 & 1225 & 736 & 1185 & 1191 & 1037 & 1268 & 1254 & 1125 & 1339 & 1125 \\
\hline 34 & 387 & 1167 & 1253 & 752 & 1218 & 1225 & 1063 & 1295 & 1283 & 1151 & 1369 & 1153 \\
\hline 37 & 397 & 1186 & 1284 & 762 & 1247 & 1255 & 1087 & 1319 & 1312 & 1177 & 1389 & 1180 \\
\hline 40 & 406 & 1201 & 1314 & 771 & 1273 & 1281 & 1109 & 1336 & 1338 & 1202 & 1405 & 1205 \\
\hline 43 & 411 & 1212 & 1339 & 777 & 1290 & 1298 & 1125 & 1345 & 1360 & 1221 & 1415 & 1220 \\
\hline 46 & 414 & 1215 & 1357 & 781 & 1301 & 1310 & 1137 & 1351 & 1376 & 1236 & 1419 & 1232 \\
\hline 49 & 416 & 1218 & 1366 & 783 & 1307 & 1316 & 1142 & 1354 & 1384 & 1243 & 1422 & 1237 \\
\hline 52 & 417 & 1218 & 1371 & 784 & 1310 & 1320 & 1146 & 1355 & 1388 & 1247 & 1423 & 1241 \\
\hline 58 & 417 & 1218 & 1373 & 784 & 1311 & 1320 & 1146 & 1355 & 1390 & 1248 & 1424 & 1241 \\
\hline
\end{tabular}

* M: Manure 
Table D. 4. Cumulative $\mathrm{CH}_{4}$ production per mass of COD added in AnCoD of TWAS/Mnaure/SSO (T: TWAS, M: Manure)

\begin{tabular}{|c|c|c|c|c|c|c|c|c|c|c|c|c|}
\hline $\begin{array}{l}\text { Time } \\
\text { (day) }\end{array}$ & TWAS & Manure & SSO & $\begin{array}{c}\mathrm{T} / \mathrm{M} / \mathrm{SSO} \\
8 / 1 / 1\end{array}$ & $\begin{array}{c}\mathrm{T} / \mathrm{M} / \mathrm{SSO} \\
1 / 8 / 1\end{array}$ & $\begin{array}{c}\mathrm{T} / \mathrm{M} / \mathrm{SSO} \\
1 / 1 / 8\end{array}$ & $\begin{array}{c}\mathrm{T} / \mathrm{M} / \mathrm{SSO} \\
0.5 / 0.25 / 0.25\end{array}$ & $\begin{array}{c}\mathrm{T} / \mathrm{M} / \mathrm{SSO} \\
0.25 / 0.5 / 0.25\end{array}$ & $\begin{array}{c}\mathrm{T} / \mathrm{M} / \mathrm{SSO} \\
0.25 / 0.25 / 0.5\end{array}$ & $\begin{array}{c}\mathrm{T} / \mathrm{M} / \mathrm{SSO} \\
0.4 / 0.4 / 0.2\end{array}$ & $\begin{array}{c}\mathrm{T} / \mathrm{M} / \mathrm{SSO} \\
0.2 / 0.4 / 0.4\end{array}$ & $\begin{array}{c}\mathrm{T} / \mathrm{M} / \mathrm{SSO} \\
0.4 / 0.2 / 0.4\end{array}$ \\
\hline 0 & 0 & 0 & 0 & 0 & 0 & 0 & 0 & 0 & 0 & 0 & 0 & 0 \\
\hline 1 & 2 & 12 & 1 & 4 & 4 & 6 & 2 & 13 & 6 & 2 & 9 & 2 \\
\hline 3 & 9 & 30 & 13 & 13 & 22 & 23 & 17 & 37 & 21 & 16 & 25 & 13 \\
\hline 4 & 21 & 54 & 28 & 23 & 48 & 46 & 37 & 67 & 41 & 34 & 49 & 33 \\
\hline 5 & 38 & 79 & 52 & 39 & 83 & 80 & 65 & 98 & 70 & 62 & 79 & 63 \\
\hline 6 & 49 & 108 & 79 & 59 & 110 & 108 & 90 & 134 & 98 & 88 & 109 & 89 \\
\hline 7 & 59 & 142 & 106 & 79 & 136 & 134 & 116 & 159 & 128 & 116 & 141 & 117 \\
\hline 8 & 65 & 162 & 135 & 99 & 157 & 154 & 140 & 185 & 158 & 144 & 174 & 144 \\
\hline 10 & 73 & 180 & 166 & 121 & 178 & 175 & 162 & 209 & 185 & 169 & 201 & 168 \\
\hline 12 & 80 & 198 & 195 & 142 & 198 & 195 & 182 & 233 & 210 & 193 & 226 & 191 \\
\hline 14 & 87 & 220 & 217 & 157 & 214 & 211 & 199 & 249 & 231 & 213 & 247 & 210 \\
\hline 16 & 92 & 234 & 231 & 168 & 228 & 226 & 215 & 265 & 249 & 230 & 267 & 227 \\
\hline 19 & 99 & 248 & 244 & 177 & 244 & 241 & 228 & 278 & 263 & 243 & 283 & 241 \\
\hline 22 & 104 & 258 & 258 & 186 & 257 & 254 & 241 & 290 & 278 & 257 & 298 & 255 \\
\hline 25 & 108 & 267 & 269 & 195 & 267 & 265 & 251 & 299 & 290 & 268 & 310 & 266 \\
\hline 28 & 111 & 275 & 277 & 200 & 275 & 272 & 259 & 314 & 299 & 276 & 320 & 274 \\
\hline 31 & 120 & 281 & 296 & 214 & 294 & 292 & 275 & 321 & 315 & 292 & 335 & 291 \\
\hline 34 & 124 & 287 & 303 & 218 & 302 & 300 & 282 & 328 & 323 & 299 & 343 & 298 \\
\hline 37 & 127 & 291 & 310 & 221 & 310 & 308 & 288 & 334 & 330 & 306 & 348 & 305 \\
\hline 40 & 130 & 295 & 317 & 224 & 316 & 314 & 294 & 338 & 337 & 312 & 352 & 312 \\
\hline 43 & 132 & 298 & 324 & 225 & 320 & 318 & 298 & 340 & 342 & 317 & 354 & 315 \\
\hline 46 & 133 & 298 & 328 & 227 & 323 & 321 & 301 & 342 & 346 & 321 & 355 & 318 \\
\hline 49 & 133 & 299 & 330 & 227 & 324 & 323 & 303 & 343 & 348 & 323 & 356 & 320 \\
\hline 52 & 134 & 299 & 331 & 228 & 325 & 324 & 303 & 343 & 349 & 324 & 356 & 321 \\
\hline 58 & 134 & 299 & 332 & 228 & 325 & 324 & 304 & 343 & 350 & 324 & 356 & 321 \\
\hline
\end{tabular}

*T: TWAS

* M: Manure 
Table D. 5. Cumulative $\mathrm{CH}_{4}$ production per mass of VSS added in AnCoD of TWAS/Mnaure/SSO (T: TWAS, M: Manure)

\begin{tabular}{|c|c|c|c|c|c|c|c|c|c|c|c|c|}
\hline $\begin{array}{l}\text { Time } \\
\text { (day) }\end{array}$ & $\begin{array}{c}\text { TWAS } \\
\text { Only }\end{array}$ & $\begin{array}{c}\text { Manure } \\
\text { Only }\end{array}$ & $\begin{array}{l}\text { SSO } \\
\text { only } \\
\end{array}$ & $\begin{array}{c}\text { T/M/SSO } \\
8 / 1 / 1 \\
\end{array}$ & $\begin{array}{c}\text { T/M/SSO } \\
1 / 8 / 1 \\
\end{array}$ & $\begin{array}{c}\mathrm{T} / \mathrm{M} / \mathrm{SSO} \\
1 / 1 / 8 \\
\end{array}$ & $\begin{array}{c}\mathrm{T} / \mathrm{M} / \mathrm{SSO} \\
0.5 / 0.25 / 0.25\end{array}$ & $\begin{array}{c}\mathrm{T} / \mathrm{M} / \mathrm{SSO} \\
0.25 / 0.5 / 0.25\end{array}$ & $\begin{array}{c}\mathrm{T} / \mathrm{M} / \mathrm{SSO} \\
0.25 / 0.25 / 0.5 \\
\end{array}$ & $\begin{array}{c}\mathrm{T} / \mathrm{M} / \mathrm{SSO} \\
0.4 / 0.4 / 0.2 \\
\end{array}$ & $\begin{array}{c}\text { T/M/SSO } \\
0.2 / 0.4 / 0.4 \\
\end{array}$ & $\begin{array}{c}\mathrm{T} / \mathrm{M} / \mathrm{SSO} \\
0.4 / 0.2 / 0.4 \\
\end{array}$ \\
\hline 0 & 0 & 0 & 0 & 0 & 0 & 0 & 0 & 0 & 0 & 0 & 0 & 0 \\
\hline 1 & 3 & 26 & 27 & 6 & 10 & 13 & 5 & 28 & 14 & 4 & 24 & 4 \\
\hline 3 & 13 & 65 & 67 & 22 & 48 & 51 & 34 & 79 & 46 & 33 & 68 & 27 \\
\hline 4 & 32 & 120 & 124 & 39 & 104 & 105 & 74 & 142 & 89 & 70 & 135 & 69 \\
\hline 5 & 57 & 175 & 181 & 68 & 181 & 180 & 129 & 209 & 151 & 125 & 217 & 129 \\
\hline 6 & 73 & 238 & 245 & 102 & 239 & 242 & 179 & 285 & 212 & 179 & 302 & 185 \\
\hline 7 & 89 & 313 & 323 & 137 & 296 & 302 & 230 & 337 & 275 & 235 & 388 & 241 \\
\hline 8 & 98 & 356 & 367 & 172 & 342 & 347 & 278 & 392 & 340 & 294 & 480 & 296 \\
\hline 10 & 110 & 398 & 410 & 210 & 387 & 394 & 321 & 443 & 398 & 345 & 553 & 347 \\
\hline 12 & 122 & 436 & 449 & 246 & 429 & 438 & 361 & 494 & 453 & 394 & 623 & 395 \\
\hline 14 & 131 & 485 & 500 & 273 & 464 & 475 & 395 & 528 & 497 & 433 & 681 & 434 \\
\hline 16 & 139 & 515 & 531 & 291 & 496 & 508 & 426 & 563 & 536 & 468 & 737 & 468 \\
\hline 19 & 149 & 546 & 564 & 307 & 530 & 543 & 454 & 591 & 567 & 496 & 779 & 498 \\
\hline 22 & 157 & 568 & 586 & 324 & 558 & 572 & 479 & 616 & 599 & 524 & 822 & 526 \\
\hline 25 & 163 & 589 & 608 & 338 & 580 & 596 & 499 & 636 & 623 & 546 & 854 & 548 \\
\hline 28 & 168 & 607 & 626 & 348 & 597 & 614 & 515 & 665 & 643 & 563 & 881 & 566 \\
\hline 31 & 182 & 620 & 640 & 371 & 639 & 658 & 545 & 681 & 679 & 596 & 924 & 601 \\
\hline 34 & 187 & 632 & 652 & 379 & 657 & 676 & 559 & 695 & 695 & 610 & 944 & 616 \\
\hline 37 & 192 & 642 & 662 & 384 & 673 & 693 & 572 & 708 & 711 & 623 & 958 & 630 \\
\hline 40 & 196 & 650 & 671 & 389 & 687 & 708 & 583 & 718 & 725 & 636 & 969 & 643 \\
\hline 43 & 199 & 656 & 677 & 392 & 696 & 717 & 592 & 722 & 737 & 647 & 976 & 651 \\
\hline 46 & 200 & 658 & 678 & 394 & 702 & 723 & 598 & 726 & 745 & 654 & 979 & 657 \\
\hline 49 & 201 & 659 & 680 & 395 & 705 & 727 & 601 & 727 & 750 & 658 & 981 & 660 \\
\hline 52 & 202 & 659 & 680 & 395 & 707 & 729 & 603 & 727 & 752 & 661 & 982 & 662 \\
\hline 58 & 202 & 659 & 680 & 395 & 707 & 729 & 603 & 728 & 753 & 661 & 982 & 663 \\
\hline
\end{tabular}

* M: Manure 
Table D. 6. Cumulative $\mathrm{CH}_{4}$ production per volume of substrate added in AnCoD of TWAS/Mnaure/SSO (T: TWAS, M: Manure)

\begin{tabular}{|c|c|c|c|c|c|c|c|c|c|c|c|c|}
\hline $\begin{array}{l}\text { Time } \\
\text { (day) }\end{array}$ & $\begin{array}{l}\text { TWAS } \\
\text { Only }\end{array}$ & $\begin{array}{l}\text { Manure } \\
\text { Only }\end{array}$ & $\begin{array}{l}\text { SSO } \\
\text { only }\end{array}$ & $\begin{array}{c}\text { T/M/SSO } \\
8 / 1 / 1\end{array}$ & $\begin{array}{c}\mathrm{T} / \mathrm{M} / \mathrm{SSO} \\
1 / 8 / 1\end{array}$ & $\begin{array}{c}\mathrm{T} / \mathrm{M} / \mathrm{SSO} \\
1 / 1 / 8\end{array}$ & $\begin{array}{c}\mathrm{T} / \mathrm{M} / \mathrm{SSO} \\
0.5 / 0.25 / 0.25\end{array}$ & $\begin{array}{c}\mathrm{T} / \mathrm{M} / \mathrm{SSO} \\
0.25 / 0.5 / 0.25\end{array}$ & $\begin{array}{c}\mathrm{T} / \mathrm{M} / \mathrm{SSO} \\
0.25 / 0.25 / 0.5\end{array}$ & $\begin{array}{c}\mathrm{T} / \mathrm{M} / \mathrm{SSO} \\
0.4 / 0.4 / 0.2\end{array}$ & $\begin{array}{c}\mathrm{T} / \mathrm{M} / \mathrm{SSO} \\
0.2 / 0.4 / 0.4\end{array}$ & $\begin{array}{c}\text { T/M/SSO } \\
0.4 / 0.2 / 0.4\end{array}$ \\
\hline 0 & 0 & 0 & 0 & 0 & 0 & 0 & 0 & 0 & 0 & 0 & 0 & 0 \\
\hline 1 & 0 & 1 & 0 & 0 & 0 & 1 & 0 & 1 & 1 & 0 & 1 & 0 \\
\hline 3 & 0 & 3 & 1 & 1 & 2 & 2 & 1 & 3 & 2 & 1 & 2 & 1 \\
\hline 4 & 1 & 5 & 3 & 1 & 5 & 5 & 1 & 6 & 4 & 3 & 4 & 3 \\
\hline 5 & 2 & 8 & 6 & 2 & 8 & 8 & 2 & 9 & 6 & 5 & 7 & 5 \\
\hline 6 & 2 & 11 & 9 & 3 & 10 & 11 & 3 & 12 & 9 & 7 & 10 & 7 \\
\hline 7 & 2 & 14 & 12 & 4 & 13 & 14 & 4 & 14 & 11 & 9 & 13 & 9 \\
\hline 8 & 3 & 16 & 15 & 5 & 15 & 16 & 5 & 16 & 14 & 11 & 16 & 11 \\
\hline 10 & 3 & 18 & 18 & 6 & 17 & 18 & 6 & 18 & 16 & 13 & 18 & 13 \\
\hline 12 & 3 & 20 & 21 & 7 & 19 & 20 & 7 & 20 & 19 & 15 & 21 & 15 \\
\hline 14 & 3 & 22 & 24 & 8 & 20 & 21 & 8 & 22 & 21 & 17 & 23 & 17 \\
\hline 16 & 4 & 23 & 25 & 9 & 22 & 23 & 8 & 23 & 22 & 18 & 24 & 18 \\
\hline 19 & 4 & 25 & 27 & 9 & 23 & 24 & 9 & 24 & 24 & 19 & 26 & 19 \\
\hline 22 & 4 & 26 & 28 & 10 & 24 & 26 & 9 & 25 & 25 & 20 & 27 & 20 \\
\hline 25 & 4 & 27 & 29 & 10 & 25 & 27 & 10 & 26 & 26 & 21 & 28 & 21 \\
\hline 28 & 4 & 28 & 30 & 11 & 26 & 27 & 10 & 27 & 27 & 21 & 29 & 22 \\
\hline 31 & 5 & 28 & 32 & 11 & 28 & 29 & 10 & 28 & 28 & 23 & 31 & 23 \\
\hline 34 & 5 & 29 & 33 & 12 & 29 & 30 & 11 & 29 & 29 & 23 & 31 & 24 \\
\hline 37 & 5 & 29 & 34 & 12 & 29 & 31 & 11 & 29 & 29 & 24 & 32 & 24 \\
\hline 40 & 5 & 30 & 34 & 12 & 30 & 32 & 11 & 29 & 30 & 24 & 32 & 25 \\
\hline 43 & 5 & 30 & 35 & 12 & 30 & 32 & 11 & 30 & 31 & 25 & 32 & 25 \\
\hline 46 & 5 & 30 & 36 & 12 & 31 & 32 & 11 & 30 & 31 & 25 & 32 & 25 \\
\hline 49 & 5 & 30 & 36 & 12 & 31 & 33 & 11 & 30 & 31 & 25 & 33 & 25 \\
\hline 52 & 5 & 30 & 36 & 12 & 31 & 33 & 12 & 30 & 31 & 25 & 33 & 25 \\
\hline 58 & 5 & 30 & 36 & 12 & 31 & 33 & 12 & 30 & 31 & 25 & 33 & 25 \\
\hline
\end{tabular}

*T: TWAS

* M: Manure 


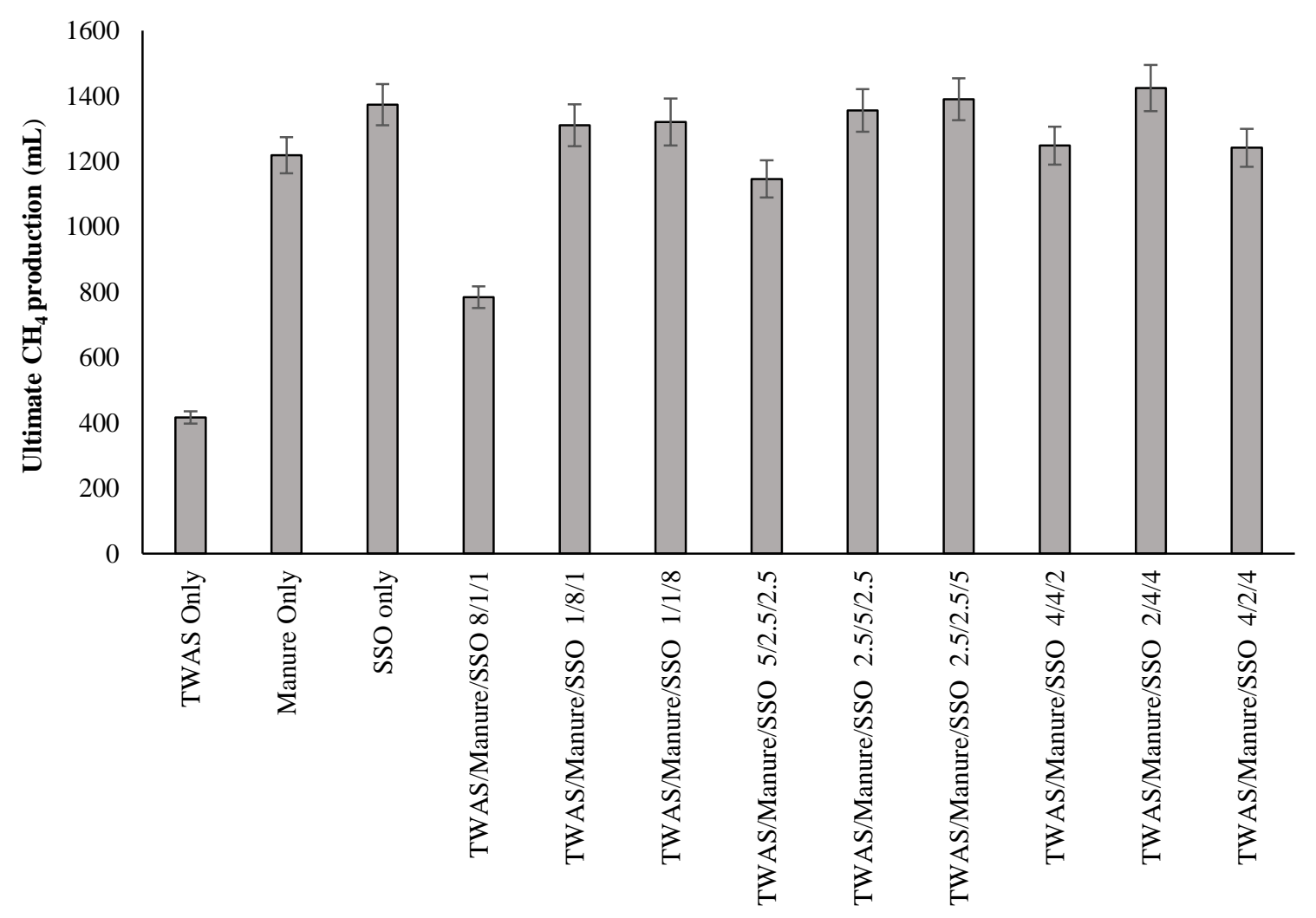

Mixing ratios

Figure D. 1. Ultimate $\mathrm{CH} 4$ production in AnCoD of TWAS/Manure/SSO 
Table D. 7. Characteristics of raw feedstocks for hydrolysis/acidification in AnCoD of TWAS/Manure/SSO

\begin{tabular}{cccccccccc}
\hline \multirow{2}{*}{ Parameters } & \multirow{2}{*}{ Units } & \multicolumn{2}{c}{ TWAS } & \multicolumn{2}{c}{ Manure } & \multicolumn{2}{c}{ SSO } & \multicolumn{2}{c}{ Inoculum } \\
& & MEAN & SD & MEAN & SD & MEAN & SD & MEAN & SD \\
\hline TCOD & $\mathrm{mg} / \mathrm{L}$ & 36875 & 2853 & 100240 & 4014 & 102180 & 4120 & 16854 & 389 \\
SCOD & $\mathrm{mg} / \mathrm{L}$ & 1931 & 26 & 10145 & 405 & 37350 & 1861 & 786 & 22 \\
TSS & $\mathrm{mg} / \mathrm{L}$ & 28934 & 1006 & 54100 & 2164 & 49510 & 2093 & 15022 & 326 \\
VSS & $\mathrm{mg} / \mathrm{L}$ & 22983 & 2216 & 45216 & 1289 & 44130 & 1743 & 10175 & 298 \\
TS & $\mathrm{mg} / \mathrm{L}$ & 35705 & 6859 & 67680 & 2707 & 76878 & 3460 & 20650 & 425 \\
VS & $\mathrm{mg} / \mathrm{L}$ & 31719 & 27 & 57830 & 2313 & 62948 & 2833 & 12988 & 396 \\
Ammonia & $\mathrm{mg} / \mathrm{L}$ & 235 & 30 & 22.3 & 1 & 1326 & 60 & 1478 & 32 \\
pH & - & 6 & 0.1 & 6.8 & 0.2 & 5.8 & 0.2 & 7.1 & 0.2 \\
Alkalinity & $\mathrm{mg} \mathrm{CaCO} / \mathrm{L}$ & 1797 & 148 & 6984 & 279 & 6415 & 289 & 3867 & 135 \\
TN & $\mathrm{mg} / \mathrm{L}$ & 2668 & 400 & 1690 & 68 & 4086 & 184 & 1983 & 69 \\
TSN & $\mathrm{mg} / \mathrm{L}$ & 386 & 64 & 119 & 4 & 1096 & 49 & 679 & 24 \\
Total Carbs & $\mathrm{mg} / \mathrm{L}$ & 849 & 112 & 25968 & 1039 & 13575 & 611 & 548 & 19 \\
Total Proteins & $\mathrm{mg} / \mathrm{L}$ & 2547 & 298 & 5126 & 205 & 2087 & 94 & 1596 & 56 \\
Total Lipids & $\mathrm{mg} / \mathrm{L}$ & 266 & 27 & 1652 & 66 & 1395 & 63 & 153 & 5 \\
\hline
\end{tabular}


Table D. 8. Measured and theoretical VFAs concentrations (mg/L) over time in hydrolysis/acidification of TWAS/Manure/SSO

\begin{tabular}{|c|c|c|c|c|c|c|c|c|c|c|c|c|}
\hline \multicolumn{13}{|c|}{ Measured VFAs concentrations (mg/L) over time } \\
\hline & TWAS & Manure & $\mathrm{SSO}$ & T/M/SSO 8/1/1 & $\begin{array}{c}\mathrm{T} / \mathrm{M} / \mathrm{SSO} \\
1 / 8 / 1\end{array}$ & $\begin{array}{c}\mathrm{T} / \mathrm{M} / \mathrm{SSO} \\
1 / 1 / 8\end{array}$ & $\begin{array}{l}\mathrm{T} / \mathrm{M} / \mathrm{SSO} \\
5 / 2.5 / 2.5\end{array}$ & $\begin{array}{l}\mathrm{T} / \mathrm{M} / \mathrm{SSO} \\
2.5 / 5 / 2.5\end{array}$ & $\begin{array}{l}\mathrm{T} / \mathrm{M} / \mathrm{SSO} \\
2.5 / 2.5 / 5\end{array}$ & $\begin{array}{c}\mathrm{T} / \mathrm{M} / \mathrm{SSO} \\
4 / 4 / 2\end{array}$ & $\begin{array}{c}\mathrm{T} / \mathrm{M} / \mathrm{SSO} \\
2 / 4 / 4\end{array}$ & $\begin{array}{c}\mathrm{T} / \mathrm{M} / \mathrm{SSO} \\
4 / 2 / 4\end{array}$ \\
\hline $\begin{array}{c}\text { Time } \\
\text { (hr) }\end{array}$ & 1 & 2 & 3 & 4 & 5 & 6 & 7 & 8 & 9 & 10 & 11 & 12 \\
\hline 0 & 428 & 1096 & 1538 & 673 & 1353 & 1660 & 977 & 1258 & 1357 & 1055 & 1402 & 1127 \\
\hline 6 & 340 & 1286 & 1914 & 657 & 1580 & 2032 & 1086 & 1459 & 1609 & 1188 & 1658 & 1297 \\
\hline 12 & 441 & 1319 & 2423 & 807 & 1690 & 2537 & 1295 & 1664 & 1949 & 1367 & 1949 & 1578 \\
\hline 24 & 672 & 1837 & 2854 & 1117 & 2296 & 3041 & 1690 & 2178 & 2424 & 1811 & 2474 & 1991 \\
\hline 48 & 744 & 1976 & 3309 & 1247 & 2503 & 3503 & 1897 & 2422 & 2755 & 2012 & 2783 & 2258 \\
\hline 72 & 925 & 2196 & 2671 & 1361 & 2667 & 2939 & 1881 & 2417 & 2497 & 2050 & 2622 & 2103 \\
\hline time $(\mathrm{hr})$ & TWAS & Manure & $\mathrm{SSO}$ & T/M/SSO 8/1/1 & $\begin{array}{l}\text { Theoretic } \\
\text { T/SSO } \\
1 / 8 / 1\end{array}$ & $\begin{array}{l}\text { VFAs concen } \\
\text { T/SSO 1/1/8 }\end{array}$ & $\begin{array}{l}\text { ations (mg/L) ove } \\
\text { T/SSO 5/2.5/2.5 }\end{array}$ & $\begin{array}{l}\text { ne } \\
\text { T/SSO } \\
2.5 / 5 / 2.5\end{array}$ & $\begin{array}{c}\text { T/SSO } \\
2.5 / 2.5 / 5\end{array}$ & $\begin{array}{c}\text { T/SSO } \\
4 / 4 / 2\end{array}$ & $\begin{array}{c}\text { T/SSO } \\
2 / 4 / 4\end{array}$ & $\begin{array}{c}\text { T/SSO } \\
4 / 2 / 4\end{array}$ \\
\hline 0 & 428 & 1096 & 1538 & 606 & 1074 & 1383 & 873 & 1040 & 1150 & 917 & 1140 & 1006 \\
\hline 6 & 340 & 1286 & 1914 & 592 & 1254 & 1694 & 970 & 1206 & 1363 & 1033 & 1348 & 1158 \\
\hline 12 & 441 & 1319 & 2423 & 727 & 1341 & 2114 & 1156 & 1375 & 1651 & 1188 & 1585 & 1409 \\
\hline 24 & 672 & 1837 & 2854 & 1007 & 1823 & 2534 & 1509 & 1800 & 2054 & 1575 & 2011 & 1778 \\
\hline 48 & 744 & 1976 & 3309 & 1124 & 1986 & 2919 & 1693 & 2001 & 2334 & 1750 & 2263 & 2016 \\
\hline 72 & 925 & 2196 & 2671 & 1227 & 2117 & 2449 & 1679 & 1997 & 2116 & 1783 & 2132 & 1878 \\
\hline
\end{tabular}


Table D. 9. Soluble and particulate COD concentrations (mg/L) over time in hydrolysis/acidification of TWAS/Manure/SSO

\begin{tabular}{|c|c|c|c|c|c|c|c|c|c|c|c|c|}
\hline \multicolumn{13}{|c|}{ Soluble COD concentrations $(\mathrm{mg} / \mathrm{L})$ ocer time } \\
\hline Time & TWAS & Manure & SSO & $\begin{array}{c}\mathrm{T} / \mathrm{M} / \mathrm{SSO} \\
8 / 1 / 1\end{array}$ & $\begin{array}{c}\text { T/M/SSO } \\
1 / 8 / 1\end{array}$ & $\begin{array}{c}\mathrm{T} / \mathrm{M} / \mathrm{SSO} \\
1 / 1 / 8\end{array}$ & $\begin{array}{l}\mathrm{T} / \mathrm{M} / \mathrm{SSO} \\
5 / 2.5 / 2.5\end{array}$ & $\begin{array}{l}\mathrm{T} / \mathrm{M} / \mathrm{SSO} \\
2.5 / 5 / 2.5\end{array}$ & $\begin{array}{l}\mathrm{T} / \mathrm{M} / \mathrm{SSO} \\
2.5 / 2.5 / 5\end{array}$ & $\begin{array}{c}\mathrm{T} / \mathrm{M} / \mathrm{SSO} \\
4 / 4 / 2\end{array}$ & $\begin{array}{c}\mathrm{T} / \mathrm{M} / \mathrm{SSO} \\
2 / 4 / 4\end{array}$ & $\begin{array}{c}\mathrm{T} / \mathrm{M} / \mathrm{SSO} \\
4 / 2 / 4\end{array}$ \\
\hline 0 & 1050 & 2803 & 9906 & 2322 & 4106 & 8975 & 4109 & 4886 & 7040 & 3945 & 5955 & 5536 \\
\hline 2 & 1700 & 3169 & 9933 & 2936 & 4548 & 9100 & 4576 & 5299 & 7353 & 4404 & 6274 & 5917 \\
\hline 4 & 1968 & 3375 & 9948 & 3198 & 4787 & 9172 & 4790 & 5507 & 7509 & 4622 & 6438 & 6094 \\
\hline 6 & 2175 & 3945 & 9956 & 3443 & 5374 & 9263 & 5065 & 5906 & 7745 & 4972 & 6745 & 6319 \\
\hline 8 & 2557 & 4243 & 9972 & 3814 & 5716 & 9350 & 5364 & 6200 & 7956 & 5280 & 6972 & 6564 \\
\hline 10 & 2868 & 4677 & 9992 & 4137 & 6184 & 9448 & 5662 & 6553 & 8189 & 5618 & 7246 & 6808 \\
\hline 12 & 3147 & 5163 & 9996 & 4437 & 6697 & 9534 & 5953 & 6923 & 8420 & 5962 & 7530 & 7044 \\
\hline 24 & 3282 & 5320 & 10009 & 4574 & 6870 & 9576 & 6075 & 7059 & 8514 & 6095 & 7636 & 7146 \\
\hline 48 & 3317 & 5757 & 10121 & 4665 & 7317 & 9725 & 6247 & 7361 & 8721 & 6332 & 7891 & 7310 \\
\hline 72 & 3368 & 6837 & 11168 & 4944 & 8516 & 10751 & 6866 & 8322 & 9681 & 7074 & 8145 & 8044 \\
\hline \multicolumn{13}{|c|}{ Particulate COD concentrations (mg/L) over time } \\
\hline Time & TWAS & Manure & SSO & $\begin{array}{c}\mathrm{T} / \mathrm{M} / \mathrm{SSO} \\
8 / 1 / 1\end{array}$ & $\begin{array}{c}\text { T/SSO } \\
1 / 8 / 1\end{array}$ & $\begin{array}{c}\text { T/SSO } \\
1 / 1 / 8\end{array}$ & $\begin{array}{c}\text { T/SSO } \\
5 / 2.5 / 2.5\end{array}$ & $\begin{array}{c}\text { T/SSO } \\
2.5 / 5 / 2.5\end{array}$ & $\begin{array}{c}\text { T/SSO } \\
2.5 / 2.5 / 5\end{array}$ & $\mathrm{~T} / \mathrm{SSO} 4 / 4 / 2$ & $\mathrm{~T} / \mathrm{SSO} 2 / 4 / 4$ & $\begin{array}{c}\mathrm{T} / \mathrm{SSO} \\
4 / 2 / 4\end{array}$ \\
\hline 0 & 23602 & 31182 & 23728 & 25372 & 29385 & 23980 & 26557 & 27793 & 25255 & 27501 & 22242 & 25756 \\
\hline 2 & 22053 & 30915 & 23581 & 24958 & 28895 & 23746 & 26090 & 27380 & 24983 & 27042 & 21923 & 25576 \\
\hline 4 & 21784 & 30659 & 23497 & 24496 & 28656 & 23573 & 25877 & 27072 & 24787 & 26824 & 21779 & 25198 \\
\hline 6 & 21577 & 30240 & 23319 & 24350 & 28265 & 23482 & 25601 & 26472 & 24451 & 26574 & 21452 & 24974 \\
\hline 8 & 21395 & 29942 & 23165 & 23990 & 27923 & 23395 & 25502 & 26279 & 24339 & 25966 & 21225 & 24329 \\
\hline 10 & 21184 & 29788 & 22885 & 23837 & 27555 & 22998 & 25375 & 25726 & 23906 & 25828 & 20951 & 24084 \\
\hline 12 & 20985 & 29586 & 22605 & 23755 & 27382 & 22770 & 25130 & 25455 & 23856 & 25584 & 20467 & 23948 \\
\hline 24 & 20780 & 28875 & 21920 & 23120 & 25960 & 22349 & 24320 & 24919 & 23182 & 24550 & 19461 & 22750 \\
\hline 48 & 20515 & 27730 & 21590 & 22776 & 25141 & 21880 & 23950 & 24108 & 22774 & 24124 & 19181 & 22110 \\
\hline 72 & 20404 & 27511 & 21496 & 22665 & 24990 & 21794 & 23830 & 23978 & 22675 & 23972 & 19055 & 21955 \\
\hline
\end{tabular}

$* \mathrm{~T}:$ TWAS
$* \mathrm{M}$ : Manure 


\begin{tabular}{|c|c|c|c|c|c|c|c|c|c|c|c|c|}
\hline \multicolumn{13}{|c|}{ Soluble COD concentrations (mg/L) over time } \\
\hline & & & & $\mathrm{T} / \mathrm{M} / \mathrm{SSO}$ & $\mathrm{T} / \mathrm{M} / \mathrm{SSO}$ & $\mathrm{T} / \mathrm{M} / \mathrm{SSO}$ & $\mathrm{T} / \mathrm{M} / \mathrm{SSO}$ & $\mathrm{T} / \mathrm{M} / \mathrm{SSO}$ & $\mathrm{T} / \mathrm{M} / \mathrm{SSO}$ & $\mathrm{T} / \mathrm{M} / \mathrm{SSO}$ & $\mathrm{T} / \mathrm{M} / \mathrm{SSO}$ & \\
\hline Time & IWAS & Manure & $\mathrm{SSO}$ & $8 / 1 / 1$ & $1 / 8 / 1$ & $1 / 1 / 8$ & $5 / 2.5 / 2.5$ & $2.5 / 5 / 2.5$ & $2.5 / 2.5 / 5$ & $4 / 4 / 2$ & $2 / 4 / 4$ & 17M/SSU 4/2/4 \\
\hline 0 & 1050 & 2803 & 9906 & 2322 & 4106 & 8975 & 4109 & 4886 & 7040 & 3945 & 5955 & 5536 \\
\hline 2 & 1700 & 3169 & 9933 & 2936 & 4548 & 9100 & 4576 & 5299 & 7353 & 4404 & 6274 & 5917 \\
\hline 4 & 1968 & 3375 & 9948 & 3198 & 4787 & 9172 & 4790 & 5507 & 7509 & 4622 & 6438 & 6094 \\
\hline 6 & 2175 & 3945 & 9956 & 3443 & 5374 & 9263 & 5065 & 5906 & 7745 & 4972 & 6745 & 6319 \\
\hline 8 & 2557 & 4243 & 9972 & 3814 & 5716 & 9350 & 5364 & 6200 & 7956 & 5280 & 6972 & 6564 \\
\hline 10 & 2868 & 4677 & 9992 & 4137 & 6184 & 9448 & 5662 & 6553 & 8189 & 5618 & 7246 & 6808 \\
\hline 12 & 3147 & 5163 & 9996 & 4437 & 6697 & 9534 & 5953 & 6923 & 8420 & 5962 & 7530 & 7044 \\
\hline 24 & 3282 & 5320 & 10009 & 4574 & 6870 & 9576 & 6075 & 7059 & 8514 & 6095 & 7636 & 7146 \\
\hline 48 & 3317 & 5757 & 10121 & 4665 & 7317 & 9725 & 6247 & 7361 & 8721 & 6332 & 7891 & 7310 \\
\hline 72 & 3368 & 6837 & 11168 & 4944 & 8516 & 10751 & 6866 & 8322 & 9681 & 7074 & 8145 & 8044 \\
\hline \multicolumn{13}{|c|}{ Particulate COD concentrations $(\mathrm{mg} / \mathrm{L})$ over time } \\
\hline Time & TWAS & Manure & SSO & $\mathrm{T} / \mathrm{M} / \mathrm{SSO}$ & $\mathrm{T} / \mathrm{SSO}$ & $\mathrm{T} / \mathrm{SSO}$ & $\mathrm{T} / \mathrm{SSO}$ & $\mathrm{T} / \mathrm{SSO}$ & $\mathrm{T} / \mathrm{SSO}$ & $\mathrm{T} / \mathrm{SSO}$ & $\mathrm{T} / \mathrm{SSO}$ & $\mathrm{T} / \mathrm{SSO} 4 / 2 / 4$ \\
\hline lime & 1 W AS & vianure & sso & 8/1/1 & $1 / 8 / 1$ & $1 / 1 / 8$ & $5 / 2.5 / 2.5$ & $2.5 / 5 / 2.5$ & $2.5 / 2.5 / 5$ & $4 / 4 / 2$ & $2 / 4 / 4$ & $1 / 3 \mathrm{SU} 4 / 2 / 4$ \\
\hline 0 & 23602 & 31182 & 23728 & 25372 & 29385 & 23980 & 26557 & 27793 & 25255 & 27501 & 22242 & 25756 \\
\hline 2 & 22053 & 30915 & 23581 & 24958 & 28895 & 23746 & 26090 & 27380 & 24983 & 27042 & 21923 & 25576 \\
\hline 4 & 21784 & 30659 & 23497 & 24496 & 28656 & 23573 & 25877 & 27072 & 24787 & 26824 & 21779 & 25198 \\
\hline 6 & 21577 & 30240 & 23319 & 24350 & 28265 & 23482 & 25601 & 26472 & 24451 & 26574 & 21452 & 24974 \\
\hline 8 & 21395 & 29942 & 23165 & 23990 & 27923 & 23395 & 25502 & 26279 & 24339 & 25966 & 21225 & 24329 \\
\hline 10 & 21184 & 29788 & 22885 & 23837 & 27555 & 22998 & 25375 & 25726 & 23906 & 25828 & 20951 & 24084 \\
\hline 12 & 20985 & 29586 & 22605 & 23755 & 27382 & 22770 & 25130 & 25455 & 23856 & 25584 & 20467 & 23948 \\
\hline 24 & 20780 & 28875 & 21920 & 23120 & 25960 & 22349 & 24320 & 24919 & 23182 & 24550 & 19461 & 22750 \\
\hline 48 & 20515 & 27730 & 21590 & 22776 & 25141 & 21880 & 23950 & 24108 & 22774 & 24124 & 19181 & 22110 \\
\hline 72 & 20404 & 27511 & 21496 & 22665 & 24990 & 21794 & 23830 & 23978 & 22675 & 23972 & 19055 & 21955 \\
\hline
\end{tabular}




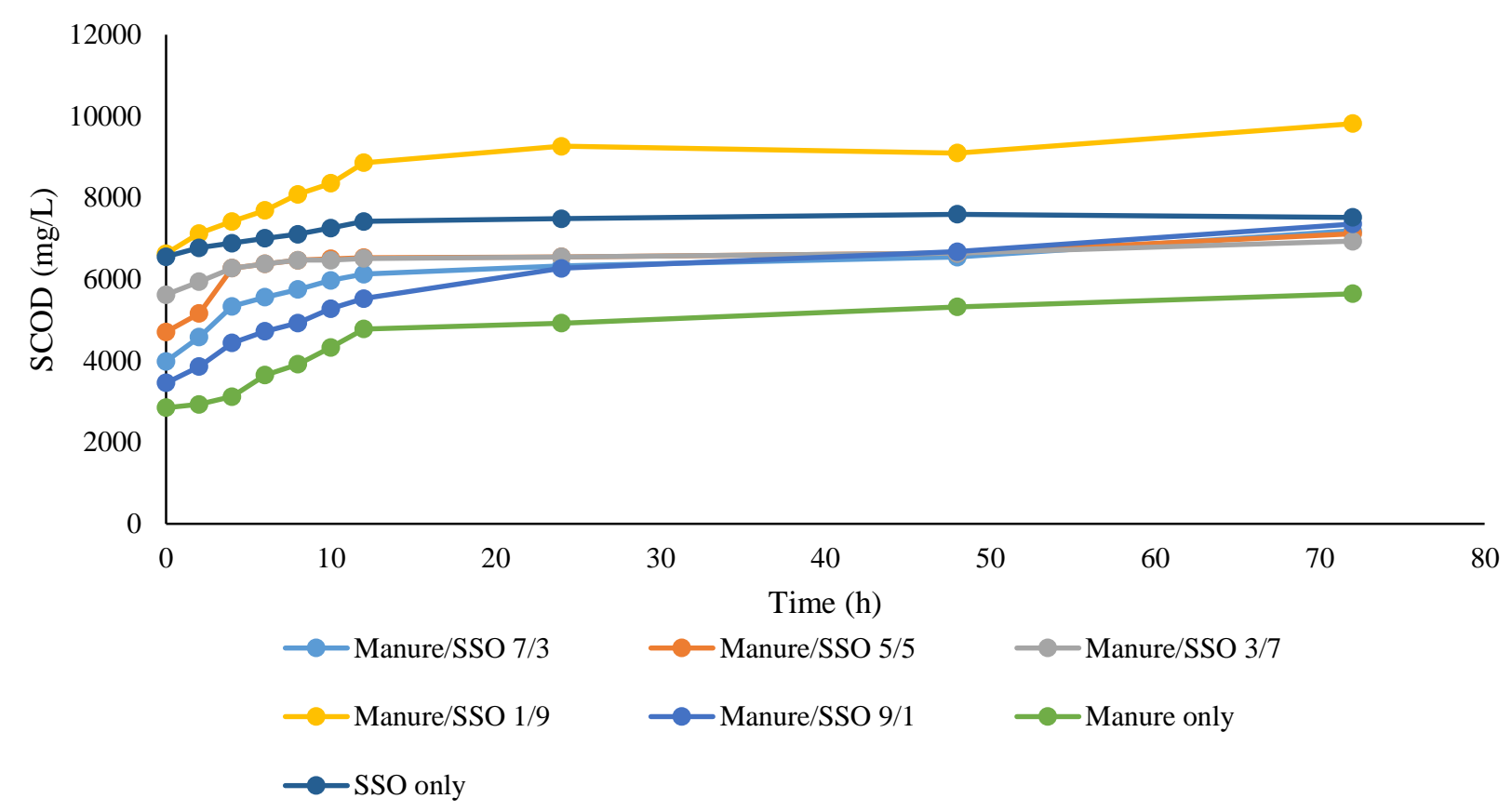

Figure D.2. Concentration of soluble COD over time at different mixing ratios 


\section{References}

Abudi, Z.N., Hu, Z., Sun, N., Xiao, B., Rajaa, N., Liu, C., Guo, D., 2016a. Batch anaerobic codigestion of OFMSW (organic fraction of municipal solid waste), TWAS (thickened waste activated sludge) and RS (rice straw): Influence of TWAS and RS pretreatment and mixing ratio. Energy. https://doi.org/10.1016/j.energy.2016.03.141

Abudi, Z.N., Hu, Z., Xiao, B., Abood, A.R., Rajaa, N., Laghari, M., 2016b. Effects of pretreatments on thickened waste activated sludge and rice straw co-digestion: Experimental and modeling study. J. Environ. Manage. https://doi.org/10.1016/j.jenvman.2016.04.028

Ahn, K.H., Park, K.Y., Maeng, S.K., Hwang, J.H., Lee, J.W., Song, K.G., Choi, S., 2002. Ozonation of wastewater sludge for reductio and recycling, in: Water Science and Technology. https://doi.org/10.1001/jama.288.11.1364

Ahring, B.K., Sandberg, M., Angelidaki, I., 1995. Volatile fatty acids as indicators of process imbalance in anaerobic digestors. Appl. Microbiol. Biotechnol. 43, 559-565. https://doi.org/10.1007/BF00218466

Akyol, Ç., Ozbayram, E.G., Ince, O., Kleinsteuber, S., Ince, B., 2016. Anaerobic co-digestion of cow manure and barley: Effect of cow manure to barley ratio on methane production and digestion stability. Environ. Prog. Sustain. Energy 35, 589-595. https://doi.org/10.1002/ep.12250

Angelidaki, I., Alves, M., Bolzonella, D., Borzacconi, L., Campos, J.L., Guwy, A.J., Kalyuzhnyi, S., Jenicek, P., Van Lier, J.B., 2009a. Defining the biomethane potential (BMP) of solid organic wastes and energy crops: A proposed protocol for batch assays. Water Sci. Technol. https://doi.org/10.2166/wst.2009.040

Angelidaki, I., Alves, M., Bolzonella, D., Borzacconi, L., Campos, J.L., Guwy, A.J., Kalyuzhnyi, S., Jenicek, P., Van Lier, J.B., 2009b. Defining the biomethane potential (BMP) of solid organic wastes and energy crops: A proposed protocol for batch assays. Water Sci. Technol. https://doi.org/10.2166/wst.2009.040

Anwar, Z., Gulfraz, M., Irshad, M., 2014. Agro-industrial lignocellulosic biomass a key to unlock the future bio-energy: A brief review. J. Radiat. Res. Appl. Sci. https://doi.org/10.1016/j.jrras.2014.02.003

Appels, L., Baeyens, J., Degrève, J., Dewil, R., 2008. Principles and potential of the anaerobic digestion of waste-activated sludge. Prog. Energy Combust. Sci. https://doi.org/10.1016/j.pecs.2008.06.002

Appels, L., Lauwers, J., Degrve, J., Helsen, L., Lievens, B., Willems, K., Van Impe, J., Dewil, R., 2011. Anaerobic digestion in global bio-energy production: Potential and research challenges. Renew. Sustain. Energy Rev. 15, 4295-4301. https://doi.org/10.1016/j.rser.2011.07.121

Azbar, N., Speece, R.E., 2001. Two-Phase, Two-Stage, and Single-Stage Anaerobic Process 
Comparison. J. Environ. Eng. 127, 240-248. https://doi.org/10.1061/(ASCE)07339372(2001)127:3(240)

Azman, S., Khadem, A., Zeeman, G., van Lier, J., Plugge, C., 2015a. Mitigation of Humic Acid Inhibition in Anaerobic Digestion of Cellulose by Addition of Various Salts. Bioengineering. https://doi.org/10.3390/bioengineering2020054

Azman, S., Khadem, A.F., Van Lier, J.B., Zeeman, G., Plugge, C.M., 2015b. Presence and role of anaerobic hydrolytic microbes in conversion of lignocellulosic biomass for biogas production. Crit. Rev. Environ. Sci. Technol. https://doi.org/10.1080/10643389.2015.1053727

Bali, G., Meng, X., Deneff, J.I., Sun, Q., Ragauskas, A.J., 2015. The effect of alkaline pretreatment methods on cellulose structure and accessibility. ChemSusChem. https://doi.org/10.1002/cssc.201402752

Bayr, S., Ojanperä, M., Kaparaju, P., Rintala, J., 2014. Long-term thermophilic mono-digestion of rendering wastes and co-digestion with potato pulp. Waste Manag. 34, 1853-1859. https://doi.org/10.1016/j.wasman.2014.06.005

Beccari, M., Majone, M., Torrisi, L., 1998. Two-reactor system with partial phase separation for anaerobic treatment of olive oil mill effluents, in: Water Science and Technology. pp. 5360. https://doi.org/10.1016/S0273-1223(98)00497-1

Benn, N., Zitomer, D., 2018. Pretreatment and Anaerobic Co-digestion of Selected PHB and PLA Bioplastics. Front. Environ. Sci. https://doi.org/10.3389/fenvs.2017.00093

Bertin, L., Grilli, S., Spagni, A., Fava, F., 2013. Innovative two-stage anaerobic process for effective codigestion of cheese whey and cattle manure. Bioresour. Technol. 128, 779-783. https://doi.org/10.1016/j.biortech.2012.10.118

Bitton, G., 2010. Wastewater Microbiology: Fourth Edition, Wastewater Microbiology: Fourth Edition. https://doi.org/10.1002/9780470901243

Björnsson, L., Murto, M., Mattiasson, B., 2000. Evaluation of parameters for monitoring an anaerobic co-digestion process. Appl. Microbiol. Biotechnol. 54, 844-849. https://doi.org/10.1007/s002530000471

Bordeleau, É.L., Droste, R.L., 2011. Comprehensive review and compilation of pretreatments for mesophilic and thermophilic anaerobic digestion. Water Sci. Technol. https://doi.org/10.2166/wst.2011.052

Bordoloi, R., Lilly, S.J., Kacprzak, G.G., Churchill, C.W., 2014. Modeling the distribution of Mg II absorbers around galaxies using background galaxies and quasars. Astrophys. J. 784. https://doi.org/10.1088/0004-637X/784/2/108

Bougrier, C., Delgenès, J.P., Carrère, H., 2007. Impacts of thermal pre-treatments on the semicontinuous anaerobic digestion of waste activated sludge. Biochem. Eng. J. https://doi.org/10.1016/j.bej.2006.11.013

Chandra, R., Vijay, V.K., Subbarao, P.M.V., Khura, T.K., 2012. Production of methane from anaerobic digestion of jatropha and pongamia oil cakes. Appl. Energy. 
https://doi.org/10.1016/j.apenergy.2010.10.049

Cioabla, A.E., Ionel, I., Dumitrel, G.A., Popescu, F., 2012. Comparative study on factors affecting anaerobic digestion of agricultural vegetal residues. Biotechnol. Biofuels. https://doi.org/10.1186/1754-6834-5-39

City of Toronto, 2019. Biosolids Management [WWW Document]. City Toronto.

City of Toronto, 2018. Ashbridges Bay Wastewater Treatment Plant: 2017 Annual Report. City.

Cornelissen, S., Koper, M., Deng, Y.Y., 2012. The role of bioenergy in a fully sustainable global energy system. Biomass and Bioenergy 41, 21-33.

https://doi.org/10.1016/j.biombioe.2011.12.049

Cuetos, M.J., Gómez, X., Escapa, A., Morán, A., 2007. Evaluation and simultaneous optimization of bio-hydrogen production using 32factorial design and the desirability function. J. Power Sources 169, 131-139. https://doi.org/10.1016/j.jpowsour.2007.01.050

Cuetos, M.J., Gómez, X., Otero, M., Morán, A., 2010. Anaerobic digestion and co-digestion of slaughterhouse waste (SHW): Influence of heat and pressure pre-treatment in biogas yield. Waste Manag. https://doi.org/10.1016/j.wasman.2010.01.034

Dareioti, M.A., Kornaros, M., 2015. Anaerobic mesophilic co-digestion of ensiled sorghum, cheese whey and liquid cow manure in a two-stage CSTR system: Effect of hydraulic retention time. Bioresour. Technol. 175, 553-562. https://doi.org/10.1016/j.biortech.2014.10.102

Demeyer, D.I., Henderickx, H.K., 1967. The effect of C18 unsaturated fatty acids on methane production in vitro by mixed rumen bacteria. Biochim. Biophys. Acta (BBA)/Lipids Lipid Metab. 137, 484-497. https://doi.org/10.1016/0005-2760(67)90130-0

Demirel, B., Scherer, P., 2008. The roles of acetotrophic and hydrogenotrophic methanogens during anaerobic conversion of biomass to methane: A review. Rev. Environ. Sci. Biotechnol. https://doi.org/10.1007/s11157-008-9131-1

Deng, Y., Dai, B., Xu, Jiaxing, Liu, X., Xu, Jiming, 2018. Anaerobic co-digestion of rice straw and soybean straw to increase biogas production by pretreatment with trichoderma reesei RUT C30. Environ. Prog. Sustain. Energy. https://doi.org/10.1002/ep.12782

Dinsdale, R.M., Premier, G.C., Hawkes, F.R., Hawkes, D.L., 2000. Two-stage anaerobic codigestion of waste activated sludge and fruit/vegetable waste using inclined tubular digesters. Bioresour. Technol. 72, 159-168. https://doi.org/10.1016/S0960-8524(99)001054

Divya, D., Gopinath, L.R., Merlin Christy, P., 2015. A review on current aspects and diverse prospects for enhancing biogas production in sustainable means. Renew. Sustain. Energy Rev. https://doi.org/10.1016/j.rser.2014.10.055

Droste, R.L., 1997. Theory and Practice of Water and Wastewater TreatmentJohn Wiley and Sons. Inc. New York, Pp754-757.

Duran, M., Tepe, N., Yurtsever, D., Punzi, V.L., Bruno, C., Mehta, R.J., 2006. Bioaugmenting 
anaerobic digestion of biosolids with selected strains of Bacillus, Pseudomonas, and Actinomycetes species for increased methanogenesis and odor control. Appl. Microbiol. Biotechnol. https://doi.org/10.1007/s00253-006-0548-6

Eastman, J.A., Ferguson, J.F., 1981. Solubilization of Particulate Organic Carbon during the Acid Phase of Anaerobic Digestion. Source J. (Water Pollut. Control Fed. Part I 53, 352 366. https://doi.org/10.2307/25041085

Eddy, M., 2003. Wastewater Engineering-Treatment, Disposal, Reuse. INC,.

Edström, M., Nordberg, ^̊., Thyselius, L., 2003. Anaerobic treatment of animal byproducts from slaughterhouses at laboratory and pilot scale, in: Applied Biochemistry and Biotechnology Part A Enzyme Engineering and Biotechnology. https://doi.org/10.1385/ABAB:109:1-3:127

Elbeshbishy, E., Dhar, B.R., Nakhla, G., Lee, H.S., 2017. A critical review on inhibition of dark biohydrogen fermentation. Renew. Sustain. Energy Rev. https://doi.org/10.1016/j.rser.2017.05.075

Elbeshbishy, E., Hafez, H., Dhar, B.R., Nakhla, G., 2011. Single and combined effect of various pretreatment methods for biohydrogen production from food waste. Int. J. Hydrogen Energy. https://doi.org/10.1016/j.ijhydene.2011.02.067

Elbeshbishy, E., Nakhla, G., 2012. Batch anaerobic co-digestion of proteins and carbohydrates. Bioresour. Technol. https://doi.org/10.1016/j.biortech.2012.04.052

Elbeshbishy, E., Nakhla, G., Hafez, H., 2012. Biochemical methane potential (BMP) of food waste and primary sludge: Influence of inoculum pre-incubation and inoculum source. Bioresour. Technol. https://doi.org/10.1016/j.biortech.2012.01.025

Esposito, G., Frunzo, L., Giordano, A., Liotta, F., Panico, A., Pirozzi, F., 2012. Anaerobic codigestion of organic wastes. Rev. Environ. Sci. Bio/Technology 11, 325-341. https://doi.org/10.1007/s11157-012-9277-8

Feng, J., Li, Y., Zhang, E., Zhang, J., Wang, W., He, Y., Liu, G., Chen, C., 2018. Solid-State Codigestion of NaOH-Pretreated Corn Straw and Chicken Manure Under Mesophilic Condition. Waste and Biomass Valorization. https://doi.org/10.1007/s12649-017-9834-z

Fernandes, T. V., van Lier, J.B., Zeeman, G., 2015. Humic Acid-Like and Fulvic Acid-Like Inhibition on the Hydrolysis of Cellulose and Tributyrin. Bioenergy Res. https://doi.org/10.1007/s12155-014-9564-z

Foladori, P., Velho, V.F., Costa, R.H.R., Bruni, L., Quaranta, A., Andreottola, G., 2015. Concerning the role of cell lysis-cryptic growth in anaerobic side-stream reactors: The single-cell analysis of viable, dead and lysed bacteria. Water Res. https://doi.org/10.1016/j.watres.2015.01.042

Frigon, J.C., Guiot, S.R., 1995. Impact of liquid-to-gas hydrogen mass transfer on substrate conversion efficiency of an upflow anaerobic sludge bed and filter reactor. Enzyme Microb. Technol. 17, 1080-1086. https://doi.org/10.1016/0141-0229(95)00040-2

Gaby, J.C., Zamanzadeh, M., Horn, S.J., 2017. The effect of temperature and retention time on methane production and microbial community composition in staged anaerobic digesters 
fed with food waste. Biotechnol. Biofuels. https://doi.org/10.1186/s13068-017-0989-4

Gelegenis, J., Georgakakis, D., Angelidaki, I., Christopoulou, N., Goumenaki, M., 2007. Optimization of biogas production from olive-oil mill wastewater, by codigesting with diluted poultry-manure. Appl. Energy 84, 646-663. https://doi.org/10.1016/j.apenergy.2006.12.001

Gerardi, M.H., 2003. The Microbiology of Anaerobic Digesters, John Wiley \& Sons, Inc. https://doi.org/10.1002/0471468967

Giordano, G.E.L.F.A., 2012. Anaerobic co-digestion of organic wastes. Rev. Environ. Sci. Bio/Technology. https://doi.org/10.1007/s11157-012-9277-8

Gonzalez-Avila, S.R., Klaseboer, E., Khoo, B.C., Ohl, C.D., 2011. Cavitation bubble dynamics in a liquid gap of variable height. J. Fluid Mech. https://doi.org/10.1017/jfm.2011.212

Gonzalez, A., Hendriks, A.T.W.M., van Lier, J.B., de Kreuk, M., 2018. Pre-treatments to enhance the biodegradability of waste activated sludge: Elucidating the rate limiting step. Biotechnol. Adv. https://doi.org/10.1016/j.biotechadv.2018.06.001

Gould, M.C., 2014. Bioenergy and Anaerobic Digestion, in: Bioenergy. https://doi.org/10.1016/b978-0-12-407909-0.00018-3

Government of Canada, 2013. Technical Document on Municipal Solid Waste Organics Processing, Ministry of the Environment in Canada.

Gujer, W., Zehnder, A.J.B., 1983. Conversion processes in anaerobic digestion. Water Sci. Technol. 15, 127-167. https://doi.org/10.2166/wst.1983.0164

Guo, J., Wang, W., Liu, X., Lian, S., Zheng, L., 2014. Effects of thermal pre-treatment on anaerobic co-digestion of municipal biowastes at high organic loading rate. Chemosphere. https://doi.org/10.1016/j.chemosphere.2013.12.007

Guo, M., Song, W., Buhain, J., 2015. Bioenergy and biofuels: History, status, and perspective. Renew. Sustain. Energy Rev. https://doi.org/10.1016/j.rser.2014.10.013

Hagos, K., Zong, J., Li, D., Liu, C., Lu, X., 2017. Anaerobic co-digestion process for biogas production: Progress, challenges and perspectives. Renew. Sustain. Energy Rev. https://doi.org/10.1016/j.rser.2016.11.184

Hanaki, K., Matsuo, T., Nagase, M., 1981. Mechanism of inhibition caused by long-chain fatty acids in anaerobic digestion process. Biotechnol. Bioeng. 23, 1591-1610. https://doi.org/10.1002/bit.260230717

He, Y., Pang, Y., Li, X., Liu, Y., Li, R., Zheng, M., 2009. Investigation on the changes of main compositions and extractives of rice straw pretreated with sodium hydroxide for biogas production. Energy and Fuels. https://doi.org/10.1021/ef8007486

Heffez, A., 2009. Our sewage plant is (unfortunately) bigger than yours [WWW Document]. Green Space, SPACINGMONTREAL, Spacing.ca.

Henze, M., van Loosdrecht, M.C.M., Ekama, G.A., Brdjanovic, D., 2015. Biological Wastewater Treatment: Principles, Modelling and Design. Water Intell. Online. 
https://doi.org/10.2166/9781780401867

Heo, N.H., Park, S.C., Kang, H., 2004. Effects of mixture ratio and hydraulic retention time on single-stage anaerobic co-digestion of food waste and waste activated sludge, in: Journal of Environmental Science and Health - Part A Toxic/Hazardous Substances and Environmental Engineering. pp. 1739-1756. https://doi.org/10.1081/ESE-120037874

Hidalgo, D., Martín-Marroquín, J.M., Sastre, E., 2014. Single-Phase and Two-Phase Anaerobic Co-Digestion of Residues from the Treatment Process of Waste Vegetable Oil and Pig Manure. Bioenergy Res. 7, 670-680. https://doi.org/10.1007/s12155-013-9396-2

Himmel, M.E., Ding, S.Y., Johnson, D.K., Adney, W.S., Nimlos, M.R., Brady, J.W., Foust, T.D., 2007. Biomass recalcitrance: Engineering plants and enzymes for biofuels production. Science (80-. ). https://doi.org/10.1126/science.1137016

Ike, M., Inoue, D., Miyano, T., Liu, T.T., Sei, K., Soda, S., Kadoshin, S., 2010. Microbial population dynamics during startup of a full-scale anaerobic digester treating industrial food waste in Kyoto eco-energy project. Bioresour. Technol. 101, 3952-3957. https://doi.org/10.1016/j.biortech.2010.01.028

Jenkins, S.R., Morgan, J.M., Zhang, X., 1991. Measuring the Usable Carbonate Alkalinity of Operating Anaerobic Digesters. Res. J. Water Pollut. Control Fed. 63, 28-34. https://doi.org/10.2307/25043948

Jihen, T., Baligh, M., Amel, F., Said, N., Moktar, H., Maher, G., Hassib, B., 2015. Microbial ecology overview during anaerobic codigestion of dairy wastewater and cattle manure and use in agriculture of obtained bio-fertilisers. Bioresour. Technol. 198, 141-149. https://doi.org/10.1016/j.biortech.2015.09.004

Kafle, G.K., Chen, L., 2016. Comparison on batch anaerobic digestion of five different livestock manures and prediction of biochemical methane potential (BMP) using different statistical models. Waste Manag. https://doi.org/10.1016/j.wasman.2015.10.021

Kangle, K.M., V, K.S., S, K. V, S, K.G., 2012. Recent Trends in Anaerobic Codigestion: A Review. Univers. J. Environ. Res. 2, 210-219.

Karagiannidis, A., Perkoulidis, G., 2009. A multi-criteria ranking of different technologies for the anaerobic digestion for energy recovery of the organic fraction of municipal solid wastes. Bioresour. Technol. 100, 2355-2360. https://doi.org/10.1016/j.biortech.2008.11.033

Kelleher Robins, 2013. Canadian Biogas Study Technical Document, Canadian Biogas Association.

Kim, J.K., Oh, B.R., Chun, Y.N., Kim, S.W., 2006. Effects of temperature and hydraulic retention time on anaerobic digestion of food waste. J. Biosci. Bioeng. 102, 328-332. https://doi.org/10.1263/jbb.102.328

Kim, S., Bae, J., Choi, O., Ju, D., Lee, J., Sung, H., Park, S., Sang, B.I., Um, Y., 2014. A pilot scale two-stage anaerobic digester treating food waste leachate (FWL): Performance and microbial structure analysis using pyrosequencing. Process Biochem. 49, 301-308. https://doi.org/10.1016/j.procbio.2013.10.022 
Kim, S., Shin, H., 2010. Enhanced Lipid Degradation in an Upflow Anaerobic Sludge Blanket Reactor by Integration with an Acidogenic Reactor. Water Environ. Res. 82, 267-272. https://doi.org/10.2175/106143009X442899

Kim, S.H., Han, S.K., Shin, H.S., 2004. Kinetics of LCFA Inhibition on Acetoclastic Methanogenesis, Propionate Degradation and ??-Oxidation, in: Journal of Environmental Science and Health - Part A Toxic/Hazardous Substances and Environmental Engineering. pp. 1025-1037. https://doi.org/10.1081/ESE-120028411

Kinnunen, M., Hilderbrandt, D., Grimberg, S., Rogers, S., Mondal, S., 2015. Comparative study of methanogens in one- and two-stage anaerobic digester treating food waste. Renew. Agric. Food Syst. 30, 515-523. https://doi.org/10.1017/S1742170514000350

Klocke, M., Nettmann, E., Bergmann, I., Mundt, K., Souidi, K., Mumme, J., Linke, B., 2008. Characterization of the methanogenic Archaea within two-phase biogas reactor systems operated with plant biomass. Syst. Appl. Microbiol. 31, 190-205. https://doi.org/10.1016/j.syapm.2008.02.003

Kwietniewska, E., Tys, J., 2014. Process characteristics, inhibition factors and methane yields of anaerobic digestion process, with particular focus on microalgal biomass fermentation. Renew. Sustain. Energy Rev. 34, 491-500. https://doi.org/10.1016/j.rser.2014.03.041

Lafitte-Trouqué, S., Forster, C.F., 2000. Dual anaerobic co-digestion of sewage sludge and confectionery waste. Bioresour. Technol. 71, 77-82. https://doi.org/10.1016/S09608524(99)00043-7

Lalman, J., Bagley, D.M., 2002. Effects of C18 long chain fatty acids on glucose, butyrate and hydrogen degradation. Water Res. 36, 3307-3313. https://doi.org/10.1016/S00431354(02)00014-3

Lay, J.J., Lee, Y.J., Noike, T., 1999. Feasibility of biological hydrogen production from organic fraction of municipal solid waste. Water Res. https://doi.org/10.1016/S00431354(98)00483-7

Lema, J.M., Suarez, S., 2017. Innovative Wastewater Treatment \&amp; Resource Recovery Technologies: Impacts on Energy, Economy and Environment. Water Intell. Online. https://doi.org/10.2166/9781780407876

Li, A.J., Li, X.Y., Yu, H.Q., 2011. Effect of the food-to-microorganism (F/M) ratio on the formation and size of aerobic sludge granules. Process Biochem. https://doi.org/10.1016/j.procbio.2011.09.007

Li, Y., Park, S.Y., Zhu, J., 2011. Solid-state anaerobic digestion for methane production from organic waste. Renew. Sustain. Energy Rev. 15, 821-826. https://doi.org/10.1016/j.rser.2010.07.042

Liew, L.N., Shi, J., Li, Y., 2012. Methane production from solid-state anaerobic digestion of lignocellulosic biomass. Biomass and Bioenergy 46, 125-132. https://doi.org/10.1016/j.biombioe.2012.09.014

Lin, J., Zuo, J., Ji, R., Chen, X., Liu, F., Wang, K., Yang, Y., 2012. Methanogenic community dynamics in anaerobic co-digestion of fruit and vegetable waste and food waste. J. Environ. 
Sci. (China) 24, 1288-1294. https://doi.org/10.1016/S1001-0742(11)60927-3

Lin, L., Xu, F., Ge, X., Li, Y., 2018. Improving the sustainability of organic waste management practices in the food-energy-water nexus: A comparative review of anaerobic digestion and composting. Renew. Sustain. Energy Rev. https://doi.org/10.1016/j.rser.2018.03.025

Lin, Y., Wang, D., Wu, S., Wang C, 2009. Alkali pretreatment enhances biogas production in the anaerobic digestion of pulp and paper sludge. J. Hazard. Mater. 170, 366-73. https://doi.org/10.1016/j.jhazmat.2009.04.086. Epub 2009 May 3.

Long, J.H., Aziz, T.N., Reyes, F.L.D.L., Ducoste, J.J., 2012. Anaerobic co-digestion of fat, oil, and grease (FOG): A review of gas production and process limitations. Process Saf. Environ. Prot. https://doi.org/10.1016/j.psep.2011.10.001

Luk, G.K., Bekmuradov, V., 2014. Energy products from source-separated organic waste. WIT Trans. Ecol. Environ. https://doi.org/10.2495/WM140411

Lynd, L.R., Weimer, P.J., van Zyl, W.H., Pretorius, I.S., 2002. Microbial Cellulose Utilization: Fundamentals and Biotechnology. Microbiol. Mol. Biol. Rev. 66, 506-577. https://doi.org/10.1128/MMBR.66.4.739.2002

Madigan, M.T., 2014. Brock Biology of Microorganisms, 14th Edition- Madigan, Pearson. https://doi.org/10.1007/s13398-014-0173-7.2

Mata-Alvarez, J., Dosta, J., Romero-Güiza, M.S., Fonoll, X., Peces, M., Astals, S., 2014. A critical review on anaerobic co-digestion achievements between 2010 and 2013. Renew. Sustain. Energy Rev. https://doi.org/10.1016/j.rser.2014.04.039

Mata-Alvarez, J., Macé, S., Llabrés, P., 2000a. Anaerobic digestion of organic solid wastes. An overview of research achievements and perspectives. Bioresour. Technol. https://doi.org/10.1016/S0960-8524(00)00023-7

Mata-Alvarez, J., Macé, S., Llabrés, P., 2000b. Anaerobic digestion of organic solid wastes. An overview of research achievements and perspectives. Bioresour. Technol. https://doi.org/10.1016/S0960-8524(00)00023-7

Mittal, A., 2011. Biological Wastewater Treatment, Chemical Engineering.

Moletta, R., Escoffier, Y., Ehlinger, F., Coudert, J.P., Leyris, J.P., 1994. On-line automatic control system. For monitoring an anaerobic fluidized-bed reactor: Response to organic overload, in: Water Science and Technology. pp. 11-20.

Monnet, F., 2003. A final report on “An Introduction to Anaerobic Digestion of Organic Wastes." Carbon N. Y.

Montusiewicz, A., 2014. Co-digestion of sewage sludge and mature landfill leachate in prebioaugmented system. J. Ecol. Eng. https://doi.org/10.12911/22998993.1125463

Moody, L., Burns, R., Wu-Haan, W., Spajić, R., 2009. Use of biochemical methane potential (BMP) assays for predicting and enhancing anaerobic digester performance. Proc. 4th Int. 44th Croat. Symp. Agric.

Naran, E., Toor, U.A., Kim, D.J., 2016. Effect of pretreatment and anaerobic co-digestion of 
food waste and waste activated sludge on stabilization and methane production. Int. Biodeterior. Biodegrad. https://doi.org/10.1016/j.ibiod.2016.04.011

Naroznova, I., Møller, J., Larsen, B., Scheutz, C., 2016. Evaluation of a new pulping technology for pre-treating source-separated organic household waste prior to anaerobic digestion. Waste Manag. https://doi.org/10.1016/j.wasman.2016.01.042

Nathao, C., Sirisukpoka, U., Pisutpaisal, N., 2013. Production of hydrogen and methane by one and two stage fermentation of food waste, in: International Journal of Hydrogen Energy. pp. 15764-15769. https://doi.org/10.1016/j.ijhydene.2013.05.047

Noike, T., Endo, G., Chang, J. -E, Yaguchi, J. -I, Matsumoto, J. -I, 1985. Characteristics of carbohydrate degradation and the rate-limiting step in anaerobic digestion. Biotechnol. Bioeng. 27, 1482-1489. https://doi.org/10.1002/bit.260271013

Nopharatana, A., Clarke, W.P., Pullammanappallil, P.C., Silvey, P., Chynoweth, D.P., 1998. Evaluation of methanogenic activities during anaerobic digestion of municipal solid waste. Bioresour. Technol. 64, 169-174. https://doi.org/10.1016/S0960-8524(97)00190-9

OECD/EIA, 2011. International Energy Outlook 2011, Center for Strategic and International Studies. https://doi.org/DOE/EIA-0484(2011)

Owen, W.F., Stuckey, D.C., Healy, J.B., Young, L.Y., McCarty, P.L., 1979. Bioassay for monitoring biochemical methane potential and anaerobic toxicity. Water Res. https://doi.org/10.1016/0043-1354(79)90043-5

P., V., D., S., M., L., 2014. Options for Reduction of Maize Silage in Biogas Plant Drazenci. Food Agric. Org. 121-130. https://doi.org/10.2507/daaam.scibook.2014.09

Paavola, T., Syväsalo, E., Rintala, J., 2006. Co-digestion of manure and biowaste according to the EC animal by-products regulation and Finnish national regulations. Water Sci. Technol. https://doi.org/10.2166/wst.2006.253

Park, Y.C., Kim, J.S., 2012. Comparison of various alkaline pretreatment methods of lignocellulosic biomass. Energy. https://doi.org/10.1016/j.energy.2012.08.010

Parra-Orobio, B.A., Torres-Lozada, P., Marmolejo-Rebellón, L.F., 2016. Influence of the mixing ratio on the anaerobic co-digestion of municipal biowaste with domestic wastewater sludge on methane production. Dyna. https://doi.org/http://10.0.60.86/dyna.v83n199.57382

Pauss, A., Andre, G., Perrier, M., Guiot, S.R., 1990. Liquid-to-Gas mass transfer in anaerobic processes: Inevitable transfer limitations of methane and hydrogen in the biomethanation process. Appl. Environ. Microbiol. 56, 1636-1644.

Peter Wright, Scott Inglis, Jianguo Ma, Curt Gooch, Brian Aldrich, Alex Meister and Norman Scott, 2013. Comparison of Five Anaerobic Digestion Systems on Dairy Farms. https://doi.org/10.13031/2013.16760

Poliafico, M., Murphy, J.., 2007. Anaerobic digestion in Ireland : Decision support system. Dep. Civil, Struct. Environ. Eng. Cork Inst. Technol. Irel.

Pouget, E.R., Hagan, H., Des Jarlais, D.C., 2012. Meta-analysis of hepatitis C seroconversion in 
relation to shared syringes and drug preparation equipment, Addiction.

https://doi.org/10.1111/j.1360-0443.2011.03765.x

R, A., K, K., R, D., M, S., 2017. Biogas Recovery from Hyper-Thermophilic Anaerobic CoDigestion of Thickened Waste Activated Sludge, Organic Fraction of Municipal Solid Waste and Fat, Oil and Grease. J. Bioremediation Biodegrad. 08. https://doi.org/10.4172/2155-6199.1000408

Rajesh Banu, J., Arulazhagan, P., Adish Kumar, S., Kaliappan, S., Lakshmi, A.M., 2015. Anaerobic co-digestion of chemical- and ozone-pretreated sludge in hybrid upflow anaerobic sludge blanket reactor. Desalin. Water Treat. https://doi.org/10.1080/19443994.2014.912156

Ratanatamskul, C., Wattanayommanaporn, O., Yamamoto, K., 2015. An on-site prototype twostage anaerobic digester for co-digestion of food waste and sewage sludge for biogas production from high-rise building. Int. Biodeterior. Biodegrad. 102, 143-148. https://doi.org/10.1016/j.ibiod.2015.03.019

Razavi, A.S., Hosseini Koupaie, E., Azizi, A., Hafez, H., Elbeshbishy, E., 2019. Hydrothermal pretreatment of source separated organics for enhanced solubilization and biomethane recovery. Bioresour. Technol. https://doi.org/10.1016/j.biortech.2018.12.024

Razavi, S.A.S., 2019. No Title. Ryerson University.

Ren, H., Jiang, N., Wang, T., Omar, M.M., Ruan, W., Ghafoor, A., 2018. Enhanced Biogas Production in the Duckweed Anaerobic Digestion Process. J. Energy Resour. Technol. 140. https://doi.org/10.1115/1.4039782

Rivière, D., Desvignes, V., Pelletier, E., Chaussonnerie, S., Guermazi, S., Weissenbach, J., Li, T., Camacho, P., Sghir, A., 2009. Towards the definition of a core of microorganisms involved in anaerobic digestion of sludge. ISME J. https://doi.org/10.1038/ismej.2009.2

Rodriguez-Verde, I., Regueiro, L., Lema, J.M., Carballa, M., 2017. Blending based optimisation and pretreatment strategies to enhance anaerobic digestion of poultry manure. Waste Manag. https://doi.org/10.1016/j.wasman.2017.11.002

Rodriguez, C., El-Hassan, Z., Olabi, A.G., 2017. Enhanced Methane Production from Waste Paper Through Anaerobic Co-Digestion with Macroalgae, in: 10th International Conference on Sustainable Energy \& Environmental Protection: Bioenergy and Biofuels. pp. 1-9. https://doi.org/https://doi.org/10.18690/978-961-286-048-6

Rozzi, A., Di Pinto, A.C., Limoni, N., Tomei, M.C., 1994. Start-up and operation of anaerobic digesters with automatic bicarbonate control. Bioresour. Technol. 48, 215-219. https://doi.org/10.1016/0960-8524(94)90149-X

Saha, M., Eskicioglu, C., Marin, J., 2011a. Microwave, ultrasonic and chemo-mechanical pretreatments for enhancing methane potential of pulp mill wastewater treatment sludge. Bioresour. Technol. 102, 7815-7826. https://doi.org/10.1016/j.biortech.2011.06.053

Saha, M., Eskicioglu, C., Marin, J., 2011b. Microwave, ultrasonic and chemo-mechanical pretreatments for enhancing methane potential of pulp mill wastewater treatment sludge. Bioresour. Technol. https://doi.org/10.1016/j.biortech.2011.06.053 
Saini, J.K., Saini, R., Tewari, L., 2015. Lignocellulosic agriculture wastes as biomass feedstocks for second-generation bioethanol production: concepts and recent developments. 3 Biotech. https://doi.org/10.1007/s13205-014-0246-5

Salminen, E., Rintala, J., 2002. Anaerobic digestion of organic solid poultry slaughterhouse waste - A review. Bioresour. Technol. https://doi.org/10.1016/S0960-8524(01)00199-7

Schievano, A., Tenca, A., Scaglia, B., Merlino, G., Rizzi, A., Daffonchio, D., Oberti, R., Adani, F., 2012. Two-stage vs single-stage thermophilic anaerobic digestion: Comparison of energy production and biodegradation efficiencies. Environ. Sci. Technol. 46, 8502-8510. https://doi.org/10.1021/es301376n

Schuetzle, D., Schuetzle, R., Kent Hoekman, S., Zielinska, B., 2015. The effect of oxygen on formation of syngas contaminants during the thermochemical conversion of biomass. Int. J. Energy Environ. Eng. 6, 405-417. https://doi.org/10.1007/s40095-015-0187-8

Shah, F.A., Mahmood, Q., Rashid, N., Pervez, A., Raja, I.A., Shah, M.M., 2015. Co-digestion, pretreatment and digester design for enhanced methanogenesis. Renew. Sustain. Energy Rev. https://doi.org/10.1016/j.rser.2014.10.053

Shen, F., Yuan, H., Pang, Y., Chen, S., Zhu, B., Zou, D., Liu, Y., Ma, J., Yu, L., Li, X., 2013. Performances of anaerobic co-digestion of fruit \& vegetable waste (FVW) and food waste (FW): Single-phase vs. two-phase. Bioresour. Technol. 144, 80-85. https://doi.org/10.1016/j.biortech.2013.06.099

Sheng, G.P., Yu, H.Q., Li, X.Y., 2010. Extracellular polymeric substances (EPS) of microbial aggregates in biological wastewater treatment systems: A review. Biotechnol. Adv. https://doi.org/10.1016/j.biotechadv.2010.08.001

Shin, S.G., Han, G., Lim, J., Lee, C., Hwang, S., 2010. A comprehensive microbial insight into two-stage anaerobic digestion of food waste-recycling wastewater. Water Res. 44, 48384849. https://doi.org/10.1016/j.watres.2010.07.019

Silvestre, G., Illa, J., Fernández, B., Bonmatí, A., 2014. Thermophilic anaerobic co-digestion of sewage sludge with grease waste: Effect of long chain fatty acids in the methane yield and its dewatering properties. Appl. Energy. https://doi.org/10.1016/j.apenergy.2013.11.075

Smith, P.H., Mah, R.A., 1966. Kinetics of acetate metabolism during sludge digestion. Appl. Microbiol.

Song, Z. lin, Yag, G. he, Feng, Y. zhong, Ren, G. xin, Han, X. hui, 2013. Pretreatment of rice straw by hydrogen peroxide for enhanced methane yield. J. Integr. Agric. https://doi.org/10.1016/S2095-3119(13)60355-X

Song, Z., Yang, G., Liu, X., Yan, Z., Yuan, Y., Liao, Y., 2014. Comparison of seven chemical pretreatments of corn straw for improving methane yield by anaerobic digestion. PLoS One. https://doi.org/10.1371/journal.pone.0093801

Sosnowski, P., Wieczorek, A., Ledakowicz, S., 2003. Anaerobic co-digestion of sewage sludge and organic fraction of municipal solid wastes. Adv. Environ. Res. 7, 609-616. https://doi.org/10.1016/S1093-0191(02)00049-7 
Sterling, M.C., Lacey, R.E., Engler, C.R., Ricke, S.C., 2001. Effects of ammonia nitrogen on H2 and $\mathrm{CH} 4$ production during anaerobic digestion of dairy cattle manure. Bioresour. Technol. https://doi.org/10.1016/S0960-8524(00)00138-3

Stuckey, D.C., McCarty, P.L., 1984. The effect of thermal pretreatment on the anaerobic biodegradability and toxicity of waste activated sludge. Water Res. https://doi.org/10.1016/0043-1354(84)90002-2

Sung, S., Contact, P., Bazylinski, D. a, Raskin, L., 2003. Biohydrogen Production from Renewable Organic Wastes. Challenges.

Sung, S., Dague, R.R., 1995. Laboratory studies on the anaerobic sequencing batch reactor. Water Environ. Res. https://doi.org/10.2175/106143095x131501

Supaphol, S., Jenkins, S.N., Intomo, P., Waite, I.S., Donnell, A.G.O., O’Donnell, A.G., 2011. Microbial community dynamics in mesophilic anaerobic co-digestion of mixed waste. Bioresour. Technol. 102, 4021-7. https://doi.org/10.1016/j.biortech.2010.11.124

Tedesco, S., Benyounis, K.Y., Olabi, A.G., 2013. Mechanical pretreatment effects on macroalgae-derived biogas production in co-digestion with sludge in Ireland. Energy 61, 27-33. https://doi.org/10.1016/j.energy.2013.01.071

Themelis, N.J., 2002. Anaerobic Digestion of Biodegradable Organics in Municipal Solid Wastes. Found. Sch. Eng. Appl. Sci. Columbia Univ. https://doi.org/10.1016/j.biotechadv.2010.10.005

Thies, E., Jenkins, T., Stutzenberger, F., 1994. Effects of the detergent Tween 80 on Thermomonospora curvata. World J. Microbiol. Biotechnol. 10, 657-663. https://doi.org/10.1007/BF00327954

Towey, F., 2013. The power of two. Lancet. Respir. Med. 1, 368. https://doi.org/10.1016/j.procbio.2004.03.007

Turovskiy, I.S., Mathai, P.K., 2005. Wastewater Sludge Processing, Wastewater Sludge Processing. https://doi.org/10.1002/047179161X

Uggetti, E., Passos, F., Solé, M., Garfí, M., Ferrer, I., 2017. Recent Achievements in the Production of Biogas from Microalgae. Waste and Biomass Valorization. https://doi.org/10.1007/s12649-016-9604-3

Varel, V.H., Hashimoto, A.G., Chen, Y.R., 1980. Effect of temperature and retention time on methane production from beef cattle waste. Appl. Environ. Microbiol.

Veeken, A., Hamelers, B., 1999. Effect of temperature on hydrolysis rates of selected biowaste components. Bioresour. Technol. https://doi.org/10.1016/S0960-8524(98)00188-6

Viotti, P., Di Genova, P., Falcioli, F., 2004. Numerical analysis of the anaerobic co-digestion of the organic fraction from municipal solid waste and wastewater: Prediction of the possible performances at Olmeto plant in Perugia (Italy). Waste Manag. Res. 22, 115-128. https://doi.org/10.1177/0734242X04043892

Wei, Y., Li, X., Yu, L., Zou, D., Yuan, H., 2015. Mesophilic anaerobic co-digestion of cattle 
manure and corn stover with biological and chemical pretreatment. Bioresour. Technol. https://doi.org/10.1016/j.biortech.2015.09.035

Wett, B., Phothilangka, P., Eladawy, A., 2010. Systematic comparison of mechanical and thermal sludge disintegration technologies. Waste Manag. https://doi.org/10.1016/j.wasman.2009.12.011

Wilcox, S.J., Hawkes, D.L., Hawkes, F.R., Guwy, A.J., 1995. A neural network, based on bicarbonate monitoring, to control anaerobic digestion. Water Res. 29, 1465-1470. https://doi.org/10.1016/0043-1354(94)00314-W

Yang, Z.H., Xu, R., Zheng, Y., Chen, T., Zhao, L.J., Li, M., 2016. Characterization of extracellular polymeric substances and microbial diversity in anaerobic co-digestion reactor treated sewage sludge with fat, oil, grease. Bioresour. Technol. 212, 164-173. https://doi.org/10.1016/j.biortech.2016.04.046

Yu, H.W., Samani, Z., Hanson, A., Smith, G., 2002. Energy recovery from grass using twophase anaerobic digestion. Waste Manag. 22, 1-5. https://doi.org/10.1016/S0956053X(00)00121-5

Yu, Z., Wen, X., Xu, M., Huang, X., 2012. Characteristics of extracellular polymeric substances and bacterial communities in an anaerobic membrane bioreactor coupled with online ultrasound equipment. Bioresour. Technol. 117, 333-340. https://doi.org/10.1016/j.biortech.2012.04.075

Yuan, X., Cao, Y., Li, J., Wen, B., Zhu, W., Wang, X., Cui, Z., 2012. Effect of pretreatment by a microbial consortium on methane production of waste paper and cardboard. Bioresour. Technol. https://doi.org/10.1016/j.biortech.2012.05.058

Zamanzadeh, M., Hagen, L.H., Svensson, K., Linjordet, R., Horn, S.J., 2017. Biogas production from food waste via co-digestion and digestion- effects on performance and microbial ecology. Sci. Rep. https://doi.org/10.1038/s41598-017-15784-w

Zhang, J., Li, W., Lee, J., Loh, K.C., Dai, Y., Tong, Y.W., 2017a. Enhancement of biogas production in anaerobic co-digestion of food waste and waste activated sludge by biological co-pretreatment. Energy. https://doi.org/10.1016/j.energy.2017.02.163

Zhang, J., Loh, K.C., Lee, J., Wang, C.H., Dai, Y., Wah Tong, Y., 2017b. Three-stage anaerobic co-digestion of food waste and horse manure. Sci. Rep. 7. https://doi.org/10.1038/s41598017-01408-w

Zhang, J., Wei, Y., Xiao, W., Zhou, Z., Yan, X., 2011. Performance and spatial community succession of an anaerobic baffled reactor treating acetone-butanol-ethanol fermentation wastewater. Bioresour. Technol. 102, 7407-7414. https://doi.org/10.1016/j.biortech.2011.05.035

Zhao, M., Wang, Y., Zhang, C., Li, S., Huang, Z., Ruan, W., 2014. Synergistic and pretreatment effect on anaerobic co-digestion from rice straw and municipal sewage sludge. BioResources. https://doi.org/10.15376/biores.9.4.5871-5882

Zhong, W., Zhang, Z., Luo, Y., Sun, S., Qiao, W., Xiao, M., 2011. Effect of biological pretreatments in enhancing corn straw biogas production. Bioresour. Technol. 
There will come a time when you believe everything is finished.

That will be the beginning...

\section{Luis L'Amour}

